
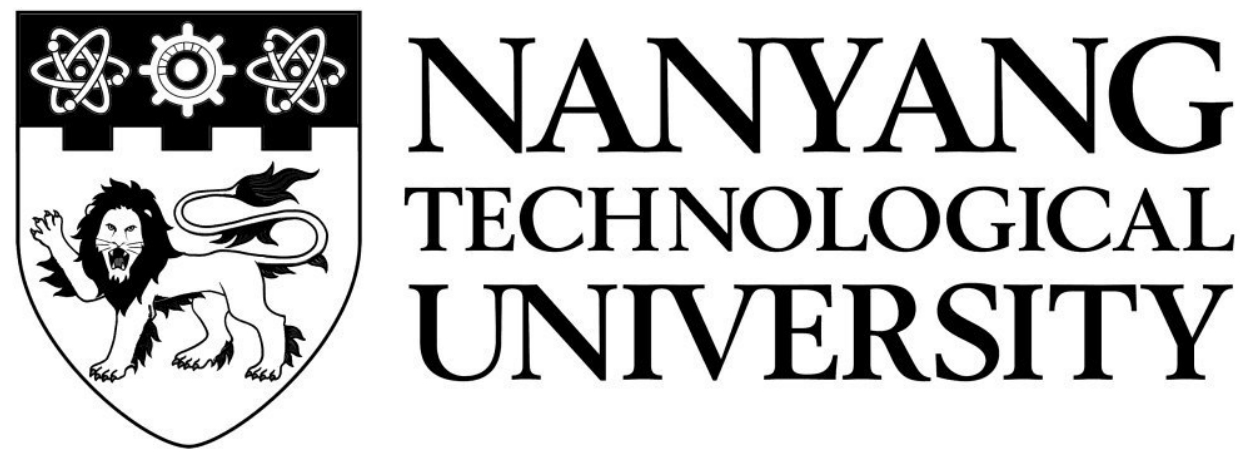
TECHNOLOGICAL UNIVERSITY

\title{
STUDIES TOWARDS THE SYNTHESIS OF
}

\author{
BIOACTIVE NATURAL PRODUCTS AND
}

DEVELOPMENT OF NEW SYNTHETIC METHODS

RABI'AH NISHA KHANIZEMAN

SCHOOL OF PHYSICAL \& MATHEMATICAL SCIENCES

NANYANG TECHNOLOGICAL UNIVERSITY 



\section{Studies towards the synthesis of bioactive natural products and development of new synthetic methods}

\section{RABI'AH NISHA KHANIZEMAN}

School of Physical and Mathematical Sciences

A thesis submitted to the Nanyang Technological University in partial fulfilment of the requirement for the degree of

Doctor of Philosophy 



\section{Acknowledgements}

The completion of this course could not have been possible without the financial support offered by NTU-Research Scholarship, and the additional financial incentive offered by NTU. Therefore, I am deeply grateful and indebted to the University for granting me the opportunity to pursue my graduate studies with a peace of mind.

Equally important is the acceptance by Professor Roderick Wayland Bates who has enabled me to carry out organic chemistry research in his laboratory. Under his patient guidance, and extremely useful advice, I managed a deeper understanding of organic chemistry and the tools used to study it. To say thank you now would merely be an understatement, regardless, I wish to express my gratitude to Professor Bates!

In addition, the supervision rendered by Professor Janine Cossy over the past two years in ESPCI research institution is invaluable. Also essential, is the close supervision provided by Dr. Amandine Guérinot.

A special thank you to the members of Professor Webster's lab for the moral support over the past 5 years.

Deepest appreciation for the help and support is extended to Mrs. Goh Ee Ling, Mr. Derek Yiren Ong, Mr. Thomas Aubineau and Dr. Attapol Pinsa for assistance with the NMR facilities, Dr. Li Yongxin, Dr. Rakesh Ganguly, Ms. Lise-Marie Chamoreau for assistance with the X-ray facilities, Ms Pui Pang-Yi and Ms Zhu Wen Wei for assistance with the mass spectrometer facilities, Mr. Claude Charvy and Dr. Cedric Przybylski for assistance with the HRMS, Mrs. Hum Wei Mei Celine, Ms. Janice Low, Professor Anna Proust, Mdm Patricia Zizzo, Mme Gael Thieullent and Mme Irene Rasoarinoro for assistance with the ESPCI-NTU collaboration, and lastly, to the members of Professor Bates' lab and members of Professor Janine's lab for assistance with the laboratory equipment and chemical reagents. 



\section{TABLE OF CONTENTS}

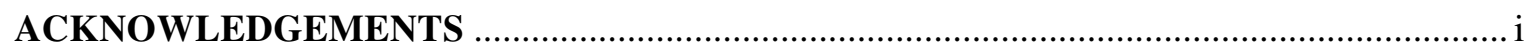

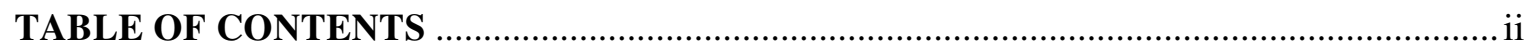

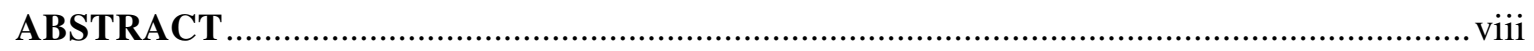

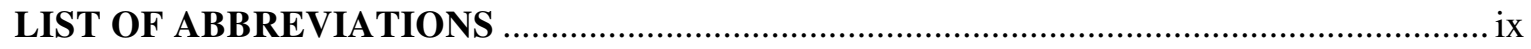

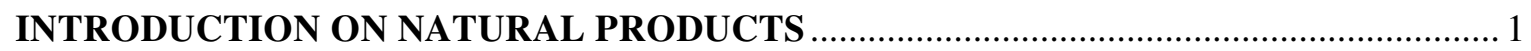

\section{CHAPTER I}

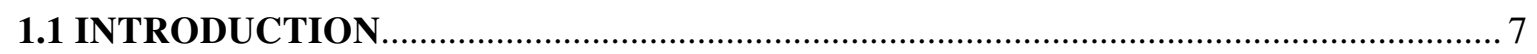

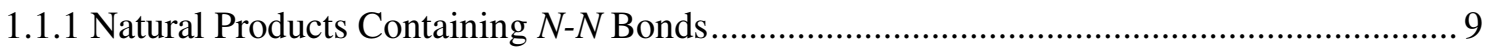

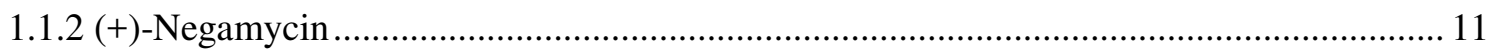

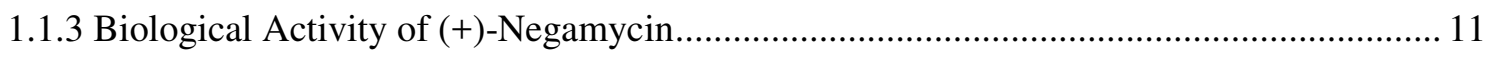

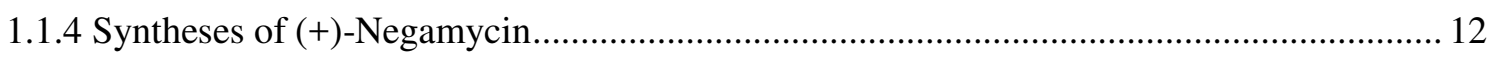

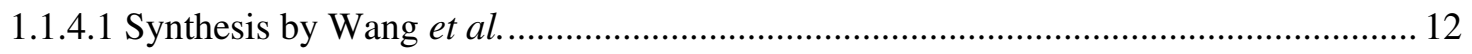

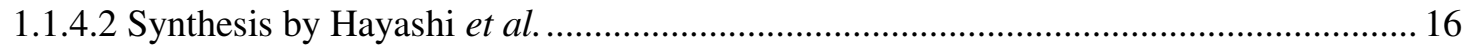

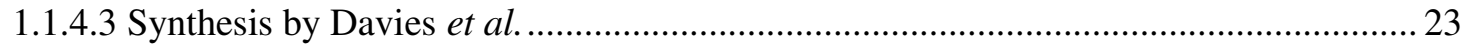

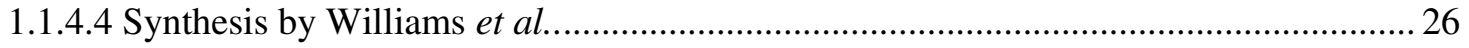

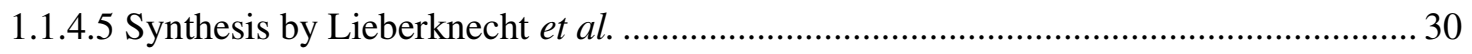

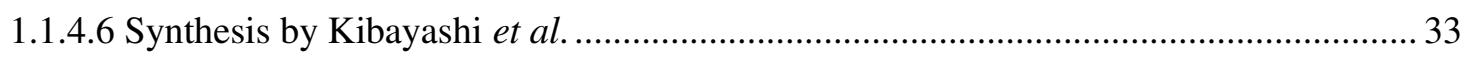

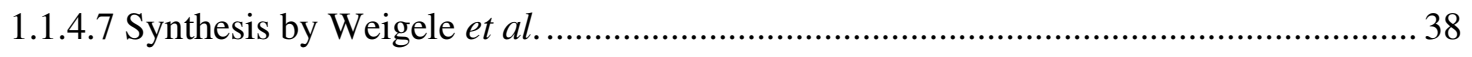

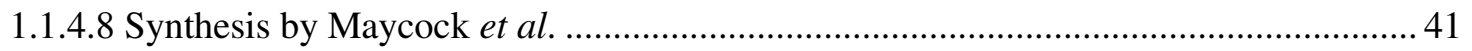

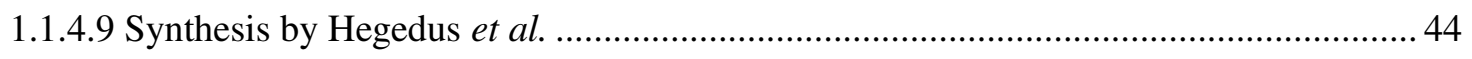

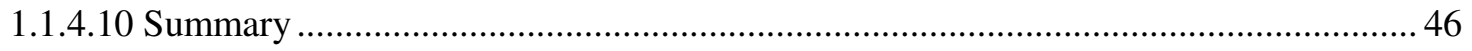




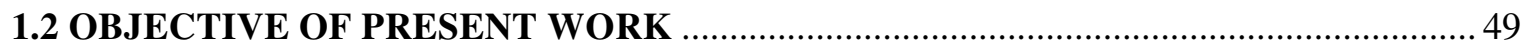

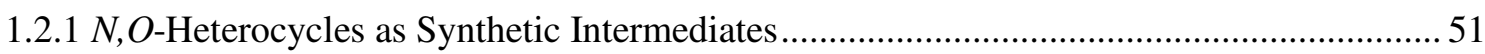

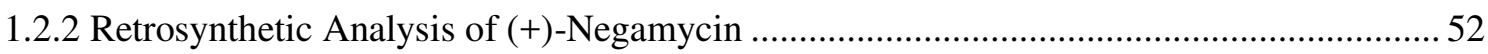

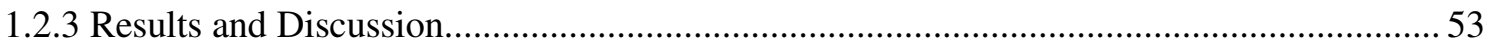

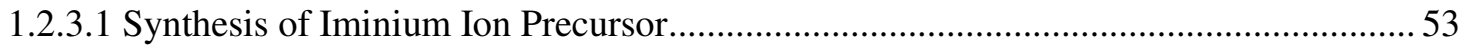

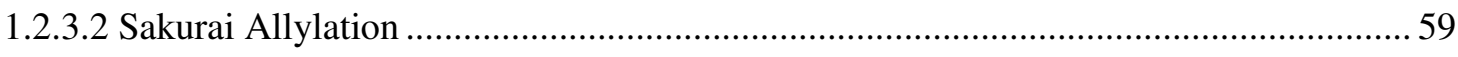

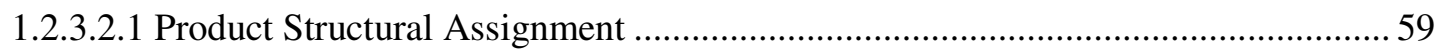

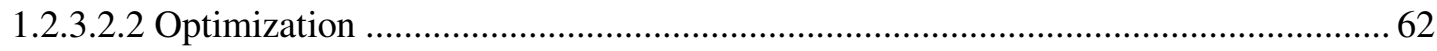

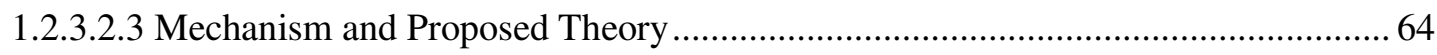

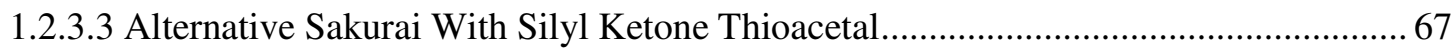

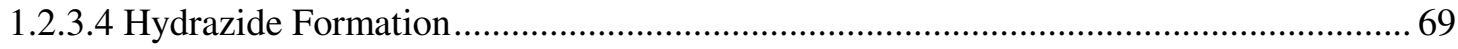

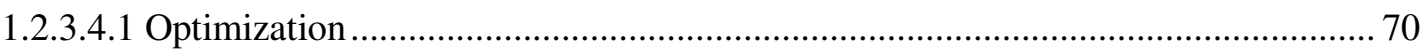

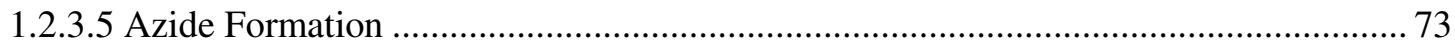

1.2.3.6 Completion of the (+)-Negamycin Synthesis............................................................ 74

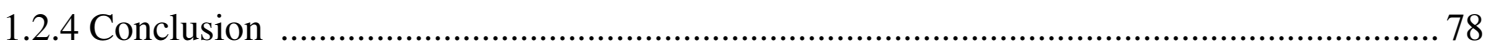

\section{CHAPTER II}

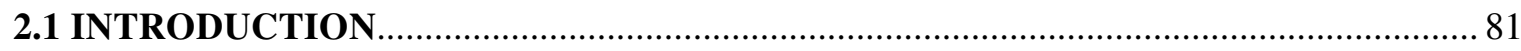

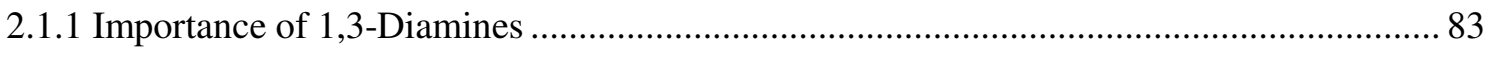

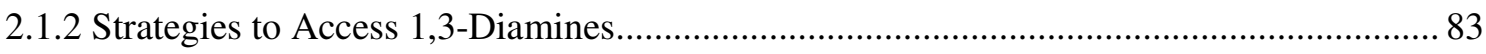

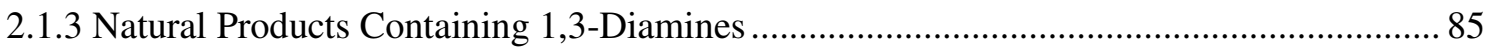

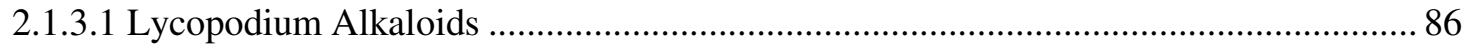

2.1.3.1.1 (-)-Cernuine: Biological Activity and Structure ................................................ 87

2.1.3.1.2 (+)-Cermizine D: Biological Activity and Structure........................................ 88

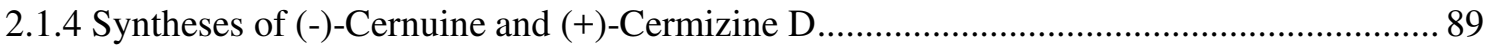




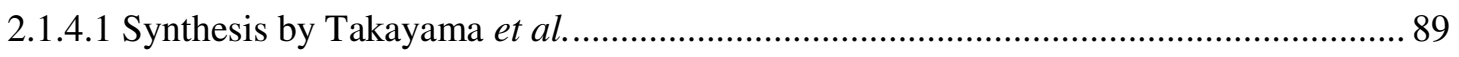

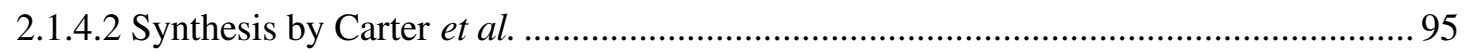

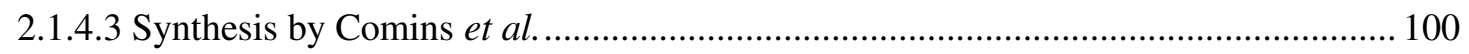

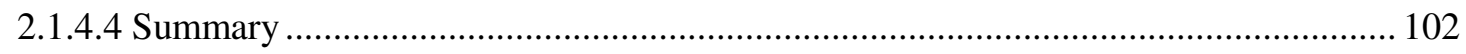

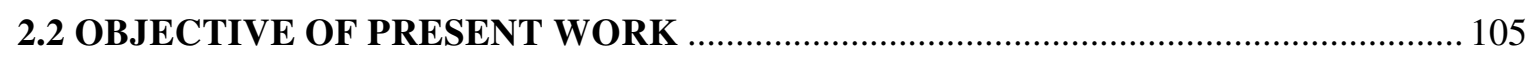

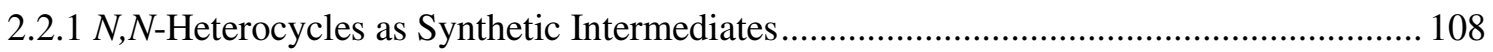

2.2.2 Retrosynthetic Analysis of (-)-Cernuine and (+)-Cermizine D ..................................... 109

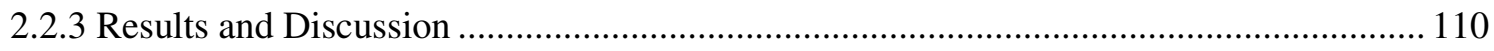

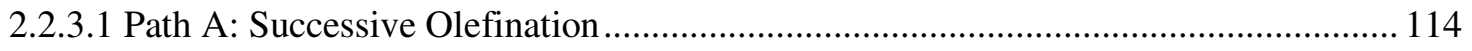

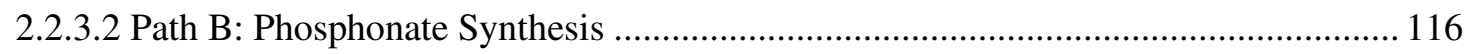

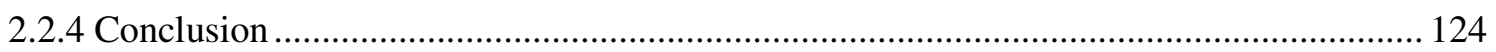

\section{CHAPTER III}

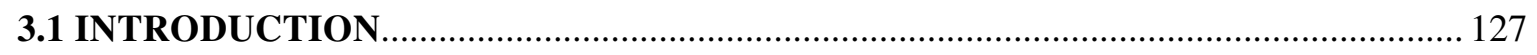

3.1.1 Natural Products Containing THP moiety …........................................................... 129

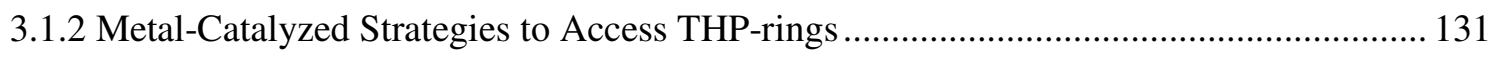

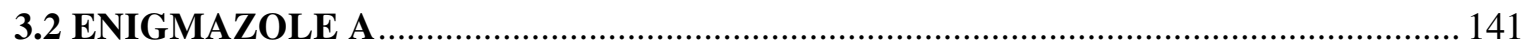

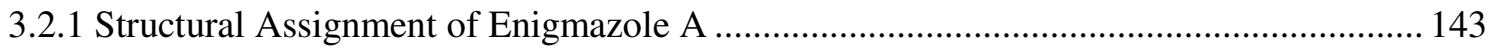

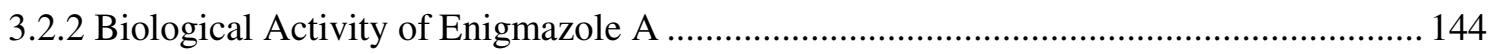

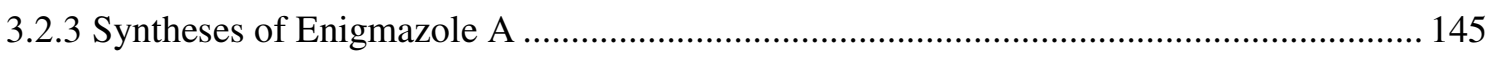

3.2.3.1 Synthesis of the C1-C7 Fragment of Enigmazole A by I.Kadota et al. ..................... 145

3.2.3.2 Synthesis of Enigmazole A by Molinski et al ....................................................... 148

3.2.3.3 Synthesis of Enigmazole A by Smith et al. ......................................................... 158 


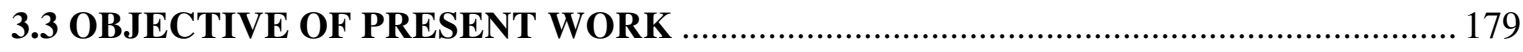

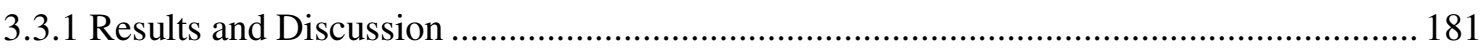

3.3.1.1 Retrosynthetic Analysis of Enigmazole A............................................................ 181

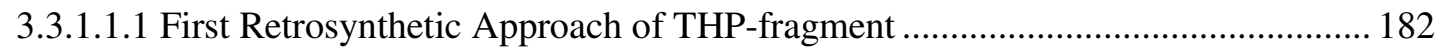

3.3.1.1.2 Second Retrosynthetic Approach of THP-fragment .......................................... 191

3.3.1.2 Other Attempts towards the Synthesis of C1-C10 Fragment of Enigmazole A ......... 202

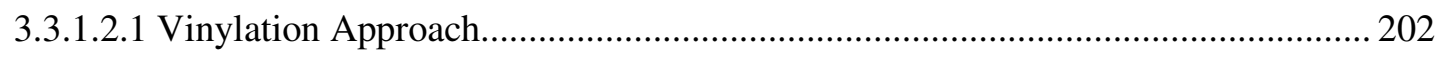

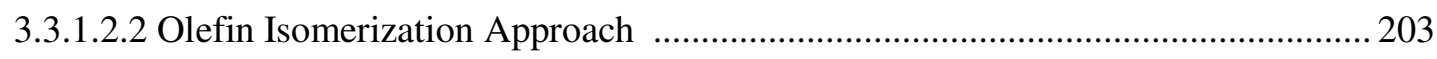

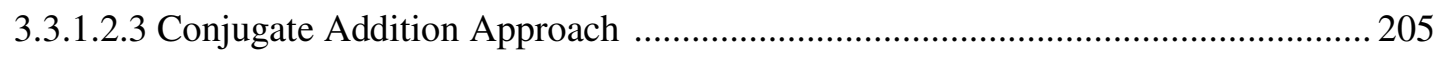

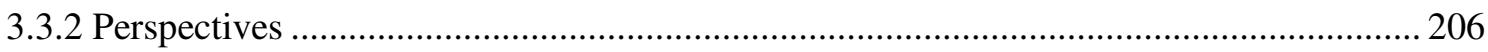

\section{CHAPTER IV}

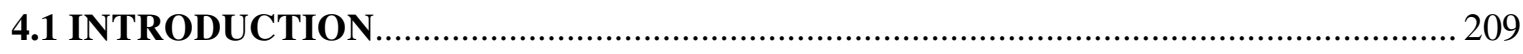

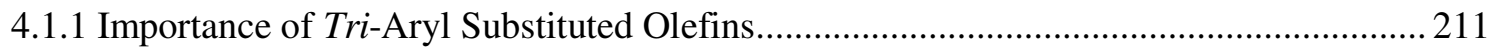

4.1.2 Methods to Access Tri-Aryl Substituted Olefins ......................................................... 213

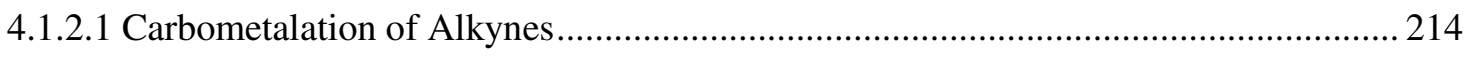

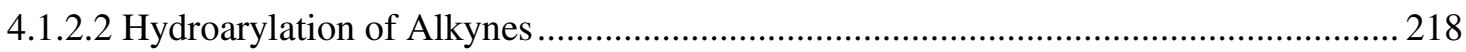

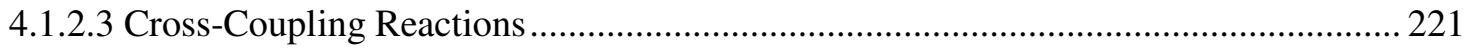

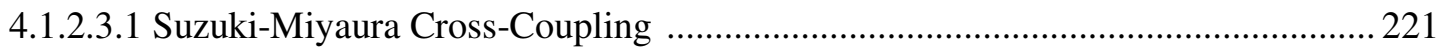

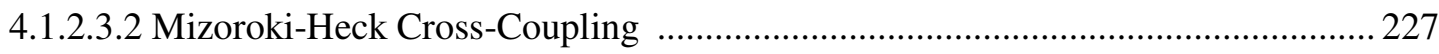

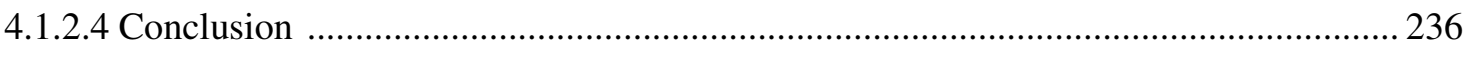




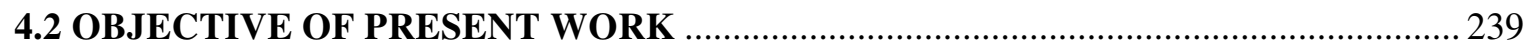

4.2.1 The Advantages of MIDA Boronate Ester ................................................................... 241

4.2.2 Heck Reaction Using Vinyl MIDA Boronate ................................................................ 242

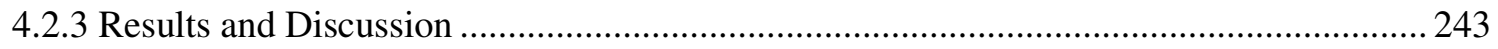

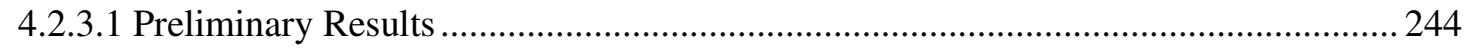

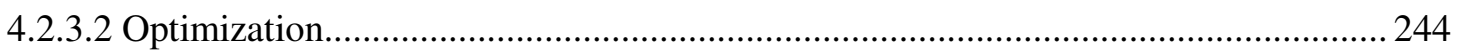

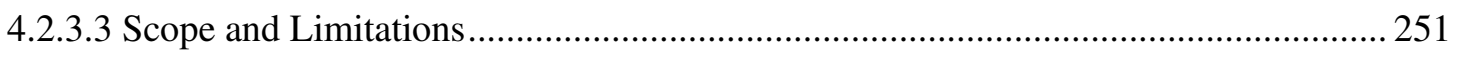

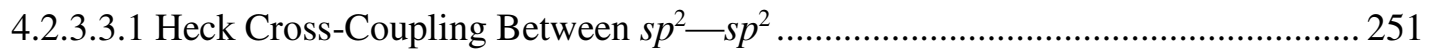

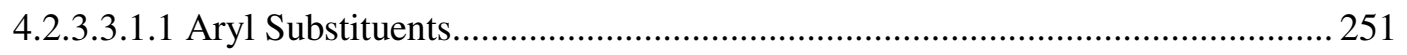

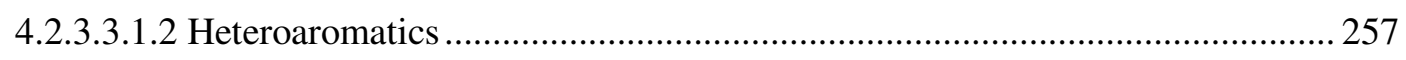

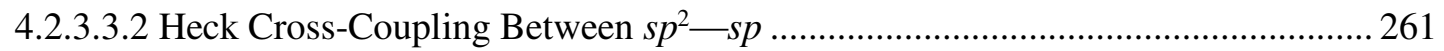

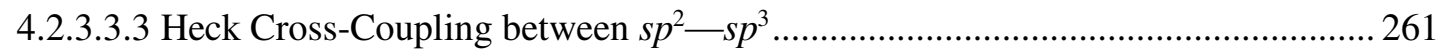

4.2.3.4 Proposed Mechanism and Structure Determination................................................ 262

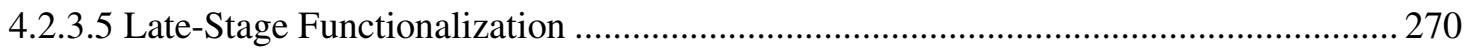

4.2.3.5.1 Suzuki-Miyaura Cross-Coupling ................................................................ 270

4.2.3.5.2 Conditions towards Tetra-Aryl Substituted Olefins ....................................... 270

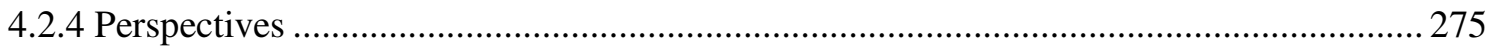

\section{CHAPTER V}

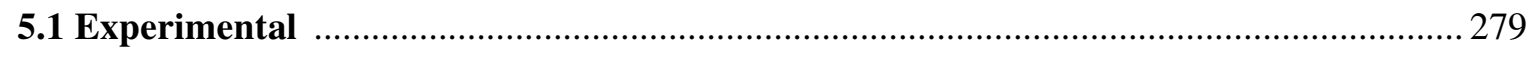

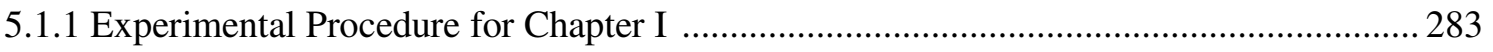

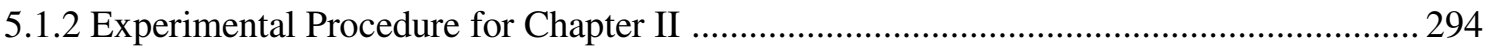

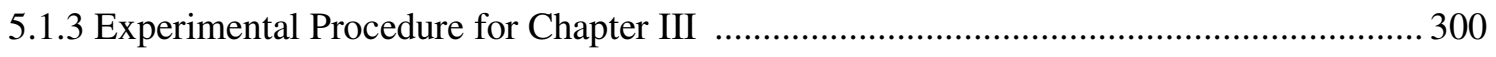

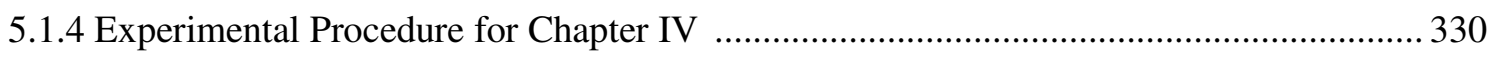




\section{CHAPTER VI}

6.1 Appendix

377

6.1.1 ${ }^{1} \mathrm{H}$ and ${ }^{13} \mathrm{C}$ NMR Spectra and Energy Calculations for Chapter I 379

6.1.2 Selected ${ }^{1} \mathrm{H}$ and ${ }^{13} \mathrm{C}$ NMR Spectra for Chapter II 395

6.1.3 Selected ${ }^{1} \mathrm{H}$ and ${ }^{13} \mathrm{C}$ NMR Spectra for Chapter III 401

6.1.4 ${ }^{1} \mathrm{H}$ and ${ }^{13} \mathrm{C}$ NMR Spectra for Chapter IV 415 




\begin{abstract}
It has been estimated that more than half of all approved drugs, from the period 1981 to 2014, are either natural products or their derivatives. This, thus, indicates that natural products (NP), together with natural product derived and natural product inspired structures are significant as sources for potential leads towards the discovery of new drugs. The introduction of this thesis thereby highlights the importance of natural products in the field of drug discovery.

In addition, the introduction emphasizes on the importance of natural products as a field of research. This is as the synthesis of natural products can result in the development of new synthetic methods which can then be applied to a broader range of applications across the field of chemistry. This new information, thus, bridges a gap in the scientific knowledge and allows for progress in science.

Therefore the content of this thesis describes the syntheses and development of new synthetic methods towards bioactive natural products containing 1,3-amino alcohol, 1,3-diamine, THP-ring as well as tri-aryl ethylene unit which represent the key themes of (+)-negamycin (Chapter 1), (-)-cernuine and (+)-cermizine D (Chapter 2), enigmazole A (Chapter 3) and tamoxifen (Chapter 4), respectively.
\end{abstract}





\section{LIST OF ABBREVIATIONS}

\begin{tabular}{|c|c|}
\hline$\AA$ & angstrom unit \\
\hline Ac & acetyl \\
\hline Acac & acetylacetonyl \\
\hline AIBN & azobisisobutyronitrile \\
\hline app & apparent \\
\hline aq. & aqueous \\
\hline $\mathrm{Ar}$ & aryl \\
\hline $\mathrm{Bn}$ & benzyl \\
\hline Boc & tert-butyloxycarbonyl \\
\hline Bom & benzyloxymethyl \\
\hline $\mathrm{bp}$ & boiling point \\
\hline $\mathrm{Bu}$ & butyl \\
\hline$n-\mathrm{Bu}$ & n-butyl \\
\hline$i$-Bu & iso-butyl \\
\hline$t$-Bu & tert-butyl \\
\hline $\mathrm{BuOH}$ & butanol \\
\hline $\mathrm{Bz}$ & benzoyl \\
\hline Calcd. & calculation \\
\hline CBS & Corey-Bakshi-Shibata \\
\hline $\mathrm{Cbz}$ & benzyloxycarbonyl \\
\hline Cod & cyclooctadiene \\
\hline COSY & correlated spectroscopy \\
\hline
\end{tabular}




\begin{tabular}{|c|c|}
\hline conc. & concentrated \\
\hline Cy & cyclohexyl \\
\hline CSA & camphorsulfonic acid \\
\hline DBU & 1,8-diazabicyclo[5.4.0]undec-7-ene \\
\hline DCC & $N . N^{\prime}$-dicyclohexylcarbodiimide \\
\hline DCE & 1,2-dichloroethane \\
\hline DDQ & 2,3-dichloro-5,6-dicyanobenzoquinone \\
\hline$d e$ & diastereomeric excess \\
\hline DEAD & diethyl azodicarboxylate \\
\hline DEPT & Distortionless Enhancement by Polarization Transfer \\
\hline DHP & dihydropyran \\
\hline DIAD & diisopropylazodicarboxylate \\
\hline DIBAL-H & diisobutylaluminium hydride \\
\hline DIPEA & diisopropylethylamine \\
\hline DMAP & $N, N$-(dimethyl-4-amino)pyridine \\
\hline DMF & dimethylformamide \\
\hline DMP & Dess-Martin periodinane \\
\hline DMSO & dimethylsulfoxide \\
\hline $\mathrm{dr}$ & diastereoselectivity \\
\hline$e e$ & enantiomeric excess \\
\hline Et & ethyl \\
\hline EtOAc & ethyl acetate \\
\hline equiv & equivalent \\
\hline $\mathrm{h}$ & hour \\
\hline HDA & Hetero-Diels-Alder \\
\hline
\end{tabular}




\begin{tabular}{|c|c|}
\hline HMPA & hexamethylphosphoramide \\
\hline HMQC & Heteronuclear Multiple Quantum Coherence \\
\hline HWE & Horner-Wadsworth-Emmons \\
\hline IBX & 2-iodoxybenzoic acid \\
\hline Imid. & imidazole \\
\hline $\mathrm{Ipc}_{2} \mathrm{BOMe}$ & Diisopinocampheylmethoxyborane \\
\hline KHMDS & Potassium $N, N$-bis(trimethylsilyl)amide \\
\hline $\mathrm{LC}_{50}$ & lethal concentration, $50 \%$ \\
\hline LiHMDS & lithium $N, N$-bis(trimethylsilyl)amide \\
\hline M & molar \\
\hline $\mathrm{Me}$ & methyl \\
\hline Mes & mesityl \\
\hline $\mathrm{MeOH}$ & methanol \\
\hline $\min$ & minutes \\
\hline MIB & 3-exo-morpholinoisoborneol \\
\hline MOM & methoxymethyl \\
\hline MPM & $p$-methoxyphenylmethyl \\
\hline MS & molecular sieve \\
\hline Ms & mesylate \\
\hline MTBD & 7-methyl-1,5,7-triazabicyclo[4.4.0]dec-5-ene \\
\hline MTBE & methyl tert-butylether \\
\hline MTPA & $\alpha$-methoxy- $\alpha$-trifluoromethylphenylacetic acid \\
\hline MW & microwave \\
\hline NBS & $N$-bromosuccinimide \\
\hline $\mathrm{NHC}$ & $N$-heterocyclic carbine \\
\hline
\end{tabular}




\begin{tabular}{|c|c|}
\hline NIS & $\mathrm{N}$-iodosuccinimide \\
\hline NMR & Nuclear Magnetic Resonance \\
\hline NMO & $N$-methyl morpholine- $N$-oxide \\
\hline NOESY & Nuclear Overhauser Effect Spectroscopy \\
\hline $\mathrm{Nu}$ & nucleophile \\
\hline PET & petroleum ether \\
\hline $\mathrm{Ph}$ & phenyl \\
\hline PPTS & pyridinium $p$-toluenesulfonate \\
\hline ppm & parts per million \\
\hline$i$-Pr & iso-propyl \\
\hline py & pyridine \\
\hline $\mathrm{rt}$ & room temperature \\
\hline Red-Al ${ }^{\circledR}$ & bis(2-methoxyethoxy)aluminium dihydride \\
\hline s & singlet \\
\hline SEGPHOS & 5,5'-bis(diphenylphosphino)-4,4'-bi-1,3-benzodioxole \\
\hline $\mathrm{t}$ & triplet \\
\hline TBAI & tetra-n-butylammonium iodide \\
\hline TBAF & tetra-n-butylammonium fluoride \\
\hline TBDPS & tert-butyldiphenylsilyl \\
\hline TBS & tert-butyldimethylsilyl \\
\hline TES & triethylsilyl \\
\hline TEMPO & 2,2,6,6-tetramethylpiperidinyloxy \\
\hline $\mathrm{Tf}$ & trifluoromethanesulfonyl \\
\hline TFA & trifluoroacetic acid \\
\hline THF & tetrahydrofuran \\
\hline
\end{tabular}


THP

TLC

TOCSY

TMS

Ts

$p$ - $\mathrm{TsOH}$ tetrahydropyran

thin layer chromatography

Total Correlation Spectroscopy

trimethylsilyl

4-toluenesulfonyl

4-toluenesulfonic acid 

Studies Towards the Synthesis of Bioactive Natural Products and Development of New Synthetic Methods 



\section{Introduction}

Introduction on Natural Products

Natural Product Synthesis as a Field of Research 



\section{Introduction on Natural Products}

In 1826, the first marketable natural product morphine was introduced by Merck for therapeutic use (Figure 1, compound 1) while in 1899 the first natural product derived semisynthetic drug aspirin (from willow Salix alba) was introduced by Bayer (Figure 1, compound 2). ${ }^{1}$ This paved the way for the isolation of a variety of early drugs such as codeine and quinine (Figure 1, compounds 3 and 4). ${ }^{1}$ Since then, many pharmaceutical drugs have continued to be derived from the use of natural products as sources of new drug leads ${ }^{2}$ and eventually go on to receive world-wide success.

Atorvastatin, for example, is a synthetic inhibitor of the enzyme HMG-CoA reductase (HMGRI) used to treat high cholesterol since 1996 and is marketed as Lipitor ${ }^{\circledR}$ by Pfizer (Figure 1, compound 5). It is a hypocholesterolimic drug that is derived from a microbial natural product $^{3}$ (fungal metabolite) and its synthesis was led by Bruce Roth in $1985 .{ }^{4}$ Atorvastatin has sold over USD 11 billion in 2004 alone, making it one of the world's best-selling drugs. ${ }^{4 a}$ Such a success could potentially be rivaled by a recently FDA approved natural product pharmacophore $^{5}$ bearing synthetic drug in 2013, sofosbuvir (Figure 1, compound 6), which is currently the only cure for hepatitis $\mathrm{C}(\mathrm{HCV})$ infections within a 2 month time period. ${ }^{6}$

Another example of a successful drug from a natural product is artemisinin (Figure 1, compound 7). It was isolated from a traditional Chinese plant Artemisia annua for the treatment of malaria. ${ }^{7}$ Its discovery and development by Professor Tu, was recognized with the 2015 Nobel Prize award, which is considered a great achievement in the area of natural products.

Therefore natural products (NP), together with natural product derived and natural product inspired structures are regarded dominant in the roles they play as sources for potential leads towards the discovery of new drugs, ${ }^{6}$ despite rational drug design, libraries of combinatorial chemistry, ${ }^{8}$ molecular modelling and other computational methods. 

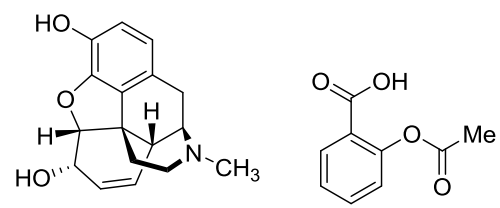

Morphine 1

Aspirin 2

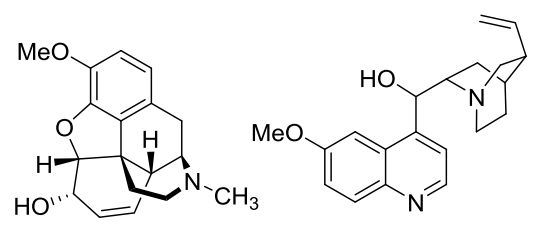

Codeine $3 \quad$ Quinine 4

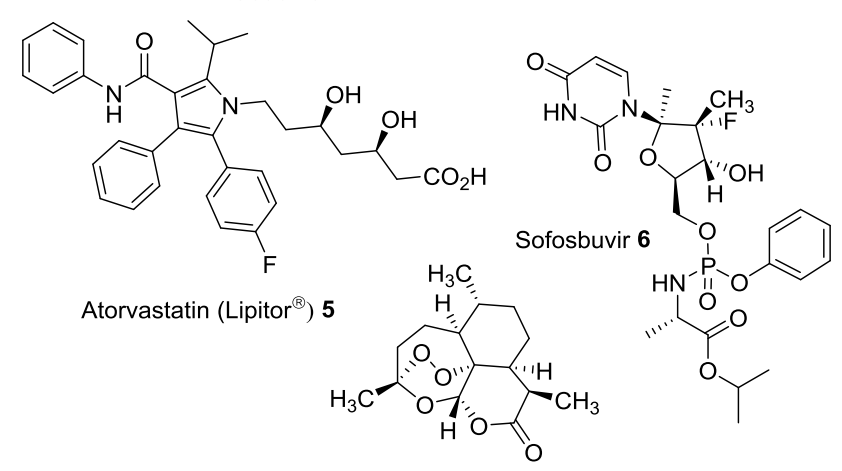

Artemisinin 7

Figure 1. Natural products inspired and derived drugs.

Figure 2 further reflects the importance and relevance of natural products in the process of drug discovery and development. It has been estimated that more than half of all approved drugs, from the period 1981 to 2014, are either natural products or their derivatives (51\%). ${ }^{6}$

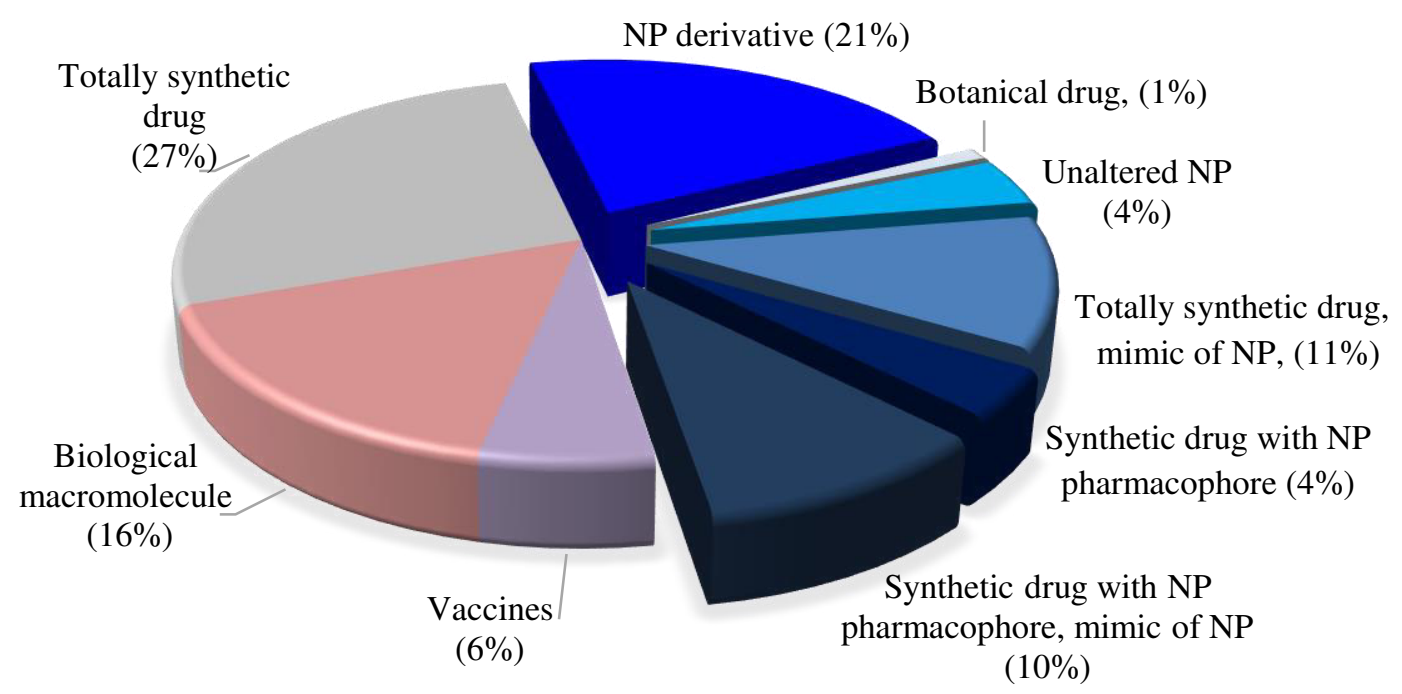

Figure 2. Approved drugs in the span of 33 years (1981 to 2014). ${ }^{6}$ 


\section{Natural Product Synthesis as a Field of Research}

The importance of natural products in chemistry, medicine as well as biology has resulted in their synthesis being an equally significant field of investigation. ${ }^{9}$ The construction of molecules of nature and/or their analogues in a laboratory has provided opportunities for discovery and invention, therefore, sharpening the tool of chemical synthesis. ${ }^{9}$

For example, the synthesis of natural products can result in the development of new synthetic methods, which can then be applied to a broader range of applications ${ }^{9}$ across the field of chemistry (Figure 3). This new information, thus, bridges a gap in the scientific knowledge and allows for progress in science. Moreover the ability to mimic nature molecules in a laboratory, particularly those with promising biological activity but scarcity in nature, enables medium to large scale production for an extensive study of its bioactivity profile and mechanism of action. This offers a cost-effective alternative to reliance on extraction techniques. More importantly, this also creates the opportunity to introduce changes in the structure of natural products for an enhancement in potency, leading to the synthesis of libraries of analogues. ${ }^{9}$ Lastly, structure validation of an assigned natural product can only be achieved by the chemical synthesis of the molecule. ${ }^{9}$

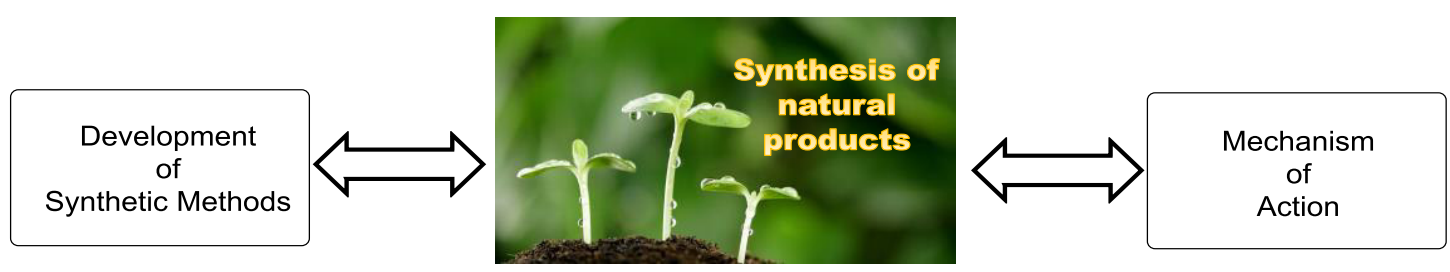

Figure 3. A summary of natural product synthesis as a research field.

Therefore this thesis describes the syntheses and development of new synthetic methods towards bioactive natural products containing 1,3-amino alcohol, 1,3-diamine, THP-ring as well as tri-aryl ethylene unit, which represent the key motifs of negamycin (chapter 1), cernuine and cermizine D (chapter 2), enigmazole A (chapter 3) and tamoxifen (chapter 4), respectively. 
This page has been intentionally left blank. 
At this juncture henceforth, the chemical compounds are labelled

following X.Y.Z system from when they are first mentioned.

Classification of X.Y.Z is as follows,

( $\mathbf{X}=$ Chapter Number $)(\mathbf{Y}=$ Scheme Number $)(\mathbf{Z}=$ Compound Number $)$ 
This page has been intentionally left blank. 


\section{Chapter 1}

\subsection{Introduction}

1.1.1 Natural Products Containing $N-N$ Bonds

1.1.2 (+)-Negamycin: Introduction

1.1.3 Biological Activity of (+)-Negamycin

1.1.4 Syntheses of (+)-Negamycin 
This page has been intentionally left blank. 


\subsection{Introduction}

\subsubsection{Natural Products Containing Nitrogen-Nitrogen Bonds}

The first naturally occurring compound bearing a nitrogen-nitrogen $(N-N)$ bond was reported in the year $1951 .{ }^{10}$ It belongs to the class of azoxy compounds and is known as toxin macrozamin (Figure 1.1).

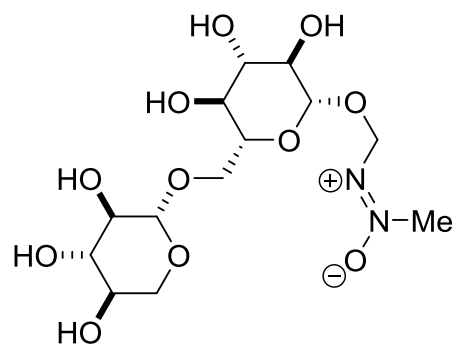

Figure 1.1. Toxin Macrozamin.

Since then, many new members of this family of compounds from a variety of structural classes have surfaced. There are at least 15 different types and over 200 natural products, up to early 2013, containing an $N-N$ bond. ${ }^{11}$ The different classes of natural products bearing the $\mathrm{N}-\mathrm{N}$ bonds are outlined in Figure 1.2, which is a reflection of the vast degree of their structural diversity. ${ }^{11 a}$ In addition, they are a remarkable source of biological activity amongst nitrogencontaining natural product. ${ }^{11 a}$ 


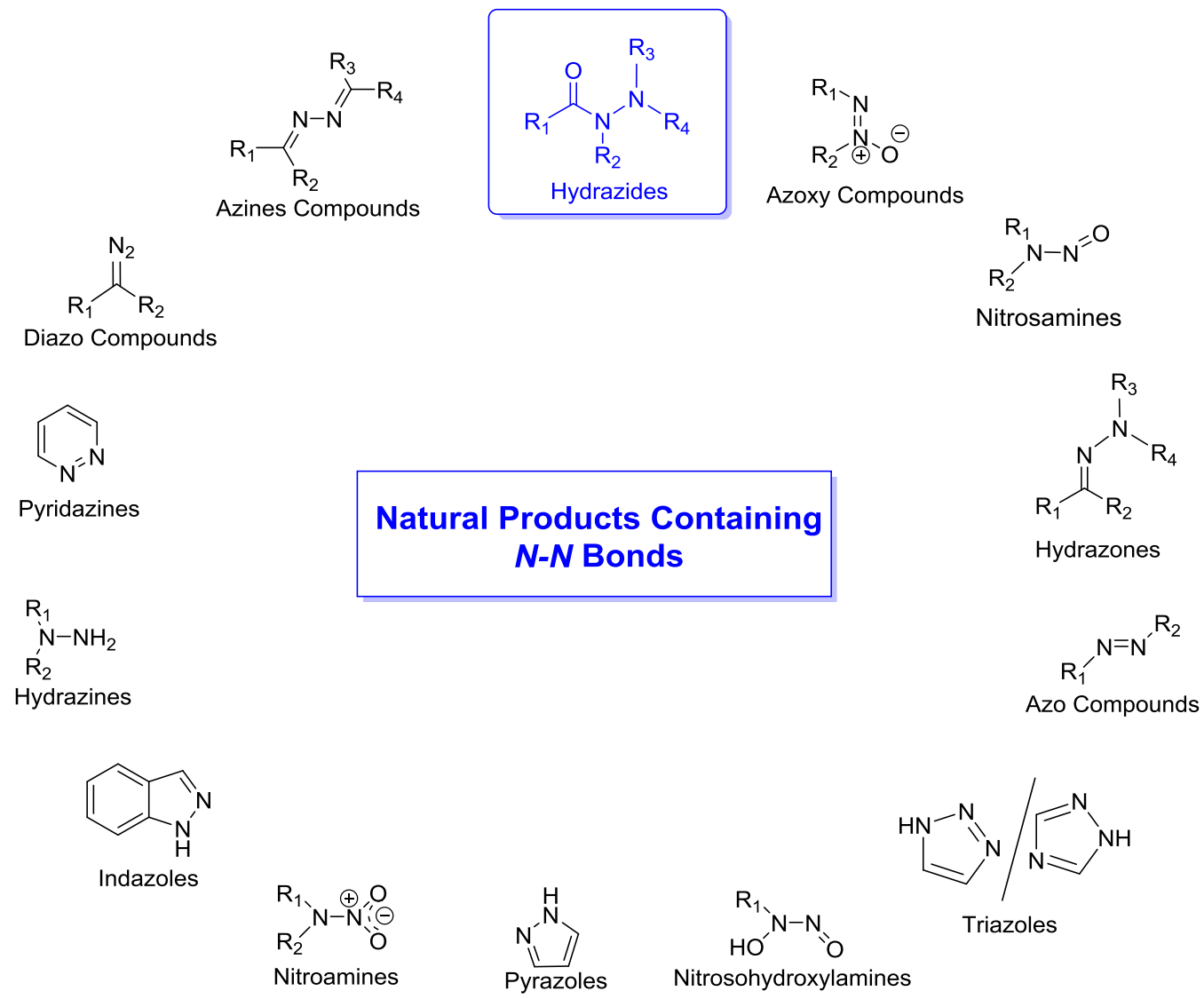

Figure 1.2. Structures of natural products containing $N-N$ bonds.

Hydrazides are highlighted in particular because of the natural product (+)-negamycin, which has been successfully synthesized and is discussed in this chapter. Hydrazides are a class of organic compounds characterized by having a nitrogen-nitrogen covalent bond with four substitutents of which, at least one bears an acyl group. They have been extracted from plants, microorganisms and marine organisms and are rare in nature. ${ }^{11 \mathrm{a}, 12}$ The enzymes responsible for the construction of $N-N$ bonds remain to be discovered. ${ }^{12}$ Hydrazides possess a great structural diversity and biological activities, ${ }^{12}$ and (+)-negamycin belongs in this category of natural compounds containing the $N-N$ bonds. 


\subsection{2 (+)-Negamycin: Introduction}

$(+)$-Negamycin is a linear natural product ${ }^{13}$ first isolated from the culture filtrates of three strains of Streptomyces Purpeofuscus in 1970 (Figure 1.3). (+)-Negamycin contains a hydrazine peptide bond and two stereogenic centers at $\mathrm{C} 3$ and $\mathrm{C} 5$ as shown in Figure 1.3. Structural elucidation was conducted by Umezawa et al. in 1970 through degradation studies ${ }^{14}$ and was confirmed by a total synthesis of the natural product from D-galacturonic acid. ${ }^{15}$

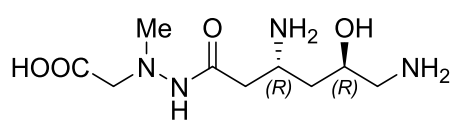

$(+)$-Negamycin

Figure 1.3. Structure of natural product (+)-negamycin.

\subsubsection{Biological Activity of (+)-Negamycin}

(+)-Negamycin is a pseudopeptide antibiotic, which shows strong inhibitory activity against multi-drug resistant Gram-positive and Gram-negative ${ }^{14 a}$ bacteria as well as Pseudomonas $^{16}$ and Pseudomonas Aerginosa, ${ }^{14 \mathrm{a}}$ with low toxicity. ${ }^{17}$ Furthermore, (+)-negamycin has been shown to specifically inhibit protein synthesis in Escherichia Coli K12. ${ }^{18}$ It has also been discovered to inhibit protein biosynthesis with miscoding activity and genetic miscoding activity on bacterial ribosome systems. ${ }^{17 b, 18}$ However, it is unlike other antibiotics with similar activity, which are categorized as aminoglycoside antibiotics, ${ }^{19}$ as (+)negamycin contains a hydrazide moiety. Also, diseases related to nonsense mutations as well as Duchenne muscular dystrophy may find treatment in $(+)$-negamycin as it has been identified as a potential viable therapy. ${ }^{11 \mathrm{a}}$ 


\subsubsection{Syntheses of (+)-Negamycin}

There have been a total of 21 syntheses of (+)-negamycin and its derivative; 5-epinegamycin. ${ }^{13,15,17 \mathrm{a}, 20}$ The most common synthetic strategy to access the natural product revolves around the key stereoselective steps for the introduction of the $3 R$ and $5 R$ chiral centers, and the hydrazine peptide bond, which follows after the key steps. The following are selected reviews of (+)-negamycin synthesis.

\subsubsection{Synthesis by Wang et al. ${ }^{17 a}$}

In 1982, Wang et al. reported the total synthesis of (+)-negamycin in allegedly five steps with an overall yield of 51\% (Scheme1.1). The retrosynthetic analysis began with the cleavage at the hydrazino peptide bond which would give the methyl hydrazine 1.1.2 and amino alcohol 1.1.3 fragments. The latter would be accessible by the key step, which is an asymmetric induction of the acyclic homoallylamine 1.1.4 in a highly stereocontrolled fashion from iodocarbamation and iodolactonization. Homoallylamine 1.1.4 would be subsequently derived from a prochiral diester precursor 1.1.6. It is worth pointing out that the preparation of diester 1.1.6 would require a multi-step reaction from dimethylmalonate following the procedures described by Fukuyama et al. ${ }^{21}$

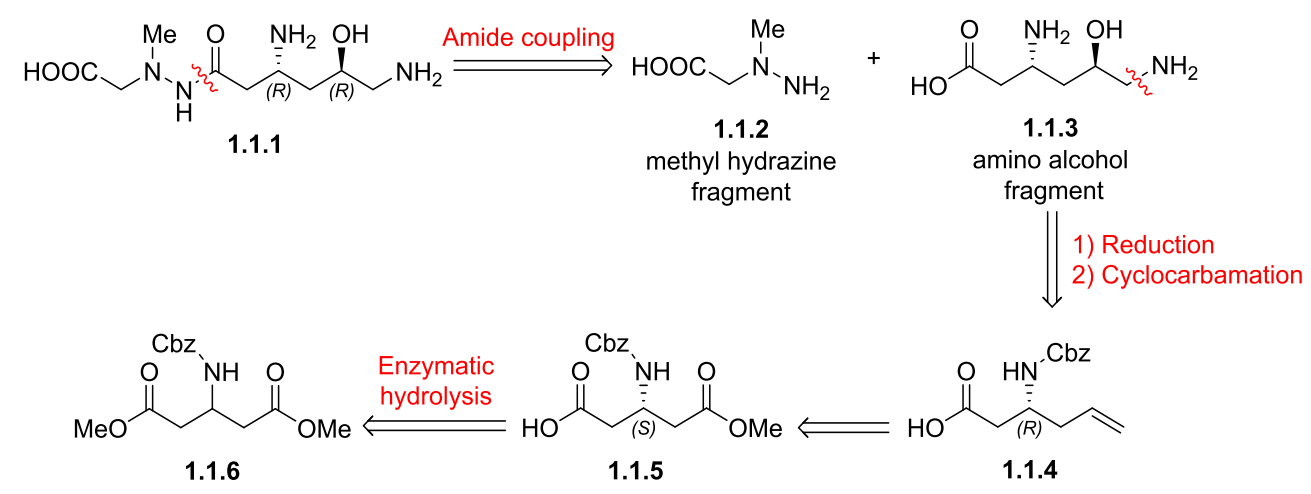

Scheme 1.1. Retrosynthetic analysis of (+)-negamycin by Wang et al. 
The synthesis began with a selective hydrolysis of 1.1.6 via an enzymatic procedure to give the chiral half ester 1.1.5 (Scheme 1.2). The efficiency of the stereoselective conversion was not reflected as there was no mention of the enantiomeric excess. The acid of 1.1.6 was then protected with a tert-butyl group followed by ester hydrolysis under basic coniditions to give the acid 1.2.1, which was reduced to the corresponding aldehyde 1.2.2 prior to a Wittig olefination to give the important homoallylamine intermediate 1.2.3. The tert-butyl ester of homoallylamine 1.2.3 was then hydrolyzed to the acid 1.1.4 prior to the two asymmetric functionalization steps; iodolactonization and iodocarbamation.

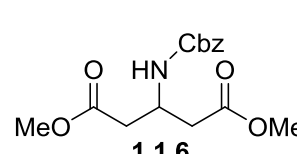

1.1.6
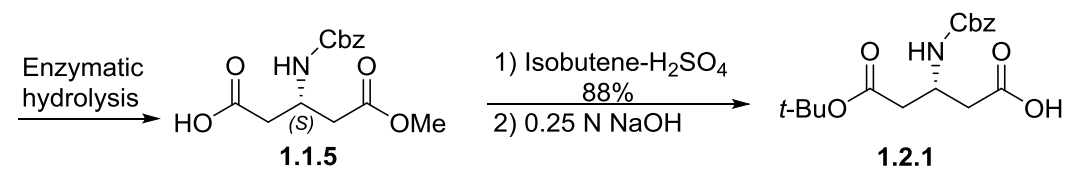

1) $\mathrm{LiAlH}_{4}$
2) $\begin{gathered}3,5-\text { Dimethyl- } \\ \text { pyrazole-DCC } \\ 76 \%\end{gathered}$

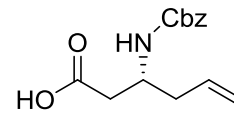

1.1.4
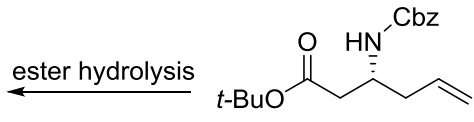

1.2 .3

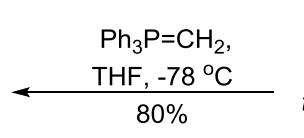

$80 \%$

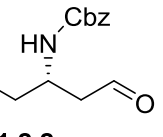

1.2.2

Scheme 1.2. Synthetic route to key intermediate 1.1.4.

Iodolactonization was carried out across the double bond of homoallylamine 1.1.4 and 1.3.3 (Scheme 1.3). The iodolactonization of $\mathbf{1 . 1 . 4}$ proceeded very poorly as it gave a mixture of trans-iodo- $\delta$-lactone-1.3.1 and cis-1.3.2 in the ratio of $1: 1.5$ respectively, with a low preference for the desired cis-1.3.2 isomer. Although there was a substantial improvement in the stereoselectivity under the same condition with the $N$-benzyl derivative $\mathbf{1 . 3 . 3}$ to a ratio of 1:6 for trans-1.3.4 and cis-1.3.5 respectively, there needed to be additional steps for the attachment and removal of the benzyl group, and this renders this approach unattractive. For this reason, the group decided to proceed with an alternative approach; iodocyclocarbamation. 


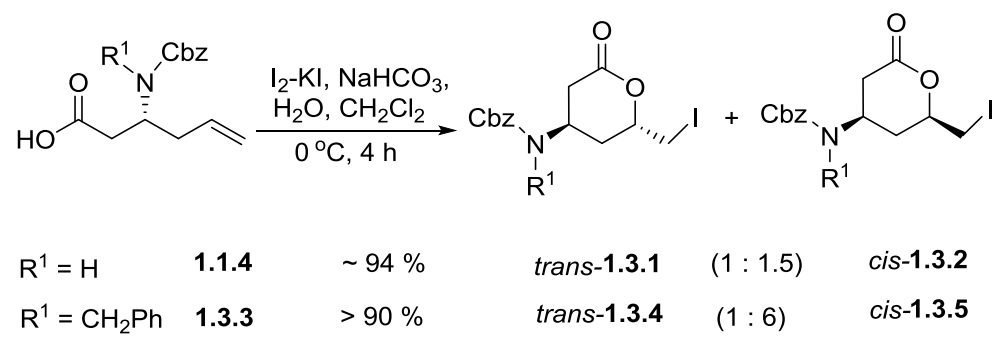

Scheme 1.3. Stereoselective iodolactonization.

Iodocyclocarbamation was employed for the functionalization of the olefin on homoallylamines 1.2.3, 1.4.3 and 1.4.6 (Scheme 1.4). The substituent on the nitrogen of homoallylamine 1.2.3 was changed from hydrogen to benzyl and the bulky tert-butyl ester groups giving 1.4.3 and 1.4.6 respectively. The objective was to influence the 1,3-asymmetric induction through the use of bulky substituents, and it was a successful attempt as it increased the yield and diastereoselectivity of the iodocyclocarbamation despite the additional steps. The iodocyclocarbamation of homoallylamine 1.2.3 produced an "excellent" yield, although it was not numerically specified. Moreover, an inseparable mixture of diastereomers was obtained, trans-1.4.1 and cis-1.4.2, in a 3:7 ratio respectively. The iodocyclocarbamation of substrate 1.4.3, on the other hand, proceeded with a high yield of $83 \%$ and it afforded the best diastereoselectivity in a 23:1 ratio with a preference for the desired trans-1.4.4 isomer. The diastereomers were reportedly separable by column chromatography. Further efforts to improve the trans/cis ratio failed with tert-butyldimethylsilane bulky group, 1.4.6. The iodocyclocarbamation of 1.4.6 gave the desired trans-1.4.7 product as the major isomer albeit at a lower yield of $69 \%$ and diastereoselectivity ratio of $14: 1$ (Scheme 1.4).

The group had previously abandoned the iodolactonization route to explore the iodocarbamation for the purpose of avoiding possible additional nitrogen-protection steps, therefore, it makes for no added advantage with this approach and their goal of reducing the number of synthetic steps, in spite of the superior stereoselectivity offered by this method. 


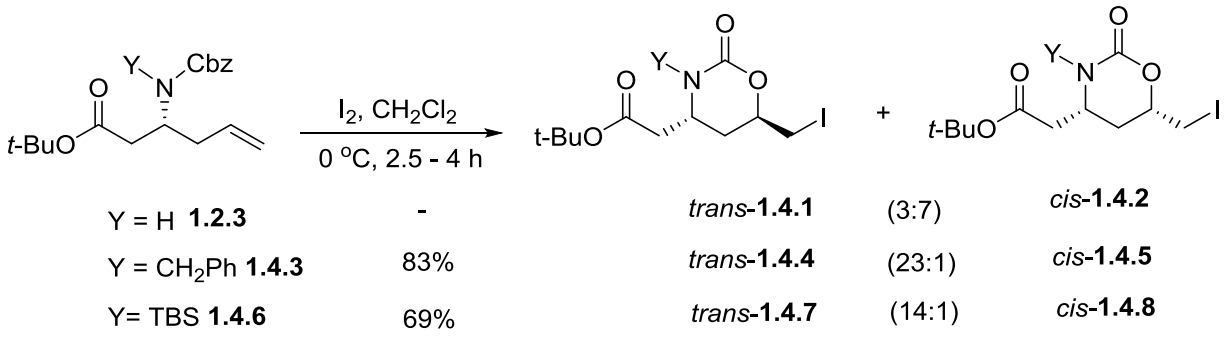

Scheme 1.4. Iodocyclocarbamation of homoallylamines.

The synthesis of (+)-negamycin did not continue from diastereomers 1.4.4/1.4.5 but from 1.4.1/1.4.2, presumably to avoid the extra step of attaching the benzyl group (Scheme 1.5). However, the benzyloxycarbamate group needed to be re-introduced upon hydrolysis with $\mathrm{Ba}(\mathrm{OH})_{2}$ that would have made using diastereoisomers 1.4.4/1.4.5 a better option given its higher reaction yield.

The stereoisomers were separated after esterification with diazomethane to afford the desired diastereomer 1.5.2. The diastereomer 1.5.2 was isolated through a reportedly easy separation via flash chromatography on silica. (Scheme 1.5). Furthermore, the free hydroxyl group in 1.5.2 needed to be protected prior to saponification of the methyl ester to prevent a potential 1,6-cyclization. This highlights the poor choice of using a cyclic carbamate functionality in the presence of an ester group that is set up for a condensation reaction. Nonetheless, (+)-negamycin was obtained in 4 steps from methyl ester 1.5.2, which included alcohol protection, methyl ester hydrolysis, peptide bond formation with 1-methylhydrazino acetate and the final hydrogenation step. In overall, (+)-negamycin was synthesized in 13 steps from the starting material 1.2.1. Wang et al. combined both chemical and enzymatic procedures in the synthesis, which differed from most syntheses of (+)-negamycin reviewed.

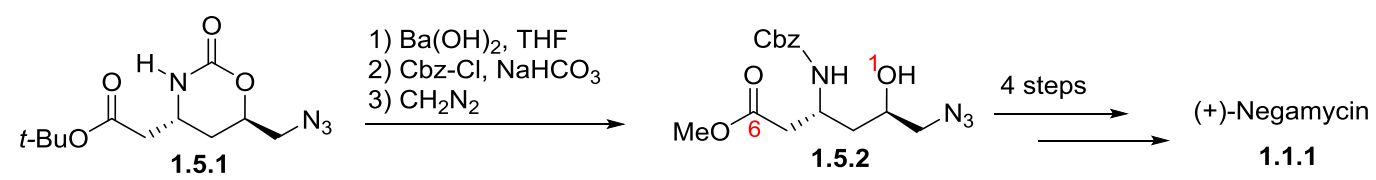

Scheme 1.5. Completion of (+)-negamycin 1.1.1 synthesis from carbamate 1.5.1. 


\subsubsection{Synthesis by Hayashi et $a .^{20 \mathrm{i}}$}

In 2008, Hayashi et al. reported a highly efficient strategy for the synthesis of (+)-negamycin (Scheme 1.6). It utilized a commercially available achiral building block $N$ Boc-2-aminoacetaldehyde 1.6.4 with an overall yield of $41 \%$ in a total of 8 steps. Retrosynthetic analysis of (+)-negamycin began with the usual cleavage of the hydrazide bond to give the methyl hydrazine fragment 1.1.2 and amino alcohol fragment 1.1.3. The amino alcohol fragment 1.1.3 would be converted into the Boc-protected oxazolidine 1.6.1, which would be accessible by the key Michael addition step through the tert-butyl $\alpha, \beta$ unsaturated ester 1.6.2. The Michael acceptor would be synthesized from the aminoacetaldehyde 1.6.4 (Scheme 1.6).
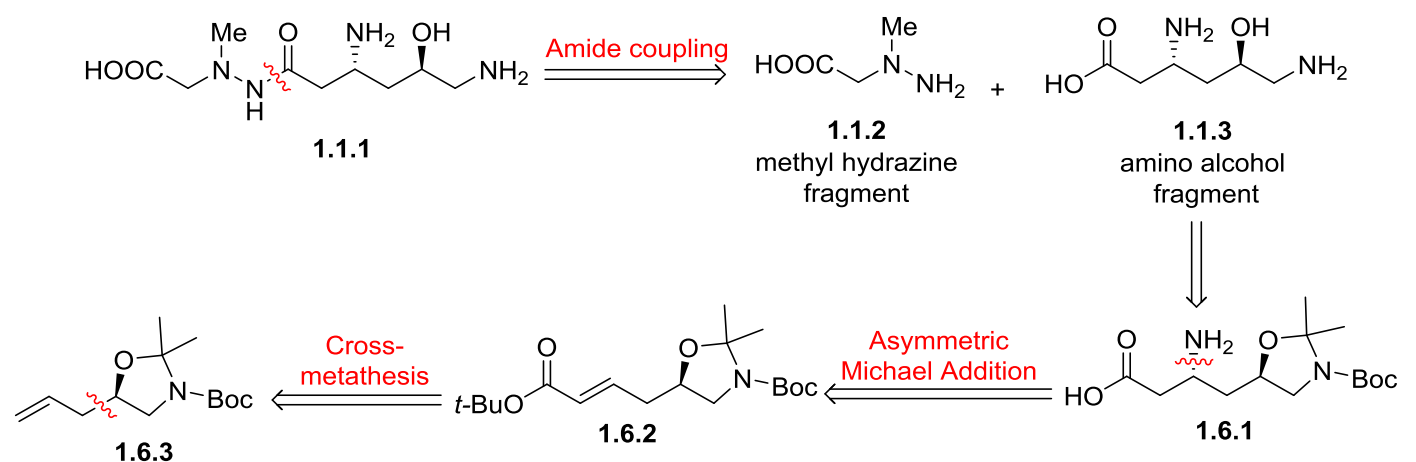

enantioselective
allylation<smiles>CC(C)(C)NCC=O</smiles>

Scheme 1.6. Retrosynthetic analysis of (+)-negamycin by Hayashi et al.

The first stereogenic center was introduced to the aldehyde 1.6.4 via Brown asymmetric allylation by employing the chiral reagent, allyldiisopinocampheyl-borane, to give $(R)$-homoallylic alcohol, ${ }^{22}$ which was directly treated with 2,2-dimethoxypropane to furnish the oxazolidine, 1.6.3 after purification in a high yield of 90\% (Scheme 1.7). However, there was no mention of the enantiomeric purity from the Brown allylation. The 
key intermediate, Michael acceptor 1.6.2, was synthesized via a cross-metathesis between oxazolidine 1.6.3 and tert-butyl acrylate with the Grubbs second-generation [Ru-II] catalyst. The expensive ruthenium-based catalyst had to be engaged in just the second step of the synthetic route, which makes the route potentially cost-inefficient depending on the catalytic loading that was not specified. Furthermore, the reaction time for the cross metathesis was stated to have been "drastically shortened" by 20 -fold when using microwave irradiation, however the overall time taken for the reaction to reach completion, microwave-assisted or otherwise, was not mentioned. Nevertheless, the cross metathesis proceeded with a good $83 \%$ yield to give the key intermediate, 1.6.2, which was engaged in the key asymmetric Michael addition step.

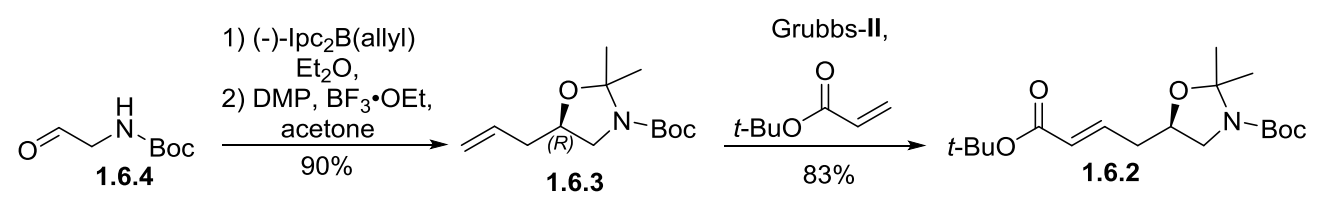

Scheme 1.7. Synthetic route to key intermediate 1.6.2.

The key stereoselective Michael addition step provided a single diastereomer 1.8.1 in $80 \%$ yield (Schemes 1.8 and 1.9 ). In this way, the second stereogenic center of $(+)-$ negamycin was incorporated efficiently, which makes the synthetic plan attractive. The diastereoselectivity could be attributed to the different aggregation forms of the lithium amide in tetrahydrofuran and diethyl ether. In THF, the proposed transition state is in a sixmembered chair conformation where the bulky $\mathrm{R}^{1}$ group of the Michael acceptor, 1.6.2, is in the equatorial position ${ }^{23}$ that leads to a si-face attack by the nitrogen of the chiral amine on the $\alpha, \beta$-unsaturated ester to give the desired diastereomer (Scheme 1.8). However, in diethyl ether, the proposed transition state does not involve a six-membered chair conformation thereby resulting in the formation of the other diastereomer 1.9.1 through a re-face nucleophilic attack (Scheme 1.9). 


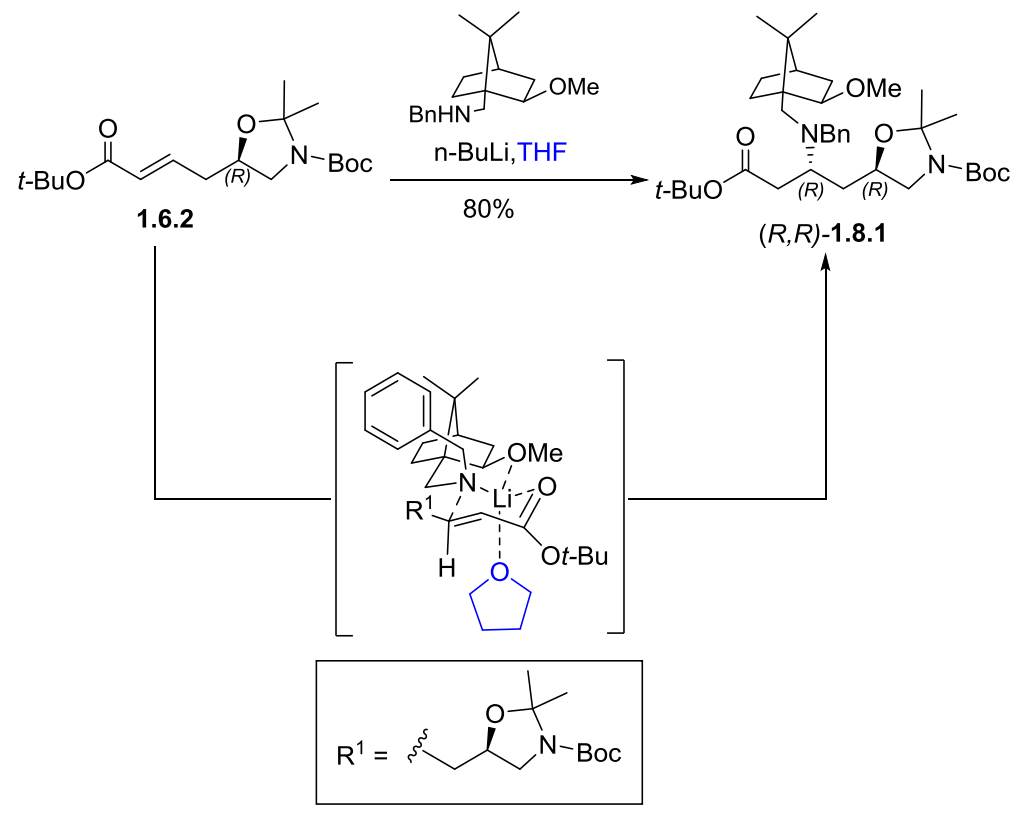

Scheme 1.8. Stereocontrol from $s$-membered chair transition state and $s i$-face attack.

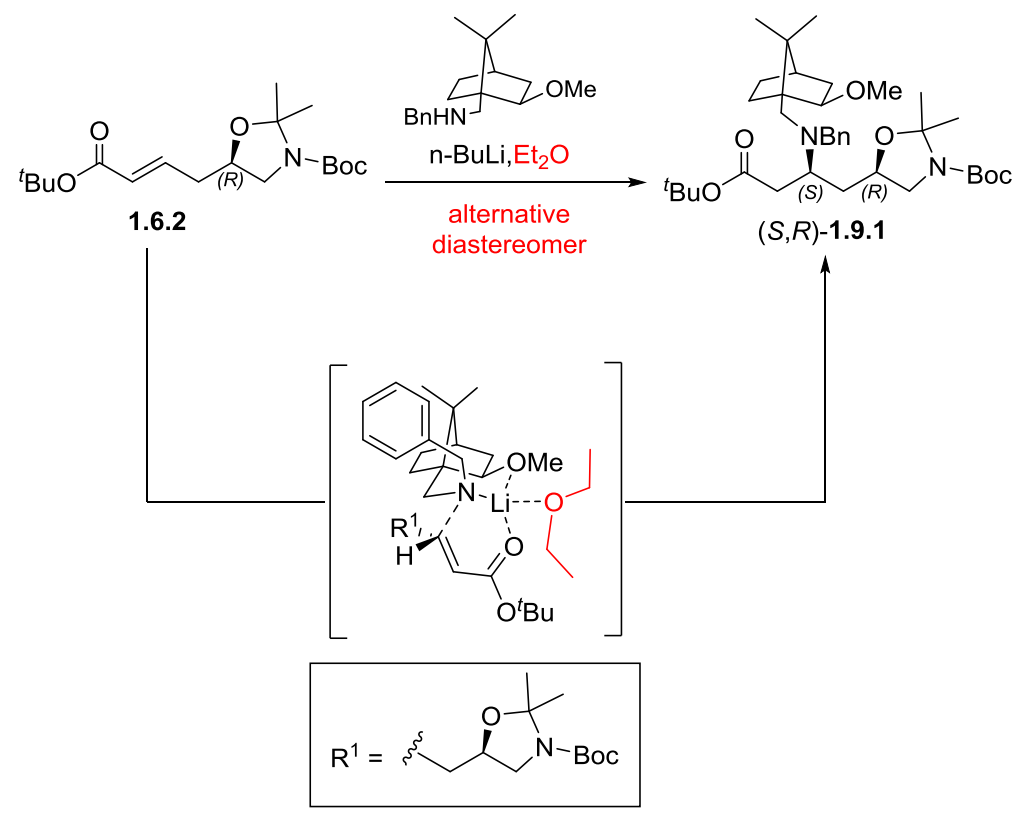

Scheme 1.9. Stereocontrol from $s$-membered transition state and $r e$-face attack.

It is noteworthy that four additional steps were required for the preparation of the bicyclic[2.2.1] chiral amine system from ketopinic acid. ${ }^{23}$ In addition, the removal of the chiral auxiliary involved the breaking of a C-N bond, which is generally difficult. ${ }^{23}$ Apart from that, an excess of $N$-iodosuccinimide (4.0 eq) was necessary for a complete removal of the chiral 
auxiliary of 1.8.1, albeit with a high $81 \%$ yield, to give the free amine 1.10.2. Although it is repeatedly emphasized as an advantage that 2-methoxy-D-bornylaldehyde $\mathbf{1 . 1 0 . 1}$ can be recycled to regenerate the chiral amine auxiliary via reductive amination with sodium borohydride, it cannot be concluded as being completely recyclable as the reductive amination reaction only gave $85 \%$ yield. ${ }^{23}$ Furthermore, the regeneration of the chiral auxiliary does not originate directly from substrate cleavage as additional steps are necessary. This lacks the versatility found in Evans' auxiliaries such as the oxazolidinone where there is a variety of cleavage methods, allowing an immediate regeneration of the auxiliary without the need for additional manipulation. In addition, the chiral amine auxiliary was only removable via NIS. ${ }^{23}$ Therefore, the recyclability aspect of the asymmetric Michael addition is a marginal advantage.

The free amine 1.10.2 was then protected by a Boc-protecting group and subjected to a hydrazine peptide bond formation with the hydrolyzed product of tert-butyl ester $\mathbf{1 . 1 0 . 3}$ to give hydrazide 1.10.4 in a high 98\% yield (Scheme 1.10). The subsequent removal of the DMP-group under acidic conditions completed the synthesis and gave the natural product $(+)$-negamycin. 


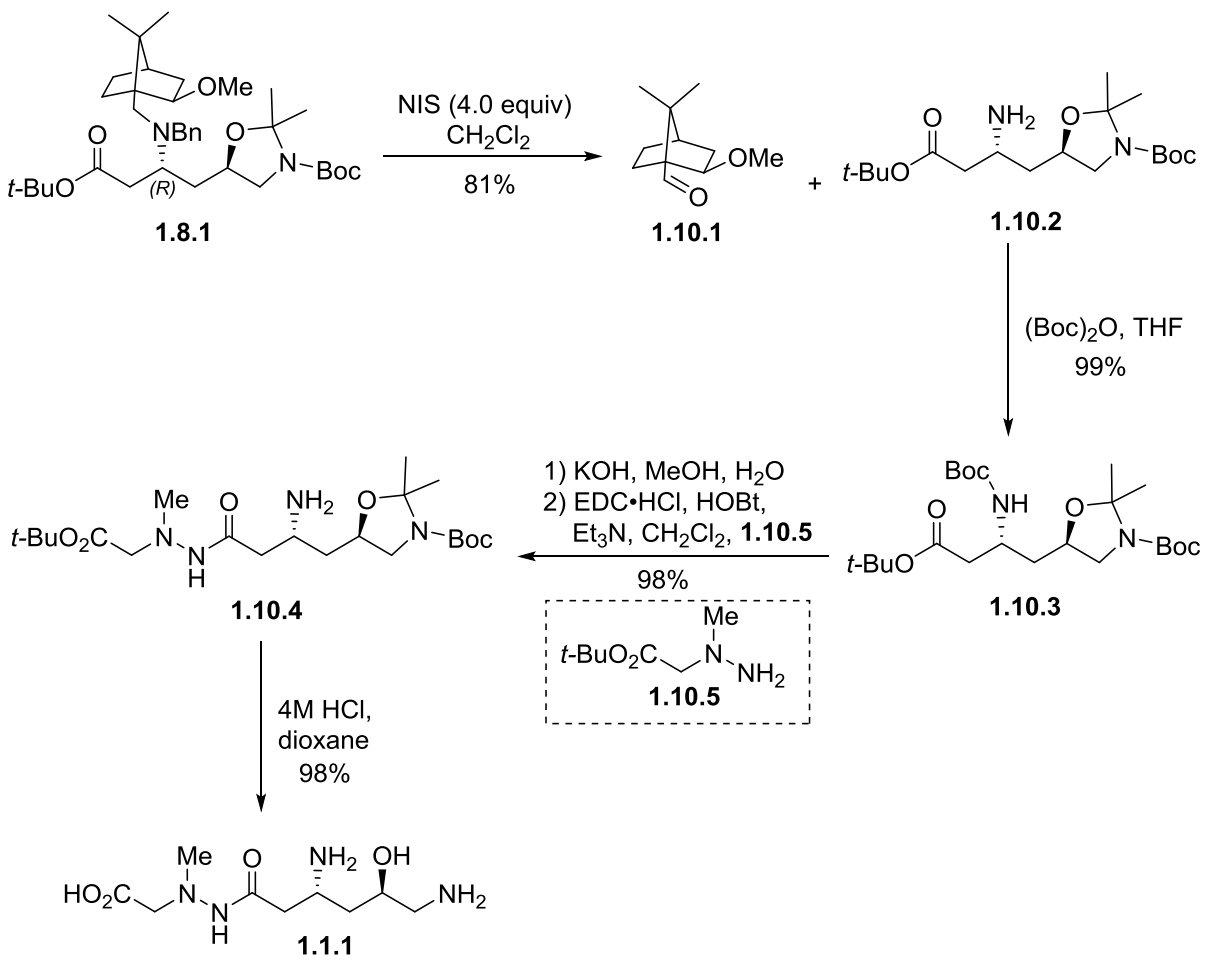

Scheme 1.10. Completion (+)-negamycin 1.1.1 synthesis from 1.8.1.

The outcome of the key step was influenced by many factors, and is thus considered a sensitive reaction. For instance, the diastereoselectivity was affected by the type and amount of additives (HMPA), and the $\beta$-carbon substituents. ${ }^{23}$ The type of solvent, polar or non-polar, was also essential to both diastereoselectivity and reaction yield. It has been found that better results were attained with polar solvents like tetrahydrofuran. ${ }^{23}$ These factors present the opportunity to optimize the key step that would allow for an improvement in the reaction outcome. Therefore this method is a better choice than the iodolactonization and iodocyclocarbamation strategies employed by Wang et al., which do not offer fine-tuning.

Two years later, Hayashi et al. contributed another synthetic route to the natural product in 13 steps with an overall $31 \%$ yield (Scheme 1.11). ${ }^{20 \mathrm{~h}}$ The retrosynthetic analysis of $(+)$-negamycin would remain accessible at the hydrazine peptide bond to give the methyl hydrazine and amino alcohol fragments. Although the key asymmetric step would be unchanged, the access to the Michael acceptor, tert-butyl unsaturated ester 1.6.2, would be from 
a Wittig olefination of aldehyde 1.11.1 instead of the previously adopted cross-metathesis strategy. Aldehyde 1.11.1 was obtained from the partial reduction of chiral ester 1.11.2 as opposed to the previously achiral $N$-Boc-2-aminoacetaldehyde 1.6.4.

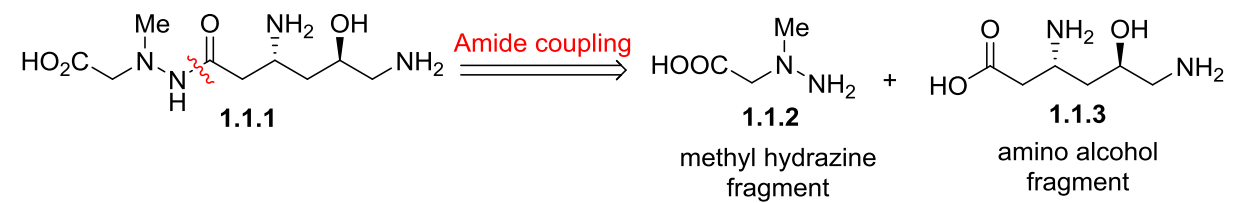

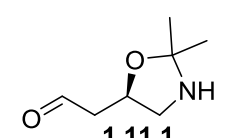

1.11.1

$\Downarrow$ Reduction

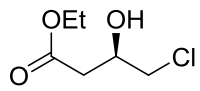

1.11.2

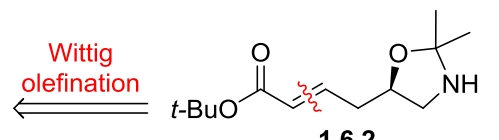

1.6.2 fragment fragment

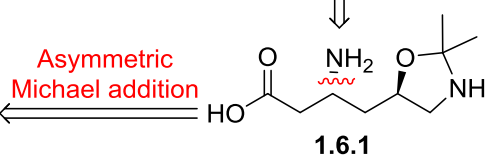

1.6.1

Scheme 1.11. Retrosynthetic analysis of (+)-negamycin by Hayashi et al.

The chloro group in the chiral ester, 1.11.2, was exchanged to an azide functionality, which was reduced during hydrogenation in the presence of $\mathrm{Boc}_{2} \mathrm{O}$ to allow for simultaneous protection of the resulting free amine (Scheme 1.12). This was followed by hydrolysis to give the carboxylic acid that was then transformed into Weinreb amide, followed by the protection of the amino alcohol as 2,2-dimethyloxazolidine 1.12.1. 


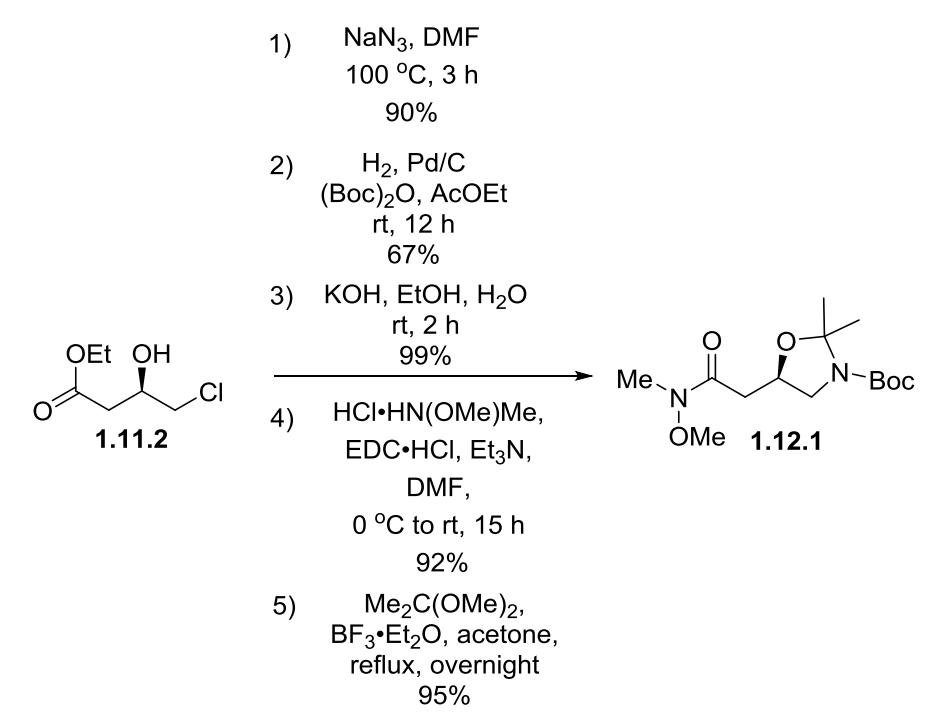

Scheme 1.12. Synthesis of Weinreb amide 1.12.1.

The Weinreb amide, 1.12.1, was treated with DIBAL at $-78{ }^{\circ} \mathrm{C}$ for $3.5 \mathrm{~h}$ to afford the aldehyde prior to a Wittig reaction (Scheme 1.13). The Wittig reaction gave the $E$-alkene 1.6.2, which is the key intermediate for the proceeding diastereoselective Michael addition step. It takes a total of 7 steps to afford the key intermediate 1.6.2, which is longer than their previously reported strategy in 2008, where it took only 3 steps. Moreover, the synthesis of bicycle[2.2.1] chiral amine system along with its removal and the subsequent protection of the free amine led to a culmination of 17 steps with an overall $31 \%$ yield. ${ }^{20 \mathrm{~h}}$ This was lower than their previously reported synthesis of the natural product (12 steps, $41 \%$ ) thus rendering this strategy of lesser significance. $^{20 \mathrm{i}}$

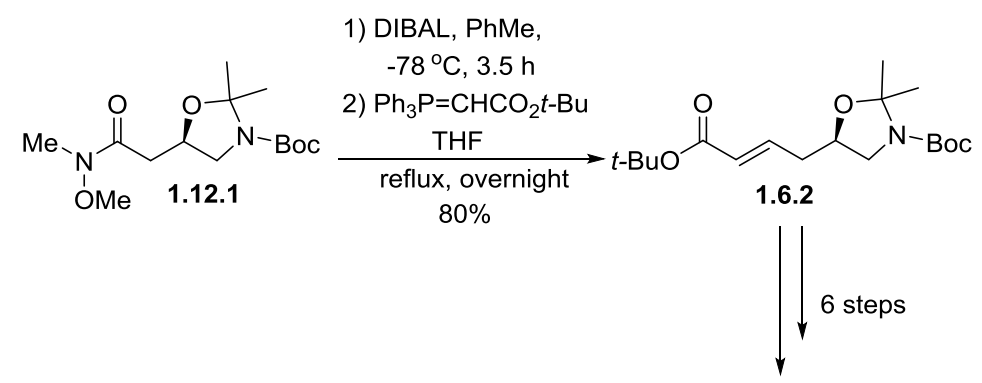

$(+)$-Negamycin

Scheme 1.13. Synthesis to access (+)-negamycin from Weinreb amide 1.12.1. 


\subsubsection{Synthesis by Davies et al. ${ }^{20 f, 20 \mathrm{~g}}$}

Similarly, in 1996 and 2011, Davies et al. employed a highly stereoselective conjugate addition as the key step in the synthesis of (+)-negamycin 1.1.1 in a total of 13 steps with $24 \%$ overall yield (Scheme 1.14). The retrosynthetic analysis began with the standard cleavage of the hydrazino peptide bond of 1.1.1. The amino alcohol fragment 1.1.3 was envisaged from 1.14.1, which would be the conjugate addition product of the key intermediate tert-ethyl- $\alpha, \beta$ unsaturated ester 1.6.2. The ester 1.6.2 would be synthesized from the achiral ethyl-4chloroacetoacetate 1.14.2 in 8 synthetic steps that are almost identical to the synthesis carried out by Hayashi et al. in 2010 .
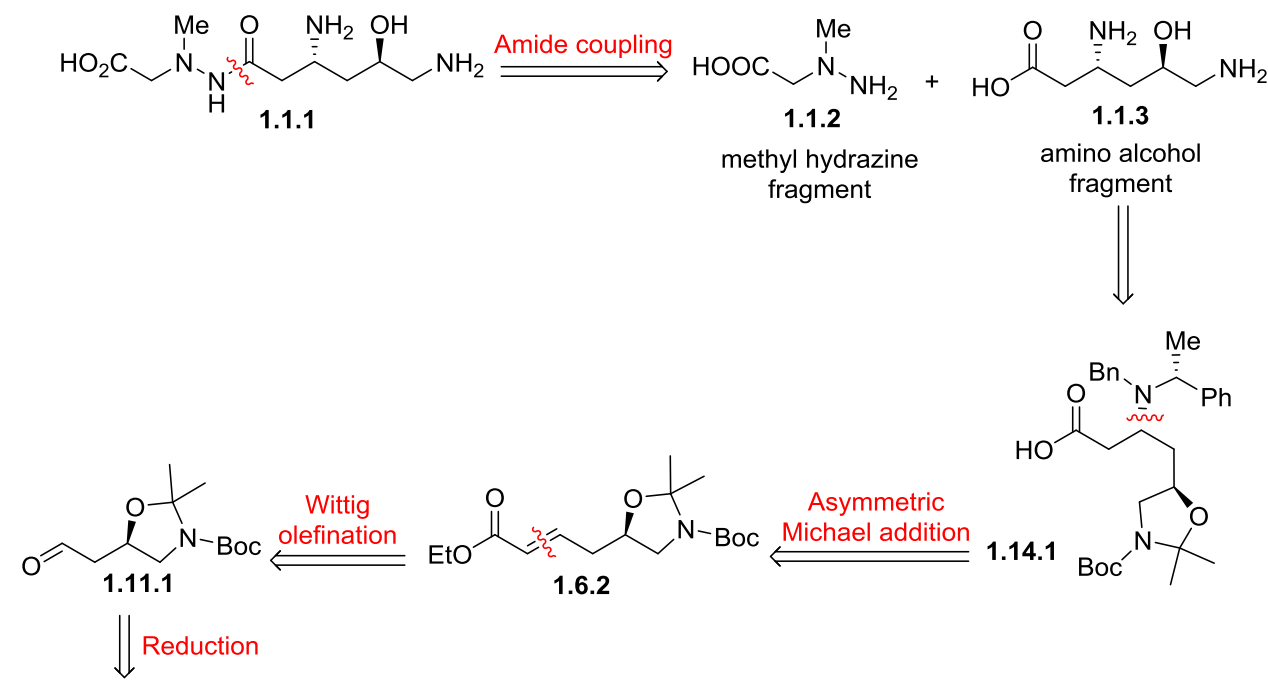<smiles>CCOC(=O)CC(=O)CCl</smiles>

Scheme 1.14. Retrosynthetic analysis of (+)-negamycin by Davies et al.

The first stereogenic center was incorporated via an asymmetric hydrogenation using the reliable and highly efficient reduction of $\mathbf{1 . 1 4 . 2}$ with $(S)$-BINAP-Ru(II) complex,${ }^{24}$ giving a $96 \%$ yield with "excellent enantioselectivity" to give $\gamma$-chloro- $\beta$-hydroxy ester, which was followed by a series of straightforward reactions to give ester 1.15.1 (Scheme 1.15). 
The partial reduction of ester 1.15.1 to aldehyde 1.11.1 prior to a Wittig reaction was not achieved through a single step with diisobutylaluminiumhydride (DIBAL-H). The reduction was reported as unsuccessful without further elucidation. It is thus unknown whether there was a possible decomposition or unreacted starting material that were recoverable. The alternatives included a two step reduction/oxidation combination of 1.15.1 to give aldehyde 1.11.1. The subsequent Wittig reaction gave $E$-alkene 1.6.2 as the major product with high stereoselectivity $(E / Z=10: 1)$. The minor stereoisomer was easily separable. The synthetic route required 8 steps to arrive at the key intermediate, $\alpha, \beta$-unsaturated ester, 1.6.2. In comparison with Hayashi et al., strategies reported in 2008 and 2010, which comprises of 3 and 7 steps respectively to afford a similar $\alpha, \beta$-unsaturated ester intermediate for the same conjugate addition, the strategy has not reflected any added advantages.

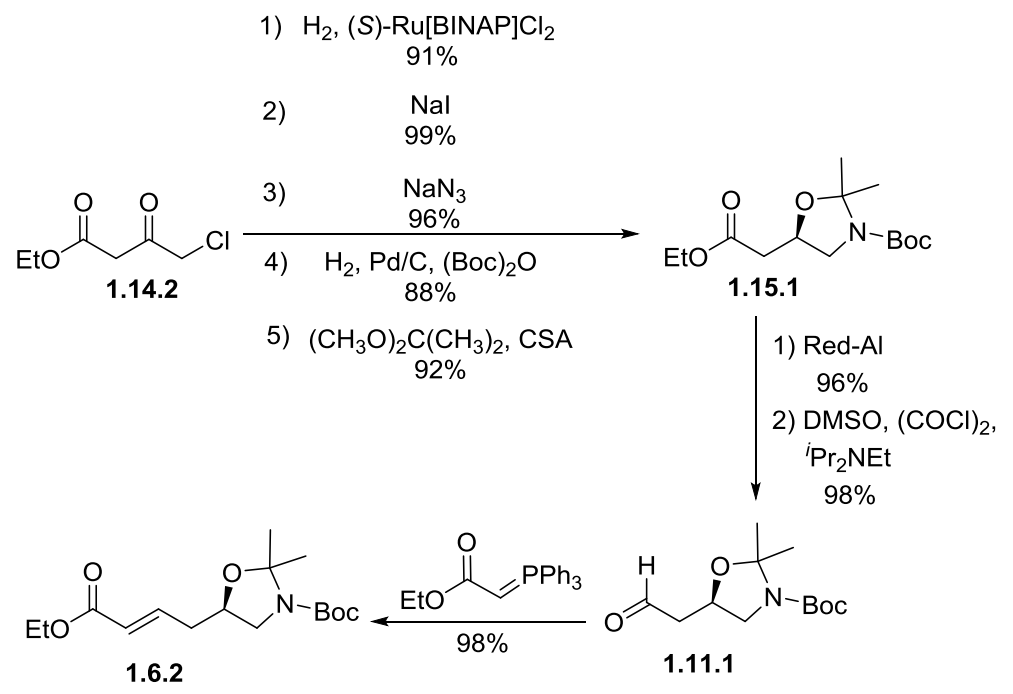

Scheme 1.15. Synthesis of key intermediate Michael acceptor 1.6.2.

The synthesis continued with the key conjugate addition of 1.6.2 with lithium amide to give a mixture of diastereomers in a ratio of $36: 1$ with $85 \%$ yield of isolated single diastereomer (> 99:1 dr) upon purification (Scheme 1.16). The absolute stereochemistry of C3, anti to the absolute configuration at C5, was determined upon the successful total synthesis of the natural product from 1.16.2 (Scheme 1.16). The hydrolysis of the ethyl ester 1.16.1 was then carried 
out to give the acid prior to the hydrazino peptide bond formation via coupling with the methyl hydrazine fragment 1.16.2 using DCC, $\mathrm{Et}_{3} \mathrm{~N}$ and additives $\mathrm{HOBt}$ in THF gave hydrazide 1.16.3 in a high $79 \%$ yield. Then, Boc-protecting groups and the oxazolidine were removed by TFA to release the amino alcohol in the anti-stereochemistry followed by a triple debenzylation to give (+)-negamycin.

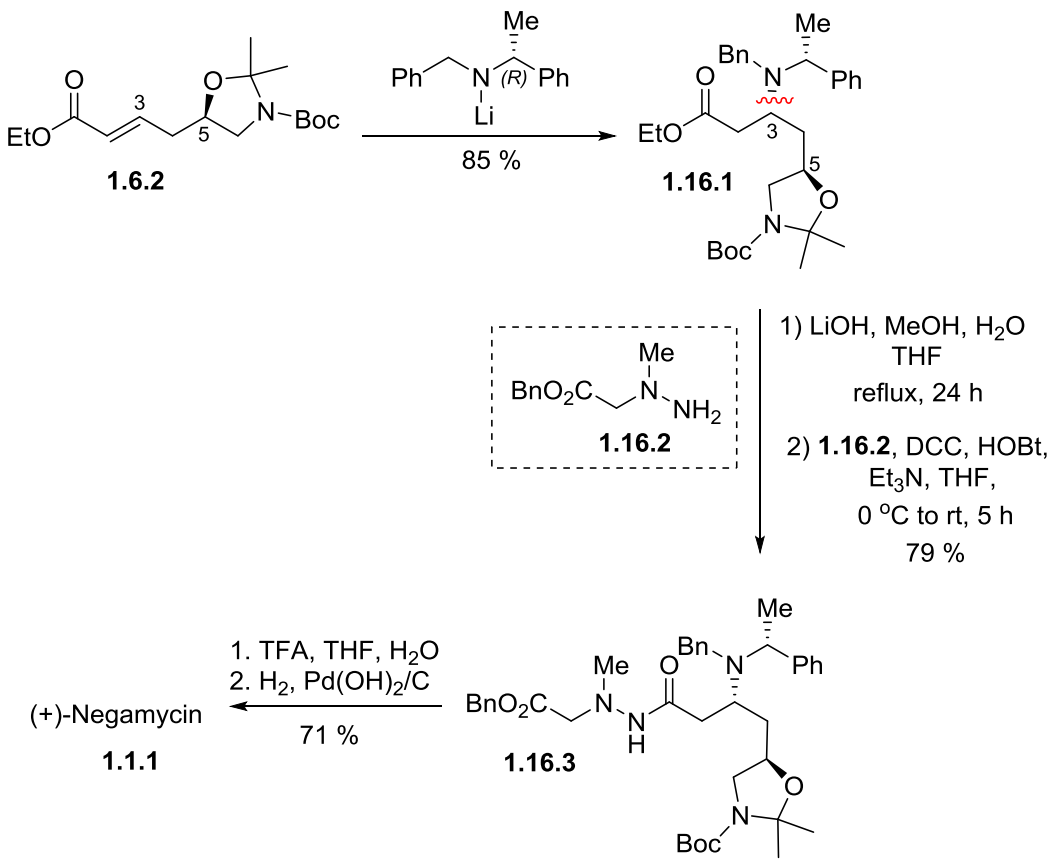

Scheme 1.16. Synthesis to access (+)-negamycin from 1.6.2.

The order of auxiliary removal and peptide coupling differs from the strategy of Hayashi et al. where, in the latter, the hydrolysis of the more robust bulky tert-butyl ester group required a strong acid such as $4 \mathrm{~N} \mathrm{HCl}$ in dioxane, which caused removal of both the oxazolidine and Boc-protecting groups (that would then needed a reintroduction) to give 1.17.1, resulting in 6exo-trig cyclization to furnish lactone 1.17.2 that could not be converted into the desired product (Scheme 1.17). Since Hayashi et al. did not attempt hydrolysis under basic conditions $\left(\mathrm{KOH}, \mathrm{MeOH}, \mathrm{H}_{2} \mathrm{O}\right)$ as did Davies' group, they resorted to auxiliary removal before the hydrolysis/peptide coupling sequence. 


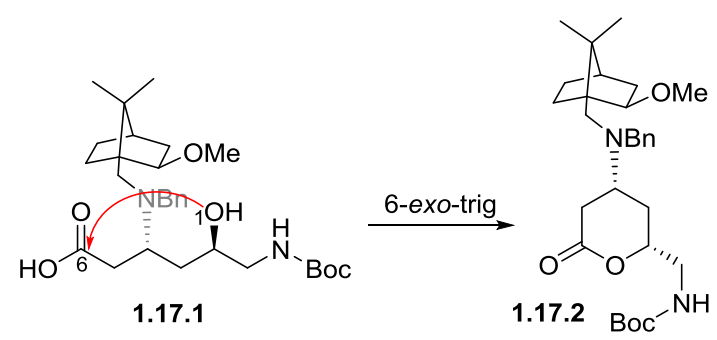

Scheme 1.17. Spontaneous lactonization of 1.17.1.

Since it is desirable for multiple chemical transformations to occur in a single step, the use of enantiopure lithium amide as Michael donor is a better option than chiral lithium amide auxiliary used by Hayashi et al. This is as the additional deprotection steps can be removed by carrying out the reduction of $N$-benzyl and $\alpha$-methylbenzyl groups in the final step of the synthesis of (+)-negamycin (global hydrogenation). In comparison, the chiral amide auxiliary utilized by Hayashi et al. requires oxidation with NIS, which does not overlap with any synthetic steps, resulting in the need for a further removal step thus reduces efficiency.

\subsubsection{Synthesis by Williams et al. ${ }^{20 \mathrm{e}}$}

In 2002, Williams et al. reported the use of $\beta$-alkoxy imine stereoselective allylation of 1.18.3 to generate the stereogenic center at $\mathrm{C} 3$ and stereoselective hydrogenation of dihydrooxazine derivative $\mathbf{1 . 1 8 . 5}$ for the introduction of chirality at $\mathrm{C} 5$ of the amino alcohol fragment 1.1.3 (Scheme 1.18). The peptide bond would be formed prior to the global hydrogenation step, which would remove protecting groups to furnish the natural product. 

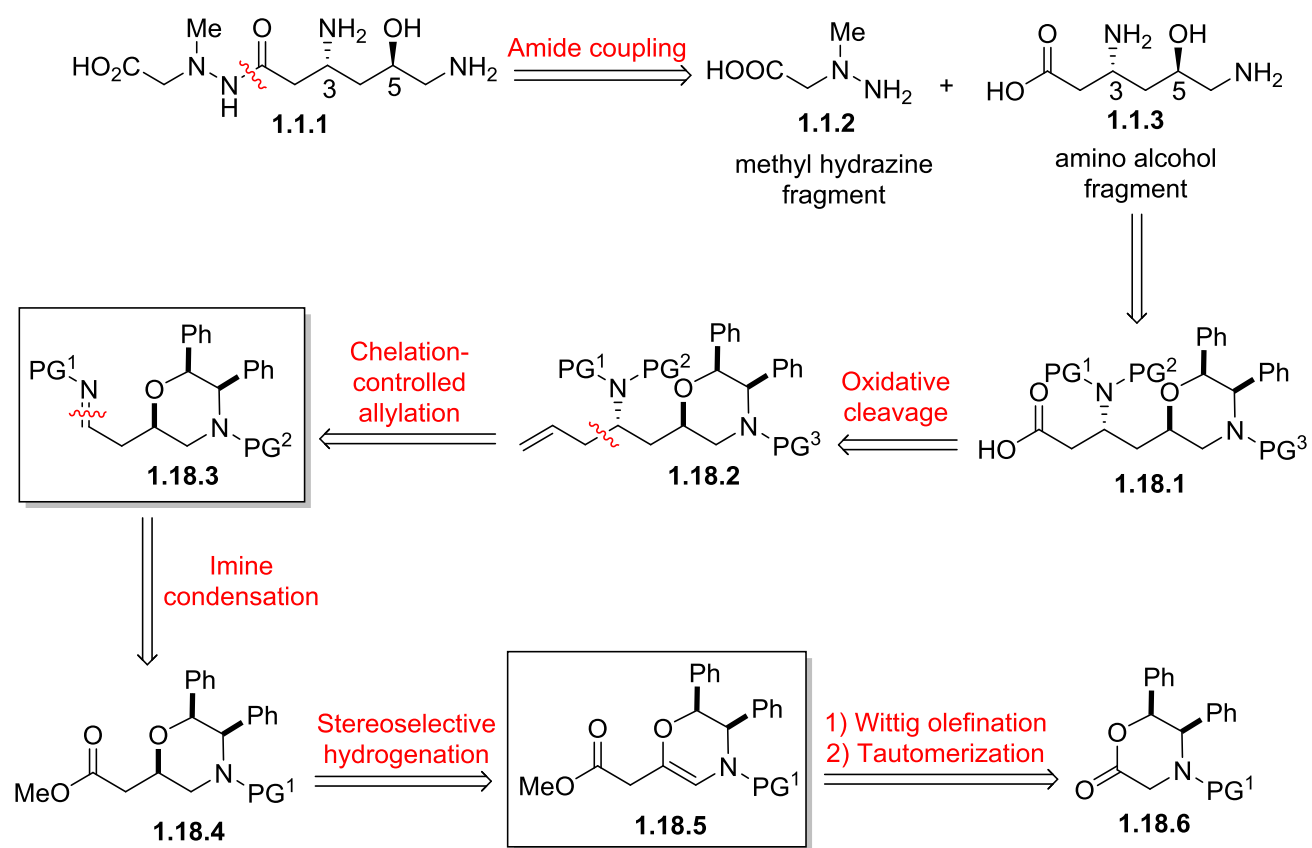

Scheme 1.18. Retrosynthetic analysis of (+)-negamycin by Williams et al.

The key intermediate 1.18.3 was prepared from $(5 R, 6 S)$-4-(benzyloxycarbonyl)-5,6diphenyl-2,3,4,6-tetrahydro-4H-1,4-oxazin-2-one $\mathbf{1 . 1 8 . 6}$ that, although is commercially available, was a complex starting material compared to starting materials from strategies reported by Davies et al., and others (Scheme 1.19).

The synthesis began with a very forcing Wittig condition to give a tri-substituted olefin, which underwent tautomerization to the thermodynamically stable tri-substituted olefin $\mathbf{1 . 1 8 . 5}$ with unsaturation within the oxazine ring (Scheme 1.19). The key stereoselective hydrogenation of 1.18.5 proceeded with a high degree of asymmetric induction to give 1.19.1 $(\mathrm{dr}=94: 6)$. The incoming pair of hydrogens were added across the double bond in a syn manner from the less hindered side, trans to the phenyl groups. The relative stereochemistry was determined by NOE measurements. This method is unlike methods such as Wang's, Santo's, Weigele's et al., where one of the two chiral centres of (+)-negamycin was introduced from chiral starting material. It is also different from Davies' and Hayashi's et al. who, in spite of exploiting achiral starting 
materials, used well established hence reliable procedures such as Brown allylation and Noyori reduction.

A disadvantage from the key stereoselective hydrogenation step was the loss of the nitrogen protecting group, 1.19.1, thus, the nitrogen of the oxazine ring needed to be re-protected to give 1.18.4 (Scheme 1.19). The ester 1.18.4 was reduced by DIBAL-H to an aldehyde that was converted to $\beta$-alkoxy imine, 1.18.3, which was the precursor to the second key stereoselective allylation step. The next stereogenic center was incorporated through a chelation-controlled allylation of $\beta$-alkoxy imine, 1.18.3 in the presence of various Lewis acids such as cerium trichloride to give homoallylamine 1.18.2 as a non-separable mixture of diastereomers. The diastereoselectivity derived from this chelation-controlled allylation to generate the second stereogenic center of $(+)$-negamycin was lower $\left(\mathrm{dr}=4.4: 1\right.$ by ${ }^{1} \mathrm{H}$ NMR) than other strategies such as diastereoselective conjugate addition with chiral amines. The origin of stereocontrol was from a combination of steric and stereoelectronic factors (Scheme 1.19). It was postulated that chelation with cerium trichloride resulted in a six-membered ring transition state with axial approach of the allylzinc reagent. Completion of the natural product validated the outcome but not the theory. 

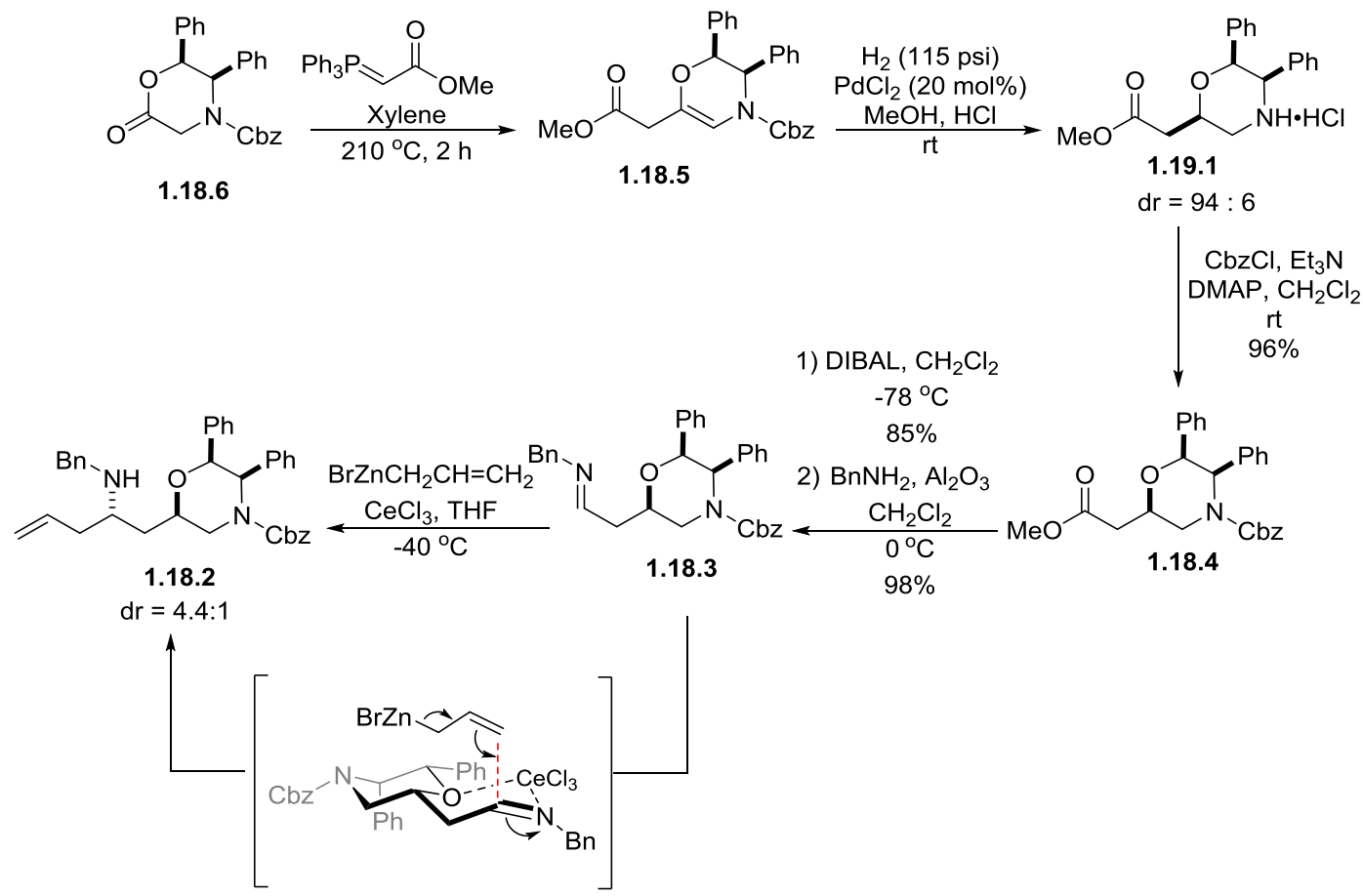

Scheme 1.19. Synthesis of 1.18.2 through chelation controlled allylation of 1.18.3.

The synthesis continued with another nitrogen protection of the homoallylamine 1.18.2 with benzylchloroformate to give 1.20.1. This was to prevent a potential attack of the nitrogen on the newly formed aldehyde from the proceeding ozonolysis step (Scheme 1.20). A further oxidation was carried out using the less aggressive pyridinium chlorochromate (PCC) to give the carboxylic acid prior to the coupling with the hydrazine moiety. This was followed by the final hydrogenation step to afford (+)-negamycin in an overall yield on $27 \%$ with a total of 8 steps. 

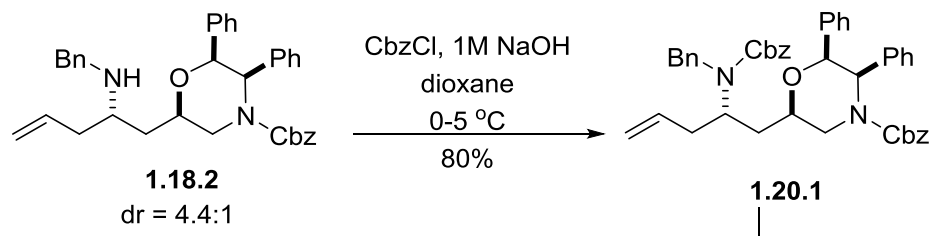

1) $\mathrm{O}_{3}, \mathrm{CH}_{2} \mathrm{Cl}_{2}, \mathrm{MeOH}$,

$-78{ }^{\circ} \mathrm{C}$

$73 \%$

2) PDC, DMF

3) 3-(3-dimethylaminopropyl)carbodiimide hydrochloride $\mathrm{Et}_{3} \mathrm{~N}, \mathrm{HOBt}, \mathrm{CH}_{2} \mathrm{Cl}_{2}$, $80 \%$

$\mathrm{H}_{2}(40 \mathrm{psi})$ $10 \% \mathrm{Pd} / \mathrm{C}(25 \mathrm{~mol} \%)$, $\mathrm{MeOH}, \mathrm{H}_{2} \mathrm{O}, \mathrm{AcOH}$, $75^{\circ} \mathrm{C}$

r $75 \%$

$(+)$-Negamycin

1.1.1

Scheme 1.20. Completion of (+)-negamycin synthesis from homoallylamine 1.18.2.

\subsubsection{Synthesis by Lieberknecht et al. ${ }^{20 \mathrm{~d}}$}

All the syntheses of (+)-negamycin have thus far consisted of key stereoselective steps to introduce the stereogenic centers at $\mathrm{C} 3$ and $\mathrm{C} 5$ of the natural product. However, the following synthetic strategy by Lieberknecht $e t$ al. in 1991 reported a Wolff rearrangement of $\alpha$ - into $\beta$ amino acids which would construct the amino alcohol fragment 1.1.3 from 1.21.1 (Scheme 1.21). This, hence, enabled them to apply well established $\alpha$-amino acid methods. The stereochemistry at $\mathrm{C} 3$, nonetheless, would be introduced using a common approach such as diastereoselective hydrogenation of enamine 1.21.2 to give 1.21.1. 


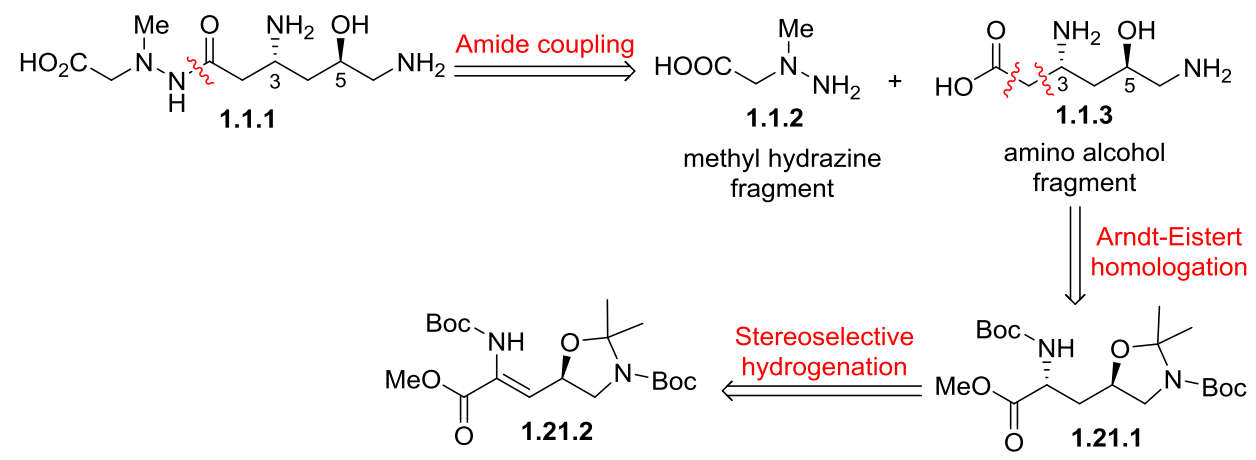

Scheme 1.21. Retrosynthetic analysis of (+)-negamycin by Lieberknecht et al.

The synthesis began with a chiral starting material 1.21.2, which accounted for the stereogenic center at C5 of (+)-negamycin (Scheme 1.22). The chirality at C3 was introduced via a catalyst-controlled diastereoselective hydrogenation using Knowles' ligand, ${ }^{25}$ in contrast to Williams et al., substrate-controlled stereoselective hydrogenation strategy, to give diastereomer 1.21.1. However, the reported diastereoselectivity was low $(\mathrm{dr}=4: 1)$ and it could be attributed to a mismatched case. The synthesis continued with a saponification of the methyl ester 1.21.1 to give the carboxylic acid, 1.22.1, which was converted to the mixed anhydride and treated with diazomethane at $0{ }^{\circ} \mathrm{C}$ to yield the diazoketone. The diazoketone then underwent proton abstraction to give the precursor to Wolff rearrangement that is the $\alpha$-diazo-methyl ketone enolate. In the presence of $\mathrm{Ag}^{+}$, the Wolff rearrangement proceeded with complete retention of the stereochemistry to give the ketene, which was susceptible to a nucleophilic attack by the methoxide to afford the homologated product $\mathbf{1 . 2 2 . 2}$ in a high $75 \%$ yield. Overall, it is an Arndt-Eistert reaction where the chirality at $\mathrm{C} 2$ of $\mathbf{1 . 2 2 . 1}$ was retained at $\mathrm{C} 3$ of $\mathbf{1 . 2 2 . 2}$ (Scheme 1.22). 

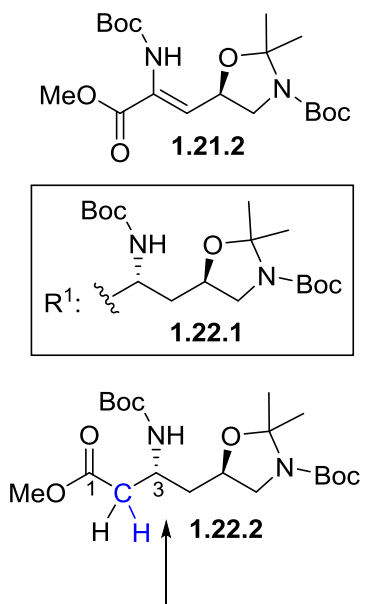

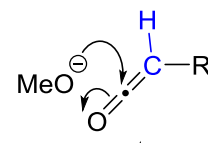
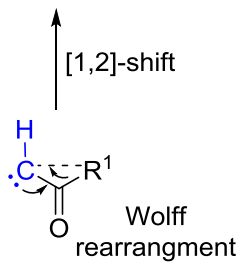

$\mathrm{N}_{2} \uparrow \mathrm{Ag}^{+}$

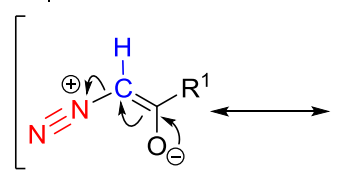

$s-(Z)$-conformation

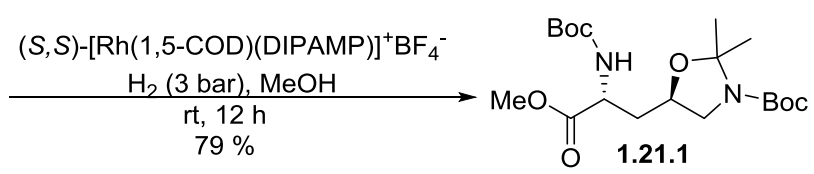

1) $\mathrm{CIC}(\mathrm{O}) \mathrm{O}\left({ }^{i} \mathrm{Bu}\right), \mathrm{Et}_{3} \mathrm{~N}, \mathrm{THF}$,

$-10{ }^{\circ} \mathrm{C}, 10 \mathrm{~min}$

2) $\mathrm{CH}_{2} \mathrm{~N}_{2}, \mathrm{Et}_{2} \mathrm{O}$

$0{ }^{\circ} \mathrm{C}, 14 \mathrm{~h}$

3) $\mathrm{PhCO}_{2} \mathrm{Ag}, \mathrm{Et}_{3} \mathrm{~N}, \mathrm{MeOH}$,

$60{ }^{\circ} \mathrm{C}, 10 \mathrm{~min}$

$75 \%$

$\mathrm{LiOH}, \mathrm{H}_{2} \mathrm{O}$
dioxane

rt, $1 \mathrm{~h}$

$100 \%$

\section{Arndt-Eistert homologation}

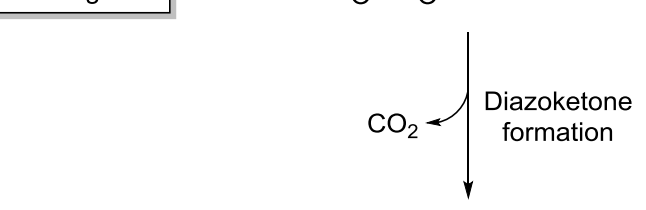

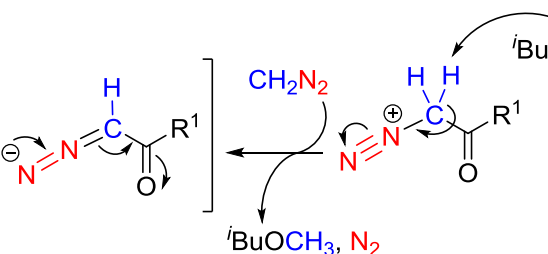

Anhydride

formation<smiles>[Z20]C[C@H](C[C@@H](NC(=O)O)C(=O)O)CN1C[C@H](C(=O)O)OC1(C)C</smiles><smiles>CC(C)C1CCCC1</smiles>

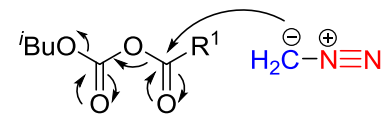

$\mathrm{BuO}^{\ominus}$

Scheme 1.22. Synthesis of methyl ester 1.22.2 via Arndt-Eistert homologation synthesis from acid 1.22.1.

The synthesis to access (+)-negamycin continued with a repeat of the methyl ester saponification of 1.22.2 to give the carboxylic acid, 1.23.1, for the coupling with hydrazine moiety (Scheme 1.23). A further 5 steps were carried out to afford (+)-negamycin in a total of 9 synthetic steps starting from 1.21.2 with an overall yield of $23 \%$. This excludes the number of steps required for the synthesis of the starting material 1.21.2.

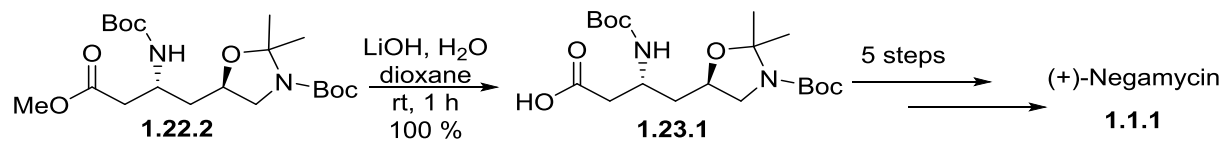

Scheme 1.23. Completion of (+)-negamycin synthesis from methyl ester 1.22.2. 


\subsubsection{Synthesis by Kibayashi et al. ${ }^{20 c, 201}$}

In 1988 and 1986, Kibayashi et al. reported the synthesis of (+)-negamycin through the use of a stereoselective 1,3-dipolar cycloaddition, which employed a nitrone modified with a variety of carbohydrate auxiliaries (Scheme 1.24). This reflects a more efficient synthetic strategy of the use of D-glucose than the strategy reported by Weigele et al. in the same year, $1988 .{ }^{26}$

The retrosynthetic analysis began with the standard disconnection at the hydrazino peptide bond. The stereochemistry at C3 and C5 carbons of (+)-negamycin were derived from the stereoselective formation of the isoxazolidine ring 1.24.1. Therefore, the key synthetic step was an enantioselective 1,3-dipolar cycloaddition between nitrones 1.24.4 and allylamine 1.24.3 (Scheme 1.24).

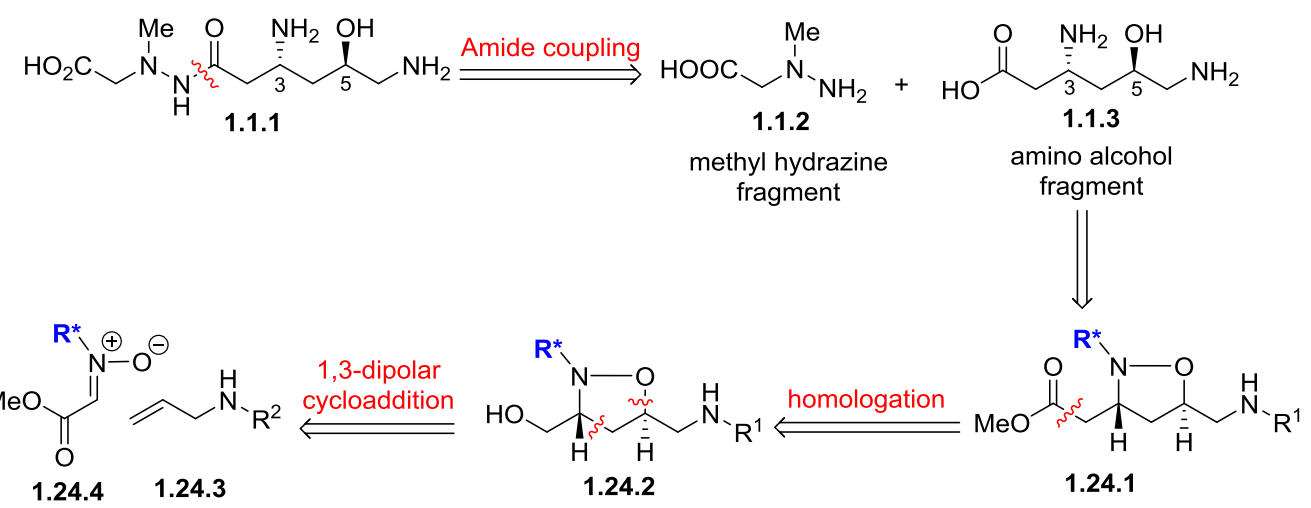

Scheme 1.24. Retrosynthesis of (+)-negamycin through 1,3-dipolar cycloaddition by Kibayashi et al.

D-Gulonic acid- $\gamma$-lactone 1.25.1 was selected as the chiral auxiliary in the synthesis of chiral nitrone 1.25.4 (Scheme 1.25). The lactone 1.25.1 underwent diol protection and partial lactone reduction to give acetal $\mathbf{1 . 2 5 . 2}$, which was treated with hydroxylamine and pyridine to afford 1.25.3. The chiral hydroxylamine 1.25 .3 generated $N$-glycosyl nitrone 1.25 .4 in situ from the treatment with methyl glyoxylate under reflux in toluene. The chiral nitrone 1.25.4 underwent a 1,3-dipolar cycloaddition with benzyloxy allylamine to give an inseparable mixture 
of isoxazolidines trans-1.25.5 and cis-1.25.6 diastereomers with a low diastereoselectivity ratio of 2:3 with the undesired cis-1.25.6 as the major diastereomer (Scheme 1.25).

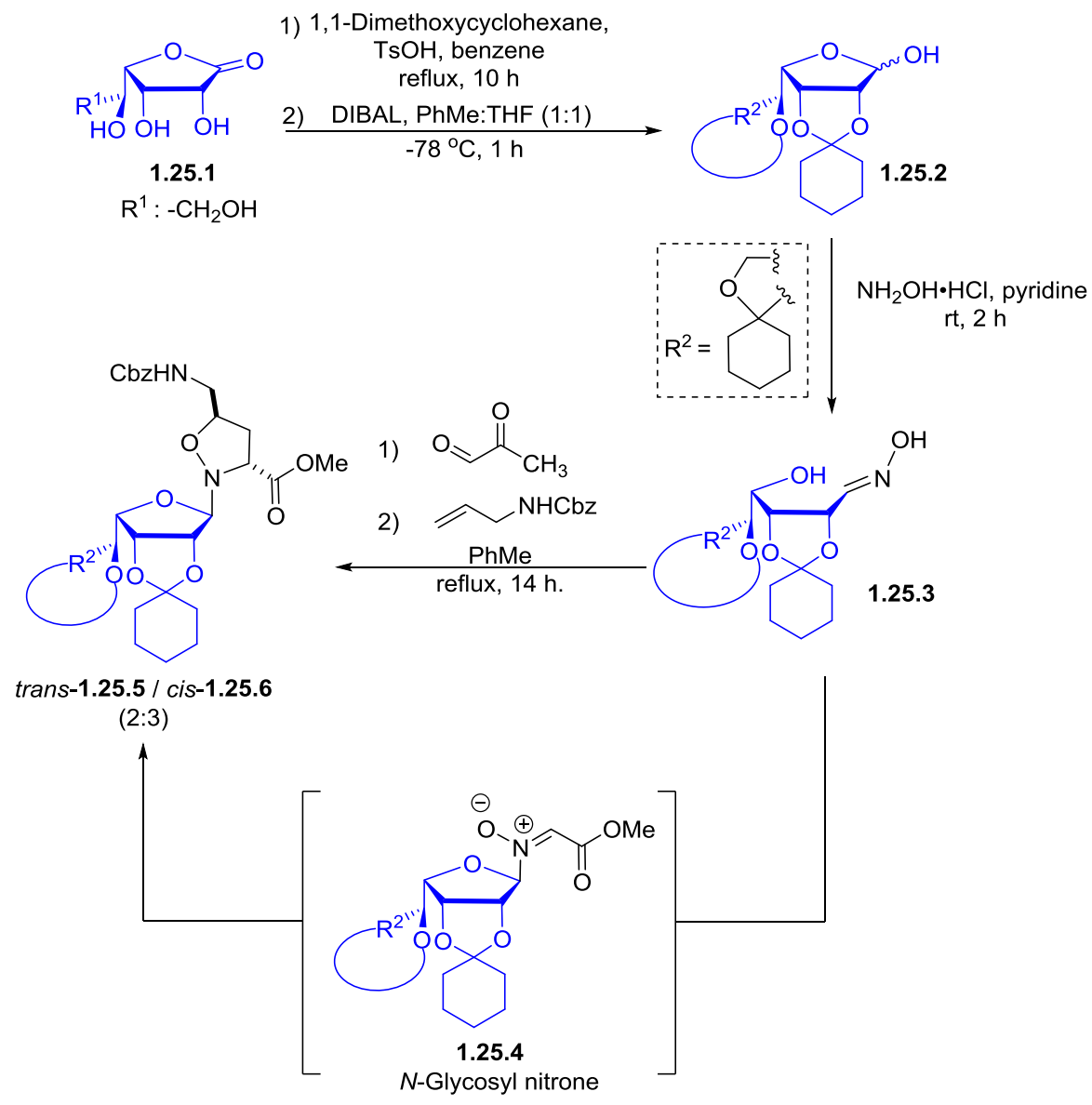

Scheme 1.25. Synthesis of isoxazolidines 1.25 .5 and 1.25 .6 .

In 1,3-dipolar cycloadditions, the conformations of both dipolarophile (s-cis or s-trans) and nitrone ( $E$ or $Z$ isomer), the approach of the nitrone towards the two faces of the dipolarophile such as exo- or endo-approach and $s i$ - or $r e$-face with respect to the dipolarophile are significant for the diastereoselectivity control. In this case, the dipolarophile is benzyloxy allyamine and thus s-cis and s-trans concept do not apply.

The first stereoselectivity issue is associated with the cis/trans isomers of isoxazolidines 1.25.5/1.25.6, which is the result of uncontrolled nitrone conformation (Figure 1.4). This, thus, enables the chiral nitrones to exist in an $E / Z$ equilibrium, giving a mixture of cis/trans 
isoxazolidines in each event of a 1,3-dipolar cycloaddition (endo- and exo-approach or re-and $s i$-face attack) leading to 4 possible stereoisomers (Figure 1.4).
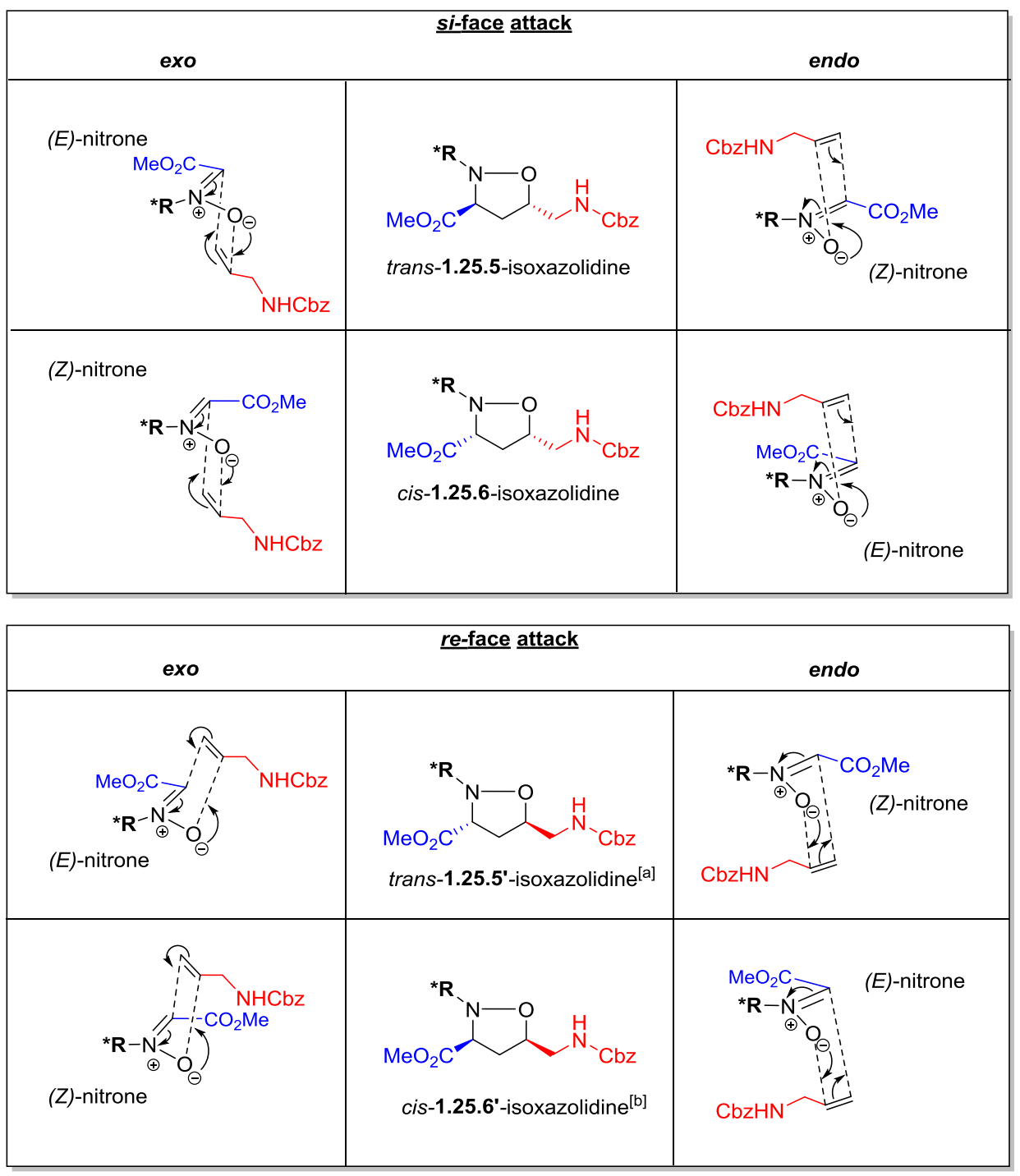

[a] 1.25.5' is the enantiomer of 1.25 .5

[b] 1.25.6' is the enantiomer of 1.25.6.

Figure 1.4. Diastereoselectivity in 1,3-dipolar cycloaddition.

The second stereoselectivity issue is with respect to the chiral auxiliary attached to the nitrone that controls both the approach and $\pi$-facial selectivity by steric and stereoelectronic factors respectively, giving rise to a highly enantioselective 1,3-dipolar cycloaddition (Figures 1.5 and 1.6). It also influences the geometry of the nitrones with respect to the furan ring, which contributes to the stereoselectivity. The use of a D-gulosyl chiral template resulted 
in a preference for the exo-approach of the nitrone towards dipolarophile. This is as steric hindrance, between the benzyl carbamate group of the dipolarophile and the furan oxygen atom of the carbohydrate moiety, could be avoided as illustrated in Figure 1.5 with Z-nitrone as an example. Similarly, steric hindrance also influences the two possible orientations of the nitrone with respect to the furan ring by favouring the endo-O-conformation of the chiral nitrone (Figure 1.6). ${ }^{27}$

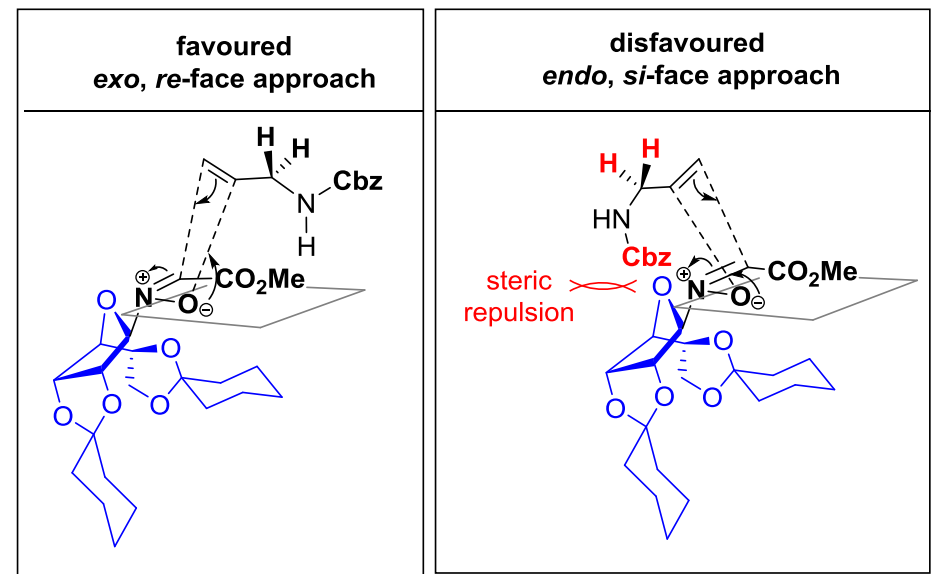

Figure 1.5. Chiral auxiliary induces exo-selective 1,3-dipolar cycloaddition.

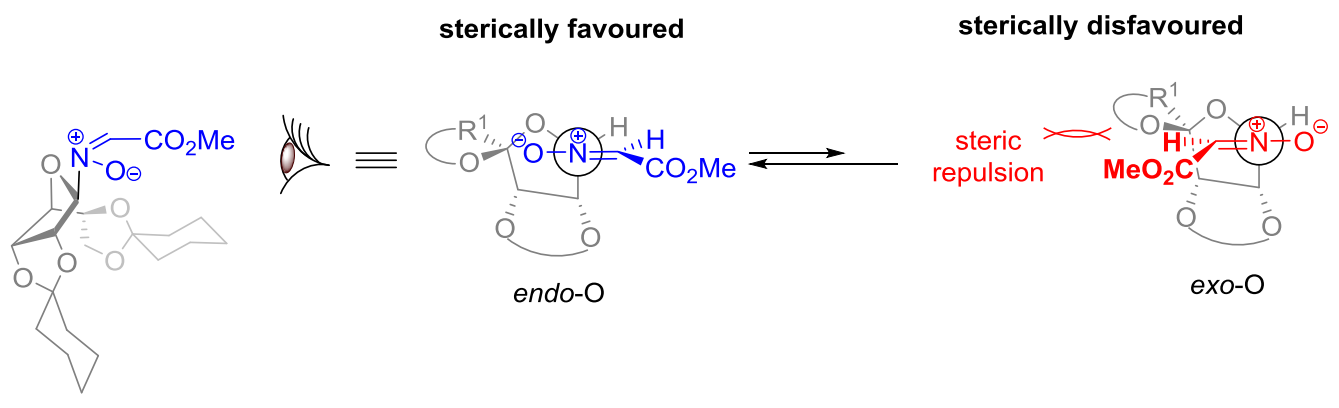

Figure 1.6. Conformation of nitrone with respect to the oxygen atom of the furan ring.

Apart from that, a highly enantioselective 1,3-dipolar cycloaddition would also require a high facial stereoinduction during the attack on dipolarophile (Figure 1.7). Since the dipolarophile is susceptible to a $r e$ - or $s i$-face attack by the chiral nitrone, with each attack giving rise to the respective transition states endo-TS-A or endo-TS-B (Figure 1.7), an introduction of a selective stabilization of one of two possible transition states would thereby enable $\pi$-facial 
selectivity of the attack on dipolarophile. This was achieved by exploiting the stereoelectronic interaction of the carbohydrate auxiliary and the cyclo-adducts. The furan oxygen atom plays a central role as the $\sigma^{*}$-orbital of the furanose $\mathrm{C}$-O bond interacts with the non-bonding lone pair of electrons on the isoxazolidine nitrogen atom. The nitrogen lone pair of electrons are in the newly formed $s p^{3}$ hybridized orbital from $s p^{2}$ orbital during the 1,3-dipolar cycloaddition. Both orbitals are anti-periplanar. ${ }^{28}$ This anomeric effect stabilizes the endo-O-TS-A, lowering its activation energy and thereby proceeds favourably over the endo-O-TS-B. ${ }^{28}$ Therefore, a $r e$ face attack of the dipolarophile is favoured by the chiral nitrone in its endo-O-conformation and in exo-approach, giving a highly enantioselective 1,3-dipolar cycloaddition (Figure 1.7).

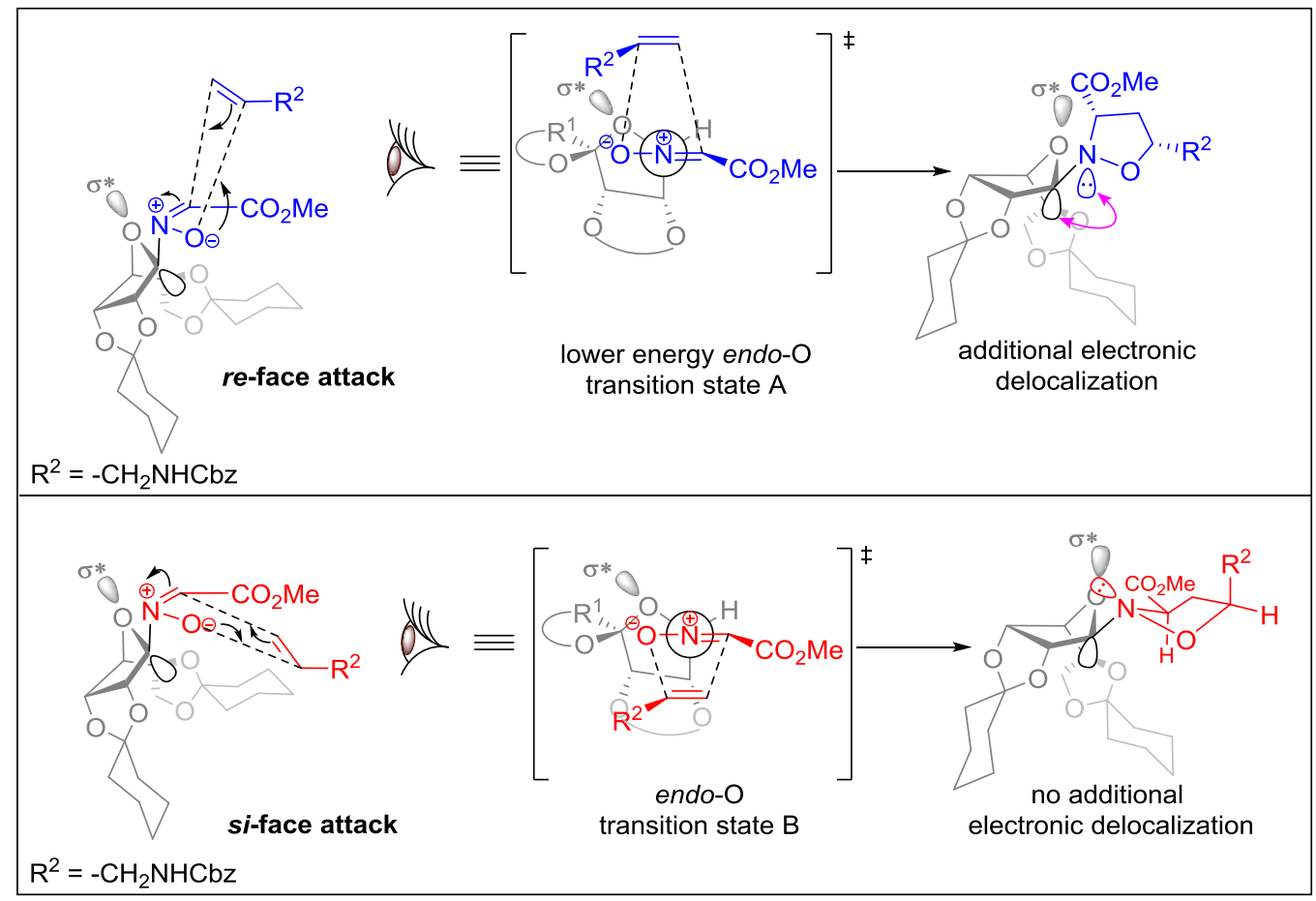

Figure 1.7. Stereoelectronic stabilization of endo-O-TS-A and endo-O-TS-B from $r e$ - and $s i$-face attack respectively.

The chiral auxiliaries were removed from the isoxazolidine diasteromers 1 .25.5/1.25.6 under acidic hydrolysis $(10 \% \mathrm{HCl} / \mathrm{MeOH})$ followed by $N$-benzylation (Scheme 1.26$)$. The protected diastereomers were reduced by $\mathrm{LiAlH}_{4}$ to give separable diastereomers 1.26.1/1.26.2. 
Their enantiomeric purity was determined by Mosher's acid analysis. the 1,3-dipolar cycloaddition gave a good enantioselectivity of isoxazolidines $1.25 .5 / 1.25 .5 '$ and 1 .25.6/1.25.6' $(>90 \%$ ee). The synthesis of (+)-negamycin was completed via six straightforward steps from isoxazolidine trans-1.26.1, which included peptide bond formation upon oxidation of the primary alcohol followed by the final global hydrogenation step (Scheme 1.26).

Overall, a highly enantioselective synthesis of (+)-negamycin was achieved by Kibayashi et al., but $60 \%$ of the generated 1,3-dipolar cycloaddition product was the incorrect cis-diastereomer, which was subsequently converted to (-)-3-epi-negamycin. This reflects the need to address the challenge of E/Z-stereocontrol of the nitrone geometry. In addition, additional auxiliary removal and nitrogen protection steps further highlights the strategy's low efficiency after obtaining only $40 \%$ desired trans-diastereomer from its key synthetic step.

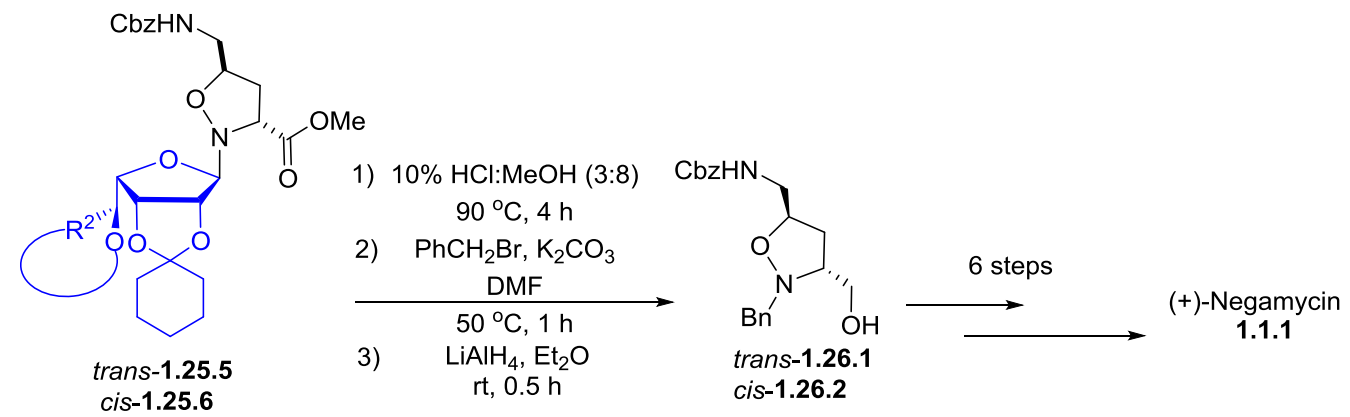

Scheme 1.26. Completion of (+)-negamycin synthesis from isoxazolidines 1.25.5 / 1.25.6.

\subsubsection{Synthesis by Weigele et al. ${ }^{26}$}

Similarly, in 1988, Weigele et al. reported the total synthesis of (+)-negamycin by using a carbohydrate starting material (Scheme 1.27). However, the purpose of the carbohydrate sets the two syntheses apart (Weigele's et al. and Kibayashi's et al.), as the D-glucose employed by Weigele et al. functioned as the skeletal structure of (+)-negamycin instead of a chiral auxiliary. Retrosynthetic analysis began with the standard disconnection at the hydrazino peptide bond followed by functional group conversion involving only the amine groups of the amino alcohol 
fragment 1.1.3 to give 1.27.1, which would be accessible from diol 1.27.2. The starting material 1,2-O-isopropylidene-D-glucose is commercially available (Scheme 1.27).

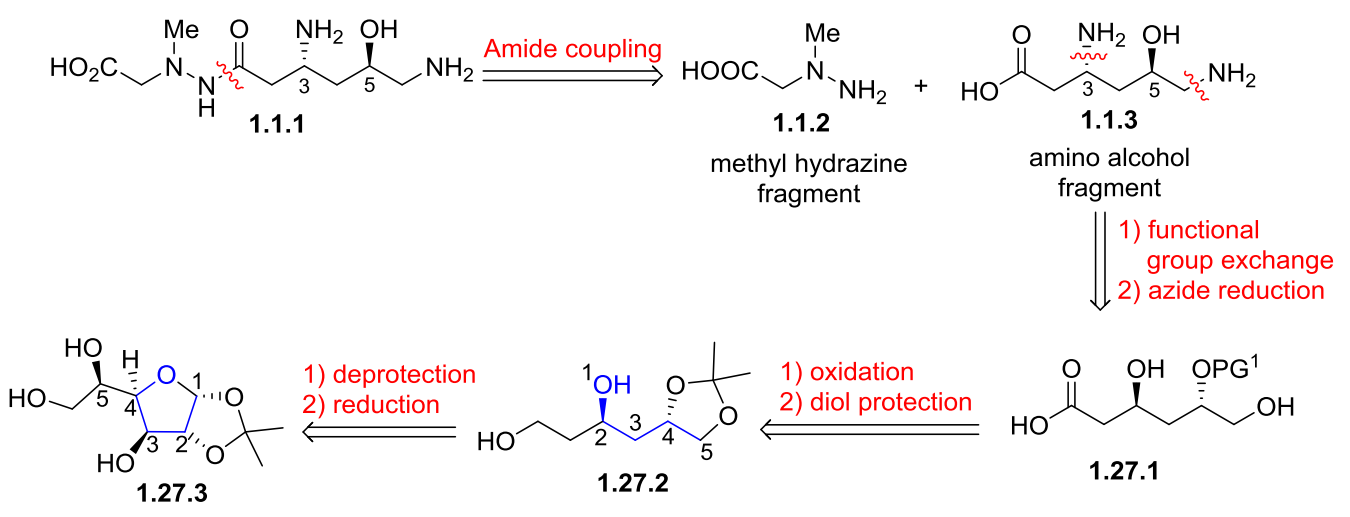

Scheme 1.27. Retrosynthetic analysis of (+)-negamycin by Weigele et al.

The synthesis began with 1,2-O-isopropylidene-D-glucose 1.27.3 (Scheme 1.28). A cyclic thiocarbonate was formed between $\mathrm{O} 5$ and $\mathrm{O} 6$, together with thiocarbamation at $\mathrm{O} 3$ of 1.28.1. The reduction of $\mathbf{1 . 2 8 . 1}$ with tributyltin hydride gave a mixture of products $1.28 .2-$ 1.28.5. Although the side products 1.28.3 - 1.28.5 were separable to give the desired compound 1.28.2 in $41 \%$ yield, the poor yield at an early point of the synthesis is considered economically unattractive. The proceeding oxidation of primary alcohol 1.28.2 to the carboxylic acid 1.28.6 was followed by peptide coupling with hydrazine via a mixed anhydride method to give 1.28.7 with unreported yield. Consequently, the efficacy of the coupling step is not known. A series of straightforward steps were carried out such as isopropylidene removal under acidic conditions from 1.28.7 and selective alcohol protection, which afforded the desired compound in a poor $50 \%$ yield as a mixture of products that were separable by column chromatography on silica gel. The diol 1.28.8 was obtained by reduction, which was carried out to release the hemiacetal function and to ultimately place the azide group by using a Mitsunobu transformation of diol 1.28.8. The natural product was obtained upon the final hydrogenation step of $\mathbf{1 . 2 8 . 9}$ that simultaneously deprotected the silyl, benzyl groups, and reduced the diazide to give the free diamine. 


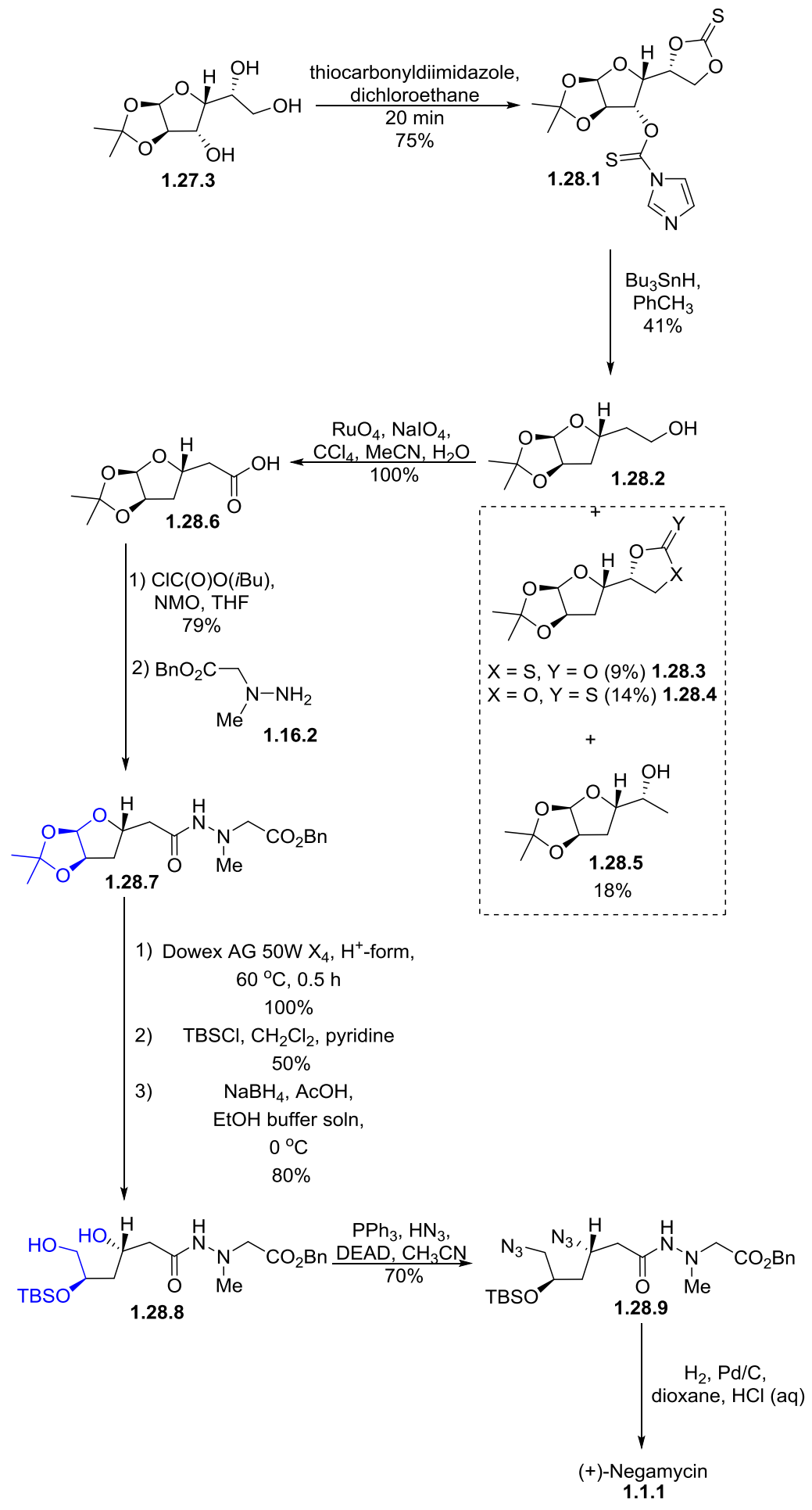

Scheme 1.28. Synthesis of (+)-negamycin from D-glucose derivative 1.27.3.

Overall, the first 8 steps of the synthesis gave a 7\% yield, which indicates low efficiency in the synthetic steps. Two of the total 9 steps gave half or less product yield together with 
mixtures of unwanted by-products due to the lack of selectivity. In addition, the precious stereogenic centers in D-glucose $(\mathrm{C} 1, \mathrm{C} 2$ and $\mathrm{C} 5$ of 1.27.3) were destroyed in a stepwise manner, failing to showcase the benefit of using a highly chiral compound as starting material. This also illustrates the common problem that carbohydrates often have too much functionality. In conclusion, this strategy proves inferior to the diastereoselective methods applied to achiral substrates.

\subsubsection{Synthesis by Maycock et al.}

Four years later, 1992, Maycock et al. reported the total synthesis of (+)-negamycin through the use of quinic acid to highlight its high versatility as starting material for the synthesis of linear chiral natural products (Scheme 1.29). ${ }^{29}$ However the synthesis relied on a lengthy route comprising of 24 steps with an overall yield of approximately $9 \%$, thereby making it unappealing. Upon disconnection at the peptide bond of (+)-negamycin, the amino alcohol fragment could be obtained from the oxidation of primary alcohol 1.29.1. The replacement of hydroxyl groups at $\mathrm{C} 4$ and $\mathrm{C} 1$ of compound 1.29.2 would lead to primary alcohol 1.29.1. The polyhydroxyl substrate 1.29.2 would be accessed by the quinic acid derivative 1.29.3 in accordance with the procedures ${ }^{[a]}$ reported by Grewe and Nolte et al. (Scheme1.29). ${ }^{30}$
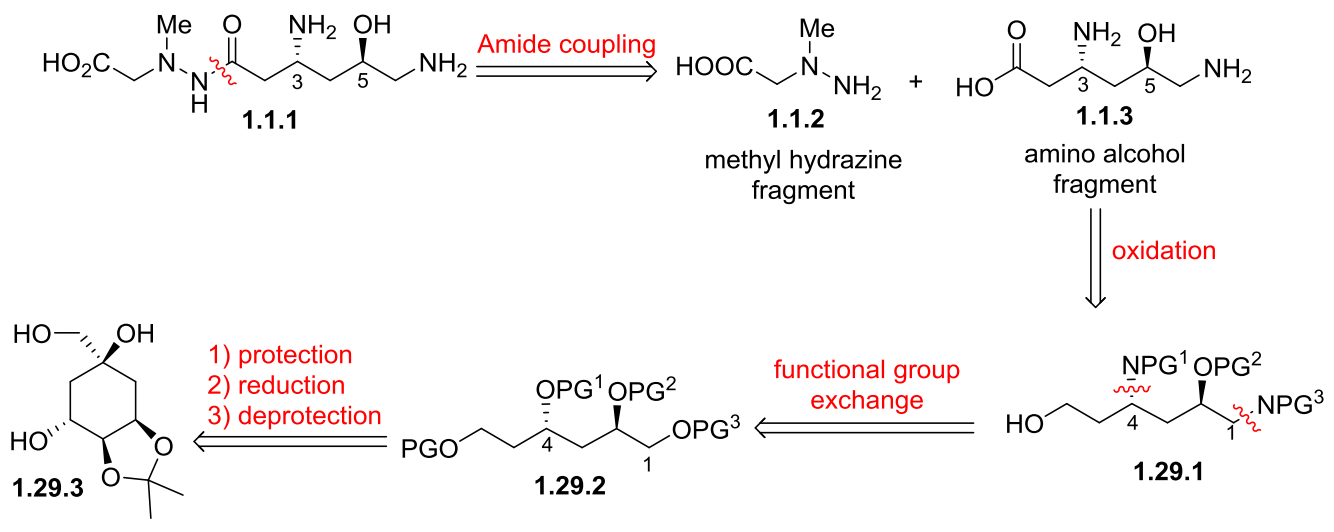

Scheme 1.29. Retrosynthetic analysis of (+)-negamycin by Maycoc et al. 
The synthesis is comprised of more than 24 steps including the additional derivation required from quinic acid 1.30.1 to the desired starting triol 1.29.3.

The triol 1.29.3, derived from quinic acid 1.30.1, was subjected to oxidation and alcohol protection to give ketone 1.30.2 in high overall yield (Scheme 1.30). The subsequent nonstereoselective reduction using sodium borohydride gave a mixture of epimers that were separable by column chromatography. The loss in product was minimized by submitting the undesired epimer to a Mitsunobu reaction to invert its stereochemistry and the resulting alcohol was benzoylated to give $\mathbf{1 . 3 0 . 3}$. The treatment of $\mathbf{1 . 3 0 . 3}$ with 1,2 -ethanedithiol and borontrifluoride diethyletherate removed the 2,2-dimethoxypropane protecting group and the resulting diol was cleaved using lead tetraacetate to give the aldehyde, which was subjected to reduction that afforded diol 1.30.4. At this stage, the two primary hydroxyl groups of $\mathbf{1 . 3 0 . 4}$ needed to be discriminated for selective protection. This was successfully done by effecting the preferred 1,4-benzoyl migration over the possible 1,5-migrations to give $\mathbf{1 . 3 0 . 5}$ upon treatment with diisopropylethylamine. The resulting diol $\mathbf{1 . 3 0 . 5}$ was then selectively protected to give 1.29.2, which was subjected to de-benzoylation followed by mesylate formation to allow for a smooth azide substitution to yield 1.30.6. The following series of 4 steps, which consisted of hydrogenation to give the free amines that underwent Boc-protection that was followed by the TBS-deprotection and oxidation of the resulting primary hydroxyl to the carboxylic acid 1.30.7, are a display of careful consideration in the choice of alcohol-protecting groups. The benefit ranging from selective azide substitution of 1.29.2 to carboxylic formation in 1.30.7 is a result of orthogonal protection strategies adopted by the group. They employed silyl, benzoyl and benzoyloxy methyl acetal protecting groups while being sensitive to the reaction conditions to avoid unwanted deprotection. For instance, the conditions for the hydrogenation of $\mathbf{1 . 3 0 . 6}$ was mild as it was carried out at $25{ }^{\circ} \mathrm{C}$, which was suitable for the reduction of azides without deprotecting the BOM group. The natural product (+)-negamycin was obtained from carboxylic acid 1.30.7 in 3 standard steps (Scheme 1.30). 

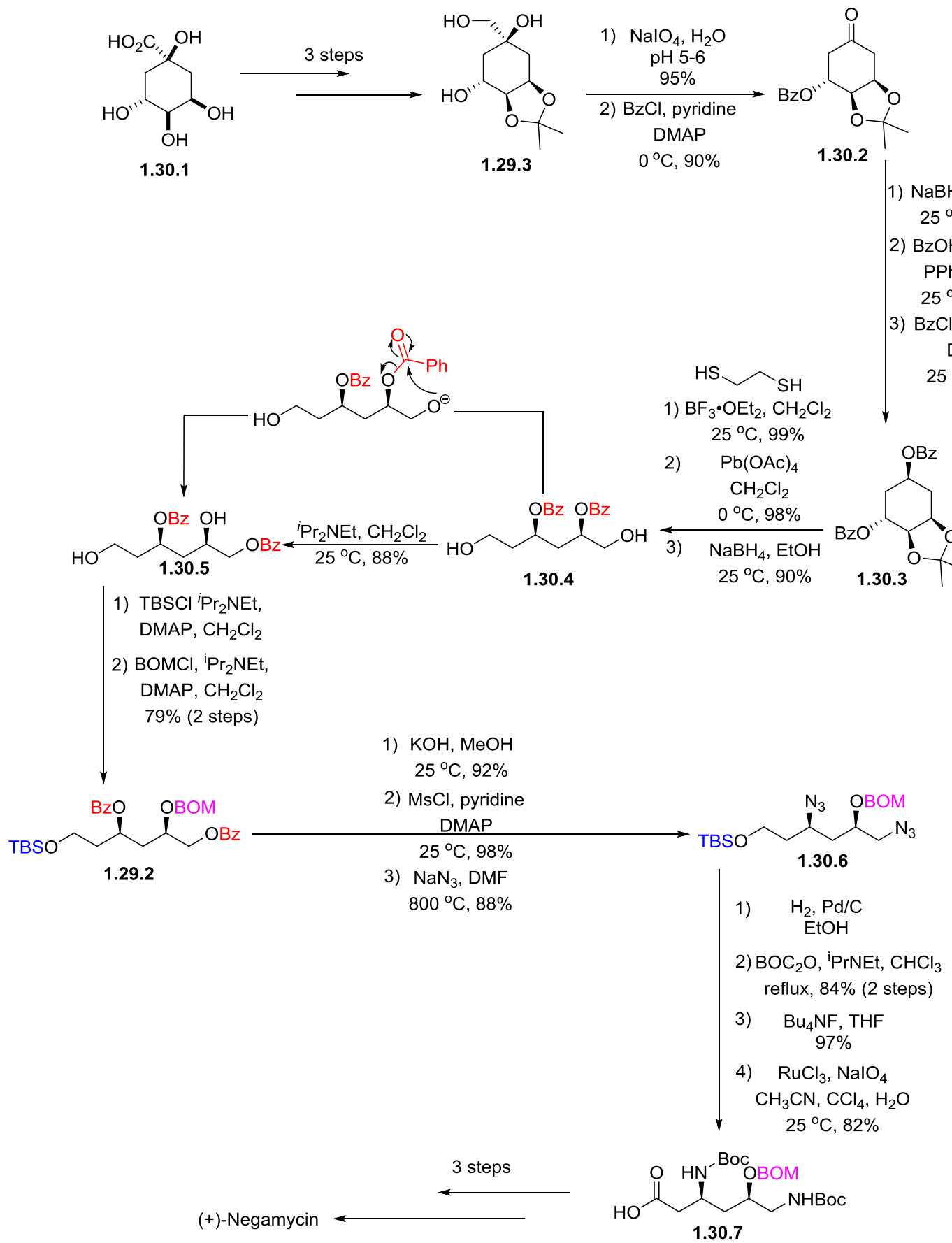

Scheme 1.30. Synthesis of (+)-negmycin from quinic acid 1.30.1.

In summary, the synthesis showcased a number of ways to overcome problems associated with organic synthesis such as the manipulation of unwanted isomer, and selective protection of primary alcohols (Scheme 1.31). 


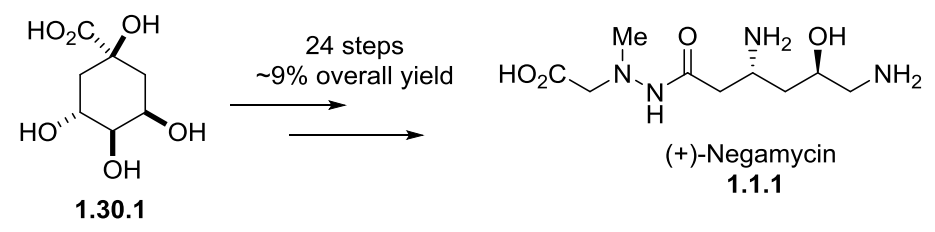

Scheme 1.31. Overall number of steps for the transformation of quinic acid to (+)-negamycin.

\subsubsection{Synthesis by Hegedus et al. ${ }^{20 \mathrm{a}}$}

In 1993, a more concise approach to the total synthesis of (+)-negamycin was reported by Hegedus et al. using palladium(II)-mediated tandem alkylation, carbonylative coupling to install the stereochemistry at C3 (Scheme 1.32). The stereogenic center at C5 would be incorporated by a selective Luche reduction of the enone 1.32.1. The enone 1.32.1 would be a decarboxylation product of diester 1.32.2, which would be derived from ene carbamate 1.32.3.
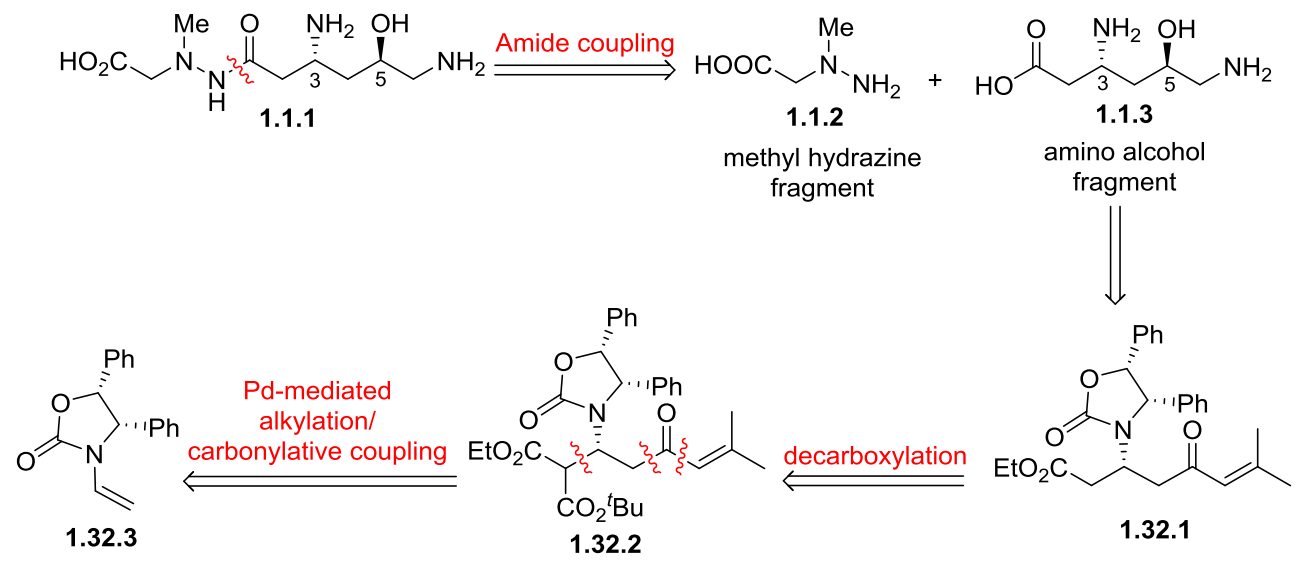

Scheme 1.32. Retrosynthetic analysis of (+)-negamycin by Hegedus et al.

The starting material ene carbamate 1.32.3 was synthesized on gram scale from the treatment of methoxy-methylpentacarbonylchromium $(0)$ carbene with $(1 R, 2 S)$-diphenylethanolamine (Scheme 1.33). The Pd(II)-mediated coupling of the ene carbamate $\mathbf{1 . 3 2 . 3}$ and the carbanion of tert-butyl ethyl malonate generated the palladacycle 1.33.1, which was subjected to carbon monoxide and isobutenyltrimethyltin to give enone $\mathbf{1 . 3 2 . 2}$ in good $77 \%$ yield. However, there was no selectivity at the malonate carbon. The stereocontrol of the diester 
group was inconsequential as decarboxylation took place in the subsequent step (Scheme 1.33). The tandem alkylation carbonylative coupling is efficient as $2 \mathrm{C}-\mathrm{C}$ bonds and $1 \mathrm{C}-\mathrm{O}$ bond were constructed in one pot. However, the method is stoichiometric in palladium that makes it less efficient compared to other strategies, which employed recyclable auxiliaries (such as the strategies reported by Kibayashi et al., Davies et al., and Hayashi et al.). Nevertheless, the method allowed for a full stereocontrol at C5 of enone 1.32.2. Although it was not immediately determined, the desired absolute stereoconfiguration at C5 was also achieved (validated upon completion of (+)-negamycin).

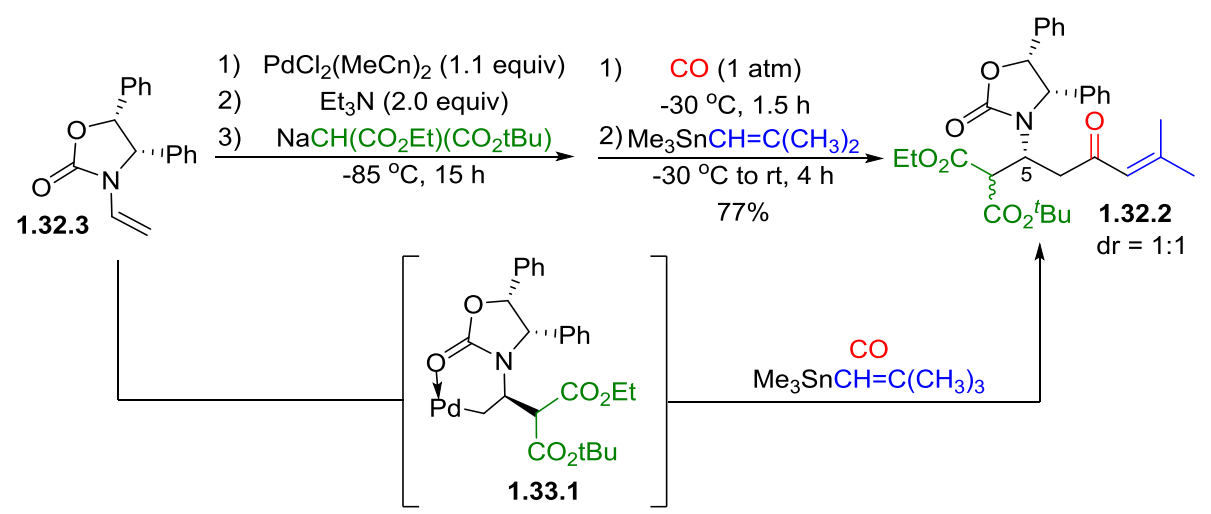

Scheme 1.33. Synthesis of 1 .32.2 from ene-carbamate 1.32.3.

The proceeding step involved a selective hydrolysis of the tert-butyl ester $\mathbf{1 . 3 2 . 2}$ to effect a decarboxylation to give the enone 1.32.1, which was then subjected to a diastereoselective Luche reduction (Scheme 1.34). The reduction gave 1.34.1 in a high $89 \%$ de and required further investigation regarding the conformation of substrate 1.32.1 in solution to understand the origin of stereoselectivity. The allylic alcohol 1.34.1 did not posses the correct configuration of (+)-negamycin and thus, was inverted by an intramolecular Mitsunobu reaction upon basic hydrolysis of the ethyl ester. The lactone was then reverted to the ester 1.34.2 as a single diastereomer. This was followed by a series of alcohol protection, ozonolysis and reduction steps to give 1.34.3. The reason for the prolonged hours for alcohol protection of 1.34.2 (40 h) was not mentioned but is likely to be due to steric hindrance. Alcohol 1.34.3 was 
taken through 5 further straightforward steps, which included peptide coupling and the final global hydrogenation step to yield (+)-negamycin in a total of 15 steps and $13 \%$ overall yield (Scheme 1.34).

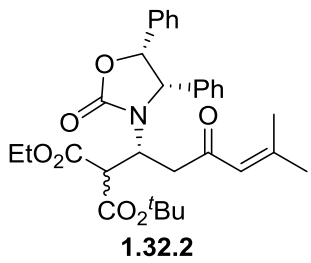

1) $\mathrm{CF}_{3} \mathrm{CO}_{2} \mathrm{H}, \mathrm{CH}_{2} \mathrm{Cl}_{2}$

2) $120^{\circ} \mathrm{C}, 2$ Torr, $0.75 \mathrm{~h}$
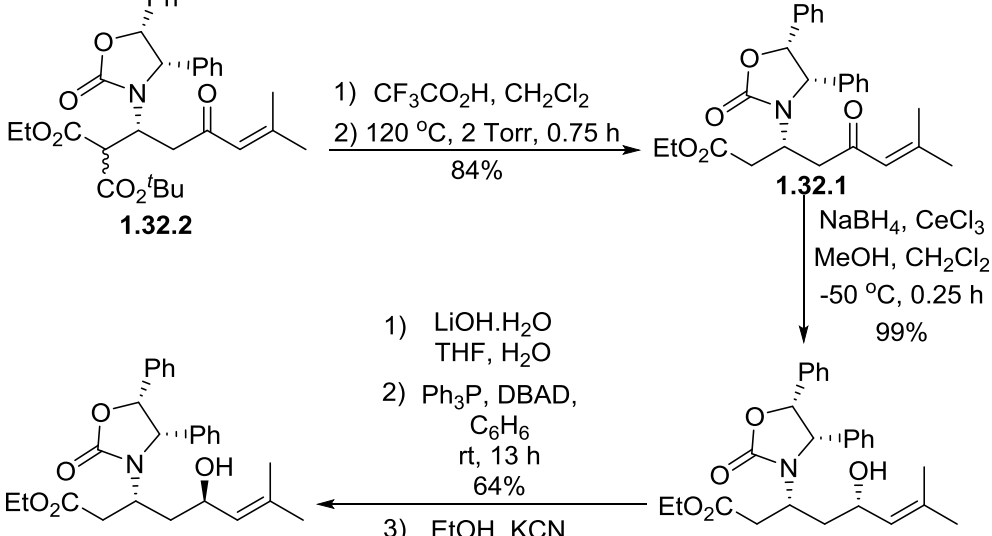

1) $\mathrm{LiOH} . \mathrm{H}_{2} \mathrm{O}$ $-50^{\circ} \mathrm{C}, 0.25 \mathrm{~h}$

2) $\mathrm{Ph}_{3} \mathrm{P}, \mathrm{DBAD}$

$\mathrm{Ph}$

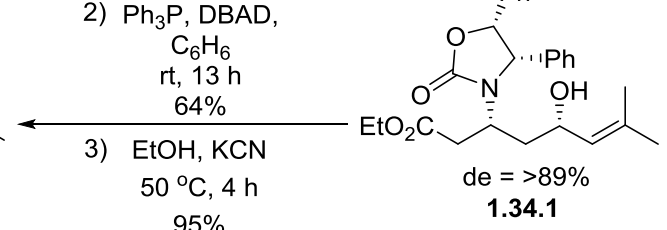

1) $\mathrm{TBSCl}$, imidazole, DMF rt, $40 \mathrm{~h}$

2) $\mathrm{O}_{3}, \mathrm{Me}_{2} \mathrm{~S}$

$\mathrm{CH}_{2} \mathrm{Cl}_{2}, \mathrm{MeOH}$

3) $\mathrm{NaBH}_{4}$ $-78{ }^{\circ} \mathrm{C}$ $95 \%$

$\mathrm{Ph}$<smiles>CCOC(=O)c1ccccc1</smiles>

$(+)$-Negamycin 15 steps, $13 \%$ overall yield

Scheme 1.34. Synthesis of (+)-negamycin from enone 1.32.2.

\subsubsection{Summary}

There were a total of 10 syntheses of (+)-negamycin discussed where various diastereoselective methods were employed to synthesize the amino alcohol fragment. These methods included, but are not limited to, substrate-controlled diastereoselective iodolactonization, ${ }^{17 a}$ 1,3-dipolar cycloaddition, ${ }^{201}$ conjugate addition with chiral amines, ${ }^{20 f}, 20 \mathrm{~g}$ chelation-controlled allylation, ${ }^{20 e}$ and palladium(II)-mediated alkylation carbonylative coupling. ${ }^{20 a}$ Hayashi et al. ${ }^{20 \mathrm{~h}, 31}$ and Davies et al., employed achiral building blocks and utilized 
diastereoselective reactions to construct the necessary stereogenic centers whereas Williams $e t$ $a l .,{ }^{20 \mathrm{e}}$ Wang et $a l .{ }^{17 \mathrm{a}}$ and Hegedus et al.,${ }^{20 \mathrm{a}}$ used chiral starting materials to establish substratecontrolled chirality induction through methods such as selective hydrogenation, iodolactonization and alkylation. Meanwhile, Kibayashi et al. ${ }^{20 \mathrm{c}}$ Weigele et al. ${ }^{26}$ and Maycock et al. ${ }^{13}$ illustrated versatility in the use of polyfunctional "chiral pool" such as quinic acid and D-glucose while Lieberknecht $e t$ al. ${ }^{20 \mathrm{~d}}$ relied on the use of amino acids.

Amongst the reviewed syntheses of (+)-negamycin, the most number of synthetic steps is 17 steps reported by Hayashi et al., and the highest overall yield is $41 \%$ that is also from the same group. ${ }^{20 \mathrm{~h}}$ The least number of synthetic steps was reported by Wiegele et al., ( 8 steps) but the group also holds the position for the lowest overall yield of $7 \% .{ }^{26}$ Overall, Hegedus et al., employed the most innovative chemistry. ${ }^{20 a}$ 
This page has been intentionally left blank. 


\section{Chapter 1}

\subsection{Objective of Present Work}

1.2.1 $\mathrm{N}, \mathrm{O}$-Heterocycles as Synthetic Intermediate

1.2.2 Retrosynthetic Analysis of (+)-Negamycin

1.2.3 Results and Discussion

1.2.4 Conclusion 
This page has been intentionally left blank. 


\subsection{Objective of Present Work}

\subsubsection{N,O-Heterocycles as Synthetic Intermediates}

A number of methods for the diastereoselective synthesis of isoxazolidines have been developed in this laboratory, including cyclocarbonylation, ${ }^{32}$ Claesson cyclization, ${ }^{33}$ and intramolecular hetero-Michael ${ }^{34}$ addition of substituted hydroxylamines. Halocyclisations ${ }^{35}$ and palladium catalyzed cyclization ${ }^{36}$ have also been reported. Apart from that, isoxazolidine rings have also been traditionally synthesized via 1,3-dipolar cycloadditions of alkenes with nitrones. ${ }^{37}$ However, this method can pose a limitation on the stereochemical and regiochemical results. $^{38}$

Our existing cycloaddition strategies in the class of $\mathrm{N}, \mathrm{O}$-heterocycles employ $O$-substituted hydroxylamines where the $\mathrm{O}-\mathrm{N}$ single bond constitutes as the tether, 1.35.B. This consists of a ring closure onto an alkene 1.35.C,${ }^{39}$ which gives either isoxazolidines $(n=1)$ or oxazines ( $\mathrm{n}=2$ ) with 1,3- or 1,4-stereocontrol, respectively (Scheme 1.35). The isoxazolidine then yields 1,3-amino alcohol upon $\mathrm{N}-\mathrm{O}$ bond cleavage .

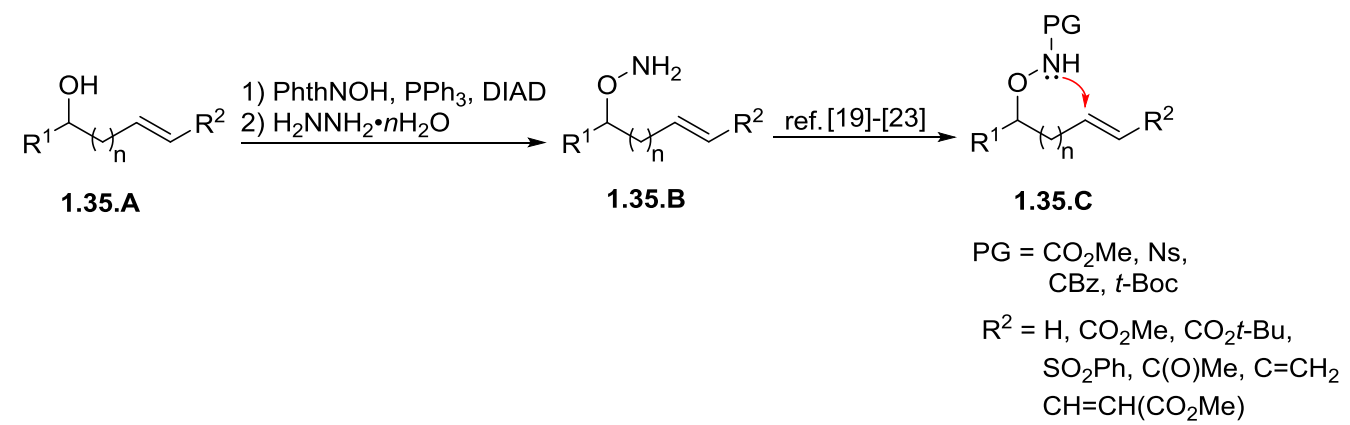

Scheme 1.35. $O$-substituted hydroxylamines employed to make $N, O$ - heterocycles.

The strategies have been very successfully applied in the synthesis of numerous alkaloids such as (-)-sedamine, ${ }^{40}(-)$-sedinine, ${ }^{33 \mathrm{~d}}(-)$-monomorine, ${ }^{41}$ and (-)-porantheridine ${ }^{33 \mathrm{~b}}$ (Figure 1.8). However, all of the processes developed in this laboratory favour formation of the 
3,5-cis-isomer, which corresponds to a syn amino alcohol upon $\mathrm{N}-\mathrm{O}$ bond cleavage that would result in epi-negamycin. Recently, workers in this laboratory have discovered that a Sakurai reaction of a 5-methoxyisoxazolidine yields the anti-isomer $\mathbf{1 . 4 1 . 3}$ as the major product (later discussed in Scheme 1.41, structure determined by X-ray crystal structure), ${ }^{42}$ a stereochemical relationship that corresponds to that of (+)-negamycin. This outcome has therefore led to the use of an isoxazolidine ring in the Sakurai reaction as the key reaction for the synthesis of (+)-negamycin.

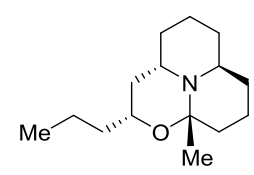

(-)-porantheridine

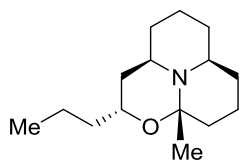

C6-epimer(porantheridine)

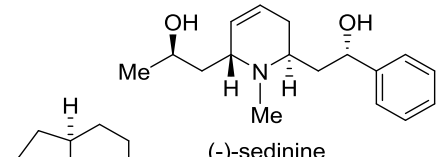

(-)-sedinine

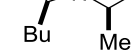

(-)-monomorine

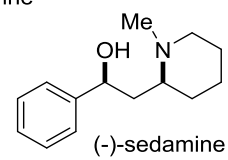

Figure 1.8. $\mathrm{N}, \mathrm{O}$ - heterocycles strategy employed to make natural products.

\subsubsection{Retrosynthetic Analysis of (+)-Negamycin}

(+)-Negamycin was disconnected at the standard hydrazide peptide bond into two smaller fragments namely, methyl hydrazine 1.1.2 and amino alcohol 1.1.3 (Scheme 1.36). The stereogenic center at C3 of (+)-negamycin was envisaged from the key Sakurai allylation step of 5-chloro-methyl-3-methoxyisoxazolidine $\mathbf{1 . 3 6 . 3}$ which would give the homoallyl isoxazolidine 1.36.2. The Sakurai precursor 1.36.3 would be accessed from the ozonolysis of benzyloxy homoallylamine 1.36.4, which would be obtained from a Mitsunobu reaction of homoallylalcohol 1.36.5. The manipulation of commercially available starting material, racemic (+/-)-epi-chlorohydrin into enantiopure (S)-epi-chlorohydrin 1.36.6 accounts for the chirality at C5. 
The anti-stereo relationship of the amino alcohol fragment at C3 and C5 would be controlled by using an isoxazolidine ring (Scheme 1.36). The $\mathrm{N}-\mathrm{O}$ bond of the isoxazolidine would be later reduced, simultaneously with an azide reduction and the removal of protecting groups, in the final global hydrogenation step to give the desired natural product. For this reason, benzyl-based protecting groups were selected as the nitrogen and carboxylic acid protecting groups. This would allow for additional deprotection steps to be obviated from the synthetic plan, and hence would provide the opportunity for an efficient synthesis.

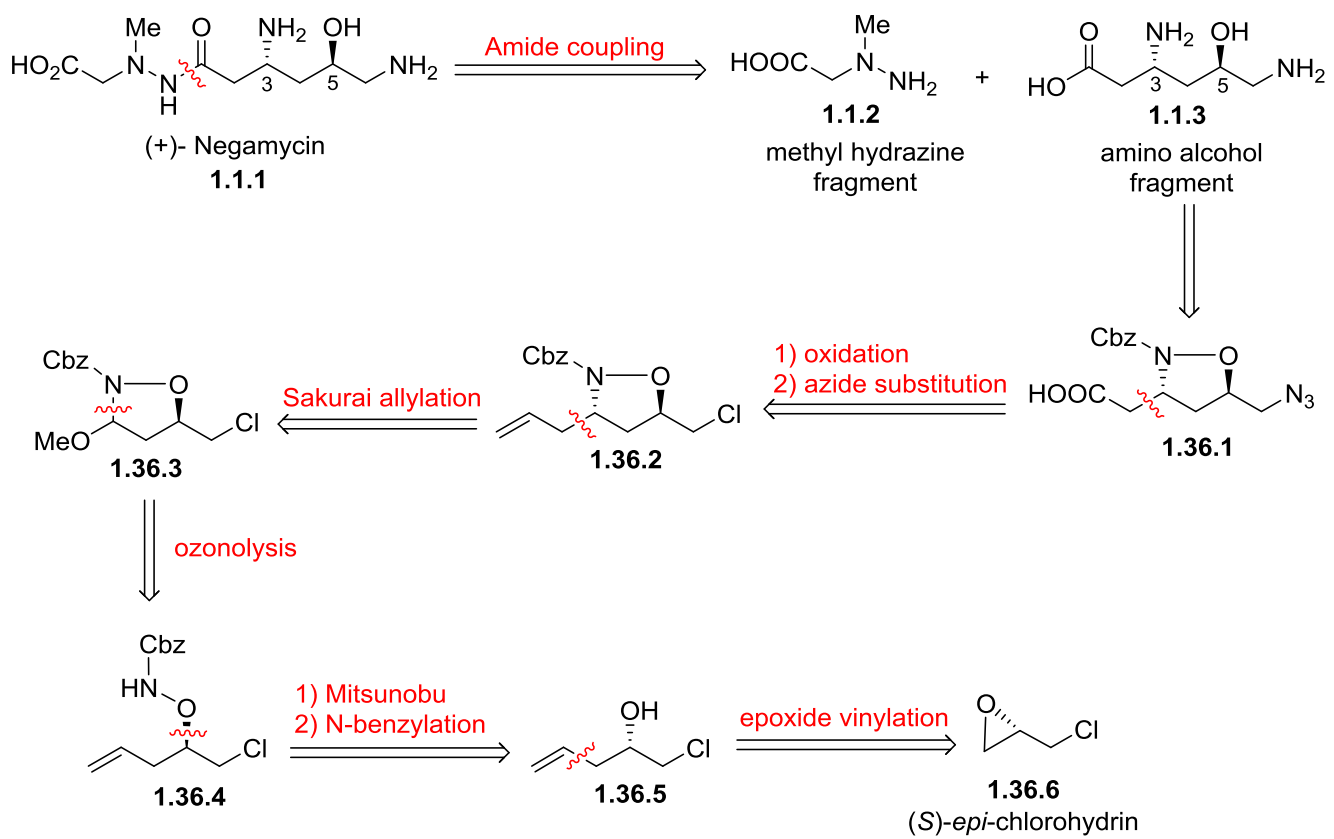

Scheme 1.36. Retrosynthesis of (+)-negamycin.

\subsubsection{Results And Discussion}

\subsubsection{Synthesis of Iminium Ion Precursor}

The enantiopure $(S)$-epi-chlorohydrin 1.36.6 was obtained through salen-mediated hydrolytic kinetic resolution of the commercially available racemate (Scheme 1.37). ${ }^{20 \mathrm{q},}{ }^{43} \mathrm{~A}$ Grignard reagent, vinyl magnesium bromide, was employed as the nucleophile to cause the epoxide ring opening of 1.36.6. Copper(I) iodide was added as a catalyst to effect a 
transmetallation that encourages the reaction through the formation of an in situ organocopper reagent. ${ }^{20 q}, 44$ The procedure was reported by Blechert and co-workers, ${ }^{45}$ and gave the desired alcohol 1.36.5 with good regioselectivity (Scheme 1.37).

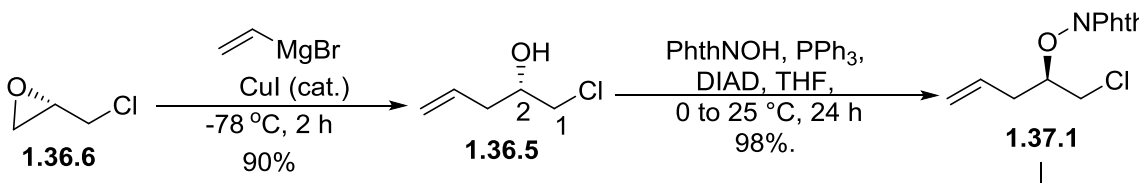
1) $\mathrm{H}_{2} \mathrm{NNH}_{2}, \mathrm{CH}_{2} \mathrm{Cl}_{2}$ $1 \mathrm{~h}, 0^{\circ} \mathrm{C}$ $90 \%$ 2) $\mathrm{CbzCl}, \mathrm{Na}_{2} \mathrm{CO}_{3}$, $\mathrm{H}_{2} \mathrm{O}, \mathrm{CH}_{2} \mathrm{Cl}_{2}$ $3 \mathrm{~h}, 0$ to $25^{\circ} \mathrm{C}$ $86 \%$
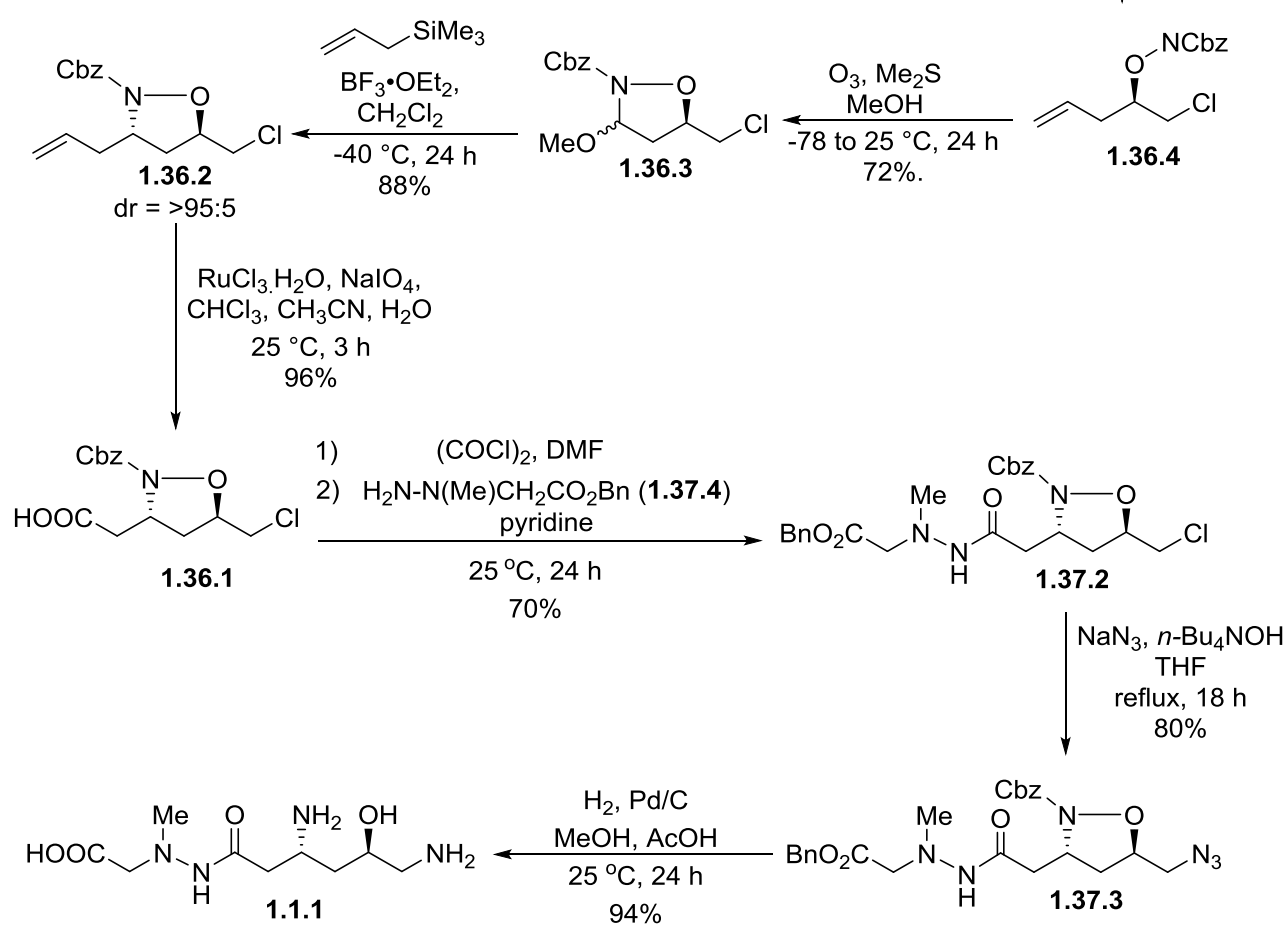

Scheme 1.37. Synthetic route to (+)-negamycin.

Although there were three possible sites of attack by the nucleophile, attack occurred exclusively from the less sterically hindered terminal end of the epoxide ring $\mathbf{1 . 3 6 . 6}$ as observed from ${ }^{1} \mathrm{H}$ NMR (Figure 1.9). The ${ }^{1} \mathrm{H}$ NMR analysis showed presence of a single characteristic multiplet at $3.88 \mathrm{ppm}$, a coupling pattern unique to the proton on carbon bearing the hydroxyl 
group. This is consistent with the desired alcohol 1.36.5. In overall, it was a fast reaction accompanied by a good $90 \%$ yield of $\mathbf{1 . 3 6 . 5}$, making it a desirable first step of the synthesis.

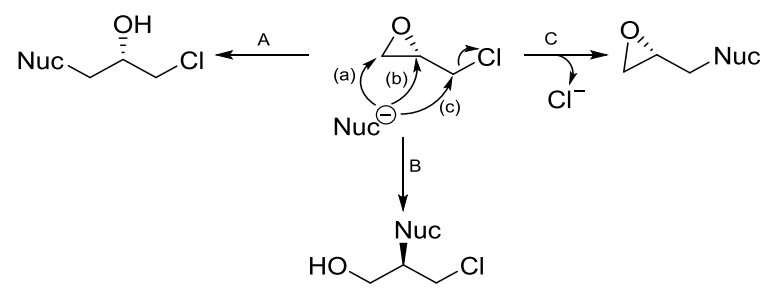

Figure 1.9. Regioselectivity of nucleophilic attack on 1.36.6.

The homoallyl alcohol 1.36.5 was then subjected to a Mitsunobu reaction using $N$-hydroxyphthalimide and triphenylphosphine in the presence of diisopropyl azodicarboxylate under Mitsunobu conditions to give $N$-phthaloyl hydroxylamine 1.37.1 in an excellent $98 \%$ yield (Scheme 1.37). Since the Mitsunobu reaction requires nucleophiles that are weakly acidic, $N$-hydroxy-phthalimide is adequately acidic to qualify as a good nucleophilic partner. ${ }^{46} \mathrm{The} \mathrm{S}_{N} 2$ attack at $\mathrm{C} 2$ of $\mathbf{1 . 3 6 . 5}$ displaced the alkyltriphenyl-phosphorane and inverted the configuration of the homoallyl alcohol $\mathbf{1 . 3 6 . 5}$ from $(S)$ to $(R)$. The high tolerance of the Mitsunobu reaction to an array of functional groups ${ }^{47}$ enabled a smooth reaction with quantitative yield. Since our approach for the incorporation of $O$-substituted hydroxylamine is through a Mitsunobu reaction, it is necessary to begin with the right enantiomer of epi-chlorohydrin 1.36.6. This is so the correct stereochemistry of the hydroxylamine, that which coincides with the natural product, can be established (Scheme 1.37).

The formation of the $N$-hydroxylamine 1.37.1 was determined by ${ }^{1} \mathrm{H}$ NMR that showed a downfield shift of proton at $\mathrm{C} 2$, previously at $3.88 \mathrm{ppm}$ to $4.46 \mathrm{ppm}$ (Figure 1.10) with a similar coupling pattern. The downfield shift is due to the phthalimide moiety exerting a deshielding effect. In addition, alcohol 1.36.5 does not bear aromatic protons whereas $N$-phthloyl hydroxylamine 1.37 .1 bears a distinct coupling pattern from the aromatic protons of the phthalimide moiety observed between 7.90 and 7.77 ppm (Figure 1.10). 


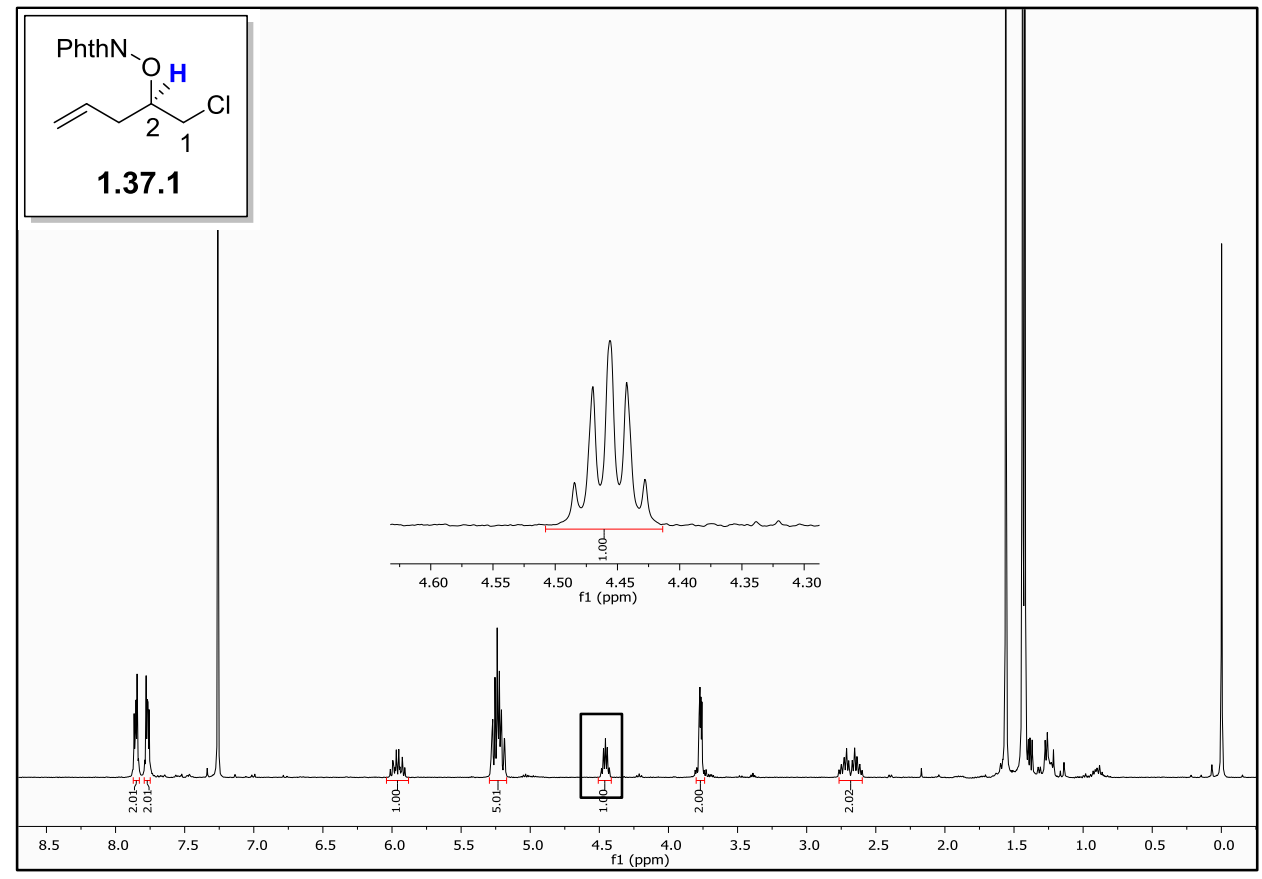

Figure 1.10. ${ }^{1} \mathrm{H}$ NMR of 1.37.1 with focus on haracteristic at $\mathrm{C} 2$.

The precursor to the 3-methoxy isoxazolidine derivative 1.36.4 was obtained from the deprotection of the phthaloyl group to give the free hydroxyl amine using hydrazine monohydrate in dichloromethane under Ing-Manske conditions (refer to Scheme 1.37). ${ }^{48}$ This was immediately followed by $N$-reprotection of the hydroxylamine with benzyl chloroformate to give benzyl carbamate derivative 1.36.4 under Schotten-Baummann conditions. The carbamate product 1.36.4 showed characteristic singlet benzyl $\mathrm{CH}_{2}$ protons at $5.18 \mathrm{ppm}$ in the ${ }^{1} \mathrm{H}$ NMR spectrum.

The synthesis continued with the ozonolysis of hydroxylamine 1.36.4 in acidic methanol to give 3-methoxyisoxazolidine $\mathbf{1 . 3 6 . 3}$ as an inconsequential mixture of diastereomers $(\mathrm{dr}=1: 1)$ in $72 \%$ yield after the reductive work up with dimethyl sulfide (refer to Scheme 1.37). It has been shown by Criegee and co-workers that an alkene 1.38.1 is susceptible to ozone cleavage to give the carbonyl ylide oxide 1.38.3 and a ketone/aldehyde 1.38.4 (Scheme 1.38). ${ }^{49}$ If the solvent employed in the process is dichloromethane, a 1,3-dipolar cycloaddition would occur between 1.38.3 and 1.38.4 to give a stable trioxolane peroxide 1.38.5. The ozonolysis in 
methanol, in contrast, do not give the trioxolane intermediate $\mathbf{1 . 3 8 . 5}$ as the methanol adds to the carbonyl ylide oxide 1.38.3 to give a methoxy hydroperoxide 1.38.7, which gives 1.38 .8 upon the reductive work-up with dimethyl sulfide. ${ }^{50}$ The purpose of using methanol during ozonolysis was to introduce the methoxy group, which is essential for the the subsequent Sakurai reaction.

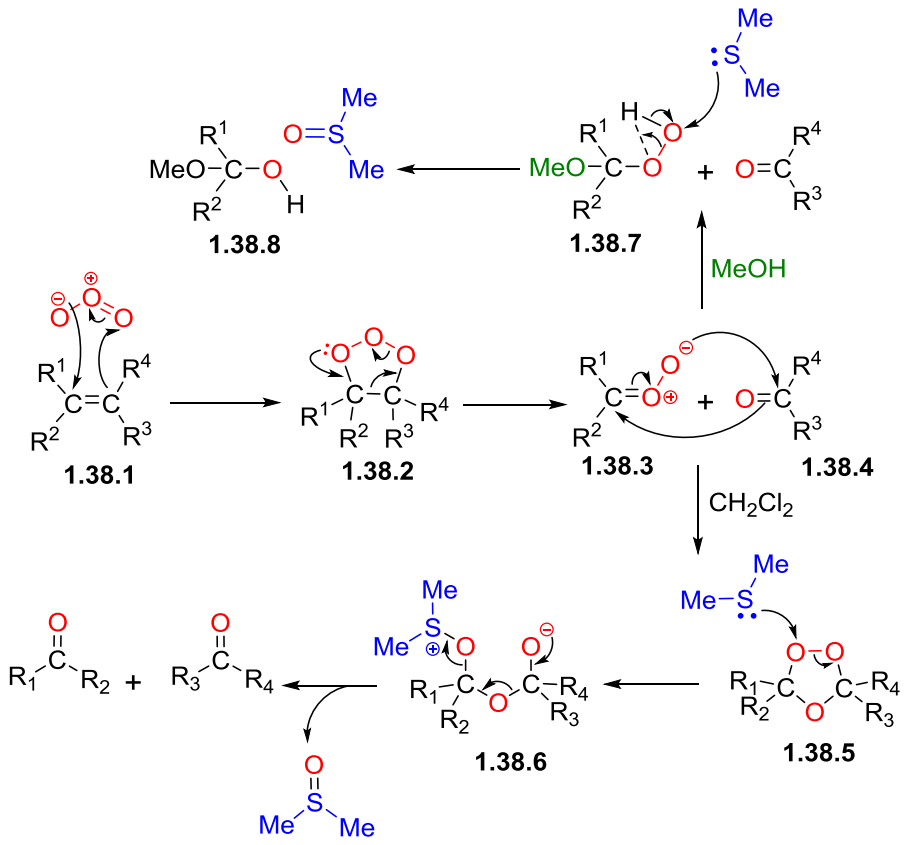

Scheme 1.38. Ozonolysis in different solvents; dichloromethane and methanol.

A proposed mechanism for the conversion benzyloxy homoallyl hydroxylamine 1.36.4 into 3-methoxyisoxazolidine 1.36.3 is outlined in Scheme 1.39. In the proposed mechanism, the 1,3-dipolar addition can proceed in two ways, path $\mathbf{A}$ and path $\mathbf{B}$. Therefore, either a carbonyl ylide oxide 1.39.2 or an aldehyde $\mathbf{1 . 3 9 . 3}$ or a combination of both could be formed from the 1,3dipolar retro-cycloaddition and then undergo intramolecular attack by the neighbouring nitrogen to create the isoxazolidine rings, 1.39.4 and 1.39.5 (Scheme 1.39). The manner of cyclization is not controlled, and hence could occur from either face to yield an inconsequential mixture of diastereomers 1.39.4, and 1.39.5 that then undergo an acid catalyzed-OMe exchange via an iminium ion 1.39.6. Under acidic conditions, an equilibrium mixture of 3-methoxyisoxazolidine 1.36.3 is expected (Scheme 1.39). 


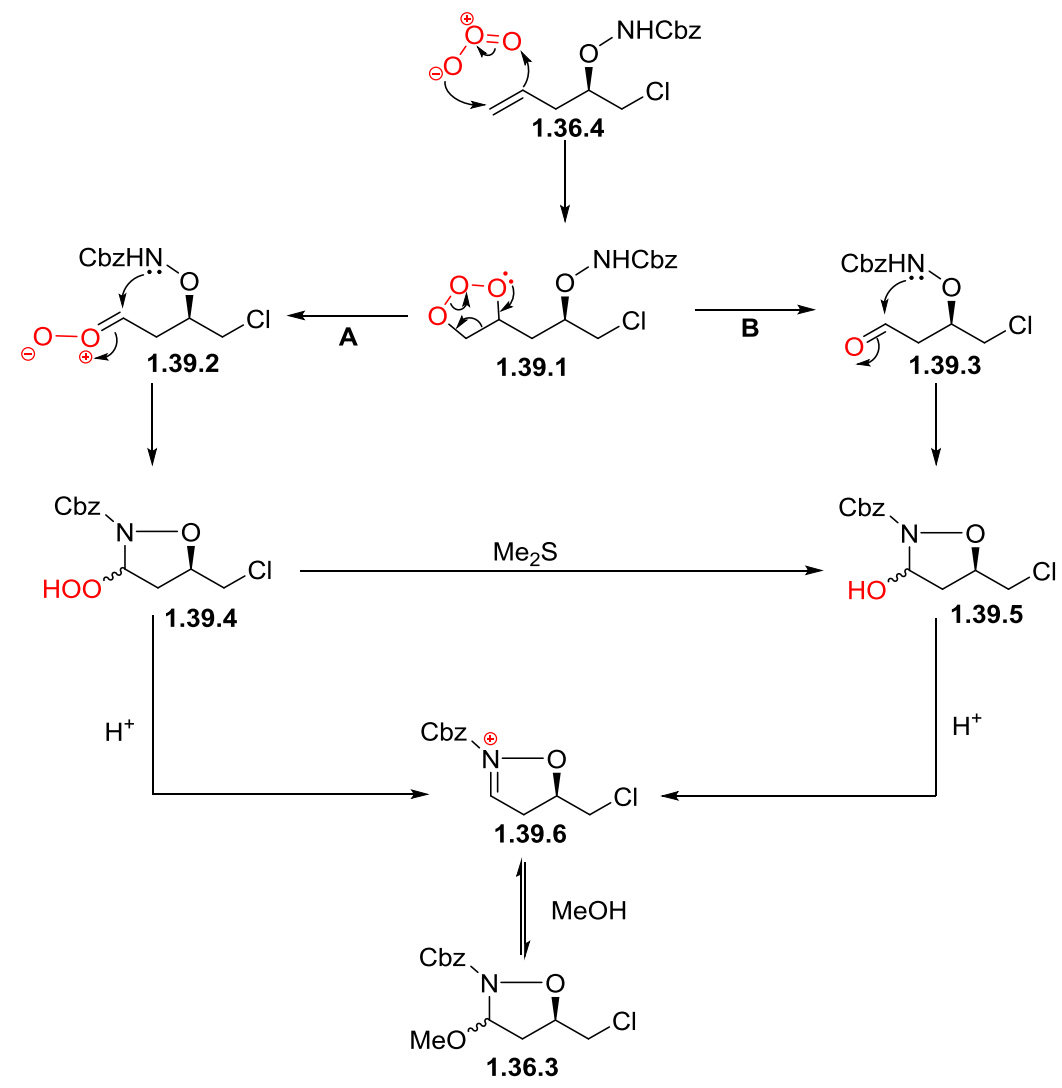

Scheme 1.39. Ozonolysis of 1.36 .4 in methanol.

It was originally intended to convert the chloro substitutent of 3-methoxyisoxazolidine 1.36.3 into an azide group 1.40.1, which would later give a free primary amine upon hydrogenation in the final global hydrogenation step (Scheme 1.40 and refer to Scheme 1.37). Unfortunately, the reaction was unsuccessful with sodium azide in dimethylformamide at $100{ }^{\circ} \mathrm{C}$ overnight. The ${ }^{1} \mathrm{H}$ NMR showed that the characteristic peaks corresponding to the benzyl protons of the benzyl carbamate group were absent, which indicated a loss of the protecting group. This may be due to decomposition of the starting material 1.36.3 since it was not recoverable. It may also be due to the low reactivity of the $\mathrm{C}-\mathrm{Cl}$ bond, which can be improved by an in situ generation of the iodo-substituent by including $\mathrm{NaI}$ in the reagent mixture. ${ }^{51}$ Nonetheless, the functional group exchange was postponed to later stages of the synthesis. 


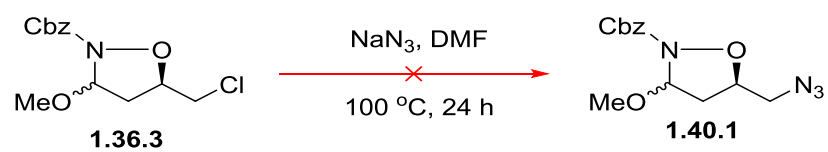

Scheme 1.40. Chloro- to azide functional group exchange.

\subsubsection{Sakurai Allylation}

The synthesis then continued with the key Sakurai allylation step to incorporate the second stereogenic center at C3 of (+)-negamycin (refer to Scheme 1.37). The 3-methoxyisoxazolidine 1.36.3 was treated with allyltrimethylsilane in the presence of boron trifluoride diethyl etherate in dichloromethane to give allylated compound 1.36.2 as a single diastereomer, surprisingly! The methoxy group $\alpha$ to nitrogen of $\mathbf{1 . 3 6 . 3}$ required Lewis acid activation because it is a poor leaving group. The use of Lewis acid was tolerated in the allylation due to the stability of the starting material $\mathbf{1 . 3 6 . 3}$ to acids. ${ }^{52}$

\subsection{Product Structural Assignment}

A rigorous ${ }^{1} \mathrm{H}$ NMR, COSY and NOESY analysis was carried out in an attempt to characterize and determine the identity of the stereoisomer obtained (cis- or trans-isomer) (Figures 1.11 and 1.12).

From the ${ }^{1} \mathrm{H}$ NMR comparison of the desired product and the starting material (Figure 1.11), there was a complete disappearance of the singlet peak at $3.39 \mathrm{ppm}$ corresponding to the methoxy protons of 3-methoxyisoxazolidine $\mathbf{1 . 3 6 . 3}$ and an emergence of characteristic alkene peaks particularly at $5.79 \mathrm{ppm}(\mathrm{H} 8)$, signifying that the desired allylated product $\mathbf{1 . 3 6 . 2}$ was synthesized (Figure 1.11). The protons of the allylated product 1.36.2 then required assignment, mainly the less obvious closely related pair of multiplets between $4.51 \mathrm{ppm}$ and $4.34 \mathrm{ppm}$ shown in Figure 1.12. The COSY NMR analysis showed a correlationship between proton accounting for the multiplet at $4.47 \mathrm{ppm}$ and protons $\alpha$ to the chloro group, H6, while no such interaction 
was observed between proton accounting for the multiplet at $4.20 \mathrm{ppm}$ and H6. This indicated that the pair of multiplet between 4.51 and $4.34 \mathrm{ppm}$ was a result of distinct protons instead of a diastereomer mixture (Figure 1.12). Therefore, the multiplet at $4.47 \mathrm{ppm}$ was labelled as H5 while the multiplet at $4.20 \mathrm{ppm}$ was identified as $\mathrm{H} 3$.

Since every proton peak in the ${ }^{1} \mathrm{H}$ NMR spectrum of the allylated product $\mathbf{1 . 3 6 . 2}$ was accounted for, with no additional set of proton peaks as observed in cases involving mixture of diastereomers, it was thus concluded that the Sakurai allylation proceeded in a completely diastereoselective manner. The initial hypothesis was that the nucleophilic attack would be initiated on either side of the ring but with a preference for the trans-isomer. Therefore, it was a serendipitious result as complete diastereoselective control was not expected.

At this stage, proton interaction through spatial orientation was investigated to try and determine the relative stereochemistry. However, NOESY NMR analysis was inconclusive as the expected correlation could not be observed. Nonetheless, validation of the antistereorelationship and absolute configuration was achieved by the elaboration of $\mathbf{1 . 3 6 . 2}$ into the natural product where comparisons could be made with the known (+)-negamycin spectroscopic data reported in the literature such as chiral optical activity and ${ }^{1} \mathrm{H}$ NMR. Upon receiving a surprising success, an optimization of the Sakurai allylation was carried out with the objective of improving the reaction yield. 


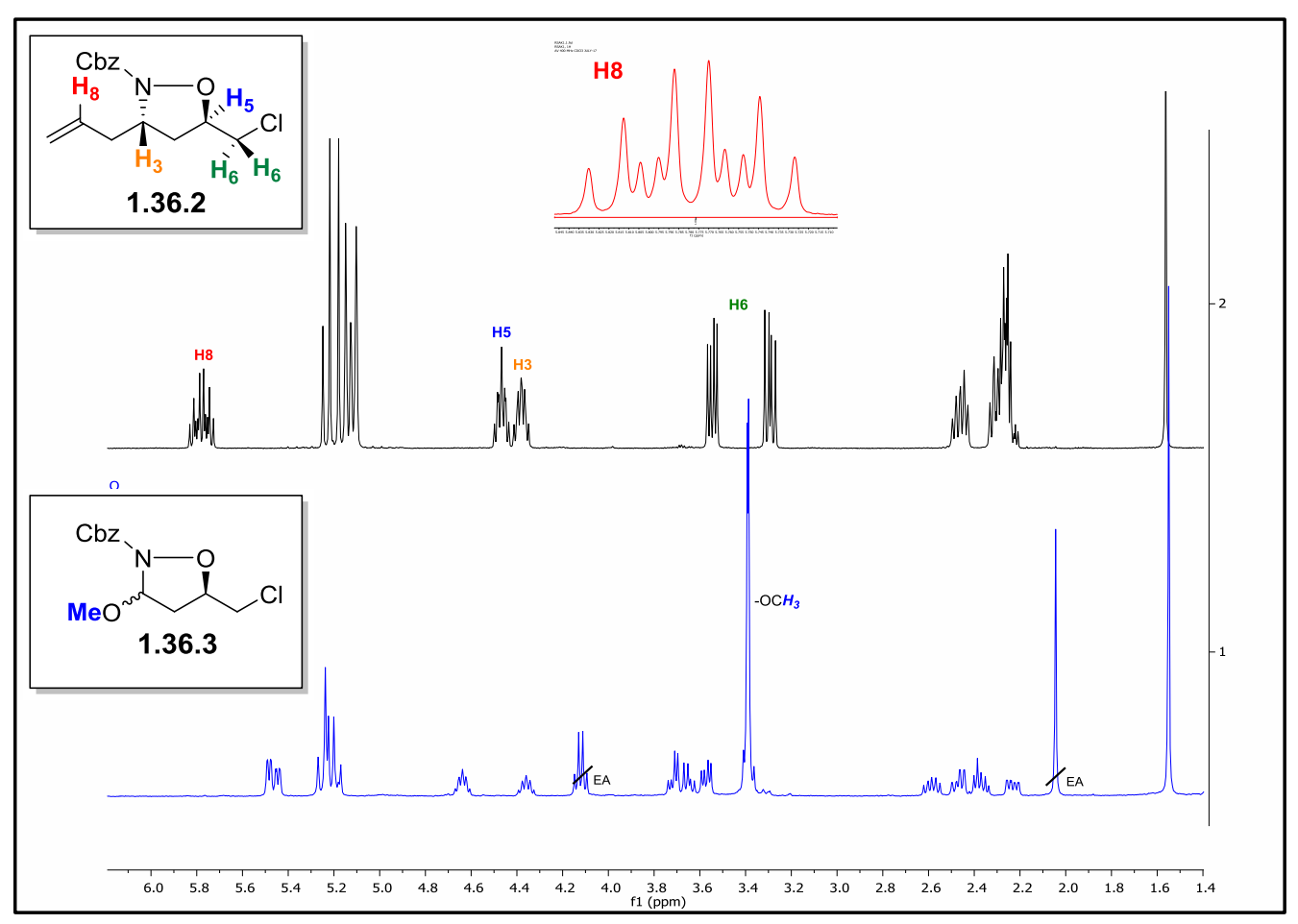

Figure 1.11. Comparison of ${ }^{1} \mathrm{H}$ NMR of 1.36.2 and 1.36.3. 


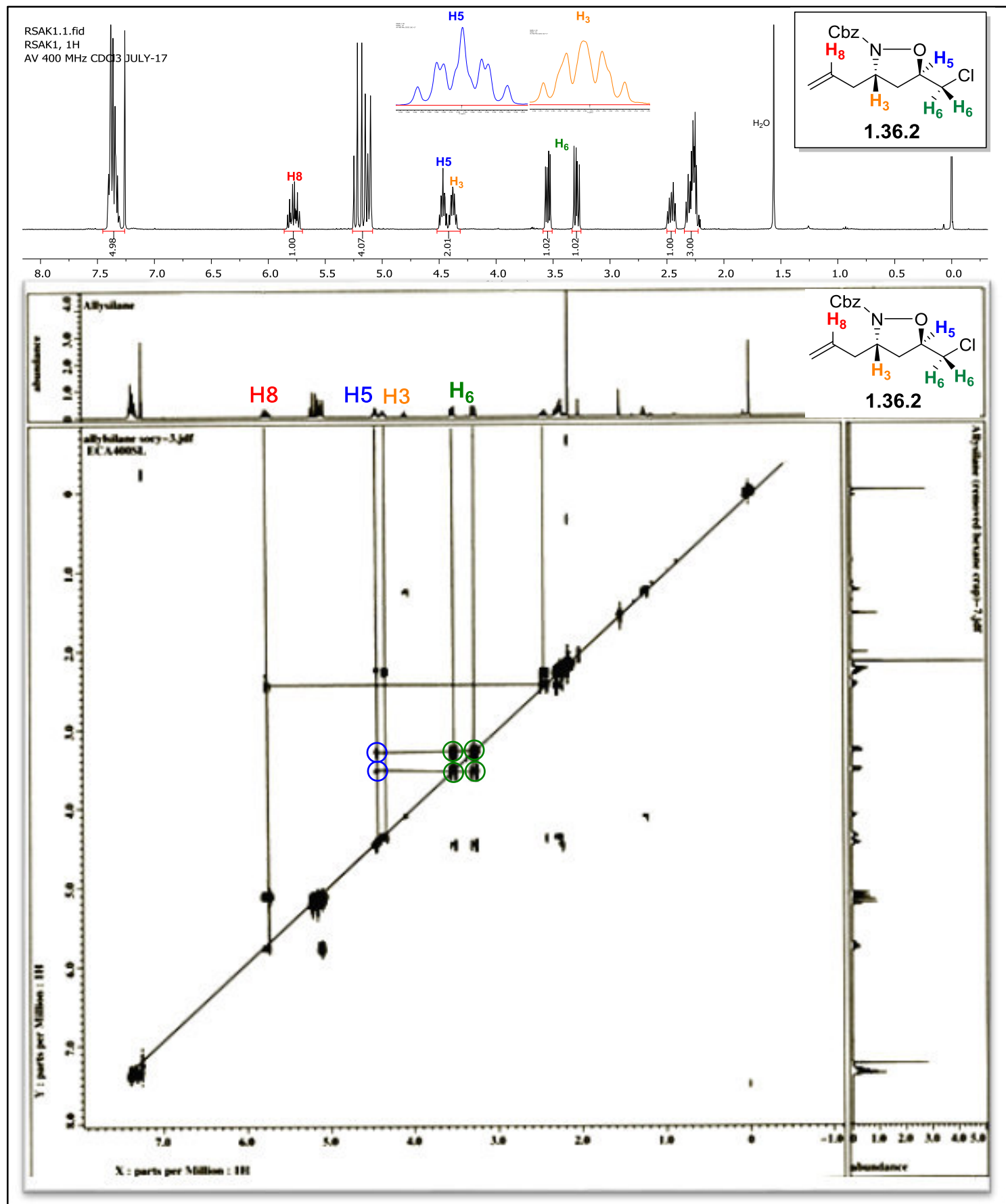

Figure 1.12. COSY NMR analysis of 1.36.2.

\subsection{Optimization}

The allylation was carried using a variety of Lewis acid while keeping other variables of the reaction condition such as allylating agent (trimethylallylsilane), reaction temperature 
$\left(-70{ }^{\circ} \mathrm{C}\right)$, reaction time $(24 \mathrm{~h})$ and solvent choice $\left(\mathrm{CH}_{2} \mathrm{Cl}_{2}\right)$ unchanged (Table 1.1). A total of four different types of Lewis acid were screened, and one was found unsuitable for the reaction. Titanium tetra-isopropoxide failed to give the allylated product as the starting material was recovered completely (Table 1.1, entry 1). Although, there was an improvement observed with use of titanium chloride and boron trifluoride, the allylation yield remained low at $45 \%$ and $46 \%$ respectively (Table 1.1, entries 2 and 3). The best allylation yield of $67 \%$ was achieved by using tin chloride as Lewis acid (Table 1.1, entry 4).

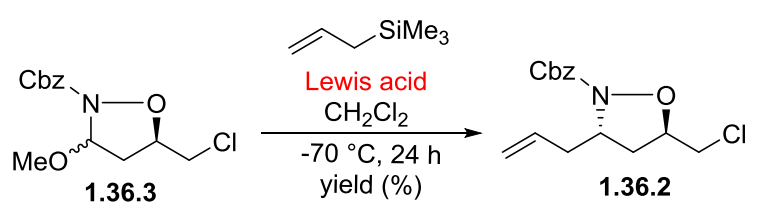

\begin{tabular}{cccccc}
\hline entry & $\begin{array}{c}\text { amount } \\
(\mathrm{mmol})\end{array}$ & $\begin{array}{c}\text { temperature } \\
\left({ }^{\circ} \mathrm{C}\right)\end{array}$ & $\begin{array}{c}\text { reaction time } \\
(\mathrm{h})\end{array}$ & $\begin{array}{c}\text { yield } \\
(\%)\end{array}$ & $\begin{array}{c}\text { Lewis acid } \\
(1.2 \text { equiv })\end{array}$ \\
\hline 1 & 0.40 & -70 & 24 & 0 & $\mathrm{Ti}\left(\mathrm{O}^{\mathrm{i} P r}\right)_{4}$ \\
2 & 0.40 & -70 & 24 & 45 & $\mathrm{TiCl}_{4}$ \\
3 & 0.40 & -70 & 24 & 46 & $\mathrm{BF}_{3} \bullet \mathrm{OEt}_{2}$ \\
$\mathbf{4}$ & $\mathbf{0 . 4 0}$ & $\mathbf{- 7 0}$ & $\mathbf{2 4}$ & $\mathbf{6 7}$ & $\mathbf{S n C l}_{4}$ \\
\hline
\end{tabular}

Table 1.1. Sakurai allylation with Lewis acid as variable factor.

Other allylation parameters such as reaction temperature and time were also modified to investigate the efficiency of the reaction (Table 1.2). The Lewis acid employed was $\mathrm{BF}_{3} \cdot \mathrm{OEt}_{2}$ and the results showed an increase in the reaction yield at higher reaction temperatures for a stirring time of $24 \mathrm{~h}$ (Table 1.2, entries 2-4). The best yield obtained for the Sakurai allylation was $88 \%$ with $\mathrm{BF}_{3} \cdot \mathrm{OEt}_{2}$ at $-40{ }^{\circ} \mathrm{C}$ for $24 \mathrm{~h}$ (Table 1.2 , entry 4 ). Since it was also the best reported yield amongst the various tested conditions, $\mathrm{BF}_{3} \cdot \mathrm{OEt}_{2}$ was selected over $\mathrm{SnCl}_{4}$ to be the choice of Lewis acid adopted for future Sakurai allylation. 


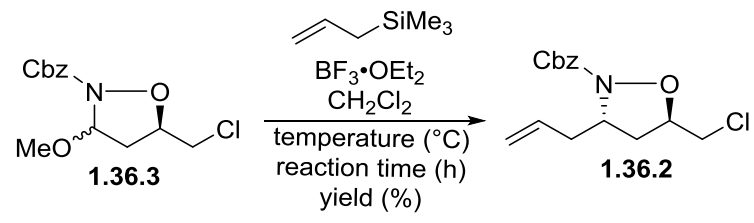

\begin{tabular}{cccccc}
\hline entry & $\begin{array}{c}\text { amount } \\
(\mathrm{mmol})\end{array}$ & $\begin{array}{c}\text { temperature } \\
\left({ }^{\circ} \mathrm{C}\right)\end{array}$ & $\begin{array}{c}\text { reaction time } \\
(\mathrm{h})\end{array}$ & $\begin{array}{c}\text { yield } \\
(\%)\end{array}$ & $\begin{array}{c}\text { Lewis acid } \\
(1.2 \text { equiv })\end{array}$ \\
\hline 1 & 0.40 & -70 & 72 & 42 & $\mathrm{BF}_{3} \bullet \mathrm{OEt}_{2}$ \\
2 & 0.40 & -60 & 24 & 46 & $\mathrm{BF}_{3} \bullet \mathrm{OEt}_{2}$ \\
3 & 0.40 & -50 & 24 & 57 & $\mathrm{BF}_{3} \cdot \mathrm{OEt}_{2}$ \\
$\mathbf{4}$ & $\mathbf{0 . 4 0}$ & $\mathbf{- 4 0}$ & $\mathbf{2 4}$ & $\mathbf{8 8}$ & $\mathbf{B F}_{3} \bullet \mathbf{O E t}_{2}$ \\
\hline
\end{tabular}

Table 1.2. Sakurai allylation with temperature and reaction time as variable factors.

\subsection{Mechanism and Proposed Theory}

Previously in this laboratory, the Sakurai allylation was performed on the 5-phenyl-3methoxy-isoxazolidine 1.41.3 to give isoxazolidine 1.41.4 as a mixture of diastereomers $(\mathrm{dr}=4: 1)$, favouring the trans-isomer (determined by X-ray crystal structure) (Scheme 1.41). ${ }^{42}$ The diastereoselectivity was significantly improved when the allylation was repeated on substrates 1.36.3 and 1.41.1. This is because analogues 1.36.3 and 1.41.1 possess a polar substituent at the $\mathrm{C} 5$ position, chloromethyl and hydroxymethyl group respectively, while 1.41.3 did not (Scheme 1.41). The stereochemical outcome can be attributed to a stereoelectronic effect.

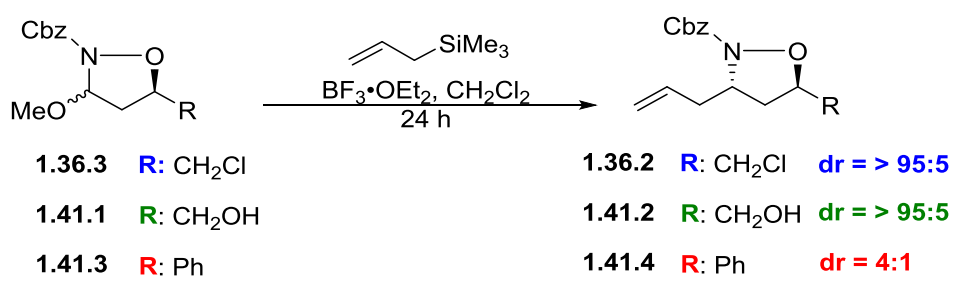

Scheme 1.41. Sakurai allylation performed on 1.36.3 - 1.41.3 gave different diastereoselectivity. 
The proposed mechanistic pathway for the Sakurai allylation begins with the activation of the methoxy group by boron trifluoride diethyletherate, which is then believed to generate an iminium ion intermediate 1.39.6 that is susceptible to nucleophilic attack by allyltrimethyl silane from either face of the isoxazolidine ring (Scheme 1.42). In the event of a polar substituent at the C5 position, we postulate that there exists an efficient shielding of one face due to an electrostatic attraction between the partial negatively charged chloro atom and the positively charged iminium ion (Scheme 1.42). This results in facial bias that accounts for the excellent stereocontrol during the allylation of substrate 1.36.3.

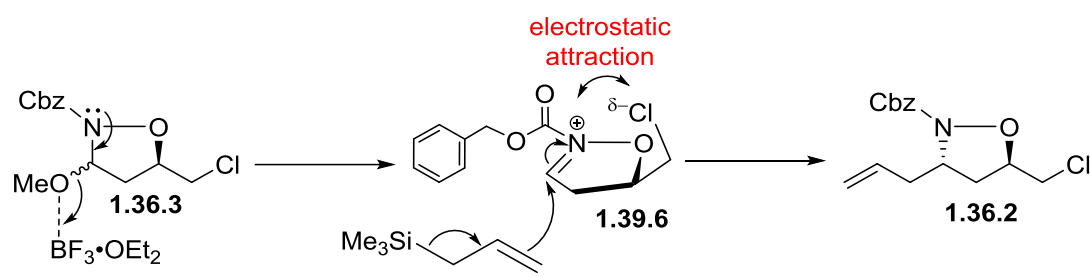

Scheme 1.42. Proposed mechanism for complete diastereoselective allylation.

The relationship between diastereoselectivity and substituents on the 3-methoxyisoxazolidine derivatives is in the extent of facial shielding provided, strong or modest, as illustrated in Figure 1.13. The shielding effect is a consequence of the electrostatic interaction, which is influenced by the type of atom present at the C5 position of the isoxazolidine ring. This, thus, reflects that the Sakurai allylation is an example of a substrate-controlled diastereoselective approach (Figure 1.13).
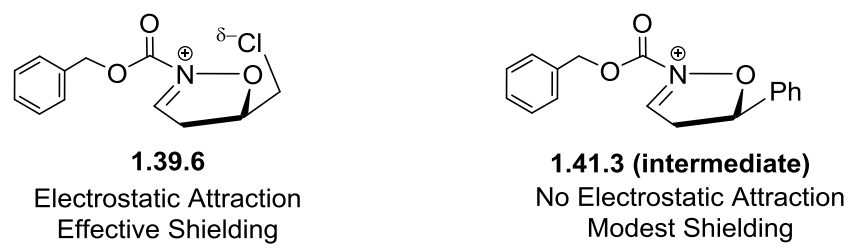

Figure 1.13. Origin of stereocontrol of Sakurai allylation. 
The energy calculation ${ }^{46}$ for the conformation of the iminium ion intermediate bearing the chloromethyl group was made and found to be the lowest when the chlorine atom was closest to the positively charged iminium ion ${ }^{53}$ (Figure 1.14, conformation B). The shielding offered in conformation B would be most effective. The alternative confomations A and C, are 2.9 $\mathrm{kcal} / \mathrm{mol}$ and $1.6 \mathrm{kcal} / \mathrm{mol}$ higher respectively. This indicates that the preferred conformation would be B, which is in agreement with our proposed theory of the allylation outcome (Figure 1.4).

In a similar energy calculation, the lowest energy conformation D of the iminium ion intermediate bearing a non-polar ethyl substituent showed the methylene group is orientated in free space and away from the ring (Figure 1.5). Consequently, the shielding effect would not be as prominent and would lead to a low stereoselectivity. This correlates with the outcome observed with 5-phenyl-3-methoxyisoxazolidine (Figure 1.5).

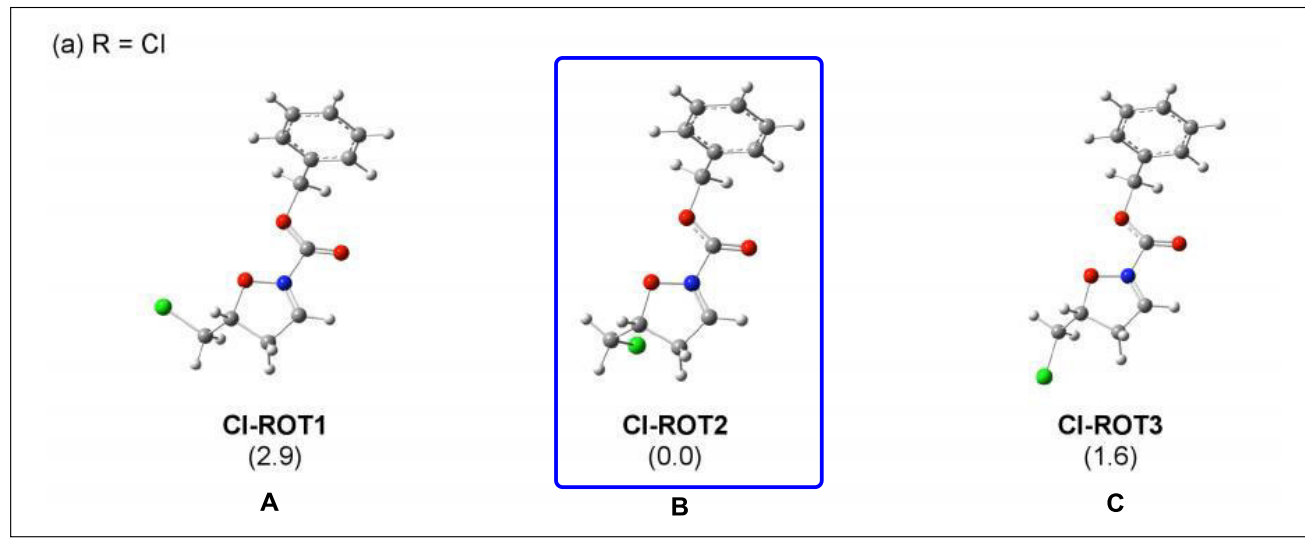

Figure 1.14. Geometry and relative energy of rotamers (in $\mathrm{kcal} / \mathrm{mol}$, in parentheses) as determined by DFT at the B3LYP/6-31G* +ZPE level. 

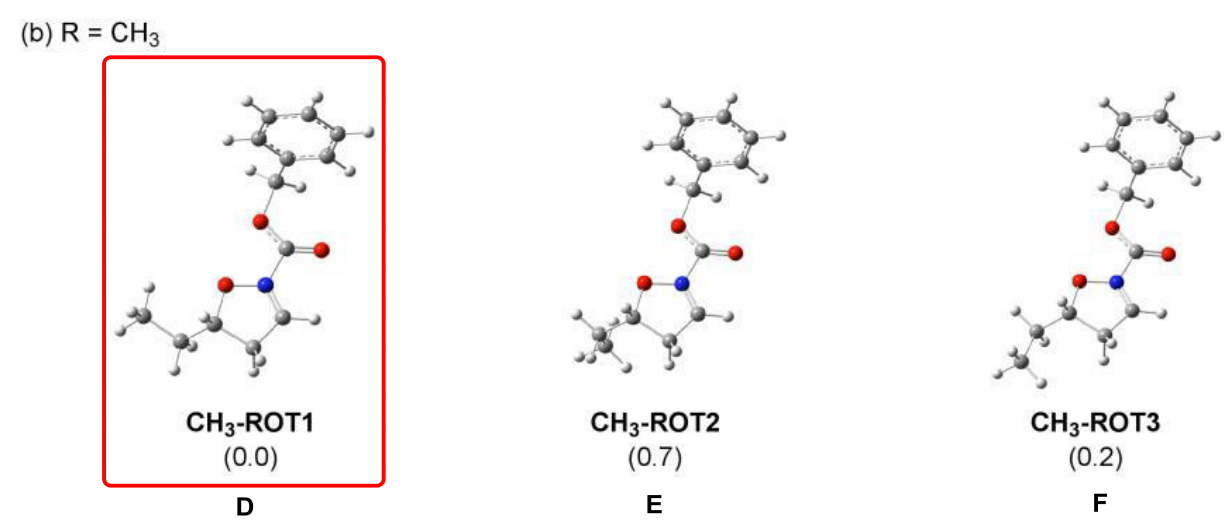

Figure 1.15. Geometry and relative energy of rotamers (in $\mathrm{kcal} / \mathrm{mol}$, in parentheses) as determined by DFT at the B3LYP/6-31G* +ZPE level.

\subsubsection{Alternative Sakurai With Silyl Ketone Thioacetal}

At this juncture, an alternative synthetic route was explored (Scheme 1.43). A silyl ketone thioacetal 1.43.1 was employed in the Sakurai step with the expectation that the product 1.43.2 could then be coupled with the hydrazine 1.16.2 fragment with the use of either $\mathrm{Cu}(\mathrm{I})$ or $\operatorname{Ag}(\mathrm{I})$ catalysis to give 1.37.2 (Scheme 1.43).

Preparation of 1.43.1 was smooth following the general procedure reported by Yang $e t$ al. and was used directly in the next step to prepare 1.43.2 (Scheme 1.43).$^{54}$ This was to avoid decomposition of $\mathbf{1 . 4 3 . 1}$ given its instability, thus, purification methods like distillation and flash chromatography were omitted. Unfortunately 1.37.2 was never obtained suggesting that 1.43.1 was not stable under the reaction conditions and hence, decomposed. The synthesis towards (+)-negamycin resumed via the initial synthetic plan, which followed the Sakurai allylation with allyltrimethylsilane (Scheme 1.43). 


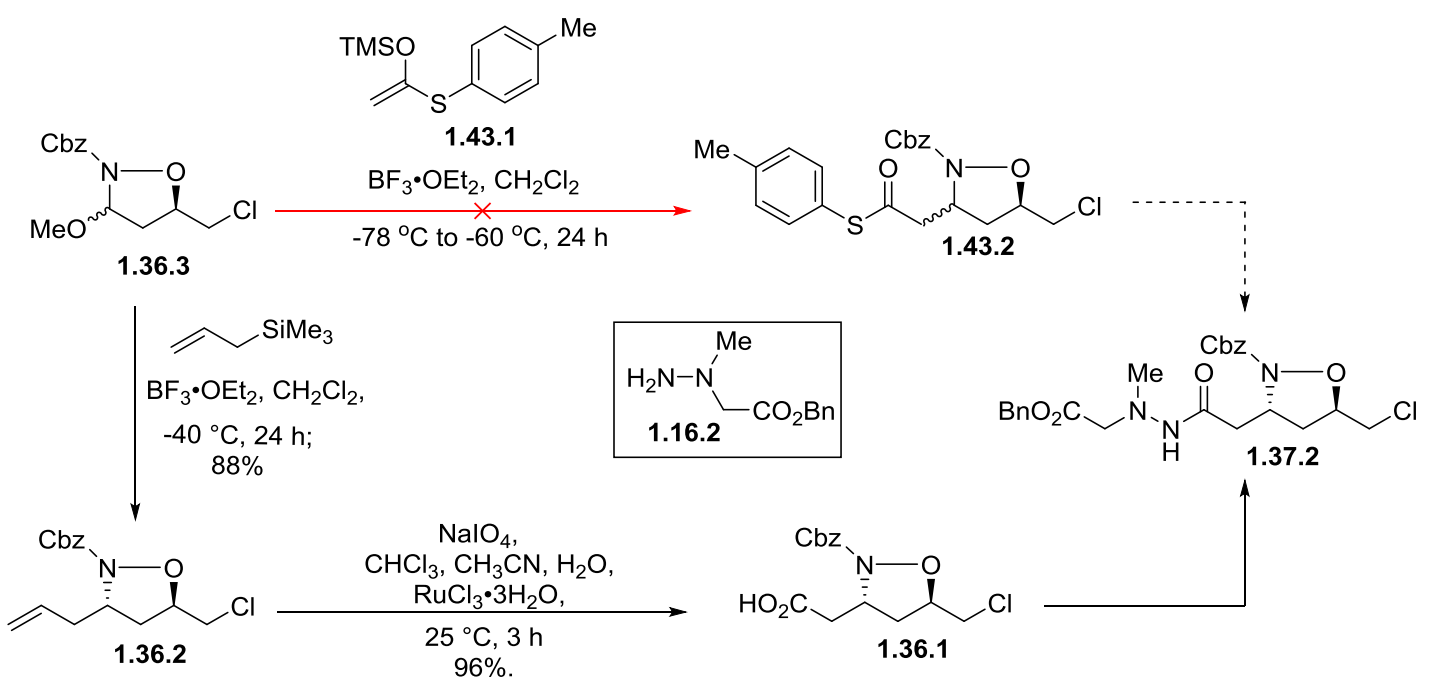

Scheme 1.43 Sakurai reaction with silyl ketone thioacetal 1.43.1.

Oxidation with $\mathrm{RuO}_{4}$

The synthesis towards (+)-negamycin continues with the $\mathrm{Ru}(\mathrm{VIII})$-catalyzed oxidative cleavage of terminal olefin 1.36.2 in the presence of ruthenium tetraoxide under Sharpless' conditions to furnish the carboxylic acid 1.36.1 in good 96\% yield (Scheme 1.44 and refer to Scheme 1.37). Ruthenium tetroxide, a powerful and aggressive oxidant, ${ }^{55}$ was generated in situ by the oxidation of ruthenium(III) trichloride $\left(\mathrm{RuCl}_{3}\right)$ with sodium metaperiodate (Scheme 1.44). ${ }^{56}$ The conditions employed are mild and the roles of $\mathrm{RuO}_{4}$ and $\mathrm{NaIO}_{4}$ are reflected in Scheme 1.44. ${ }^{57}$ Acetonitrile was used as a solvent along with the $\mathrm{CHCl}_{3} / \mathrm{H}_{2} \mathrm{O}$ system because it is believed to act as a ligand for ruthenium and stabilize the intermediates hence improving the effectiveness of catalytic action of $\mathrm{RuO}_{4}$ is reported. ${ }^{57}$ Although $\mathrm{RuO}_{4}$ can oxidize other functional groups such as arenes, the reactivity can be controlled by careful selection of reaction conditions. Alternatively, carboxylic acid 1.36.1 might be obtained by a combination of ozonolysis followed by PDC-oxidation of the resulting aldehyde but it would be two steps. 

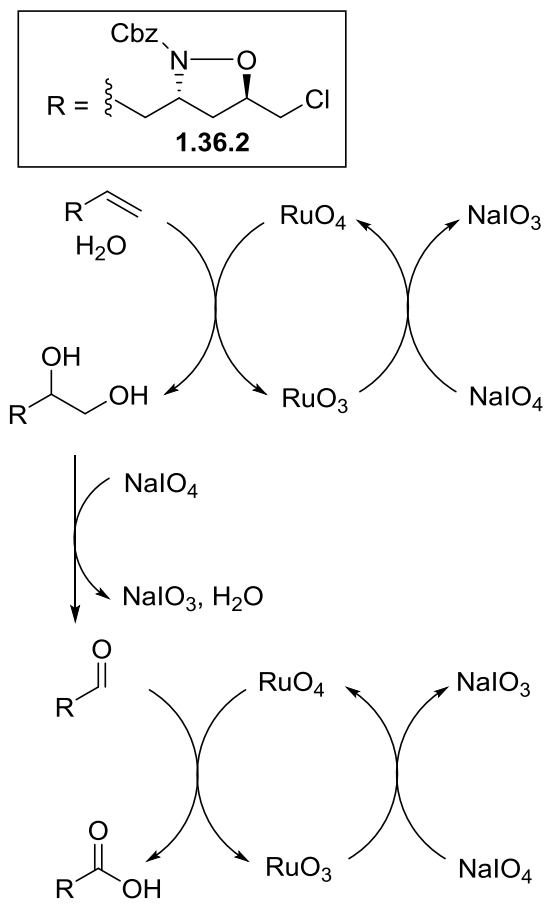

Scheme 1.44. Ruthenium(VIII) catalysed $\mathrm{C}=\mathrm{C}$ bond cleavage.

\subsubsection{Hydrazide Formation}

The coupling of the carboxylic acid 1.36.1 to the methylhydrazine fragment 1.16.2 to give hydrazide 1.37.2 was realized after several attempts (refer to Scheme 1.37). The methyl hydrazine was unavailable due to shipping restrictions, but the hydrazine sulfate salt $\mathbf{1 . 4 5 . 1}$ could be obtained (Scheme 1.45). The free methyl hydrazine was liberated under basic conditions. The initial challenge in preparing fragment 1.16.2 was the insolubility of the $\mathrm{N}$-methylhydrazine salt 1.45.1 in organic solvent such as dichloromethane, giving rise to a nonhomogenous reaction mixture. The problem was resolved by the change to a more polar solvent, dimethylsulfoxide. However, its subsequent removal proved difficult as multiple extractions were required that caused a significant lost of the polar product 1.16.2 since it is soluble in the aqueous layer. In addition, flash chromatography required a very polar eluent for the purification of 1.16.2, which resulted in the co-elution of DMSO. Therefore, in search of a compromise, the 
choice of solvent reverted to dichloromethane and the reaction time was extended to $24 \mathrm{~h}$ stirring in triethylamine prior to the treatment with bromo benzylacetate (Scheme 1.45).

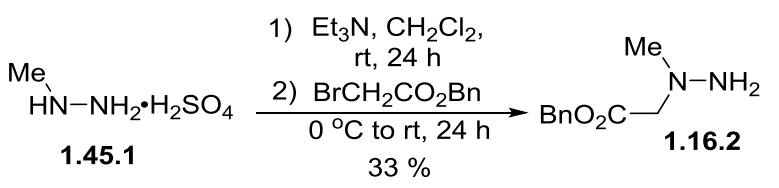

Scheme 1.45. Synthesis of N-methylhydrazine fragment 1.16.2.

After many attempts, it was found that the most effective peptide coupling could be carried out by the conversion of carboxylic acid 1.36.1 to the acid chloride prior to coupling with methyl hydrazine 1.16.2 in the presence of pyridine to give 1.37.2 in the best yield of $70 \%$ (refer to Scheme 1.37).

\subsection{Optimization}

Several coupling strategies were explored to optimize the yield by reducing the amount of by-products, and to facilitate the purification of the desired hydrazide 1.37.2 (Table 1.3). The use of coupling agent 1,3-dicyclohexylcarbodiimide (DCC) was reported by Hegedus et al. to have caused purification problems due to the insoluble dicyclohexylurea by-product, which was inseparable from the amide product. ${ }^{20 a}$ Nonetheless, DCC was employed but gave a low yield of $44 \%$ (Table 1.3, entry 1). Therefore the reaction was repeated with the addition of acylation catalyst such as dimethylaminopyridine (DMAP) ( $3 \mathrm{~mol} \%$ and $10 \mathrm{~mol} \%$ ) in the presence of triethylamine as a base (Table 1.3, entries 2 and 3). DMAP is known to be a powerful acylation catalyst ${ }^{58}$ which can dramatically increase the reaction rate ${ }^{58}$ by assisting the nucleophilic amine attack through a charge-separated adduct formation. ${ }^{58}$ Unfortunately, the outcome was not aligned with expectations as the use of DMAP did not result in a marked improvement in the yield. When DMAP was employed in a 1:1 molar equivalent with the substrate, the yield only marginally improved by $7 \%$ from $51 \%$ to $58 \%$ (Table 1.3 , entry 4 ). It was decided to activate 
the carboxy component as anhydrides using $\operatorname{EtOC}(\mathrm{O}) \mathrm{Cl}$ and $i$ - $\mathrm{BuOC}(\mathrm{O}) \mathrm{Cl}$ while keeping the base $\left(\mathrm{Et}_{3} \mathrm{~N}\right)$ unchanged (Table 1.3, entries 5 and 6). Unfortunately, after overnight stirring at room temperature, the amide yield was found to decrease to as low as $44 \%$. The intermediate acylation agent was switched to the use of acid chlorides. The acid 1.36.1 was treated with oxalyl chloride $\mathrm{CO}_{2} \mathrm{Cl}_{2}$ in the preformation of the acid chloride from (Table 1.3, entries 7-9). There was a substantial yield improvement from $40 \%$ to $60 \%$ with the addition of DMAP (Table 1.3, entries 7 and 8). Gratifyingly, the desired result was obtained by changing the base to pyridine while keeping other parameters the same (Table 1.3,entry 9).

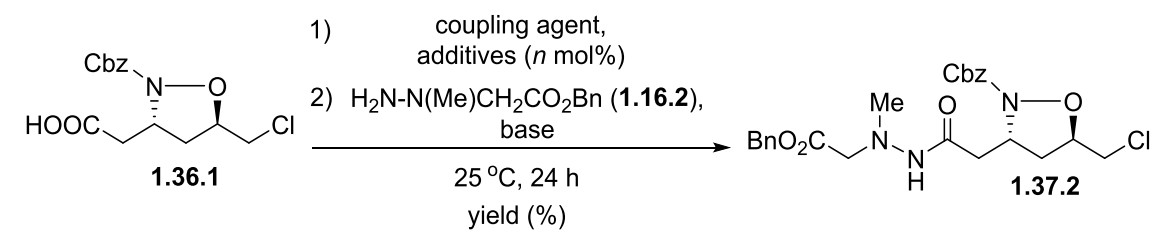

\begin{tabular}{|c|c|c|c|c|}
\hline entry & $\begin{array}{l}\text { coupling method/ } \\
\text { additives }(n \mathrm{~mol} \%)\end{array}$ & $\begin{array}{c}\text { temperature } \\
\left({ }^{\circ} \mathrm{C}\right)\end{array}$ & $\begin{array}{l}\text { reaction } \\
\text { time }(\mathrm{h})\end{array}$ & $\begin{array}{c}\text { yield } \\
(\%)\end{array}$ \\
\hline 1 & DCC & 0 to 25 & 24 & 44 \\
\hline 2 & DCC/DMAP (3 mol\%), $\mathrm{Et}_{3} \mathrm{~N}$ & 0 to 25 & 24 & 48 \\
\hline 3 & DCC/DMAP (10 mol\%), Et ${ }_{3} \mathrm{~N}$ & 0 to 25 & 24 & 51 \\
\hline 4 & DMAP (1.0 equiv), $\mathrm{Et}_{3} \mathrm{~N}$ & 25 & 24 & 58 \\
\hline 5 & ${ }^{i} \mathrm{BuOCOCl}, \mathrm{Et}_{3} \mathrm{~N}$ & 25 & 24 & 48 \\
\hline 6 & $\mathrm{EtOCOCl}, \mathrm{Et}_{3} \mathrm{~N}$ & 25 & 24 & 44 \\
\hline 7 & $\mathrm{CO}_{2} \mathrm{Cl}_{2}, \mathrm{Et}_{3} \mathrm{~N}$ & 25 & 24 & 40 \\
\hline 8 & $\mathrm{CO}_{2} \mathrm{Cl}_{2}$, DMAP (3 mol\%), $\mathrm{Et}_{3} \mathrm{~N}$ & 25 & 24 & 60 \\
\hline 9 & $\mathrm{CO}_{2} \mathrm{Cl}_{2}$, Pyridine & 25 & 24 & 70 \\
\hline
\end{tabular}

Table 1.3. Conditions adopted for coupling between 1.36.1 and 1.16.2. 
This can be attributed to the milder basicity of pyridine than triethylamine, which circumvent the tendency of ketene formation under basic conditions (Scheme 1.46). ${ }^{59}$ In addition, pyridine acts as a nucleophilic catalyst and undergoes an addition-elimination reaction with the acyl chloride to give a reactive trigonal acylpyridinium salt intermediate, accelerating the reaction. ${ }^{59}$

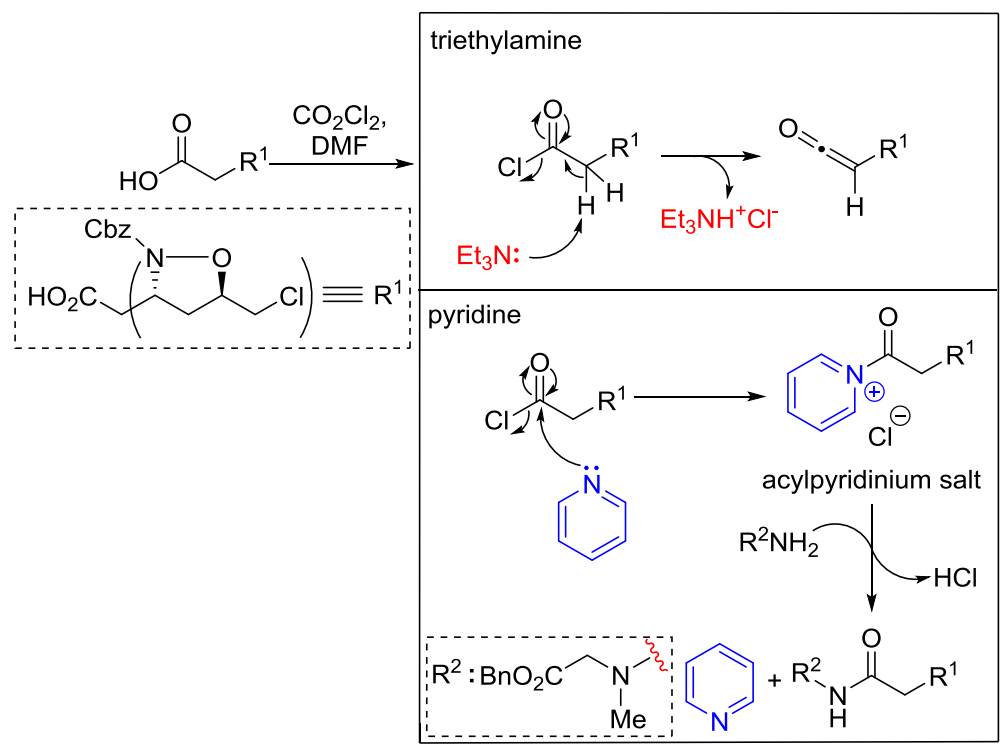

Scheme 1.46. Ketene formation by triethylamine treatment of acid chloride and the role of pyridine.

The success of the coupling step was confirmed by ${ }^{1} \mathrm{H}$ NMR as the peaks corresponding to the two protons at $\mathrm{C} 2$ of $\mathbf{1 . 3 7 . 2}$ was shifted downfield (Figure 1.16). Additionally, the highlighted protons have become diastereotopic after coupling. 


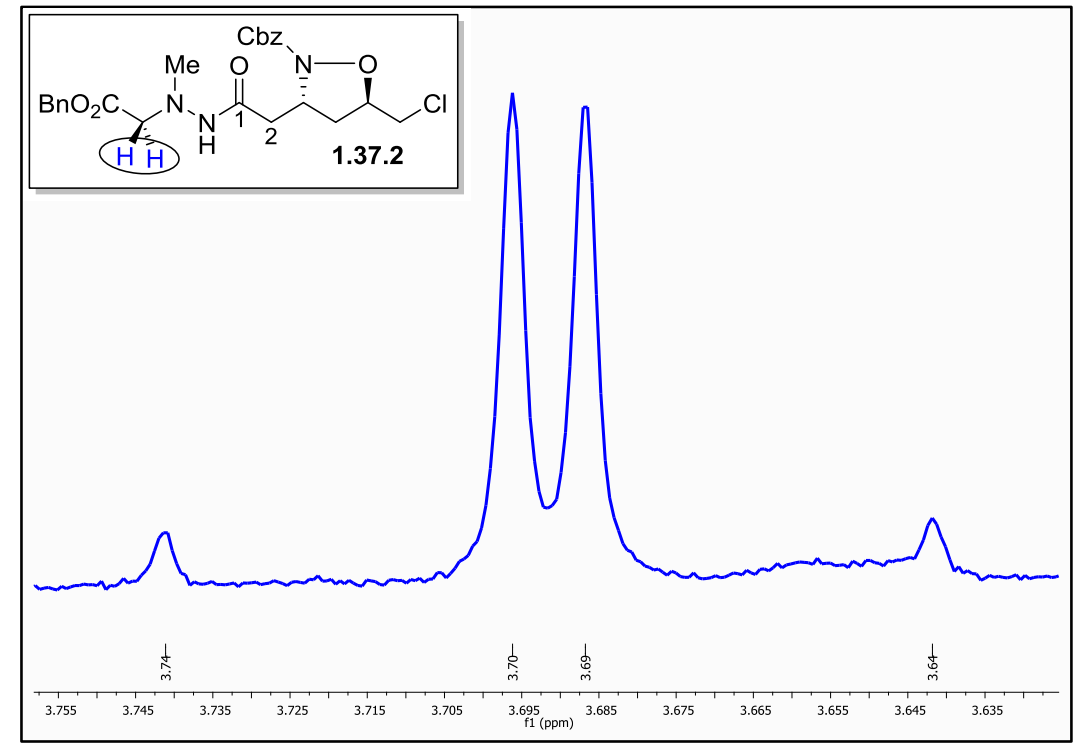

Figure 1.16. ${ }^{1} \mathrm{H}$ NMR of diastereotopic protons of 1.37.2.

\subsubsection{Azide Formation}

With the hydrazide 1.37.2 at hand, the azide group was finally incorporated (refer to Scheme 1.37). The previously failed attempts of installing the azide functionality in place of the chloro substituent led to the use of of a different azide source such as tetra- ${ }^{n}$ butylammonium azide. ${ }^{60}$ The substrate $\mathbf{1 . 3 7 . 2}$ and tetra- $^{n}$ butylammonium azide mixture in tetrahydrofuran was brought to reflux for 24 hours to afford the desired azide $\mathbf{1 . 3 7 . 3}$ with a high $84 \%$ yield. The success prompted questions regarding the azide reactivity in $\mathrm{NaN}_{3}$ and ${ }^{n} \mathrm{Bu}_{4} \mathrm{~N}^{+} \mathrm{N}_{3}{ }^{-}$in DMF.

It is postulated that, in DMF, $\mathrm{NaN}_{3}$ exists as an ion pair thus reducing the reactivity of the azide anion. ${ }^{61}$ The azide anion remains close to the counterion instead of the electrophile 1.37.2 leading to no nucleophilic initiation. On the contrary, tetra- ${ }^{n}$ butylammonium azide is not ion paired in DMF and hence the azide anion is naked, resulting in the successful azide substitution. 


\subsubsection{Completion of the (+)-Negamycin Synthesis}

The final global hydrogenation of $\mathbf{1 . 3 7 . 3}$ was achieved smoothly to produce the natural product (+)-negamycin after purification using ion-exchange chromatography with a weak cationic exchange media amberlite CG-50 in the $\mathrm{NH}_{4}{ }^{+}$form eluting with water followed by $0.5 \%$ aqueous ammonium hydroxide (Figure 1.17). The ninhydrin active fractions were collected and water was removed via distillation to afford (+)-negamycin, confirmed by ${ }^{1} \mathrm{H}$ NMR where the chemical shifts matched the literature ${ }^{20 a}$ reported for the natural product (Figure 1.17 and Table 1.4). However, in the reported literature of the ${ }^{1} \mathrm{H}$ NMR of (+)-negamycin by Hegedus et al., protons $\alpha$ to-CONH group $\left(\mathrm{H}_{2}\right)$ are reported as an app.doublet of doublet (dd) with coupling constants 1.9 and $7.0 \mathrm{~Hz}$ while other literature cited a doublet with a coupling constant of $7.0 \mathrm{~Hz} \cdot{ }^{13,14 \mathrm{~b}, 15,20 \mathrm{a}, 20 \mathrm{c}, 20 \mathrm{~g}, 20 \mathrm{~h}, 201}$ Additionally, in 2014, Olivier et al. reported the $\mathrm{H}_{2}$ protons as a multiplet. ${ }^{62}$ These did not coincide with the ${ }^{1} \mathrm{H}$ NMR analysis of our synthesized (+)-negamycin as two sets of doublet of doublet $(2 \mathrm{x}$ dd) were observed (Figure 1.17). 


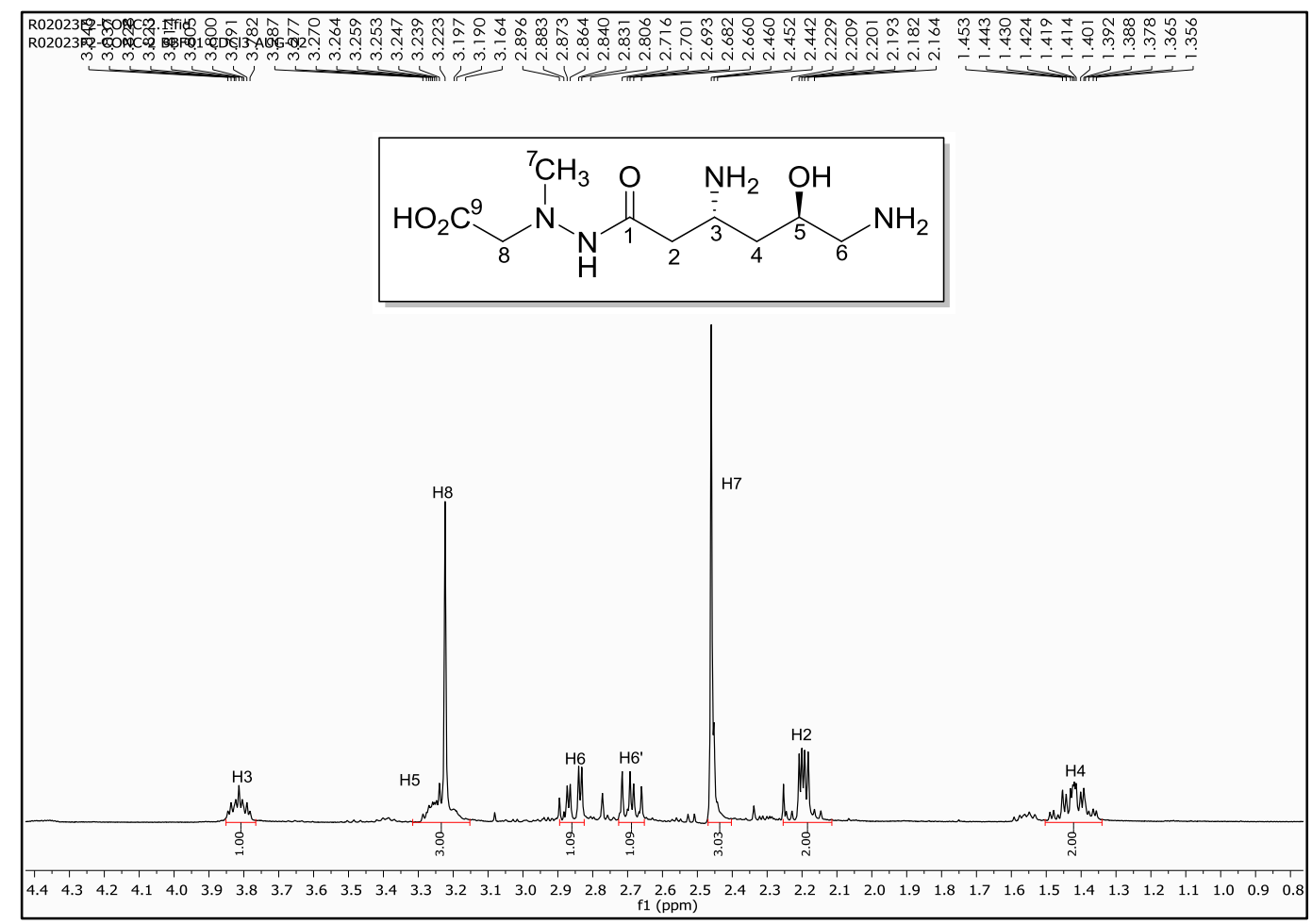

Figure 1.17. ${ }^{1} \mathrm{H}$ NMR of natural product negamycin.

\begin{tabular}{|c|c|c|c|c|c|c|}
\hline \multicolumn{4}{|c|}{ Literature $^{20 \mathrm{a}}(+)-$ Negamycin } & \multicolumn{3}{|c|}{ Synthesized (+)-Negamycin } \\
\hline no. & $\delta_{\mathrm{H}}$ & integral/mult & $J(\mathrm{~Hz})$ & $\delta_{\mathrm{H}}$ & integral/mult & $J(\mathrm{~Hz})$ \\
\hline H4 & 1.44 & $2 \mathrm{H}(\mathrm{m})$ & - & $1.48-1.37$ & $2 \mathrm{H}(\mathrm{m})$ & - \\
\hline $\mathbf{H} 2$ & 2.22 & $2 \mathrm{H}(\mathrm{dd})$ & $1.9,7.7^{[a]}$ & 2.40 & $1 \mathrm{H}(\mathrm{dd})$ & $6.4,14.6$ \\
\hline & & & & 2.15 & $1 \mathrm{H}(\mathrm{dd})$ & $7.3,14.5$ \\
\hline H7 & 2.46 & $3 \mathrm{H}(\mathrm{s})$ & - & 2.46 & $3 \mathrm{H}(\mathrm{s})$ & - \\
\hline H6 & 2.88 & $1 \mathrm{H}(\mathrm{dd})$ & $3.1,13.2$ & 2.85 & $1 \mathrm{H}(\mathrm{dd})$ & $3.5,13.2$ \\
\hline H8 & 3.23 & $2 \mathrm{H}(\mathrm{s})$ & - & 3.23 & $2 \mathrm{H}(\mathrm{s})$ & - \\
\hline H5 & 3.29 & $1 \mathrm{H}(\mathrm{m})$ & - & $3.27-3.22$ & $1 \mathrm{H}(\mathrm{m})$ & - \\
\hline H3 & 3.84 & $1 \mathrm{H}(\mathrm{m})$ & - & $3.84-3.79$ & $1 \mathrm{H}(\mathrm{m})$ & - \\
\hline
\end{tabular}

[a] misassignment of $\mathrm{H} 2$ protons by Hegedus et al.

Table 1.4. ${ }^{1} \mathrm{H}$ NMR of natural product negamycin. 
Therefore, a homodecoupling experiment was carried out on the proton region 3.84$3.79 \mathrm{ppm}$ of proton $\beta$ to $-\mathrm{CONH}$ group $\left(\mathrm{H}_{3}\right)$ with the expectation of observing two sets of doublet $\left(2 \mathrm{x} \mathrm{d}\right.$ ) corresponding to $\mathrm{H}_{2}$ (Figures 1.18 and 1.19). The result was consistent with the expectation in that the coupling pattern of two sets of doublets $(2 \mathrm{x} \mathrm{d})$ were observed at 2.34 ppm and 2.33 ppm with an average coupling constant of $6.6 \mathrm{~Hz}$ (Figure 1.19).

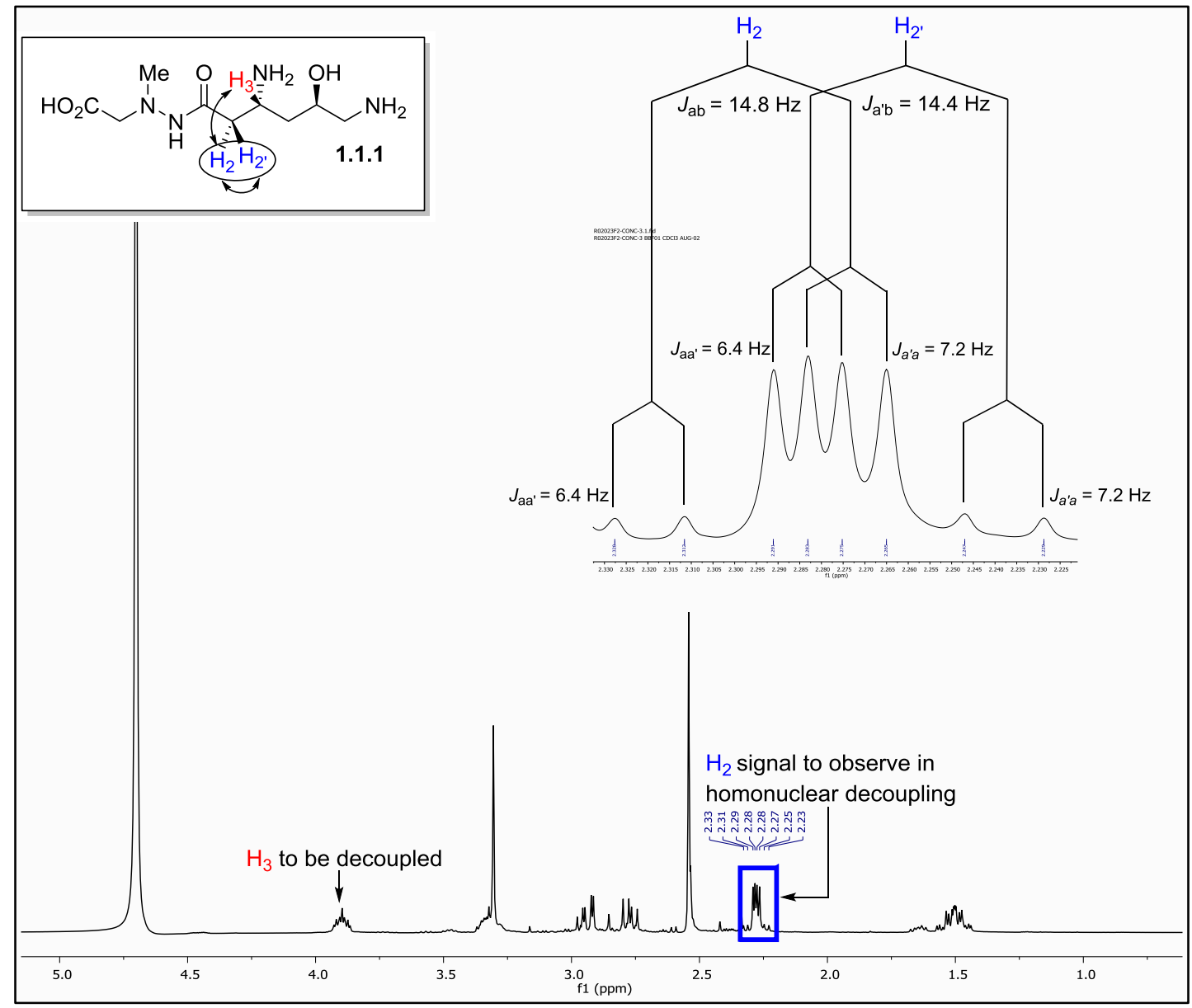

Figure 1.18. Homodecoupling of proton $\beta$ to $-\mathrm{CONH}$ group. 


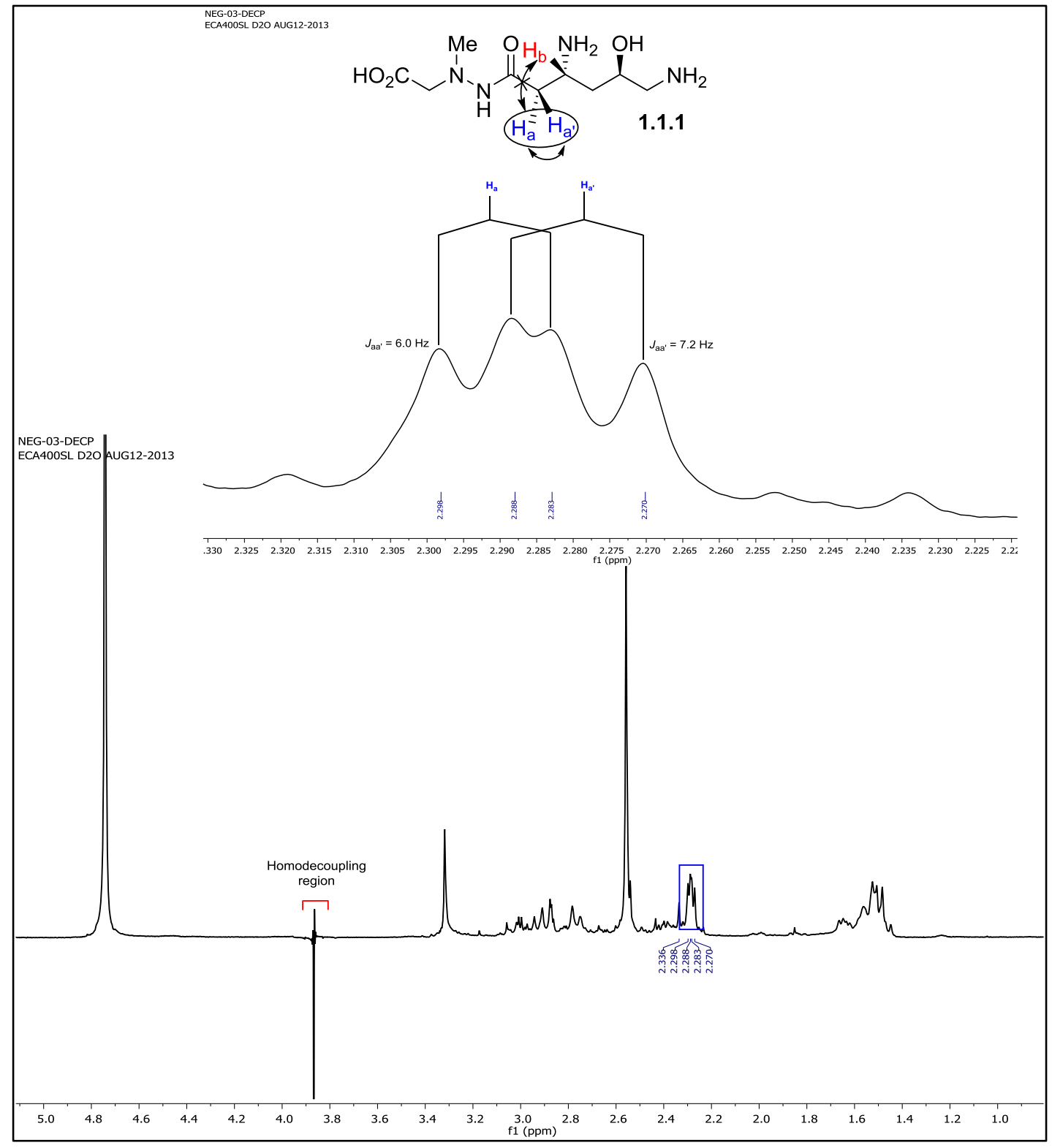

Figure 1.19. Homodecoupling of proton $\beta$ to $-\mathrm{CONH}$ group. 


\subsubsection{Conclusion}

In conclusion, (+)-negamycin was prepared from $(S)$-epi-chlorohydrin in a total of 10 steps and $23 \%$ overall yield. This contributes as an improvement in both synthetic steps and overall yield in contrast to some of the reported lengthy syntheses of (+)-negamycin. ${ }^{20 a}$, $20 \mathrm{~g}$ Moreover, the physical properties of (+)-negamycin compared favorably with the reported literature. ${ }^{20 \mathrm{~g}}$ and the spectroscopic data obtained were in excellent agreement with those reported by Umezawa et al. ${ }^{14 \mathrm{a}}\left\{[\alpha]_{\mathrm{D}}^{23.1}+2.5^{\circ}\left(c 2.0\right.\right.$ in $\left.\mathrm{H}_{2} \mathrm{O}\right) ;$ lit. $^{14 \mathrm{~b}}[\alpha]_{\mathrm{D}}^{29.0}+2.5^{\circ}\left(c 2.0, \mathrm{H}_{2} \mathrm{O}\right)$. 
This page has been intentionally left blank. 
This page has been intentionally left blank. 


\section{Chapter 2}

\subsection{Introduction}

2.1.1 Importance of 1,3-Diamines

2.1.2 Strategies to Access 1,3-Diamines

2.1.3 Natural Products Containing 1,3-Diamines

2.1.4 Lycopodium Alkaloids 
This page has been intentionally left blank. 


\subsection{Introduction}

\subsubsection{Importance of 1,3-diamines}

Chiral 1,3-diamines with syn- or anti-configuration are ubiquitous structural features in natural products ${ }^{63}$ as well as various bioactive compounds. ${ }^{64}$ In addition, they are a significant substructure present in chiral reagents, ${ }^{65}$ ligands, ${ }^{66}$ and catalysts in asymmetric synthesis,${ }^{67}$ enabling the synthesis of chiral compounds in high yields and selectivity. ${ }^{68}$ And they contribute in field of medicine for instance, in the area of cytostatic cancer ${ }^{64 c, 64 d}$ as well as in the research associated with protease inhibitors (AIDS). ${ }^{69}$ These reflect the many application of 1,3-diamines, and thus highlighting the imperative need in methods for its preparation.

\subsubsection{Strategies to access 1,3-diamines}

The common strategies for the synthesis of 1,3-diamines consist of reduction of pyrazolidines 2.1.2 (Scheme 2.1, eq. 1), ${ }^{70}$ diimines, ${ }^{71}$ azides 2.1.7 (Scheme 2.1, eq. 2), ${ }^{72}$ pyrimidines 2.1.9 and 2.1.12 (Scheme 2.1, eq. 3 and eq. 4 ), ${ }^{73}$ quaternary imminium salts 2.1.16 generated in situ by aminoalkylation of enamines 2.1.14 (Scheme 2.1, eq. 5) ${ }^{74}$ and the reduction of $\beta$-amino imines 2.1.20 (Scheme 2.1, eq. 6). ${ }^{75}$ There is also the stereodivergent cyclization effected by intermolecular allylic substitution of urea analogues 2.1.24 and 2.1.25 (Scheme 2.1, eq. 7) ${ }^{76}$ as well as an asymmetric allylic amination of $\mathbf{2 . 1 . 2 8}$ followed by an amino aziridine formation and opening to give 2.1.30 (Scheme 2.1, eq. 8) ${ }^{77}$ Apart from that, there is also an independent introduction of the two amines ${ }^{78}$ and the proline-catalyzed $\alpha$-amination ${ }^{79}$ recently developed by Kumar et al. 
2) $\mathrm{PhMgBr}, \mathrm{PhMe}$
$\mathrm{H} \mathrm{H}_{2} \mathrm{O}$

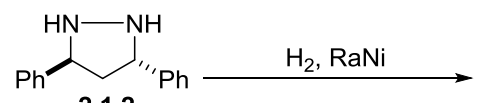
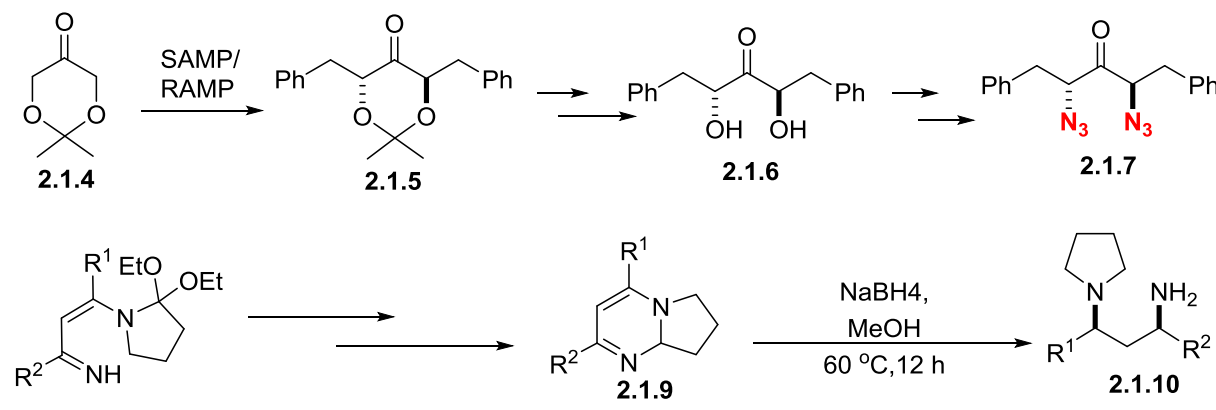

2.1.8

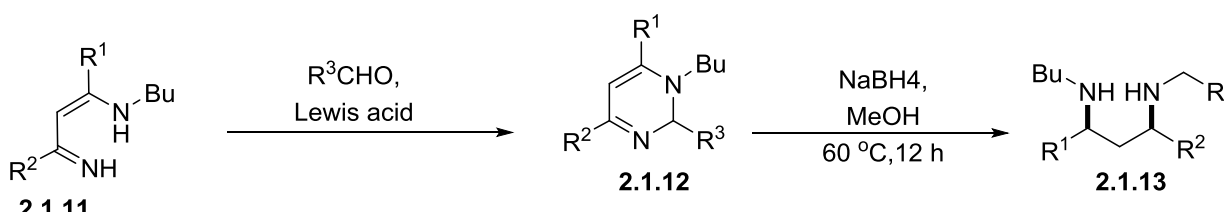

2.1.11
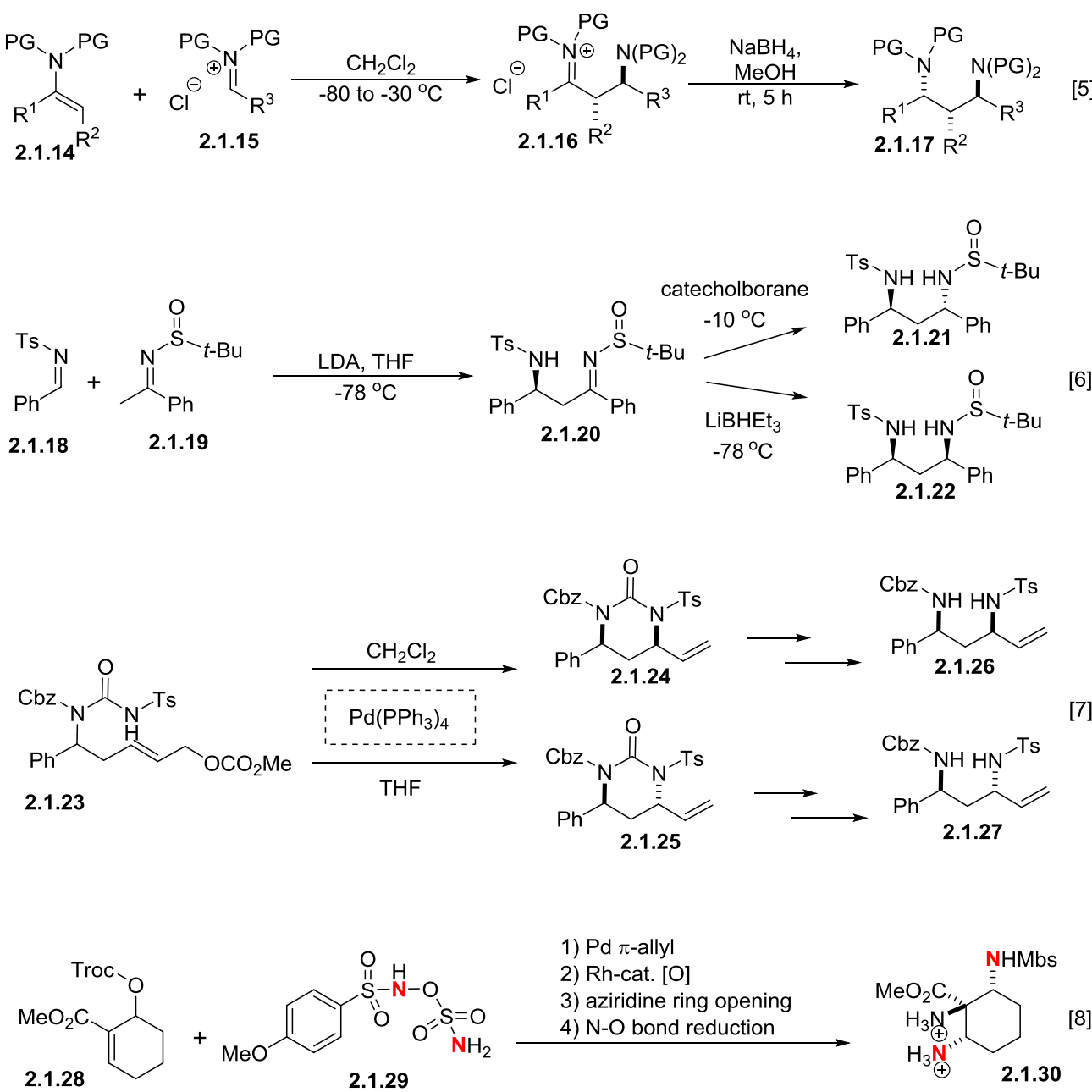

Scheme 2.1. Strategies to access 1,3-diamines. 


\subsubsection{Natural Products Containing 1,3-diamines}

Enantiomerically enriched 1,3-diamines play a crucial role in the total synthesis of pharmacologically active molecules such as batzelladine $\mathrm{F}$, which was isolated from a red Jamaican sponge (Figure 2.1). ${ }^{64 \mathrm{~h}}$ The batzelladines alkaloids are a part of the growing family of guanidine alkaloids, ${ }^{64 g}$ which may emerge as a possible lead for AIDS treatment because the majority of the batzelladines alkaloids could be the remedy for an autoimmune disorder. ${ }^{6 \mathrm{~g}}$, $64 \mathrm{~h}$, ${ }^{80}$ Saxitoxin, a potent neurotoxin, is also known as paralytic shellfish poison. It is a potent inhibitor of voltage-gated sodium channels and helps in the understanding of protein function of electrical transmission in cells. ${ }^{81}$ The structural diversity of 1,3-diamines continues in spectinomycin where the diamines are not a part of the fused tricyclic ring system (Figure 2.1). Spectinomycin, isolated from Streptomyces spectabilis, is a member of the aminoglycoside antibiotics $^{82}$ and it possesses potent activity against the penicillin-resistant gonorrhea bacteria. ${ }^{83}$ Lanesoic acid, another marine-derived substance bearing a tetra-hydropyrimidine ring and isolated from sponges of Theonella swinhoei in Indo-Pacific, shows cytotoxicity against human tumor cell lines. ${ }^{84}$
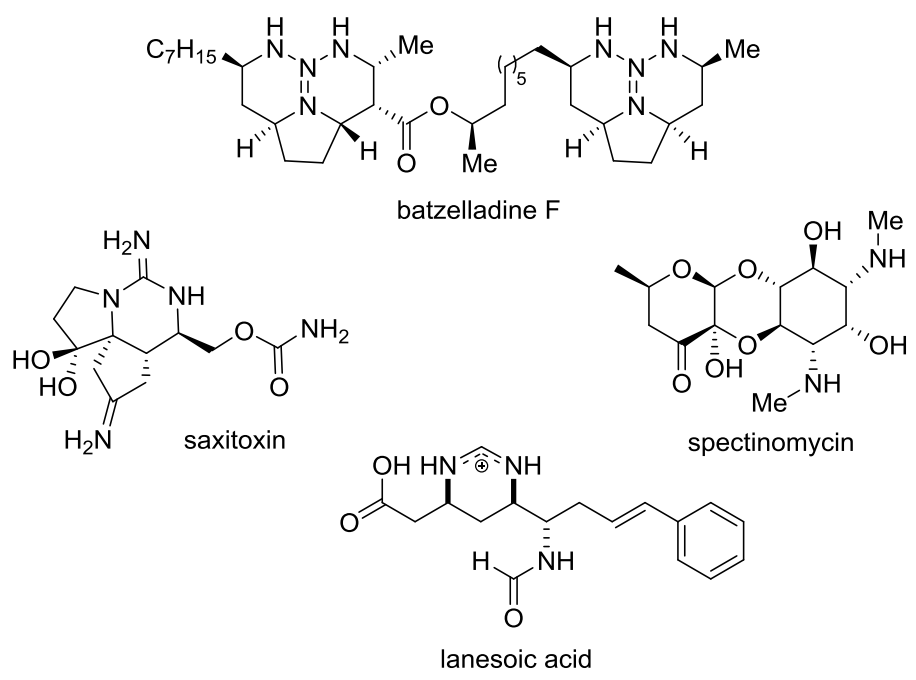

Figure 2.1. Bioactive natural products bearing 1,3-diamines. 


\subsubsection{Lycopodium Alkaloids}

The Lycopodiaceae family is a group of vascular plants, and over 500 species can be found around the world with a majority flourishing in tropical regions (Figure 2.2). ${ }^{85}$ They produce biologically active polycyclic alkaloids with complex and unique structures that have ignited a widespread interest in the field of medicinal, biogenetics and biology ${ }^{86}$ as well as in synthetic chemistry. For instance, in 1881, Karl Bödeker isolated lycopodine from lycopodium complanatum $^{87}$ in Germany that has since seen an array of biogenetically active natural products unearthed in the lycopodium club mosses (Figure 2.2). Huperzine A, which was isolated from Lycopodium serratum Thunb is a potent acetylcholine esterase (AChE) inhibitor ${ }^{88}$ It has also been shown to aid in memory disorders related to Alzheimer's disease and to promote neurite outgrowth..$^{85 \mathrm{~b}, 89}$

Lycopodium alkaloids make for an attractive natural product target in total synthesis because of the opportunity to develop new chemical methods from the stereochemical challenges presented in the highly diverse skeletal structure. ${ }^{85 \mathrm{~b}}$ For instance, the classic tetracyclic skeleton of the lycopodane family has a rich history in organic chemistry and has been a proving ground for new approaches to the synthesis of polycyclic alkaloid targets. ${ }^{85 a}$, 90 The majority of contributions to the syntheses of lycopodium alkaloids by various research groups were carried out in linear fashion with cyclic starting materials. ${ }^{91}$ Similarly, stereoselective synthesis of alkaloid Lycoposerramine-W has also been elaborated by Takayama et al. ${ }^{85 b}$ (Figure 2.2)

The continued efforts in lycopodium research led to the eventual discovery of new and more complex alkaloids such as (-)-cernuine and (+)-cermizine D, which possess syn-1,3diamine structural motif and are classified as cernuane-type alkaloids (Figure 2.2). (-)-Cernuine and (+)-cermizine D emerged to be ideal synthetic targets for our lab because of the potential 
application of tetra-hydropyrazolidine chemistry to overcome issues related to the synthesis of syn-1,3-diamines.
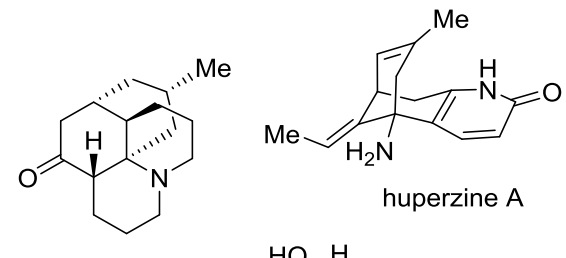

huperzine $\mathrm{A}$

lycopodine

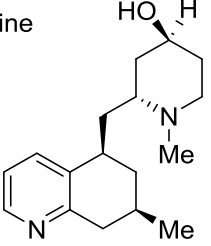

lycoposerramine-W
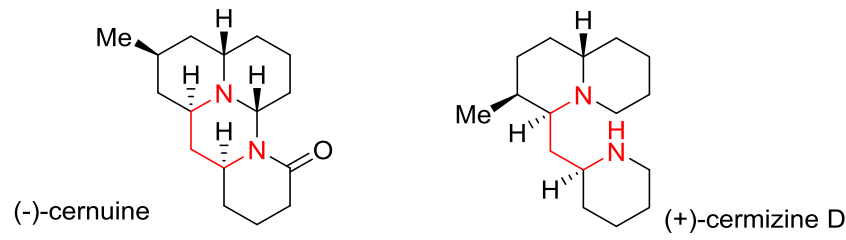

Figure 2.2. Bioactive Lycopodium alkaloids.

\subsubsection{1 (-)-Cernuine: Biological Activity and Structure}

(-)-Cernuine is part of the cernuane-type family and was isolated from the extracts of lycopodium cernuum (Figures 2.3 and 2.4) by Marion and Manske in $1948{ }^{92}$ However, it was not until 1967 that its chemical structure, a fused tetracyclic ring system bearing an aminal moiety, ${ }^{93}$ and its stereochemistry both absolute and relative was elucidated by Ayer et al. (Figure 2.4).${ }^{94}$ This was done through a combination of chemical and physical techniques, ${ }^{95}$ and the scaffold of (-)-cernuine is considered rare in the family of Lycopodium alkaloids. ${ }^{94-96}$ (-)-Cernuine alkaloids are also known to have therapeutic potential ${ }^{97}$ and biological activities such as enzyme-inhibition to acetylcholine esterease (AchEe) ${ }^{93}$

(-)-Cernuine consists of four-fused rings that include $\delta$-lactam and syn-1,3-diamines as part of the hexahydropyrimidine moiety (Figure 2.4). It has a total of five chiral centers located at C5, 
C7, C9, C13 and C15 (Figure 2.4). The first and only stereocontrolled synthesis of (-)-cernuine was reported in 2008 by Takayama et al. ${ }^{93}$

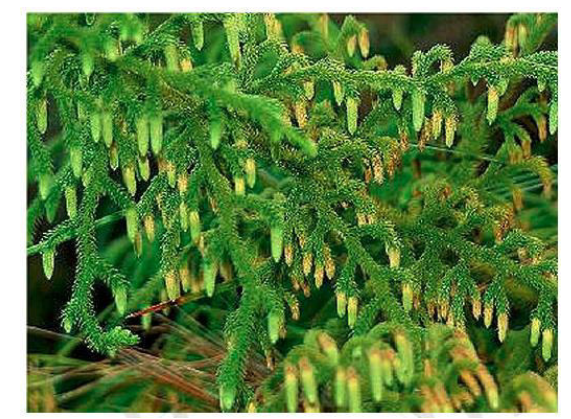

Figure 2.3. Club moss lycopodium cernuum.

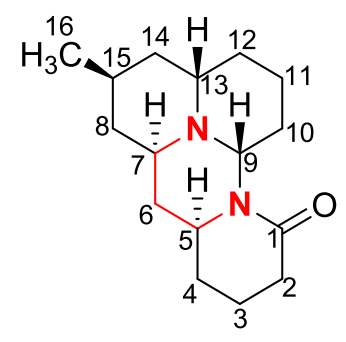

(-)-cernuine

Figure 2.4. Structure of (-)-cernuine.

\subsubsection{2 (+)-Cermizine D: Biological Activity and Structure}

In 2004, Kobayashi et al. investigated the extracts of $2.75 \mathrm{~kg}$ of lycopodium cernuиm. ${ }^{98}$ The lycopodium cernuum was collected in Okinawa Prefecture in $2001,{ }^{96 a}$ and led to the isolation of a family of cermizines alkaloids. Amongst the cermizines identified, $5.5 \mathrm{mg}$ of (+)-cermizine D was isolated and has shown to exhibit cytotoxicity against murine lymphoma L1210 cells at $7.5 \mu \mathrm{g}$ per $\mathrm{mL} .{ }^{86} \mathrm{It}$ is also categorized as a cernuane-type alkaloid because of its cernuane skeleton structure.

(+)-Cermizine D contains a quinolizidine scaffold and a piperidine ring joined together at C6. There are a total of 4 chiral centers at C5, C7, C13 and C15 (Figure 2.5). The relative 
stereochemistry was derived from NOESY interactions between protons $\mathrm{H} 7$ and $\mathrm{H} 15$ while proton correlations from TOCSY and COSY NMR and proton-carbon correlations from HMBC NMR analysis were employed to establish the quinolizidine and piperidine ring. There have been three previously reported syntheses of (+)-cermizine D by Takayama et al..${ }^{99}$ in 2009 , by Carter et al., ${ }^{100}$ in 2012 and a recent synthesis by Comins et al., in $2015 .{ }^{101}$

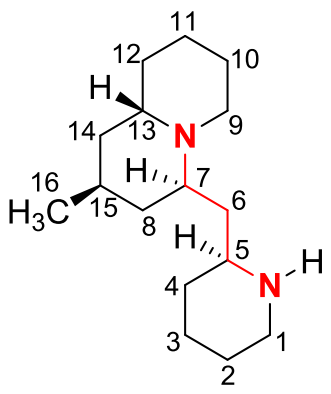

(+)-cermizine D

Figure 2.5. Structure of (+)-cermizine D.

\subsubsection{Syntheses of (-)-Cernuine and (+)-Cermizine D}

\subsubsection{Synthesis by Takayama et $a l .{ }^{93}$}

\section{(-)-Cernuine}

In 2008, Takayama et al. reported the first stereocontrolled total synthesis of (-)-cernuine by employing chiral starting material (+)-citronellal (Scheme 2.2). The retrosynthesis began with two disconnections, at the $\mathrm{N}-\mathrm{C} 9$ bond and $\mathrm{C} 2-\mathrm{C} 3$ bond, to give the bicylic lactam 2.2.1 bearing the homoallylamine handle. The aminal function was expected from the reductive cyclization of the free amine in $\mathbf{2 . 2 . 1}$ with carbonyl at $\mathrm{C} 1$. The subsequent ringclosing metathesis to establish $\mathrm{C} 2-\mathrm{C} 3$ would complete the tetracyclic ring construction of (-)-cernuine. Homoallylamine 2.2.1 could be accessed by aminoallylation of aldehyde 2.2.2 that could be prepared from aminoacetal 2.2.3 through asymmetric allylation and ring-closing 
metathesis. The oxazolidinone $\mathbf{2 . 2 . 4}$ can be synthesized from $\alpha$-amination of 2.2.5, which is a known derivative of (+)-citronellal (Scheme 2.2).

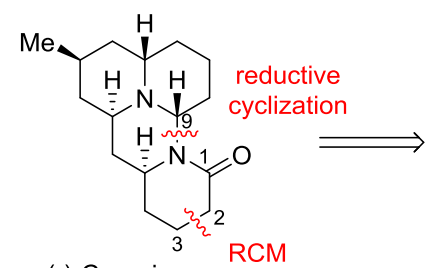

$(-)$-Cernuine

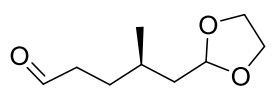

2.2.5

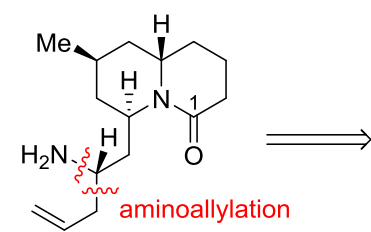

2.2.1

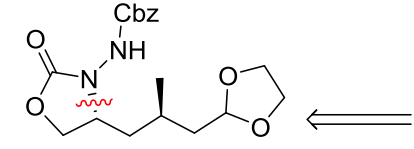

2.2.4

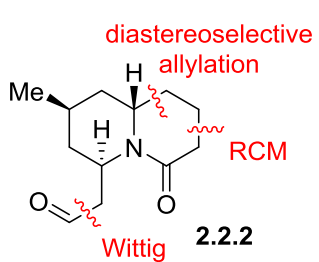

$\Downarrow$<smiles>CO[C@H]1C[C@H](C)C[C@@H]2COC(=O)N12</smiles>

Scheme 2.2. Retrosynthesis of (-)-cernuine by Takayama et al., to give smaller fragment 2.2.5.

The synthesis began with the organocatalytic $\alpha$-amination of aldehyde $\mathbf{2 . 2 . 5}$, simultaneously reduced and then treated with potassium carbonate to give oxazolidinone $\mathbf{2 . 2 . 4}$ with moderate diastereoselectivity excess $(\mathrm{de}=75 \%$ ) (Scheme 2.3 ). The following steps included the $N-N$ bond cleavage that was found most efficient with Raney nickel after deprotection of the benzyl carbamate group. The subsequent treatment of the intermediate in acidic methanol released the acetal function at C6 and resulted in 1,6-cyclization of the nitrogen on the newly formed aldehyde to give aminoacetal 2.2.3. The following Sakurai allylation of aminoacetal 2.2.3 was completely diastereoselective to give substrate 2.3.1 as a single diastereomer, which was determined by NOESY experiments. The result could be attributed to the bottom face axial attack on the acyliminium intermediate and the Fürst-Platner concept (Scheme 2.3). ${ }^{102}$ 


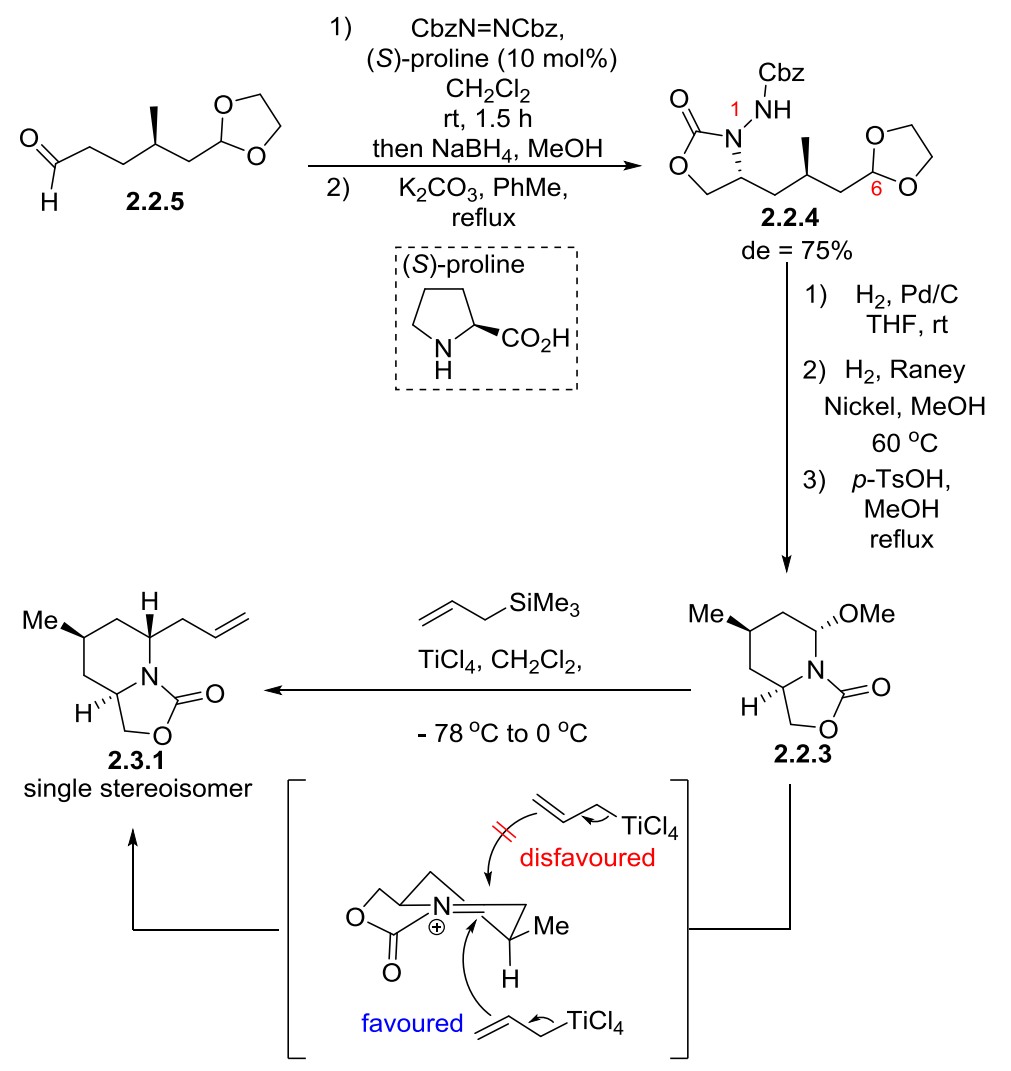

Scheme 2.3. Synthesis of substrate 2.3.1 from 2.2.5.

The synthesis continued from diastereomer 2.3.1 towards the formation of quinolizidinone 2.4.2 (Scheme 2.4). The oxazolidinone was hydrolysed under basic conditions followed by acryloylation of the newly formed amine to give acrylamide 2.4.1. Ring closing metathesis of 2.4.1 followed by hydrogenation gave the crucial intermediate quinolizidinone 2.4.2. It is significant as it offers access to other lycopodium alkaloids (Scheme 2.4).

The primary hydroxyl group of 2.4.2 was subjected to IBX oxidation to furnish aldehyde, which was homologated through Wittig olefination and followed by hydrolytic cleavage of the enol ether with hydrochloric acid to give aldehyde 2.2.2. The aldehyde 2.2 .2 underwent stereoselective aminoallylation transfer with chiral amine 2.4 .3 to give the 2-azaCope rearrangement product homoallylamine 2.2.1 with high $94 \%$ diastereomeric excess. ${ }^{103}$ The stereochemistry at $\mathrm{C} 5$ was proposed through the mechanism driven by chair-transition states 
(Scheme 2.4). The relative stereoconfiguration received validation in the later stages through NOESY experiments.
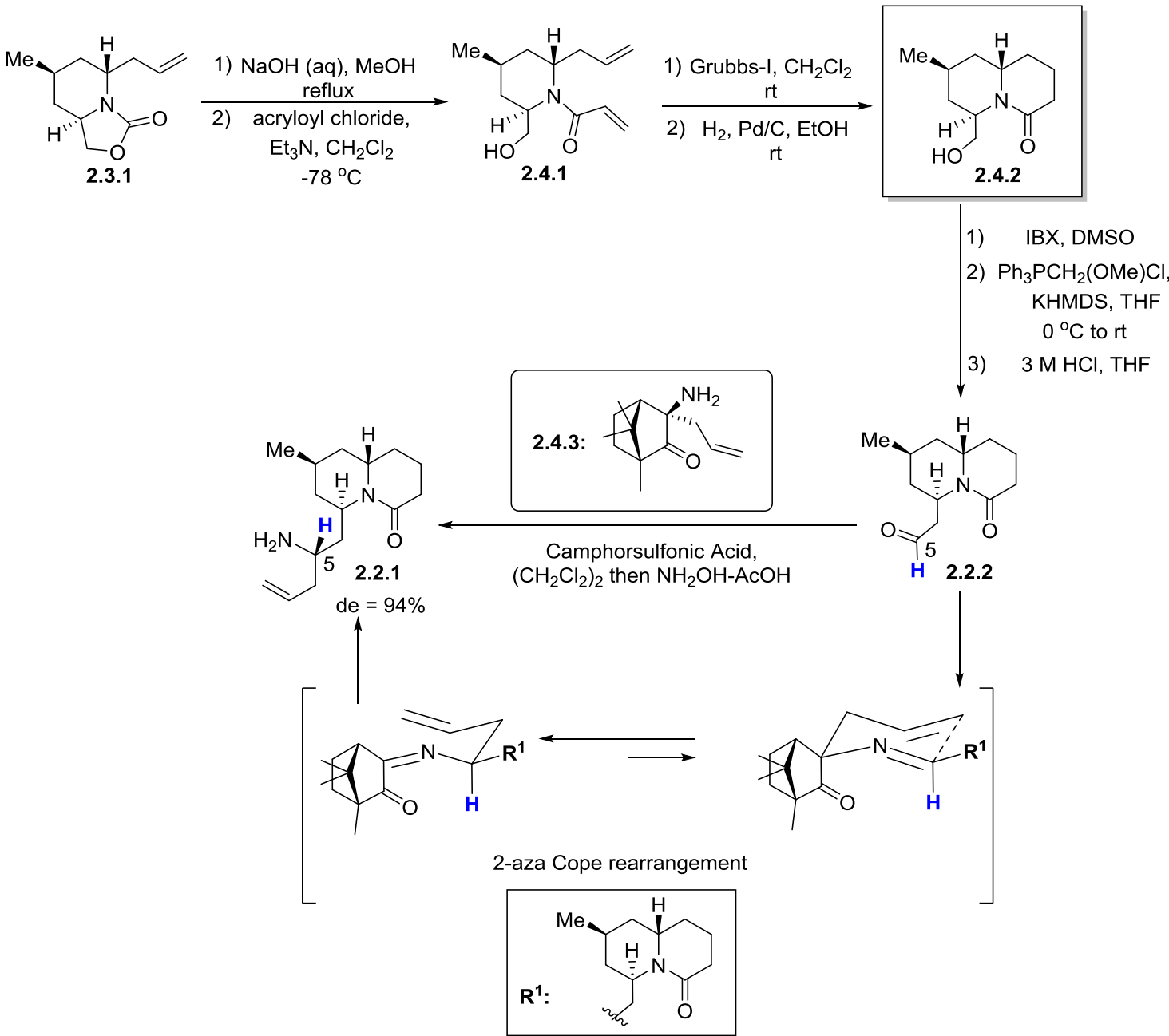

Scheme 2.4. Synthesis of key intermediate quinolizidine 2.4.2 and homoallylamine 2.2.1.

The aminal function in (-)-cernuine was constructed from a reductive cyclization of 2.2.1 in the presence of titanium(IV) chloride and sodium borohydride (Scheme 2.5). Other reductants such as DIBAL-H and Red-Al led to over reduction. Stereoselective reduction of amidine 2.5.1 was possible as it was proposed that the hydride attack occurs preferentially from the convex face. The aminal 2.5.2 was directly exposed to acryloyl chloride in the presence of triethylamine to give the acrylamide intermediate, which was treated with Grubbs-II followed 
by hydrogenation to furnish (-)-cernuine and conclude the total synthesis of 19 steps (Scheme 2.5).

In overall, (-)-cernuine was synthesized in a diastereoselective fashion by employing substrate controlled asymmetric reactions such as the Sakurai allylation and reduction of amidine 2.5.1. The group's strategy of using the Grubbs-II and hydrogenation combination to construct 2 of the 4 rings of (-)-cernuine, namely, the quinolizidine and pyridinone rings was efficient as it consistently gave high yields with little by-products.

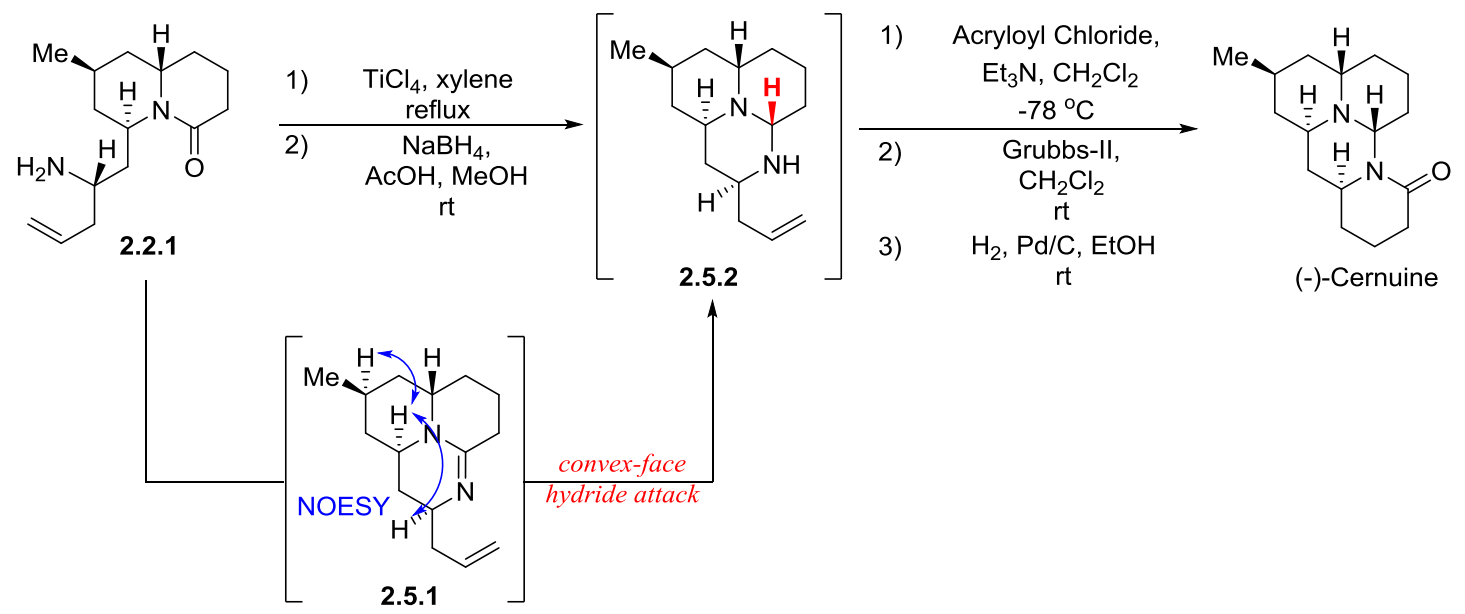

Scheme 2.5. Synthesis of (-)-cernuine from homallylamine 2.2.1.

\section{$\underline{(+)-C e r m i z i n e ~ D}$}

Takayama et al., reported the first total synthesis of (+)-cermizine D in 2008 by using the same intermediate that led to the synthesis of (-)-cernuine. ${ }^{93}$ The quinolizidinone $\mathbf{2 . 4 . 2}$ has been used by the group to derive cermizine $\mathrm{C}$ and senepodine $\mathrm{G}$ (Figure 2.6).

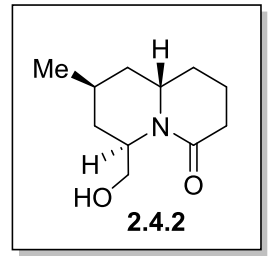

Figure 2.6. Important common intermediate quinolizidinone 2.4.2. 
The retrosynthetic analysis of (+)-cermizine $\mathrm{D}$ began with the reduction of the piperidinone ring of 2.6.1 (Scheme 2.6). The piperidinone ring of 2.6.1 was envisaged from a ring-closing metathesis which would be the result from the acryloylation of homoallylalmine 2.2.1. The synthesis of (+)-cermizine D required only 5 steps from 2.2.1 (Scheme 2.6).

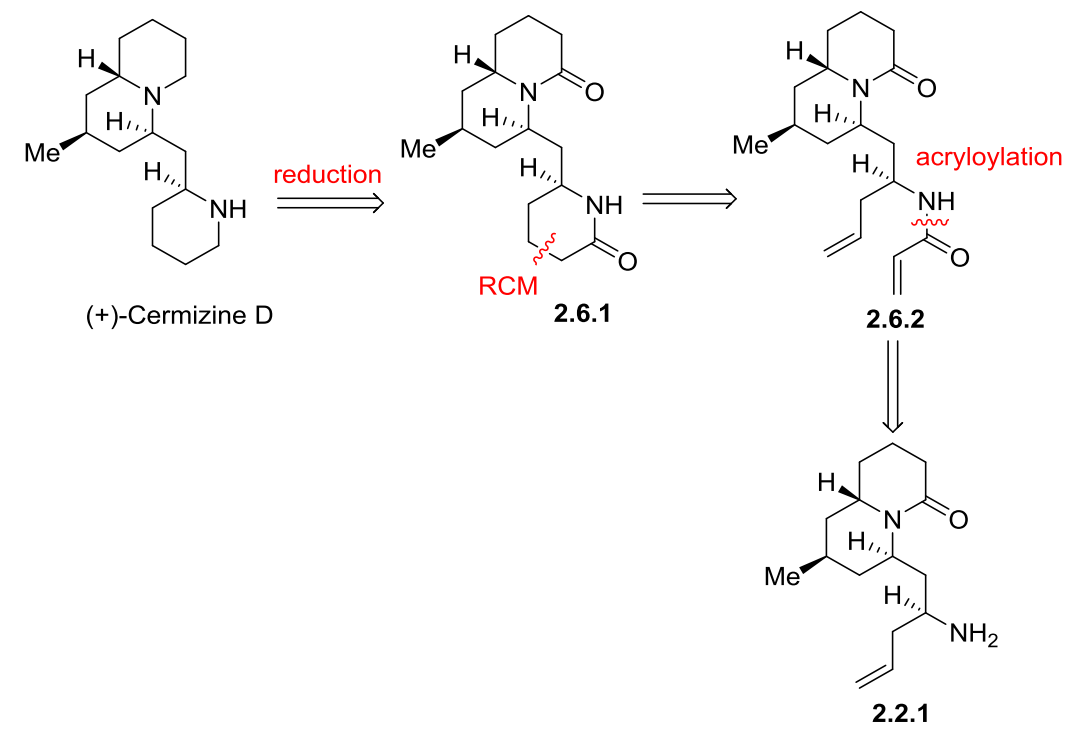

Scheme 2.6. Retrosynthetic analysis of (+)-cermizine D by Takayama et al.

The acryloylation of homoallylamine 2.2.1 was followed by the ring-closing metathesis using Grubbs-II and the subsequent hydrogenation gave the 2-piperidinone 2.6.1 in excellent 95\% yield over two steps (Scheme 2.7). The final steps involved reduction of bisamide 2.6.1 using lithium aluminium hydride and trifluoroacetic acid to give the (+)-cermizine D salt (Scheme 2.7). The salt obtained matched the spectroscopic data reported by Kobayashi. However, the optical rotation was different between synthetic and natural cermizine D. Therefore, the absolute configuration of (+)-cermizine $\mathrm{D}$ could not be entirely confirmed 
<smiles>C=CCC(N)CC1CC(C)C[C@H]2CCCC(=O)N12</smiles>

2.2.1

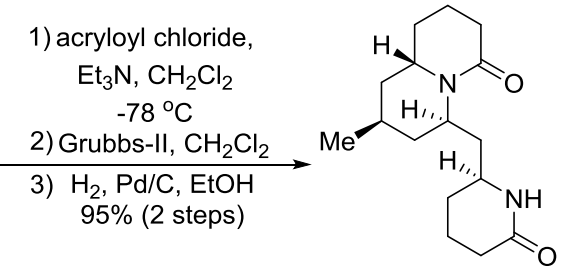

2.6.1
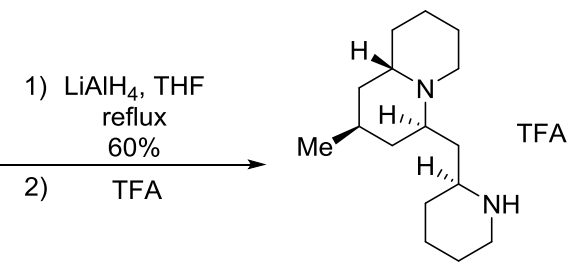

$(+)$-Cermizine $D$

Scheme 2.7. Synthesis of (+)-cermizine D from homoallylamine 2.2.1.

\subsubsection{Synthesis by Carter et al. $^{100}$}

In 2012, Carter et al. reported the diastereoselective synthesis of (+)-cermizine D in a total of 10 steps (Scheme 2.8). The group had also introduced a common sulfone intermediate 2.8.3 that enabled access to other lycopodium alkaloids. The retrosynthesis differs from Takayama's et al., as the final step would involve an intramolecular cyclization over a lactam reduction. The bispiperidine 2.8.1 would be cleaved at C4-C5 to give two smaller fragments, aldehyde 2.8.2 and sulfone 2.8.3. The sulfone 2.8.3 would be derived from alcohol 2.8.4, which would be accessible from stereoselective alkylation of 2.8.5 upon homologation. The piperidine 2.8.5 would be synthesized from stereocontrolled intramolecular conjugate addition of known aldehyde 2.8.6 (Scheme 2.8).

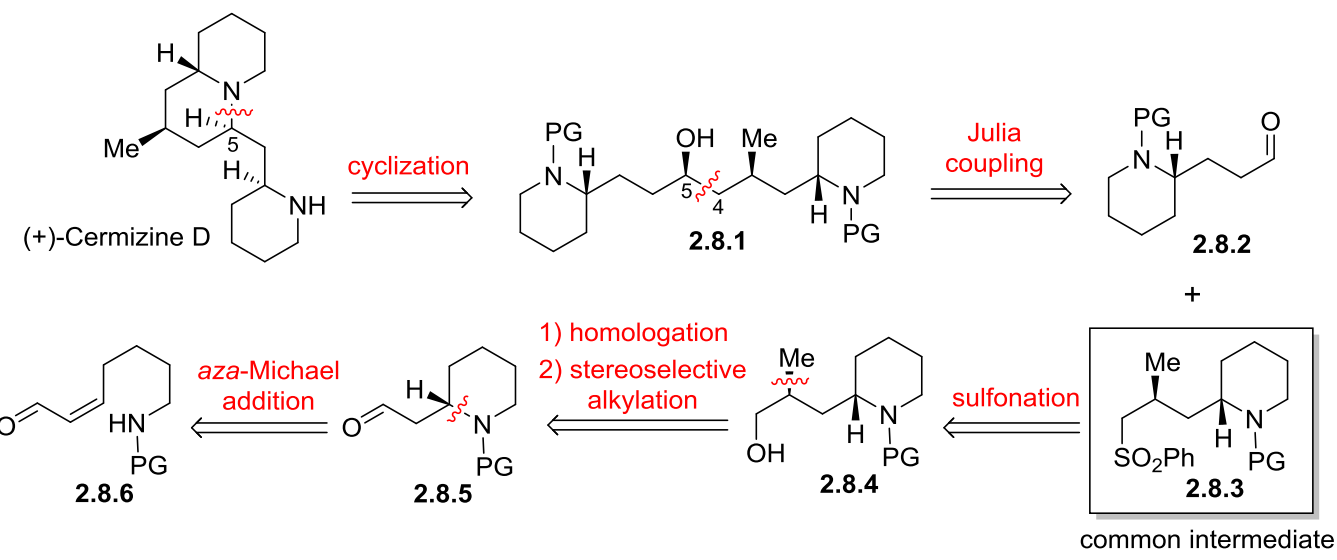

Scheme 2.8. Retrosynthetic analysis of (+)-cermizine D by Carter et al. 
The heteroatom conjugate addition of Boc-protected nitrogen of 2.9.1 was effected stereoselectively using chiral catalyst $\mathbf{2 . 9 . 3}$ to give piperidine $\mathbf{2 . 9 . 2}$ in high $96 \%$ enantioselectivity and good $85 \%$ yield (Scheme 2.9). However, the reaction required 10 days. It is unclear whether the ten days were necessary to achieve the desired outcome under mild conditions. The subsequent homologation of 2.9.2 followed by acid treatment led to the aldehyde intermediate, which was subjected to a Pinnick oxidation prior to the amidation with oxazolidinone $(S)$-2.9.4 or $(R)-2.9 .4$ ' to give 2.9.5 / 2.9.5' respectively. The choice of Evan's auxiliaries have a great impact on the diastereoselectivity. This is as the oxazolidinone $(S)$-2.9.4 gave a poor 1.5:1 diastereoselectivity at $\mathrm{C} 3$ of $\mathbf{2 . 9 . 6}$ due to a mismatched case with respect to the C5 hydrogen of the piperidine ring. An improvement was observed with the use of oxazolidinone $(R)-$ 2.9.4' $^{\prime}(\mathrm{dr}=20: 1)($ Scheme 2.9$)$

The group eventually reported alternative pathways by using Horner-WadsworthEmmons olefination of 2.9.2 followed by methylation through Gilman cuprate reagent of the $\alpha, \beta$-unsaturated sulfone $\mathbf{2 . 1 0 . 1}$ that failed to give any selectivity (Scheme 2.10). They also experimented with less-established procedures by employing halomethylphenylsufone as the electrophile for the alkylation of 2.9.6 to give 2.10.2, but it was done at the expense of increasing the number of synthetic steps without an obvious advantage. ${ }^{104}$ The alternative alkylation routes are reflected in Scheme 2.10.

The synthesis progressed from 2.9.6 with subsequent straightforward steps comprising of reductive removal of the auxiliary, sulfurization and sulfide oxidation to give the sulfone 2.9.7 (Scheme 2.9). Next, Julia coupling was performed to combine sulfone 2.9.7 and piperidine 2.9.2 to give alcohol 2.9.8 with a low diastereoselectivity $(\mathrm{dr}=1.5: 1)$. However, the undesired diastereomer-2.9.8' was transformed using a two-step oxidation and reduction method to give the same selectivity. Although the objective of recycling the undesired isomer 2.9.8' was to minimize loss of material, it appears that more resources were ultimately utilized in the process. 
In addition, at this stage, the relative configuration at C5 of 2.9.8 was not established. Nonetheless, the synthesis continued with the desulfurization of 2.9.8, deprotection of Bocgroups and the final intramolecular cyclization to give (+)-cermizine $\mathrm{D}$. The spectroscopic data agrees with those reported by Takayama et al. 

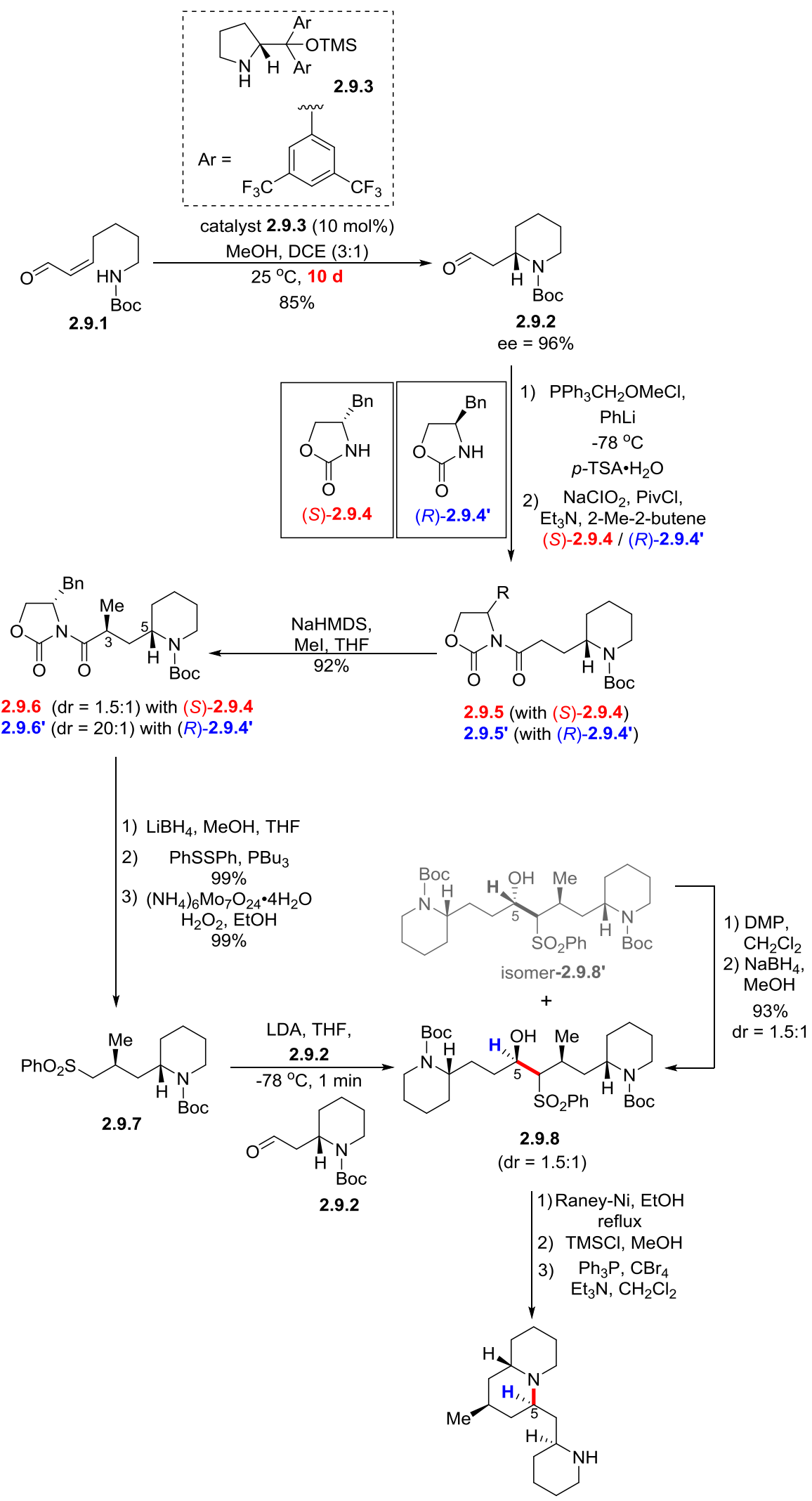

(+)-Cermizine D

Scheme 2.9. Synthesis of (+)-cermizine D from $\alpha, \beta$-unsaturated aldehyde 2.9.1. 


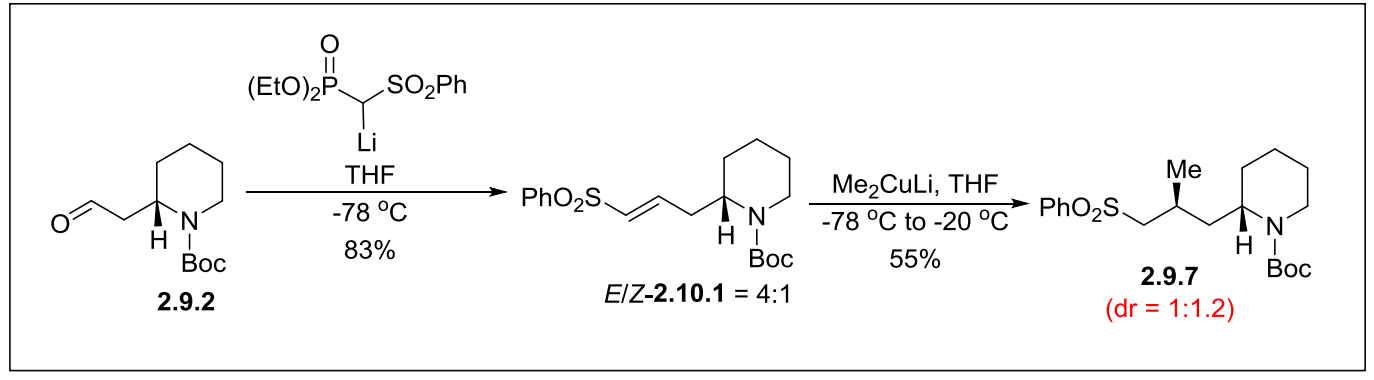

Alternative alkylation with $\alpha$-halomethvlphenvlsulfone

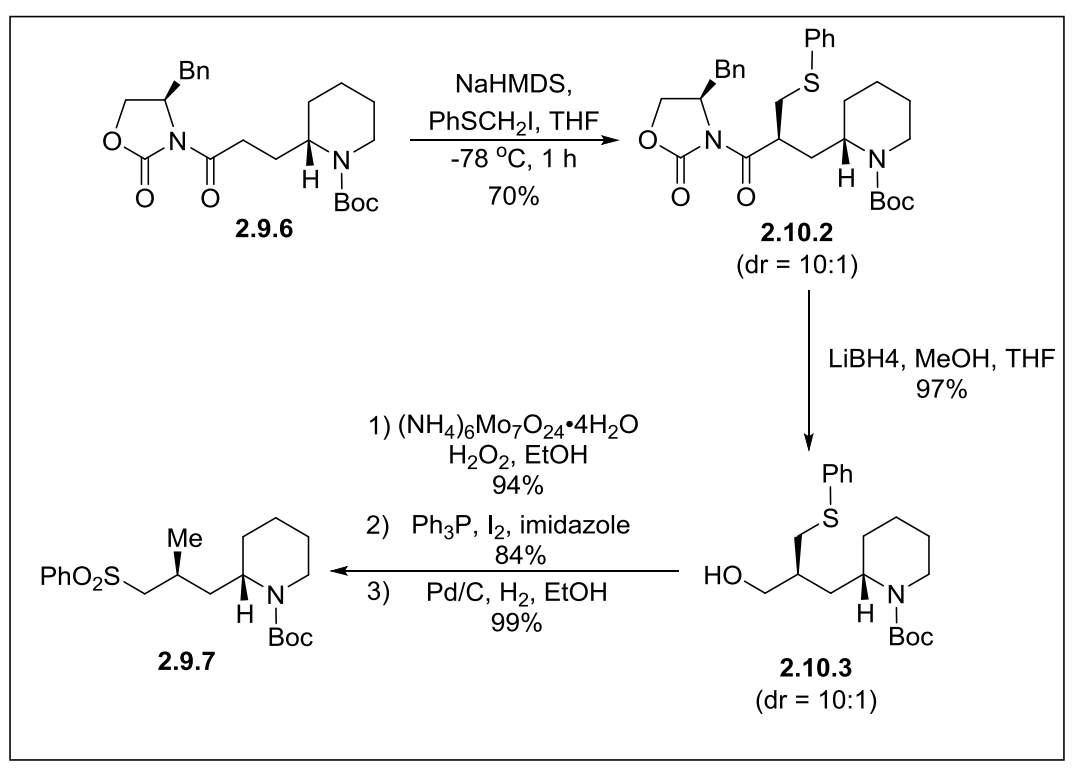

Scheme 2.10. Alternative alkylations reactions explored by Carter et al.

In summary, (+)-cermizine D was synthesized in a total of 11 steps. The starting material employed was achiral, and all five stereogenic centers were incorporated through asymmetric manipulations. These included the use of chiral catalysts and auxiliaries. The group had claimed the synthesis to be practical. This could be due to the shorter 11 steps strategy than the 19-steps route reported by Takayama's et al., but the poor diastereoselectivity in at least two of the synthetic steps, and 10-days reaction time in the first step cannot be dismissed. Therefore, it is not as practical as Takayama's et al. approach in spite of its brevity. 


\subsubsection{Synthesis by Comins et al. ${ }^{101}$}

In 2015, Comins et al. reported the stereoselective synthesis of cermizine D by utilizing the key pyridinone intermediate 2.11.2 that had been developed by the group and employed to synthesize other natural products such as (+)-lasubine (Scheme 2.11). ${ }^{105}$ The quinolizidine of cermizine $\mathrm{D}$ was envisaged from the addition of Grignard reagents to 1-acyl-4methoxypyridinium salts of 2.11.3. Cermizidine D would be expected from the reduction of pyridine of 2.11.1, which would be derived from dihydropyridone 2.11.2 through 1,4-conjugate addition, Wittig olefination and reduction. Compound 2.11.2 would be accessible from auxiliary-controlled alkylation, exploiting $N$-acylaminium chemistry of $\mathbf{2 . 1 1 . 3}$ (Scheme 2.11).
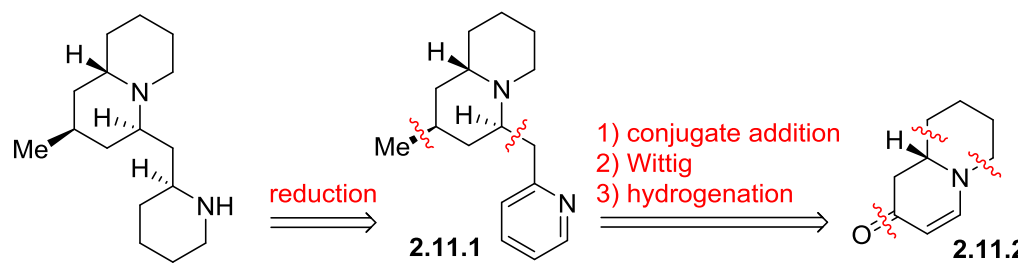

$(+)$-Cermizine D
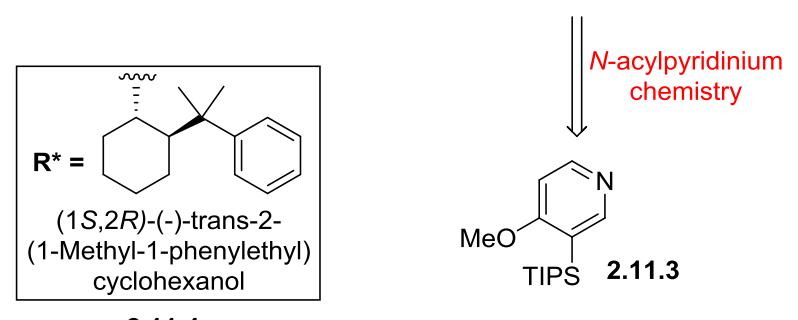

2.11.4

Scheme 2.11. Retrosynthesis of (+)-cermizine D by Comins et al.

The synthesis began with the auxiliary attachment of (-)-TCCOCOCl 2.11.4 on 4methoxy-3-TIPS-pyridine $\mathbf{2 . 1 1 . 3}$, which was followed by the stereoselective alkylation with Grignard reagent to give 2.12.1 without indication of the selectivity (Scheme 2.12). Although the same reaction had been reported by the group in 1990, the chiral auxiliaries employed then were menthol-derived auxiliaries such as (-)-menthyl or (-)-8-phenylmethyl, which gave good diastereo- and enantioselectivity (HPLC). The use of a different auxiliary such as $\mathbf{2 . 1 1 . 4}$ may 
not be regarded to give the same selectivity. Therefore, the diastereoselectivity excess of enaminone 2.12.1 should be mentioned in the current synthesis (Scheme 2.12).

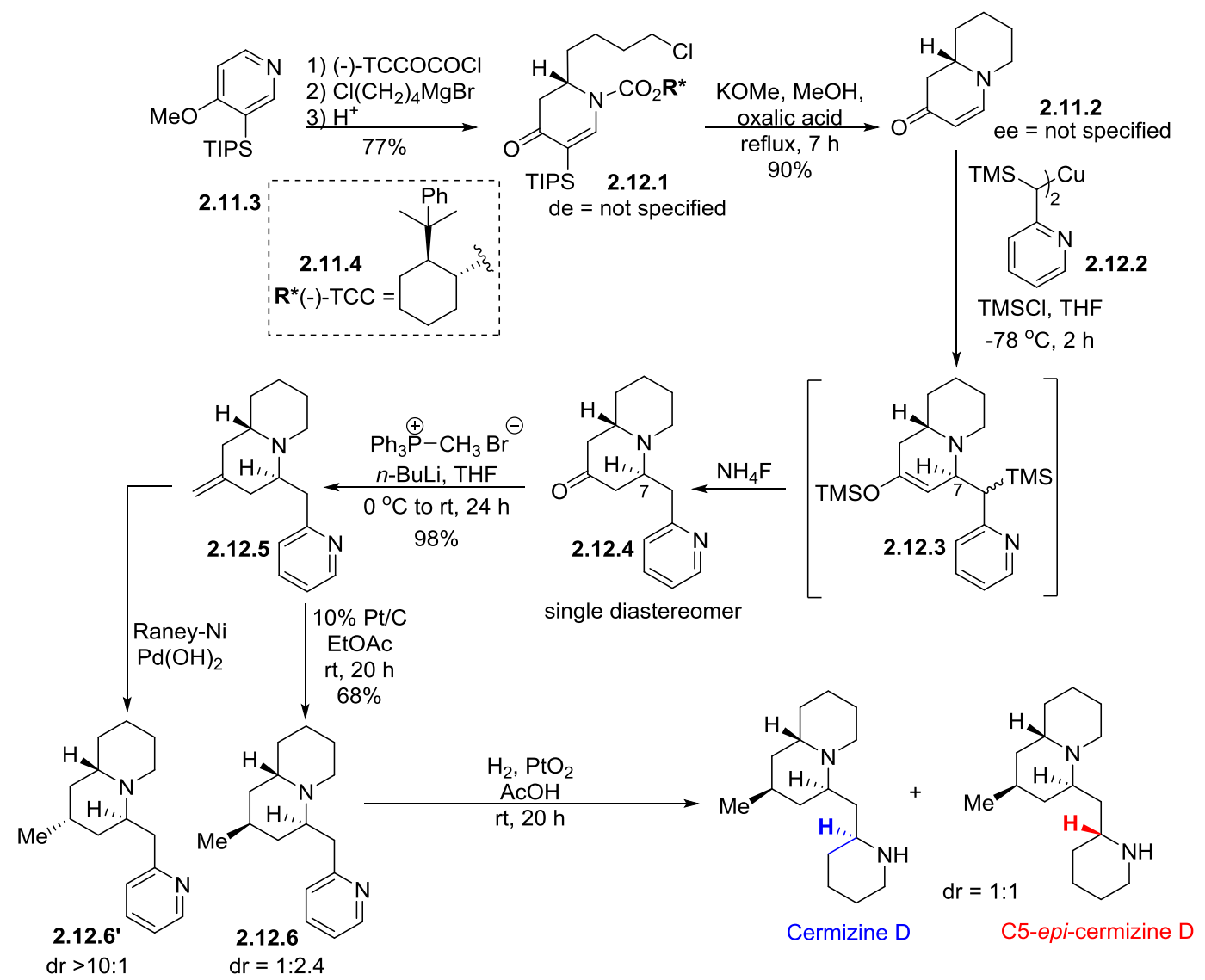

Scheme 2.12. Synthesis of (+)-cermizine D and C5-epi-cermizine D from 2.11.3.

The proceeding treatment of $\mathbf{2 . 1 2 . 1}$ with sodium methoxide in methanol gave $90 \%$ yield of the dihydropyridone $\mathbf{2 . 1 1 . 2}$. The auxiliary was recovered and recyclable (Scheme 2.12). The enantiopurity of 2.11.2 was not provided. Nonetheless, 2.11.2 was subjected to the 1,4-conjugate addition in the presence of trimethylsilyl chloride and cuprate reagent 2.12.2 to incorporate the pyridine at $\mathrm{C} 7$ of $\mathbf{2 . 1 2 . 3}$. The Lewis acid role of TMSCl was essential to promote the addition through carbonyl activation of 2.11.2. The organocuprate reagent 2.12.2 was prepared in 2 steps using commercially available 2-((trimethylsilyl)methyl)pyridine. The TMS substituent was necessary to introduce stereo-selectivity through steric hindrance and was smoothly cleaved upon aqueous work-up of 2.12.3 using tetra- ${ }^{n}$ butylammonium fluoride to give pyridine 2.12.4 
as a single diastereomer, making it an efficient step. The solid product 2.12.4 was recrystallized, giving rise to a possible X-ray crystal structure analysis. However, there was no mention regarding the relative configuration examination at $\mathrm{C} 7$ of $\mathbf{2 . 1 2 . 4}$ by means of X-ray crystallography or NOESY experiments.

After the Wittig olefination of ketone 2.12.4 to give exo-methylene 2.12.5 in high 98\% yield, the choice of reducing agent becomes key in furnishing the desired isomer 2.12.6 (Scheme 2.12). The final hydrogenation of 2.12.6 in the presence of platinum dioxide gave cermizine D as a 1:1 mixture of separable epimers. This makes the reduction step unattractive because of the maximum $50 \%$ yield. Nonetheless, the total synthesis of cermizine D was completed in 9 steps and 13\% yield from 4-methoxy-3-TIPS-pyridine 2.11.3.

\subsubsection{Summary}

In summary, the three groups have succeeded in the synthesis of lycopodium alkaloids through a flexible common intermediate. However, only the intermediate introduced by Takayama's et al. was successful in providing access to both (-)-cernuine and (+)-cermizine D. 
This page has been intentionally left blank. 
This page has been intentionally left blank. 


\section{Chapter 2}

\subsection{Objective of Present Work}

2.1.1 N,N-Heterocycles as Synthetic Intermediates

2.1.2 Retrosynthetic Analysis of Cernuine and Cermizine D

2.1.3 Results and Discussion

2.1.4 Conclusion 
This page has been intentionally left blank. 


\subsection{Objective of Present Work}

The formation of an isoxazolidine ring via an intramolecular Michael addition of the nitrogen of an $O$-substituted hydroxylamine followed by a reductive cleavage of the $\mathrm{N}-\mathrm{O}$ bond to generate $s y n-1,3$-aminoalcohols ${ }^{39}$ has inspired us to apply a similar strategy and synthesize syn-1,3-diamines via the formation of syn-tetra-hydropyrazolidines followed by a reductive cleavage of the $N-N$ bond (Scheme 2.13).

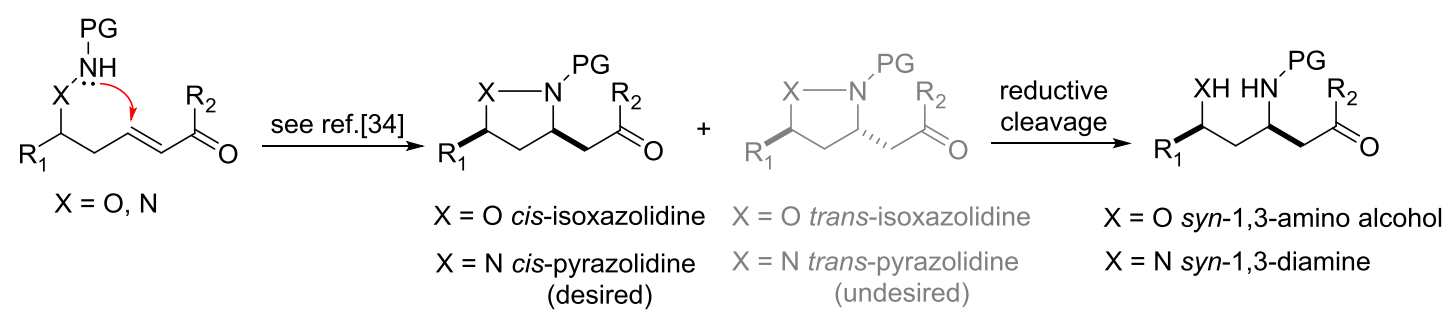

Scheme 2.13. From $N, O$-heterocycles to $N, N$-heterocycles.

This, thus, led us to focus on the reduction of pyrazolidines as the synthetic route of choice to access 1,3-diamines, among other aforementioned strategies. The purpose of our study is to develop a method for the formation of 1,3-diamines, involving hydrazines in contrast to hydroxylamines, as an extension of our work on 1,3-aminoalcohols.

Our goal is also driven by the potential application that could find itself in using $\mathrm{N}, \mathrm{N}$-tetra-hydropyrazolidines as a common key synthetic intermediate for both an efficient and practical development of chemical syntheses. ${ }^{98}$ This includes an eventual total synthesis of lycopodium alkaloids such as (-)-cernuine and (+)-cermizine D. The promise of being able to generate a library of organic compounds through a common intermediate underlines the significance of our study in the generation of new knowledge that finds its relevance in the application of total synthesis. 


\subsection{1 $\quad N, N$-Heterocycles As Synthetic Intermediates}

Substituted pyrazolidine ring can be stereoselectively synthesized through varying methods (Figure 2.8). This comprises of [3+2] cycloaddition reactions, ${ }^{106}$ or hetero-DielsAlder, ${ }^{107}$ azomethine-imine 1,3-dipolar cycloaddition, ${ }^{108}$ and that of cyclization between a dienophile with an aldazine or ketazine. ${ }^{108 a}$ Other methods include metal-catalyzed stereocontrolled synthesis of 3,5-disubstituted pyrazolidines. ${ }^{67 c, 109}$ The vastly available synthetic strategies to pyrazolidines do not eliminate the requirement for specifically engineered starting materials, and harsh reaction conditions. Therefore, we hypothesize a synthetic route towards formation of stereoselective pyrazolidines by carrying out a tandem deprotection-aza-Michael addition reaction, utilizing simple commercially available starting material and under mild conditions. Figure 2.8 reflects an overall view regarding the connection between 1,3-diamines and our objective.

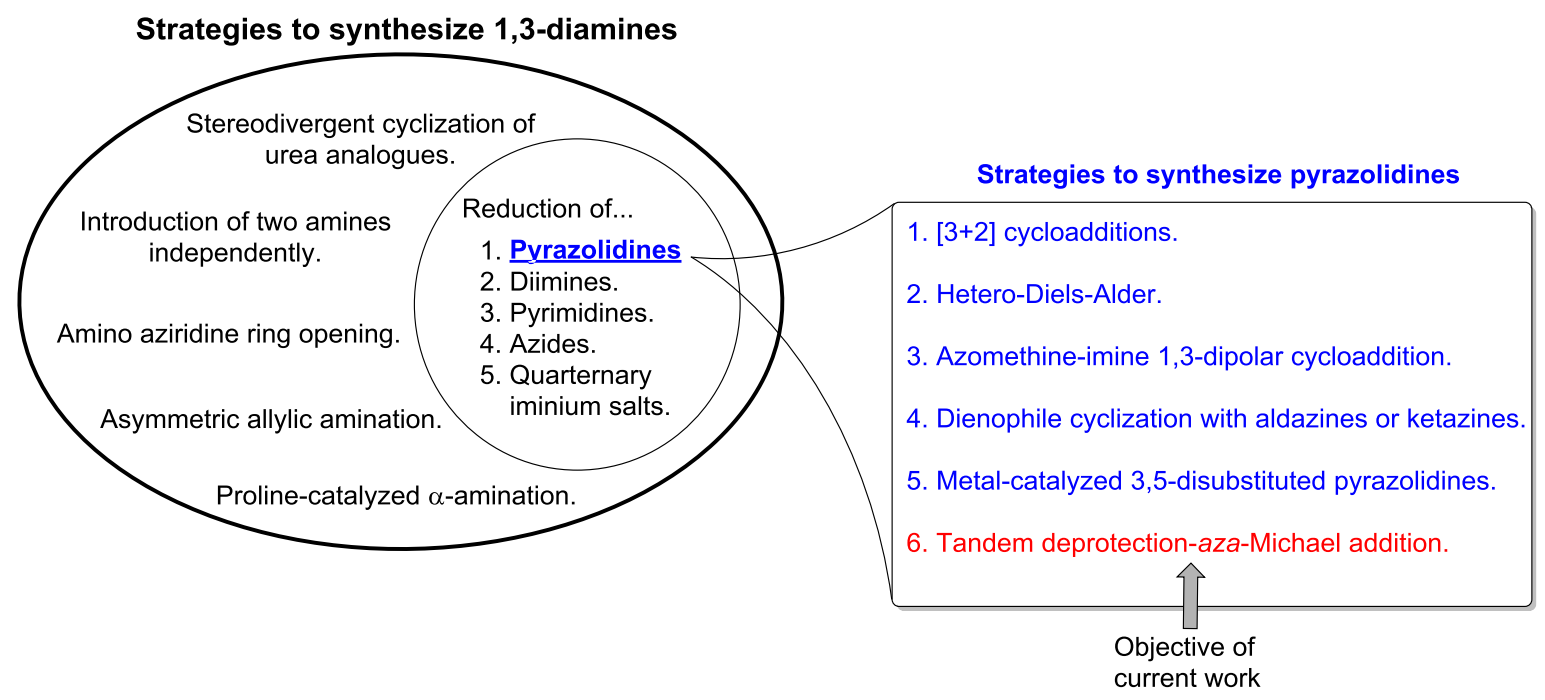

Figure 2.8. An overview of the objective. 


\subsubsection{Retrosynthetic Analysis of (-)-Cernuine and (+)-Cermizine D}

Our retrosynthetic analysis of (-)-cernuine began with disconnection at the $\mathrm{C} 16$ aminal function (Scheme 2.14). The aminal carbon would be introduced through hydroformylation after allylation and $N-N$ bond cleavage of the common intermediate 2.14.1. This would effect a spontaneous cyclization through condensation of both nitrogen atoms on newly formed aldehyde upon hydroformlyation. The precursor of the common intermediate $\mathbf{2 . 1 4 . 1}$ was envisaged from the stereoselective substrate controlled hydrogenation of unsaturated thioester 2.14.2, which would be followed by a one-pot Fukuyama reduction and acyl substitution by neighbouring nitrogen of the pyrazolidine at C7. The pyrazolidine 2.14.2 was expected from the tandem nitrogen deprotection $a z a$-Michael cyclization of the Horner-Wadsworth-Emmons (HWE) olefin product between aldehyde $\mathbf{2}$.14.3 and phosphonate 2 .14.4. The piperidinone 2 .14.3 would then be accessed by the conjugate addition of the cross-metathesis product of $\mathbf{2 . 1 4 . 5}$. The amidation of hydrazine 2.14.6 with hexenoic acid 2.14.7 would enable access to 2.14.5 (Scheme 2.14).

The retrosynthesis of (+)-cermizine $\mathrm{D}$ was devised with the purpose of exploiting the common intermediate 2.14.1 (Scheme 2.14). This could be achieved through Sakurai allylation of 2.14.1 and the cross-metathesis/reduction sequence of the consequent allylated product which would give 2.14.8. The removal of the carbonyl groups in bisamide 2.14 .8 would ideally yield the natural product (+)-cermizine (Scheme 2.14). 


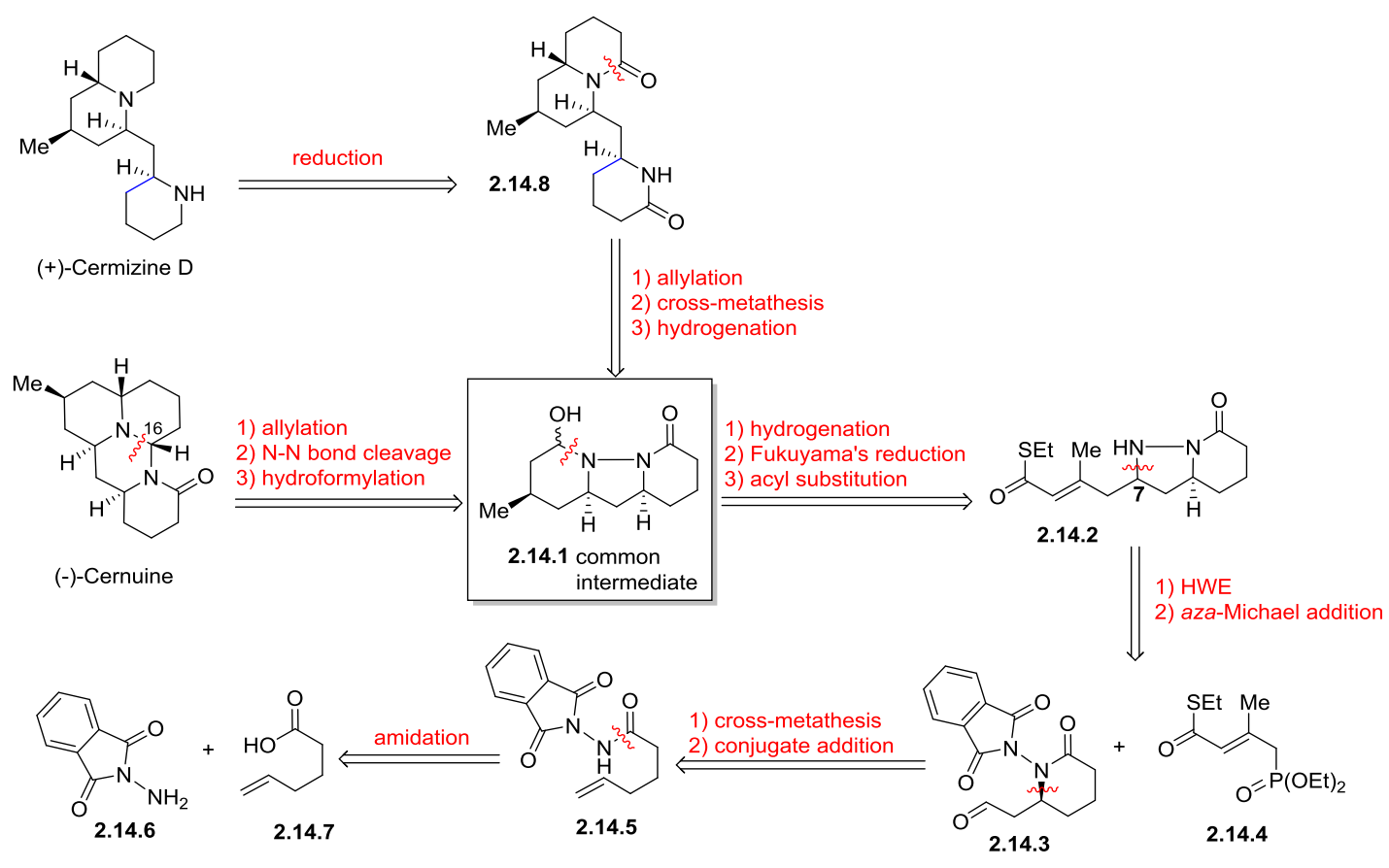

Scheme 2.14. Retrosynthetic analysis of (-)-cernuine and (+)-cermizine D.

\subsubsection{Results and Discussion}

The target cyclization towards the formation of a stereoselective tetrahydropyrazolidine 2.14.2 was projected to spontaneously proceed via an intramolecular $a z a-$ Michael addition of the nitrogen upon treatment of the $\alpha, \beta, \gamma, \delta$-unsaturated ethylthioester 2.15.1 with hydrazine monohydrate for the removal of its phthaloyl group (Scheme 2.15).

Hypothesis:

Tandem nitrogen deprotection $a z a$-Michael addition

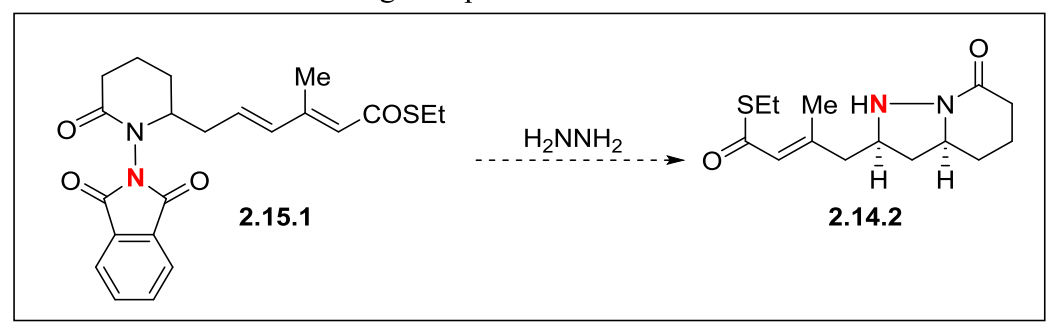

Scheme 2.15. Target cyclization of diene 2.15.1 to give 2.14.2 
The starting materials required for the preparation of the precursor to tetrahydropyrazolidine $\mathbf{2 . 1 5 . 1}$ began with three commercially available compounds; hydrazine monohydrate, phthalimide 2.16.1 and cyclohexanone 2.16.2 (Scheme 2.16).

Firstly, the two nitrogens of hydrazine were made distinguishable in terms of their reactivity through a mono-protection with phthalimide in ethanol to give $\mathrm{N}$-aminophthalimide 2.14.6, which was obtained in $75 \%$ yield and used directly in the subsequent amidation step with 5-hexenoic acid 2.14.7 (Scheme 2.16). Although, 5-hexenoic acid 2.14.7 is commercially available for purchase, large quantity of the 5-hexenoic acid 2.14.7 required in the early stages of the synthesis made it cost-effective to acquire it through the oxidation of cyclohexanone 2.16.2 $\left(\mathrm{H}_{2} \mathrm{O}_{2}, \mathrm{MeOH}\right)$, which gave the acid 2.14 .7 in $65 \%$ yield after distillation. ${ }^{110}$ Besides that, amongst the many nitrogen-protecting groups such as tert-butyl carbazate, the phthaloyl group was used due to the physical properties of the product $N$-aminophthalimide. It is a nonhygroscopic white solid with a high melting point $\left(200-202^{\circ} \mathrm{C}\right)$, allowing for easy handling.

The subsequent amide bond formation in 2.14.5 was carried out via nucleophilic acyl substitution of the acyl chloride with the free amine of the $N$-aminophthalimide 2.14.6 to give the desired hydrazide $\mathbf{2 . 1 4 . 5}$, which was isolated in $72 \%$ overall yield (Scheme 2.16 ). The acyl chloride was derived from exposure of 5-hexenoic acid 2.14.7 to oxalyl chloride in dichloromethane $\left(\mathrm{CH}_{2} \mathrm{Cl}_{2}\right)$ with a catalytic amount of dimethylformamide (DMF).

The terminal olefin of hydrazide $\mathbf{2 . 1 4 . 5}$ was subjected to a cross-metathesis with crotonaldehyde (G-II, DCE, $50{ }^{\circ} \mathrm{C}$ ) in an attempt to furnish the Michael acceptor 2.16.3. This was not observed from ${ }^{1} \mathrm{H}$ NMR analysis, only aldehyde $r a c-2.14 .3$ was exclusively isolated upon purification on silica gel (Scheme 2.16). The premature cyclization was initially believed to occur during flash chromatography, catalyzed by the acidic silica gel. Therefore, a neutralized flash column chromatography was employed in the subsequent purification of the crossmetathesis product where the silica gel was doped with triethylamine. ${ }^{111}$ This was unsuccessful 
and led to the analysis of the crude mixture instead where ${ }^{1} \mathrm{H}$ NMR confirmed in-situ intramolecular $a z a$-Michael cyclization. The characteristic olefinic protons of the Michael acceptor were absent in the ${ }^{1} \mathrm{H}$ NMR of the crude mixture, while the multiplet corresponding to the single proton $\beta$ to the aldehyde was visible $(\delta 4.51$, tt, $J=7.0,5.3 \mathrm{~Hz})$ and thereby validated the cyclization. The unsuccessful attempt of obtaining Michael acceptor 2.16.3 prevented the intended stereocontrolled intramolecular conjugate addition of the nitrogen on the prochiral carbon C5 through MacMillan's imidazolidinone chiral catalyst 2.16.4, which reportedly gives high enantioselectivity. ${ }^{111-112}$ Consequently, an enantioselective synthesis could not be achieved at this point.

There were two pathways from aldehyde $r a c-\mathbf{2 . 1 4 . 3}$ that were investigated to arrive to diene 2.15.1 (Scheme 2.16). Pathway A was a two-step olefination that exploited simple phosphonates and it involved $\alpha, \beta$-unsaturated ketone intermediate $(E)$-2.16.9. Meanwhile, pathway B consisted of a single step HWE olefination utilizing phosphonate 2.14.4, which needed to be prepared (Scheme 2.16). 

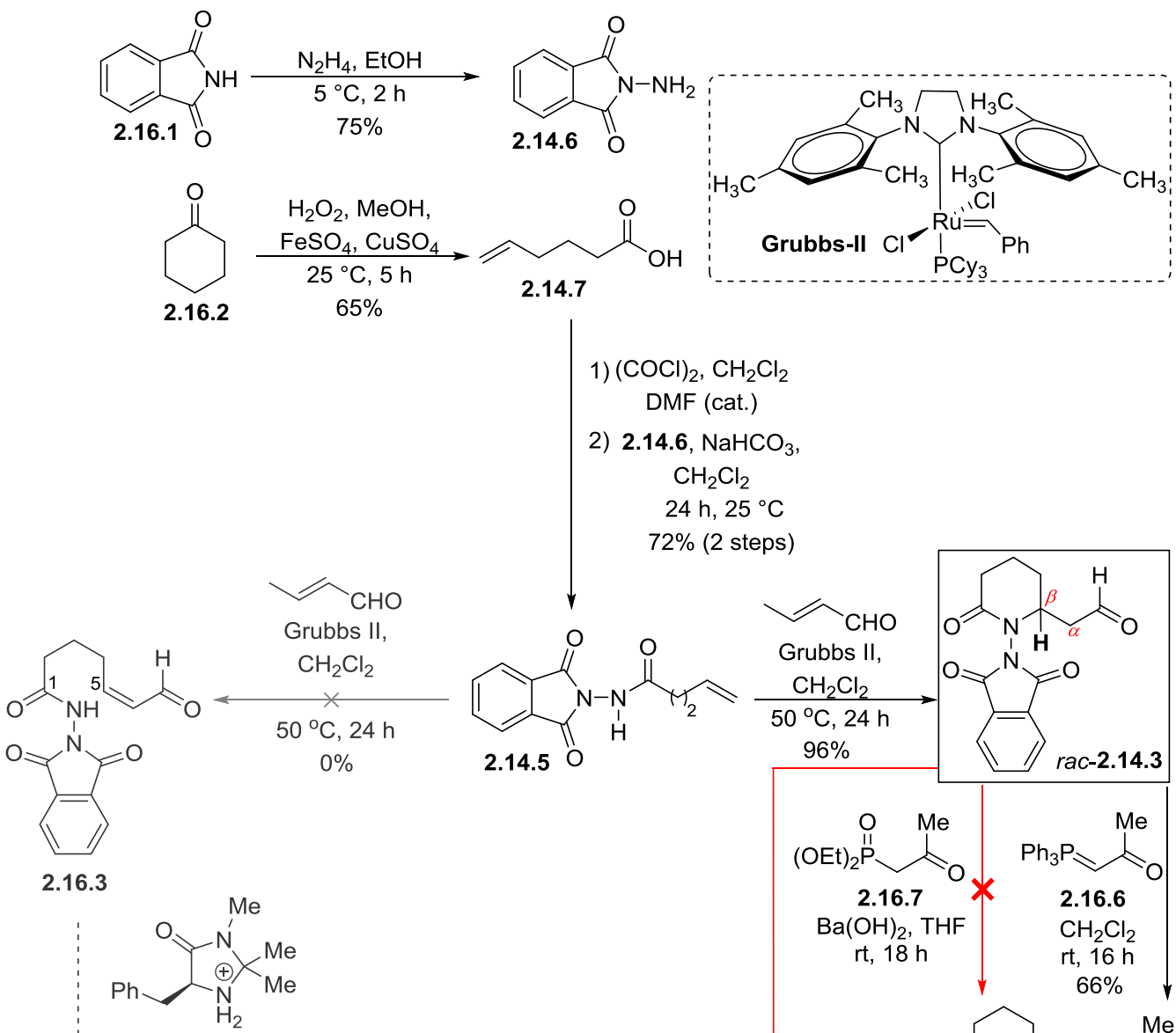

Macmillan's Catalyst 2.16.4 intended route
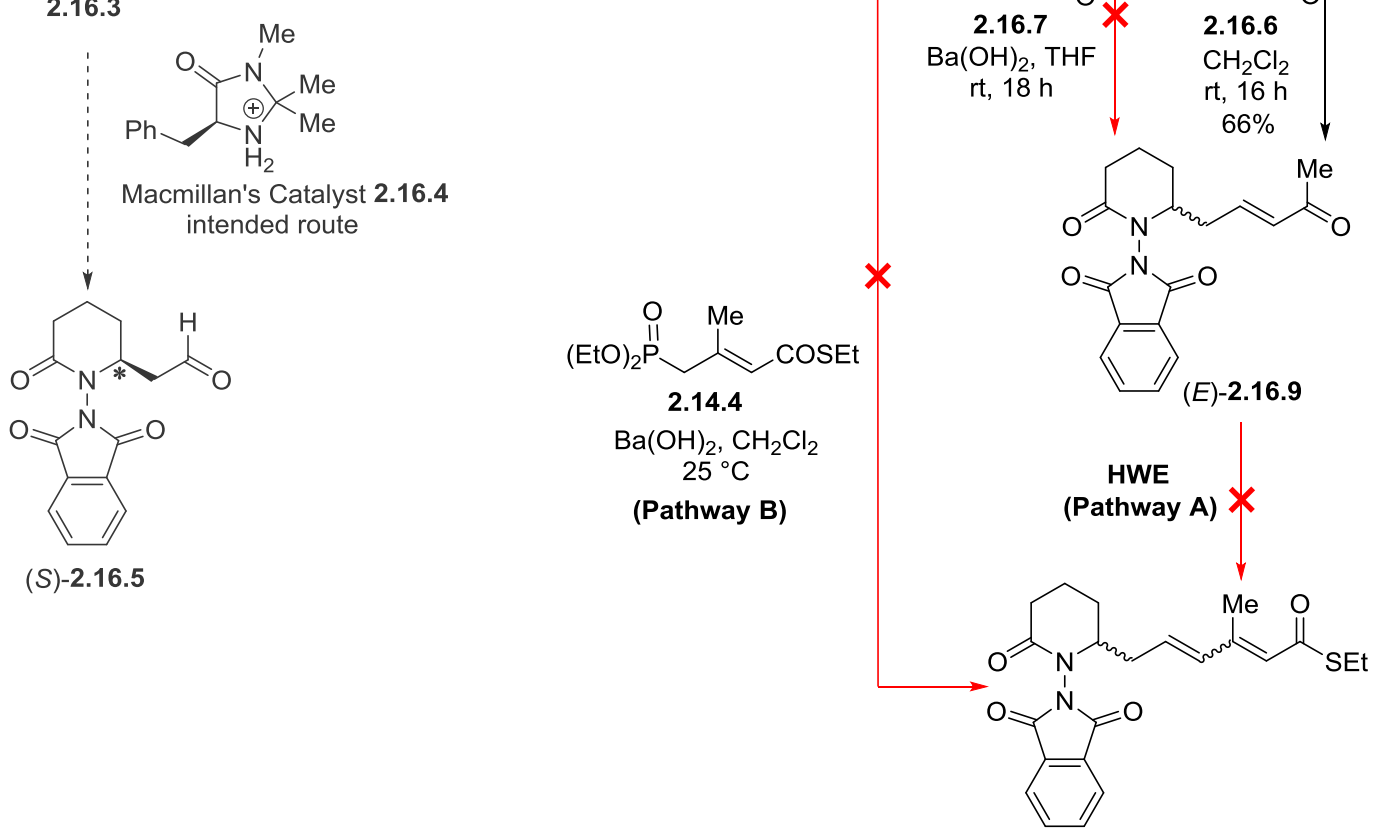

$(S)-2.16 .5$

2.15.1
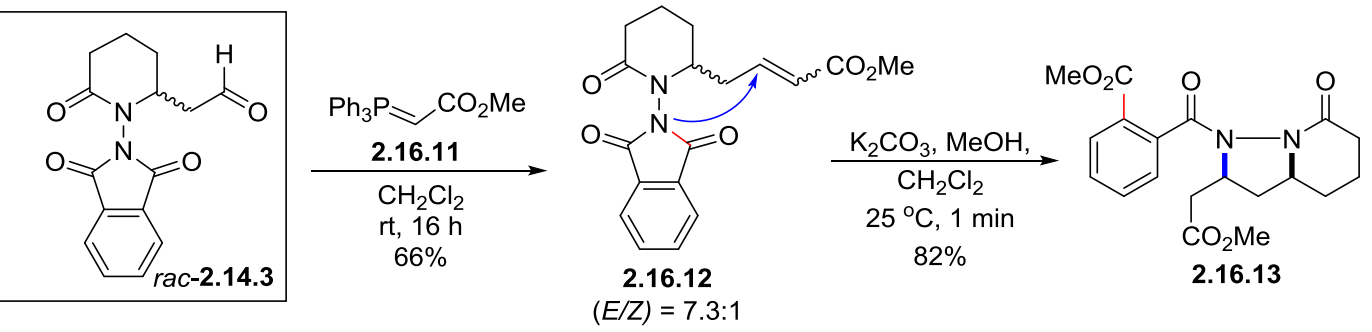

Scheme 2.16. Synthesis of objective fragment 2.16.13. 


\subsubsection{Path A: Successive Olefination}

The aldehyde rac-2.14.3 was submitted to a Wittig olefination using methyl ketone phosphonate 2.16.6 in dichloromethane at room temperature to furnish the desired $\alpha, \beta$ unsaturated methyl ketone $(E)$-2.16.9, which was only partly separable from the resulting triphenylphosphine oxide due to the similar polarities of the two compounds (Figure 2.9). The success of the Wittig olefination was confirmed by the presence of the methyl peak and olefinic protons from ${ }^{1} \mathrm{H}$ NMR analysis (Figure 2.9).

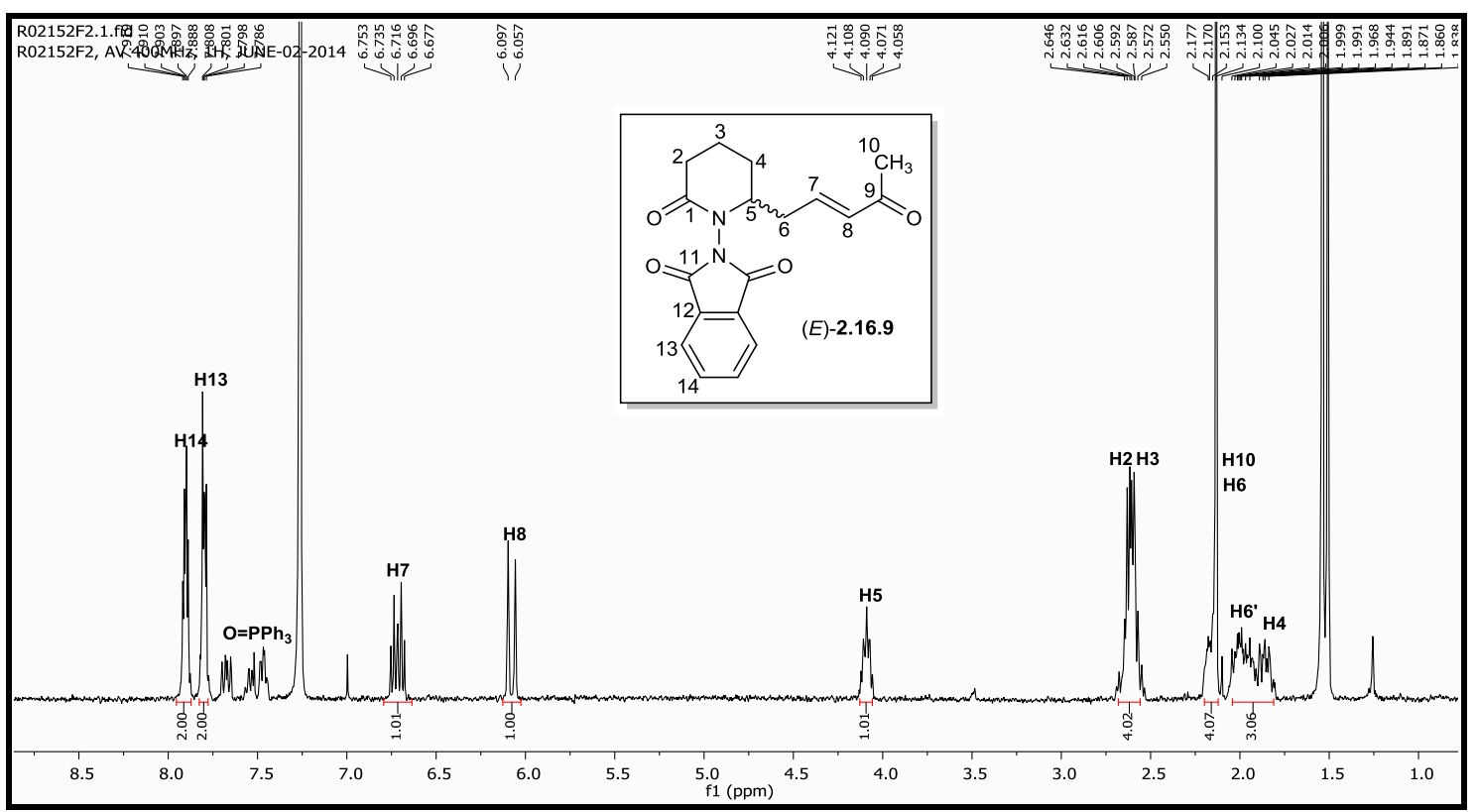

Figure 2.9. ${ }^{1} \mathrm{H}$ NMR analysis of Wittig product $(E)-\mathbf{2 . 1 6 . 9}$

An alternative olefination method such as the HWE reaction was carried out in the attempt to remove the co-elution issue associated with triphenylphosphine oxide and the Wittig product (Scheme 2.17). Therefore, a revised version of phosphonate 2.16.6 was synthesized beginning with the commercially available diethyl phosphite 2.17.1. The diethyl phosphite 2.17.1 was converted into the corresponding methanephosphonate $\mathbf{2 . 1 7 . 2}$ by deprotonation with sodium hydride $(\mathrm{NaH}, \mathrm{THF})$, and methylation with methyl iodide $\left(\mathrm{CH}_{3} \mathrm{I}, \mathrm{THF}, 88 \%\right.$ isolated yield). The anion of methanephosphonate 2.17.2 failed to undergo the acylation with acetyl 
chloride in catalytic amount of copper(I) iodide but proceeded smoothly upon exposure to $n$-BuLi and ethyl acetate to give phosphonate 2.17.3. Unfortunately, the alternative HWE between phosphonate 2.17.3 and $\mathrm{rac}-\mathbf{2 . 1 4 . 3}$ failed to furnish the desired $\alpha, \beta$-unsaturated methyl ketone (E)-2.16.9 (Scheme 2.17). Therefore, Wittig olefination remained as the primary synthetic route to access $(E)$-2.16.9.

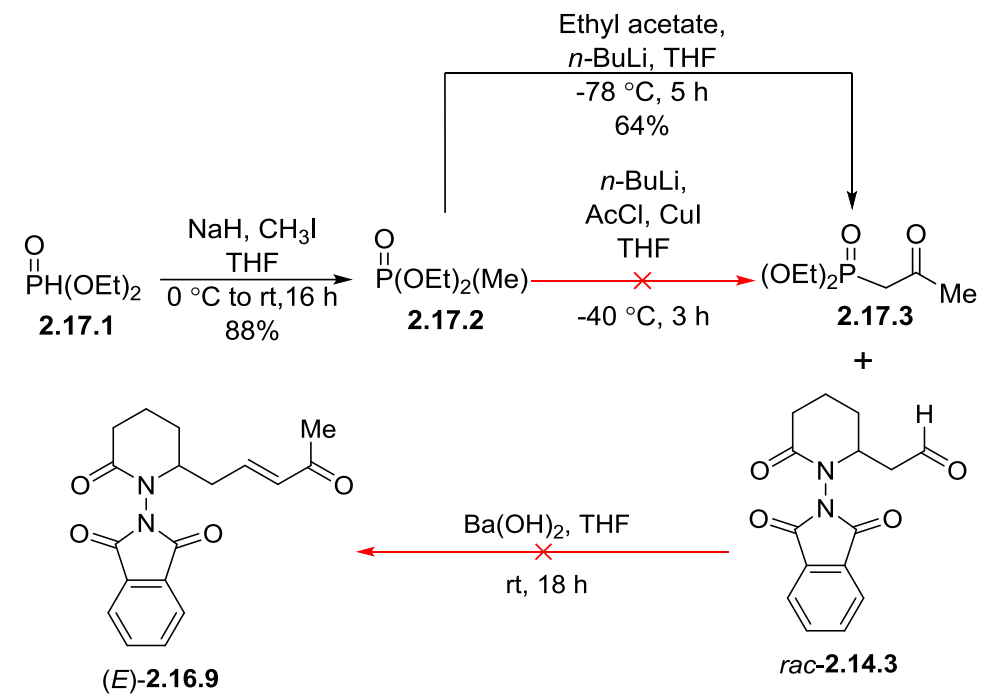

Scheme 2.17. Unsuccessful alternative route to $(E)$-2.16.9 using HWE.

The synthesis continued with the proceeding HWE olefination of $(E)$-2.16.9 using phosphonate ethanethioate 2.18.3 (Scheme 2.18). The phosphonate ethanethioate 2.18.3 was synthesized from bromoacetic acid 2.18.1 with ethanethiol $\left(\mathrm{CH}_{2} \mathrm{Cl}_{2}, \mathrm{DCC}\right)$ and catalytic amount of 4-dimethylaminopyridine (DMAP) to give the $\alpha$-bromothioester 2.18.2, which was purified via cold-finger distillation and subjected to substitution with diethyl phosphite in the presence of $\mathrm{NaH}$. Unfortunately, desired phosphonate 2.18.3 was not obtained. The alternative was the Michaelis-Arbuzov reaction ${ }^{113}$ with neat triethylphosphite to give the desired ethanethioatephosphonate 2.18.3, which was in quantitative yield after distillation (Scheme 2.18).

It was anticipated that, at this stage, the triphenylphosphine oxide contamination issue of the Wittig product $(E)$-2.16.9 could be removed after the formation of diene 2.15.1 as there 
would be a bigger polarity difference that may allow for a better separation on silica-gel column chromatography (Scheme 2.18). Nonetheless, the presence of triphenylphosphine oxide in the starting material, $\alpha, \beta$-unsaturated methyl ketone $(E)$-2.16.9, would not affect the intended olefination due to its stability. The HWE olefination with phosphonate ethanethioate $\mathbf{2 . 1 8 . 3}$ did not give the desired $\alpha, \beta, \gamma, \delta$-unsaturated thioester as no conversion of the starting material was observed. The lack of reactivity of the ketone compared to an aldehyde may be accountable for the unsuccessful olefination. Therefore, efforts were diverted to pathway B where a HWE olefination with aldehyde $r a c$ - 2.14.3 to afford $\alpha, \beta$-unsaturated thioester 2.15.1 in a single step was carried out. The synthesis of phosphonate 2.14 .4 became the primary focus (refer to previous Scheme 2.16).

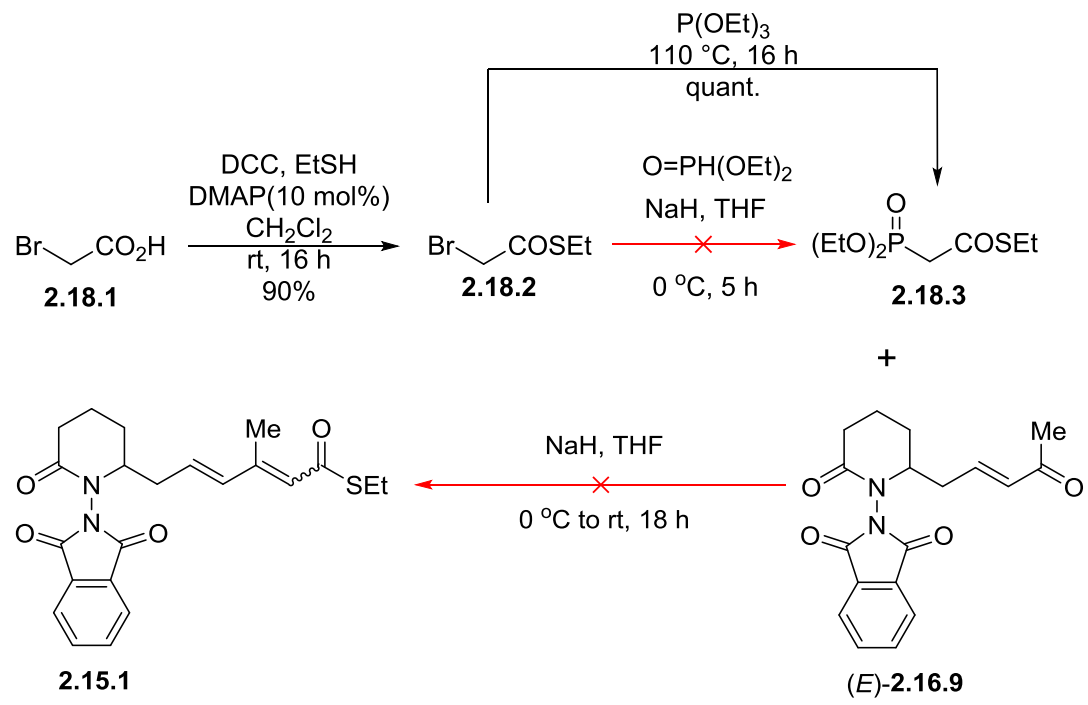

Scheme 2.18. Unsuccessful HWE between 2.18.3 and (E)-2.16.9.

\subsubsection{Pathway B: Synthesis of phosphonate 2.14.4 for HWE with aldehyde rac-2.14.3.}

The synthesis of phosphonates 2.14.4 and 2.19.6 was achieved through Arbuzov reaction of bromide 2.19.1 that would be derived from dehydrohalogenation of dibromide 2.19.2 (Scheme 2.19). The regioselectivity of the elimination reaction would favour deprotonation at $\mathrm{C} 2$ over $\mathrm{C} 4$, following an $\mathrm{E}_{1} \mathrm{cB}$ mechanism, to give the desired product 2.19.1. Several methods 
were explored in the synthesis of dibromo acid 2.19.2. Alternatively, bromide 2.19 .1 could be prepared from the Reformatsky reaction between $\alpha$-bromoester 2.19.3 and $\alpha$-chloroketone 2.19.4 in addition to the radical bromination of 3-methylcrotonic acid 2.19.5 (Scheme 2.19).

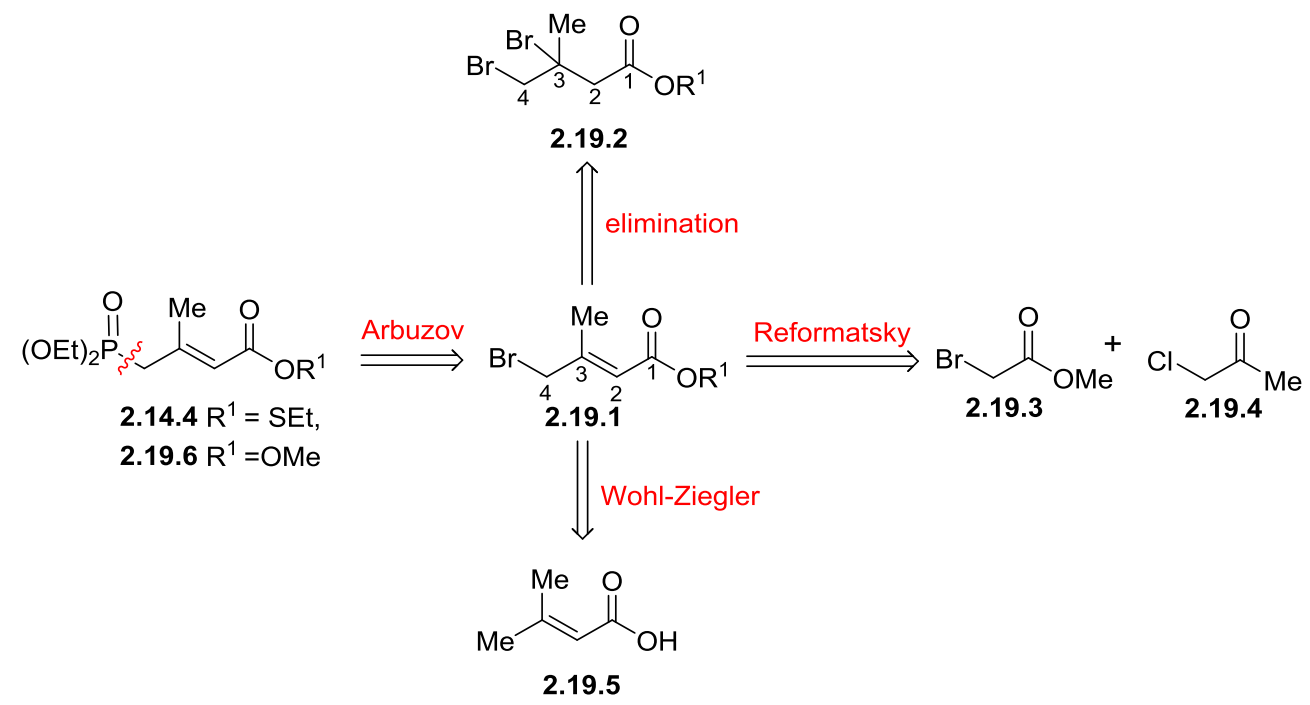

Scheme 2.19. Retrosynthesis of phosphonates 2.14.4 and 2.19.6.

\section{Arbuzov-Elimination}

The first method explored a palladium-catalyzed carbonylation of allyl chloride $\mathbf{2 . 2 0 . 1}$ in the presence of ethanol and potassium carbonate in the attempt to generate ester $\mathbf{2 . 2 0 . 2}$ that would have been dibrominated to give 2.19.2 (Scheme 2.20). ${ }^{114}$ Unfortunately, the carbonylation was unsuccessful as ester 2.20.2 was not formed.

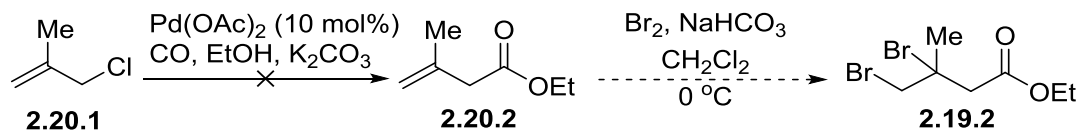

Scheme 2.20. Unsuccessful carbonylation of 2.20.1

The alternative bromination strategy employed isopentenyl alcohol 2.21.1 in the presence of bromine solution and sodium bicarbonate to give dibromide $\mathbf{2 . 2 1 . 2}$ in high $94 \%$ yield (Scheme 2.21). ${ }^{115}$ The primary alcohol was submitted to oxidation under Sharpless 
conditions $^{56}$ to afford carboxylic acid 2.21.3, which was then subjected to thioesterification using ethanethiol with DCC and DMAP. Unfortunately, the thioesterification failed to generate thioester 2.21.5. A more reactive acyl chloride 2.21.4 was used but the outcome remained the same. Therefore, the carboxylic acid 2.21.3 was transformed into methyl ester 2.19.2 through thionyl chloride in methanol. The esterification of $\mathbf{2 . 2 1 . 3}$, in contrast to the thioesterication, proceeded smoothly with no complication. The subsequent dehydrohalogenation of ester 2.19.2 failed to give the desired olefin 2.19.1 under a variety of mild organic and inorganic bases (Table 2.1, entries $1-5$ ). There was no deprotonation observed from the use of potassium carbonate in THF and butanone (Table 2.1, entries 1 and 2). Similarly, there was no conversion of the starting material 2.19.2, in the use of organic bases such as DIPEA and TEA in dichloromethane (Table 2.1, entries 3 and 4). Instead, the major product isolated in the treatment of 2.19.2 with sterically hindered base DBU was unsaturated lactone 2.21.6 (Table 2.1, entry 5). The absence of bromine isotopes peaks on GCMS analysis confirmed the result. This could be attributed to the basic conditions used for the hydrolysis of methyl ester 2.19.2, which promoted an in-situ cyclization followed by dehydrohalogenation to give 2.21.6. Perhaps, a bulkier ester group such as tert-butyl ester would provide the stability needed. Nonetheless, other alternatives were explored to arrive to the desired substrate 2.19.1. 


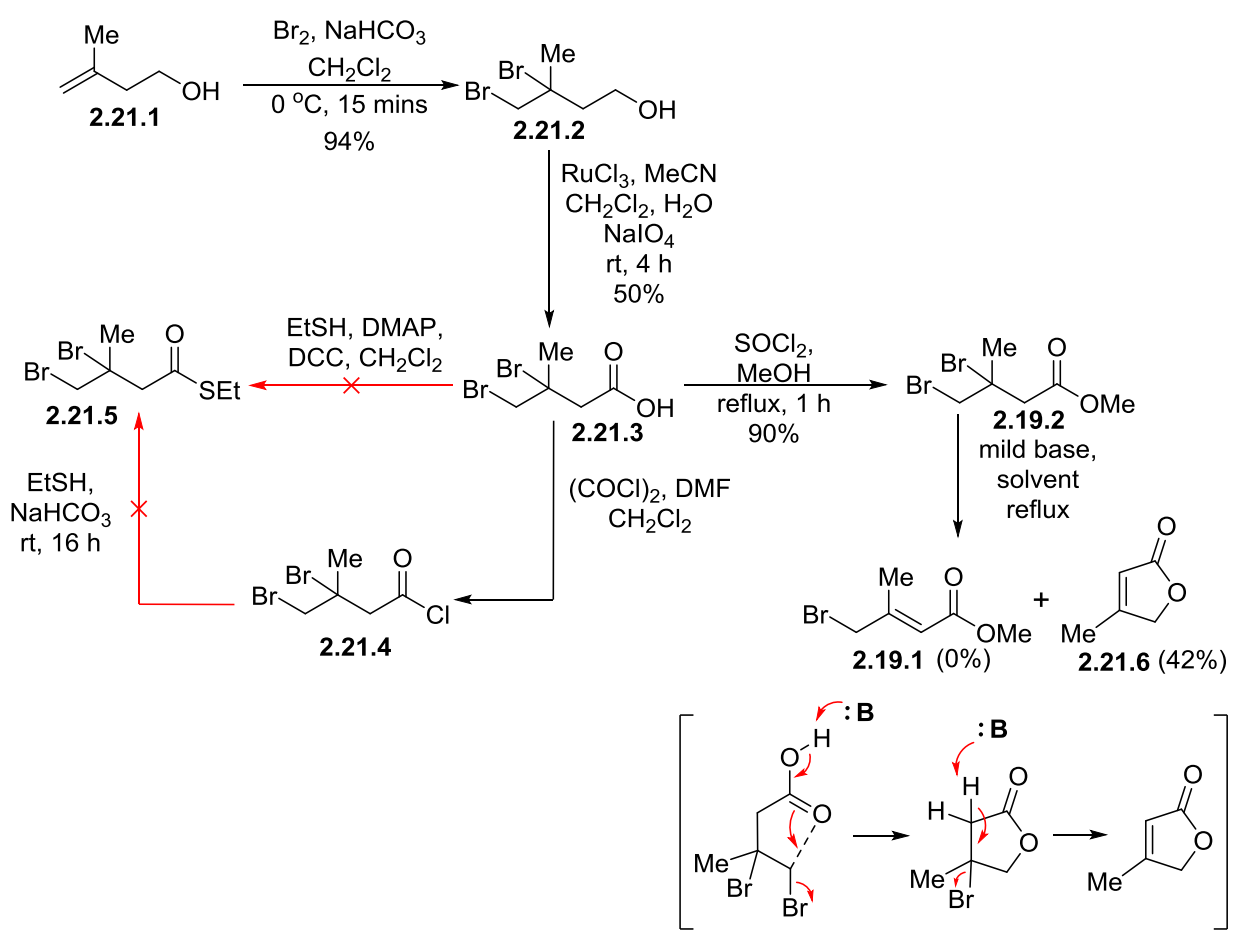

Scheme 2.21. Various methods towards desired bromide 2.19.1.

\begin{tabular}{ccccc}
\hline entry & solvent & base & $\begin{array}{c}\text { conversion of } \\
\mathbf{2 . 1 9 . 2}(\%)\end{array}$ & $\begin{array}{c}\text { yield (\%) } \\
\text { of 2.21.6 }\end{array}$ \\
\hline 1 & THF & $\mathrm{K}_{2} \mathrm{CO}_{3}$ & 0 & - \\
2 & butanone & $\mathrm{K}_{2} \mathrm{CO}_{3}$ & 0 & - \\
3 & $\mathrm{CH}_{2} \mathrm{Cl}_{2}$ & DIPEA & 0 & - \\
4 & $\mathrm{CH}_{2} \mathrm{Cl}_{2}$ & $\mathrm{Et}{ }_{3} \mathrm{~N}$ & 0 & - \\
5 & toluene & $\mathrm{DBU}$ & 100 & 42 \\
\hline
\end{tabular}

Table 2.1. Conditions towards 2.19.1.

\section{Arbuzov-Reformatsky / Wohl-Ziegler}

The ester 2.22.1 was obtained by the generation of zinc-enolate from the exposure of $\alpha$ bromoester 2.19.3 to activated zinc in toluene and ether at reflux (Scheme 2.22). The nucleophilic substitution of $\alpha$-chloroketone 2.19.4 with zinc enolate resulted in the formation of $\beta$-hydroxyl ester 2.22.2, which was then treated with methanolic potassium hydroxide to 
promote the formation of epoxide $\mathbf{2 . 2 2 . 3}$ followed by its ring opening with methanolic hydrochloric acid to give unsaturated ester 2.22.1. The subsequent bromination of ester 2.22 .1 with $\mathrm{PBr}_{3}$ furnished the desired bromide 2.19.1 in excellent 96\% yield. However, the low $20 \%$ yield of the Reformatsky reaction led to the exploration of a third synthetic strategy involving radical bromination of 3-methylcrotonic acid 2.19.5 (Scheme 2.22).

Wohl-Ziegler reaction was performed to yield allylic bromide $\mathbf{2 . 2 2 . 4}$ using $N$-bromosuccinimide with radical initiator azobis(cyclohexanecarbonitrile) (ACHn) (Scheme 2.22). It was reported that the best yield for a Wohl-Ziegler bromination requires the use of carbon tetrachloriden $\left(\mathrm{CCl}_{4}\right)$ as solvent of choice given the insolubility issue associated with $\mathrm{N}$-bromosuccinimide. However, environmental concerns pertaining to the use of $\mathrm{CCl}_{4}$ has led to acetonitrile as a substitute. Acetonitrile, from results gathered by Carenno et al., ${ }^{116}$ can be exploited because, unlike most solvents, it is not reactive towards NBS. The bromination gave trans-bromide 2.22.4 that was esterified to give methyl ester 2.19.1, which underwent Arbuzov substitution with triethylphosphite to give the key phosphonate 2.19.6. The HWE olefination was attempted using two different conditions. The first condition employed barium hydroxide in aqueous tetrahydrofuran and it gave no reaction. ${ }^{117}$ From ${ }^{1} \mathrm{H}$ NMR analysis, $(Z)$-isomer of phosphonate 2.19.6 was obtained. It is thus postulated that, in the presence of a base, alkene isomerization of the phosphonate 2.19.6 occurred. The second attempt to generate the phosphonium ylide using sodium hydride ${ }^{118}$ was futile because there was no conversion of the starting material, aldehyde rac-2.14.3, into the desired olefin $\mathbf{2 . 1 5 . 1}$. Therefore, the target diene 2.15.1 was modified to $\alpha, \beta$-unsaturated ester $\mathbf{2 . 1 6 . 1 2}$ in the effort to test the hypothesis of the tandem nitrogen deprotection aza-Michael addition (Figure 2.10 in the following section 2.1.3). 


\section{A) Reformatskv}

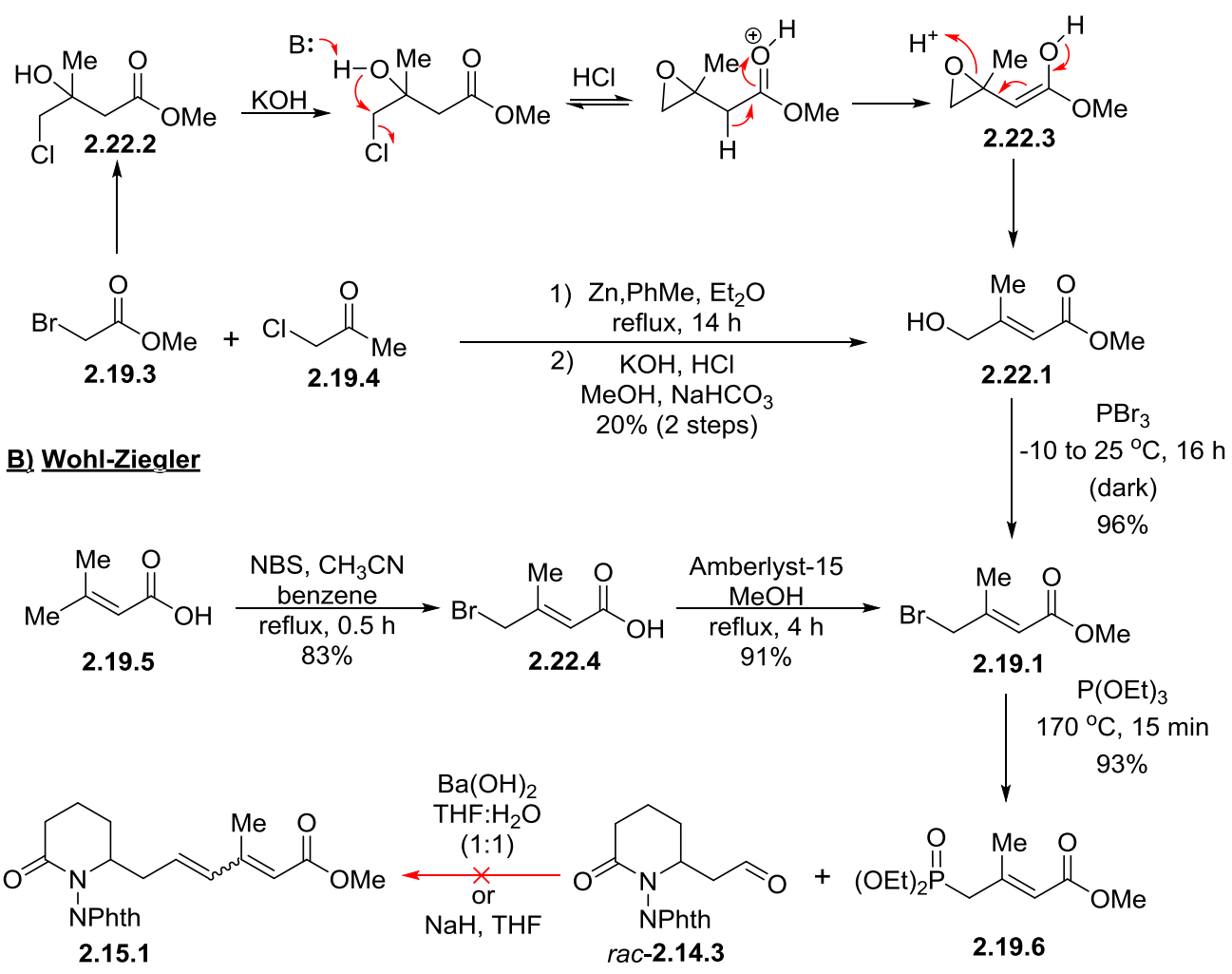

Scheme 2.22. Reformatsky and Wohl-Ziegler reactions to access 2.19.6.

Tetrahydropyrazolidine Formation

The modification from the old model to a new model signified that the cyclization precursor $\alpha, \beta, \gamma, \delta$-unsaturated ester 2.15.1 has changed to $\alpha, \beta$-unsaturated ester $\mathbf{2 . 1 6 . 1 2}$ for the hypothesis test (Figure 2.10). The ester was prepared from the Wittig olefination of rac-2.14.3 with phosphonate 2.16.11 $\left(\mathrm{Ph}_{3} \mathrm{P}=\mathrm{CH}_{2} \mathrm{CO}_{2} \mathrm{Me}\right)$, which proceeded smoothly at room temperature to furnish 2.16.12 in modest 66\% yield $(E / Z=7.3: 1) \quad$ (refer to Scheme 2.16).

Hypothesis: aza-Michael addition

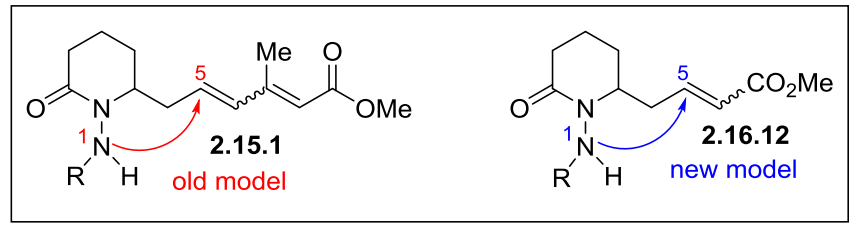

Figure 2.10. Comparison between old and new model. 
The unsaturated ester 2.16.12 was treated with hydrazine monohydrate to deprotect the phthaloyl group and effect a spontaneous cyclization of aminopiperidinone to form cis-tetrahydropyrazolidine 2.23.1 (Scheme 2.23 and Figure 2.11). This condition $\left(\mathrm{N}_{2} \mathrm{H}_{4}, \mathrm{CH}_{2} \mathrm{Cl}_{2},{ }^{\circ}{ }^{\circ} \mathrm{C}\right.$ ) were previously adopted in the case of hydroxylamines. However, a decomposition was observed from ${ }^{1} \mathrm{H}$ NMR analysis. This could be the consequence of a highly reactive free amino group upon deprotection, hence, a partial deprotection was carried out using postassium carbonate in methanol. Gratifyingly, the product isolated in high $82 \%$ yield was the desired syn-pyrazolidine $\mathbf{2 . 1 6 . 1 3}$ as a single diastereomer, which was confirmed by X-ray crystallography analysis (Figure 2.11).
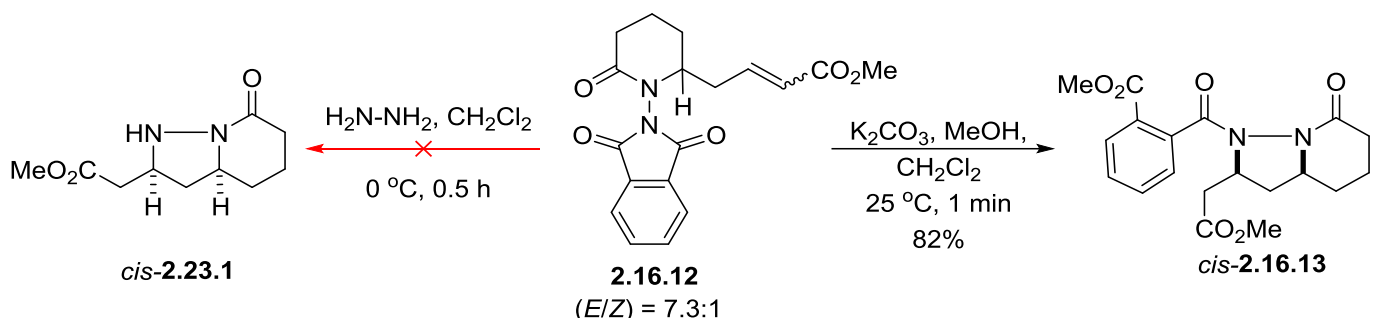

Scheme 2.23. Different cyclization conditions. 


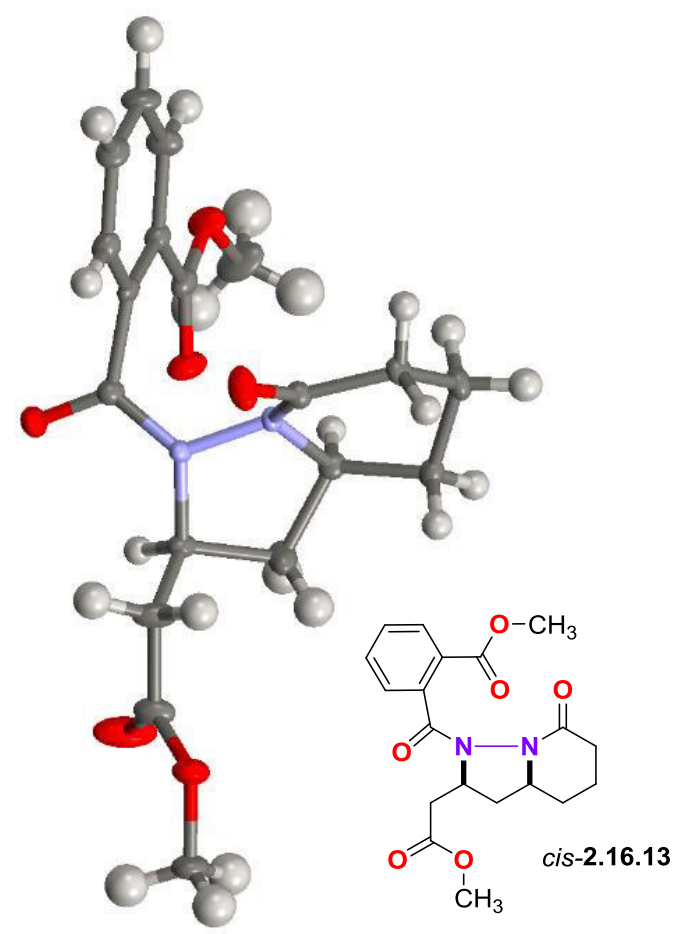

Figure 2.11. X-Ray crystal structure of cis-pyrazolidine 2.16.13.

The proposed reaction mechanism is elaborated in Scheme 2.24, methoxy substitution at one of the two available carbonyl groups of the cyclic imide breaks the ring to give the semiprotected nitrogen 2.24.1 (Scheme 2.24). The spontaneous nitrogen cyclization gave the syn-tetra-hydropyrazolidine $\mathbf{2 . 1 6 . 1 3}$. This could be attributed to the envelope-like conformation of the five-membered ring where the pseudo-equatorial position of the $Z$ substituent does not give rise to a steric repulsion in the transition state (TS-I), which is likely with the pseudo-axial transition state (TS-II) (Scheme 2.24). 


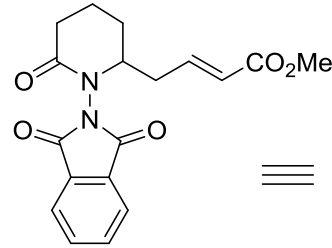

2.16.12

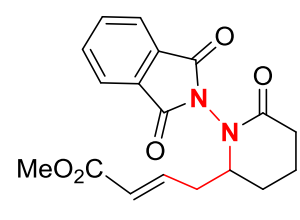

2.16.12

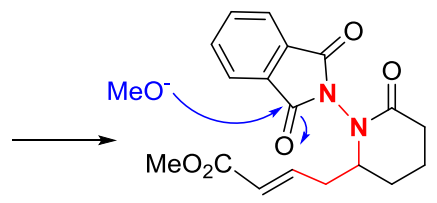

$$
\downarrow
$$
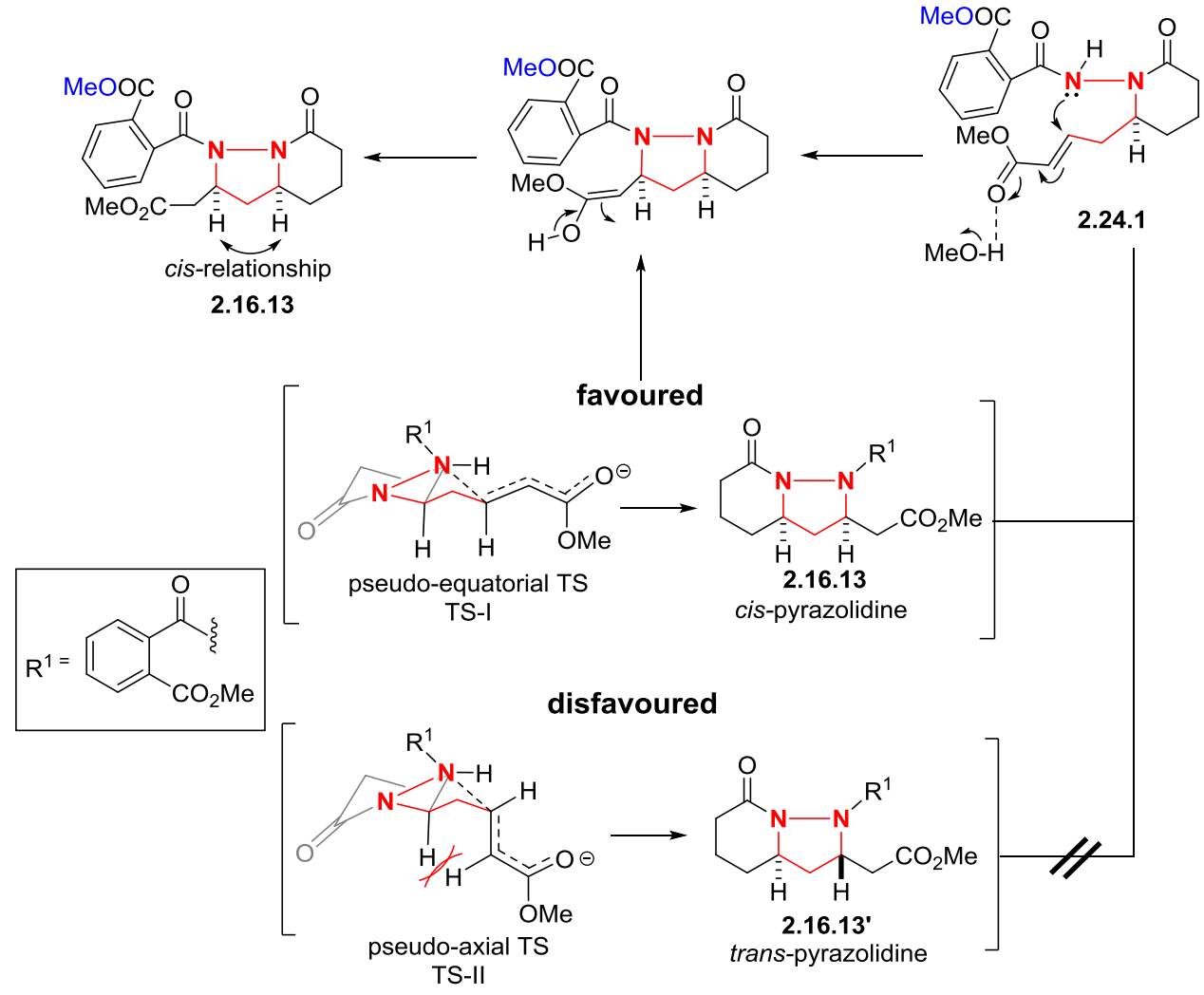

Scheme 2.24. Proposed mechanism for the cyclization of 2.16.12.

\subsubsection{Conclusion}

The challenges involving diene formation 2.15 .1 and phosphonate 2.14 .4 prompted the exploration of a variety of alternative synthetic routes and reaction conditions. Although, the advancement towards the completion of the alkaloids synthesis was halted due to time constraints, the hypothesis towards the 1,3-diamine formation was realized (Scheme 2.25). In overall, the retrosynthetic approach of the pyrazolidine preparation offers its novelty particularly 
in the construction of the syn-pyrazolidine ring 2.16.13 employing mild conditions through a tandem deprotection-cyclization strategy with control in stereochemistry. This would make for an essential contribution in the synthesis of pyrazolidines and hence 1,3-diamines.

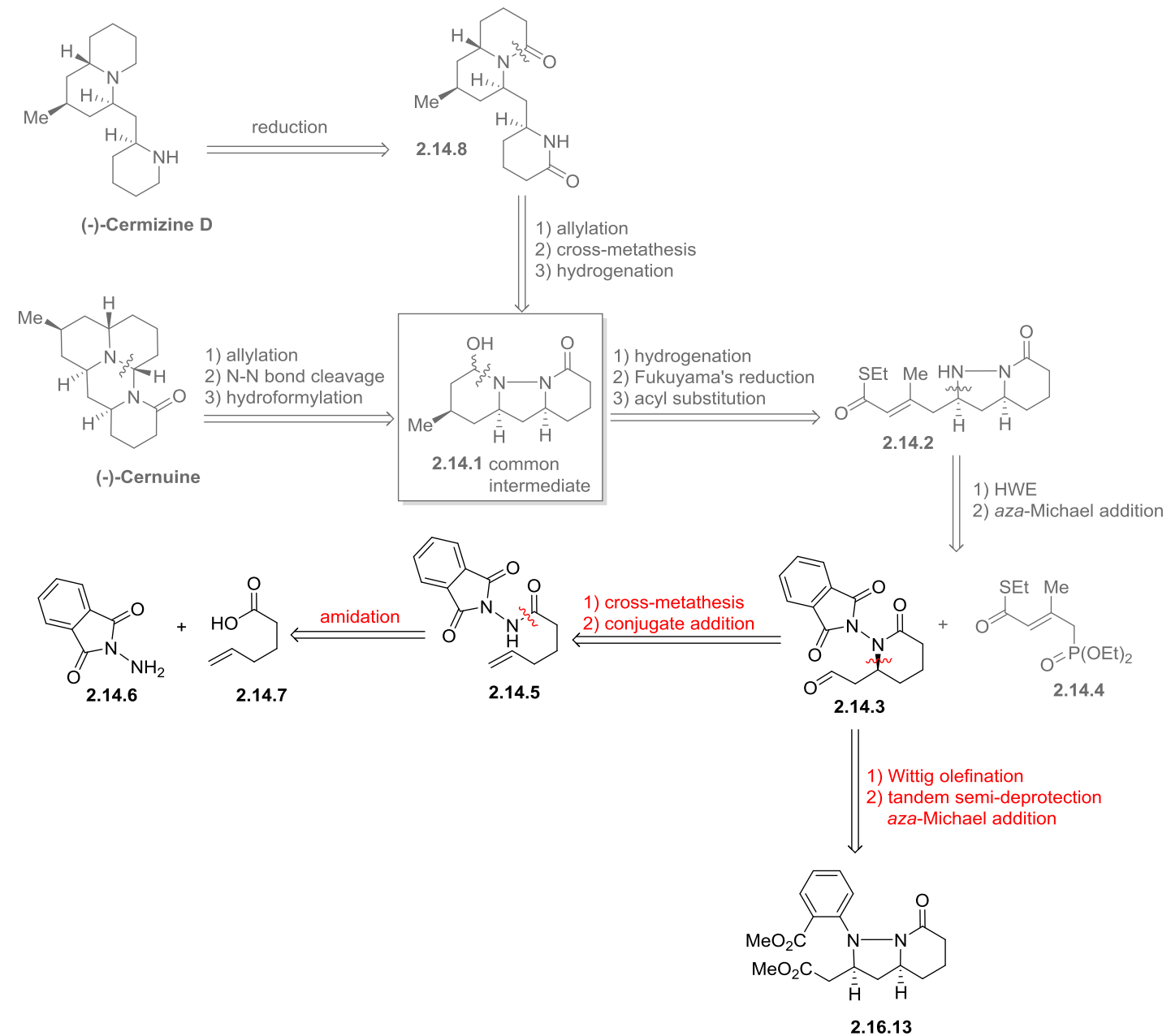

Scheme 2.25. The extent of work fulfilled towards the synthesis of lycopodium alkaloids. 
This page has been intentionally left blank. 


\title{
Chapter 3
}

\author{
3.1 Introduction
}

3.1.1 Natural Products Containing THP-Ring

3.1.2 Metal-Catalyzed Strategies to Access THP-Ring 
This page has been intentionally left blank. 


\subsection{Introduction}

\subsubsection{Natural Products Containing THP-Moiety}

The presence of saturated oxygen heterocycles is ubiquitous in biologically active natural products (Figure 3.1). ${ }^{119}$ They can therefore emerge as a platform for the discovery of new drugs. There have been a substantial amount of contributions in the development of chemo-, regio-, and stereoselective reactions for the construction of saturated oxygen heterocycles. ${ }^{119 \mathrm{c}}$ The tetrahydropyran motif, particularly, is found in synthetic targets such as marine macrolides enigmazole and phorboxazole A, which were identified as powerful cytotoxic agents (Figure 3.1). ${ }^{119 a, 120} 119 \mathrm{~b}, 121$ Similarly, marine metabolites bistramide and leucascandrolide A are known to exhibit countless biological properties of which bistramide A was further involved in a unique protein kinase $\mathrm{C} \delta$-activation ${ }^{122}$ while leucascandrolide $\mathrm{A}$ is classified as a potent antifungal that inhibits the growth of Candida albicans (Figure 3.1). ${ }^{123}$ 

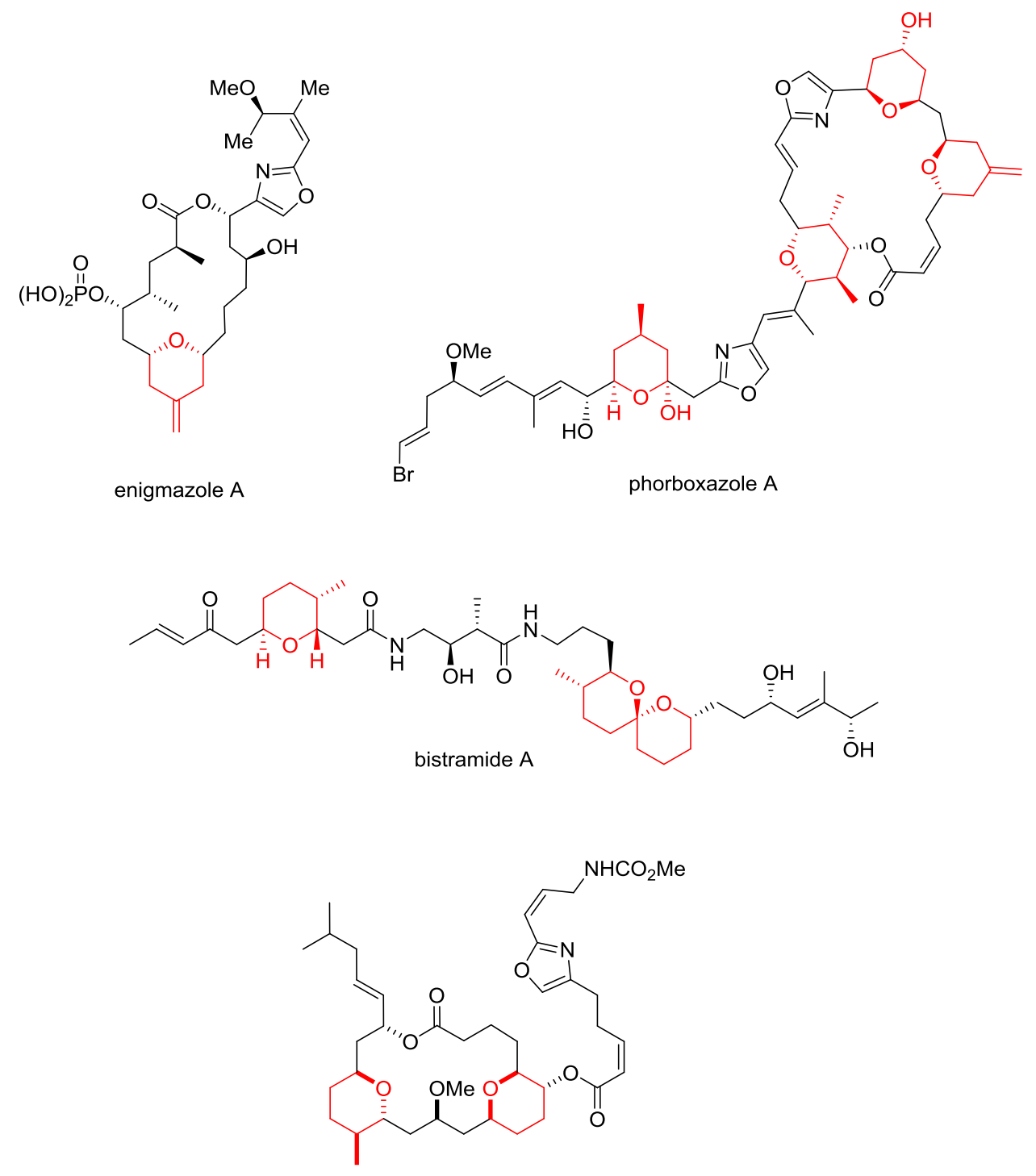

leucascandrolide A

Figure 3.1. Biologically active natural products bearing common structural THP feature.

Enigmazole $\mathrm{A}$ is a phosphate-containing macrolide, and it appears to exhibit a rare phenotypic activity, ${ }^{124}$ its cytotoxic properties are thus of particular interest for the advances in new potential anticancer agents. Hence, further investigation of its bioactivity via in cellulo click reaction may lead to the identification of the biological target(s). The designs of analogues to access powerful anti-tumor agents that can become anticancer agents can then be achieved. 


\subsubsection{Metal-Catalyzed Strategies to Access THP-ring}

The abundant presence of the optically active substituted tetrahydropyran moiety (Figure 3.2) in a range of classes of natural products has resulted in extensive and refined studies in the construction of its structural motif in a stereocontrolled fashion. There are many methodologies that have been developed to increase the stereochemical complexity in the synthesis of tetrahydropyrans, ${ }^{119 b}$ such as iodocyclization, ${ }^{125}$ Williamson ether synthesis, ${ }^{126}$ radical cylizations, ${ }^{127}$ anodic cylizations, ${ }^{128}$ reduction of cyclic hemi-ketals, ${ }^{129}$ cyclization onto epoxides, ${ }^{130}$ Michael reactions, ${ }^{126 c,} 131$ hetero-Diels-Alder cyclizations, ${ }^{132}$ and cyclizations onto oxocarbenium ions. ${ }^{133}$

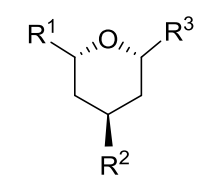

Figure 3.2. 2,4,6-trisubstituted tetrahydropyran ring.

Amongst them are the advantages of a metal-catalyzed intramolecular alkoxy cyclization of $\delta$-hydroxyl alkenes (Scheme 3.1). This requires electrophilic activation of the olefin by a stoichiometric or catalytic amount of metal to form a $\pi$-allyl complex, which is followed by a nucleophilic substitution. ${ }^{134}$ Alternatively, the corresponding heterocycle could be obtained indirectly through an onium intermediate prior to the nucleophilic addition. ${ }^{130 \mathrm{~b}}$ The strategies described focus on the catalytic process of metal-activated alkene additions.

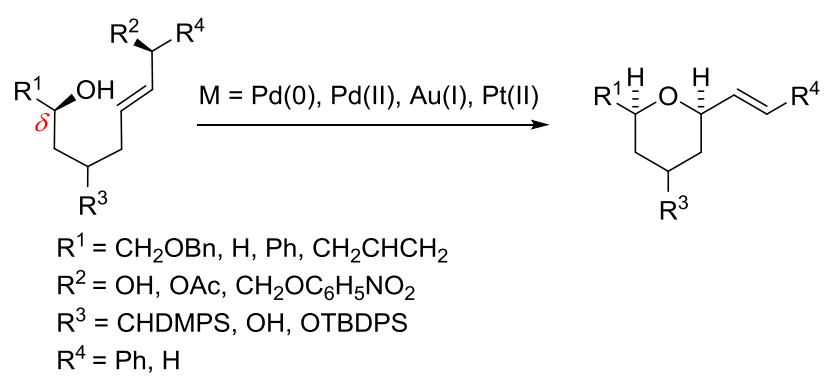

Scheme 3.1. Metal-catalyzed hydroalkoxylation of $\delta$-hydroxyl alkenes. 


\section{Metal-catalyzed Intramolecular Cyclization nto $\mathrm{C}-\mathrm{C}$ Double Bonds}

Palladium(0)-catalysis

In 2003, Burke et al. reported the ligand-controlled Pd(0)-mediated intramolecular alkoxy cyclizations of tetraol 3.2.1 to establish the bis-THP units in the total synthesis of phorboxazoles $\mathrm{A}$ and $\mathrm{B}$. The use of $(R, R)$-diphenylphosphino benzamide (DPPBA) 3.2.2, Trost's ligand system ${ }^{135}$ for the concurrent two-directional desymmetrization tetraol 3.2.1 afforded the desired bis-oxane 3.2.3 in high enantiomeric excess (Scheme 3.1).

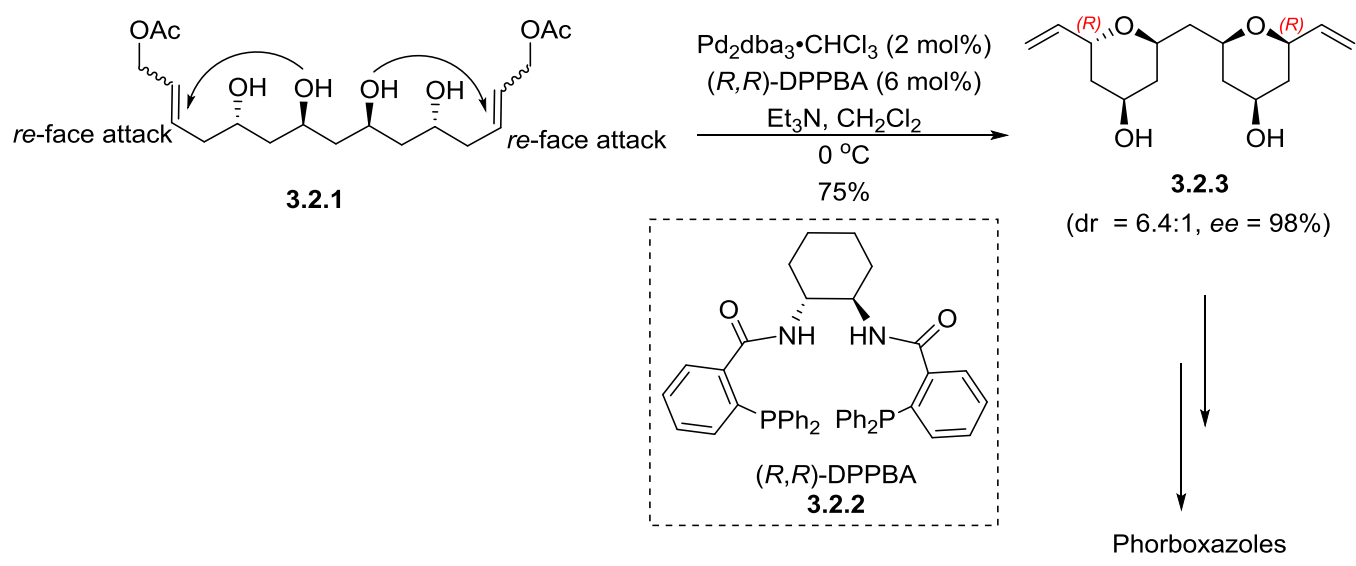

Scheme 3.2. $\operatorname{Pd}(0)$-catalyzed, ligand-controlled hydroalkoxylation of 3.2.1

In 2004, Lee et al. reported the stereocontrolled synthesis of 2-alkenyl-4-methylene THP in the studies towards the total synthesis of zampanolide (Scheme 3.3). ${ }^{136}$ The group showed that high stereocontrol was achieved by using $\mathrm{Pd}(0)$-mediated cyclization to give a high diastereomeric ratio. For instance, the alkene cyclization of carbonate 3.3.1 afforded exclusively the cis-THP 3.3.3 in high 71\% under mild conditions (Scheme 3.3). The substrate-controlled diastereoselectivity originates from the formation of the $\pi$-allylpalladium cation complex that involves the coordination of the palladium to the alkene from the less-hindered face that is opposite to the leaving group. The following nucleophilic attack would then proceed from the opposite side of the metal leading to high diastereoselectivity. Similarly, when using the epimer3.3.4, a single trans-THP 3.3.5 diastereomer was obtained (Scheme 3.3). 

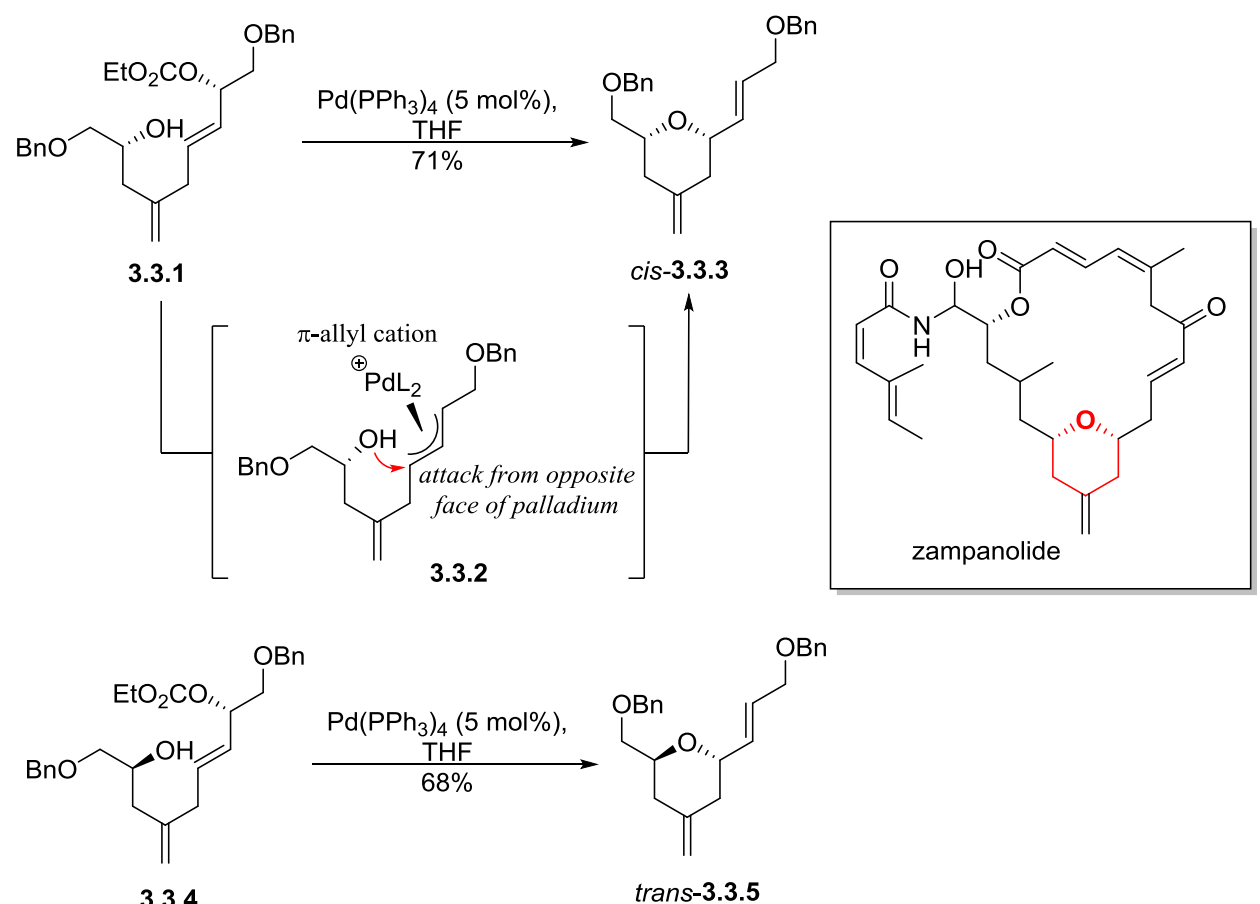

Scheme 3.3. $\operatorname{Pd}(0)$-catalyzed hydroalkoxylation of 3.3.1

In 2005, Trost et al. reported the total synthesis of (+)-hippospongic acid A, which demonstrated the highly enantioselective synthesis of THP-unit through the use of DPBA-based chiral ligand 3.2.2 (Scheme 3.4). The treatment of hydroxyl alkene 3.4.1 with $\mathrm{Pd}_{2}(\mathrm{dba})_{3}$ ( $2 \mathrm{~mol} \%)$ in the presence of chiral diphosphine and triethylamine in dichloromethane gave the THP-3.4.2 enantiomer in high 80\% yield and enantiomeric excess of $94 \%$. 


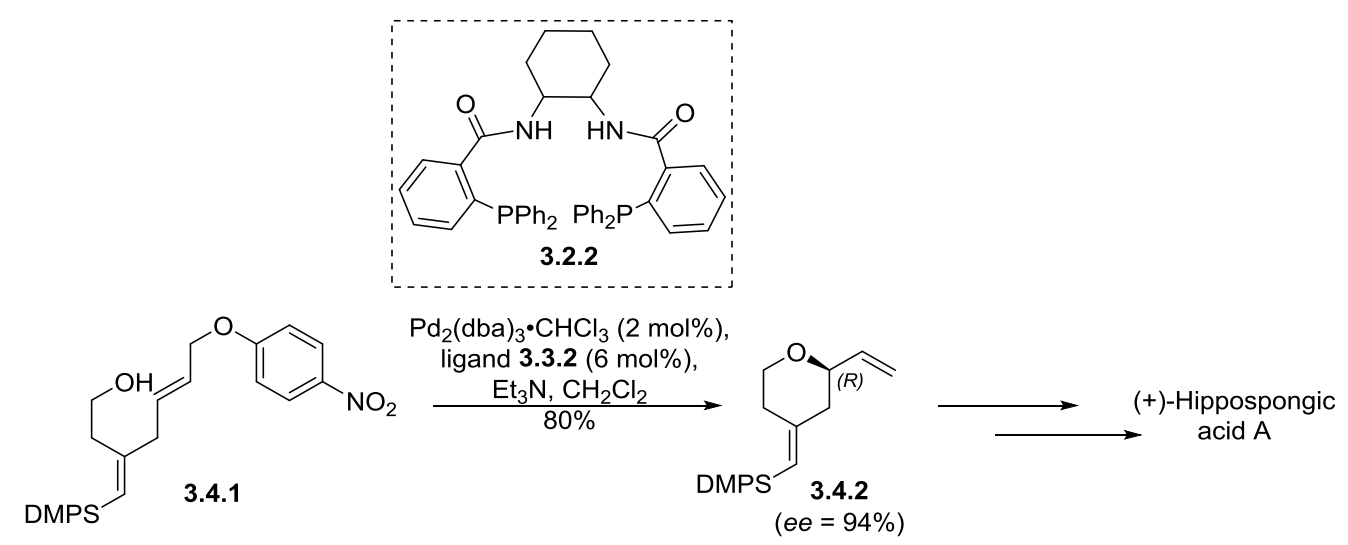

Scheme 3.4. $\operatorname{Pd}(0)$-catalyzed, ligand-controlled hydroalkoxylation of 3.4.1.

Palladium(II)-catalysis

In 2006, White et al. reported the synthesis of the C9-C19 containing the THP-unit in phorboxazole A through Semmelhack-type conditions (Scheme 3.5). ${ }^{137}$ This involved the use of Pd(II)-mediated intramolecular 1,2-alkoxycarbonylation of hydroxyl alkene 3.5.1 (Scheme 3.5). ${ }^{138}$ The single diastereomer trisubstituted THP 3.5.2 was isolated in a modest yield of $58 \%$ from the recoverable starting material ( $15-20 \%$ unreacted 3.5.1). Although the reaction was carried out at room temperature, it requires three days to give a substantial conversion of 3.5.1. The use of stoichiometric amount of oxidant such as benzoquinone ${ }^{139}$ is necessary to maintain a catalytic process of $\mathrm{Pd}(\mathrm{II})$ species by the regeneration of $\mathrm{Pd}(\mathrm{II})$ from $\mathrm{Pd}(0) .{ }^{138}$

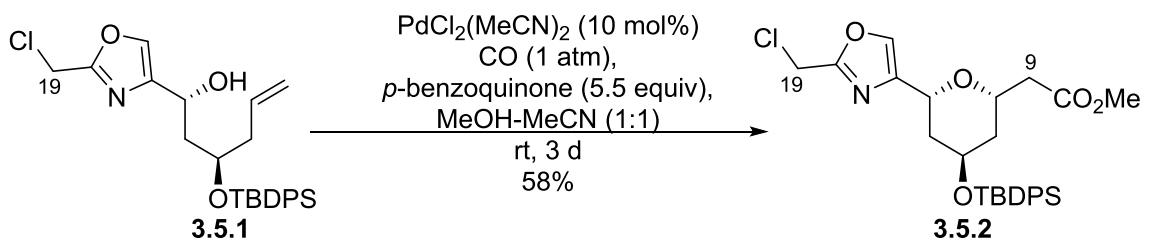

Scheme 3.5. Pd(II)-catalyzed alkoxycarbonylation of 3.5.1.

Similarly, in 2000, this method was adopted by Leighton et al. in the total synthesis of leucascandrolide A (Scheme 3.6). The intramolecular alkoxycarbonylation of diol 3.6.1 provided the desired cis-THP 3.6.2 in high 75\% yield and diastereoselectivity (dr $>10: 1)$. The 
reaction required the use of benzonitrile as cosolvent for a cleaner and more efficient reaction. It is worth noting that the cyclization was highly selective in that the two alcohols and alkenes in 3.6.1 were differentiated, and thus removed the need for additional protection steps. Additionally, the use of copper salt $\left(\mathrm{CuCl}_{2}\right)$ was required as an oxidant for the regeneration of the active Pd(II) species.

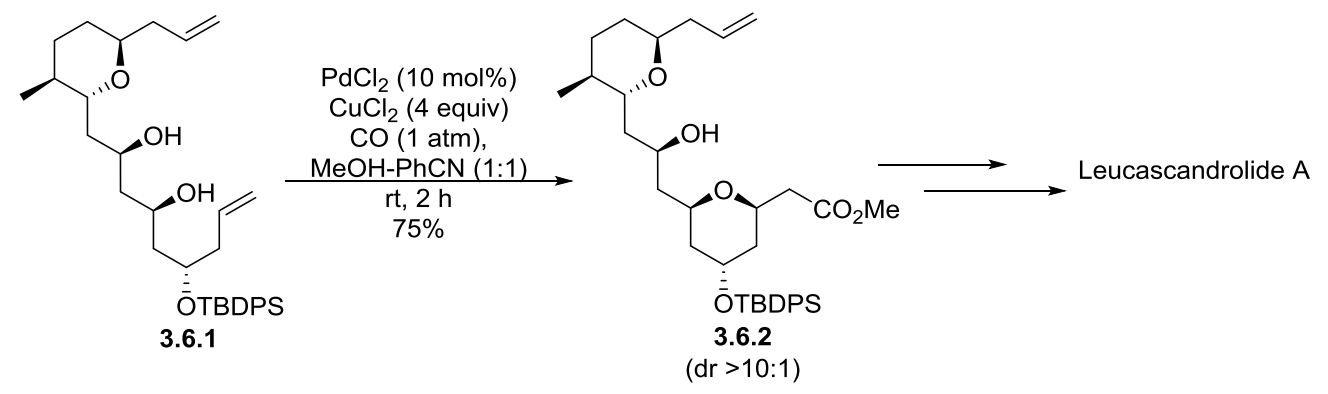

Scheme 3.6. Pd(II)-catalyzed alkoxycarbonylation of 3.6.1 towards synthesis of leucascandrolide A.

In addition, Kitching et al., had adopted a similar Pd(II)-catalyzed alkoxycarbonylation strategy to construct one of the 2 THP-rings present in the family of bistramide (Scheme 3.7). ${ }^{140}$ Although it was mentioned that other isomers were obtained together with desired THP-3.7.2, the diastereoselectivity was not specified.

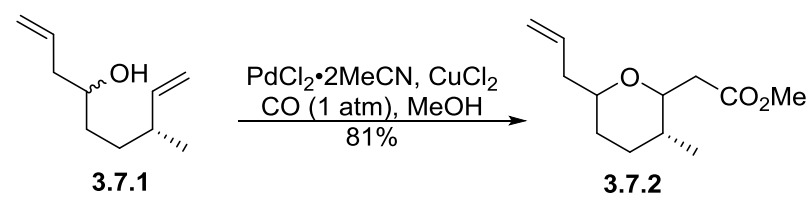

Scheme 3.7. Pd(II)-catalyzed alkoxycarbonylation of 3.7.1.

In 2007, Uenishi et al. reported the use of electrophilic intramolecular Pd(II)-catalyzed, 1,3-chirality transfer reaction of the linear 1,7-monoallylic alcohols 3.8.1 and 3.8.3 (Scheme 3.8). The method is key in the stereospecific construction of cis-THP 3.8.2 (Scheme 3.8, eq 1) and trans-THP core 3.8.4 (Scheme 3.8, eq 2) in the total syntheses of natural product diospongins A and B, respectively. ${ }^{141}$ The high diastereoselectivity is attributed to the reaction 
mechanism that involves a syn-coordination of the palladium to the allylic alcohols 3.8 .1 and 3.8.3 and a subsequent syn-oxypalladation and syn-elimination.<smiles>OC(F)/C=C/CCCC(O)c1ccccc1</smiles>

ŌH

3.8.1
$\underset{\mathrm{PdCl}_{2}\left(\mathrm{CH}_{3} \mathrm{CN}\right)_{2}(10 \mathrm{~mol} \%)}{\mathrm{THF}}$
$\stackrel{0}{ }{ }^{\circ} \mathrm{C}, 20 \mathrm{~min}$

$92 \%$

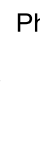

Y

3.8.2

$(\mathrm{dr}=11.5: 1)$

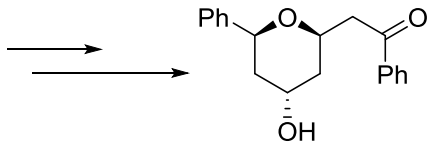

Diospongin A

[1]

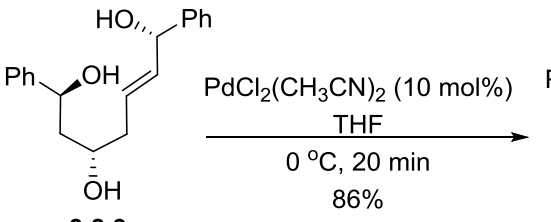

3.8.3

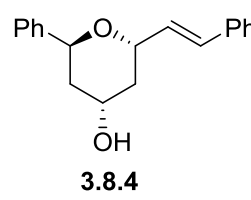

$(\mathrm{dr}=11.5: 1)$

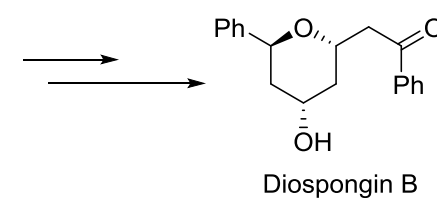

[2]

Scheme 3.8. Pd(II)-catalyzed hydroalkoxylation of 3.8.1 and 3.8.3.

Other metal-mediated cyclizations

In 2007, as an alternative to palladium metal, Aponick et al. reported the use of $\mathrm{Au}(\mathrm{I})$ complexes to promote the cyclization of monoallylic diols 3.9.1 to cis-2,6-tetrahydropyran 3.9.2 with a high diastereoselectivity of up to 25:1 (Scheme 3.9). ${ }^{119 a}$ Moreover, the method has a wide tolerance to a variety of substrates under mild conditions with catalyst loading as low as $0.1 \mathrm{~mol} \%{ }^{119 \mathrm{a}}$ and low temperatures were employed.

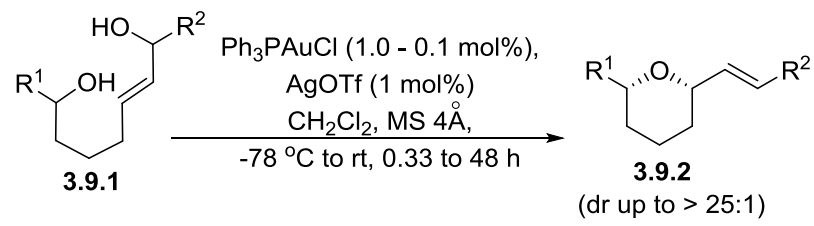

Scheme 3.9. Au(I)-catalyzed hydroalkoxylation of diol 3.9.1.

In 2005, He et al. reported the use of silver(I) triflate in the intramolecular hydroalkoxykation of inert olefins such as 3.10.1 to access THP-units (Scheme 3.10, eq 1). ${ }^{142}$ In the same year, Duñach et al. reported the cycloisomerisation of non-activated alkenes by 
tin(IV) trifluoromethane-sulfonate as catalyst. ${ }^{143}$ In the case of trans-5-phenyl-4-penten-1-ol 3.10.3, the reaction gave only the THP-3.10.4 regioisomer without competition from the 5-membered ring THF-isomer (Scheme 3.10, eq 2). Other metals such as cerium and platinum have also been employed. For instance, in 2002, Rosini et al., reported the use of cerium(III) chloride heptahydrate and sodium iodide in acetonitrile, which delivered 6-substituted THPesters 3.10.6 from alcohol 3.10.5 (Scheme 3.10, eq 3). ${ }^{144}$ The reaction proceeds with complete retention of stereochemistry of the starting material 3.10.5. Platinum-catalyzed $\delta$-hydroalkoxylation, on the other hand, was reported by Widenhoefer et al. in $2004 .{ }^{145}$ The reaction of hydroxyl alkene 3.10.7 was carried out using platinum(II) complexes, $\left[\mathrm{PtCl}_{2}\left(\mathrm{H}_{2} \mathrm{C}=\mathrm{CH}_{2}\right)_{2}\right]_{2}$, in the presence of phosphine ligands at $70{ }^{\circ} \mathrm{C}$ for $24 \mathrm{~h}$ to give desired THP-3.10.8 in $60 \%$ yield with high diastereoselectivity ( $>50: 1)$ (Scheme 3.10, eq 4).

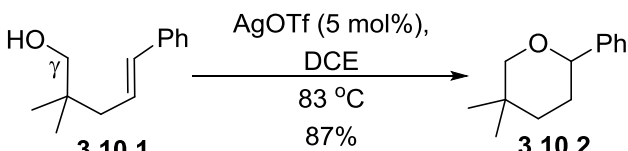

[1]

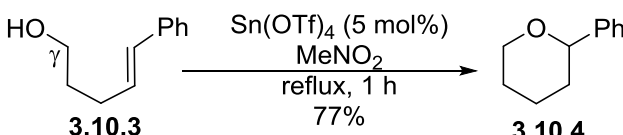

[2]
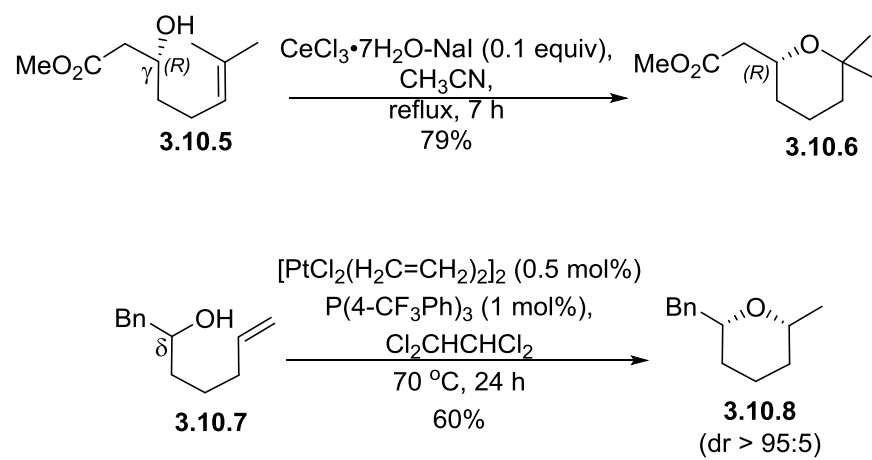

Scheme 3.10. Hydroalkoxylation catalyzed by silver, tin, cerium and platinum.

Similarly, a milder and eco-friendly method using iron(III)-promoted hydroalkoxylation was described by Cossy et al. (Scheme 3.11). ${ }^{146}$ The strategy developed exploited 
the use of inexpensive iron metal to catalyze the cyclization of allylic alcohols $\mathbf{3 . 1 1 . 1}$ to furnish cis-2,6-tetrahydropyrans 3.11.2 with high diastereoselectivity, which are the thermodynamically stable THP-product. The origin of stereocontrol derives from the proposed zwitterionic intermediate where the proposed carbocation is supported by observations of epimerization, which occurs in the 1:1 mixture of isomers where the trans-isomer epimerizes to give the single most stable $c i s$-isomer. Additionally, formation of a $\pi$-allyl cation reflects the significance of having both an activated allylic leaving group and allylic substituent capable of carbocation stabilization at C7. It is worth noting that, the relative positions of the carbon double bond and the allylic leaving group is interchangeable, and generates the same $\pi$-allyl intermediate. The cyclization proceeds under mild catalytic conditions where no heating is required as the cyclization occurs at room temperature with $5 \mathrm{~mol} \%$ catalytic loading. This, thus, inspires the key step in the synthesis of enigmazole A.

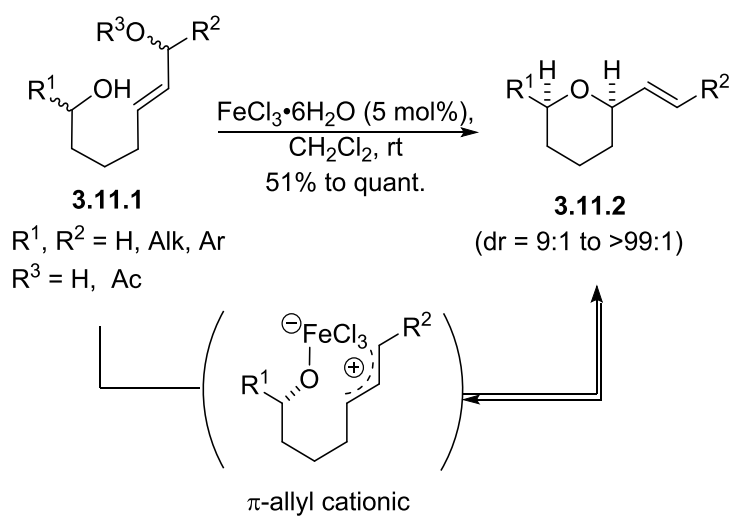

Scheme 3.11. Iron(III)-mediated cyclization of alcohols 3.11.1. 
This page has been intentionally left blank. 
This page has been intentionally left blank. 


\section{Chapter 3}

\subsection{Enigmazole A}

3.2.1 Structural Assignment of Enigmazole A

3.2.2 Biological Activity of Enigmazole A

3.2.3 Syntheses of Enigmazole A 
This page has been intentionally left blank. 


\subsection{Enigmazole A}

Enigmazole $\mathrm{A}$ is an 18-membered ring cytotoxic phosphomacrolide consisting of the embedded cis-2,6-disubstituted tetrahydropyran ring isolated from the south coast of Papua New Guinea in 1998 and it belongs to the collection of marine sponge cinachyrella enigmatica. ${ }^{124}$ The aqueous extract (46.6 g) was obtained from the extraction of the marine sponge (202 $\mathrm{g})$ with water at low temperature $\left(4{ }^{\circ} \mathrm{C}\right)$ and the organic extract $(4.6 \mathrm{~g})$ was obtained from repeated soaking of the animal residue in a mixture of methanol and dichloromethane. The aqueous extract $(16.5 \mathrm{~g})$ was subjected to partitioning between water and $n-\mathrm{BuOH}$ where the $n$ - $\mathrm{BuOH}$ soluble fractions were combined with the organic extract (3.3 g). The subsequent partitioning with aqueous dichloromethane, desalting process, size-exclusion chromatography and cytotoxicity test against IC-2 murine mast cell line resulted in active fractions that were then submitted to purification by reverse-phase HPLC, which led to the isolation of enigmazole A (21.2 mg).

\subsubsection{Structural Assignment of Enigmazole A by Gustafson et al.}

The different type and numbers of protons were analysed through ${ }^{1} \mathrm{H}$ NMR and HSQC (Figure 3.3). The analysis showed 3 aliphatic methyls (C24, C26 and C27), olefinic methyl (C25), a methoxy group at $\mathrm{C} 23$, an exo-methylene at $\mathrm{C} 9$, eight methylenes $(\mathrm{C} 3, \mathrm{C} 6, \mathrm{C} 8, \mathrm{C} 10$, C12, C13, C14 and C16), two aliphatic methines (C2 and C4), six oxy-methines (C5, C7, C11, $\mathrm{C} 15, \mathrm{C} 17$ and $\mathrm{C} 23)$, one olefinic methane (C21) and aromatic proton that was isolated (C19). From these small units, the group assembled three bigger fragments by using COSY and TOCSY experiments. The three identified fragments are fragment A (C5 - C19), fragment B $(\mathrm{C} 21-\mathrm{C} 25)$ and fragment $\mathrm{C}(\mathrm{C} 26-\mathrm{C} 27)$. The connections between these fragments such as fragment $\mathrm{A}$ and $\mathrm{C}$ were established on the basis of $\mathrm{HMBC}$ cross peaks analysis. The connection between fragment A and B, involving a heavily functionalized 2,4-disubstituted oxazole ring, 
were joined using the same technique but were provided further confirmation by a characteristically large heteronuclear coupling between C19 and H19 at $209.2 \mathrm{~Hz}$. The phosphate ester at $\mathrm{C} 5$ was determined by ${ }^{31} \mathrm{P}$ NMR, which showed a single resonance at $\delta 2.17$ ppm. This is considered unusual given the rarity of the phosphate ester group being present in polyketides. Enigmazole A has a total of eight stereogenic centers (Figure 3.3).

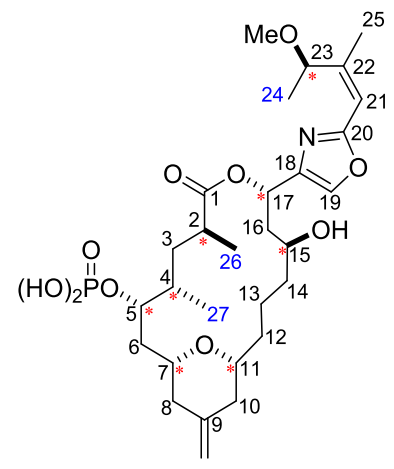

Enigmazole A

Figure 3.3. Numbered structure of enigmazole A.

\subsubsection{Biological Activity of Enigmazole A}

This marine natural product showed significant cytotoxic activity in the NCI 60-cell antitumor screen and IC-2 mast cells $\left(\mathrm{IC}_{50} 0.37 \mu \mathrm{g} / \mathrm{mL}\right) .{ }^{147}$ Moreover, the structurally unique core of enigmazole bears a potential scaffold for the discovery and the development of new compounds with selectivity for aberrant c-Kit signalling cells. ${ }^{124} \mathrm{c}-\mathrm{Kit}$ is a gene that encodes for the cytokine receptor, which is a type-III transmembrane protein-tyrosine kinase belonging to the immunoglobulin superfamily. ${ }^{148}$ It plays a dominant role in the management of gametogenesis, haematopoiesis, mast cell development, migration and function, stem cell maintenance as well as melanogenesis. ${ }^{149}$ Gastrointestinal stromal cell tumours (GISTs) and acute myelogenous leukaemia ${ }^{150}$ in cancer patients with a specific c-Kit genotype are consequently implicated because c-Kit is also involved in a number of gain-of-function mutations in humans ${ }^{124}$ that transfer a constitutive, ligand-independent kinase activity. ${ }^{124}$ 
Therefore, kinase inhibitors with c-Kit as one of its molecular targets proved successful as drugs (Imatinib, Gleevec ® by Novartis).

Enigmazole A does not behave as a kinase inhibitor but it does play a role in the c-Kit signalling cascade in a manner not yet elucidated. The natural product and two other isolated related congeners do not exhibit selectivity for mutant c-Kit. ${ }^{150}$ The reverse is true for the minor side fractions of the crude sponge extract as high selectivity for malignant cells expressing mutant c-Kit was observed. ${ }^{150}$ Therefore the scaffold of enigmazole A bears potential for the discovery of a pharmacophore.

\subsubsection{Syntheses of Enigmazole A}

There have been a total of three complete syntheses of enigmazole A succeeded by Molinski et al., ${ }^{124}$ Fürstner et al., ${ }^{150}$ and Smith et al., ${ }^{121}$ between the years 2010 and 2016. In addition, the synthesis of $\mathrm{C} 1-\mathrm{C} 7$ fragment of enigmazole $\mathrm{A}$ was reported by Kadato et al. in 2014. ${ }^{151}$ Each synthesis reflects distinctively diverse routes undertaken to arrive to the target natural product.

\subsubsection{Synthesis of $\mathrm{C} 1-\mathrm{C} 7$ Fragment of Enigmazole A by Kadato et al. ${ }^{151}$}

Kadato et al. reported the stereoselective synthesis of part of enigmazole A by employing Evans' chiral auxiliary 3.12.1 to perform stereocontrolled aldolization to form the chiral centers at $\mathrm{C} 4$ and $\mathrm{C} 5$ while asymmetric methylation to introduce the stereogenic center at C2 (Scheme 3.12). 


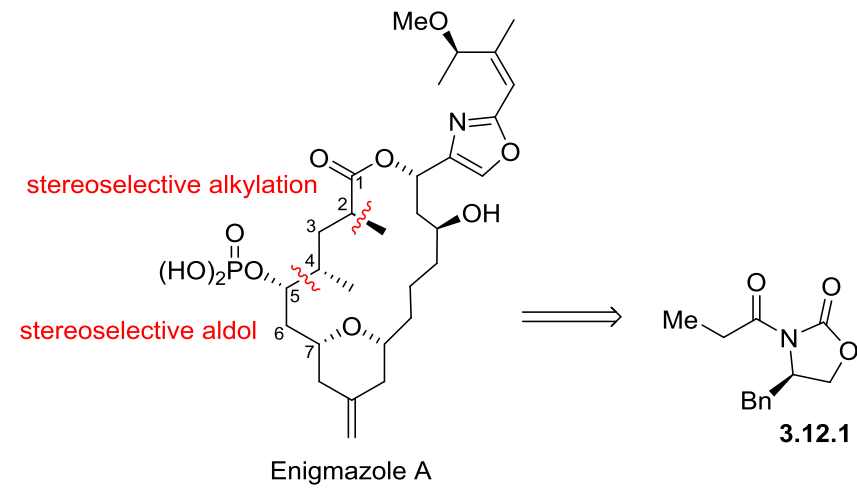

Scheme 3.12. Retrosynthetic analysis of enigmazole A by Kadato et al.

The chiral starting material was submitted to an aldol reaction with aldehyde 3.13.1 to give single diastereomer 3.13.2 in high 97\% yield (Scheme 3.13). The resulting alcohol was protected, followed by auxiliary cleavage and carbon homologation with Wittig olefination and subsequent hydrogenation gave 3.13.3. The group expected to create the third chiral center at C2 from substrate-controlled introduction of the methyl group. This was carried out after lactonization of 3.13.3 under acidic conditions (CSA, benzene, reflux) where subsequent treatment of resulting lactone with KHMDS and DMPU generated nucleophile 3.13.4, which underwent substitution with methyl iodide. However, a lack in selectivity resulted in an inseparable mixture of isomers 3.13 .5 and 3.13.5' $(\mathrm{dr}=5: 1)$. The relative stereochemistry was determined by ${ }^{1} \mathrm{H}$ NMR analysis and NOESY experiments. This led to an alternative auxiliarycontrolled methylation method utilizing Evans' oxazolidinone (Scheme 3.13). 


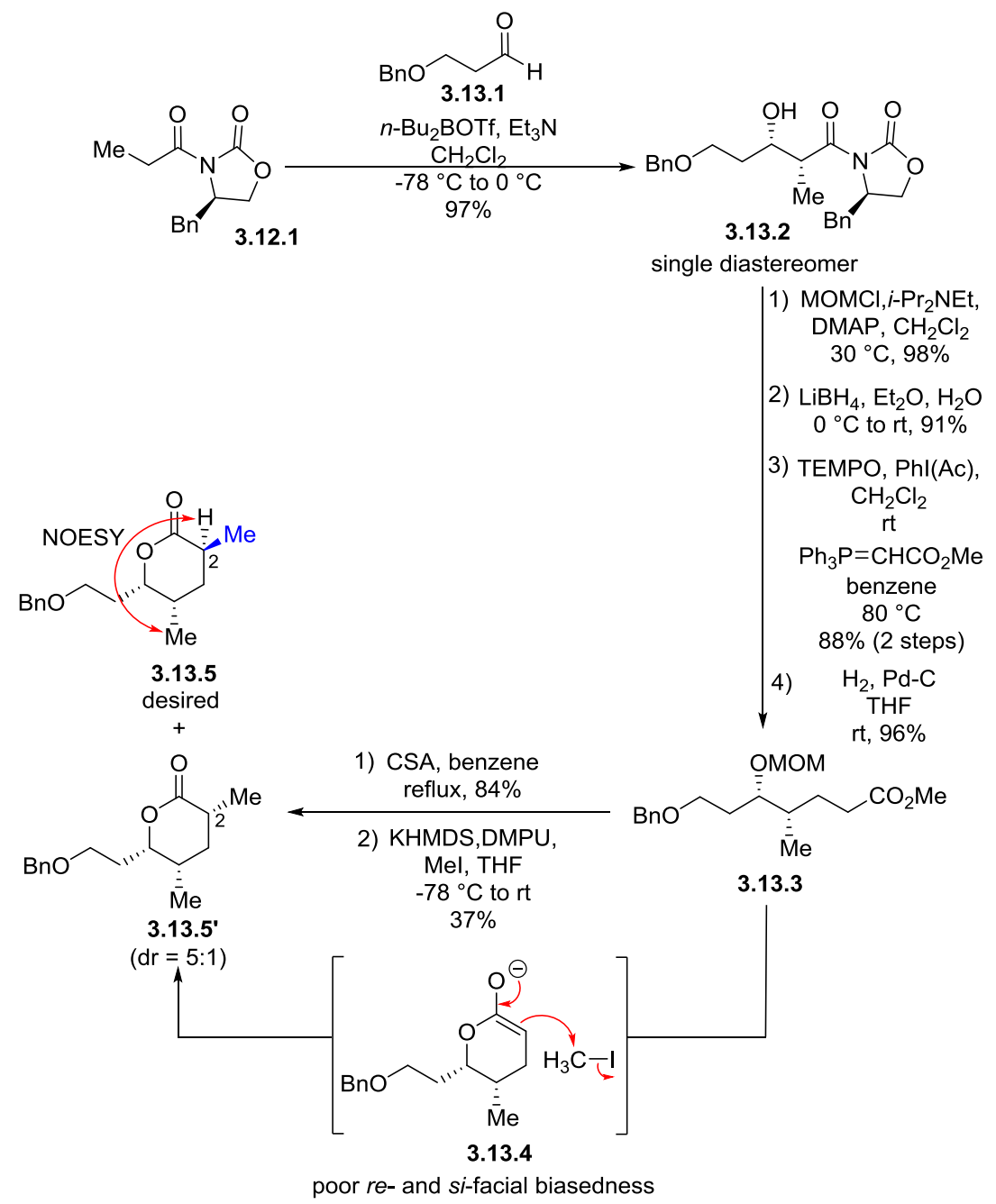

Scheme 3.13. Synthesis of lactone isomers 3.13 .5 and 3.13.5' from 3.12.1.

The oxazolidinone 3.14.1 was prepared from the saponification of methyl ester 3.13.3 to give the corresponding carboxylic acid, which was converted into acid anhydride ( $\mathrm{PivCl}$, $\mathrm{Et}_{3} \mathrm{~N}$ ) (Scheme 3.14). The resulting anhydride was employed in the acylation of (S)-4-benzyl2-oxazolidinone in the presence of lithium chloride to give 3.14.1. The following stereoselective alkylation proceeded to give a single diastereomer of 3.14.2. The oxazolidinone 3.14.2 was converted into lactone 3.13.5 to determine the relative stereochemistry. The diastereomer 3.14.2 was subjected to reductive cleavage of the chiral auxiliary, followed by TBS-protection of the resulting alcohol and deprotection of the benzyl group by hydrogenation to give the desired C1 - C7 fragment 3.14.3 of enigmazole A (Scheme 3.7) 


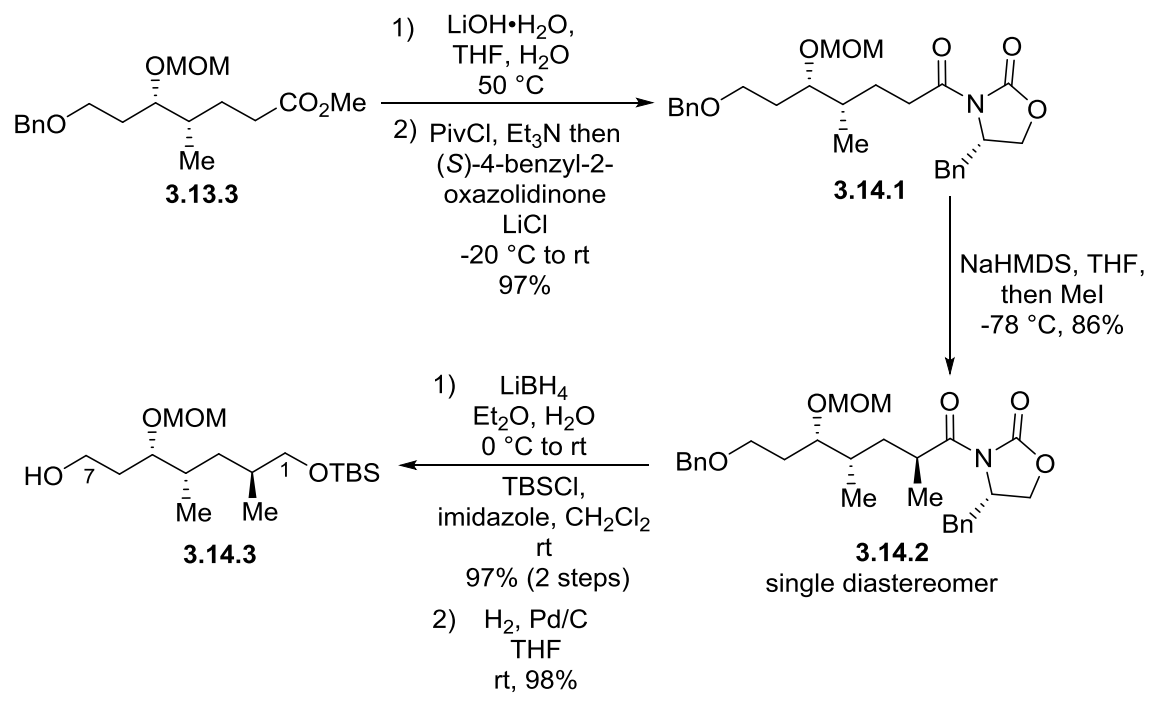

Scheme 3.14. Completion of $\mathrm{C} 1-\mathrm{C} 7$ fragment from ester 3.13.3.

Kadato et al. has illustrated the advantages of using an auxiliary-controlled stereoselective alkylation through the use of Evans' oxazolidinone. The synthetic plan included repetitions of the same well-established procedures, and thus did not reflect elements of novelty or a substantial extension in the work of selective alkylation.

\subsubsection{Synthesis of Enigmazole A by Molinski et al. ${ }^{124}$}

The first total synthesis of enigmazole A was completed in the year 2010 by Molinski et al. ${ }^{124}$ It was carried out in the longest linear sequence comprising of 22 steps. The global retrosynthetic analysis of enigmazole A is reflected in Scheme 3.15. There are four key steps involved in the approach to enigmazole A reported by Molinski et al. The first is an efficient Negishi-type coupling between 3.15.6 and 3.15.5 that would form the $\mathrm{C} 21-\mathrm{C} 22$ bond. The other key steps include a hetero-Diels-Alder (HAD) cycloaddition between aldehyde 3.15.1 and diene 3.15.2 that would establish the central THP-ring, the Wittig olefination between 3.15.3 and 3.15.2 would allow the formation of the $\mathrm{C} 12-\mathrm{C} 13$ bond. The third key step would consists of a late stage macrolactonization that would complete the 18-membered ring of enigmazole A (Scheme 3.15). 


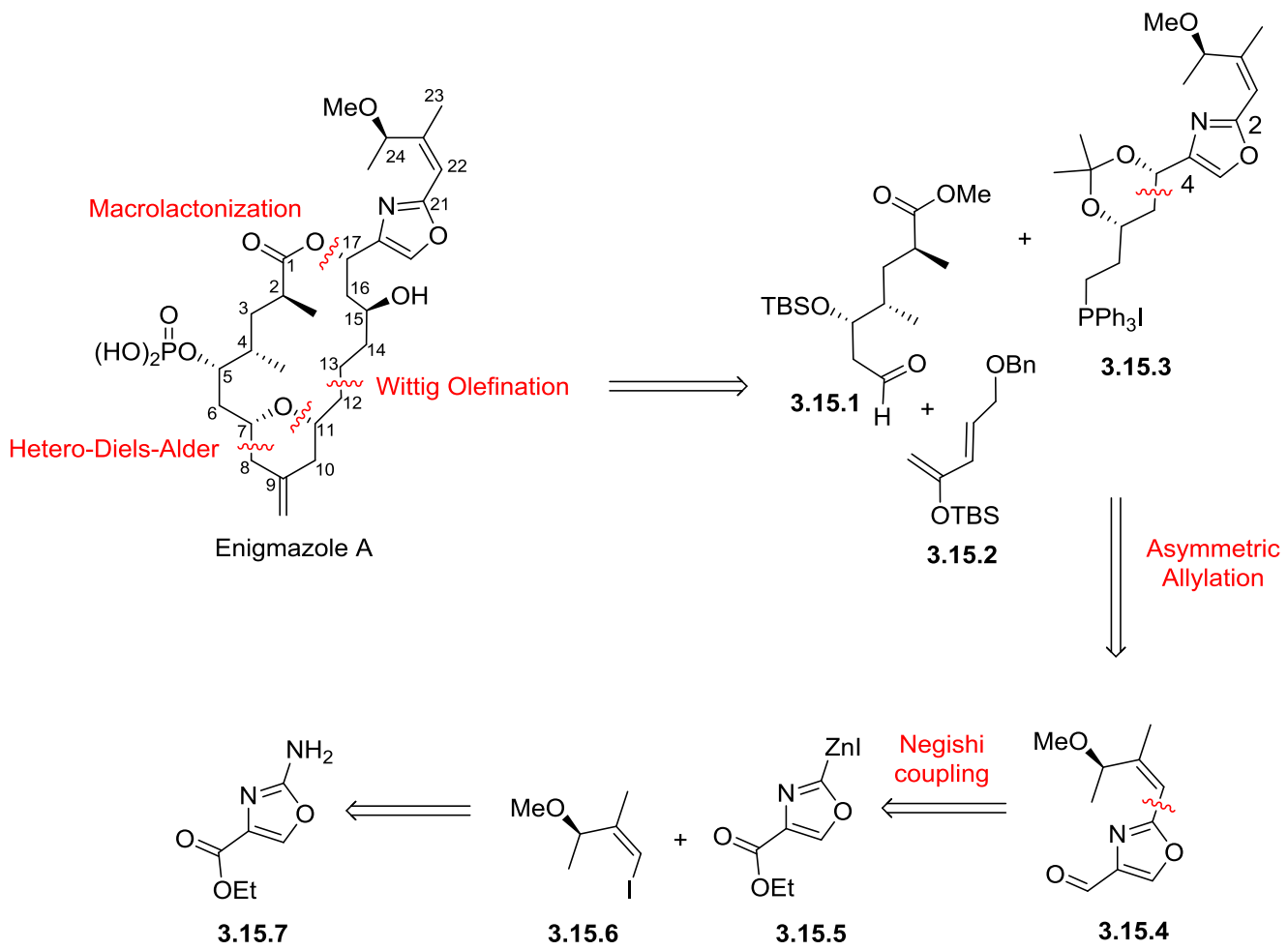

Scheme 3.15. Retrosynthetic analysis of enigmazole by Molinski et al.

One of the major fragments, the fragment 3.16.5, contained a functionalized oxazole at $\mathrm{C} 2$ and $\mathrm{C} 4$ (Scheme 3.16). The C2 functionalization was achieved by using an oxazol-2-ylzinc reagent 3.15.5, which enabled coupling with vinyl iodide 3.15.6 through a Negishi coupling. ${ }^{152}$ However, the preparation of oxazol-2-ylzinc reagents by transmetalation of 2-lithiooxazole with $\mathrm{ZnCl}_{2}$ required basic conditions that removed other reactive substituents on the oxazole like an ester group. Although the Negishi coupling can be achieved by using vinyl zincate and 2-chlorooxazole (shown by Smith et al. $){ }^{121}$ the use of metalated oxazoles specifically attracted the group. This is as metalated zincates are not regularly employed in natural product synthesis. ${ }^{153}$ Therefore, Molinski et al. decided to use the ambident 2-aminooxazole carboxylate ester 3.15.7 that grants control of its reactivity despite increasing the number of synthetic steps (Scheme 3.16). 
The nucleophilicity of $\mathbf{3 . 1 5 . 5}$ at $\mathrm{C} 2$ can be revealed through diazonium salt formation of 3.15.7, followed by its conversion to iodide 3.16.1 and a direct $\mathrm{Zn}(0)$ metalation to give oxazol-2-yl zincate 3.15.5 under Knochel's conditions (Scheme 3.16). ${ }^{154}$ The fragment 3.15.5 was submitted to a Negishi coupling with its vinyl iodide partner 3.16.4, which was prepared in 3 steps beginning with the oxidation of allylic alcohol 3.16.2. ${ }^{155}$ The stereochemistry at C3 of allylic alcohol 3.16.4 was introduced by an asymmetric alkylation of aldehyde 3.16.3 using Nugent's chiral catalyst $(+)$-morpholinoisoborneol (MIB) $)^{156}$ with dimethylzinc reagent, giving 3.16.4 in a high enantiomeric excess of 93\%. Upon methylation of 3.16.4 under standard conditions, the Negishi cross-coupling of fragments 3.15.5 and 3.15.6 was carried out to give multigram quantities of oxazole 3.16.5. It is noteworthy that the oxazole zincate 3.15 .5 is considerably stable to storage in the dark under $\mathrm{N}_{2}$ in THF at $4{ }^{\circ} \mathrm{C}$ prior to use (Scheme 3.16).
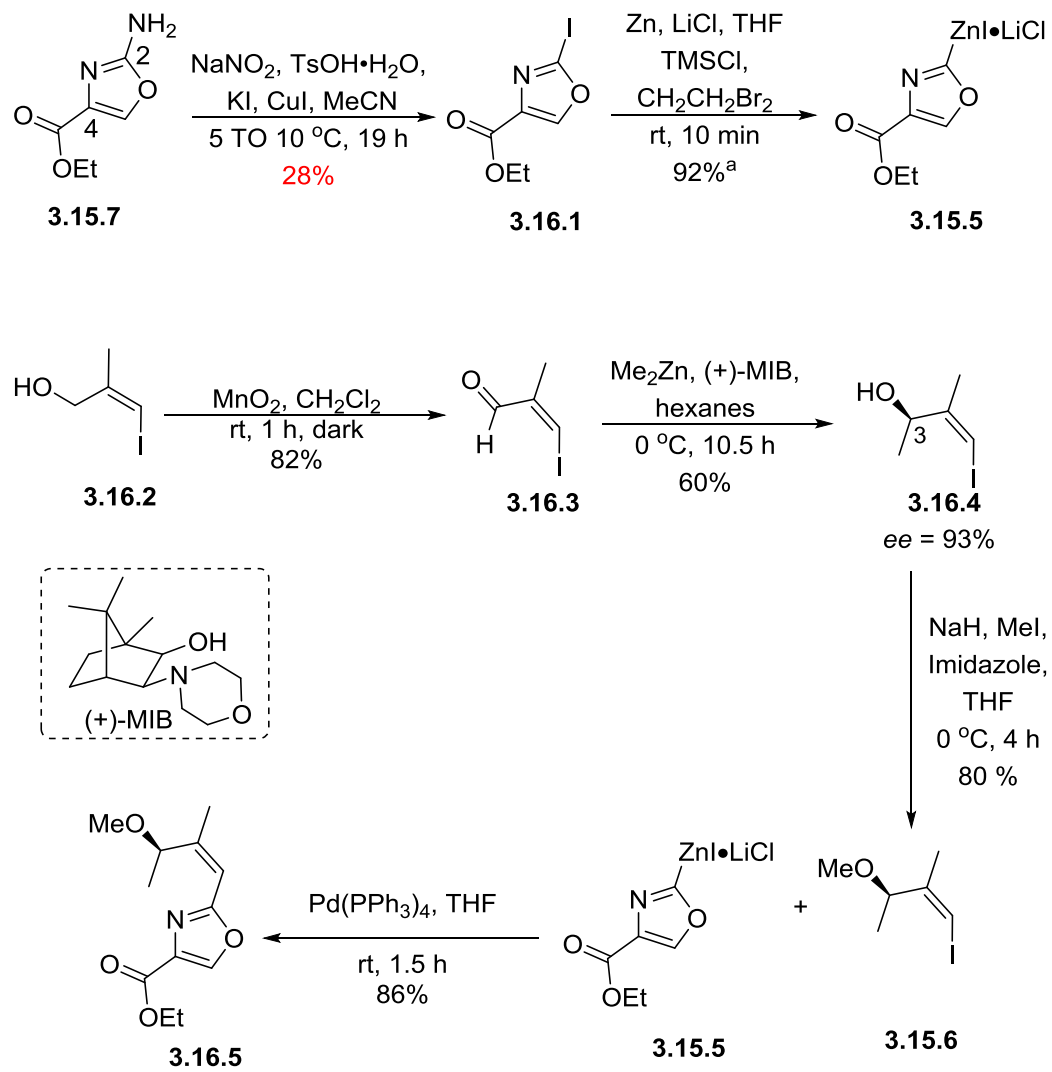

Scheme 3.16. Efficient Negishi coupling to obtain oxazole 3.16.5. 
The ester of oxazole 3.16.5 was reduced by a DIBAL-H reduction to give aldehyde 3.15.4 that was subjected to an asymmetric allylation (Scheme 3.17). The diastereoselective allylation demonstrated by Molinski et al. employed Corey's C2-symmetric catalyst 3.17.2. ${ }^{157}$ The ligand 3.17.1 reacts with $\mathrm{BBr}_{3}$ followed by tributyltin to give the allylation agent 3.17.2. The prepared allylborane 3.17.2 was then added to aldehyde 3.15.4 to produce 3.17.3 with a high diastereoselectivity $(\mathrm{dr}=24: 1)$. The diastereocontrol can be explained by chair-like transition state TS-I formed between the $(S, S)$-allylborane reagent 3.17.2 and aldehyde 3.15.4, which takes into account the optimum requirement of both the stereoelectronic and steric repulsion factors (Scheme 3.17).

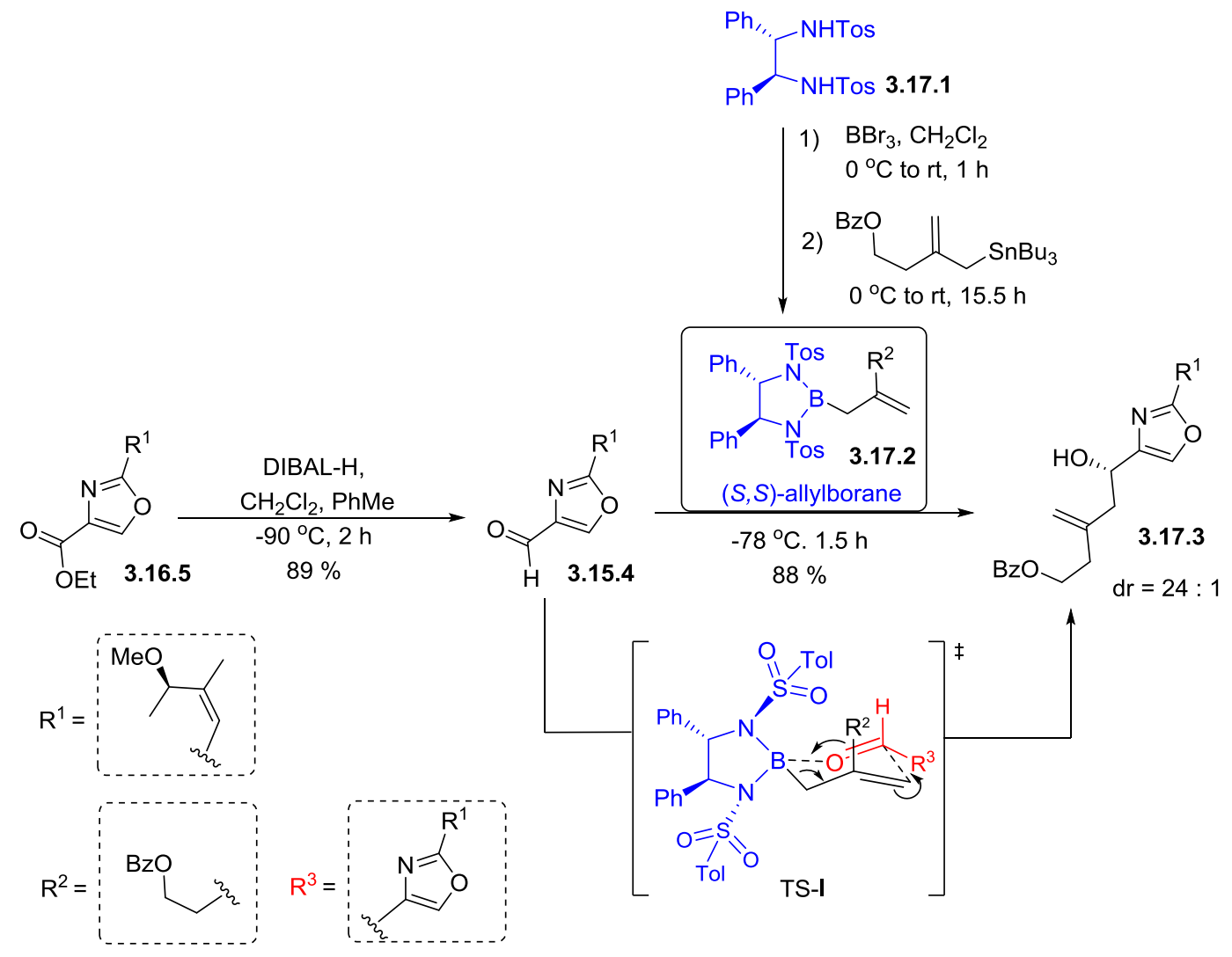

Scheme 3.17. Key diastereoselective allylation of aldehyde 3.15.4.

The homoallylic alcohol 3.17.3 was subjected to an oxidative cleavage to afford ketone 3.18.1 (Scheme 3.18). Ketone 3.18.1 was selectively reduced under Narasaka's conditions ${ }^{158}$ to 
produce syn-1,3-diols ( $\mathrm{dr}>95: 5)$. This reflects the third example of stereoselectivity control in the synthesis of enigmazole A. All of which involves manipulation of the carbonyl functionality. The phosphonium salt 3.15.3 was then derived through standard procedures including benzoate deprotection and acetonide protection ${ }^{159}$ of diols 3.18.2 to give 3.18.3 followed by phosphine salt formation (Scheme 3.18).

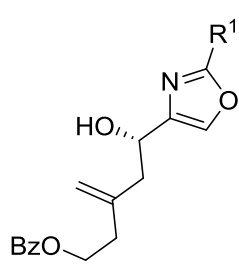

3.17.3

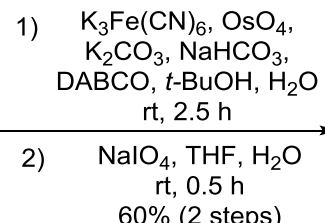
$60 \%$ (2 steps)

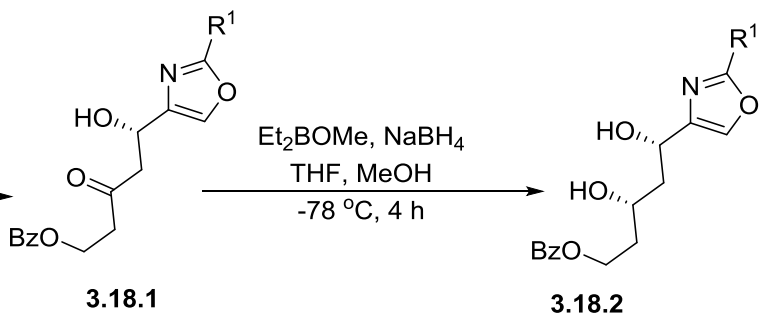

3.18 .2

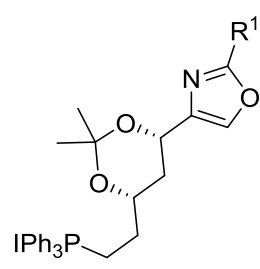

3.15 .3

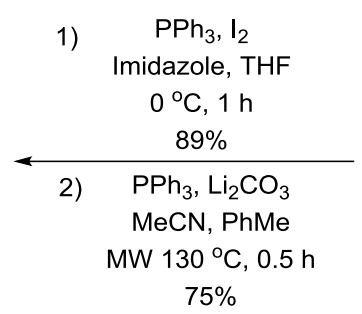

3.18.3

Scheme 3.18. Synthesis towards phosphonium salt 3.15.3.

The $\mathrm{C} 1-\mathrm{C} 7$ fragment, namely the dienophile 3.15.1, contains three stereogenic centers (Scheme 3.19). Molinski et al. managed two of the three by employing a chiral starting material 3.19.1. The third chiral center was introduced by the diastereoselective Roush allylation ${ }^{160}$ on aldehyde 3.19.2, which is the second allylation step and both of which are reagent-controlled allylation (Scheme 3.19). The $\alpha$-chiral substituted aldehyde 3.19.2 was synthesized in 4 steps. Substrate controlled allylation was attempted using a variety of Lewis acid but with a poor 
diastereoselectivity ratio. This signifies inadequate innate diastereofacial selectivity of aldehyde 3.19.2. Therefore, Barbier, ${ }^{161}$ Brown $^{22 b}$ and Roush allylation became a natural alternative for reagent-directed chirality transfer.

The Roush allylation condition on aldehyde 3.19.2 gave reasonable yield and diastereoselectivity $(\mathrm{dr}=9: 1)$. This is as the Brown allylation, although afforded a good diastereoselectivity $(\mathrm{dr}>10: 1)$ was plagued by isolation problems from byproducts, whereas Barbier allylation did not give high diastereoselectivity $(\mathrm{dr}=3.9: 1)$. Duthaler-Hafner allylation ${ }^{162}$ could be a promising alternative, but was not explored by the group.

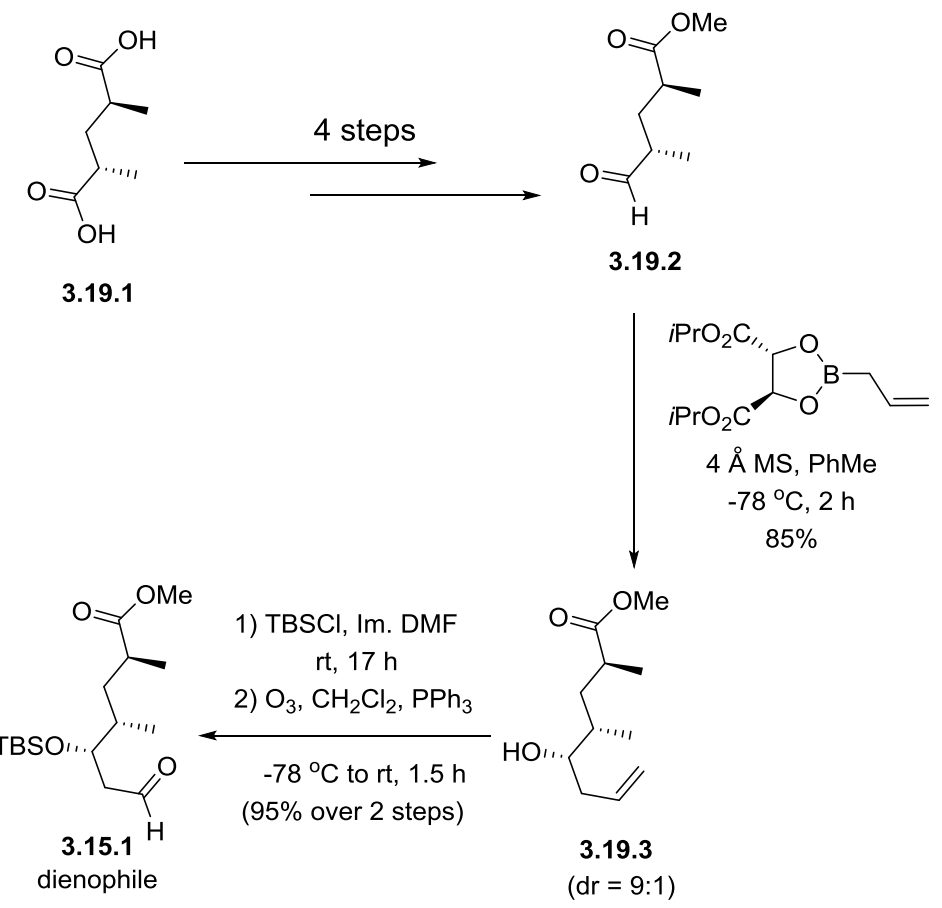

Scheme 3.19. Synthesis towards dienophile 3.15.1 from diacid 3.19.1.

The dienophile 3.15.1 were then combined with diene 3.15 .2 through a hetero-DielsAlder (HDA) cycloaddition to construct the THP-ring of enigmazole A, which is one of the key steps (Figure 3.4). There are 4 possible stereoisomers derivable from HDA that are a result from the nature of attack (endo- or exo-) and the approach of diene with respect to the dienophile to initiate either a $r e$ - or $s i$-face attack (Figure 3.4). 

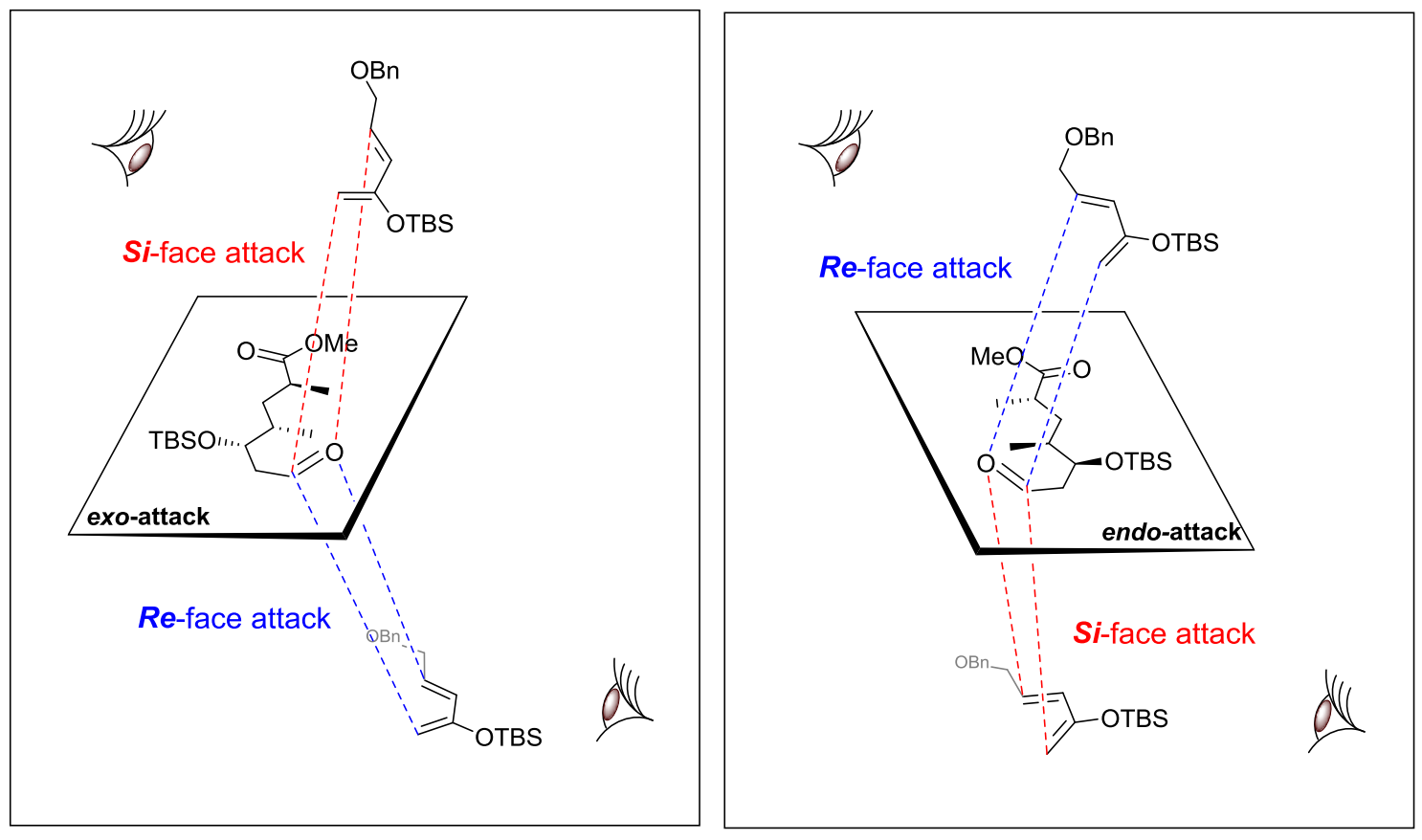

Figure 3.4. Asymmetric depiction of Hetero-Diels-Alder reaction leading to 4 possible diastereomers.

There were 3 separable isomers, 3.20.1 - 3.20.3 were isolated out of the possible four (Scheme 3.20). While the yield of $81 \%$ is high, it is not indicative of the isolated yield of the desired product 3.20.1 but a global yield of the mixture of diastereomers 3.20.1 - 3.20.3

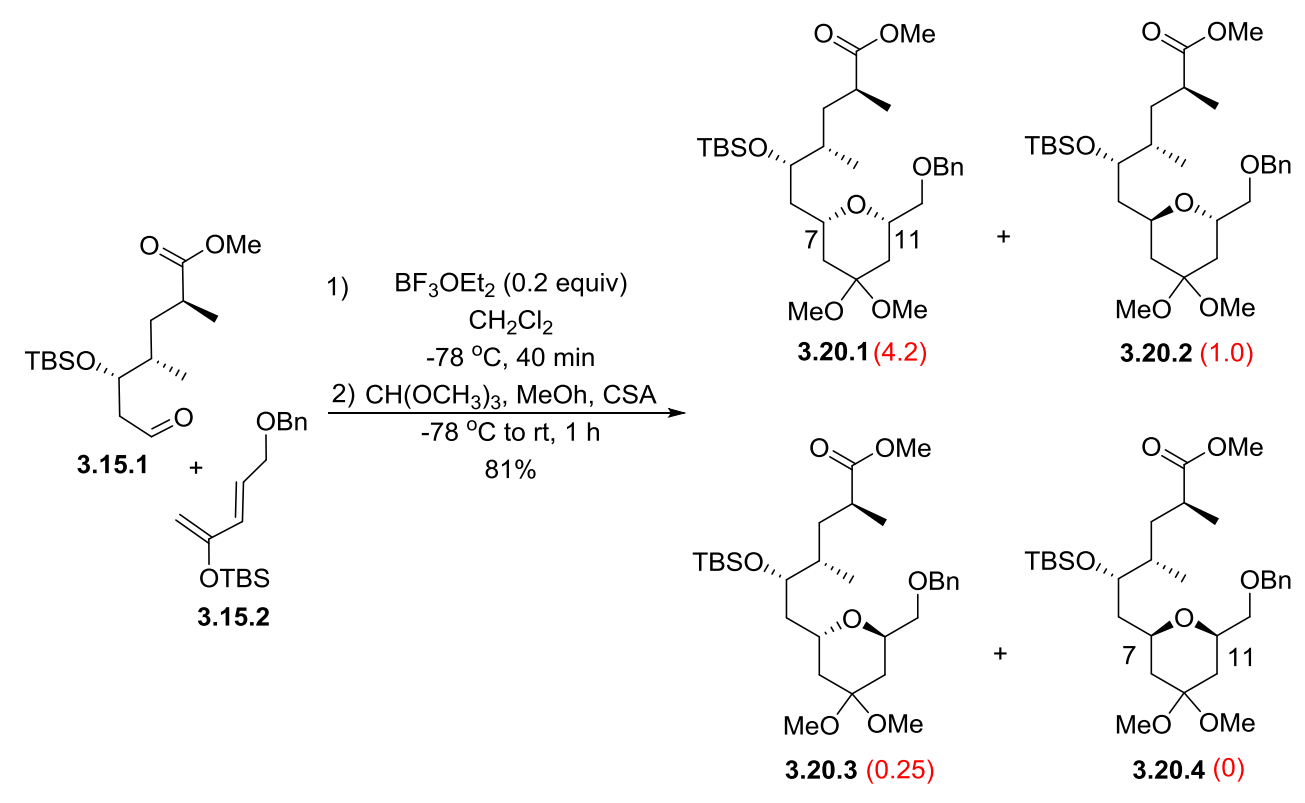

Scheme 3.20. HDA of dienophile 3.15.1 and diene 3.15.2 followed by a one-pot dimethylketal formation. 
The relative configuration of diastereomers were justifiably assigned by NOESY experiments based on the correlation of axial protons $\mathrm{H} 7$ and H11 (Scheme 3.21). This signifies a syn-THP configuration amongst the mixture of diastereomers obtained. The absolute configuration of $(7 R, 9 S, 11 R)-\mathbf{3 . 2 1} .3$, was assigned by a parallel analysis of analogue 3.21.1 synthesized under identical reaction conditions (Scheme 3.20). This is unreliable as the analogue 3.21.1 contains a 3-carbon-long alkyl chain with a terminal alkene instead of an $\alpha$-heteroatom of the benzyl group as in substrate 3.20.1, which may compromise the outcome. The actual substrate 3.20.1 should be used to assign the absolute configuration through Mosher's acid analysis. Nonetheless, the data obtained from the eventual completion of the natural product matches the physical properties of isolated enigmazole A by Gustafson. ${ }^{147}$

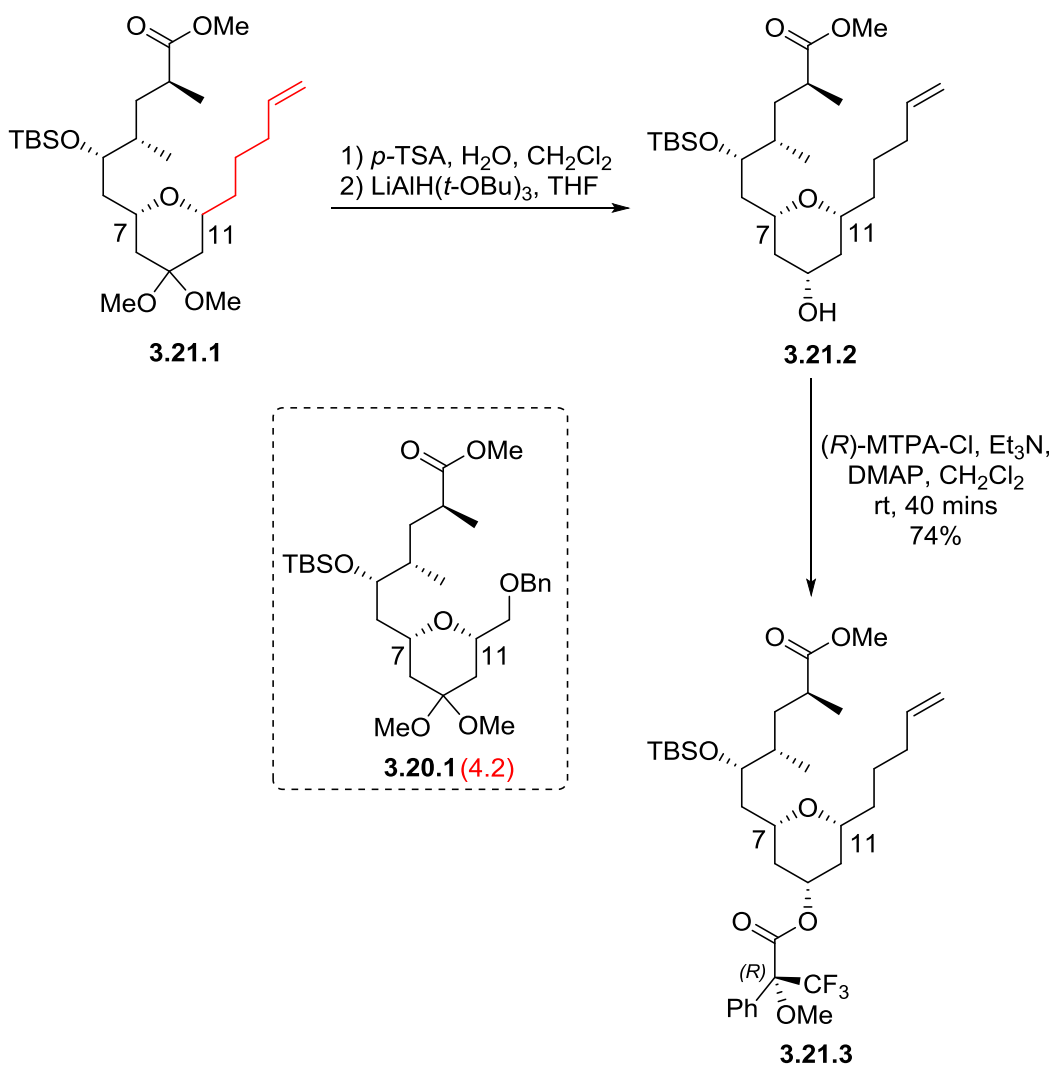

Scheme 3.21. Absolute configuration assignment of analogue 3.21.3 using Mosher's acid analysis.

Although the HDA constitutes as one of the key steps, there was no discussion associated with the failed HDA involving Jacobsen's chiral catalyst ${ }^{163}$ as an attempt to induce a 
reagent controlled HDA. It was stated that the reaction "failed to give product" with no remark on the contents of the failed reaction nor a mention of any recovered starting material.

The HDA product 3.20.1 was converted to aldehyde 3.22.1 in two steps (Scheme 3.22). After benzyl deprotection of the primary hydroxyl, a Swern oxidation produced the aldehyde 3.22.1. This aldehyde 3.22.1 was then involved in a Wittig olefination with phosphonium salt 3.15.3. The merger of these two fragments 3.22.1 and 3.15.3 forms the open chain scaffold of enigmazole $\mathrm{A},(Z)$-isomer 3.22.2. There was no reported alkene stereoselectivity in the Wittig olefination step and thus no ratio of the geometric isomers was given.

The Keck macrolactonization, amongst other macrolactonization conditions that were tested such as Mukaiyama, ${ }^{164}$ Yamaguchi, ${ }^{165}$ Yonemitsu, ${ }^{166}$ Shiina, ${ }^{167}$ was found effective when applied to compound 3.22.2 to give the 18-membered ring macrolide 3.18.1 in 35\% overall yield over 3 steps (Scheme 3.22). It is worth pointing out that, due to the bond geometry of hydroxyl groups at $\mathrm{C} 15$ and $\mathrm{C} 17$ of 3.22.2 after acetonide deprotection, the macrolactonization of 3.22.2 was faced with ring-selectivity issues when performed after the alkene hydrogenation. The competing 16-membered macrolide formation (as opposed to the expected 18-membered ring), was obtained as the major product under a range of conditions. Therefore, the 18-membered macrolide 3.18.1 was formed prior to hydrogenation.

The alcohol 3.22.3 was prepared in 4 steps from alkene 3.18.1. The selective reduction of (Z)-isomer of 3.18.1 using Wilkinson's catalyst, followed by Lombardo's olefination upon the removal of the ketal functionality at $\mathrm{C} 9$ incorporated the exo-methylene group, and the subsequent TBS-deprotection furnished the alcohol 3.22.3 (Scheme 3.22). Enigmazole A was obtained after the introduction of the phosphate ester group, in $0.41 \%$ overall yield over a total of 22 steps. 

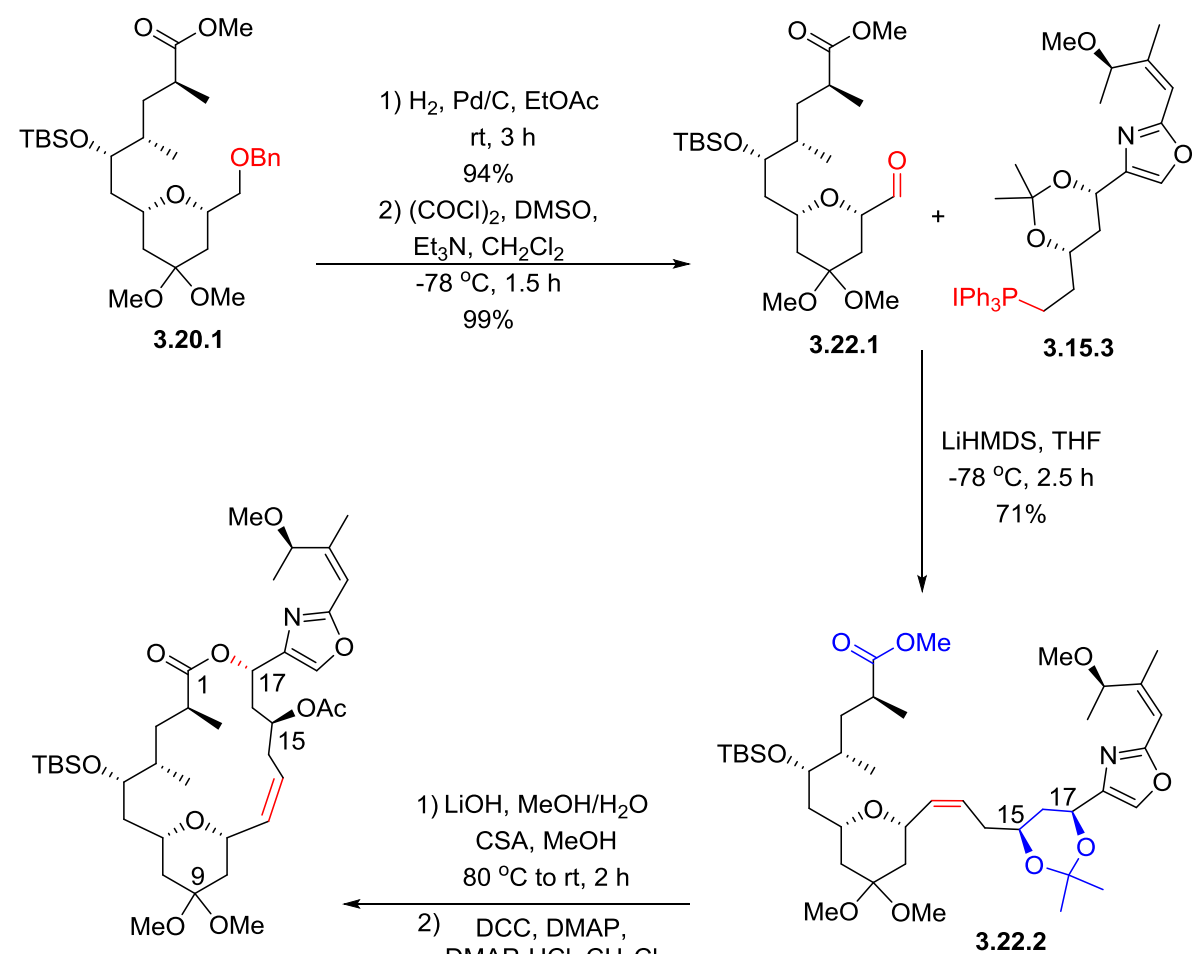

1) $\mathrm{LiOH}, \mathrm{MeOH} / \mathrm{H}_{2} \mathrm{O}$ CSA, $\mathrm{MeOH}$ $80^{\circ} \mathrm{C}$ to rt, $2 \mathrm{~h}$

3.18.1

2) DCC, DMAP, DMAP $\cdot \mathrm{HCl}, \mathrm{CH}_{3} \mathrm{Cl}$ reflux, $15.5 \mathrm{~h}$

then $\mathrm{AcOH}, \mathrm{MeOH}$

3.22.1

3.15.3

LiHMDS, THF $-78^{\circ} \mathrm{C}, 2.5 \mathrm{~h}$ $71 \%$

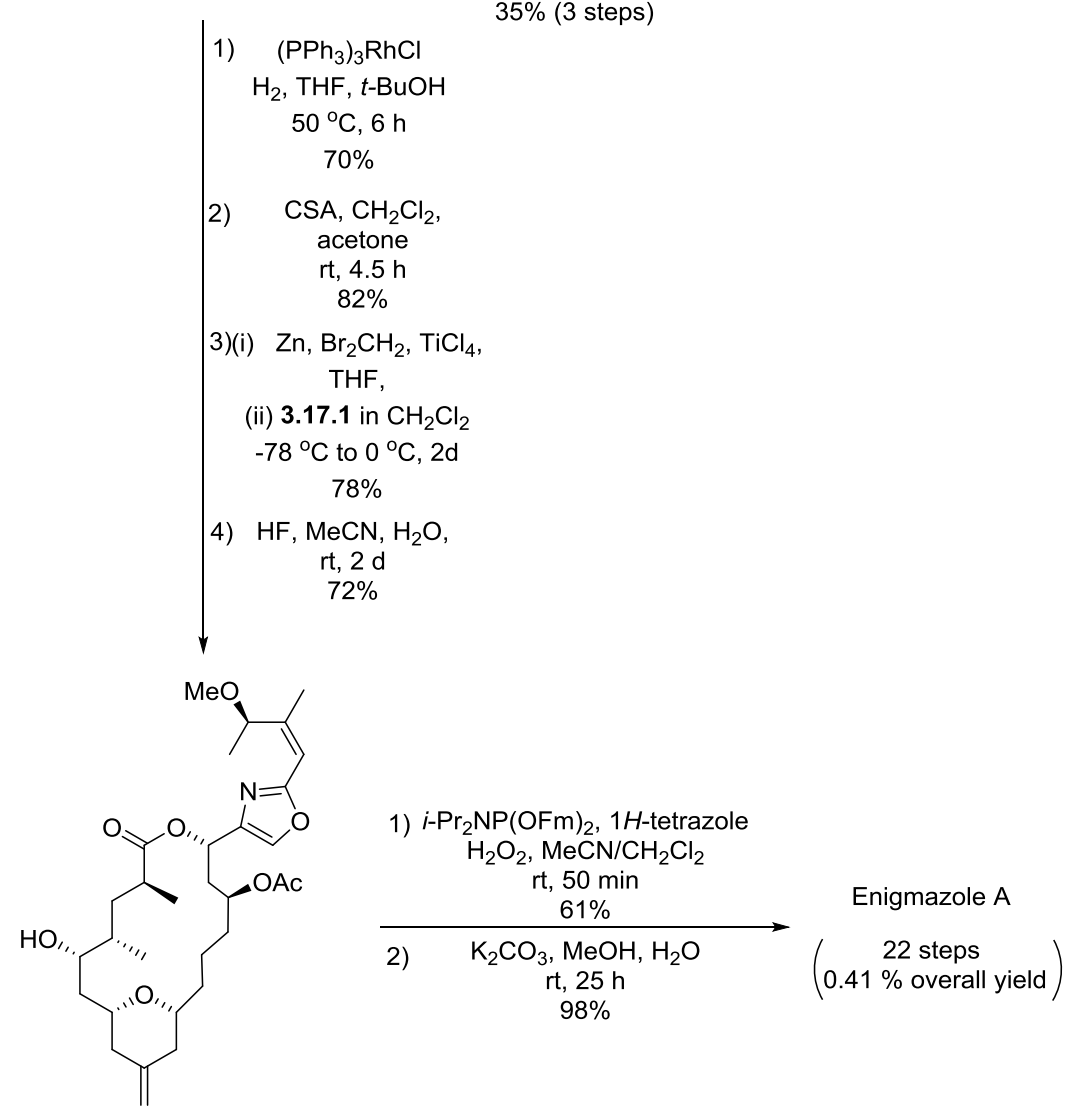

3.22.3

Scheme 3.22. The total synthesis of enigmazole A from HDA product 3.20.1. 


\subsubsection{Synthesis of Enigmazole A by Smith et al.}

A different approach to access enigmazole A that extensively exploits the use of dithiane couplings was undertaken by Smith et al. in 2015 (Scheme 3.23). ${ }^{121}$ The natural product was envisaged from two fragments, the aldehyde 3.23.1 and the $\beta$-hydroxyacid 3.23.2, together with a Petasis-Ferrier rearrangement. Although this is a contrast from the retrosynthesis reported by Molinski et al., which involved 3 smaller fragments, both syntheses employed the macrolactonization strategy to induce ring-closure as a final step.

The larger fragment 3.23.2 would be accessed by a dithiane-epoxide union involving dithiane 3.23.3 and epoxide 3.23.6 (Scheme 3.23). The dithiane fragment 3.23.3 would be synthesized from 2-chlorooxazole 3.23.5 and vinyl zincate would derive from vinyl iodide 3.23.4 using Negishi coupling. ${ }^{152}$ The epoxide fragment 3.23.6 would be constructed from epoxides 3.23.9 and 3.23.8 with dithiane 3.23.7 by a multicomponent Type I Anion Relay Chemistry (ARC) $)^{168}$ (Scheme 3.23). 

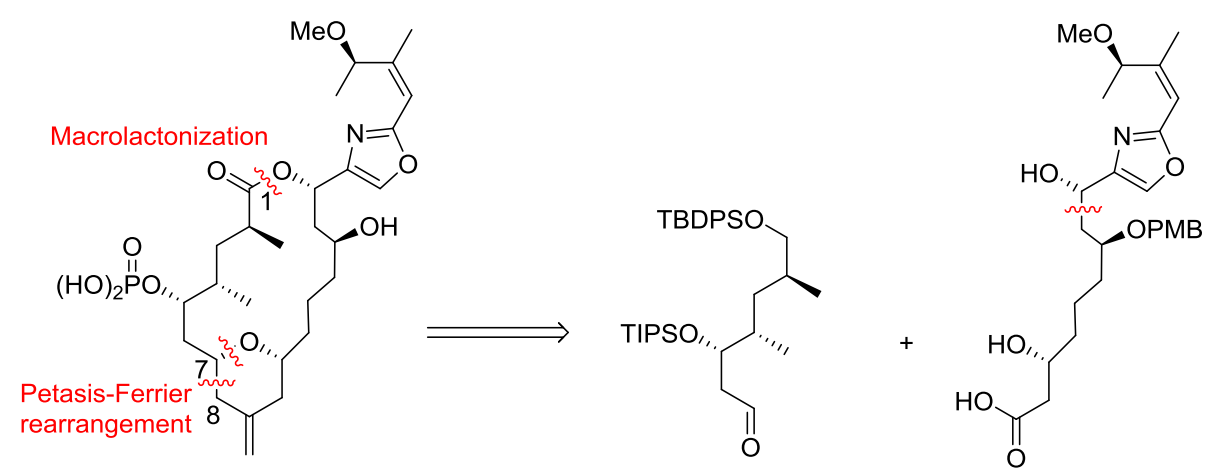

Enigmazole A

Fragment $\mathrm{C} 1-\mathrm{C} 7$

3.23.1

Fragment $\mathrm{C} 8 \mathrm{-C} 18$

3.23.2

Dithiane-Epoxide union

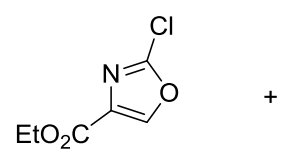

3.23 .5<smiles>COC(C)/C(C)=C\I</smiles>

3.23.4

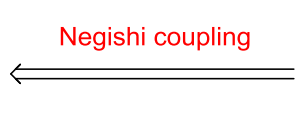

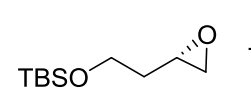

3.23 .9<smiles></smiles>

3.23.8

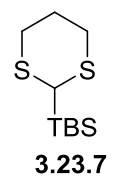

3.23.7

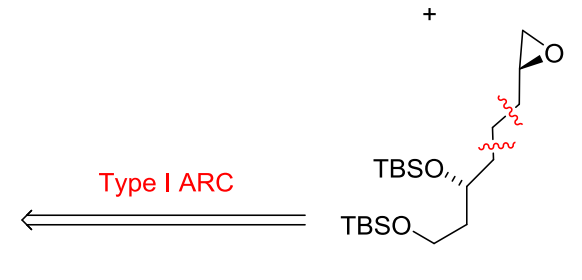

3.23.6

Scheme 3.23. Retrosynthetic analysis of enigmazole A by Smith et al.

Synthesis of Fragment C8-C18

The assembly of epoxide 3.23.6 began with two enantio-enriched starting epoxides 3.23.9 and 3.23.8 with dithiane 3.23.7. Treatment of 3.23.7 with $n$-BuLi and then the addition of epoxide 3.23.9 followed by epoxide 3.23.8, compound 3.24.1 was obtained as a single diastereomer in $90 \%$ yield (Scheme 3.24). This strategy is highly efficient as there are $2 \mathrm{C}-\mathrm{C}$ and $\mathrm{O}-\mathrm{Si}$ bond formations in a one pot reaction. The simultaneous TBS-protection of the hydroxyl upon its formation from the first coupling of dithiane 3.23.7 with epoxide 3.23.9 proceeds through a $[1,4]$-Brook rearrangement. The intramolecular silyl group migration 
proceeds with a complete retention of the stereogenic center. This strategy of a multicomponent anion relay chemistry is advantageous as it enables rapid construction of structurally complex scaffolds in a single step. ${ }^{169}$ Smith et al., unlike Molinski and Fürstner et al., used enantiomerically pure starting materials to acquire the 2 stereogenic centers in 3.24.1. The following PMB-reduction and dithiane group removal steps resulted in diol 3.24.2, which furnished epoxide 3.23.6 under Fraser-Reid conditions (Scheme 3.24). ${ }^{170}$

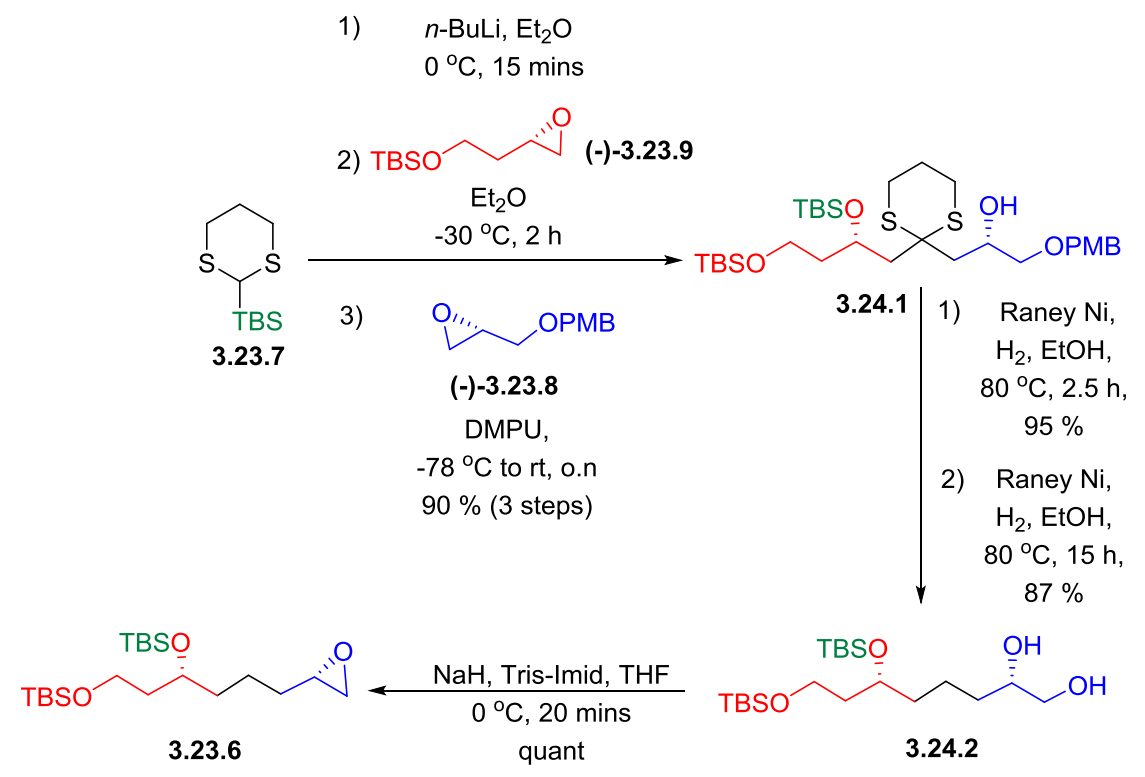

Scheme 3.24. Type I ARC multicomponent coupling towards epoxide 3.23.6.

The synthesis continues with the construction of the dithiane 3.23.3. The enantiopure starting material $(R)$-3-butyn-2-ol 3.25.1 was subjected to a copper-assisted carbometalation /iodination sequence that was followed by methylation to give vinyl iodide $\mathbf{3 . 2 3 . 4}$ in $77 \%$ yield over 2 steps (Scheme 3.25). The vinyl iodide was transformed into an organozinc reagent to undergo the Negishi coupling with 2-chlorooxazole 3.23.5 to give the ester 3.25.2. The dithiane 3.23.3 was obtained from the DIBAL-H reduction of ester 3.25.2 followed by carbonyl protection with 1,3-propanethiol in two steps (Scheme 3.25). 


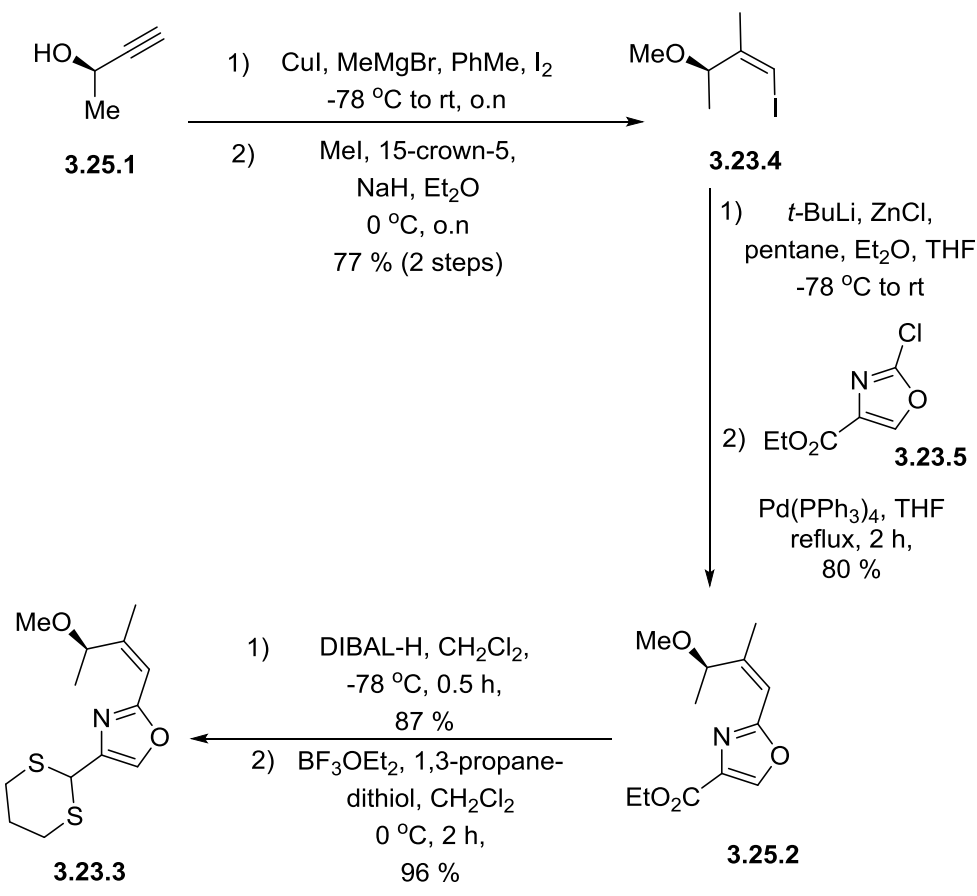

Scheme 3.25. Construction of dithiane fragment 3.23.3 from enantiopure $(R)$-3-butyn-2ol 3.25.1.

The dithiane 3.23.3 was assembled with epoxide 3.24.3 to give hydroxyl 3.26.1 in 77\% yield (Scheme 3.26). The functional group interconversion from dithiane of $\mathbf{3 . 2 6 . 1}$ to a chiral hydroxyl group at C17 in 3.26.2 proceeded by a diastereoselective reduction of the $\beta$-hydroxylketone. By using Narasaka-Prasad 1,3-directed reduction ${ }^{158}$ to give syn-diol 3.26.2, a high diastereoselectivity ( $\mathrm{dr}>20: 1)$ was achieved. This reduction proceeds through a 6-membered ring transition state (TS-I and TS-II) where hydride delivery favours path I (Scheme 3.26) as it leads to the more stable chair-like transition state, a concept that is known as Fürst-Plattner rule. ${ }^{102}$ 


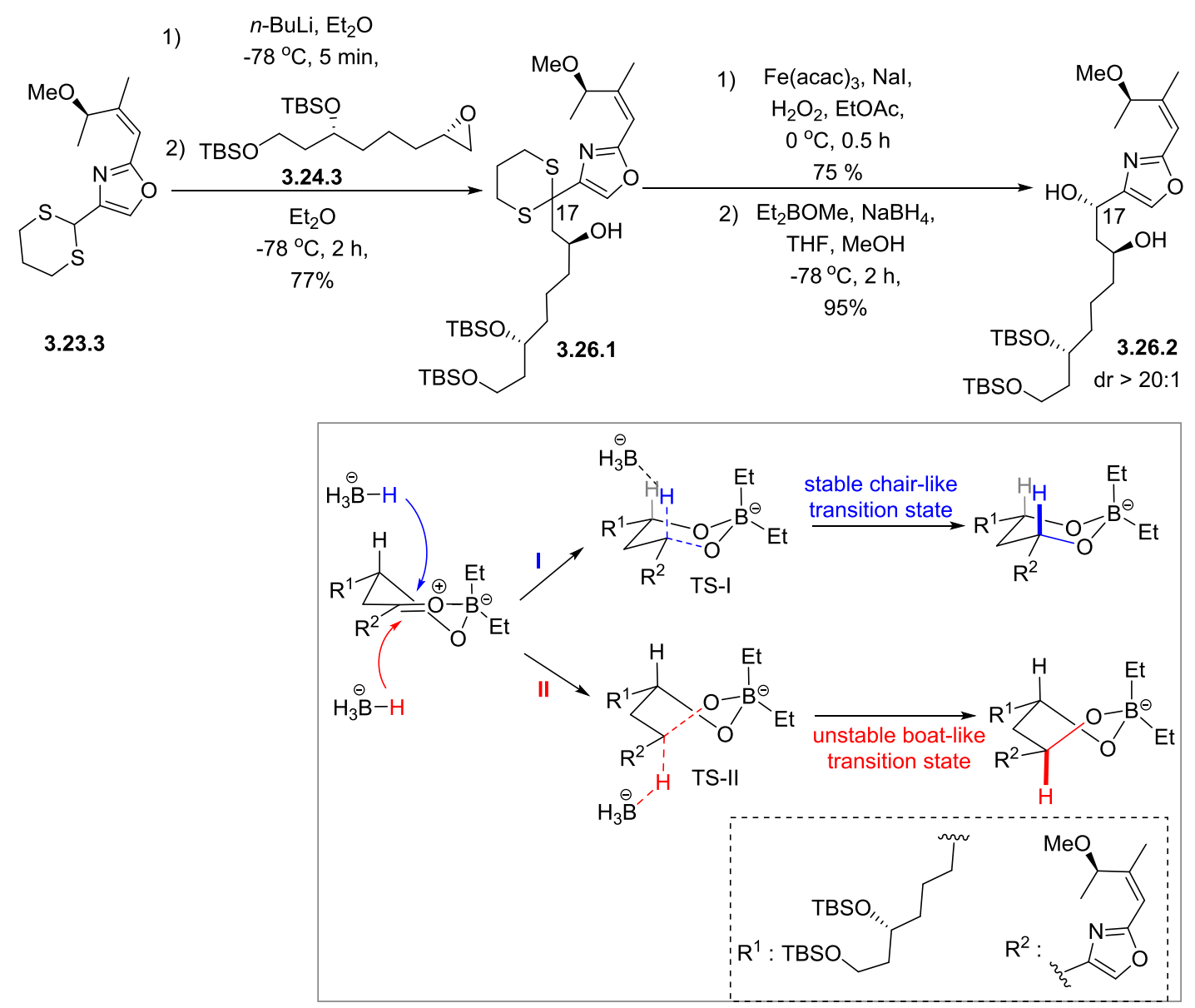

Scheme 3.26 Trans-diaxial hydride attack on $\beta$-hydroxylketone to favour syn-diol 3.26.2

The syn-diol 3.26.2 was protected as PMP acetal followed by a sequence of deprotection steps and an oxidation to give the acid fragment 3.27.1 (Scheme 3.27). The proceeding step was elegantly done as the selective reduction of the PMP-acetal by DIBAL-H proceeded in the presence of a carboxylic acid, which was not implicated in the reduction. This was justified by the strong binding ability of the oxazole moieties to aluminium Lewis acids. ${ }^{126 b,}{ }^{132 c,}, 171$ Moreover, the hydroxyl at C7 of 3.23.2 remains as a PMB-protected alcohol. The chemoselective reduction aids in minimizing the number of unnecessary steps associated with protection/deprotection of alcohols (Scheme 3.27). 

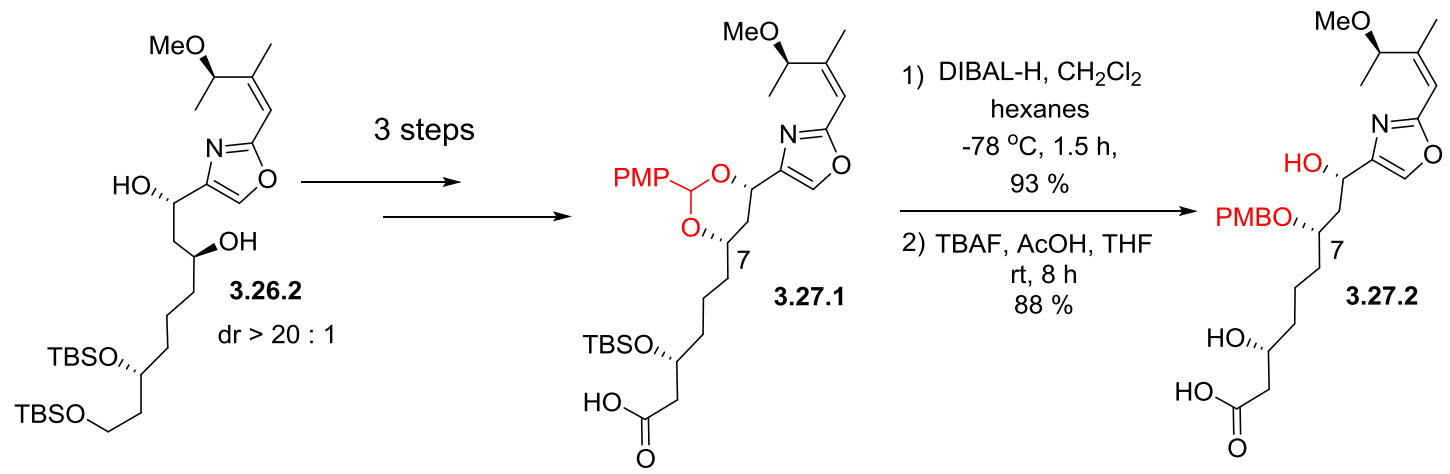

Scheme 3.27. Construction of fragment C $8-\mathrm{C} 18$ of $\beta$-hydroxyacid 3.27.2

Synthesis of Fragment $\mathrm{C} 1-\mathrm{C} 7$

The synthesis continued with the focus on fragment 3.23.1. The Myer's alkylation of 3.28.1 delivered hydroxyl 3.28.2 in quantitative yield (Scheme 3.28). Smith et al. used commercially available enantiopure $(R)$-Roche ester to arrive to alkyl iodide 3.28.1. This accounts for the fourth center of chirality arriving from a chiral pool source. The diastereoselective Myer's alkylation proceeded with a high diastereofacial biased because of the $(R, R)$-pseudoephedrine employed as a chiral auxiliary, which influences the approach of the electrophile and hence the resultant stereochemistry of the product. 


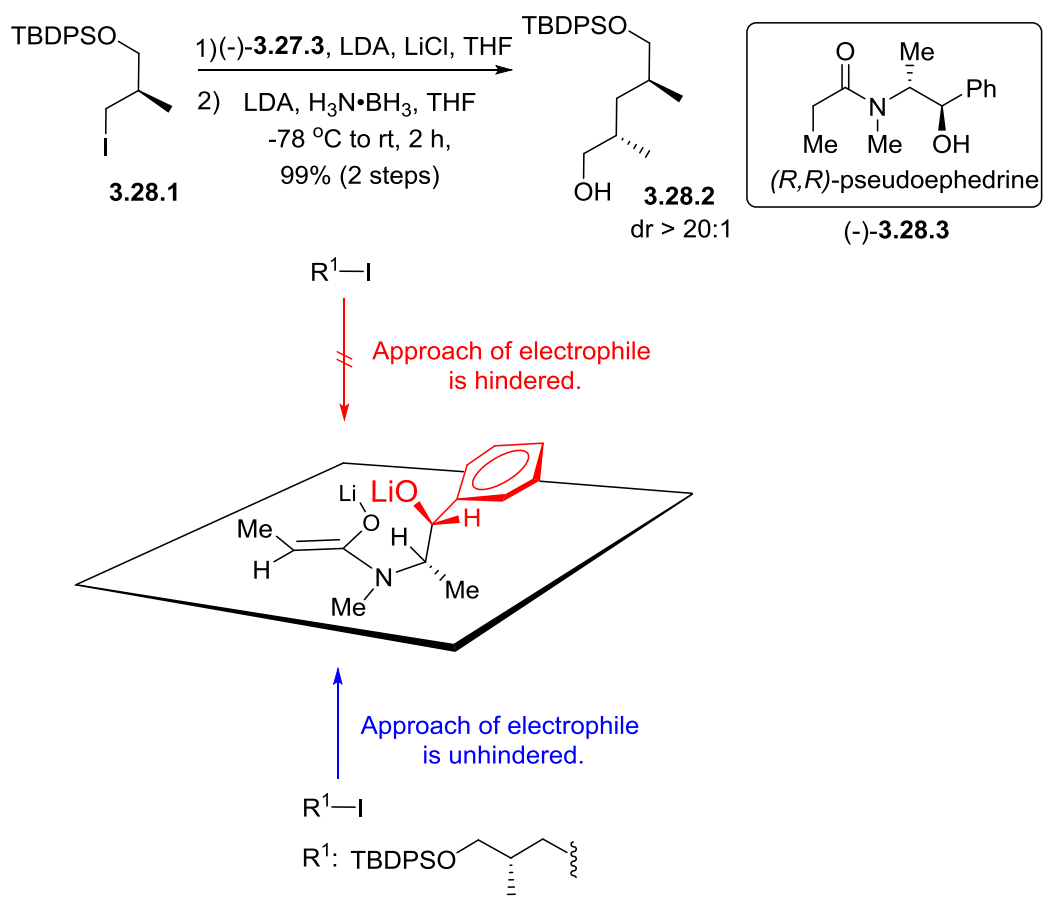

Scheme 3.28. Diastereoselective alkylation achieved through facial biasedness.

The Parikh-Doering oxidation of alcohol 3.28.2 to the aldehyde was followed by a reagent controlled diastereoselective Brown allylation (Scheme 3.29). Allylic 3.29.1 was then obtained from the TIPS-protection of the resulting homoallylic alcohol. The terminal alkene then underwent an oxidative cleavage to provide the aldehyde 3.23 .1 in $75 \%$ overall yield over 2 steps.

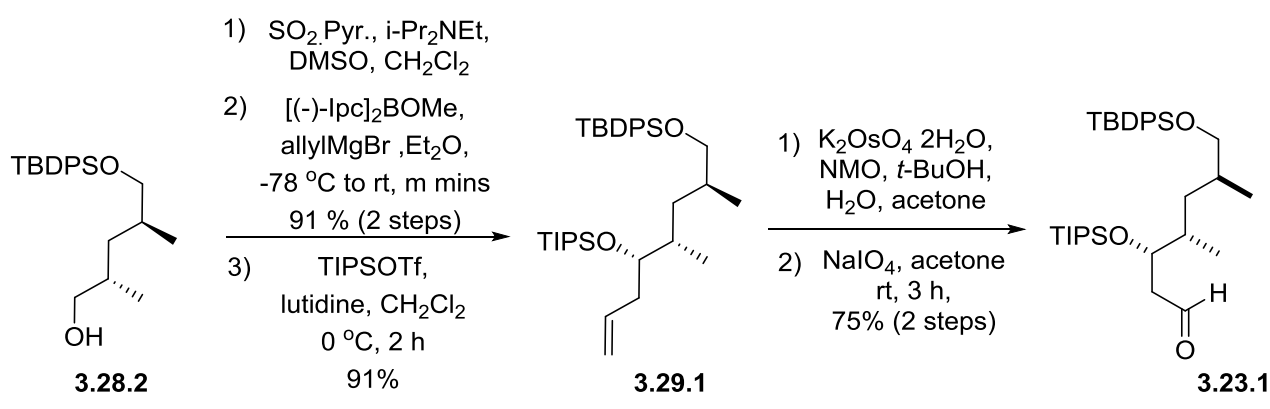

Scheme 3.29. Construction of western hemisphere aldehyde 3.23.1. 
Synthesis of Enigmazole A

The fragments 3.23.2 and 3.23.1 were combined through a Petasis-Ferrier rearrangement (Scheme 3.30). Firstly, aldehyde 3.23.1 was treated with a bulky base followed by exposure to $\beta$-hydroxyacid 3.23.2 to give the dioxanone 3.30.1 in a high yield of $95 \%$. The exo-methylene unit was then formed through the treatment of dioxanone 3.30.1 with $\mathrm{Cp}_{2} \mathrm{TiMe}_{2}$ to give the dioxane 3.30.2. The Petasis-Ferrier rearrangement gave the desired THP-substrate 3.30.4 with a high diastereoselectivity $(\mathrm{dr}>20: 1)$. The key step took place rapidly and gave a high $87 \%$ yield of the desired product. The one-pot olefination of the resulting tetrahydropyranone 3.30.3 from Petasis-Ferrier rearrangement is mandatory to prevent a retroMichael fragmentation of the ketone 3.30.3 catalyzed by $\mathrm{AlMe}_{2} \mathrm{Cl}^{121}$ In the Petasis-Ferrier rearrangement, an unconventional quenching strategy was therefore employed by the group involving 'the addition of methylene ylide to the rearrangement reaction mixture'. ${ }^{121}$ 

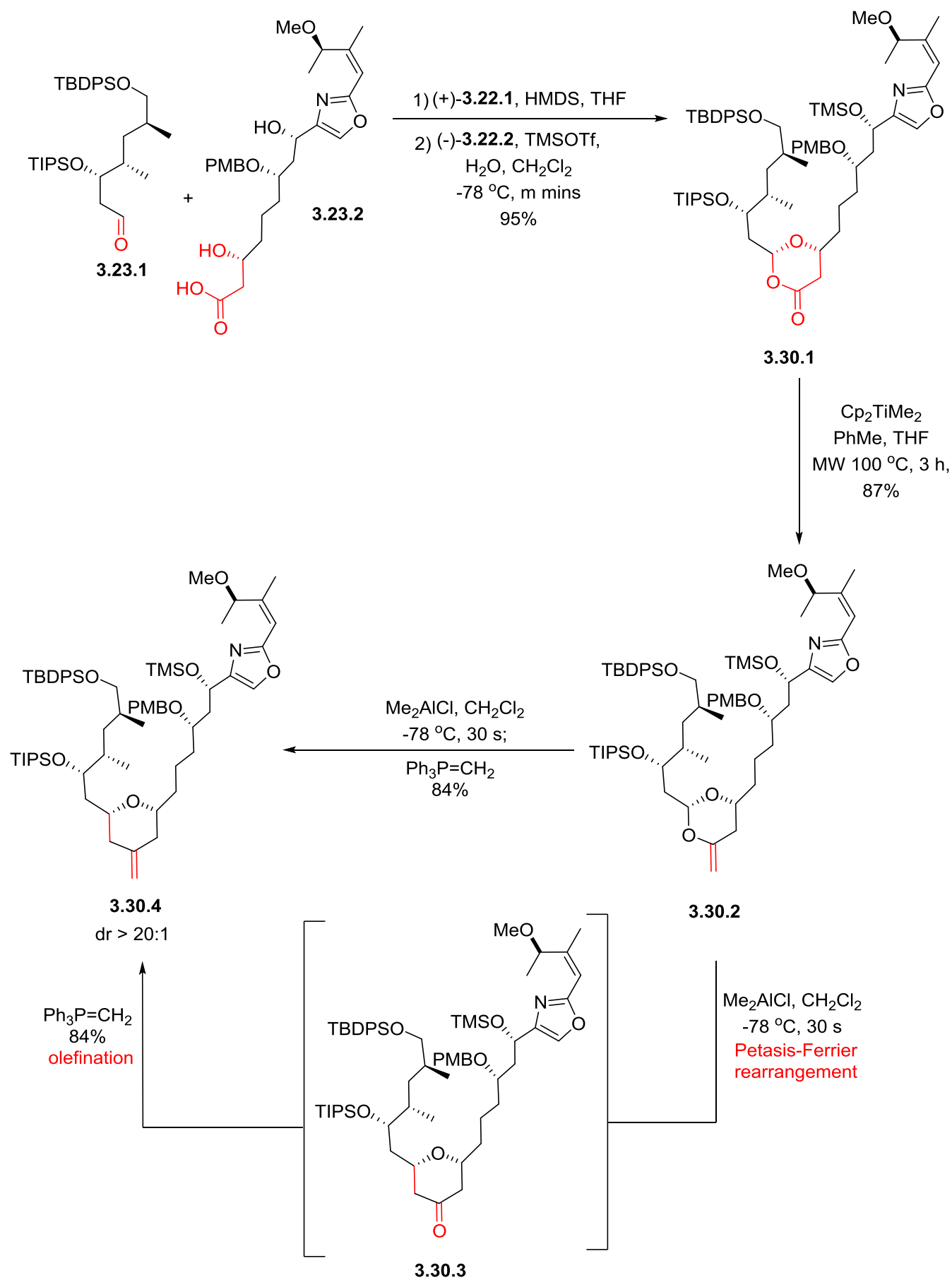

Scheme 3.30. Petasis-Ferrier rearrangement of dioxane 3.30.2 to give THP-ring 3.30.4.

The treatment of THP-fragment 3.30.4 with potassium hydroxide led to the removal of the TBDPS and TMS protecting groups simultaneously (Scheme 3.31). Then the oxidation of primary alcohol using TEMPO gave the acid 3.31.1, which under Yamaguchi 
macrolactonization conditions, produced the 18-membered macrolide 3.31.2. Compound 3.31.2 was then transformed to enigmazole A through deprotection and phosphorylation steps (Scheme 3.31). It is noteworthy that an intramolecular cyclization of the C5 hydroxyl with C1 carbonyl was reported when the TIPS deprotection was carried out before the ring-closure step.

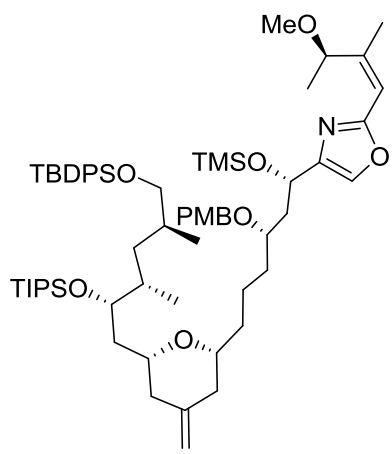

3.30 .4
1)

$\mathrm{KOH}, 18-$ crown-6 $\mathrm{H}_{2} \mathrm{O}, \mathrm{THF}$,

$\mathrm{rt}, 3 \mathrm{~h}$,

$84 \%$

2) TEMPO, $\mathrm{NaClO}, \mathrm{NaClO}_{2}$ $t$ - $\mathrm{BuOH}, \mathrm{CH}_{3} \mathrm{CN}, \mathrm{H}_{2} \mathrm{O}$ rt, $3 \mathrm{~h}$ $75 \%$

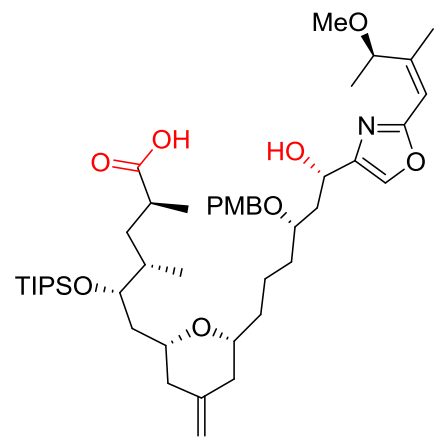

3.31.1

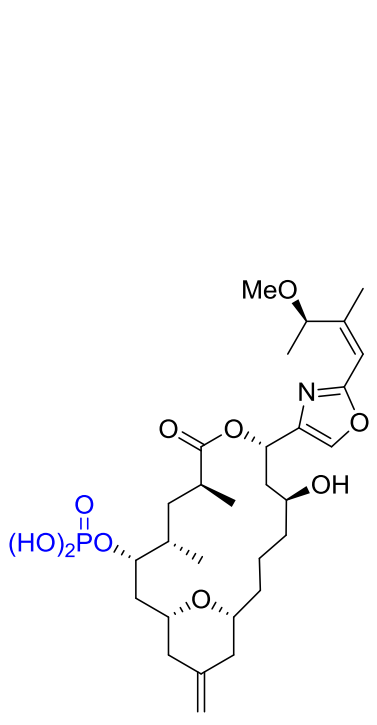

1) HF/Pyr. (1:6), THF,

$45{ }^{\circ} \mathrm{C}, 48 \mathrm{~h}$ $70 \%$ i- $\mathrm{Pr}_{2} \mathrm{NP}(\mathrm{OFm})_{2}$

2) $1 \mathrm{H}$-tetrazole, $\mathrm{MeCN} / \mathrm{CH}_{2} \mathrm{Cl}_{2}, \mathrm{H}_{2} \mathrm{O}_{2}$ $0^{\circ} \mathrm{C}, 1.2 \mathrm{H}$

3) $\mathrm{DDQ}, \mathrm{pH} 7$ buffer, $\mathrm{CH}_{2} \mathrm{Cl}_{2}$ $\mathrm{rt}, 0.5 \mathrm{~h}$ $61 \%$ (2 steps)

4) $\mathrm{Na}_{2} \mathrm{CO}_{3}, \mathrm{H}_{2} \mathrm{O}, \mathrm{MeOH}$ then r.FAr. A.H Mar.N HaO

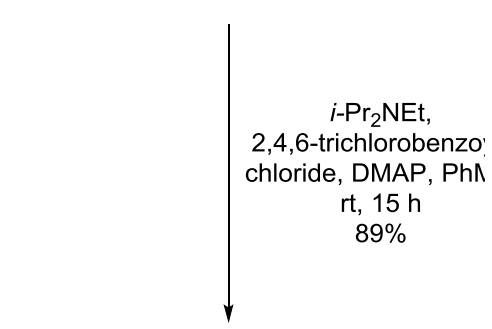

Scheme 3.31. Total synthesis of enigmazole A. 


\subsubsection{Synthesis of Enigmazole A by Fürstner et al.}

Recently, Fürstner et al., reported a concise total synthesis of enigmazole A adopting a fundamentally distinctive approach from all previously reported syntheses of the natural product by Molinski et al., and Smith et al. It is the first synthesis of enigmazole A in which the cis-2,6tetrahydropyran center was constructed after the ring closure to give the macrolide. Apart from that, the strategies employed for the sequences leading to the THP-construction are elegant and succinct.

In the retrosynthesis, Fürstner et al., envisaged that access to enigmazole A from 3.32.1 would allow the introduction of the phosphate ester (Scheme 3.32). The THP would be constructed from the metal-mediated transannular hydroalkoxylation of macrolide 3.32.2. Compound 3.32.2 bears an allene unit at $\mathrm{C} 5$ and $\mathrm{C} 7$ and the crucial hydroxyl group at $\mathrm{C} 11$ that would be responsible for the cyclization giving the THP ring. The allene 3.32.2 would be the result of the [3,3]-sigmatropic shift of the propargylic acetate of alkyne 3.32.3, which would be the product of ring-closing alkyne metathesis (RCM) of 3.32.4. The preparation of 3.32.4 would take place from three smaller fragments 3.32.5, 3.32.6 and the oxazole unit 3.32.7. The oxazole 3.32.7 would be accessed from the ester 3.32.8, which would be synthesized through $\mathrm{C}-\mathrm{H}$ bond activation at $\mathrm{C} 2$ position of the oxazole unit. This is a different approach from Molinski et al., and Smith et al., both of whom adopted the Negishi cross-coupling (Scheme 3.32). 


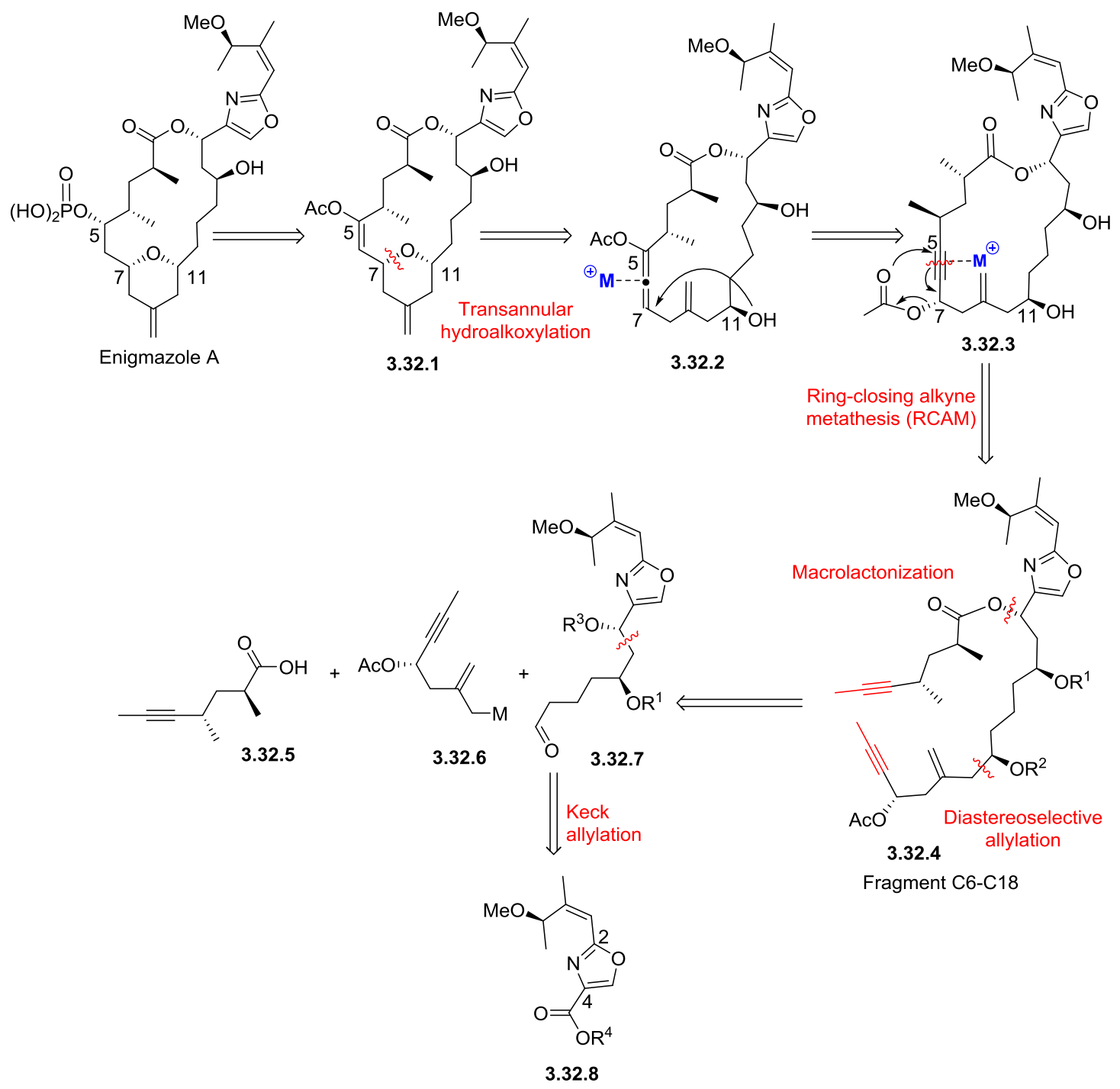

Scheme 3.32. Retrosynthetic analysis of enigmazole A by Fürstner et al.

The oxazole ester 3.32.8 was prepared from the direct coupling of vinyl iodide 3.33.1 and oxazole $\mathbf{3 . 3 3 . 2}$ by a $\mathrm{Pd}$-catalyzed $\mathrm{C}-\mathrm{H}$ activation in high $74 \%$ yield (Schemes 3.33 and 3.34). This method is attractive because of the expunged need for prefunctionalization. Moreover, gram amounts of oxazole ester 3.32.8 were attainable. The ester of 3.32.8 was subjected to a reduction (DIBAL-H) to the aldehyde, which was submitted to the Keck allylation (Scheme 3.33). The Keck allylation achieves diastereofacial discrimination through the chiral $(S) /(R)$-Binol ligands, and proceeds via a 6-membered chair transition state outlined in Scheme 3.34. The resulting homoallylic alcohol 3.33.3 was obtained with high diastereoselectivity 
$(\mathrm{dr}>95: 5)$ and was protected using a Boc group. In treatment of the carbonate with $\mathrm{IBr}$, a substrate-controlled asymmetric iodolactonization took place to give the carbonate 3.33.4. The alkene was activated by the electrophilic IBr, inviting a nucleophilic attack from the Boc handle from the $\beta$-protected alcohol. This method of introducing a chiral center was not observed in the previous two syntheses of enigmazole A. This is due to the preference of using the carbonyls of aldehydes or ketones through diastereoselective reduction, or allylation to introduce chirality.

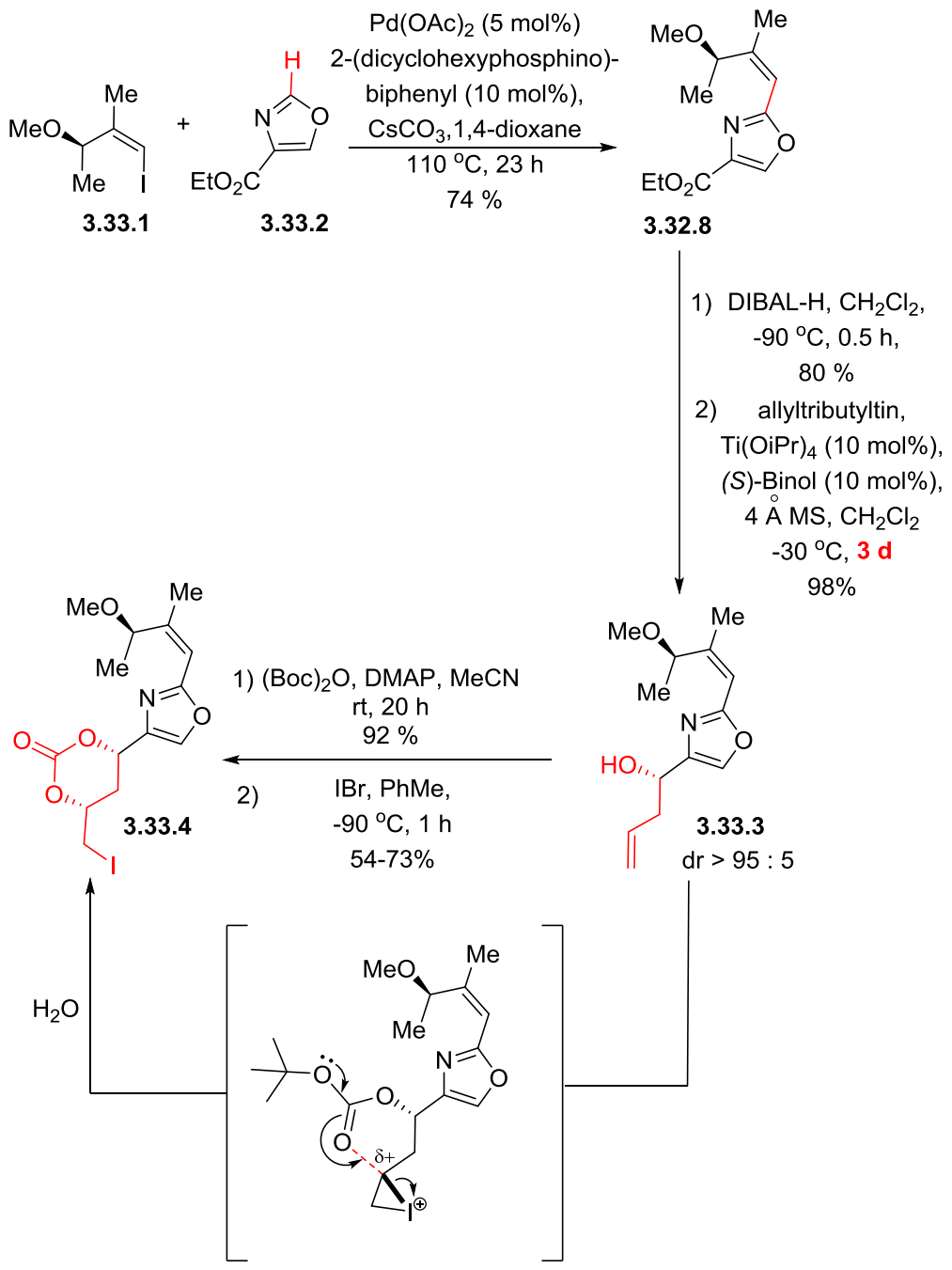

Scheme 3.33. $\mathrm{Cis}$-, vicinal functionality of carbonate 3.33.4 introduced by $O$-cyclization. 


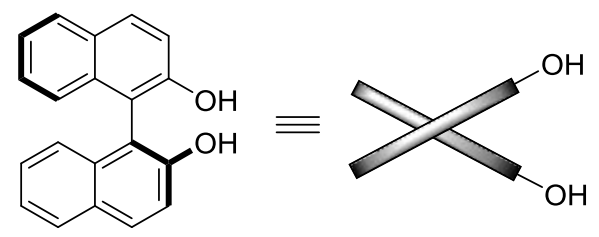

(S)-Binol (S)-Binol (top view)

3.34.1
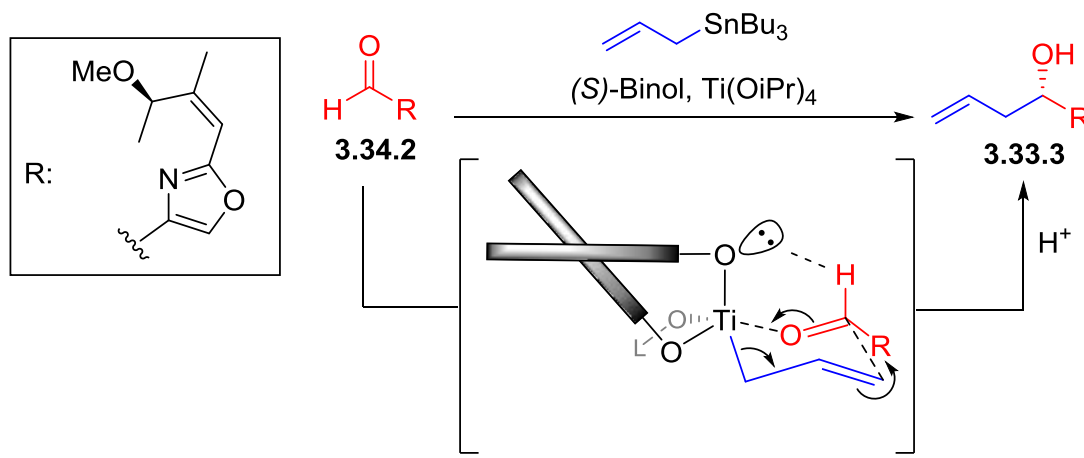

Scheme 3.34. Proposed mechanism of diastereoselective Keck allylation by Corey et al. ${ }^{172}$

The carbonate 3.33.4 was exposed to potassium carbonate in methanol to hydrolyze the cyclic carbonate and to form the terminal epoxide upon displacement of the iodide (Scheme 3.35). A routine hydroxyl protection of the remaining alcohol gave 3.35.1. Aldehyde fragment 3.32.7 was obtained in three further steps that involved the oxirane ring-opening of

\subsection{1.}
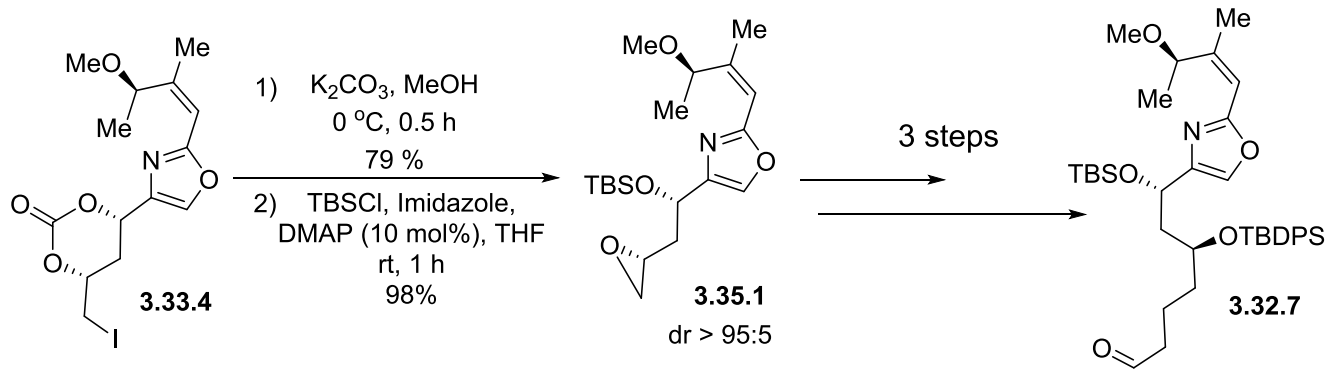

Scheme 3.35. Synthesis of aldehyde 3.32.7 from iodo-carbonate 3.33.4.

In order to build up the $\mathrm{C} 6-\mathrm{C} 18$ fragment, the allyltributyltin reagent 3.32.6 that bears a chiral propargylic acetate group was prepared (Scheme 3.36). This was achieved by diastereoselective Keck allylation of aldehyde 3.36.1 and allyltributyltin 3.36.2 to give 3.36.3 
(Scheme 3.36). The stereochemistry of the acetate group plays an instrumental role towards the success of the key step in the later part of the synthesis. The optically active allyltributyltin reagent 3.32.6 was then obtained from the iodination of propargylic acetate 3.36.3 followed by coupling with tributyltin in two steps.

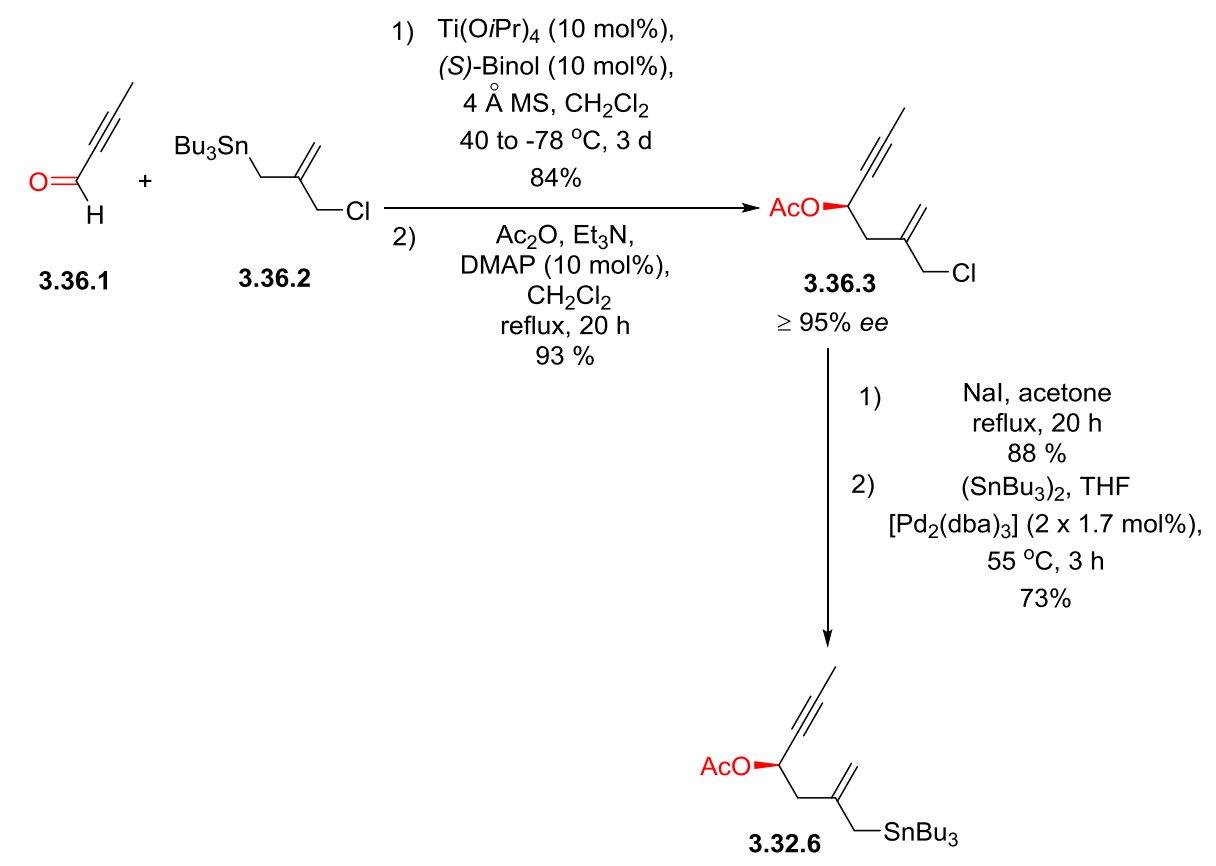

Scheme 3.36. Preparation of allyltributylltin 3.32.6.

The allyltributyltin 3.32.6 was then engaged in the third catalyst-controlled diastereoselective allylation of aldehyde 3.32.7 (Scheme 3.37). An alternative from Keck allylation, the functionalized allyltin is mediated by Corey's chiral bromoborane to give the homoallylic alcohol 3.37.1 with high diastereoselectivity ( $\mathrm{dr}>10: 1)$ (Scheme 3.37). The alkyne 3.37.2 was obtained after two alcohol protecting steps from 3.37.1. 

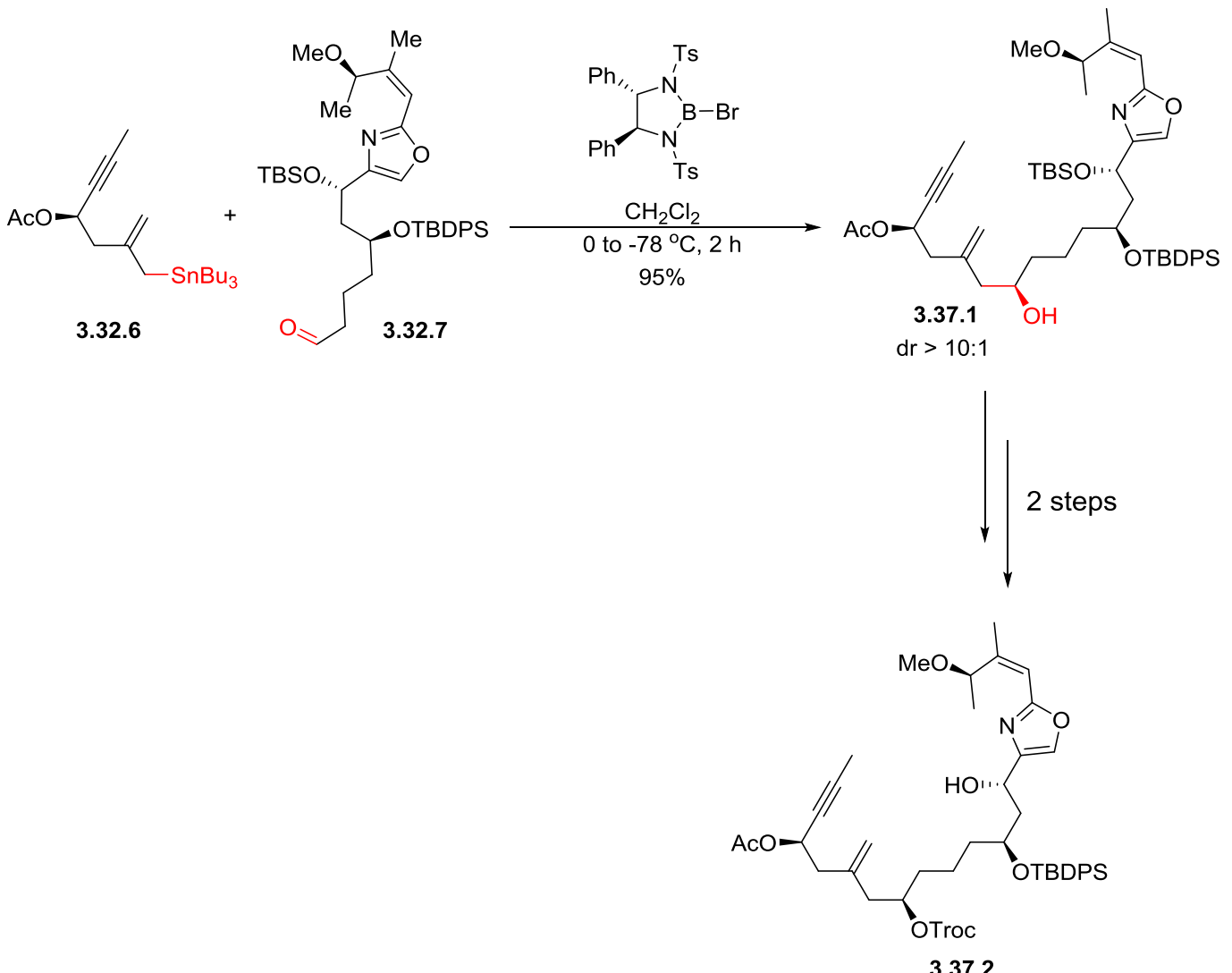

Scheme 3.37. Diastereoselective allylation of 3.32.6 using Corey's chiral catalyst and synthesis of alkyne 3.37.2.

The precursor of the RCM 3.32.4 had to be prepared from an auxiliary-controlled diastereoselective Myer's alkylation of 3.38.1 to give the alcohol 3.38.2, which was transformed to the alkyne 3.32.5 in five straightforward steps (Scheme 3.38). The alkyne 3.32.5 has a carboxylic acid appended on the opposite end that sets up the esterification with fragment 3.37.2. The esterification proceeded smoothly under Yamaguchi's conditions to give the alkyne ring closing metathesis precursor 3.32.4 in quantitative yield (Scheme 3.38).

The alkyne ring-closing key step was then carried out in the presence of molybdenum ate-complex 3.38.4 with a catalytic loading of $31 \mathrm{~mol} \%$ to give a high yield of $79 \%$ of the metathesis product 3.32.2 (Scheme 3.38). The high catalytic loading is required because of the multiple available decomposition pathways. Hence, a clean and fast alkyne metathesis with a $100 \%$ conversion of the starting material 3.32.4 can only be achieved with a $31 \mathrm{~mol} \%$ catalytic 
loading. The scarcely available examples of successful ring-closing alkynes metathesis bearing derivatives of propargylic esters adds value to this method in spite of its low efficiency.

The next major step of the synthesis was the $\pi$-acid-catalyzed tandem reactions leading to the formation of the embedded cis-pyran ring of 3.32.1 (Scheme 3.38). Fürstner et al. employed the dinuclear Biphep complex $(R)-\mathbf{3 . 3 8 . 5}$ to form the chiral gold(I) complex with $\mathrm{AgSbF}_{6}$ (Scheme 3.37). When alkyne substrate 3.32.2 is exposed to the chiral gold(I) complex, the [3,3]-sigmatropic rearrangement took place prior to the $\mathrm{C} 11$ hydroxyl attack on $\mathrm{C} 7$ to form the $c i s$-THP ring. This key step showcased a tedious process that was executed with the proper phasing of the two reactions, which is a product of refined planning. This is evident in the selection of the Troc-alcohol protecting group in 3.32.4 that is orthogonal in reactivity to the TBS-protected alcohols. Thereby, a selective removal of the Troc group on 3.32.4 was possible prior to the $\pi$-acid catalyzed cascade reaction. The total synthesis of enigmazole A was completed in just 4 steps from substrate 3.32.1 with the highest overall yield amongst the three reported syntheses at $3.3 \%$. 


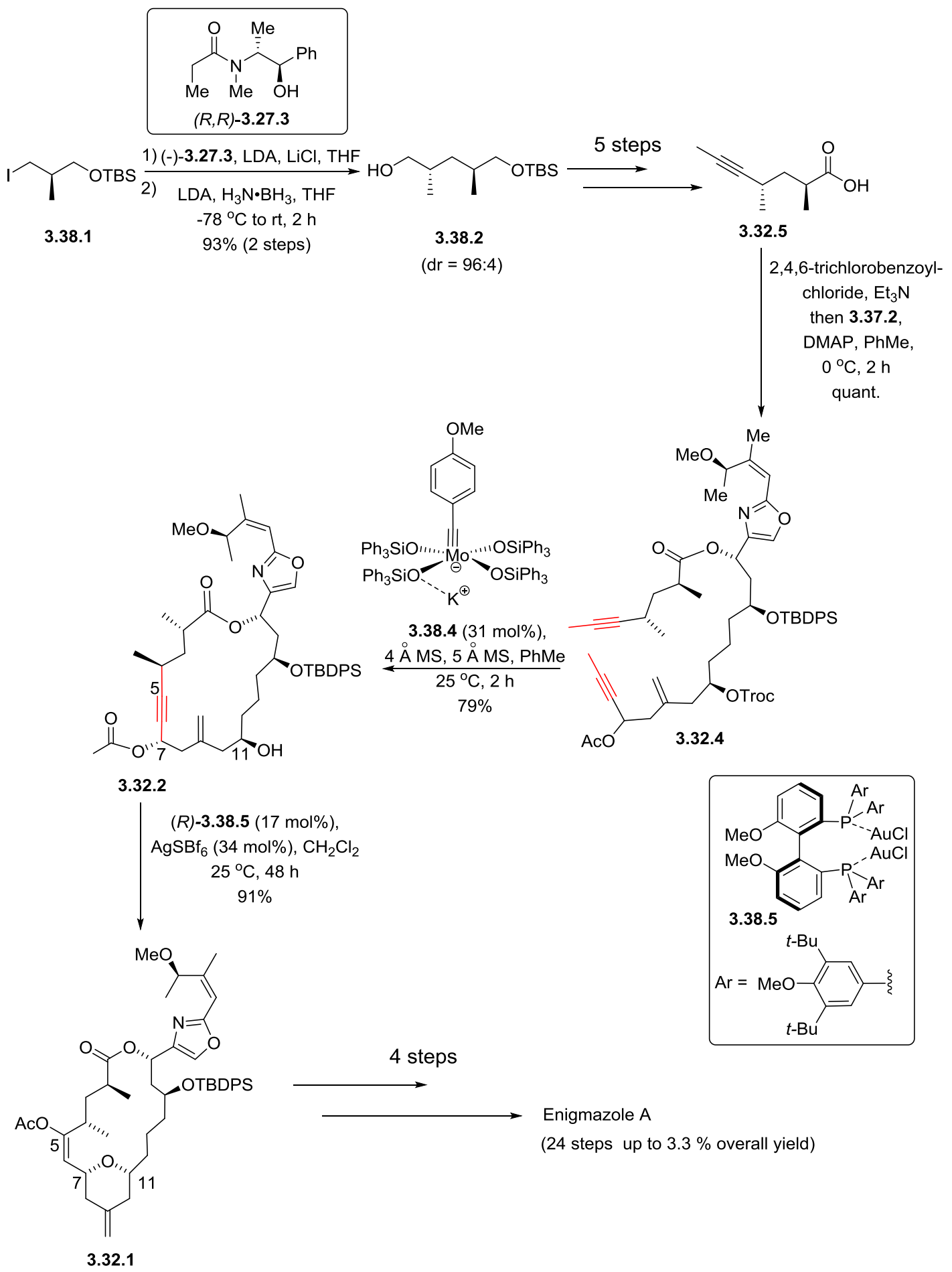

Scheme 3.38 Key transannular hydroalkoxylation step leading to 3.32.1 and to the completion of enigmazole A. 


\subsubsection{Summary}

The syntheses reflect a variety of existing methods to introduce a stereogenic center using auxiliary-, substrate- and catalyst-controlled strategies. The common diastereoselective methods employed were Keck allylation, Myer's alkylation, Brown and Roush allylation, the use of Corey's chiral bromoborane ligand, Narasaka-Prasad reduction, Wilkinson's reduction of $\mathrm{C}=\mathrm{C}$ double bonds, intramolecular alkene cyclofunctionalization, hetero-Diels-Alder and more.

The THP-core of enigmazole A was constructed from HDA cycloaddition, PetasisFerrier rearrangement and metal-catalyzed transannular hydroalkoxylation (Figure 3.6). The best method would offer high diastereoselectivity of the cis-THP ring, and good yield, this was achieved by Smith et al. through the Petasis-Ferrier rearrangement, which gave the highest yield for the desired diastereomer compared to the other two strategies. The HDA resulted in a mixture of diastereomers with low selectivity while the gold(I)-catalyzed hydroalkoxylation was erratic and did not offer a reliable yield. Although the transannular hydroalkoxylation method does not compare favourably with others in terms of result, it remains as the only strategy to create the THP-ring after macrolactonization and thereby emerges as the more innovative strategy.

Apart from that, there were unexpected challenges confronted in each of the established synthetic path towards enigmazole A and were resolved in an efficient manner. While Molinski et al. overcame the macrolactonization challenges by the reversal of synthetic steps, Smith et al. overcame the Petasis-Ferrier rearrangement inconsistency by adopting an unconventional quenching. Fürstner et al., on the other hand, was careful with the selection of alcohol protecting groups for the selective deprotection in the later stages of the synthesis. 
In summary, the total syntheses of enigmazole A have been successfully carried out by Molinski et al., Smith et al. and Fürstner et al., together with the asymmetric synthesis of C1 - C7 fragment by Kadota et al.
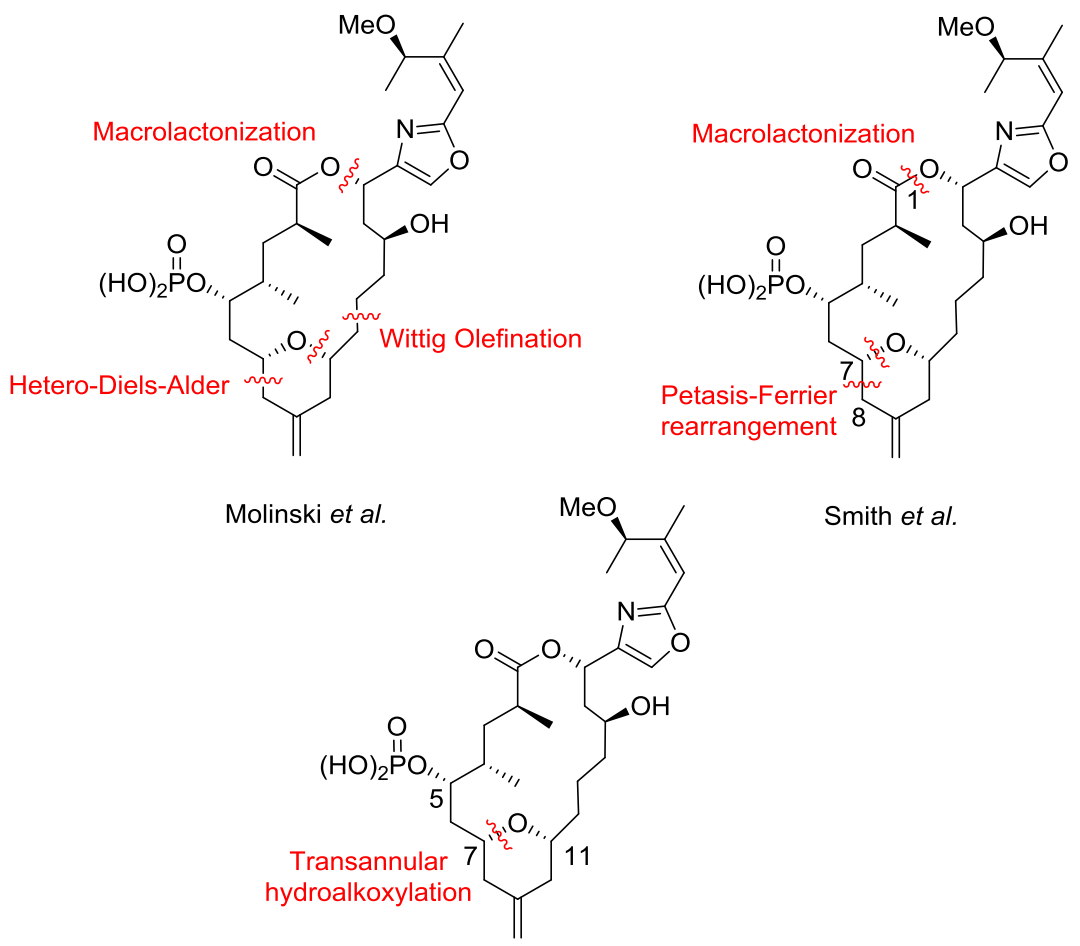

Smith et al.

Fürstner et al.

Figure 3.6. Distinctive retrosynthetic analysis for the total synthesis of enigmazole A. 
This page has been intentionally left blank. 


\title{
Chapter 3
}

3.3 Objective of Present Work

\author{
3.3.1 Results and Discussion
}

3.3.2 Perspectives 
This page has been intentionally left blank. 


\subsection{Objective of Present Work}

The primary objective of the total synthesis towards enigmazole $\mathrm{A}$ is the construction of the cis-1,2-tetrahydropyran ring by using the method that has been developed by Cossy et al., namely, the iron(III)-mediated cyclization (refer to Section 3.1.2, Scheme 3.10). In consequence, a retrosynthetic analysis including this key step has been designed.

\subsubsection{Results and Discussion}

\subsubsection{Retrosynthetic Analysis of Enigmazole A}

Enigmazole A could be accessed by performing a ring-closing metathesis applied to 3.39.1 to form the $\mathrm{C} 12-\mathrm{C} 13$ bond followed by a selective hydrogenation to produce the saturated 18-membered ring (Scheme 3.39). In the final step, the exo-methylene group at C9 would be installed using neutral olefination conditions such as Tebbe's $\left(\mathrm{Cp}_{2} \mathrm{Ti}=\mathrm{CH}_{2}, \mathrm{AlMe}_{3}\right.$, $\left.\mathrm{PhMe},-78^{\circ} \mathrm{C}\right)$ or Lombardo's olefination $\left(\mathrm{CH}_{2} \mathrm{Br}_{2}, \mathrm{Zn}, \mathrm{TiCl}_{4}, \mathrm{THF},-40^{\circ} \mathrm{C}\right)$ applied to the ketone present at the C9 position. Tetrahydropyranone 3.39.1 would be synthesized using Krische et al. stereoselective allylation of the aldehyde 3.39.2. Esterification between the THP and oxazole units, 3.39.3 and 3.39.4 respectively, would yield 3.39.2. For our part, we focused on the synthesis of THP-fragment 3.39.3 (Scheme 3.39). 

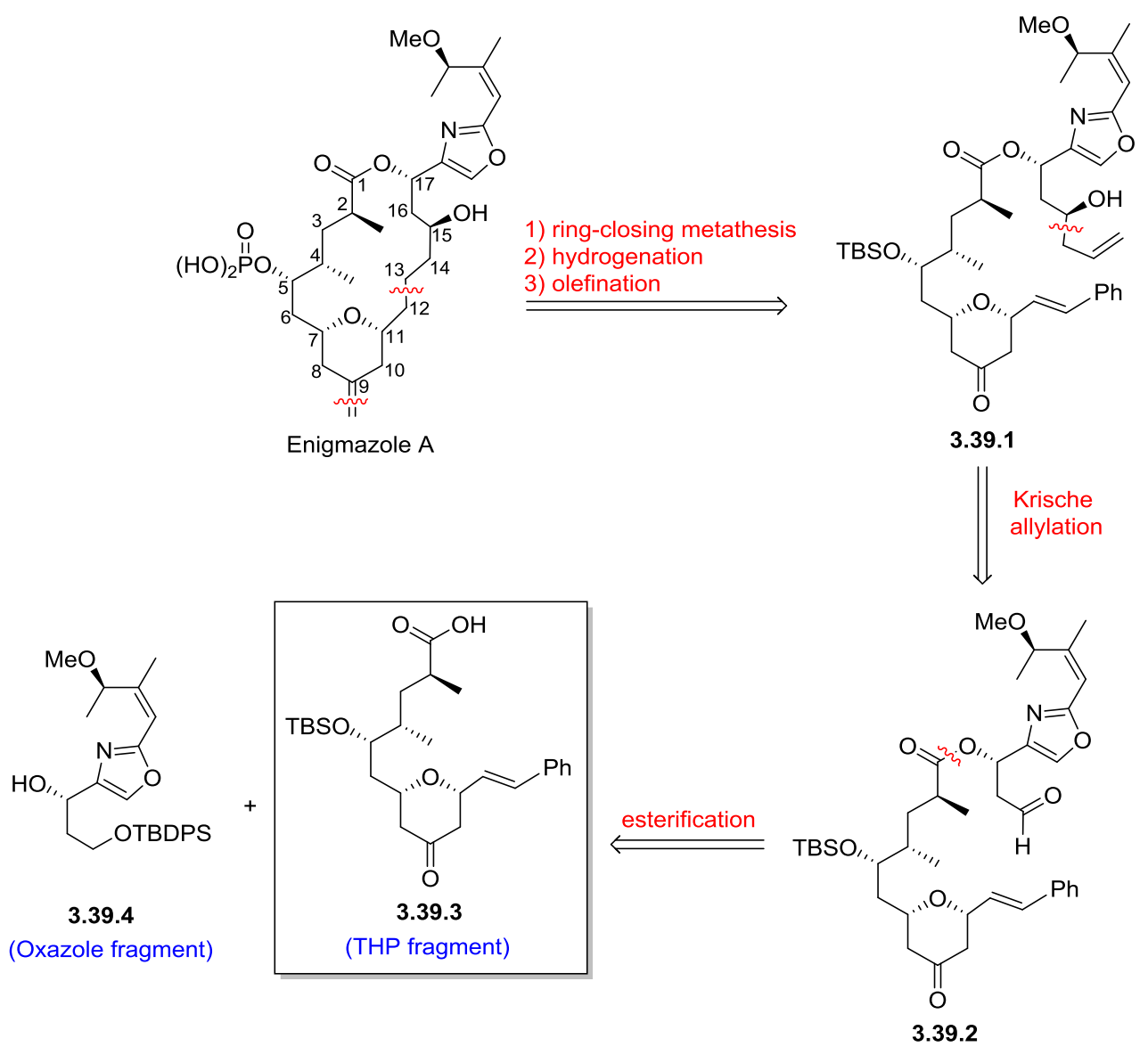

Scheme 3.39. Retrosynthetic analysis of enigmazole A.

\subsection{First Retrosynthetic Approach of THP-fragment}

The retrosynthetic analysis of the THP-fragment 3.39.3 continues with a Myer's alkylation $\left((R, R)\right.$-pseudoephedrine, $\left.\mathrm{LDA}, \mathrm{LiCl},-78^{\circ} \mathrm{C}\right)$ that would be used to establish the required absolute configuration at the $\mathrm{C} 2$ position of the THP fragment 3.39.3 (Scheme 3.40). The key step is the formation of cis-2,6-disubstituted tetrahydropyranone 3.40.1, which would be constructed by the iron(III) chloride-catalyzed cyclization of 1,5-allylic alcohol 3.40.2 $\left(\mathrm{FeCl}_{3} \bullet 6 \mathrm{H}_{2} \mathrm{O}, \mathrm{CH}_{2} \mathrm{Cl}_{2}\right.$, rt). Allylic alcohol 3.40.2 was envisaged from the Mukaiyama aldol reaction of aldehyde 3.40.3, which would be prepared from the ozonolysis of olefin 3.40.4 $\left(\mathrm{O}_{3}, \mathrm{CH}_{2} \mathrm{Cl}_{2} .-78{ }^{\circ} \mathrm{C}\right)$. Protected homoallylic alcohol 3.40.4 was envisaged from the reduction (DIBAL-H, THF, $\left.-40^{\circ} \mathrm{C}\right)$ and stereoselective Duthaler-Hafner allylation $((R, R)-$ or $(S, S)$ - Ti 
catalyst, $\mathrm{Et}_{2} \mathrm{O},-78{ }^{\circ} \mathrm{C}$ ) would be applied to the commercially available $(S)$-Roche ester $\mathbf{3 . 4 0 . 5}$ (Scheme 3.40).

\section{IHP fraqument}

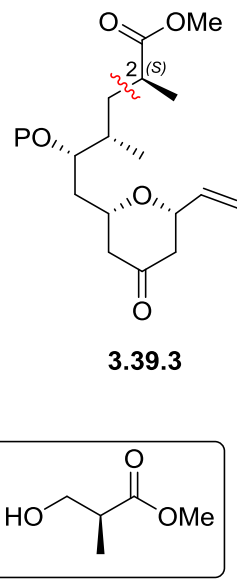

(S)-Roche Ester,

3.40.5
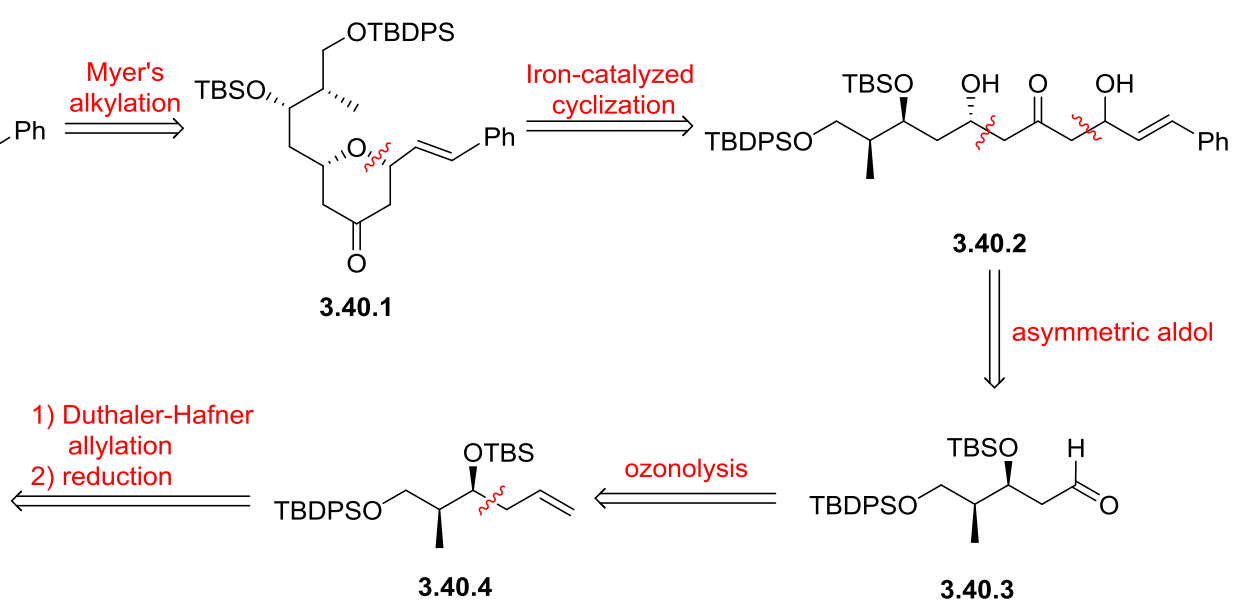

Scheme 3.40. Retrosynthetic analysis of the THP-fragment 3.39.3.

\section{Synthesis towards the C1 - C10 Fragment of Enigmazole A}

The aim is to attain the crucial fragment 3.40.2, which will be treated with $\mathrm{FeCl}_{3}$ to obtain the desired pyranone 3.40.1 (Scheme 3.41).
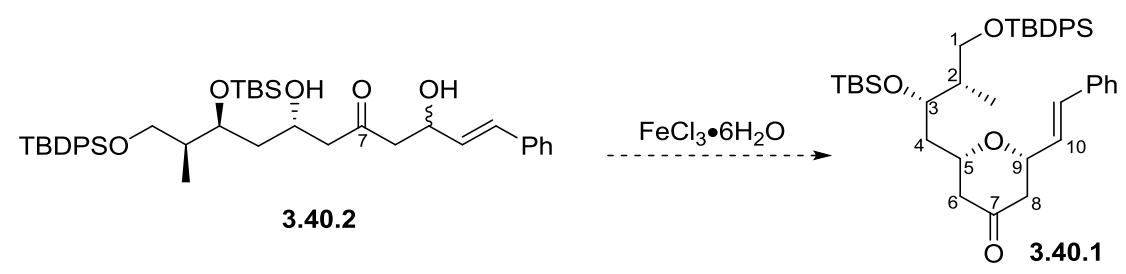

Scheme 3.41. Towards desired pyranone 3.40.1 from diol 3.40.2.

The synthesis of THP-fragment 3.39.3 started with the protection of the commercially available (S)-Roche ester 3.40.5 using TBDPSCl (imid., DMF, $0{ }^{\circ} \mathrm{C}$ ) (Scheme 3.42). Compound 3.42.1 was isolated in quantitative yield. The TBDPS-protected methyl ester 3.42.1 was then 
converted into Weinreb amide 3.42.2 with $\mathrm{N}, \mathrm{O}$-dimethylhydroxylamine at $-40{ }^{\circ} \mathrm{C}$ and was purified by column chromatography on silica gel to give stable and easy to handle white crystals. The Weinreb amide 3.42.2 enabled access to the sensitive $\alpha$-chiral substituted aldehyde 3.42.3 by reduction with DIBAL-H in tetrahydrofuran at $-78{ }^{\circ} \mathrm{C}$. The aldehyde $\mathbf{3 . 4 2 . 3}$ was of sufficient purity (based on ${ }^{1} \mathrm{H}$ NMR) to be directly engaged in the proceeding Duthaler-Hafner allylation with $(R, R)$-Ti to give an overall yield of 76\% of the desired allylated alcohol 3.42.4 with a good diastereoselectivity $(\mathrm{dr}=19: 1)$ (Scheme 3.42). The anti-diastereomer was identified by comparisons of ${ }^{1} \mathrm{H}$ NMR data with the known anti-isomer sample of alcohol 3.42.6. ${ }^{173}$

The homoallylic secondary alcohol 3.42.4 was protected using TBSOTf (2,6-lutidine, $\mathrm{CH}_{2} \mathrm{Cl}_{2},-78{ }^{\circ} \mathrm{C}$ ), and compound $\mathbf{3 . 4 0 . 4}$ was isolated in $70 \%$ yield (Scheme 3.42). The terminal alkene of 3.40.4 was subjected to an oxidative cleavage over two steps using osmium(VIII) oxide followed by the addition of sodium metaperiodate to yield aldehyde $\mathbf{3 . 4 0 . 3}$ in $90 \%$ yield. ${ }^{[a]}$

A diastereoselective Mukaiyama aldol ${ }^{174}$ reaction was explored to access the $\mathrm{C} 1-\mathrm{C} 7$ fragment, and control the C5 stereogenic center. Aldehyde 3.40.3 was subjected to the addition of acetone trimethylsilylenol ether in the presence of 2.5 equiv of $\mathrm{Me}_{2} \mathrm{AlCl}\left(-78{ }^{\circ} \mathrm{C}\right.$ in $\left.\mathrm{CH}_{2} \mathrm{Cl}_{2}\right)$, which gave rise to $\beta$-hydroxymethyl ketone $\mathbf{3 . 4 2 . 5}$ as a 3:1 mixture of inseparable diastereomers in favour of the desired anti-isomer by literature precedence (56\% yield) (Scheme 3.42). The poor diastereoselectivity from the Mukaiyama-type aldol reaction led to a temporary alternative by using allyltitanium complex $(S, S)$-Ti to furnish the optically active homoallylic alcohol 3.42.6 with excellent diastereomeric purity $(\mathrm{dr}>95: 5)$ and a modest overall yield of 53\%. The $53 \%$ yield could be attributed to the insufficiently dry aldehyde, thus, aldehyde $\mathbf{3 . 4 0 . 3}$ was azeotropically dried with toluene prior to the allylation (Scheme 3.42). Unfortunately, no significant improvement in the allylation yield was observed.

\footnotetext{
${ }^{\text {[a] }}$ the ozonolyzer was unavailable.
} 

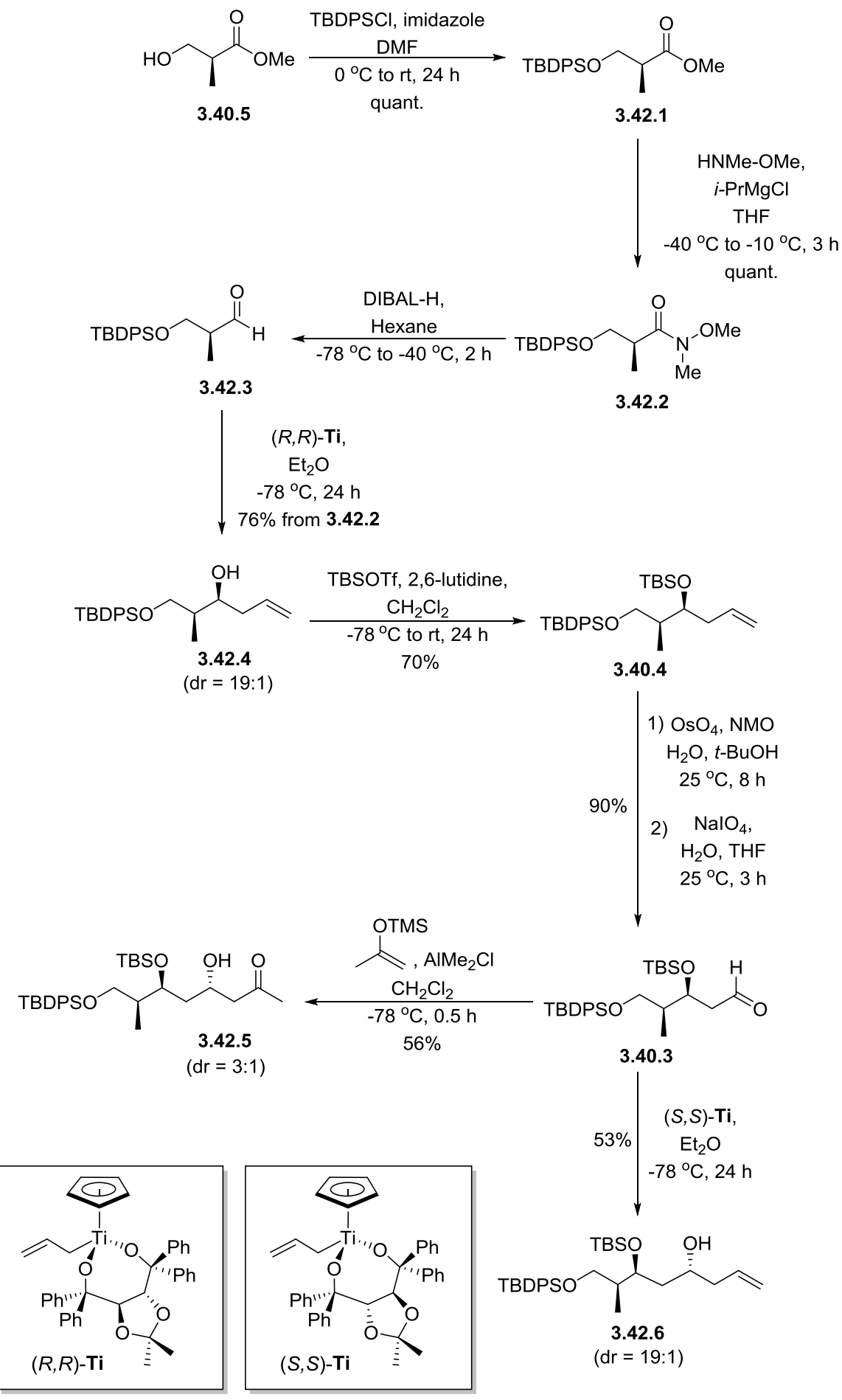

Scheme 3.42. Towards synthesis of fragment C1 - C7 3.42.6.

The Wacker oxidation was then attempted on homoallylic alcohol 3.42.6 in order to transform the $\mathrm{C} 1-\mathrm{C} 7$ fragment to the $\mathrm{C} 1-\mathrm{C} 10$ fragment 3.42.5 (Scheme 3.43). Although the 
Wacker oxidation was carried out under different conditions, a complete conversion of the starting material 3.42.6 was never achieved (Table 3.1). The set-up of Wacker oxidation is important as the manner in which oxygen was introduced to the system affected the outcome of the reaction. No reaction occurred when the oxygen was not bubbled through the reaction mixture (Table 3.1, entry 1). Apart from that, some improvements of the oxidation yield was achieved through extended reaction times (Table 3.1, entries $1-5$ ). The Wacker oxidation eventually gave a best yield of $73 \%$ when carried out in $\mathrm{THF} / \mathrm{H}_{2} \mathrm{O}$ solvent system using palladium(II) and copper(I) chloride with oxygen bubbling through the mixture for $18 \mathrm{~h}$ (Table 3.1, entry 5). The formation of side products such as the aldehyde 3.43.2 and lactol 3.43.1 was isolated in small amounts (Table 3.1, entry 5). Other solvent systems such as $\mathrm{DMF} / \mathrm{H}_{2} \mathrm{O}$ (Table 3.1, entry 6) or using a single solvent $\mathrm{H}_{2} \mathrm{O}$ (Table 3.1, entry 7) were tested but did not give a good yield in spite of the substantial conversion of the starting material 3.42.6.

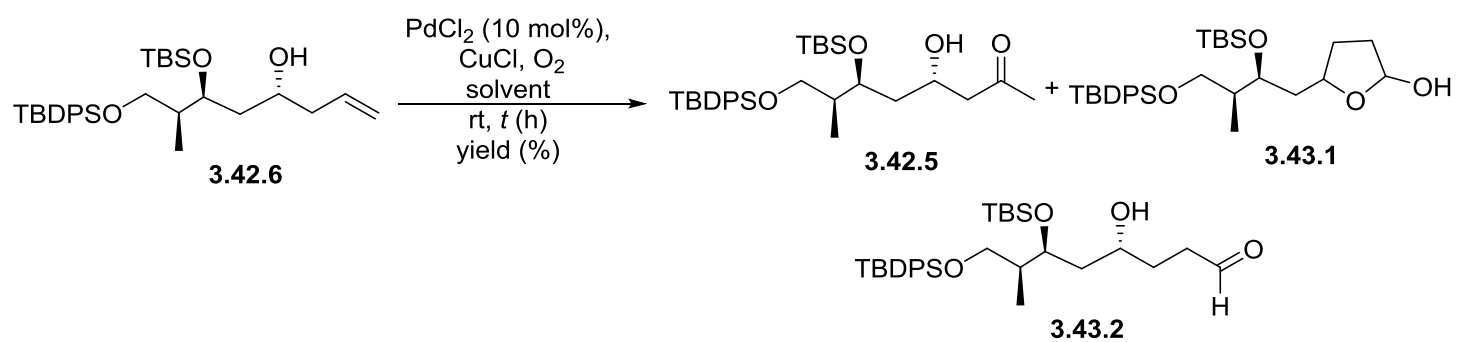

Scheme 3.43. Wacker oxidation of 3.42.6.

\begin{tabular}{ccccccc}
\hline entry & $\begin{array}{c}\text { solvent } \\
(\text { ratio })\end{array}$ & $\begin{array}{c}\text { reaction } \\
\text { time }(\mathrm{h})\end{array}$ & $\begin{array}{c}\text { conversion of } \\
\mathbf{3 . 4 2 . 6}(\%)^{[\mathrm{cc}]}\end{array}$ & $\begin{array}{c}\text { yield of } \\
\mathbf{3 . 4 2 . 5}(\%)\end{array}$ & $\begin{array}{c}\text { yield of } \\
\mathbf{3 . 4 3 . 2}(\%)\end{array}$ & $\begin{array}{c}\text { yield of } \\
\mathbf{3 . 4 3 . 3}(\%)\end{array}$ \\
\hline & $\begin{array}{c}\mathrm{THF} / \mathrm{H}_{2} \mathrm{O} \\
(1.5: 1)\end{array}$ & 5 & 0 & - & - & - \\
& & & & & & - \\
2 & $\begin{array}{c}\mathrm{THF} / \mathrm{H}_{2} \mathrm{O} \\
(1.5: 1)\end{array}$ & 7 & 76 & 44 & - & - \\
& $\begin{array}{c}\mathrm{THF} / \mathrm{H}_{2} \mathrm{O} \\
3\end{array}$ & 9 & 81 & 57 & - & - \\
$(1.5: 1)$ & & & & &
\end{tabular}




\begin{tabular}{|c|c|c|c|c|c|c|}
\hline 4 & $\begin{array}{c}\mathrm{THF} / \mathrm{H}_{2} \mathrm{O} \\
(1.5: 1)\end{array}$ & $12^{[\mathrm{b}]}$ & 96 & 66 & 12 & - \\
\hline 5 & $\begin{array}{c}\mathrm{THF} / \mathrm{H}_{2} \mathrm{O} \\
(1.5: 1)\end{array}$ & $18^{[\mathrm{b}]}$ & 88 & 73 & 10 & 2 \\
\hline 6 & $\begin{array}{c}\mathrm{DMF} / \mathrm{H}_{2} \mathrm{O} \\
(1.5: 1)\end{array}$ & 10 & 10 & 4 & - & - \\
\hline 7 & $\mathrm{H}_{2} \mathrm{O}^{[\mathrm{a}]}$ & 10 & 88 & 15 & - & - \\
\hline
\end{tabular}

[a] substrate was dissolved in minimum DMF prior to reaction. [b] reaction mixture was bubbled with $\mathrm{O}_{2}$ using balloon. [c] based on isolated yield of recovered starting material.

Table 3.1. The Wacker oxidation of $\mathbf{3 . 4 2 . 6}$ under varied conditions.

With substrate 3.42.5 in hand, its transformation to the crucial compound 3.44.1 was then carried out (Scheme 3.44). The methyl ketone 3.42.5 was treated with 2.2 equivalents of LiHMDS $\left(-78^{\circ} \mathrm{C}\right.$ in $\mathrm{CH}_{2} \mathrm{Cl}_{2}$ ) and cinnamaldehyde was added to the reaction mixture. However, under these conditions, the aldol product 3.44.1 was not obtained (Scheme 3.44). It is suspected that under basic conditions, migration of the tert-butyldimethylsilyl protecting group at the $\beta$-position of 3.42.5 may occur and may lead to the formation of compound 3.45.1 (Scheme 3.45).

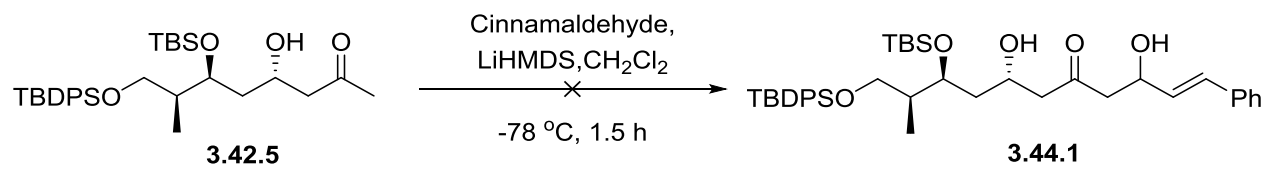

Scheme 3.44. Aldolization attempt of 3.43.1. 


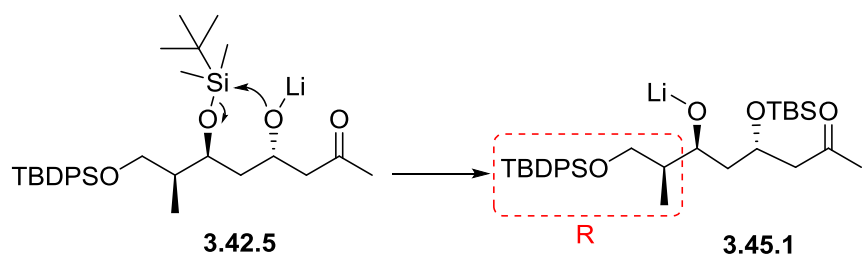

Scheme 3.45. Potential TBS migration in 3.42.5.

Therefore, protection of the secondary hydroxyl group in $\beta$-hydroxymethyl ketone 3.42.5 is necessary prior to the aldol reaction (Scheme 3.46). The protection of $\beta$-hydroxymethyl ketone 3.42.5 using $\mathrm{TESCl}\left(\mathrm{Et}_{3} \mathrm{~N}, \mathrm{CH}_{2} \mathrm{Cl}_{2}, 0{ }^{\circ} \mathrm{C}\right)$ in the presence of catalytic amount of DMAP was unsuccessful. On the contrary, the protection of $\beta$-hydroxymethyl ketone 3.42.5 using TESOTf (2,6-lutidine, $\mathrm{CH}_{2} \mathrm{Cl}_{2},-78{ }^{\circ} \mathrm{C}$ ), produced compound 3.46.1 in $85 \%$ yield (Scheme 3.46).

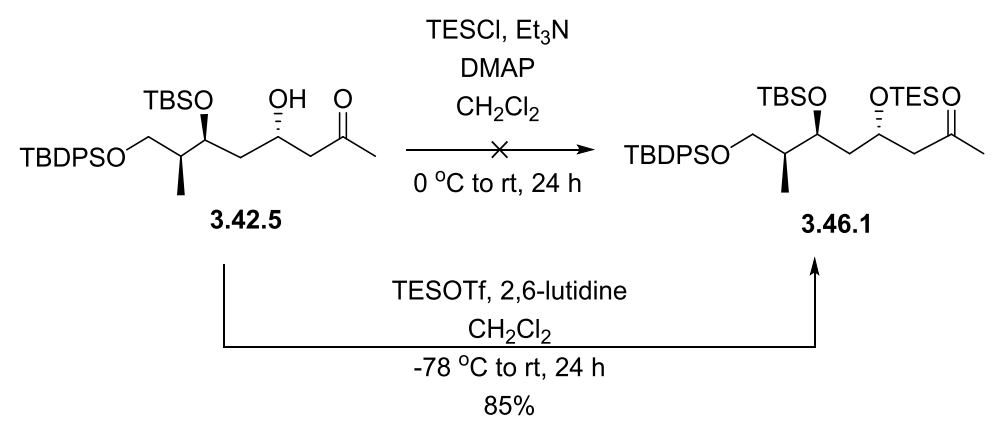

Scheme 3.46. Protection $\beta$-hydroxymethyl ketone 3.42.5.

The aldol reaction was repeated with the TES-ether protected secondary alcohol 3.46.1 under a range of reaction conditions (Table 3.2, entries 1-6). Initially, the aldolization was performed with cinnamaldehyde in the presence of 1.2 equiv of LiHMDS $\left(-78{ }^{\circ} \mathrm{C}\right.$ in $\left.\mathrm{CH}_{2} \mathrm{Cl}_{2}\right)$ but under these conditions only the starting material 3.46.1 was recovered (Table 3.2, entry 1). Similarly, the outcome remained unchanged in spite of an increase in the equivalence of base used (Table 3.2, entries 2 and 3). The aldol reaction was thus repeated with a different base such as LDA (1.2 equiv) followed by an increased in base equivalence (Table 3.2, entries 4 and 5). The unchanging outcome led us to suspect that the scale of the reaction was responsible for the consecutively unsuccessful aldolization. Therefore, the scale of the reaction was substantially 
increased and the aldolization was repeated (Table 3.2, entry 6). The desired aldol product 3.47.2 was not obtained, instead the undesired enone 3.47.1 that corresponds to the elimination product was isolated (Scheme 3.46).

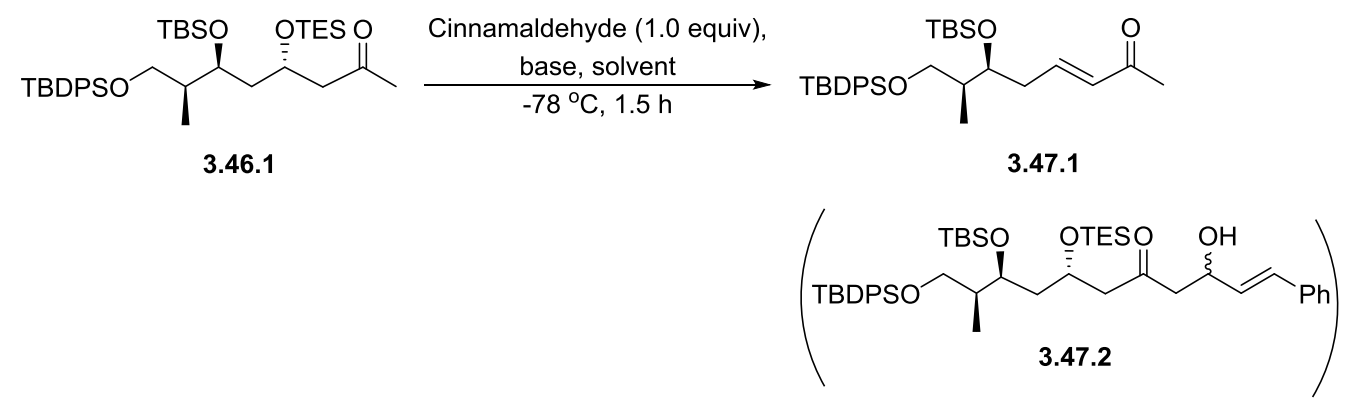

Scheme 3.47. Aldolization of methyl ketone 3.46.1.

\begin{tabular}{ccccccc}
\hline entry & $\begin{array}{c}\text { scale } \\
(\mathrm{mg})\end{array}$ & base $(n$ equiv) & solvent & $\begin{array}{c}\text { conversion } \\
(\%) \mathbf{3 . 4 6 . 1}\end{array}$ & $\begin{array}{c}\text { recovered } \\
(\%) \mathbf{3 . 4 6 . 1}\end{array}$ & $\begin{array}{c}\text { yield } \\
(\%) \mathbf{3 . 4 7 . 1}\end{array}$ \\
\hline 1 & 21 & LiHMDS (1.2) & $\mathrm{CH}_{2} \mathrm{Cl}_{2}$ & 90 & 10 & 0 \\
2 & 25 & LiHMDS (2.2) & $\mathrm{CH}_{2} \mathrm{Cl}_{2}$ & 90 & 10 & 0 \\
3 & 24 & LiHMDS (2.2) & $\mathrm{THF}$ & 87 & 13 & 0 \\
4 & 91 & LDA (1.2) & THF & 30 & 70 & 0 \\
5 & 65 & LDA (2.2) & THF & 81 & 19 & 0 \\
6 & 441 & LDA (1.2) & THF & 96 & 4 & 12 \\
\hline
\end{tabular}

Table 3.2. Various conditions for aldol reactions on ketone 3.46.1.

Due to the dehydration of 3.43.1 in the presence of a strong base, the formation of the titanium and boron enolate was attempted to make the aldol component allylic alcohol 3.47.2 from the adolization of 3.46.1 with cinnamaldehyde (Scheme 3.48). The reaction was carried out on a model substrate, methyl ketone 3.48.1. A variety of conditions were tested to determine 
the best conditions for the aldolization. The aldolization proceeded in each of the tested conditions to give aldol adducts 3.48.2 and 3.48.3. It was found that the use of 1.0 equiv of titanium tetrachloride in the presence of DIPEA gave the undesired thermodynamic product as well as the desired kinetic product in a 2:1 ratio (Table 3.3, entry 1). This was not the outcome obtained with other bases such as LDA, or with dicyclohexylboron chloride and $\mathrm{Et}_{3} \mathrm{~N}$ (Table 3.3, entry 2). With LDA (1.2 equiv), 3.48.2 was isolated in $61 \%$ yield and with the use of 1.5 equiv of dicyclohexylboron chloride in the presence of $\mathrm{Et}_{3} \mathrm{~N}$, desired aldol adduct 3.48.2 was obtained in $91 \%$ (Table 3.3, entry 3 ).

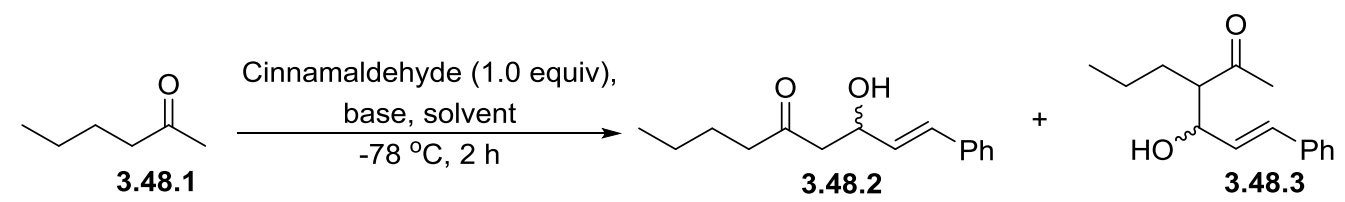

Scheme 3.48. Aldolization of model ketone 3.48.1 with Lewis acids.

\begin{tabular}{ccccccc}
\hline entry & $\begin{array}{c}\text { substrate } \\
\text { scale }(\mathrm{mg})\end{array}$ & $\begin{array}{c}\text { base } \\
(n \text { equiv) }\end{array}$ & $\begin{array}{c}\text { Lewis acid } \\
(n \text { equiv })\end{array}$ & solvent & \multicolumn{3}{c}{ yield } \\
1 & 21 & DIPEA (1.1) & $\mathrm{TiCl}_{4}(1.0)$ & $\mathrm{CH}_{2} \mathrm{Cl}_{2}$ & $48 \%$ & $\mathbf{3 . 4 8 . 3}$ \\
\hline 2 & 25 & LDA (1.2) & $\mathrm{TiCl}_{4}(1.0)$ & $\mathrm{THF}$ & $61 \%$ & - \\
3 & 24 & $\mathrm{Et}_{3} \mathrm{~N}(1.7)$ & $\mathrm{Cy}_{2} \mathrm{BCl}(1.5)$ & $\mathrm{Et}_{2} \mathrm{O}$ & $91 \%$ & - \\
\hline
\end{tabular}

Table 3.3. Various conditions for aldol reactions on ketone 3.48.1 with Lewis acids.

The best conditions $\left(\mathrm{Cy}_{2} \mathrm{BCl}, \mathrm{Et}_{3} \mathrm{~N}\right)$ were then selected to perform the aldolization of methyl ketone 3.46.1 with cinnamaldehyde (Scheme 3.49). Unfortunately, when these better conditions were applied to methyl ketone 3.46.1, the desired aldol adduct 3.47.2 was not obtained and 53\% of the starting material 3.46.1 was recovered. 


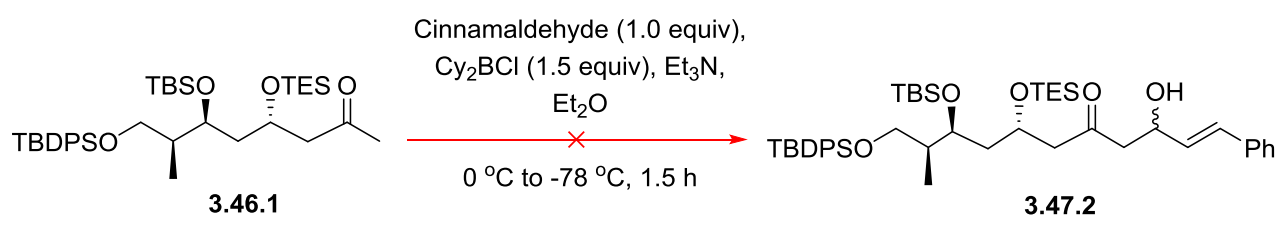

Scheme 3.49. Failed aldolization of methyl ketone 3.46.1.

The unsuccessful attempts to couple methyl ketone 3.46.1 with cinamaldehyde to arrive to fragment 3.47.2 led to a modification of the synthetic approach towards the desired pyranone 3.39.3 and its precursor.

\subsection{Second Retrosynthetic Approach of THP-fragment}

The first modification, in the synthesis of 3.50.2, was to replace the C7-ketone with a C7-alcohol. The second and final amendment was in the switching of positions between the $\mathrm{C}=\mathrm{C}$ and hydroxyl group of the allylic alcohol, thus, we planned to synthesize $\mathbf{3 . 5 0 . 1}$ to access 3.50.2 (Scheme 3.50). The incorporation of all these changes established the new synthetic approach.

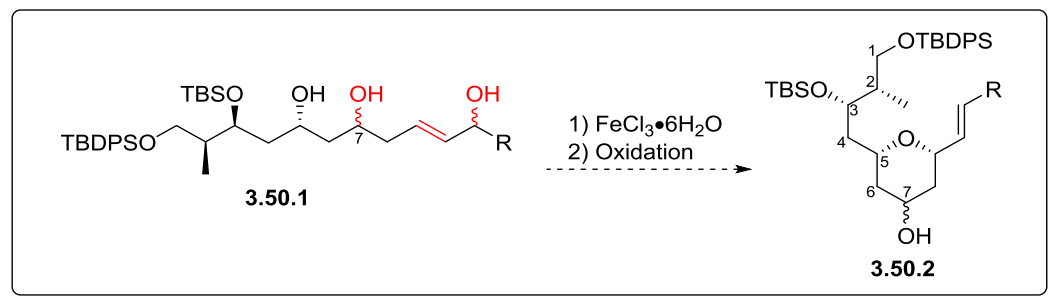

Scheme 3.50. Modifications of previous synthetic approach to give the new synthetic approach.

In this new retrosynthetic approach, the pyranone 3.39.3 would be formed by the Fe(III)-catalyzed hydroalkoxylation of triols 3.50.1a and 3.50.1b (Scheme 3.51). Triols 3.50.1a and 3.50.1b were expected to be synthesized from two different olefination techniques such as cross-metathesis and Wittig olefination. In path A, it was expected that the cross-metathesis (G-II, $\mathrm{CH}_{2} \mathrm{Cl}_{2}$ ) between homoallylic alcohol 3.51.1 and $\alpha$-vinyl benzyl alcohol 3.51.2 would 
furnish the desired product 3.50.1a. Meanwhile, in path B, the aldehyde would be subjected to a Wittig olefination with phosphonate 3.51.4 to give triol 3.50.1b. Both paths were explored beginning with the cross-metathesis (Scheme 3.51).

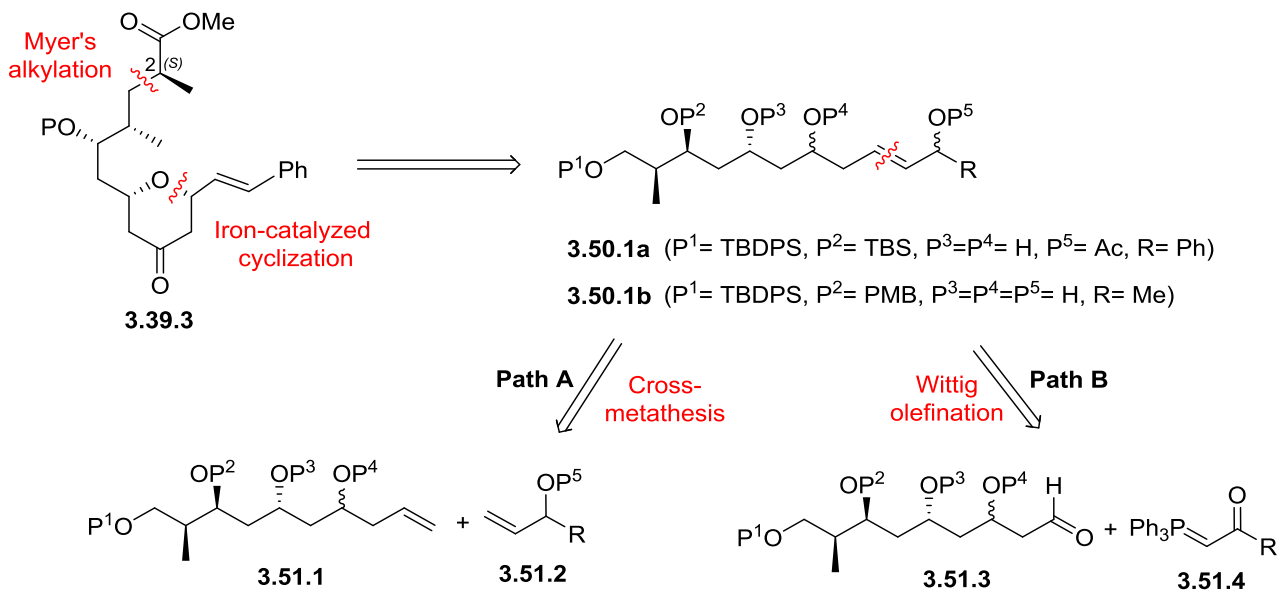

Scheme 3.51. Retrosynthetic analysis of 3.39.3.

\section{Cross-Metathesis Approach:}

The synthesis of 3.51.1 has been envisaged from the homoallylic alcohol 3.42.6, and thus, the carbon homologation would be achieved by an oxidative cleavage followed by an allylation of the ensuing aldehyde (Scheme 3.52).

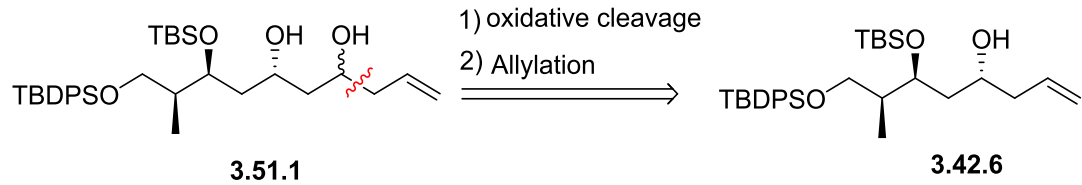

Scheme 3.52. Access to cross-metathesis precursor diol fragment 3.51.1.

The terminal alkene of homoallylic alcohol 3.42.6 was subjected to an oxidative cleavage over two steps using osmium(VIII) oxide followed by the addition of sodium metaperiodate to yield aldehyde 3.53.1 with a high yield of $80 \%$ (Scheme 3.53). Two 
equivalence of allylmagnesium bromide was then employed to anticipate the deprotonation of the free alcohol as well as the nucleophilic attack on the aldehyde. Under these conditions, the outcome of the reaction showed a migration of the neighbouring TBS alcohol protecting group was observed upon the deprotonation of the hydroxyl, which resulted in a myriad of observed products. This highlighted the need to protect the $\beta$-hydroxyl group of aldehyde 3.53.1.

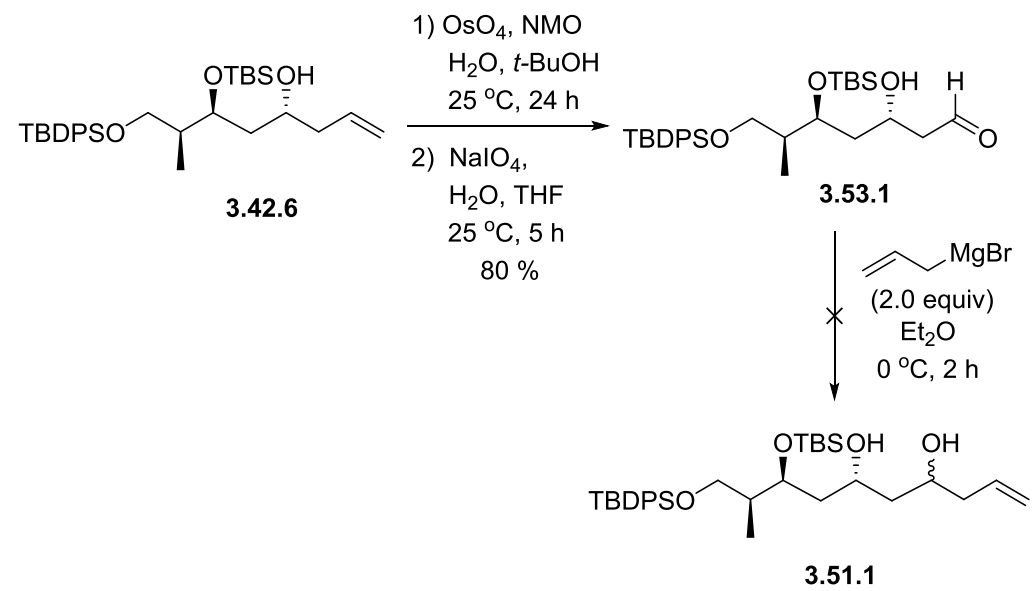

Scheme 3.53. Failed allylation of unprotected $\beta$-hydroxy aldehyde 3.53.1.

Therefore, the homoallylic alcohol 3.42.6 was protected using acetic anhydride (pyridine, DMAP, $\mathrm{CH}_{2} \mathrm{Cl}_{2}, 0^{\circ} \mathrm{C}$ ) and compound 3.54.1 was isolated in $78 \%$ yield (Scheme 3.54). The proceeding oxidation step gave aldehyde $\mathbf{3 . 5 4 . 2}$ in a $93 \%$ overall yield. 


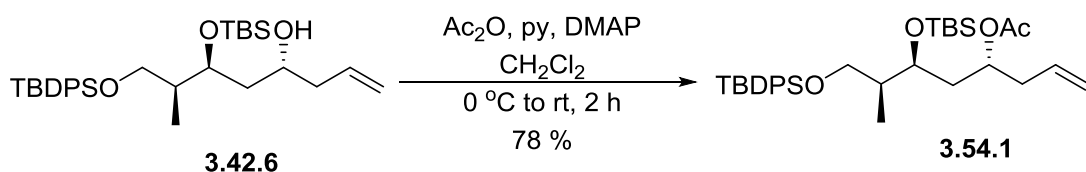

3.42 .6

$78 \%$

3.54.1

1) $\mathrm{OsO}_{4}, \mathrm{NMO}$

$\mathrm{H}_{2} \mathrm{O}, t-\mathrm{BuOH}$

$25^{\circ} \mathrm{C}, 24 \mathrm{~h}$

2) $\mathrm{NaIO}_{4}$, $\mathrm{H}_{2} \mathrm{O}$, THF

$25^{\circ} \mathrm{C}, 5 \mathrm{~h}$ $93 \%$

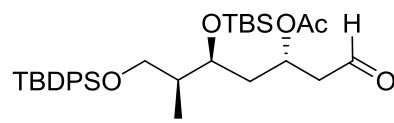

3.54 .2

Scheme 3.54. Protection and oxidation routes to yield 3.54.1 and 3.54.2 respectively, from 3.42.6.

The aldehyde 3.54.2 was then subjected to an allylation using 1.0 equiv of allylmagnesium bromide to prevent the cleavage of the acetyl group in 3.54.2 and to effect an exclusive attack on the more reactive aldehyde in order to produce the desired homoallylic alcohol 3.55.1 (Scheme 3.55). However, compound 3.55.1 was not obtained as the acetyl group was removed during the allylation to give the desired 1,3-diol fragment 3.51 .1 in $61 \%$ yield, which was a pleasant outcome (Scheme 3.55).

Nonetheless, to better understand the reaction, the allylation was repeated at a lower temperature $\left(-78^{\circ} \mathrm{C}\right)$ in an attempt to obtain the desired homoallylic alcohol $\mathbf{3 . 5 5 . 1}$ but the allylation was unsuccessful (Scheme 3.55). This is as the allylmagnesium bromide acts as a base to effect an elimination of the acetate protecting group through a proton abstraction $\alpha$ to the aldehyde and the major product, $\alpha, \beta$-unsaturated aldehyde $\mathbf{3 . 5 5 . 2}$ was obtained in $17 \%$ yield 
(Scheme 3.55). This outcome was not obtained previously in the allylation with unprotected homoallylic alcohol 3.42.6 because the alcohol is a poor leaving group than the acetate.

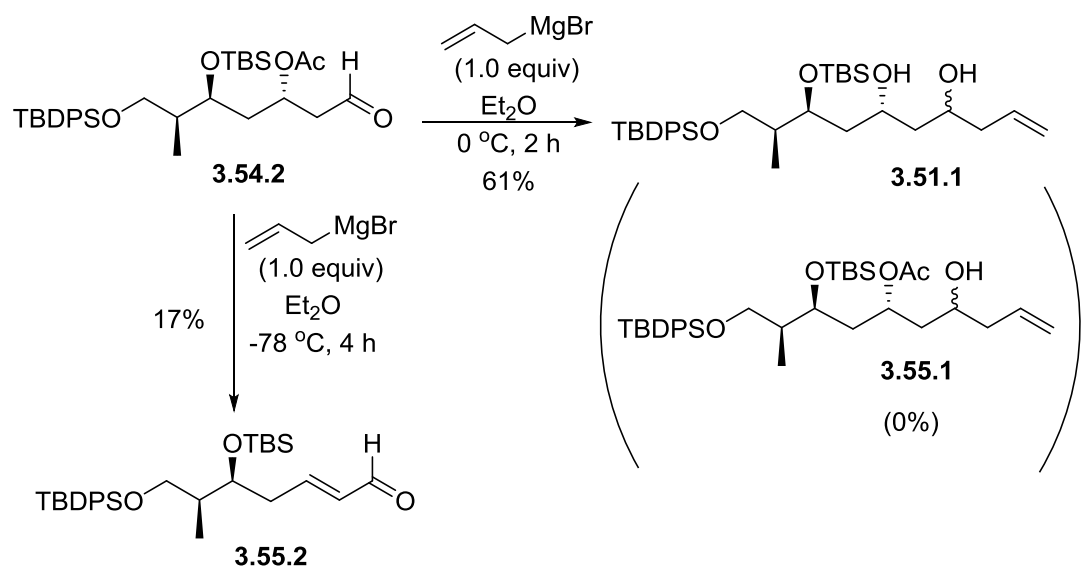

Scheme 3.55. Allylation at different temperatures of aldehyde 3.54.2.

The effects of the Grignard allylation conditions employed on other alcohol protecting groups were also investigated (Scheme 3.56). Therefore, the homoallylic alcohol 3.42.6 was protected using 3,4-dihydropyran (PPTS, $\mathrm{CH}_{2} \mathrm{Cl}_{2}, 25^{\circ} \mathrm{C}$ ) and compound 3.56.1 was isolated in a $53 \%$ yield, with a recovered $27 \%$ of the starting material 3.42.6. The terminal alkene in 3.56.1 was then subjected to an oxidative cleavage over two steps using osmium(VIII) oxide and sodium metaperiodate, to give the expected aldehyde $\mathbf{3 . 5 6 . 2}$ in a $95 \%$ overall yield. The allylation was then carried out on the THP-protected $\beta$-alkoxy aldehyde 3.56.2 in an attempt to avoid the elimination pathway. Although the reaction gave the desired homoallylic alcohol 3.56.3, it was isolated in a low yield of $27 \%$. The purification was problematic as there were several purified fractions obtained, all of which showed similar proton NMR spectra due to the presence of a mixture of diastereomers resulting from both the allylation and the THP-protecting group (that bears a stereogenic carbon). Nonetheless, it was evident that a milder allylation method was required and thus, the route was disregarded (Scheme 3.56). 

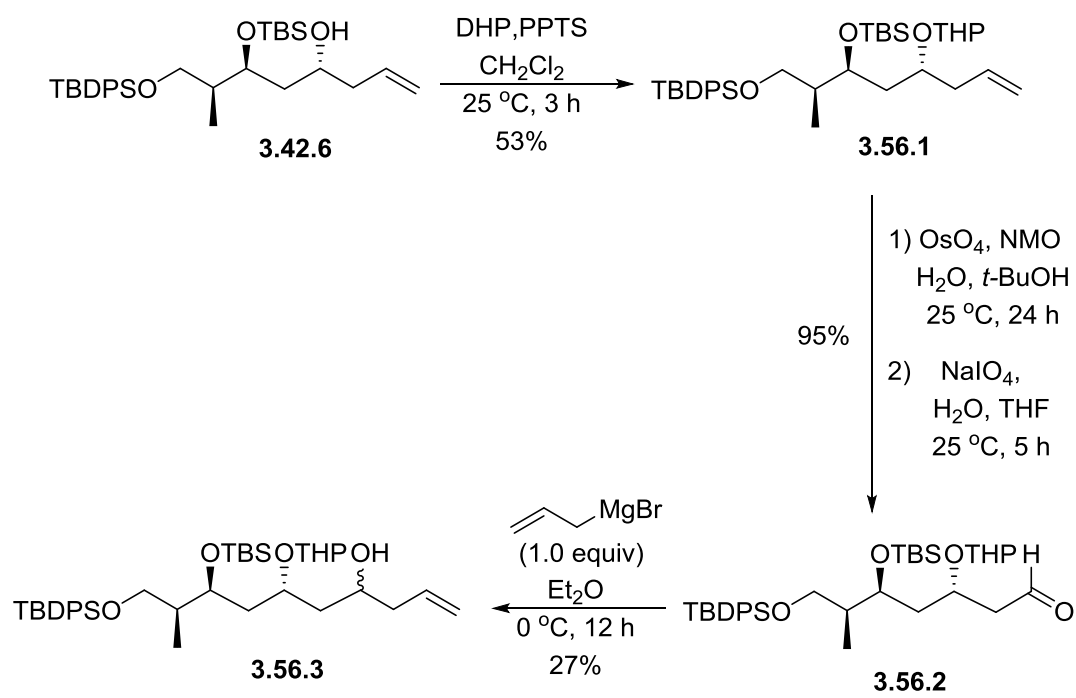

Scheme 3.56. Protection with DHP followed by oxidative cleavage and allylation to give 3.56.3.

A mild allylation alternative method such as the Barbier allylation ( $\mathrm{In}, \mathrm{THF}, \mathrm{H}_{2} \mathrm{O}$ ) of 3.57.1 was therefore carried out to arrive to the desired diol 3.51.1 (Scheme 3.57). The unprotected homoallylic alcohol 3.42.6 was subjected to an oxidative cleavage of the terminal double bond $\left(\mathrm{OsO}_{4}, \mathrm{NaIO}_{4}\right)$ to provide the corresponding aldehyde 3.57.1 that was successfully transformed into diol 3.51.1 in the presence of indium. Compound 3.51.1 was isolated with an overall yield of $46 \%$ from 3.42 .6 .

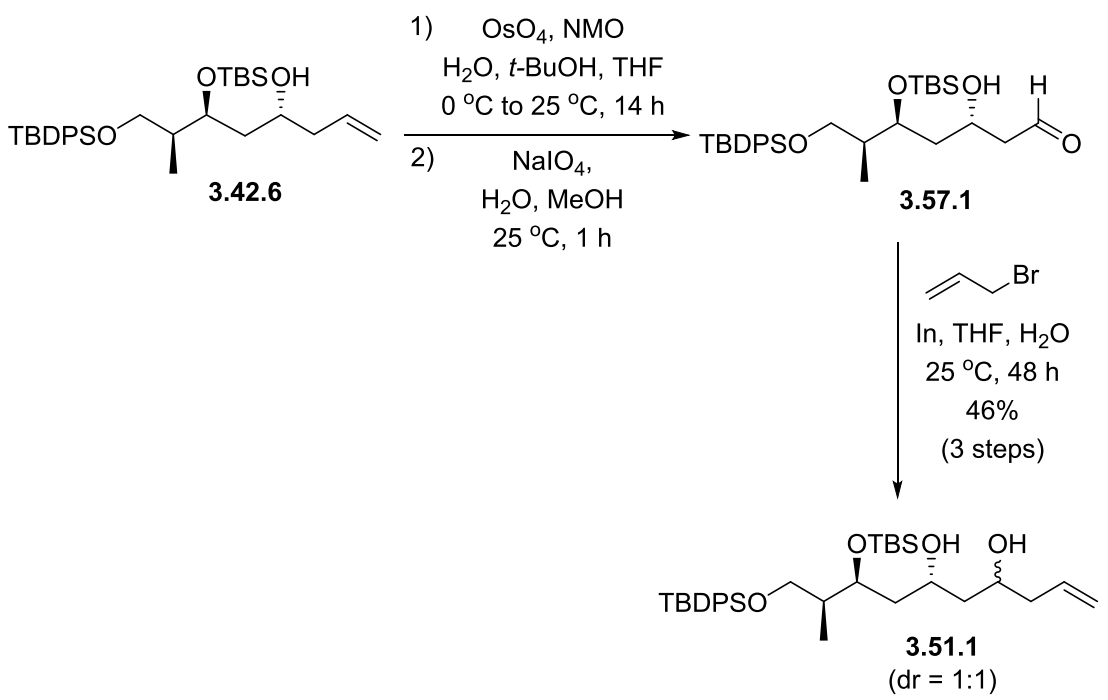

Scheme 3.57. Oxidative cleavage of terminal olefin 3.42.6 followed by allylation of 3.57.1 to yield 3.51.1. 
The 1,3-diol 3.51.1 was subsequently engaged in a cross-metathesis with allylic acetate 3.51.2 using Hoveyda-Grubbs catalyst $\left(10 \mathrm{~mol} \%, 50{ }^{\circ} \mathrm{C}, \mathrm{CH}_{2} \mathrm{Cl}_{2}\right)(\mathrm{Scheme} 3.58)$. The desired diol 3.50.1a was not obtained and thus the intended Fe(III)-catalyzed cyclization supposed to lead to 3.58.1 could not be investigated.

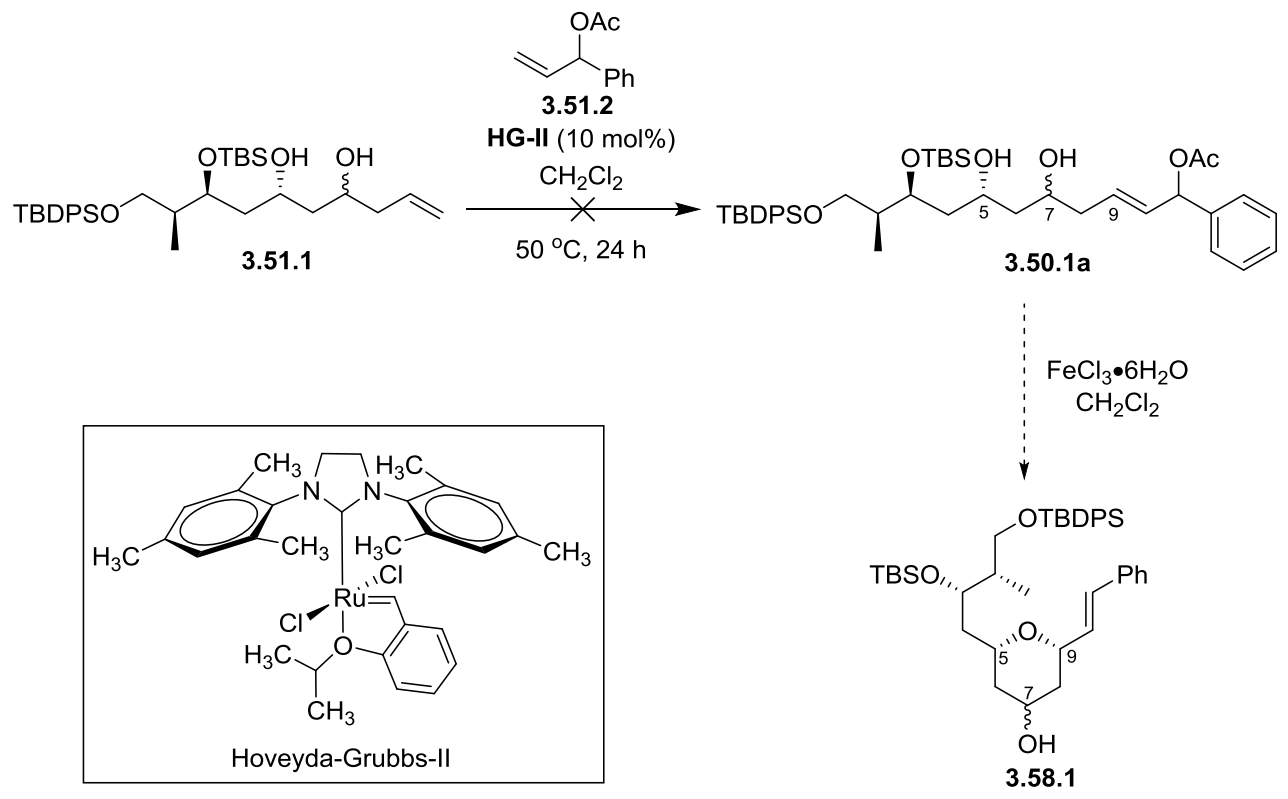

Scheme 3.58. Failed cross-metathesis between 1,3-diol 3.51.1 and 3.51.2.

The lack of success associated with this strategy led us to a switch of the secondary alcohol protecting group from TBS to a PMB-ether so as to circumvent issues associated with the silyl-group migration.

\section{Wittig Olefination Approach:}

By using the Wittig reaction, compound 3.59.7 was prepared (Scheme 3.59). The synthesis began with the TBDPS-alcohol protected methyl ester 3.42.1, which was quantitatively converted into Weinreb amide 3.42.2 with $N, O$-dimethylhydroxylamine at $-40^{\circ} \mathrm{C}$. The amide 3.42.2 was then reduced with DIBAL-H in tetrahydrofuran at $-78{ }^{\circ} \mathrm{C}$ to furnish aldehyde 3.42.3, which was directly engaged in the proceeding diastereoselecive Duthaler- 
Hafner allylation with $(R, R)$-Ti to give homoallylic alcohol 3.42.4. The homoallylic alcohol 3.42.4 was then protected as a PMB-ether 3.59.1 under acidic conditions in the presence of PMB-trichloroacetimidate. The protecting step was followed by an oxidative cleavage over two steps using osmium(VIII) oxide and sodium metaperiodate to give the desired aldehyde 3.59.2. The latter was directly treated with the Duthaler allyltitanium complex $(S, S)$-Ti to furnish homoallylic alcohol 3.59.3 (30\% yield over three steps, $\mathrm{dr}=19: 1)($ Scheme 3.43). The second Duthaler-Hafner allylation, evolving to the homoallylic alcohol 3.59.3 proceeded with a low isolated $30 \%$ yield because of purification problems caused by the remains of excess (S,S)-TADDOL from the titanium complex. Consequently, the homoallylic alcohol 3.59.3 was contaminated by $(S, S)$-TADDOL (33\% contamination by ${ }^{1} \mathrm{H}$ NMR analysis based on the extra aromatic protons present), but it was obtained with $64 \%$ purity and good diastereoselectivity of $19: 1$.

The synthesis continued with homoallylic alcohol 3.59.3 that was subjected to an oxidative cleavage over two steps using osmium(VIII) tetraoxide and sodium metaperiodate to give the desired aldehyde 3.59.4 that was directly employed in the proceeding third Duthaler allyltitanation with allyltitanium complex $(R, R)$-Ti to furnish the homoallylic alcohol $\mathbf{3 . 5 9 . 5}$ as a single diastereomer in a low overall yield of $21 \%$ over 3 steps due to the remaining TADDOL contamination present with 3.59.3 (Scheme 3.59). It is noteworthy that the trans-acetonide was desired over the cis-acetonide because the latter was found to be more robust towards the acidic deprotection conditions. ${ }^{124}$

Also, in the protection of the diol 3.59.5 using 2,2-dimethoxypropane (PPTS, $\mathrm{CH}_{2} \mathrm{Cl}_{2}$ ), the trans-acetonide 3.59.6 was isolated in $72 \%$ yield (determined by ${ }^{13} \mathrm{C}$ NMR of acetal carbon at $100.6 \mathrm{ppm}$ and dimethyl carbons at $25.0 \mathrm{ppm}$ ) (Scheme 3.59). ${ }^{175}$ The terminal double bond of 3.59.6 was oxidatively cleaved to give the corresponding aldehyde 3.51.3 that was directly submitted to a Wittig reaction with 1-(triphenylphosphoranylideneacetone) 3.51 .4 in 
tetrahydrofuran. The key Wittig olefination step had to be performed under reflux over $10 \mathrm{~h}$ to furnish the desired $\alpha, \beta$-unsaturated methyl ketone 3.59.7. We have to point out that no conversion of the starting aldehyde $\mathbf{3 . 5 1 . 3}$ was observed when the reaction was carried out at 25 ${ }^{\circ} \mathrm{C}$. The resulting desired $\alpha, \beta$-unsaturated methyl ketone 3.59.7 was isolated in $36 \%$ overall yield from 3.59.6 over three steps (Scheme 3.58) and determined by ${ }^{1} \mathrm{H}$ NMR where the $E$-stereoisomer double bond $(J=16.0 \mathrm{~Hz})$ was observed.

The $\alpha, \beta$-unsaturated ketone $\mathbf{3 . 5 9 . 7}$ was then subjected to the Luche reduction $\left(\mathrm{CeCl}_{3}, \mathrm{MeOH}, \mathrm{NaBH}_{4}\right)$ that proceeded smoothly and cleanly to give allylic alcohol 3.59.8 (Scheme 3.59). ${ }^{176}$ The allylic alcohol 3.59.8 was then deprotected using dilute aqueous hydrochloric acid ( $1 \mathrm{~N} \mathrm{HCl}, 0.1$ equiv) to give the pyranone precursor 3.50.1b that was isolated in $69 \%$ overall yield over two steps (Scheme 3.59). 


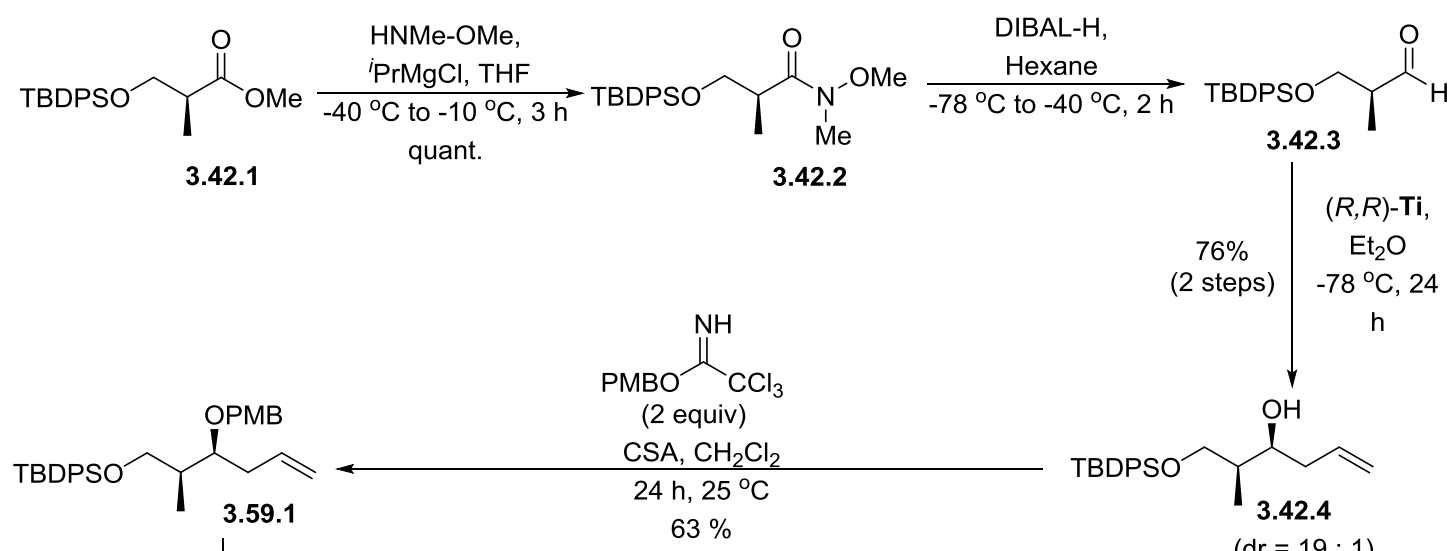

1) $\mathrm{OsO}_{4}, \mathrm{NMO}$

$(\mathrm{dr}=19: 1)$

$\mathrm{H}_{2} \mathrm{O}, t-\mathrm{BuOH}, \mathrm{THF}$

$0{ }^{\circ} \mathrm{C}$ to $25^{\circ} \mathrm{C}, 14 \mathrm{~h}$

2) $\mathrm{NalO}_{4}$,

$\mathrm{H}_{2} \mathrm{O}, \mathrm{MeOH}$

$25^{\circ} \mathrm{C}, 1 \mathrm{~h}$
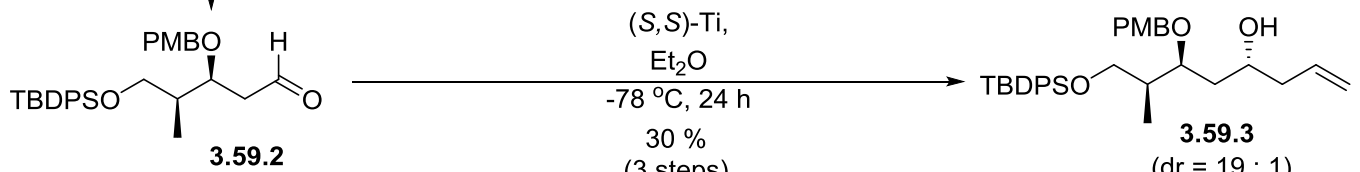

59.2

(3 steps)

$r=19: 1$

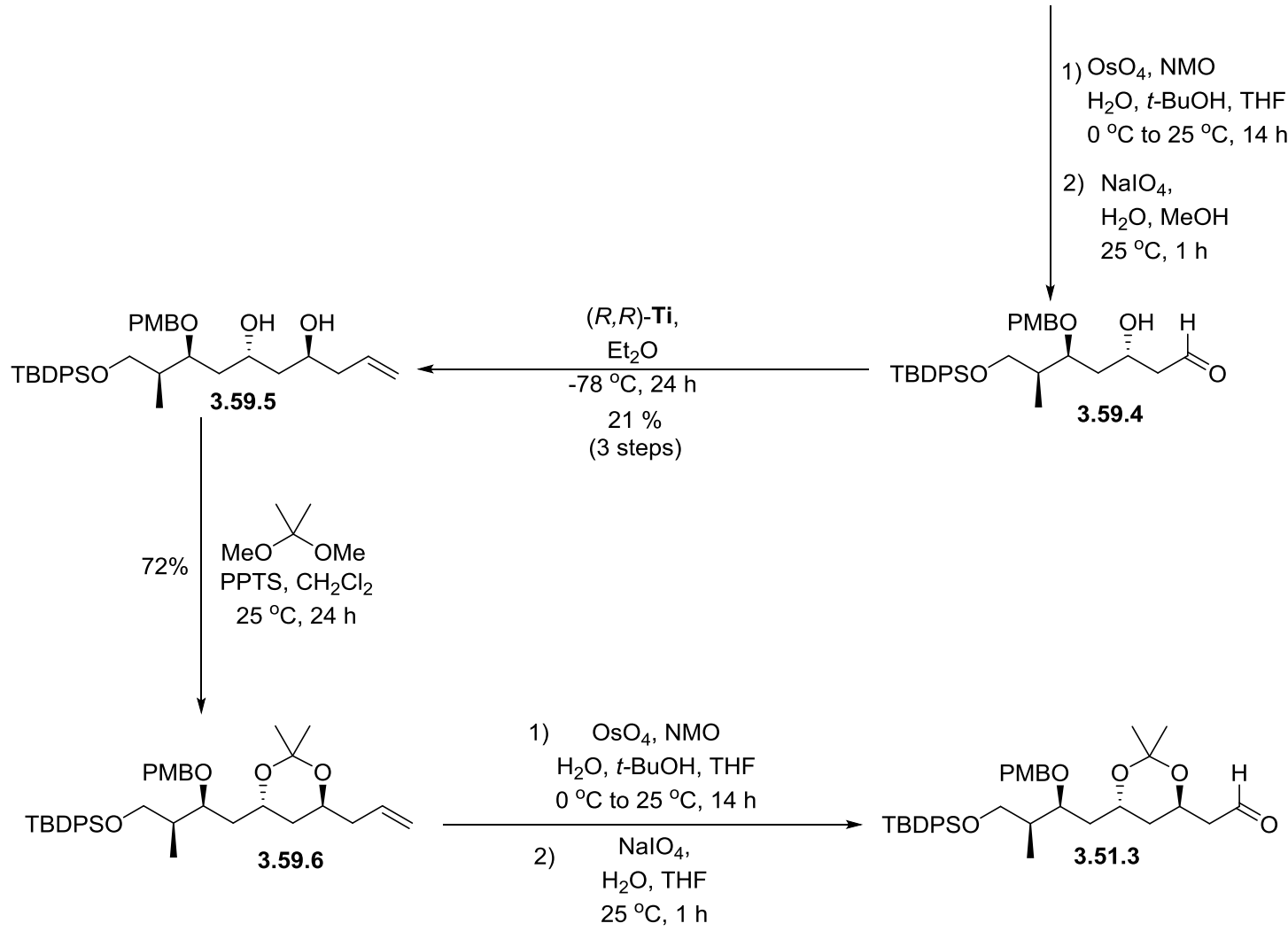




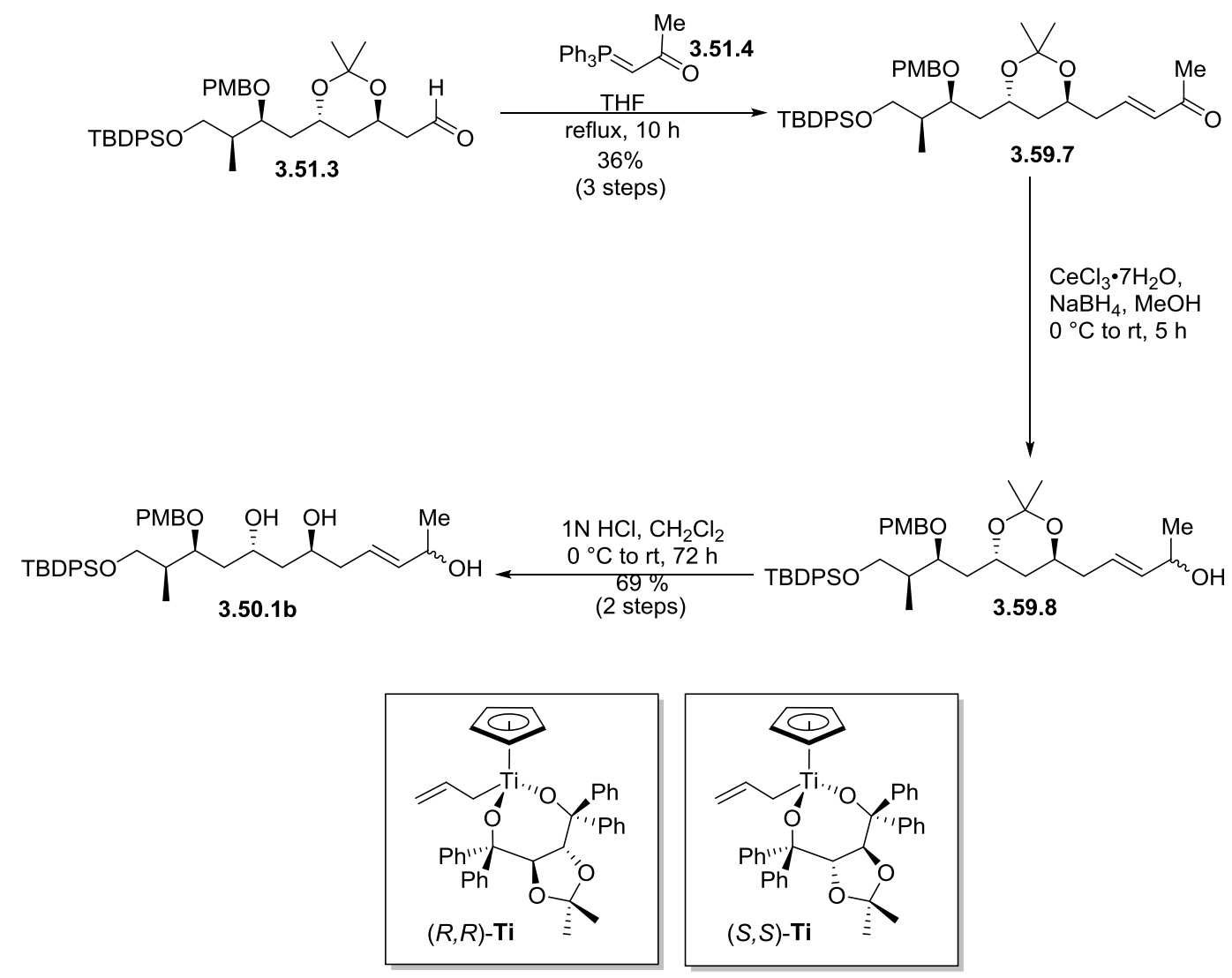

Scheme 3.59. Synthesis towards objective triol fragment 3.50.1b.

The $\mathrm{FeCl}_{3}$ cyclization was then carried out on triol 3.50.1b at room temperature overnight in the attempt to form the desired cis-tetrahydropyran 3.60.1 (Scheme 3.60). The $\mathrm{FeCl}_{3}$ cyclization was proposed to proceed via the iron(III)-activated allylic alcohol $\mathbf{3 . 6 0 . 2}$ and $\pi$-allyl cation intermediate 3.60.3. Unfortunately, the cyclization was unsuccessful as there were no characteristic signals corresponding to the protons at the C5 and C9 positions of 3.60.1 (Scheme 3.60).

The analysis of ${ }^{1} \mathrm{H}$ NMR spectra reflected a decomposition of triol 3.50.1b. Although the TLC showed a major non-polar spot compared to the starting material $\mathbf{3 . 5 0 . 1 b}$, it was accompanied by a myriad of compounds present in the reaction mixture (Scheme 3.60). After an overnight stirring, the conversion of $\mathbf{3 . 5 0 . 1 b}$ remained incomplete. 


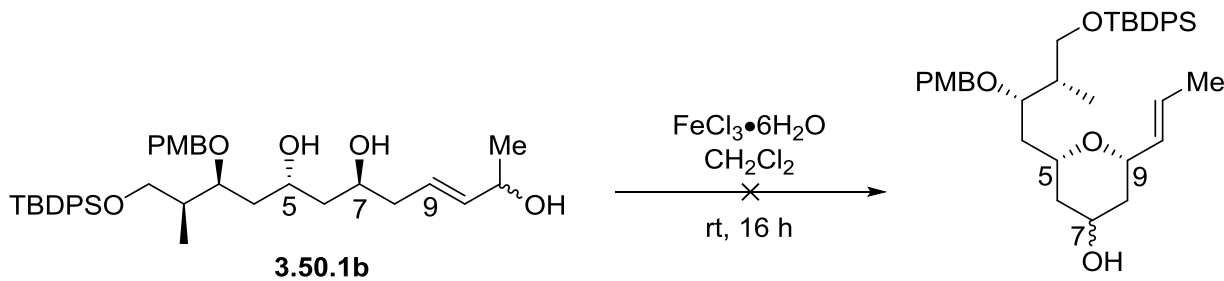

$3.50 .1 b$

3.60.1

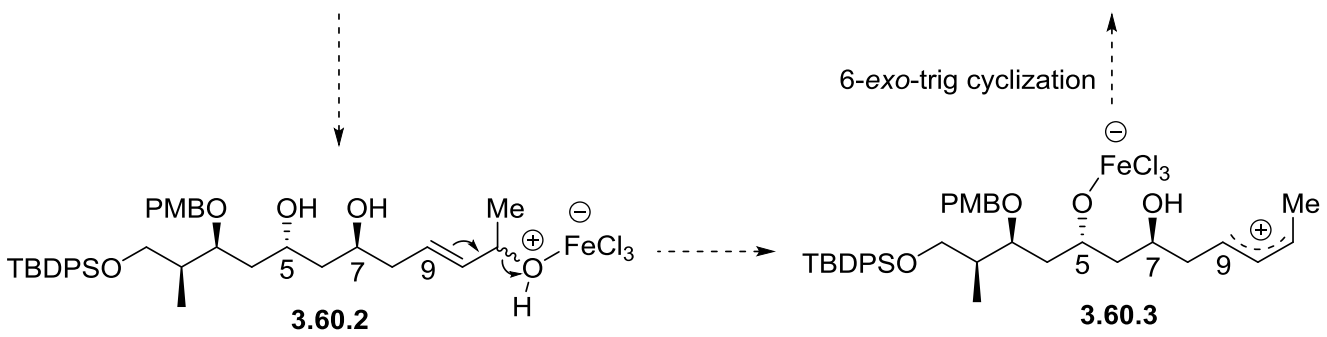

Scheme 3.60. Unsuccessful attempt of Fe(III)-promoted cyclization of 3.50.1b to give 3.60.1.

\subsubsection{Other Attempts towards the Synthesis of C1-C10 Fragment of Enigmazole A}

\subsection{Vinylation Approach}

Alternative attempts to access the tetrahydropyran precursor, triol 3.61.5, were examined through the cis-acetonide, which was synthesized from the already available aldehyde 3.59.4 via Duthaler-Hafner allylation to give cis-diol 3.61.1 (Scheme 3.61). Under acidic conditions in 2,2-dimethoxypropane, 3.61.2 was obtained in moderate $54 \%$ yield. The following oxidative bond cleavage of 3.61.2 furnished the aldehyde 3.61.3 in high $79 \%$ yield. The aldehyde was treated with 1-propenylmagnesium bromide, to yield the allylic alcohol 3.61.4, which was directly submitted to the acetonide deprotection step. Unfortunately, triol 3.61.5 was not obtained under acidic conditions $(1.0 \mathrm{M} \mathrm{HCl}) .{ }^{124}$ A subsequent increase in the $\mathrm{HCl}$ concentration led to the decomposition of 3.61.4 (Scheme 3.61). 


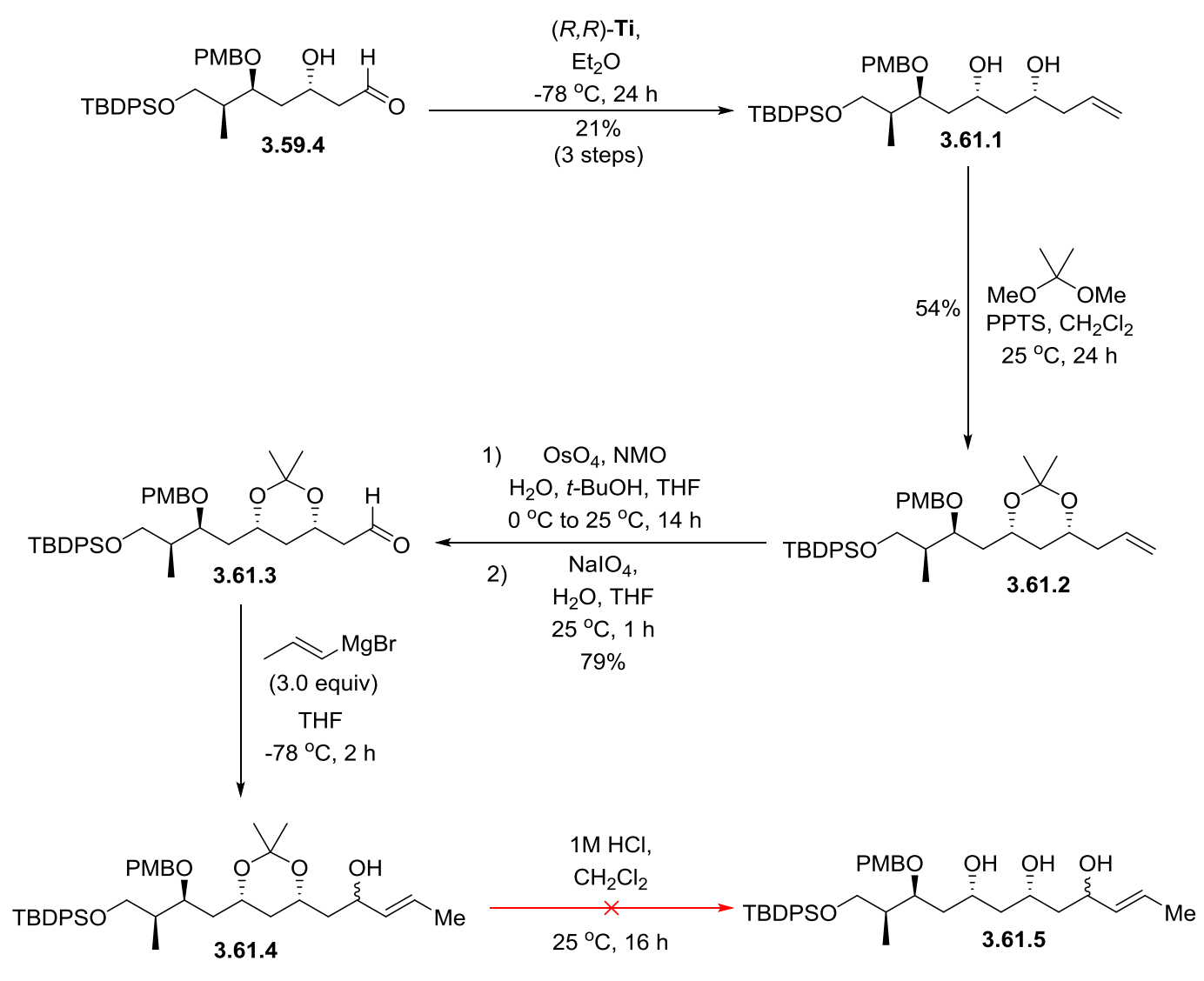

Scheme 3.61. Efforts towards the synthesis of triol 3.61.5 from aldehyde 3.59.4.

\subsection{Olefin Isomerization Approach}

Since the relative position of the olefin (C9-C10) and the allylic leaving group (C11) is interchangeable, the precursor to the Fe(III)-cyclization was envisaged from allyl alcohol 3.62.1, which would be expected to generate the same $\pi$-allyl cation intermediate 3.60.2 as allyl alcohol 3.50.1b upon treatment with $\mathrm{FeCl}_{3} \cdot 6 \mathrm{H}_{2} \mathrm{O}$ (Scheme 3.62).

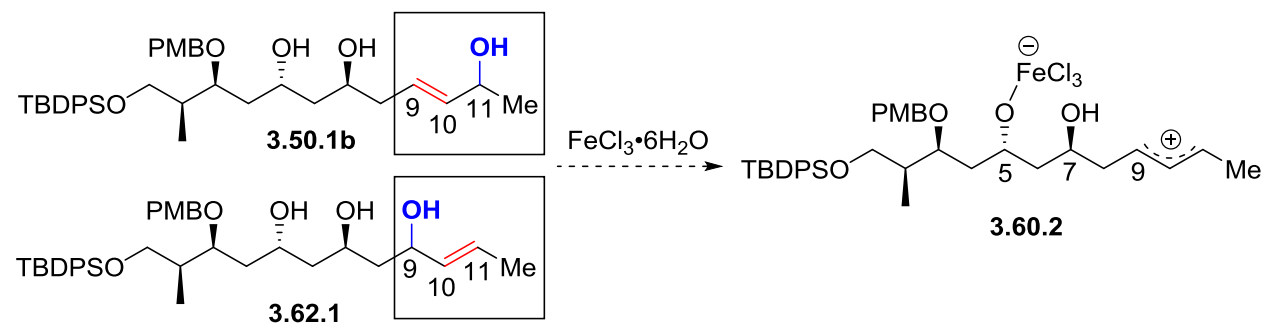

Scheme 3.62. Triol 3.62.1 and 3.50.1b would generate the same $\pi$-allyl cation 3.60.2 
The trans-acetonide 3.51.3 was subjected to allyltitanation with Duthaler-Hafner complex, $(S, S)$-Ti, to give homoallylic alcohol 3.63.1(Scheme 3.63). The alkene isomerization of homoallylic alcohol 3.63.1 was thus carried out under Wipf et al. conditions to achieve the allyl alcohol 3.63.3. ${ }^{177}$ The product isolated corresponds to saturated alcohol 3.63.2, the expected allylic alcohol 3.61.3 was not observed (determined by analysis of ${ }^{1} \mathrm{H}$ NMR). The possible source of hydrogen, responsible for the formation of the saturated product, could be from a $\beta$-hydride elimination when a trialkylamine is coordinated to the ruthenium metal. Consequently, the subsequent acetonide deprotection of $\mathbf{3 . 6 3 . 3}$ could not be carried out and the key cyclization step towards desired THP-3.60.1 could not be investigated.
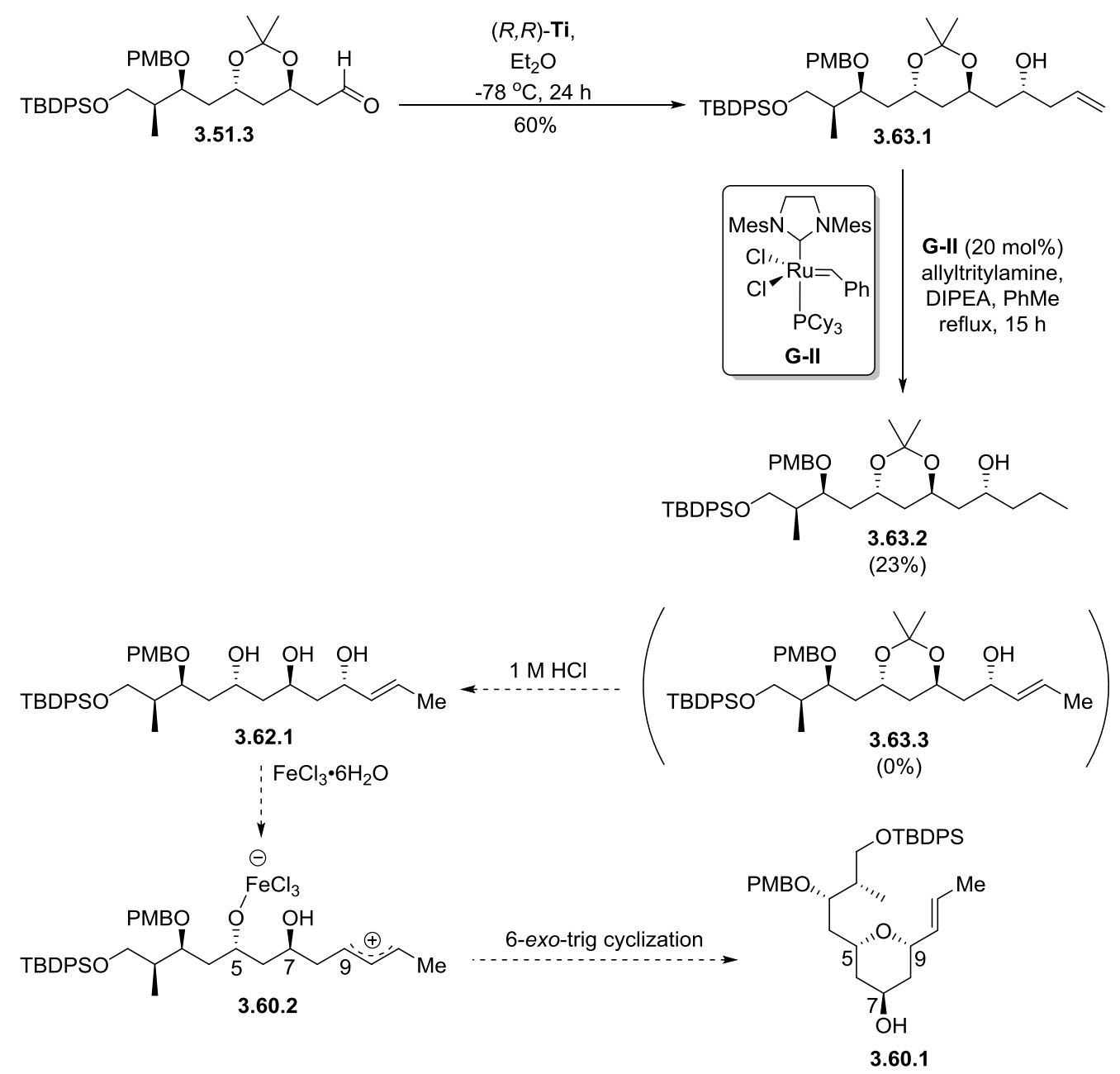

Scheme 3.63. Attempt towards desired allyl alcohol 3.63.3 from aldehyde 3.51.3 towards desired THP-3.60.1. 


\subsection{Conjugate Addition Approach}

Alternatively, an approach towards the tetrahydropyran 3.64.3 was envisaged from phenyl vinyl ketone 3.64.1 with 3.59.5 using another cross-metathesis reaction (Scheme 3.64). The Michael acceptor $\alpha, \beta$-unsaturated phenyl ketone 3.64.2 was obtained in low $25 \%$ yield. The following oxo-1,4-addition reaction was carried out under mild acidic conditions with CSA at room temperature for $4 \mathrm{~h}$ to $24 \mathrm{~h}$ but no conversion of 3.64.2 was observed (Scheme 3.64). Therefore, the reaction was repeated with Amberlyst-15 overnight to give a complete consumption of 3.64.2. However, peaks corresponding to the desired product 3.64.3 were not observed in the ${ }^{1} \mathrm{H}$ NMR spectrum.

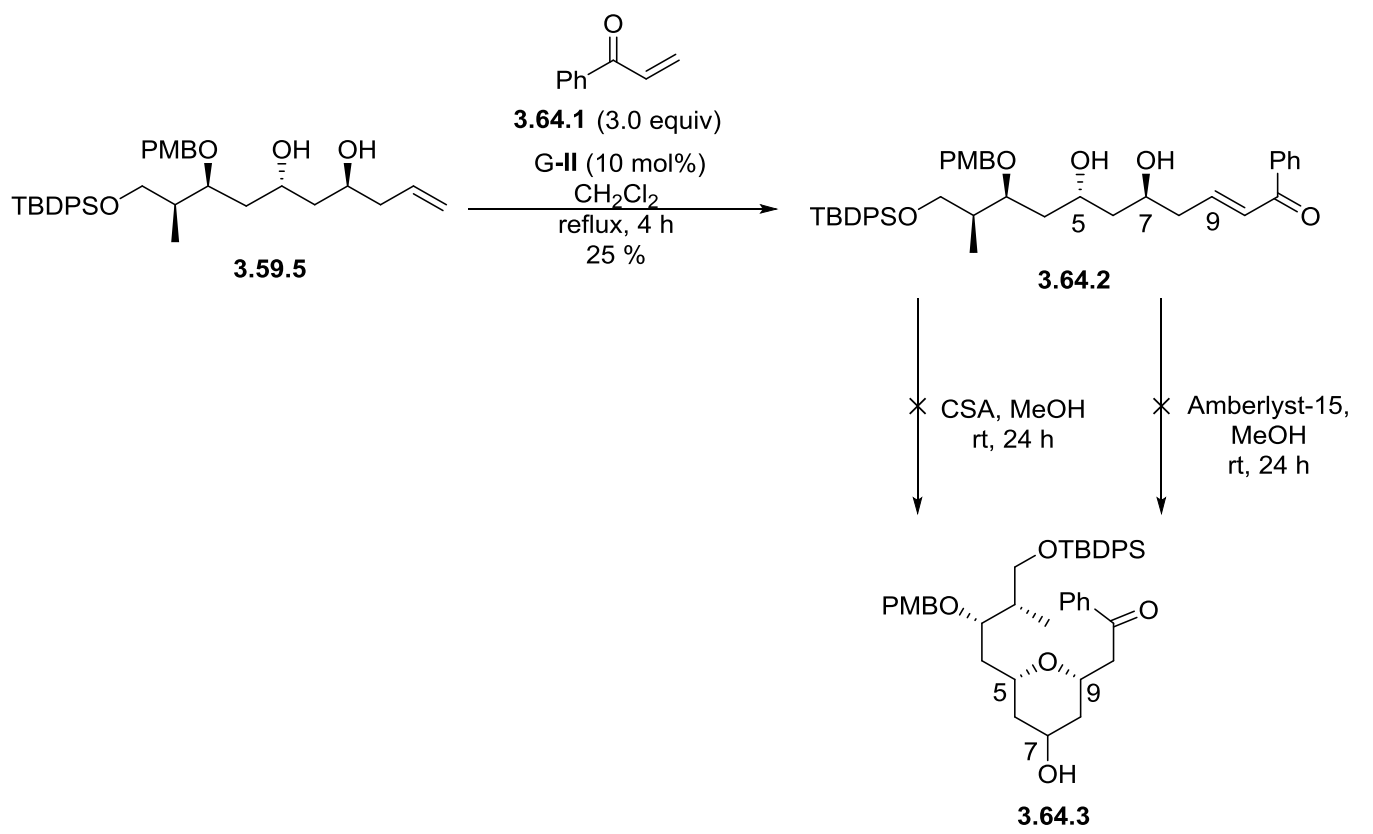

Scheme 3.64. Alternative approach towards THP-fragment 3.64.3 through intramolecular conjugate addition of 3.64.2. 


\subsubsection{Perspectives}

There is a need to isolate the major product from the $\mathrm{FeCl}_{3}$ catalyzed cyclization to better understand the issue. The triol 3.50.1b needs to be synthesized in greater quantity so as to allow for experimentations with other cyclization conditions such as employing a softer Lewis acid like indium(III) trichloride, this will enable progress in the natural product synthesis. Other non-Fe(III)-catalyzed approaches towards the synthesis of the THP-fragment such as the Prinscyclization ${ }^{178}$ has to be explored. 
This page has been intentionally left blank. 
This page has been intentionally left blank. 


\title{
Chapter 4
}

\author{
4.1 Introduction
}

4.1.1 Importance of Tri-Aryl Substituted Olefins

4.1.2 Transition-Metal Catalyzed Strategies to Access TriAryl Substituted Olefins 
This page has been intentionally left blank. 


\subsection{Introduction}

\subsubsection{Importance of Tri-Arylethylene Unit}

Stilbene derivatives offer a wide range of biological activities, photophysical and photochemical properties, ${ }^{179}$ implying a broad range of application in chemistry including life and materials sciences (Figure 4.1). ${ }^{180}$ The highly conjugated $\pi$-systems can also be found in $\pi$-conjugated dendrimers that are molecular architectures bearing the potential for new functional materials. ${ }^{181}$ They can also be classified as stilbene-based derivatives and possess a broad range of biological activities. ${ }^{182}$ An example of a tri-arylethylene framework associated with a pharmaceutical application is the well-known estrogen receptor antagonist (Z)-tamoxifen, which is used for the treatment of metastatic breast cancer (Figure 4.1). ${ }^{183}$ The structural motif is also in analogues $(Z)$-ospemifene and $(Z)$-toremifene. ${ }^{183 e}$ The tri-arylethylenes framework was specifically inferred as an estrogen agonist whilst the attached alkyl chains are responsible for the antagonist behaviour. ${ }^{183 e}$ Another example of a bioactive tri-arylethylene compound is the COX-2 inhibitor. ${ }^{184}$ The COX-2 is predominately expressed in activated cells as a response to mediators of inflammation and thus, a selective inhibitor of COX-2 would lead to a reduction in ulceration and dyspepsia (Figure 4.1). ${ }^{184}$ 


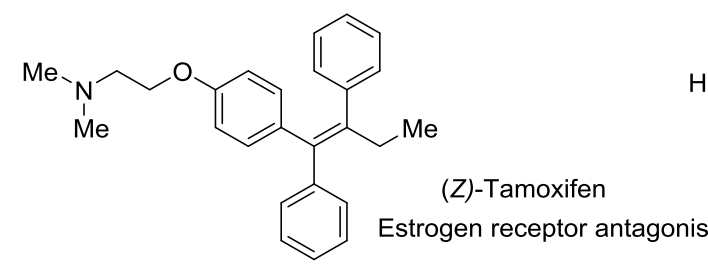<smiles>OCCOc1ccc(C(=C(CCCl)c2ccccc2)c2ccccc2)cc1</smiles>

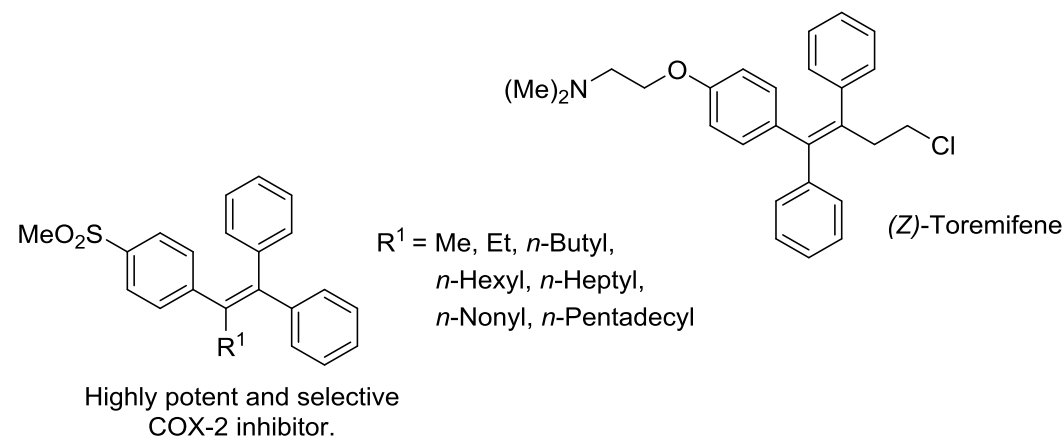

Figure 4.1. Tri-arylethylene framework present in receptor antagonists of breast cancer therapeutic agents and COX-2 inhibitor bearing a tri-arylethylene unit.

Apart from the area of medical application, the tri-arylethylene with its extended $\pi$-systems provide attractive optical properties in dyes and pigments (Figure 4.2). ${ }^{185}$ Some organic dyes with the tri-arylethylene design can be used as organic sensitizers in solar cells. ${ }^{186}$ Additionally, the tri-arylethylenes can be recognized as organic luminophores due to their aromatic chromophores. ${ }^{186}$ This enables displays of rare light emissions such as the blue-light emission that can be utilized as biological probes, chemical sensors and display technology. ${ }^{186}$ Furthermore, the tri-aryethylene unit, in their aggregation state, can emit aggregation-induced emissions (AIE), ${ }^{187}$ which are highly luminescent and thus enable these units to be used in OLED devices as fluorescent chemosensors (Figure 4.2). ${ }^{186}$ 


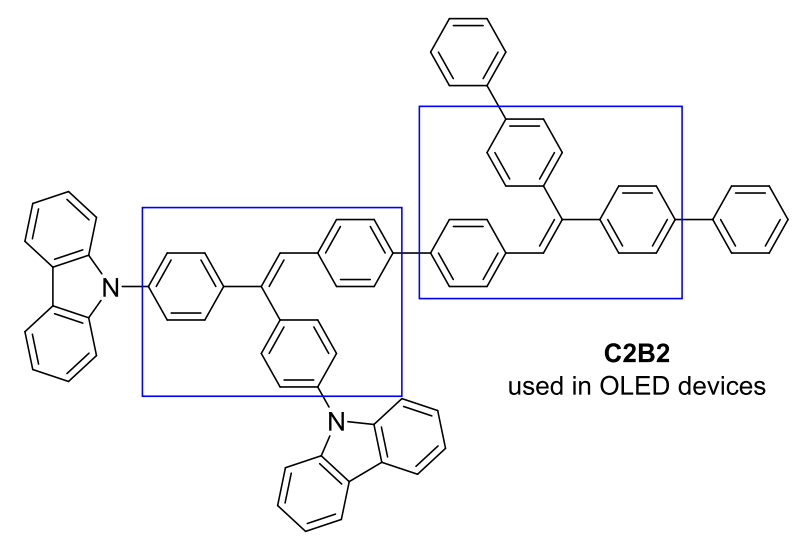

Figure 4.2. An example of an extended $\pi$-system through tri-arylethylene backbone.

These examples reflect that the tri-arylethylene unit is finding increasing applications in dynamic fields, and for this reason, a range of methods have been developed to access the structural motif. ${ }^{188}$

\subsubsection{Methods to Access Tri-Aryl Substituted Olefins}

The existing strategies for the construction of multisubstituted olefins are vast. They include McMurry reaction, ${ }^{189}$ Meerwin reaction, ${ }^{190}$ Wittig olefination, ${ }^{191}$ Horner-Wadsworth Emmons (HWE) olefination, ${ }^{192}$ Julia reactions, ${ }^{193}$ alkyne carbolithiation, ${ }^{194}$ and reactions that use $\mathrm{CF}_{3}$-bearing oxiranes, ${ }^{195}$ organosilanes, ${ }^{196}$ electrotelluration ${ }^{197}$ and ynolate anions ${ }^{198}$ and more. ${ }^{199}$ Nonetheless, it is the transition-metal catalyzed reactions ${ }^{200}$ that emerge as the most versatile reactions for the task because of its wide tolerance to a range of functional groups. ${ }^{201}$ Additionally, one of the most powerful tools for the construction of $\mathrm{C}-\mathrm{C}$ bonds is the transition metal catalyzed cross-coupling reactions. For instance, Suzuki-Miyaura, ${ }^{202} \mathrm{Heck},{ }^{203}$ Stille, ${ }^{204}$ Kumada, ${ }^{205}$ Negishi, ${ }^{206}$ Hiyama ${ }^{207}$ and Sonogashira ${ }^{208}$ cross-coupling reactions have resulted in a paradigm shift in retrosynthetic analysis planning. 


\subsubsection{Carbometalation of Alkynes}

The combination of carbometalation and cross-coupling sequence to afford a diversityoriented library of arylated alkenes is an attractive strategy. ${ }^{209}$ The stereodefined synthesis of substituted olefins can be carried out using carbometalation of alkynes, a method that has proven attractive..$^{210}$

Carbometallation of alkynes $\left(\mathrm{Ar}^{1}-\mathrm{C} \equiv \mathrm{C}-\mathrm{Ar}^{2}\right)$ with Nickel followed by Negishi-type coupling

In 2006, Sato et al. reported the $\mathrm{Ni}(0)$-catalyzed arylative carboxylation of alkynes (Scheme 4.1). The alkyne 4.1.1 underwent an oxidative cyclization to afford the oxanickelacycle intermediate 4.1.2 in the presence of $\mathrm{CO}_{2}$ and $\mathrm{Ni}(0)$. This is followed by the introduction of a third aryl group specifically through transmetalation by an organozinc reagent in a Negishi-type coupling. The reductive elimination step from the alkenylnickel complex 4.1.3 then gives the multisubstituted olefin 4.1.4. This method was used to access tamoxifen. ${ }^{211}$

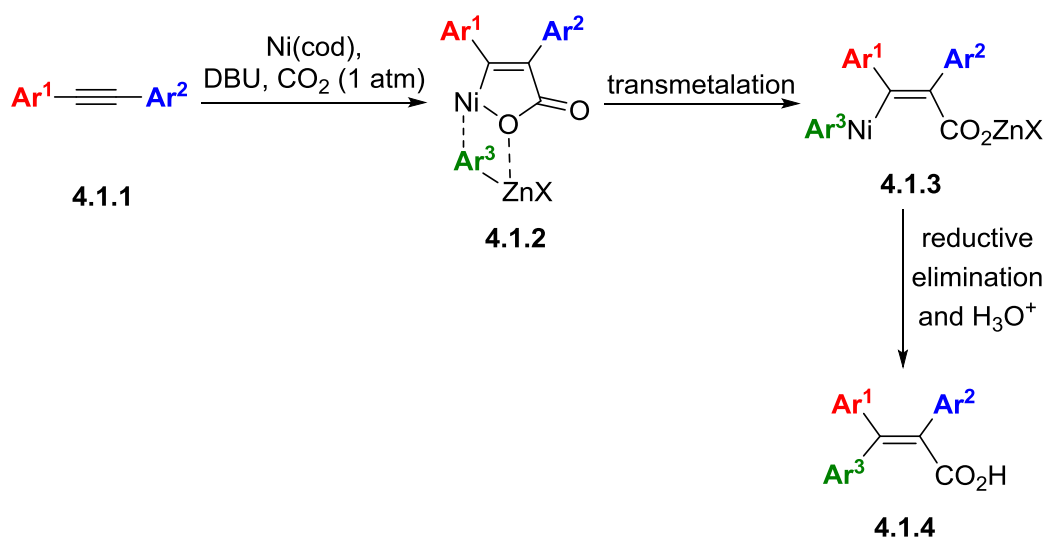

Scheme 4.1. Ni(0)-catalyzed cabometallation of alkynes.

Carbometallation of alkynes $\left(\mathrm{Ar}^{1}-\mathrm{C} \equiv \mathrm{C}-\mathrm{Ar}^{2}\right)$ with Zirconium followed by Heck or Suzuki coupling

Apart from nickel, metalation of alkynes can also be initiated by zirconium metal as reported by Nakajima et al (Scheme 4.2). ${ }^{210}$ In the first metallo-esterification of alkynes 4.1.1 
using $\mathrm{Cp}_{2} \mathrm{ZrEt}_{2}$ and chloroformate (Scheme 4.2). The zircono-esterification product 4.2.2 is then transformed into functionalized stereodefined halogenated di-arylethylenes using NCS and $\mathrm{NBS}^{212}$ as electrophiles. The obtained alkenyl halides 4.2.3 were subjected to a further crosscoupling such as Suzuki-Miyaura or Heck-Mizoroki to give the tri-aryl substituted alkenes 4.2.5 (Scheme 4.2).

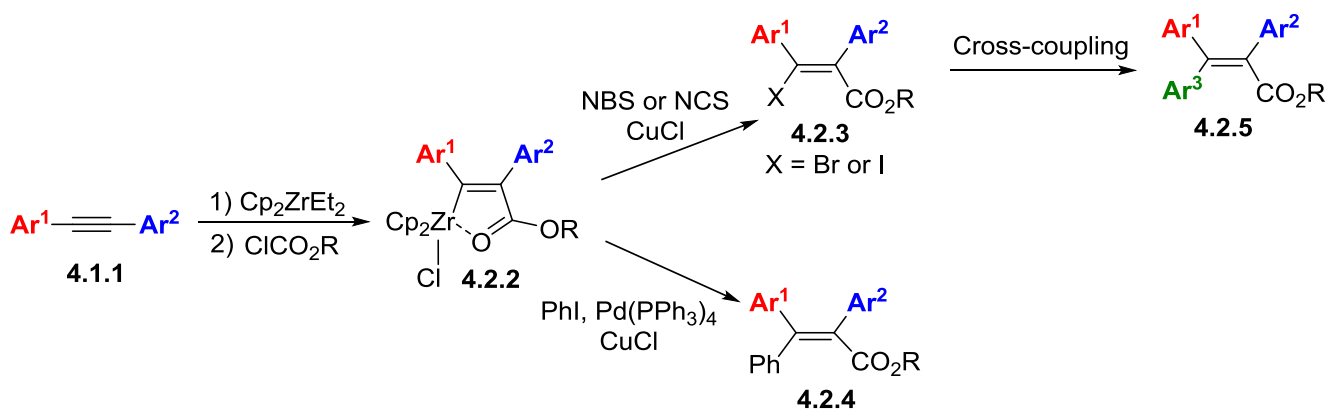

Scheme 4.2. Zirconoesterification of alkynes towards tri-arylethylene unit.

In 2004, a similar combination of carbometalation/cross-coupling (Scheme 4.3) was been reported by Yoshida et al., directly from the zirconium intermediate 4.3.2. ${ }^{213}$ It is worth noting that the use of $\mathrm{CuCl}$ is necessary for the transmetalation of alkenylzirconocene to occur and result in an organocopper substrate. ${ }^{214}$ Although the reaction conditions are mild, and the yields are modest, the final product 4.3.3 contains three identical aryl substituents that obviates stereoselectivity issues associated with the reaction when employing unsymmetrical alkynes, in which, was not explored.
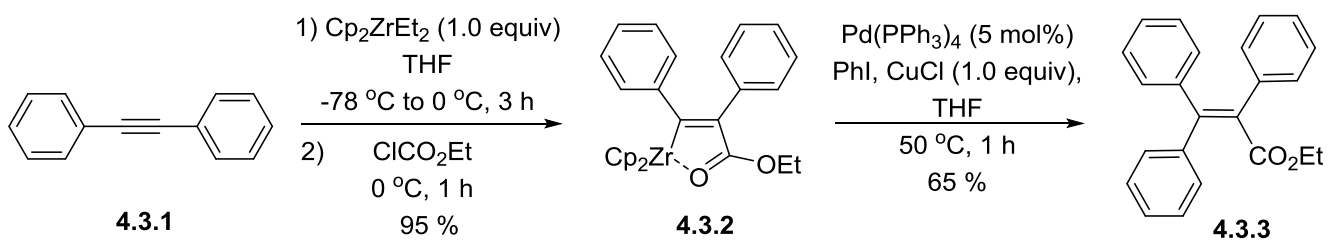

Scheme 4.3. Zirconoesterification of 1,2-diphenylacetylene 4.3.1 towards tri-arylethylene unit. 
Carbometallation of alkynes of type $\mathrm{Ar}^{1}-\mathrm{C} \equiv \mathrm{C}-\mathrm{Ar}^{2}$ with tin followed by Migita-Kosugi-Stille or $\underline{\text { Negishi Coupling }}$

Similarly, in 2010, the synthesis of tamoxifen and tamoxifen-type aryl ethylenes through stereo- and regioselective stannyllithiation of diarylacetylenes followed either by a Negishi or Stille cross-coupling were reported by Nakamura et al. (Scheme 4.4). ${ }^{215}$ The work showed high tolerance towards a wide range of aryl substituents in para-positions in spite of the electronic nature of the substituents and the substitution patterns. For instance, ortho-substituted aromatic compounds are also accommodated by the method, which is rare compared to some previously reported examples, and can thus be advantageous. More beneficial to the synthesis of tri-aryl ethylenes is the high selectivity, with the exception of two out of nine examples, observed in the fully trans-metallometallation of the starting alkyne with lithium trimethyltin to give vinyllithium intermediate up to $>99: 1$ regioselectivity in the absence of catalyst-control or directing-group assistance. However, a proposed theory for the origin of the selectivity requires further investigation by the group.

Following the synthesis of 4-hydroxytamoxifen 4.4.5, the alkyne 4.4.1 was subjected to stannyllithiation to give 4.4.2, which was immediately trapped by ethyl iodide to give 4.4.3 and 4.4.4 as an inseparable mixture of regioisomers (95:5, determined by ${ }^{1} \mathrm{H}$ NMR analysis) (Scheme 4.4). The proceeding Stille coupling with aryl iodide 4.4.3 / 4.4.4 and the removal of MOM group gave 4-hydroxytamoxifen 4.4.5 as a mixture of isomers and in good 91\% yield from the starting alkyne. Additionally, 4-hydroxytamoxifen 4.4.5 was prepared by the group in gram-scale. Alternatively, when the vinyllithium intermediate 4.4.2 was treated with zinc chloride, the corresponding organozinc compound was obtained. The following Negishi crosscoupling provided an array of tri-aryl ethylene 4.4.6 in a generally high yields between $80 \%$ and $95 \%$. Although the method is adaptable, the purity of isolated product is limited to the preceding 
regioselectivity of the carbometallation step that makes improvement on regiocontrol difficult given its unclear origin of selectivity.
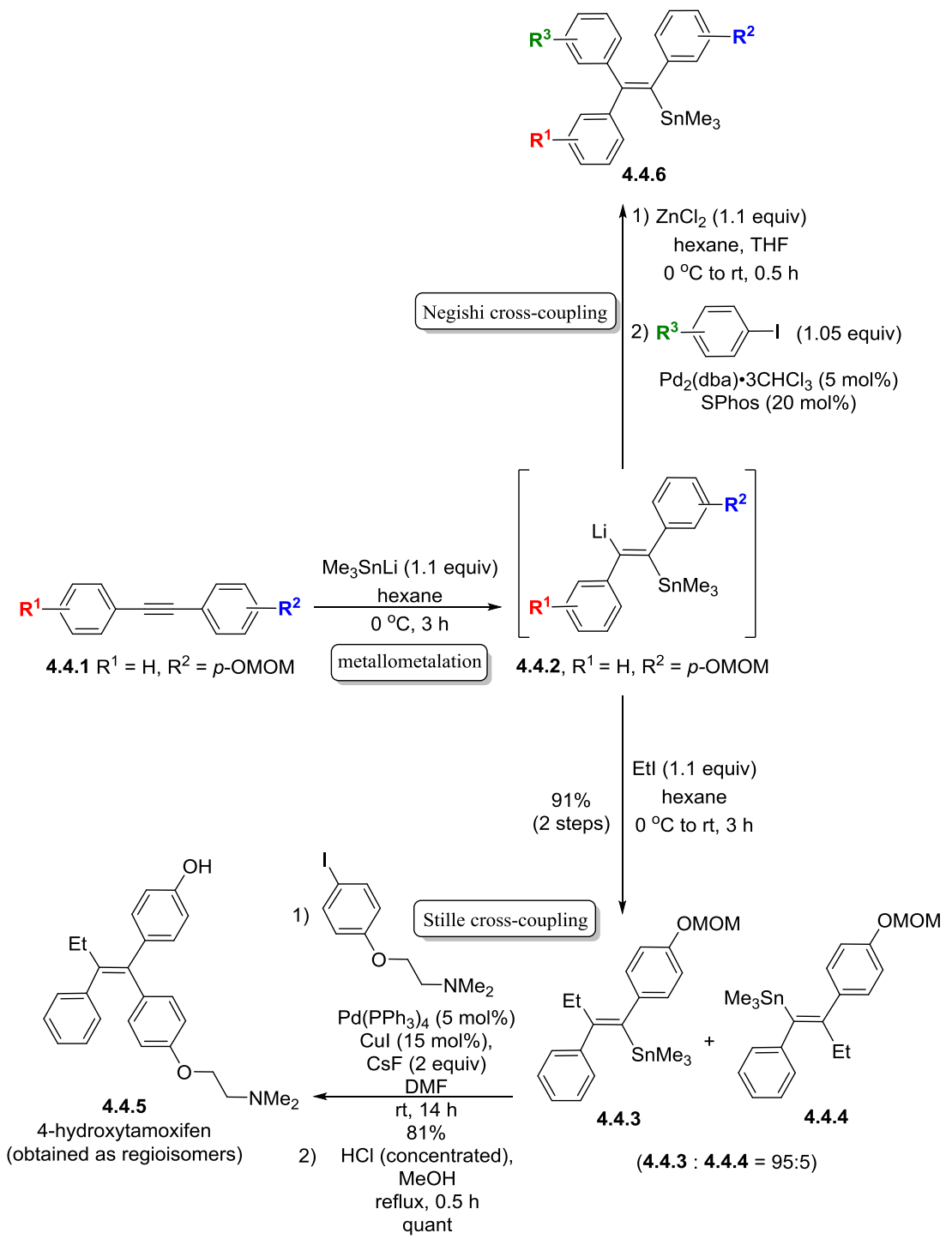

Scheme 4.4. Trans-stannyllithiation followed by Negishi or Stille cross-coupling reported by Nakamura et al.

Carbometallation of alkynes $\left(\mathrm{Ar}^{1}-\mathrm{C} \equiv \mathrm{C}-\mathrm{M}\right)$ with magnesium followed by Kumada-TamaoCorriu coupling

In 2004, Yoshida et al. reported a general synthetic scheme for the synthesis of tamoxifen-type tri-aryl ethylenes based on the $\mathrm{Cu}$-catalyzed carbomagnesation of alkynes of type 4.5.1 followed by Pd-catalyzed cross-coupling ${ }^{213}$ (Scheme 4.5). 
The stereo- and regioselectivity control in the carbometalation step were achieved by "a complex-induced proximity effect" of the pyridyl group 4.5.1 (Scheme 4.5). The proceeding Kumada-Tamao-Corriu-type ${ }^{205,216}$ coupling of the alkenylmagnesium substrate with aryl iodide was carried out in one-pot with good yields to give 4.5.2. Suzuki-Miyaura coupling was then employed after the stereoselective borodesilylation of 4.5.2 to give 4.5.3. The $\mathrm{Si} / \mathrm{B}$ exchange was carried out in the presence of $\mathrm{BCl}_{3}$ followed by a one-pot treatment with pinacol to produce 4.5.3. In the following Suzuki reaction using aryl idodide, 4.5 .4 was obtained. The method accommodates a broad selection of electronically different aryl groups, it also offers a good stereo- and regioselectivity, reaction yield and most significantly, enables the synthesis of olefins with three distinctive aryl substituents. However, its limitation is in the starting alkyne, 1-butynyldimethl(2-pyridyl)silane, where an ethyl group remains as the fourth alkene substituent in all the examples provided. (Scheme 4.5).

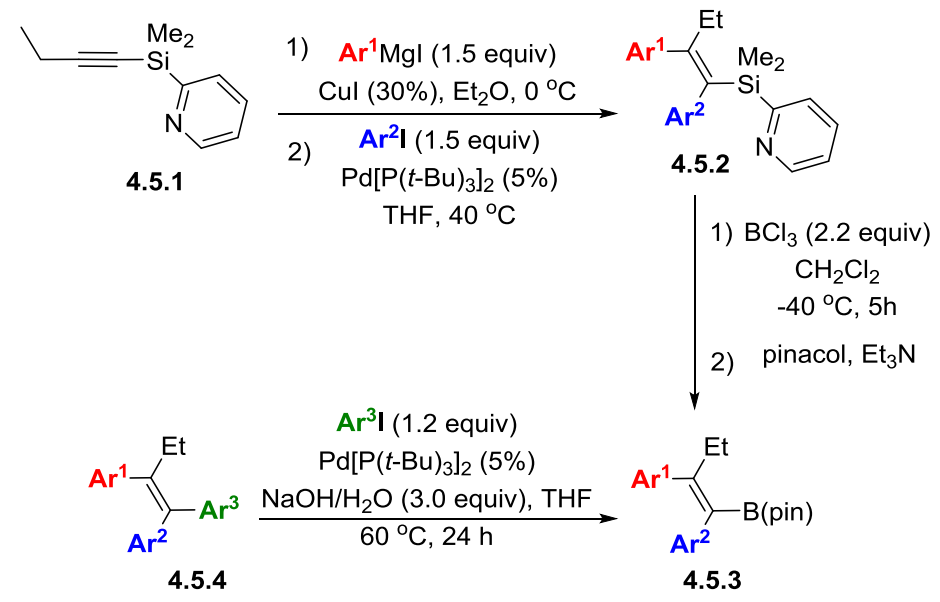

Scheme 4.5. Carbometalation/Coss-coupling combination strategy by Yoshida et al.

\subsubsection{Hydroarylation of Alkynes}

Alkenyl arenes can also be efficiently synthesized by transition-metal catalyzed alkyne hydroarylation from easily accessible arenes and alkynes (Scheme 4.6). ${ }^{217}$ Catalysts such as $\mathrm{Pd}(0), \quad \mathrm{Pd}(\mathrm{II}), \mathrm{Pt}(\mathrm{II}), \mathrm{Pt}(\mathrm{IV}), \mathrm{Au}(\mathrm{III}), \mathrm{Au}(\mathrm{I}), \mathrm{Ru}(\mathrm{II})$ can catalyze the intermolecular 
hydroarylation of alkynes. ${ }^{218}$ We have to point out that, the broad choice of aryl groups $\left(\mathbf{R}^{1}\right)$ for hydroarylation of alkynes also includes arylboronic acids (Scheme 4.6). ${ }^{219}$

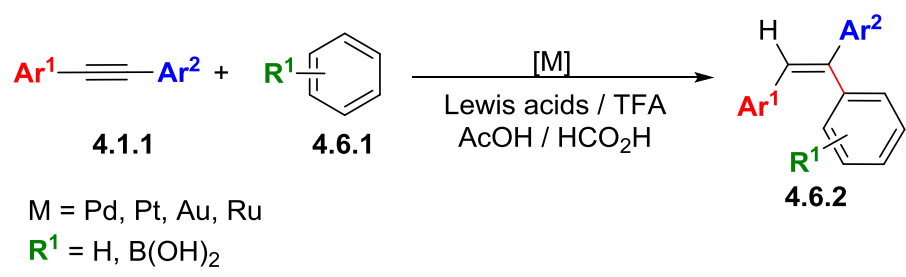

Scheme 4.6. Hydroarylation of alkynes.

\section{$\underline{\text { Hydroarylation of Alkynes of type } \mathrm{Ar}^{1}-\mathrm{C} \equiv \mathrm{C}-\mathrm{Ar}^{2} \text { : Friedel-Craft type reactions }}$}

For example, Fujiwara et al. reported the use of electrophilic $\mathrm{Pd}(\mathrm{II})$ and $\mathrm{Pt}(\mathrm{II})$ cationic complexes that are generated in situ in trifluoroacetic acid (TFA), ${ }^{218}$ at room temperature, to give tri-arylethylenes in high yields in spite of a prolonged reaction time of $48 \mathrm{~h}$ (Scheme 4.7). For instance, the reaction between diarylacetylene 4.3.1 with arene 4.7.1 in the presence of $\mathrm{PtCl}_{2}$ (5 mol\%) to give olefin 4.7.2 (Scheme 4.7). ${ }^{220}$ Furthermore, the scope of the reaction successfully included heteroaromatic substrates. ${ }^{220}$

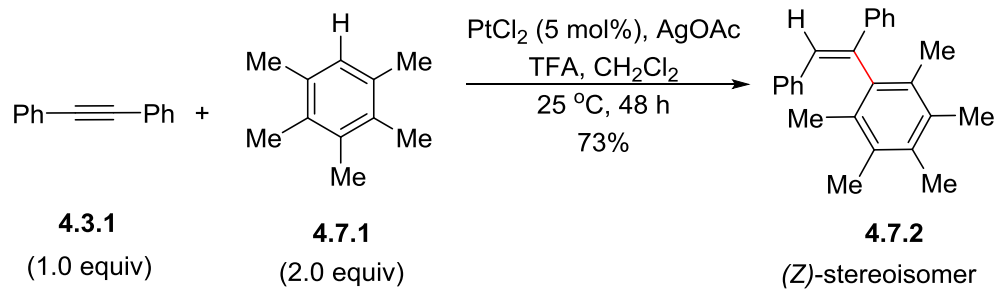

Scheme 4.7. Pt(II)-catalyzed hydroarylation of diphenylacetylene.

Similarly, in 2003, Reetz et al. reported the use of $\mathrm{Au}(\mathrm{I}) / \mathrm{Au}(\mathrm{III})$ catalytic process in the alkenylation of arenes (Scheme 4.8). ${ }^{221}$ It is noteworthy that the method is more efficient towards the construction of 1,1-disubstituted aryl olefins than the tri-substituted aryl ethylenes. However, a selected example of the [Au(III)]-catalyzed hydroarylation of diphenylacetylene 4.3.1 with mesitylene 4.8.1 shows a much reduced reaction time of $4 \mathrm{~h}$ to furnish the desired trisubstituted aryl ethylene 4.8.2 albeit with a poor yield of 5\% (Scheme 4.8). 

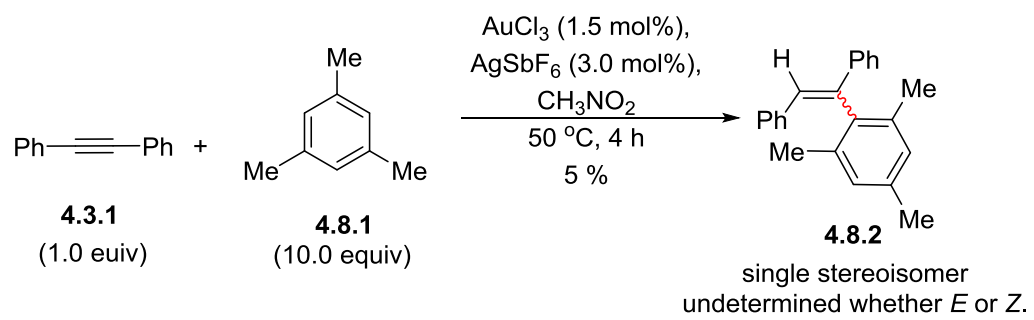

Scheme 4.8. Au(III)-catalyzed hydroarylation of diphenylacetylene.

On the other hand, the work of Murai, Chatani, Kakiuchi since 1993 documented the significant contributions of $\mathrm{Ru}(0)$ as a catalyst achieved the hydroarylation of bis-aryl acetylenes (Scheme 4.9). Moreover, in 2001, the pioneering work of Oi and Inoue et al., Ackermann et al., Bruneau et al. and Dixneuf et al. reflect the significant use and success of $\mathrm{Ru}(\mathrm{II}) .{ }^{217 \mathrm{~b}} \mathrm{~A}$ recent reviews of advances in the ruthenium-catalyzed hydroarylation of alkynes with arenes and aromatic sulfoxides ${ }^{222}$ have been published by Jeganmohan et al. ${ }^{223}$

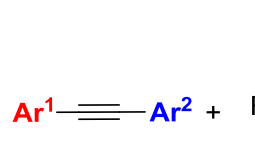

4.1.1<smiles></smiles>

4.9.1
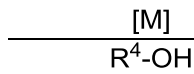

$\mathrm{R}^{4}-\mathrm{OH}$ $\mathrm{M}=\mathrm{Pd}, \mathrm{Rh}, \mathrm{Cu}, \mathrm{Co}, \mathrm{Ni}$

Scheme 4.9. Directing group assisted hydroarylation of alkynes.

Recently, Szostak et al. reported the first $\mathrm{Ru}(0)$-catalyzed aryl ketones directed hydroarylation of alkynes 4.1.1 to give multisubstituted alkenes 4.10.2 through in situ generation of $\mathrm{Ru}(0)$ catalyst (Scheme 4.10). ${ }^{224}$ The catalyst $\mathrm{Ru}(0)$ catalyst is generated in situ from the bench-stable $\mathrm{Ru}(\mathrm{II})$ precursors such as the commercially available $\left[\mathrm{Ru}(p \text {-cymene }) \mathrm{Cl}_{2}\right]_{2}$ under reductive conditions in the presence of sodium formate and phosphine ligands. The reductive conditions are allegedly "mild" but the optimized conditions revealed a $15 \mathrm{~h}$ heating at $180{ }^{\circ} \mathrm{C}$ in toluene. Moreover, 12 out of the 15 examples were from the use of symmetrical alkyne diphenylacetylenes are used and the $E / Z$-stereoselectivity varied from 1.5:1 to 6.1:1. 
Nonetheless, it should be highlighted that the directing group assisted hydroarylation of alkynes demonstrates ortho-alkenylation of aryl ketones, ${ }^{225}$ which often emerge as a synthetic challenge due to steric factors.

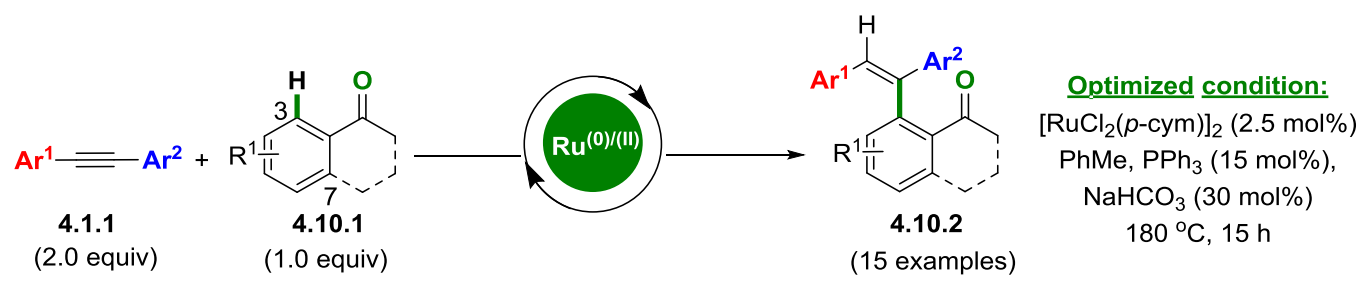

Scheme 4.10. $\mathrm{Ru}(0)$-catalyzed ketone-directed hydroarylation of alkynes.

\subsubsection{Cross-Coupling}

\subsection{Suzuki-Miyaura Cross-Coupling from Alkynes and a Sequential Two or Three Component Coupling}

\section{From Alkynes of Type R-C $\equiv C$-Ar ${ }^{1}$}

In 2003, Larock et al. exploited the versatility of palladium metal to synthesize triarylsubstituted alkenes (Scheme 4.11). ${ }^{226}$ The three component $\operatorname{Pd}(0)$-catalyzed intermolecular cross-coupling consisting of an aryl iodide 4.11.1, an internal alkyne 4.1.1 and an arylboronic acid 4.11.2 is an example of two $\mathrm{C}-\mathrm{C}$ bond formation in a one-pot reaction (Scheme 4.11). The regiochemistry is primarily governed by steric factors. ${ }^{227}$ This is evident in the cis-addition of the aryl halide to the less sterically hindered or electron-rich carbon (electronic factor) of the alkyne, as the arylboronic acid transfers its aryl group to the other remaining alkyne carbon. It is worth noting that the results obtained by the group concur with the work of Cacchi et al. ${ }^{228}$ thereby reinforcing the factors that influence the regioselectivity. 


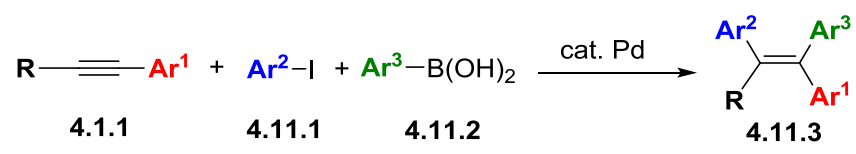

Scheme 4.11. Pd(0)-catalyzed three component intermolecular cross-coupling.

The three component coupling of 1-phenylpropyne 4.12.1, iodotoluene 4.12.2 and phenylboronic acid 4.12.3 gave the tri-arylethylene, 4.12.4 and 4.12.5, of a mixture of inseparable isomers 6.5:1 under optimized conditions (Scheme 4.12, eq 1). Although the method allows for the synthesis of multisubstituted aryl alkenes in a one-pot operation, the regioselectivity challenge remains unresolved. Consequently, the desired product 4.12.4 remains contaminated by the other regioisomeric 4.12.5 that renders this method less attractive. In addition, the reaction conditions are not considered mild as it requires prolonged heating at $100{ }^{\circ} \mathrm{C}$ (Scheme 4.12, eq 1). It is noteworthy that a mild room temperature $\mathrm{Pd}(\mathrm{II})$-catalyzed addition of two arylboronic acids 4.12.7 to internal alkynes 4.12.6 was reported by Larock et al. 2 years later (Scheme 4.12 , eq 2). ${ }^{229}$ 


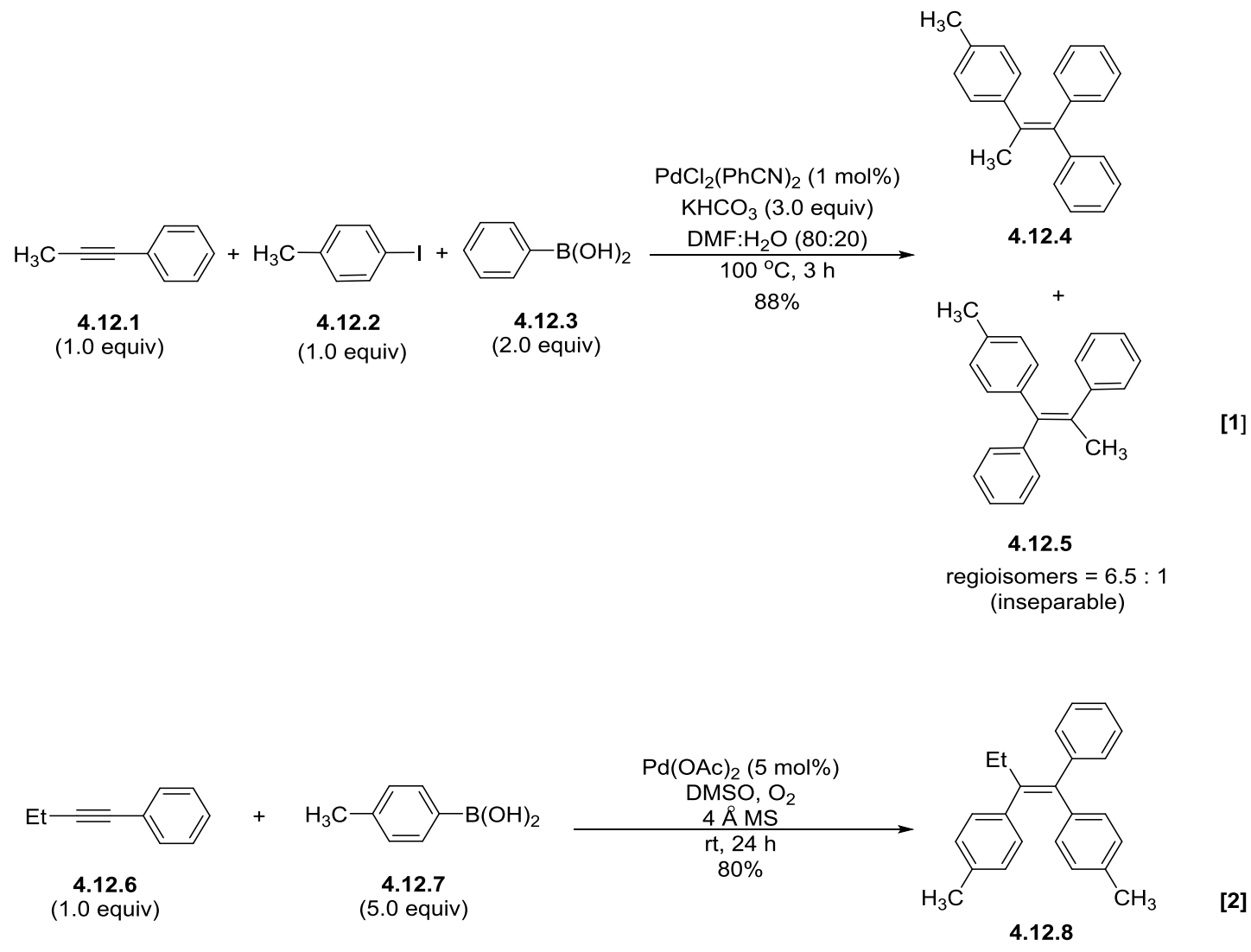

Scheme 4.12. Pd-catalyzed intermolecular cross-coupling.

\section{From Alkynes of Type $\mathrm{Ar}^{1}-\mathrm{C} \equiv \mathrm{C}-\mathrm{M}$}

Another example to illustrate the supremacy of cross-coupling reactions was reported by Nishihara et al. in 2014 (Scheme 4.13). ${ }^{230}$ The stepwise Suzuki-Miyaura cross-coupling of vic- and gem-diborylated vinylsilanes 4.13.1 and 4.13.5 alkenes, respectively, offers a regioand stereodefined synthesis towards tri-arylethylenes 4.13.4 4.13.8 (Scheme 4.13). The precursors for the cross-coupling, 4.13.2 and 4.13.6, were prepared from the stereoselective diborylation and silylborylation of simple alkynes 4.13.1 and 4.13.5 respectively.

The vicinal borylated olefin 4.13.2 was subjected to the Pd(II)-mediated key coupling with aryl iodides in the presence of excess potassium hydroxide, and the reaction proceeded with perfect discrimination between the two vicinal boryl groups to give exclusively the 
1,2-diarylated vinylsilane, (Z)-4.13.3 regioisomer (Scheme 4.13). The regioselectivity was consistent with twelve different substrates and gave moderate to good yields. The desired triarylated olefins 4.13.4 were then obtained under the conditions $\left(\mathrm{Pd}_{2} \mathrm{dba}_{3} \cdot \mathrm{CHCl}_{3}(5 \mathrm{~mol} \%)\right.$, $\mathrm{P}$-Bu $\mathrm{Bu}_{3}(20 \mathrm{~mol} \%), \mathrm{KOH}$ aq.) described in the second Suzuki coupling. The origin of the regioselectivity is attributed to the $\alpha$-effect of the silicon group (electronic effect) ${ }^{230}$ as opposed to the steric effect. ${ }^{231}$

Apart from the high regioselectivity, another advantage in using the method is that the electronic nature of the aromatic of aryl iodides does not have any influence on the stereoselectivity as the method tolerates a variety of electron rich and electron deficient (including $-\mathrm{NO}_{2}$, and -CN groups) $\mathrm{Ar}^{2}-\mathrm{I}$ (Scheme 4.13). More importantly, the method enables access to the desired structural isomers of tri-substituted olefins by the introduction of appropriate aryl groups in the correct order of the sequential Suzuki cross-coupling. Furthermore, the $\mathrm{C}-\mathrm{Si}$ bond present in the final product $\mathbf{4 . 1 3 . 4}$ could be exploited in further functionalization through Hiyama cross-coupling to provide complex $\pi$-conjugated substrates. However, the effects of ortho- and meta-substituted aryl iodides were not investigated. Therefore further studies on the influences of aromatic substitution patterns should be conducted, which could qualify the protocol as a reliable general and practical synthetic scheme for the synthesis of multiarylated olefins.

In contrast, under identical conditions used for the cross-coupling of vicinal diborylated olefins 4.13.2, the geminal diborylated olefins 4.13.6 did not perform as well (Scheme 4.13). A mixture of $(E / Z)$-4.13.7 stereoisomers were obtained in the Suzuki cross-coupling of 4.13.6 (Scheme 4.13). The major (Z)-4.13.7 stereoisomer was identified by X-ray crystallography analysis. Subsequently, the tri-arylated ethylene 4.13.8 was furnished through another Suzuki cross-coupling of olefin $(Z)$-4.13. The origin of the observed stereoselectivity of $(E / Z)-\mathbf{4 . 1 3 . 7}$ remains unclear currently, and it demands further studies for clarifications. 
A similar approach employing gem-diborylalkenes was reported by Hiyama et al. ${ }^{232,233}$ in 2005 and Sawamura et al. in 2015. ${ }^{234}$ Apart from gem-diborylakenes, gem-dibromoalkenes was also employed as reported by McCombie et al., ${ }^{235}$ in 2001.
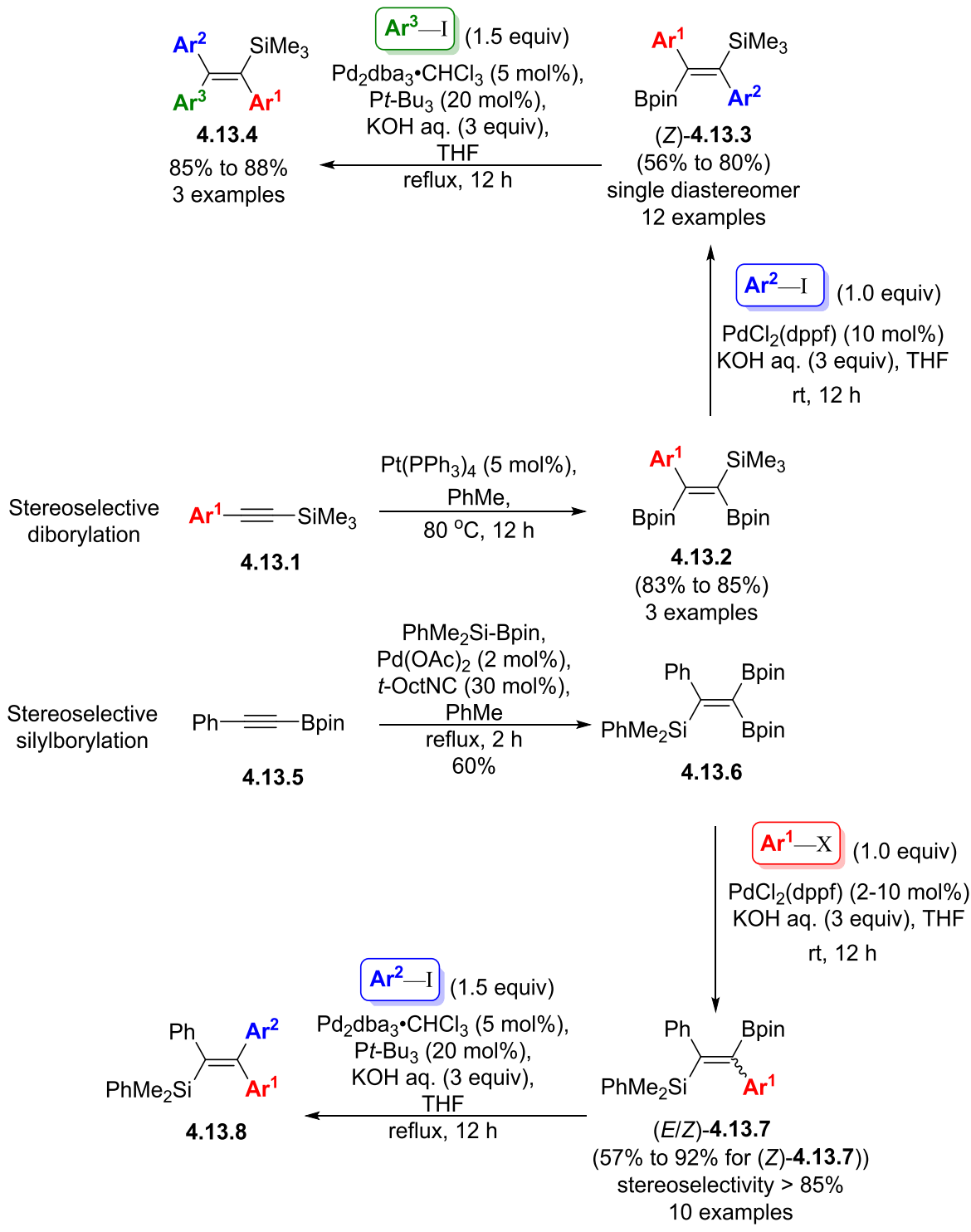

Scheme 4.13. Sequential Suzuki cross-coupling of gem- and vic-diborylalkenes 4.13.2 and 4.13.6.

\section{From Alkynes of Type $A r^{1}-C \equiv C-H$}

In 2015, Torbman et al. reported the use of gem-dibromoalkenes 4.14.2 in the sequential Suzuki cross-coupling functionalization of 1,1-dibromoenol phosphate 4.14.2 (Scheme 4.14). ${ }^{236}$ 
For instance, the Suzuki reaction of enol phosphate 4.14.2 with electron-poor arylboronic acid 4.14.3 showed a selective arylation to give the single $(Z)-4.14 .4$ isomer in moderate yield. This was followed by a second Suzuki reaction with an electron-rich arylboronic acid 4.14.5 to give the $(E)$-4.14.6. The tolerance of both electron-rich and electron-deficient aromatics reflect the versatility of the method (Scheme 4.14). Although the starting material, functionalized 1,1-dibromoenol phosphate, is not commercially available and requires two preparation steps from alkyne 4.14.1, the synthesis is efficient and provided 4.14.2 in high $93 \%$ yield. ${ }^{237}$ Alternatively, enolphosphate 4.14.2 can be prepared from dibromoketone as described by the group. $^{237}$

The method demonstrated full chemo- and stereocontrol that is advantageous in the synthesis towards unsymmetrical arylated olefins. However, only six examples were reported. More examples to widen the scope of the reaction could aid in the claim of this method as a general strategy towards a total chemo- and stereoselective sequential Suzuki cross-coupling using 4.14.2 to access tri-arylethylenes.

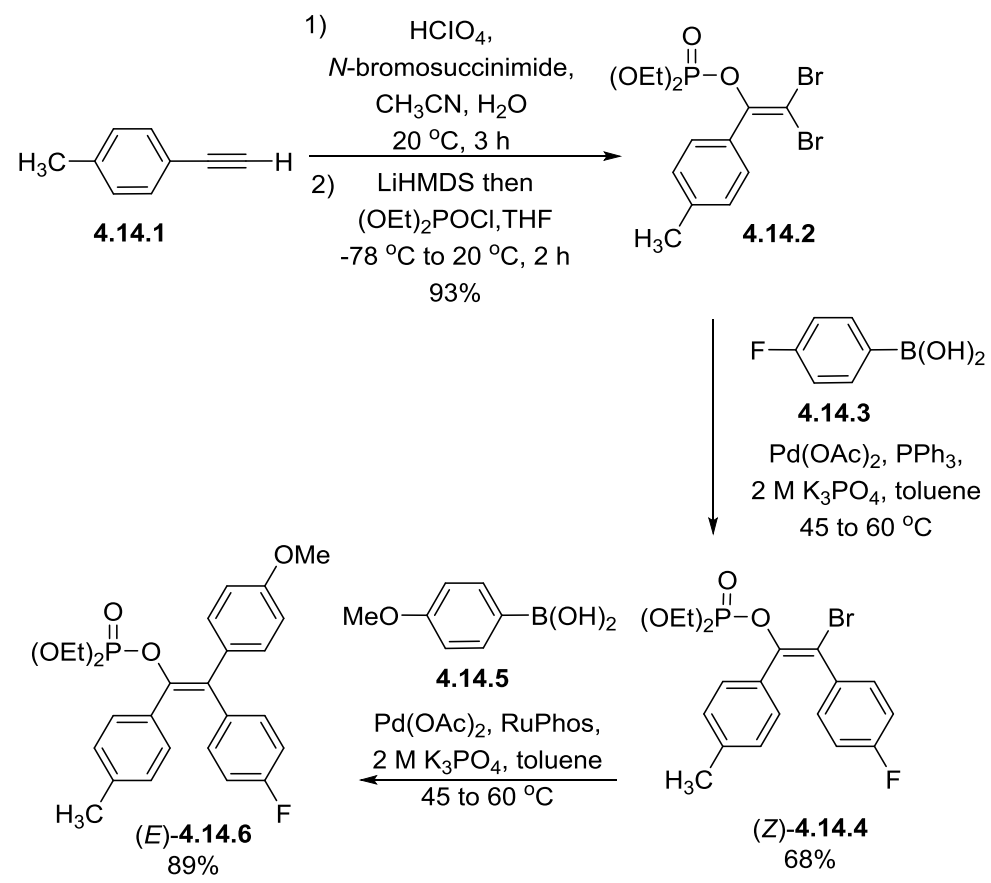

Scheme 4.14. Suzuki-Miyaura cross-coupling functionalization of enolphosphate dibromide 4.14.2. 


\section{$\underline{\text { Other Cross-Coupling Reactions }}$}

Apart from palladium, other metals such as nickel and copper have been employed in the metal-catalyzed cross-coupling to access tri-substituted aryl ethylenes such as the recently published work of Qiu et al., ${ }^{238}$ and Cahiez et al. ${ }^{239}$

\subsection{Mizoroki-Heck Cross-Coupling from Alkenes and a Sequential Two Component Coupling}

The constant search for new and improved Heck catalytic systems to achieve an enhanced catalytic activities has led to the use of a variety of ligands, commercially available or engineered, and development of better catalysts. ${ }^{240}$

\section{Heck Vinylation of Aryl Halides Using PCP-ligands}

In 2000, Jensen et al. reported the use of palladium phosphinito PCP pincer complex for the Heck vinylation of aryl halides to construct a highly efficient and regioselective tri-aryl ethylenes (Scheme 4.15). ${ }^{241}$ This is an expansion of the work developed by Milstein et $^{2 .{ }^{242}}$ and Bedford et al. ${ }^{243}$ in the late 1990s. The PCP ligand precursor was prepared in a straightforward manner from resorcinol 4.15.1 and chlorodiisopropylphosphine 4.15.2 with DMAP. A $5 \mathrm{~h}$ reflux of the precursor with $\mathrm{Pd}(\mathrm{COD}) \mathrm{Cl}_{2}$ afforded the palladium complex 4.15.3 that was then employed in the akenylation of aryl bromides and iodides (Scheme 4.15).

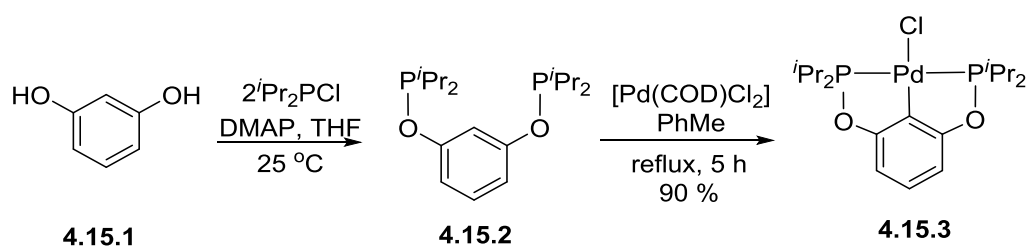

Scheme 4.15. Preparation of phosphinito PCP pincer palladium complex 4.15.3. 
The Heck arylation of styrene 4.16.1 with aryl halides 4.16.2 / 4.16.3 in the presence of 4.15.3 in DMF exclusively gave the one-pot double arylation product in excellent yield between 97-99\% (Scheme 4.16). The low catalytic loading of $0.0007 \mathrm{~mol} \%$ reflects the high turnover number, and hence efficiency of the catalytic system with the use of the phosphinito PCP pincer palladium complex (Scheme 4.16). Moreover, the high turnover number was not restricted to the type of aryl halide employed $(\mathrm{X}=\mathrm{Br}$, I). In addition, the palladium PCP pincer complex is stable to heat as it can withstand over $200{ }^{\circ} \mathrm{C}$ of heating in DMF for $36 \mathrm{~h}$ in air. However, we have to point out that this technique reflects a narrow scope due to the lack of examples provided. In addition, the reaction limitation in terms of stereoselectivity and regioselectivity were not explored. This is as the aryl source employed in the double Heck arylation was not varied to give tri-arylethylenes with different aryl groups.

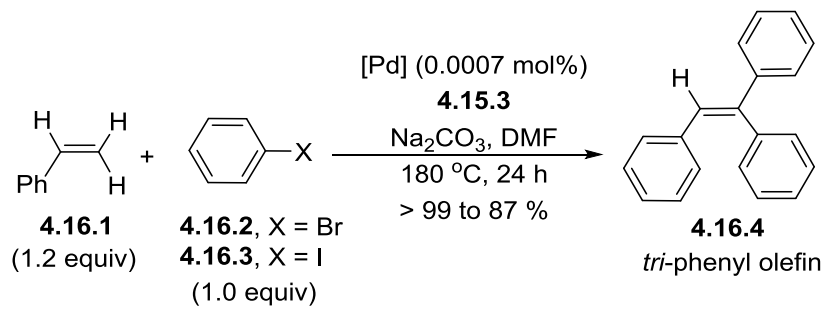

Scheme 4.16. Diarylation of styrene 4.16.1.

\section{Heck Arylation Using Complex-Ligands}

In 2014, Shang et al., reported the use of 1,3-bis-(5-ferrocenylisoxazoline-3-yl)benzene 4.17.2 in a palladium-catalyzed Heck reaction in water as the solvent (Scheme 4.17). For instance, the Heck reaction between stilbene 4.17.1 and 4-iodotoluene 4.12.2 to give (E)-4.17.3 (Scheme 4.17). The ligand 4.17.2 can be synthesized in four steps and is thermally stable. The method features a low catalyst loading of $0.2 \mathrm{~mol} \%$, the use of an environmentally friendly and economical solvent like water, and a stereocontrolled access to $(E)-\mathbf{4 . 1 7 . 3}$ from a 1,2disubstituted olefin starting material 4.17.1, and is carried out in air and under $6 \mathrm{~h}$. Nonetheless, the regioselectivity issue was not addressed with the use of stilbene 4.17.1. Additionally, there 
was no mention on the recyclability of ligand 4.17.2 that may render the approach as non-atom economical since the same coupling product can be obtained under ligand-free conditions.

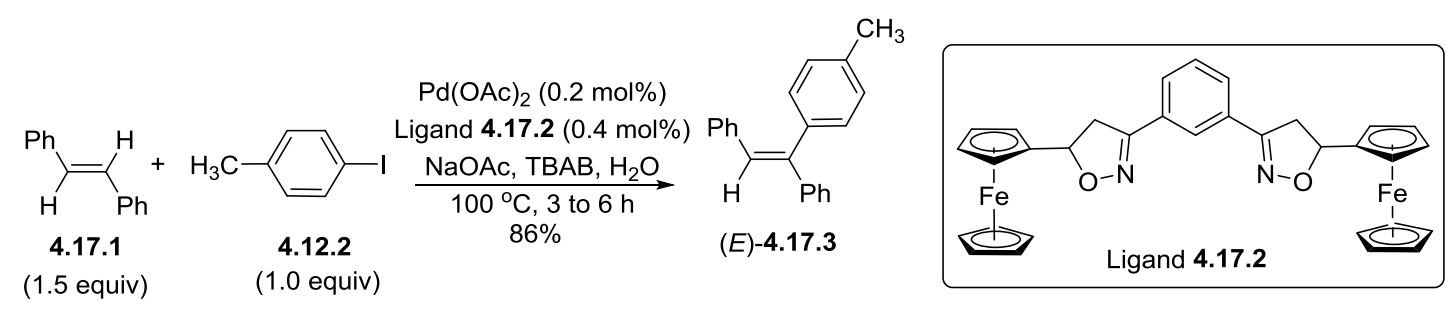

Scheme 4.17. Diarylation of 1,2-diphenylacetylene using ligand 4.17.2.

Similarly, the benzimidazolium-palladium-NaOAc system developed by Tang et al. utilized the in-situ generation of an electron-rich benzimidazolylidene 4.18.1, which acts as a ligand support to the palladium during the Heck vinylation of aryl halides (Scheme 4.18). For instance, the arylation of styrene 4.16.1 with aryl bromide 4.16.2 in the presence of Pd-4.18.1 (0.2 mol\%) to give 4.16.4 (Scheme 4.18). Although the method was successful to construct triarylethylene unit 4.16.4, this strategy is insufficient to be regarded as a general platform towards the construction of tri-substituted aryl ethylenes. The recurring challenges of regio- and stereocontrol in the construction of unsymmetrical alkenes remain unexplored in the method.
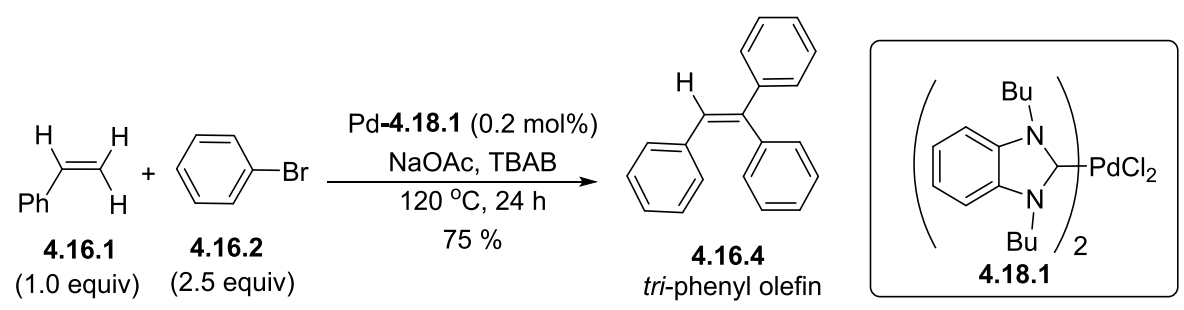

Scheme 4.18. Diarylation of styrene using ligand Pd-complex 4.18.1.

On the other hand, the work of Santelli et al. in 2003, provided more examples of Heck arylation of stilbene and 1,1-diphenylethylene to give tri-arylethylenes. ${ }^{244}$ This was done by using an efficient catalyst system capable of producing a highly unprecedented level of catalytic turnover number of as high as 28000000 which is more than a PCP pincer-type palladium 
complex. Santelli et al. reported the use of a tetrapodal phosphane ligand cis,cis,cis-1,2,3,4 tetrakis(dphenylphosphanylmethyl)-cyclopentane (Tedicyp) (Figure 4.4).

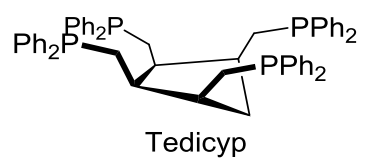

Figure 4.3. Structure of Tedicyp.

The Heck arylation of stilbene or 1,1-diphenylethylene with aryl halides $(\mathrm{X}=\mathrm{Br}, \mathrm{I})$ requires a low catalyst loading of between $0.2-1 \mathrm{~mol} \%$ and heating at $130{ }^{\circ} \mathrm{C}$ in DMF for $20 \mathrm{~h}$ (Scheme 4.19). The Heck product 4.16.4 was obtained from the reaction between 4.17.1 with iodobenzene 4.16.3 in good 78\% yield. Similarly, the reaction between stilbene 4.17.1 with 4bromoanisole 4.19.3 afforded 4.19.6 in 75\% yield. This reflects flexibility of the method by high tolerance of different aryl halides. In addition, the method included the use of the less common internal alkene 4.17.1 as Heck substrate, ${ }^{203,245}$ however, only two examples were provided.

The method also accommodates the use of both electron rich, 4.19.3, and electron poor aryl halides, 4.19.2, 4.19.4 and 4.19.5 (Scheme 4.19). Additionally, the use of meta-substituted aryl bromide 4.19.2 was successfully employed to give 4.19.8 in good 69\% yield. However, the use of ortho-substituted aryl halogen was not evaluated. Also, in spite of good compatibility of the method with aryl bromides, the use of 4-bromoanisole 4.19.3 gave a low stereoselectivity of 4.19.6 $(E / Z=61: 39)$. Although the major product was reported as $(E)-4.19 .6$, the technique by which the major and minor stereoisomers were determined was not reported. 


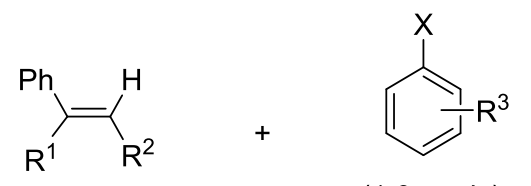

(1.3 equiv)
(1.0 equiv)

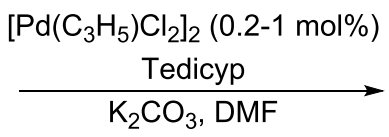

$130^{\circ} \mathrm{C}, 20 \mathrm{~h}$

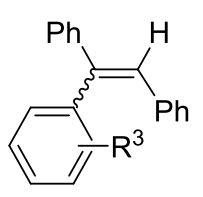

4.16.4, $78 \%, \mathrm{R}^{3}=\mathrm{H}$ from 4.17 .1

(E)-4.19.6, 75\%, E/Z = 61:39, $\mathrm{R}^{3}=p$-OMe

or

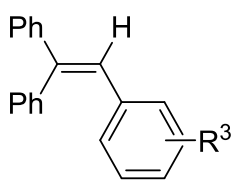

4.19.7, $\quad 62 \%, \mathrm{R}^{3}=p-\mathrm{OMe}$

4.19.8, $69 \%, \mathrm{R}^{3}=m-\mathrm{CF}_{3}$

4.19.9, $51 \%, \mathrm{R}^{3}=p-\mathrm{CF}_{3}$

4.19.10, 70\%, $\mathrm{R}^{3}=p-\mathrm{F}$

4.16.4, $63 \%, R^{3}=H$ from 4.19 .1

Scheme 4.19. Heck vinylation of unsymmetrical alkenes.

The use of unhindered terminal or monosubstituted alkenes are common for the Heck reaction, ${ }^{246}$ thereby increasing the value of methods using sterically demanding Heck substrate like the 1,2-disubstituted alkenes. Another group that employed the use of internal alkenes in the Heck reaction is Rafiee et al. in $2009{ }^{247}$ where a 1,2-diphenylethylene and a triphenylsubstituted alkene were used in the vinylation of bromobenzene catalyzed by the palladacycle 4.20.2 (Scheme 4.20). The single step access to phosphopalladacycle 4.20.2 is carried out by combining 2-diphenylphosphino-2'-methyl-biphenyl 4.20.1 with palladium(II) acetate. ${ }^{248}$ The palladacycle 4.20.2 is efficacious at promoting the Heck coupling reactions. ${ }^{247}$

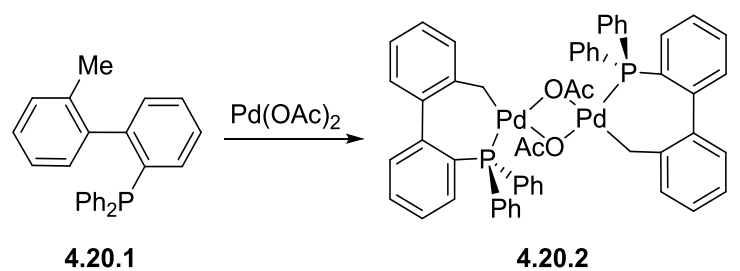

Scheme 4.20. Preparation of phosphopalladacycle 4.20.2. 
Although the Heck arylation of $d i$ - and tri-substituted phenyl olefins with bromobenzene 4.16.2 was successful, conversion of the starting materials and the total yield are less than ideal (Scheme 4.21). The 65\% yield of the coupling between ( $Z$ )-stilbene 4.21.1 and bromobenzene 4.16.2 is better than the $40 \%$ yield for the tri-substituted aryl ethylene 4.16.4 despite heating at $130{ }^{\circ} \mathrm{C}$ for $24 \mathrm{~h}$ (Scheme 4.21). It has been proposed that multiple substituted olefins are reluctant to undergo the Heck migratory insertion step, ${ }^{249}$ therefore, the conversion of $d i$ - and tri-substituted olefins into Heck products is low.

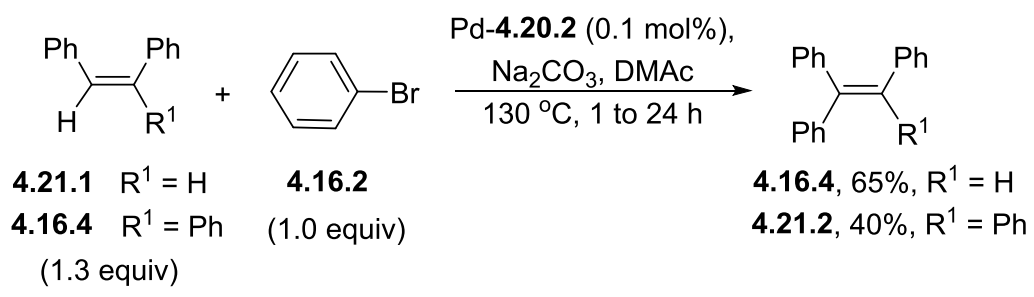

Scheme 4.21. Heck cross-coupling with Pd-4.20.2 catalytic system.

In 2013, Shi et al. reported the controlled double-Heck arylation of styrene 4.16.1 catalyzed by $d i$-NHC dipalladium complex 4.4 .5 (Figure 4.4) in a one-pot reaction to achieve unsymmetrical arylated olefins (Scheme 4.22). The use of a precatalyst 4.4.5 under controlled reaction temperatures enable a phosphine-free and sequential one-pot Heck arylation. Although the catalytic efficiency of $d i$-NHC dipalladium complex 4.4.5 is reflected through a low catalytic loading of $1 \mathrm{~mol} \%$, the catalytic system remains limited to the use of aryl bromides and aryl iodides.

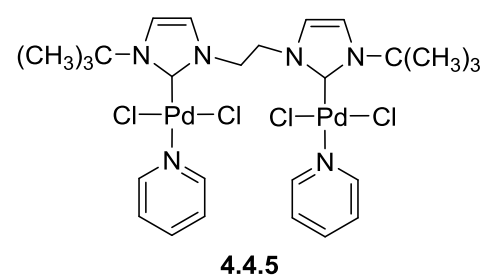

Figure 4.4. $d i$-NHC dipalladium complex 4.5.2. 
The first arylation of styrene 4.16.1 was carried out with bromobenze 4.16.2 to give the monoarylated product exclusively, which was followed by a second arylation with a range of aryl halides 4.16.2, 4.19.3, 4.22.1 $-\mathbf{4 . 2 2 . 3}$ in a sequential manner (Scheme 4.22). It is noteworthy that a mixture of mono- and diarylated styrene was not obtained. Furthermore, the method tolerates both para-substituted electron-rich and electron-poor aryl groups since tri-aryl olefins 4.19.4 and 4.22.5 were obtained with a consistently high $89 \%$ and $85 \%$ yield respectively. The stereoselectivity of the reaction was determined by GCMS and was reported to be high for tri-arylethylenes 4.22.4, 4.19.4 and 4.22.5 but declined in the case of 4.22.6 $(E / Z=63: 37)$. Although the method reflects some stereocontrol and high yield, the major isomer ( $E$ or $Z$ ) was not identified through X-ray or NOESY experiments, also, the employed reaction conditions are harsh. For instance, the prolonged conventional heating at $150{ }^{\circ} \mathrm{C}$ over $15 \mathrm{~h}$ could be exchanged for microwave-assisted heating to reduce the reaction time, and mitigate the forcing conditions. The narrow scope comprising of only para-substituted aryl groups would prevent the method from a general application towards tri-arylated olefins. In addition, the influence of the phenyl ring substitution pattern on the outcome of the Heck cross-coupling was not investigated. Therefore, the scope should be broadened with more examples of unsymmetrical tri-aryl ethylenes.

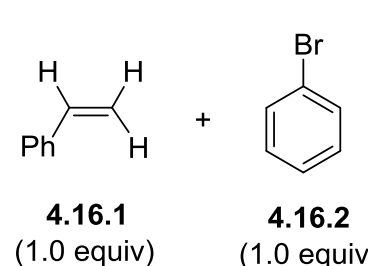

(1.0 equiv) $\quad$ (1.0 equiv)
1) $[\mathrm{Pd}]-4.4 .5(1 \mathrm{~mol} \%)$, TBAB, NaOAc, DMAC
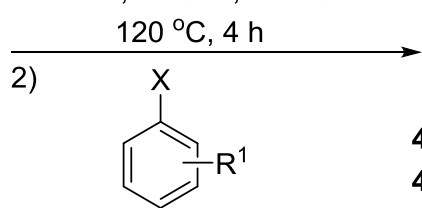

(1.0 equiv)

$150{ }^{\circ} \mathrm{C}, 15 \mathrm{~h}$

4.16.2 $\mathrm{R}^{1}=\mathrm{H}, \mathrm{X}=\mathrm{Br}$

4.19.3 $\mathrm{R}^{1}=p$-OMe, $\mathrm{X}=\mathrm{Br}$

4.22.1 $\mathrm{R}^{1}=p-\mathrm{CH}_{3}, \mathrm{X}=\mathrm{Br}$

4.22.2 $\mathrm{R}^{1}=p-\mathrm{F}, \mathrm{X}=\mathrm{I}$

4.22.3 $\mathrm{R}^{1}=p-\mathrm{Cl}, \mathrm{X}=$ I

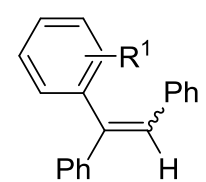

4.16.4, $87 \%, \mathrm{R}^{1}=\mathrm{H}$

4.19.4, 89\%, $E / Z=83: 17, \mathrm{R}^{1}=p-\mathrm{OMe}$

4.22.4, 79\%, $E / Z=80: 20, \mathrm{R}^{1}=p-\mathrm{CH}_{3}$

4.22.5, 85\%, $E / Z=90: 10, \mathrm{R}^{1}=p-\mathrm{F}$

4.22.6, $81 \%, E / Z=63: 37, \mathrm{R}^{1}=p-\mathrm{Cl}$

Scheme 4.22. Heck cross-coupling with a dipalladium complex $\mathbf{4 . 4 . 5}$ catalytic system. 


\section{Heck Reaction without Complex Ligands}

In 2004, Yoshida et al., reported a one-pot sequential arylation of vinyl metals to access the extended $\pi$-system of tri-arylethylenes (Scheme 4.23). ${ }^{185}$ Since arylation of a vinyl metal can be exploited through a $\mathrm{C}-\mathrm{H}$ Heck reaction or a $\mathrm{C}-\mathrm{M}$ Suzuki-Miyaura cross-coupling and the two reactions are orthogonal, hence by principle, it is possible under redefined conditions to carry out both reactions sequentially in a one-pot reaction. The challenge was in controlling the two different cross-coupling reactions so that one may begin after the other ends instead of both occurring simultaneously. This is done by the arylation of 1.0 equivalent of the starting material 4.23.1 in the presence of excess aryl halides. Double arylation product, tri-substituted alkene 4.23.2, is no longer a Heck candidate due to the sterically congested double bonds (Scheme 4.23). Therefore, the second Suzuki-Miyaura pathway can be established by exploiting the $\mathrm{C}-\mathrm{B}$ bond of the diarylation product to give the desired olefin. The use of aryl bromides followed by the more reactive aryl halides, the problem of Suzuki-Miyaura cross-coupling with the remaining aryl halide from the first step can be prevented. Moreover, the successful incorporation of heterocycles in the second stage by the Suzuki-Miyaura cross-coupling proves as an added advantage.

The elegant idea allows for a rapid construction of multisubstituted aryl bonds that can be used to design conjugated dendrimers as reported by Yoshida et al. two years later (Scheme 4.23) ${ }^{181}$ Although the idea is elegant, an obvious limitation is in the double Heck arylation to furnish the $\beta, \beta$-disubstituted alkene 4.23.2 intermediate bearing the same aryl groups.

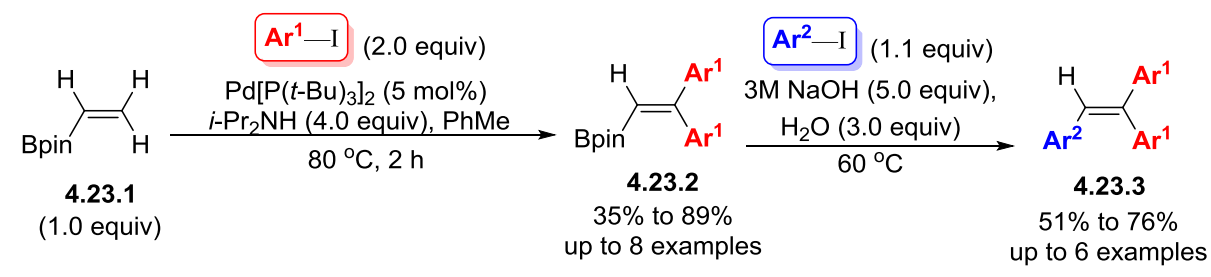

Scheme 4.23. One-pot diarylation towards tri-arylethylenes 4.23.3. 
In 2012, a similar elegance was demonstrated by the work of Chen et al. in the synthesis of unsymmetrical tri-arylethylenes in one pot under ligand-free Heck diarylation between styrene 4.16.1 and aryl iodide (Scheme 4.24). ${ }^{246 \mathrm{~b}}$ Styrene 4.16.1 was arylated in the excess of iodobenzene 4.16.3 in the presence of $\mathrm{Pd}(\mathrm{OAc})_{2}$ and $\mathrm{AgOAc}$ in acetic acid at $110{ }^{\circ} \mathrm{C}$ for $10 \mathrm{~min}$ followed by the addition of a second aryl iodide (Scheme 4.24). The method is flexible because it accommodates both electron rich and poor phenyl groups. It also tolerates ortho-substituted aryl iodide however only one experiment was conducted to ascertain this. The yield for the second arylation is reportedly higher if performed on the isolated $(E)$-stilbene. The stereoselectivity control of the final $(E)$-arylated 4.24.1 remains moderate to good but not excellent. This is as the stereoselectivity ratio of more than 50:1 (E/Z>50:1) was not achieved in any case. Nevertheless, the stereoisomers are separable and this coupled with the short reaction time makes for an attractive methodology towards the rapid access of tri-arylethylene unit.

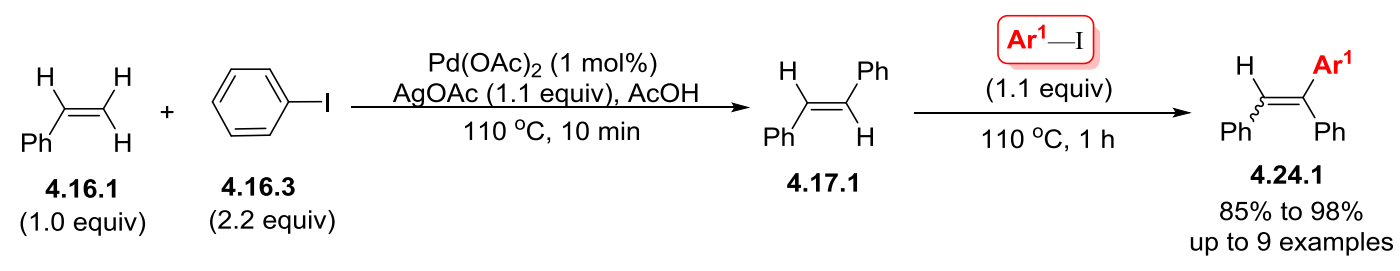

Scheme 4.24. Access to unsymmetrical tri-arylethylenes in one pot under ligand-free Heck diarylation.

\section{Other Heck Catalytic System}

Thus far, the synthetic methods reviewed regarding the Heck cross-coupling reaction towards the desired tri-arylethylenes have revealed a heavy reliance on the use of a homogenous palladium catalyst. Other metals such as indium trichloride can also be employed as shown by Liang et al. in 2013 through indium(III)-catalyzed Heck under ligand free conditions. ${ }^{250}$ Besides that, a different phase catalytic system can also be employed towards the synthesis of multisubstituted aryl ethylenes. ${ }^{251}$ This includes the biopolymer wool-Pd complex reported by 
Lei et al. ${ }^{252}$ in 2011 and the recyclable solid-supported palladium(0) microparticle developed by Das et al. in 2012. ${ }^{253}$ In addition to palladium microparticles, Heck reactions are also conducted using palladium nanoparticles in ionic liquids..$^{254}$

\subsubsection{Conclusion}

Overall, the existing metal-catalyzed reactions towards tri-arylethylenes have exposed issues associated with regio-, chemo- and stereoselectivity, which led to purification problems. Furthermore, it is accompanied by the need to adopt forcing conditions including prolonged heating at high temperatures. Also, the instability of alkenyl metal reagents leads to difficult preparation, while its potential toxicity, ${ }^{255}$ prevents the existing reactions from emerging as a universal application to access stereoselective construction of tri-arylethylenes. More importantly, the review reflects on the tedious ability to attach three distinctive aromatic groups around the $\mathrm{C}=\mathrm{C}$ double bond. Although some were successfully rectified by optimization of reaction conditions, other issues like stereoselectivity were not as successfully resolved. Consequently, the synthesis of tri-substituted olefins bearing distinct aryl groups remain a

challenge. Amongst the many methods, the transition-metal catalyzed $s p^{2}-s p^{2}$ cross-coupling reactions in particular have emerged as the most promising because of their ability to introduce stereocontrol and a wide tolerance towards a range of functional groups. However, low or no reactivity of some of the discussed catalytic system towards aryl chlorides as the coupling partner remains unaddressed. Nonetheless, the Heck-Mizoroki reaction was elected as the synthetic strategy to access tri-substituted aryl ethylenes unit in a stereospecific manner. 
This page has been intentionally left blank. 
This page has been intentionally left blank. 


\section{Chapter 4}

\subsection{Objective of Present Work}

4.2.1 The Advantages of MIDA Boronate Ester

4.2.2 Heck Reaction Using Vinyl MIDA Boronate

4.2.3 Results and Discussion

4.2.4 Perspectives 
This page has been intentionally left blank. 


\subsection{Objective of Present Work}

There is no report of the use of $N$-methyliminoacetic acid (MIDA) boronate (Figure 4.5) in the sequential Heck and Suzuki cross-coupling strategy to achieve tri-arylethylenes from 1,2disubstituted olefins thus far.

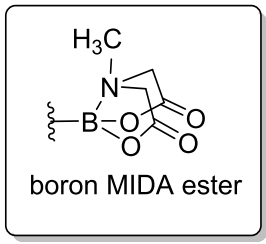

Figure 4.5. $N$-methyliminoacetic acid esters of boronic acids (MIDA boronate).

Therefore, the objective of the present work was to develop an approach towards these arylated olefins 4.25 .2 by applying the Heck coupling conditions on phenyl vinyl MIDA boronate ester 4.25.1 with a variety of iodo-aryl substituents 4.25.2 (Scheme 4.25).

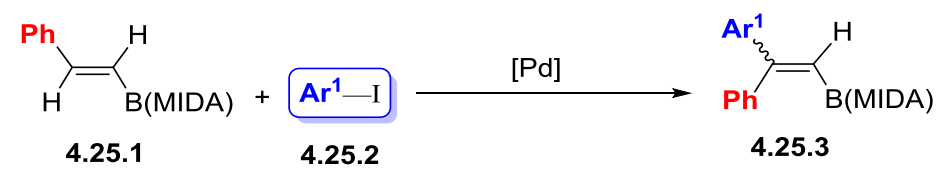

Scheme 4.25. Heck reaction between 1,2-phenyl vinyl MIDA boronate ester 4.25.1 and iodo-aryls 4.25.2.

\subsubsection{The Advantages of MIDA Boronate}

Some of the limitations when employing boronic acid functional group are issues associated with purification such as the incompatibility of trifluoroborates with silica gel column chromatography. ${ }^{256}$ Other problems include the handling of sensitive substrates that are prone to decomposition, narrow functional group tolerance, and difficulty in the removal of bulky boronic esters. ${ }^{255}$ These issues have been rectified by Burke et al. ${ }^{257}$ by using $N$-methyliminoacetic acid (MIDA) boronates. The N-coordinated MIDA boronates feature benchstability due to the absence of a reactive p-orbital on the boron, ${ }^{256}$ compatibility with silica gel 
chromatography, convenient handling in its crystalline form, inert towards a variety of common synthetic reagents ${ }^{258}$ yet susceptible to an easy and mild cleavage. ${ }^{259}$

The boronic MIDA esters have thus emerged as an increasingly general platform for the construction of small-molecules based on building blocks. ${ }^{259 \mathrm{k}}$ The involvement of boron MIDA esters in the combination of Heck and Suzuki cross-coupling strategies to access multiarylated olefins is underdeveloped compared to cross-coupling reactions employing other boronic acids such as pinacol boronic acids. Therefore, the utility of boronate MIDA ester should be broadened to further uncover the potential of the boronate.

\subsubsection{Heck Reaction Using Vinyl-MIDA Boronate}

The Heck reaction between iodobenzene 4.16.3 and vinylboronic MIDA ester 4.26.1 was carried out under reaction conditions that were developed in the laboratory ${ }^{259 \mathrm{j}}$ to give the expected olefin 4.25.1 as the minor product, the unexpected major olefin 4.26.2, and the starting material as an inseparable mixture (Scheme 4.26). The major olefin 4.26.2 appears to be the result from a second Heck reaction of the initially desired product 4.25.1 with iodobenzene 4.16.3. Therefore, we wanted to take advantage of this results and synthesize tri-aryl substituted olefins.

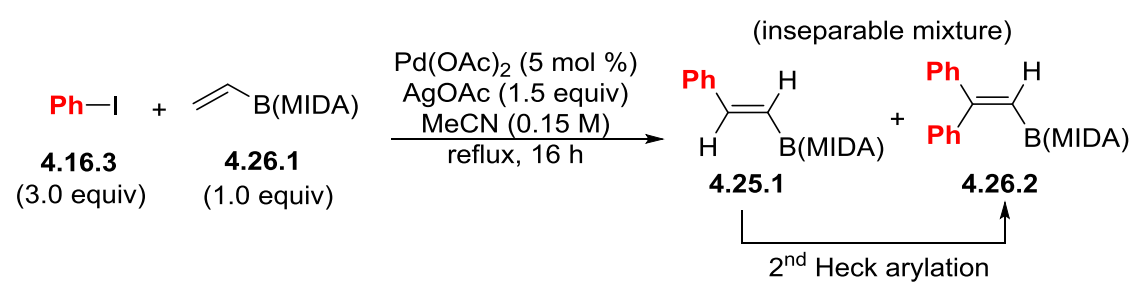

Scheme 4.26. Heck one-pot diarylation of 4.26.1.

The $d i$-substituted olefin starting material 4.25.1 has to be prepared in order to carry out the Heck reaction towards the desired unsymmetrical tri-substituted olefin $\mathbf{4 . 2 5 . 3}$ (Scheme 4.27). This was done by first obtaining the $d i$-substituted olefin 4.25.1 through olefin 
cross-metathesis of styrene 4.16.1 and vinyl boronic MIDA ester 4.26.1 followed by the crosscoupling reaction. The presence of the MIDA boronate group in the final olefin product is advantageous as it can be exploited for a late-stage functionalization through coupling reactions such as Suzuki-Miyaura coupling to give tri-arylethylenes with three different phenyl groups.

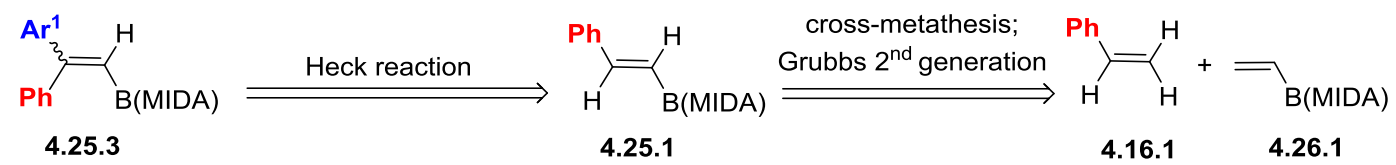

Scheme 4.27. Retrosynthetic analysis of tri-substituted olefin 4.25.3.

\subsubsection{Results and Discussion}

\section{Preparation of phenyl vinyl MIDA boronate}

The starting material 4.25.1, from cross-metathesis between styrene 4.16.1 and commercially available vinylboronic(MIDA) ester 4.26.1 published by Burke et al., ${ }^{255}$ was readily obtained in quantitative yield (Scheme 4.28). With the starting material at hand, an optimization of the reaction conditions was carried out. It is noteworthy that the choice of the aromatic at the $\mathrm{C} 2$ position of the organoboron coupling olefin 4.25 .1 is not limited to a simple phenyl ring as Watson et al. has recently developed a library of diverse boronate derivatives. ${ }^{260}$
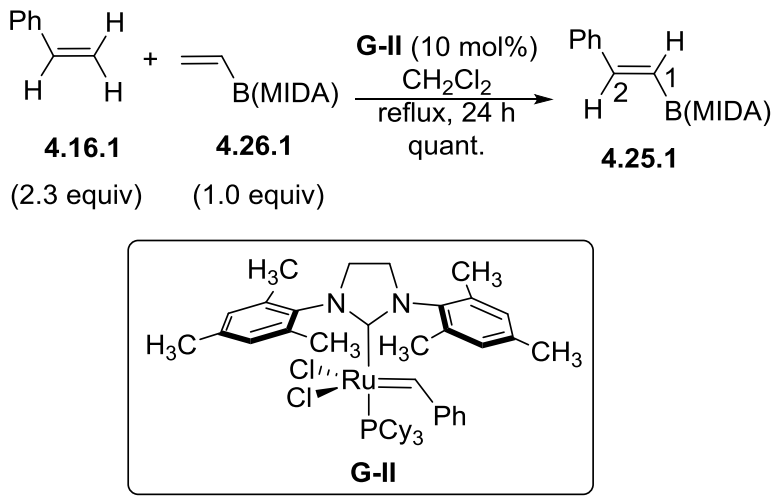

Scheme 4.28. Cross-metathesis between styrene 4.16.1 and vinylboron(MIDA) ester 4.26.1. 


\subsubsection{Preliminary Results}

The treatment of phenyl vinyl MIDA boronate 4.25.1 with excess 1-iodo-4methylbenzene 4.12.2 in the presence of $\mathrm{Pd}(\mathrm{OAc})_{2}(10 \mathrm{~mol} \%)$ and $\mathrm{AgOAc}$ (1.5 equiv) delivered the expected coupling product 4.29.1 and its regioisomer 4.29.2 (Scheme 4.29). In addition, the starting material underwent complete conversion after $18 \mathrm{~h}$ at $80{ }^{\circ} \mathrm{C}$ in acetonitrile. The two regioisomers, 4.29.1 and 4.29.2, were separable by column chromatography on silica gel to give an isolated yield of $85 \%$ and $14 \%$ respectively. It is noteworthy that the major regioisomer 4.29.1 was obtained as a single stereoisomer crystal (from ${ }^{1} \mathrm{H}$ NMR analysis).

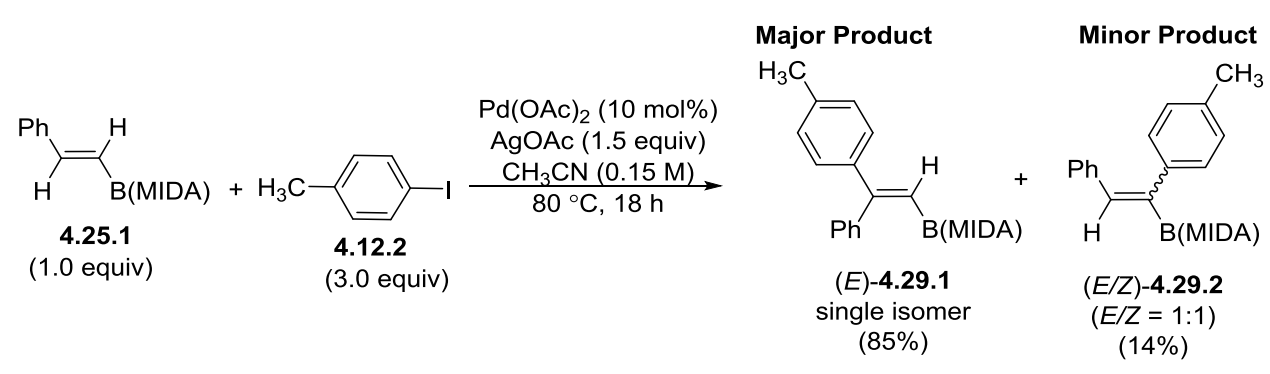

Scheme 4.29. Heck reaction between MIDA boronate 4.25.1 and 4-iodotoluene 4.29.1.

\subsubsection{Optimization of the Heck Reaction Conditions}

The optimization of the Heck reaction began with a primary aim of decreasing the reaction time of $18 \mathrm{~h}$ and the equivalent of aryl iodides used (Schemes 4.30 and 4.31, Tables 4.1 and 4.2). The secondary aim was to perform the Heck reaction using the less expensive aryl bromides or aryl chlorides in place of aryl iodides. Since microwave-assisted heating enables solvents to reach elevated temperatures rapidly, ${ }^{261}$ experiments were thus carried out with microwave irradiation for a reduction in reaction times (Table 4.1) prior to investigation using conventional heating (Table 4.2). The optimization was carried out using iodotoluene 4.12.2 with the desired internal olefin 4.25.1 with $\mathrm{Pd}(\mathrm{OAc})_{2}(10 \mathrm{~mol} \%), \mathrm{AgOAc}$ (1.5 equiv) and acetonitrile $(c=0.15 \mathrm{M})$ (Table 4.1). 


\section{The Effects of Microwave-Assisted Heating on the Heck Reaction}

The Heck reaction gave the expected arylated olefin as a mixture of $(E / Z)-4.29 .1$ isomers and the separable regioisomer as a mixture of (E/Z)-4.29.2 (Scheme 4.30). Unfortunately, there was a trade-off between shorter reaction times and a high conversion of 4.25.1 (Table 4.1, entries 1 and 2). The reaction was limited to a 93\% conversion when heated at $100{ }^{\circ} \mathrm{C}$ for $1 \mathrm{~h}$ (Table 4.1, entry 3 ). Neither an increase in the reaction time nor temperature provided an improvement to the reaction conversion (Table 4.1, entries 4 and 5). It is suspected that the catalyst $\mathrm{Pd}(\mathrm{OAc})_{2}$ was becoming deactivated during the process. Therefore, the catalyst was added in two portions $(5 \mathrm{~mol} \% \times 2)$ at intervals of $30 \mathrm{~min}$ to avoid a potential catalyst decomposition that may account for the incomplete conversion of 4.25.1. The conversion was monitored between the two 30 min intervals through ${ }^{1} \mathrm{H}$ NMR analysis (Table 4.1, entry 6). Unfortunately, complete conversion of 4.25.1 was not observed. It was then suspected that the incomplete conversion could derive from the lower equivalents of aryl iodide used. Therefore, the reaction was repeated with 3 mol equiv of aryl iodide, however no improvement was observed (Table 4.1, entry 7).

In terms of stereoselectivity of the major tri-substituted olefin product $(E / Z)-\mathbf{4 . 2 9 . 1}$, there was a slight compromise ( $3 \%$ decrease) when the reaction was heated under microwave irradiation at $120{ }^{\circ} \mathrm{C}$ (Table 4.1, entry 5). The optimization efforts reverted to conventional heating with the objective of a complete conversion of the starting material 4.25.1.

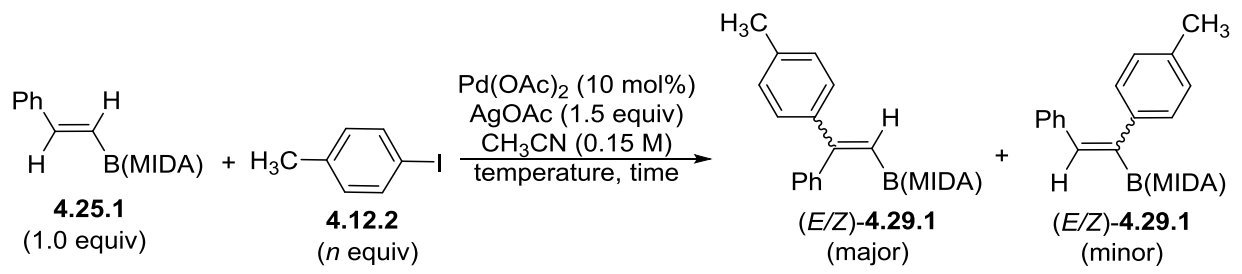

Scheme 4.30. Optimization of the Heck coupling with MIDA boronate 4.25.1 using 4-iodotoluene 4.29.1. 


\begin{tabular}{|c|c|c|c|c|c|c|}
\hline \multirow[b]{2}{*}{ entry } & \multicolumn{6}{|c|}{ Microwave-Assisted } \\
\hline & $\begin{array}{l}\text { equivalent } \\
\qquad(n)\end{array}$ & $\begin{array}{l}\text { temperature } \\
\quad\left({ }^{\circ} \mathrm{C}\right)\end{array}$ & $\begin{array}{l}\text { reaction } \\
\text { time }(\mathrm{h})\end{array}$ & $\begin{array}{c}\text { conversion } \\
(\%)\end{array}$ & $\begin{array}{c}\text { 4.29.1 } \\
\left(E / Z \text { isomer }^{a}\right)\end{array}$ & $\begin{array}{l}4.29 .1 / \\
4.29 .2\end{array}$ \\
\hline 1 & 1.5 & 85 & 0.5 & 30 & $15: 1$ & $4: 1$ \\
\hline 2 & 1.5 & 85 & 1.0 & 79 & $15: 1$ & $4: 1$ \\
\hline 3 & 1.5 & 100 & 1.0 & 93 & $15: 1$ & $4: 1$ \\
\hline 4 & 1.5 & 100 & 1.5 & 93 & $14: 1$ & $4: 1$ \\
\hline 5 & 1.5 & 120 & 1.0 & 75 & $12: 1$ & $4: 1$ \\
\hline \multirow{2}{*}{$6^{b}$} & \multirow{2}{*}{1.5} & \multirow{2}{*}{100} & 0.5 & 52 & $19: 1$ & $3: 1$ \\
\hline & & & 0.5 & 92 & $13: 1$ & $4: 1$ \\
\hline 7 & 3.0 & 100 & 1.0 & 93 & $15: 1$ & $4: 1$ \\
\hline
\end{tabular}

Table 4.1. Optimization of reaction temperature and time through microwave-assisted heating.

The Effects of Conventional Heating on The Heck Reaction

The use of conventional heating enabled one of the two primary aims to be achieved, that is, the use of a lower aryl iodide equivalent from 3.0 to 1.0 equiv (Table 4.2, entries 1-3). However, improvements to the reaction time remained unsuccessful. There was a decrease in both the conversion of $\mathbf{4 . 2 5 . 1}$, from $87 \%$ to $78 \%$, and stereoselectivity of major regioisomer 4.29.1 from 10:1 to 5:1, when heated at $130{ }^{\circ} \mathrm{C}$ (Table 4.2, entries 4-6). The ideal temperature and reaction time to achieve an optimum stereoselectivity with a complete conversion was between $80^{\circ} \mathrm{C}$ to $90{ }^{\circ} \mathrm{C}$ for $18 \mathrm{~h}$ (Table 4.2, entries 1 and 2).

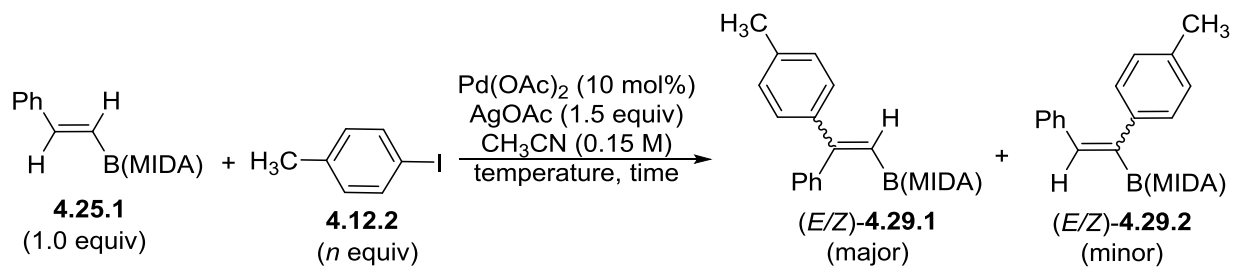

Scheme 4.31. Optimization of the Heck coupling with MIDA boronate 4.25.1 using 4-iodotoluene 4.29.1. 


\begin{tabular}{ccccccc}
\hline entry & $\begin{array}{c}\text { Conventional } \\
\text { equivalent } \\
(n)\end{array}$ & $\begin{array}{c}\text { temperature } \\
\left({ }^{\circ} \mathrm{C}\right)\end{array}$ & $\begin{array}{c}\text { reaction } \\
\text { time }(\mathrm{h})\end{array}$ & $\begin{array}{c}\text { conversion } \\
(\%)\end{array}$ & $\begin{array}{c}\mathbf{4 . 2 9 . 1} \\
\left(E / Z \text { isomer }{ }^{a}\right)\end{array}$ & $\begin{array}{c}\mathbf{4 . 2 9 . 1} / \\
\mathbf{4 . 2 9 . 2}\end{array}$ \\
\hline 1 & 3.0 & 80 & 18 & 100 & $>95: 5$ & $4: 1$ \\
2 & 1.5 & 90 & 18 & 100 & $14: 1$ & $4: 1$ \\
3 & 1.0 & 80 & 18 & 86 & $14: 1$ & $5: 1$ \\
4 & 1.5 & 130 & 5 & 87 & $10: 1$ & $4: 1$ \\
5 & 1.5 & 130 & 7 & 82 & $9: 1$ & $4: 1$ \\
6 & 1.5 & 130 & 13 & 78 & $5: 1$ & $3: 1$ \\
\hline
\end{tabular}

${ }^{a}$ Ratio isomers of major product.

Table 4.2. Optimization of reaction temperature and time through conventional heating.

\section{The Effects of Different Catalysts and Reaction Conditions on The Heck Reaction}

Other similar conditions of the Heck reaction previously reported in the literature by Whiting et al. ${ }^{262}$ and Burke et al., ${ }^{257}$ were also explored prior to establishing the optimized reaction conditions. The condition employed by Whiting et al., used the same catalyst, with a lower catalytic loading $\mathrm{Pd}(\mathrm{OAc})_{2}(5 \mathrm{~mol} \%)$, and an additional ligand $\mathrm{P}(o \text {-tolyl })_{3}(10 \mathrm{~mol} \%)$, at $50{ }^{\circ} \mathrm{C}$ in the presence of AgOAc. The conversion of phenyl vinyl MIDA boronate was very low, $5 \%$, under these conditions. The poor result led us to maintain our optimized conditions (Scheme 4.32).

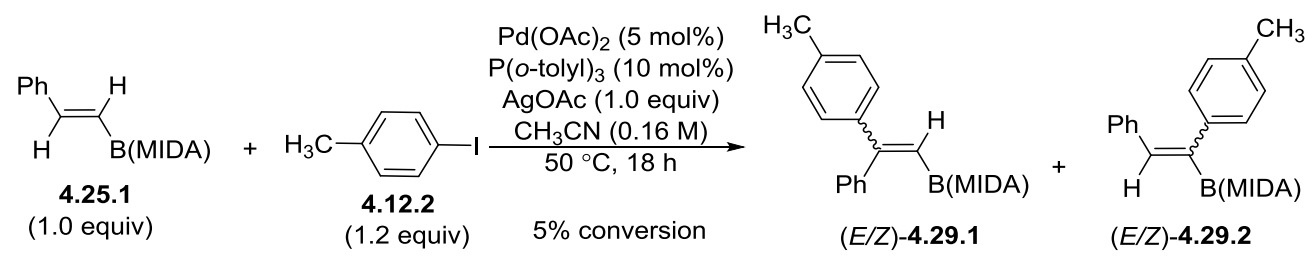

Scheme 4.32. Whiting's condition with MIDA boronate 4.25.1 and 4-iodotoluene 4.12.2. 
The conditions employed by Burke et al. were also tested and we found that the use of $\mathrm{Pd}\left(\mathrm{PPh}_{3}\right)_{4}$ in the presence of $\mathrm{Ag}_{3} \mathrm{PO}_{4}$ in THF at $100{ }^{\circ} \mathrm{C}$ delivered no reaction between 4iodotoluene 4.12.2 and MIDA boronate ester 4.25.1 (Scheme 4.33, eq 1). Nonetheless a 32\% conversion (by the analysis of ${ }^{1} \mathrm{H}$ NMR spectra) of the vinylboronic(MIDA) ester 4.26.1 was estimated in the reaction with 4-iodotoluene 4.12.2 (Scheme 4.33, eq 2).
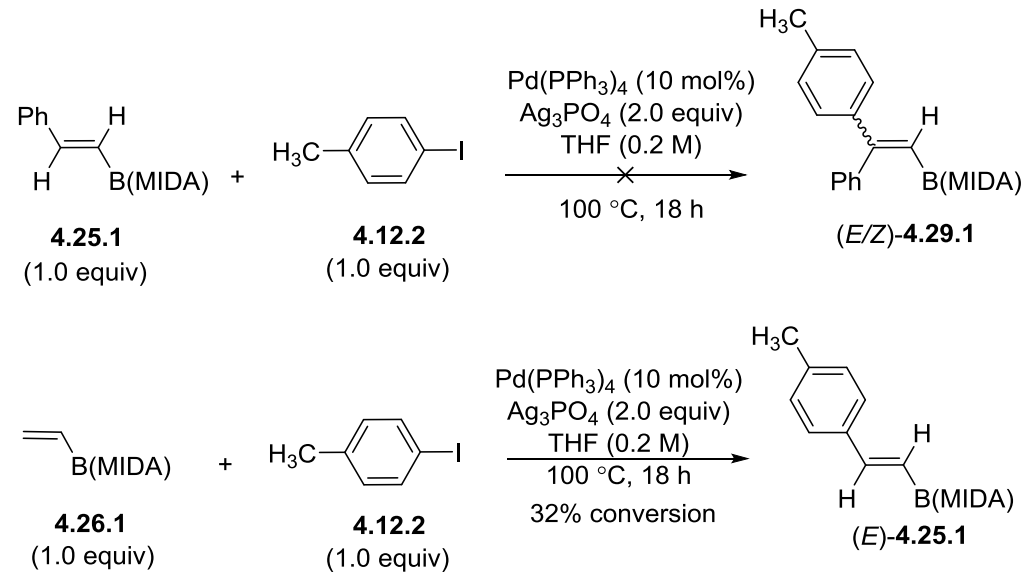

[2]

Scheme 4.33. Burke's condition with MIDA boronate 4.25.1 and 4-iodotoluene 4.12.2.

\section{The Effects of Concentration on the Heck Reaction}

The concentration of the reaction appears to be inversely proportional to yield of the major regioisomer product $(E / Z)-\mathrm{C}$ (Table 4.3 , entries $1-3)$. There was complete conversion of the starting material 4.25.1 in each event. Under the conditions when $c=0.15 \mathrm{M}$, the major regioisomeric product was isolated in $85 \%$ yield as a single diastereomer of $(E)-\mathbf{4 . 2 9 . 1}$ while the minor regioisomer was obtained as a 1:1 mixture of stereoisomers $(E / Z)-4.29 .2$ (Table 4.3, entry 1). A similar trend was observed under the conditions when $\mathrm{c}=0.12 \mathrm{M}$ with a modest increase in the isolated yield of (E)-4.29.1 (Table 4.3, entry 2). The Heck reaction delivered (E)-4.29.1 in the lowest yield of 66\% when the concentration was increased to $c=1.0 \mathrm{M}$ (Table 4.3, entry 3). Therefore, the Heck reactions described henceforth was carried out in $c=0.12 \mathrm{M}$. 


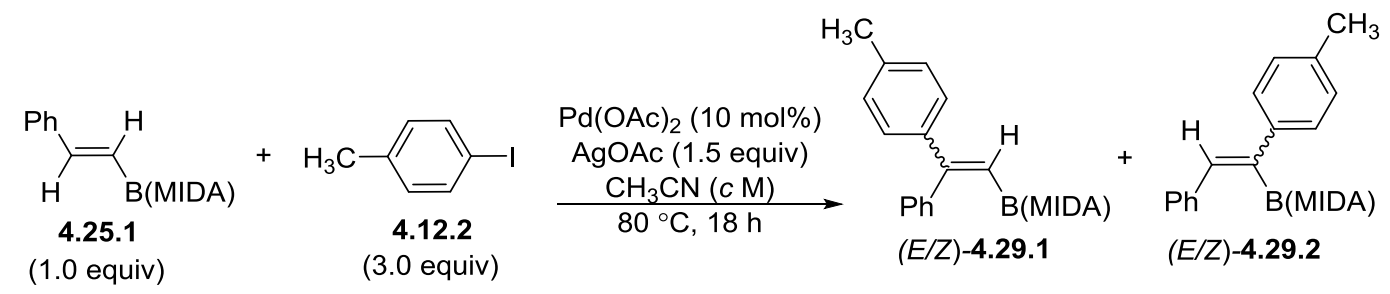

Scheme 4.34. Heck reaction between MIDA boronate 4.25.1 and 4-iodomethylketone 4.34.1.

\begin{tabular}{cccccc}
\hline entry & $\begin{array}{c}\text { concentration } \\
\left(\mathrm{mmol} \mathrm{cm}^{-3}\right)\end{array}$ & $\begin{array}{c}\text { conversion }(\%) \\
\text { of } \mathbf{4 . 2 5 . 1}\end{array}$ & $\begin{array}{c}\text { yield (\%) } \\
\text { of 4.29.1 }\end{array}$ & $\begin{array}{c}E / Z- \\
\text { selectivity } \\
\text { of 4.29.1 }\end{array}$ & $\begin{array}{c}E / Z- \\
\text { selectivity } \\
\text { of 4.29.2 }^{\mathrm{a}}\end{array}$ \\
\hline 1 & 0.15 & 100 & 85 & $>95: 5$ & $1: 1$ \\
2 & 0.12 & 100 & 91 & $>95: 5$ & $1: 1$ \\
3 & 1.00 & 100 & 66 & $>95: 5$ & $1: 1$ \\
\hline
\end{tabular}

Table 4.3. Effects of concentration on the Heck reaction between MIDA boronate 4.25.1 and 4-iodotoluene 4.12.2.

The Effects of a Different Boronate on the Heck Reaction

At this point, the objective was to showcase the advantages of using boron MIDA ester 4.25.1. This prompts the question of whether a comparable Heck cross-coupling result under the optimized conditions can be obtained by using a simple boron group such as the commonly employed bis(pinacolato)diboron 4.36.1 instead of the boron MIDA ester 4.25.1 (Scheme 4.35). The switch from using a MIDA boronate ester to a simple boron group such as the bis(pinacolato)diboron on the $\mathrm{C} 1$ position was investigated to better understand the reaction limitation. Therefore, the Heck coupling was also carried out between 1,2-phenyl vinyl boronic acid pinacol ester 4.36.1 and 4-iodotoluene 4.12.2 to give the desired tri-substituted olefin (E)-4.36.2 in a low yield of $16 \%$ despite a full consumption of the starting Bpin reagent 4.36.1 (Scheme 4.35, eq 2). This suggests other reaction pathways may have occurred such as the possible polymerization of $\mathbf{4 . 3 6 . 1}$ that may lead to solid by-products. Alternatively it suggests a possible decomposition of the Bpin reagent 4.36.1 under the optimized Heck reaction conditions, which would reflect the advantage of employing the more stable MIDA boronate 
4.25.1. Overall, the outcome showed a preference for the MIDA boronate ester group at the $\mathrm{C} 1$ position, suggesting a presence of some activating properties offered by the MIDA boronate ester group on the $\beta$-carbon that may be lacking with the $\mathrm{B}(\mathrm{pin})$ group.
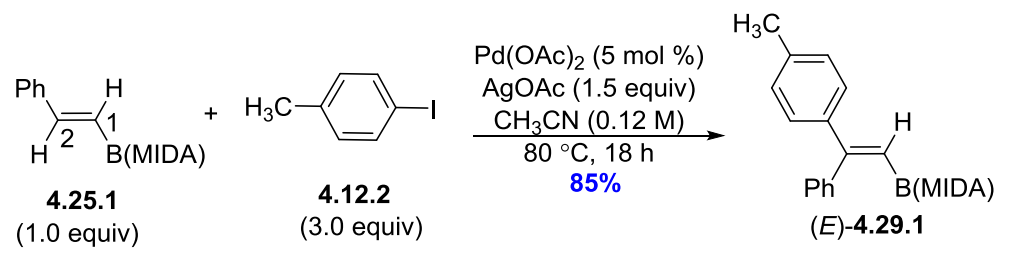

[1]

$(E)-4.29 .1$
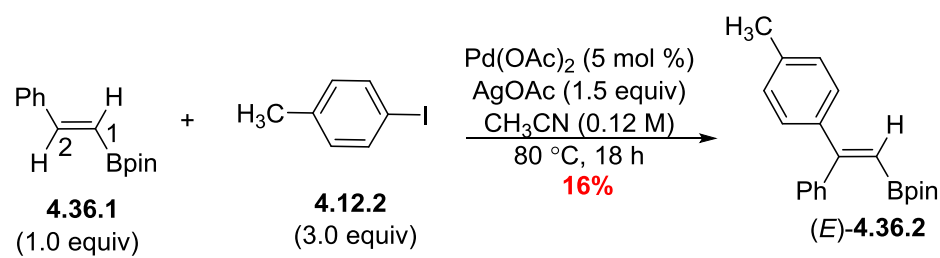

Scheme 4.35. Comparison of reaction outcomes when using two different types of boron groups.

The Effects of Different Aryl Halides on the Heck Reaction

Therefore with the established optimized reaction conditions, $\mathrm{Pd}(\mathrm{OAc})_{2}(5 \mathrm{~mol} \%)$ with $\mathrm{AgOAc}$ (1.5 equiv) in $\mathrm{CH}_{3} \mathrm{CN}\left(c=0.12 \mathrm{M}\right.$ ), at $80{ }^{\circ} \mathrm{C}$ for $18 \mathrm{~h}$, we embarked on the secondary goal, that is, the use of other aryl halides $(\mathrm{X}=\mathrm{Br}, \mathrm{Cl})$ (Scheme 4.36). The Heck reaction was thus carried out using an aryl bromide, 4-bromoanisole 4.19.3 (4-bromotoluene was unavailable) in place of 4-iodotoluene 4.12.2 (Scheme 4.36). Unfortunately, there was no conversion of 4.25.1 to the desired olefin $(E)$-4.37.1. This suggests that, under our optimized reaction conditions, the Heck reaction with MIDA boronate 4.25.1 proceeds with a full conversion only with the use of aryl iodides. 


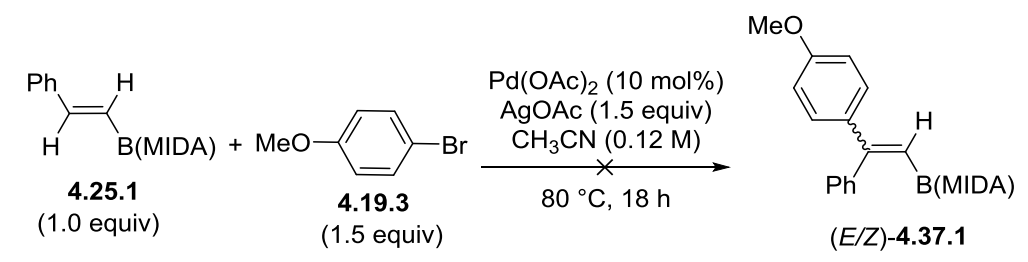

Scheme 4.36. No Heck coupling reaction occurred when using aryl bromide.

\subsubsection{Scope and Limitation}

\subsection{Heck Cross-Coupling Between $s p^{2}-s p^{2}$}

The scope began with the Heck cross-coupling of MIDA boronate ester 4.25.1 with a panel of substituted aryl iodides 4.25.2 to give tri-substituted olefins as major regioisomers 4.25.3 and the separable minor regioisomers 4.25.4 (Scheme 4.37).

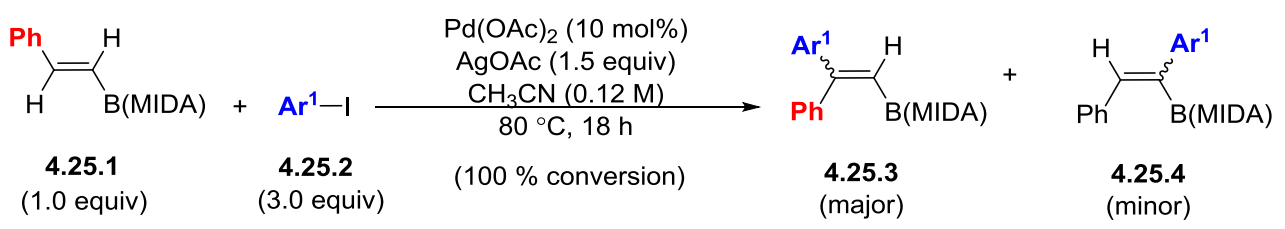

Scheme 4.37. Optimized condition for the Heck coupling with MIDA boronate 4.25.1.

\subsection{Aryl Substituents}

Heck Reaction with Ortho-Substituted Aryl Iodides

The ortho-substituted iodo substrates did not perform well in the Heck coupling as neither substrates gave a full conversion (Table 4.4, entries 1-2). This is regardless of the electronic nature of the aryl substituents on the benzene ring, which indicates that steric congestion around the iodide would hinder the reaction. It is likely that the steric hindrance significantly affects the oxidative addition step of the catalytic cycle. ${ }^{263}$ Therefore, further investigation on the scope of the reaction ensured no substitution near the iodide. 


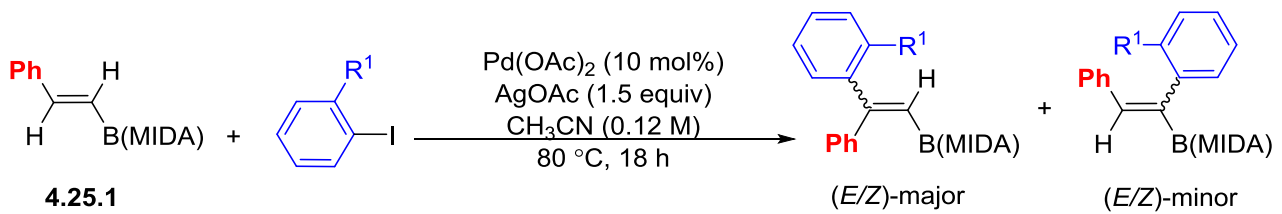

(1.0 equiv) (3.0 equiv)

Scheme 4.38. Heck vinylation of $o$-substituted aryl iodides.

\begin{tabular}{ccccc}
\hline entry & aryl iodide & $\mathrm{R}^{1}$ & $\begin{array}{c}\text { conversion } \\
\text { of } \mathbf{4 . 2 5 . 1}(\%)^{\mathrm{a}}\end{array}$ & $\begin{array}{c}(\text { E/Z)-stereoselectivity } \\
\text { of major regioisomer }\end{array}$ \\
\hline 1 & $\mathbf{4 . 3 9 . 1}$ & $\mathrm{OMe}$ & 39 & $>95: 5$ \\
2 & $\mathbf{4 . 3 9 . 2}$ & $\mathrm{CO}_{2} \mathrm{Me}$ & 53 & $>95: 5$ \\
\hline
\end{tabular}

[a] Determined by NMR with respect to 4.25.1. [b] Determined by NMR with respect to 4.25.1.

Table 4.4. Ortho-substituted aryl compounds employed in Heck coupling with 4.21.1.

Heck Reaction with Para-Substituted Aryl Iodides

A wide range of para-susbtituted aryl iodides were evaluated in the Heck reaction with 4.25.1 under the previously optimized conditions (Scheme 4.39 and Table 4.5). Gratifyingly, the two regioisomers product obtained were separable by flash chromatography on silica gel. The yield of the desired major regio-isomer product is high for substrates bearing electrondonating aryl substituents such as methyl 4.12.2 (85\%) and methoxy 4.39.13 (93\%). More importantly, the stereoselectivity of tri-substituted olefins 4.29 .1 and 4.39.14 is excellent $(E / Z>95: 5)$. Apart from that, the small amount of isolated minor regioisomers, 4.29.2 (14\%) and 4.39.14 (7\%) (Table 4.5, entries 1 and 2), indicate high reaction efficiency because the mass balance of the reaction is near quantitative.

However, when 4-iodoaniline 4.39.2 was employed, there was no conversion of 4.25.1 observed by ${ }^{1} \mathrm{H}$ NMR analysis (Table 4.5, entry 3), a consequence that could be attributed to a catalyst poisoning by the free amine group bearing a lone pair of electrons that may implicate the reaction by means of complexation with the palladium catalyst, forcing the reaction to a halt. 
Nonetheless, in protection of 4-iodoaniline as a carbamate using the $t$-Boc group 4.39.3, the Heck reaction then gave a full conversion to the regioisomers 4.39.15 and 4.39.16 with excellent stereoselectivity and a high isolated yield of the major isomer $\mathbf{4 . 3 9 . 1 5}$ at $73 \%$ (Table 4.5 , entry 4). This reflects that the shielding property offered by the protecting group is instrumental for the reaction to proceed.

Electron deficient aryl iodides were then employed, and it was observed that for aryl iodide bearing the trifluoromethyl substituent 4.39.4, the yield of the major regioisomer 4.39.17 was slightly lower at $78 \%$ while the yield for the minor regioisomer 4.39 .18 was substantial (21\%) (Table 4.5, entries 5). Apart from that, the reaction conditions are tolerant of halogen substituents on the aromatic ring, $p$-iodo-fluorobenzene 4.22.2 and 1-iodo-4-bromobenzene 4.39.5 (Table 4.5, entries 6 and 7). Interestingly, the use of 1-iodo-4-bromobenzene 4.39.5 in the Heck reaction demonstrated a chemoselective coupling on the iodide to furnish major and minor regioisomers Heck products $\mathbf{4 . 3 9 . 2 1}$ in $70 \%$ and $\mathbf{4 . 3 9 . 2 2} 17 \%$ yield respectively. (Table 4.5, entry 7). The presence of a bromine atom on the final Heck products provides an opportunity for further functionalization.

The presence of a carbonyl para-substituent was then evaluated. The substrate 4iodoacetophenone 4.39.6 was utilized in the presence of phenyl vinyl boronate 4.25.1 to give the expected tri-substituted olefin $\mathbf{4 . 3 9 . 2 3}$, which was isolated in $72 \%$ yield and excellent stereoselectivity of over $95 \%$ (Table 4.5 , entry 8 ). Similarly, the presence of a carboxylic acid 4.39.7 at the para-position did not interfere with the efficiency of the Heck coupling but affected the purification process, resulting in an inseparable mixture of regioisomers 4.39.25 and 4.39.26 (Table 4.5, entry 9). Therefore, the estimated yields were based on the ${ }^{1} \mathrm{H}$ NMR ratio and the mass of the combined regioisomers 4.39.25 and 4.39.26. The purification problem was readily addressed with the conversion of the acid to an ester group 4.39.8, affording the desired major regioisomer 4.39.27 in a good yield of 73\% (Table 4.5, entry 10). However, when the ester 
group was changed to an amide functionality such as the secondary amide substrate 4.39.9, the Heck coupling reaction showed no conversion of phenyl vinyl boronate 4.25.1 (Table 4.5, entry 11). Fortunately, the use of tertiary amide $\mathbf{4 . 3 9 . 1 0}$ provided the major regioisomer Heck product 4.39.29 in a high isolated yield of $86 \%$ with good stereoselectivity (Table 4.5 , entry 12 ).

Although the reaction showed tolerance to both electron-donating and electronwithdrawing aryl groups, there are some identified limitations of the method associated with the latter. The strongly electron-withdrawing cyano 4.39.11 and nitro 4.39.12 substituents, gave a poor conversion of $15 \%$ and $19 \%$ respectively (Table 4.5 , entry 13 and 14). Consequently, isolation of the desired product via column chromatography on silica gel failed due to the coelution of the starting material 4.25.1 with the products and thus, the tri-substituted olefin products were not cleanly isolated. The negative outcome may suggest a deactivation of the catalyst.

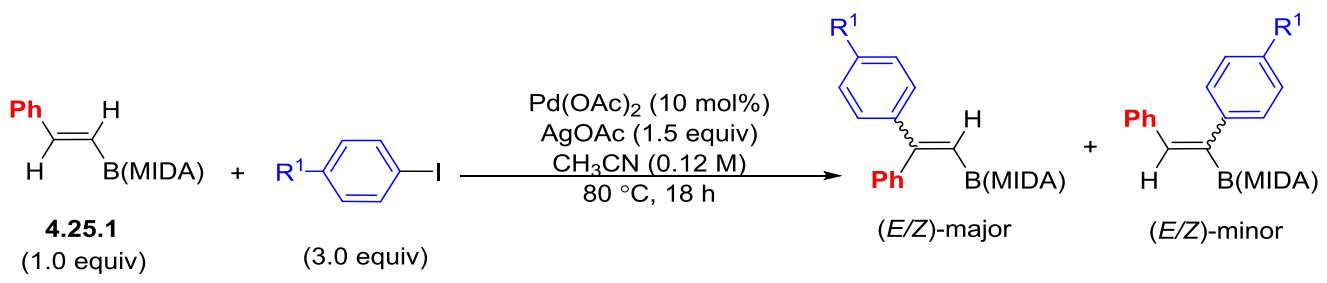

Scheme 4.39. Heck arylation of MIDA boronate 4.25.1 with $p$-substituted aryl iodides.

\begin{tabular}{ccccccc}
\hline entry & $\begin{array}{c}\text { aryl } \\
\text { iodide }\end{array}$ & $\mathrm{R}^{1}$ & $\begin{array}{c}\text { conversion } \\
(\%) \text { of } \mathbf{4 . 2 5 . 1}\end{array}$ & $\begin{array}{c}\text { yield }(\%) \text { of } \\
\text { major } \\
\text { regioisomer }\end{array}$ & $\begin{array}{c}\text { yield }(\%) \text { of } \\
\text { minor } \\
\text { regioisomer }\end{array}$ & $\begin{array}{c}(E / Z)- \\
\text { selectivity of } \\
\text { major } \\
\text { regioisomer }\end{array}$ \\
\hline 1 & $\mathbf{4 . 1 2 . 2}$ & $\mathrm{Me}$ & 100 & $\mathbf{4 . 2 9 . 1 - 8 5}$ & $\mathbf{4 . 2 9 . 2 - 1 4}$ & $>95: 5$ \\
2 & $\mathbf{4 . 3 9 . 1}$ & $\mathrm{OMe}$ & 100 & $\mathbf{4 . 3 9 . 1 3 - 9 3}$ & $\mathbf{4 . 3 9 . 1 4 - 7}$ & $>95: 5$ \\
3 & $\mathbf{4 . 3 9 . 2}$ & $\mathrm{NH}_{2}$ & 0 & - & - & - \\
4 & $\mathbf{4 . 3 9 . 3}$ & $\mathrm{NH} t$-Boc & 100 & $\mathbf{4 . 3 9 . 1 5 - 7 3}$ & $\mathbf{4 . 3 9 . 1 6 - 8}$ & $>95: 5$ \\
\hline
\end{tabular}




\begin{tabular}{ccccccc}
\hline 5 & $\mathbf{4 . 3 9 . 4}$ & $\mathrm{CF}_{3}$ & 100 & $\mathbf{4 . 3 9 . 1 7 - 7 8}$ & $\mathbf{4 . 3 9 . 1 8}-21$ & $20: 1$ \\
6 & $\mathbf{4 . 2 2 . 2}$ & $\mathrm{F}$ & 100 & $\mathbf{4 . 3 9 . 1 9 - 7 3}$ & $\mathbf{4 . 3 9 . 2 0}-8$ & $11: 1$ \\
7 & $\mathbf{4 . 3 9 . 5}$ & $\mathrm{Br}$ & 100 & $\mathbf{4 . 3 9 . 2 1 - 7 0}$ & $\mathbf{4 . 3 9 . 2 2}-17$ & $17: 1$ \\
8 & $\mathbf{4 . 3 9 . 6}$ & $\mathrm{C}(\mathrm{O}) \mathrm{Me}$ & 100 & $\mathbf{4 . 3 9 . 2 3}-72$ & $\mathbf{4 . 3 9 . 2 4} 26$ & $20: 1$ \\
$9^{\mathrm{a}}$ & $\mathbf{4 . 3 9 . 7}$ & $\mathrm{COOH}$ & 100 & $\mathbf{4 . 3 9 . 2 5}-70^{\mathrm{b}}$ & $\mathbf{4 . 3 9 . 2 6}-28^{\mathrm{b}}$ & $11: 1$ \\
10 & $\mathbf{4 . 3 9 . 8}$ & $\mathrm{CO}_{2} \mathrm{Et}$ & 100 & $\mathbf{4 . 3 9 . 2 7}-73$ & $\mathbf{4 . 3 9 . 2 8}-16$ & $>95: 5$ \\
11 & $\mathbf{4 . 3 9 . 9}$ & $\mathrm{CONHBn}^{2}$ & 0 & - & - & - \\
12 & $\mathbf{4 . 3 9 . 1 0}$ & $\mathrm{CONBn}_{2}$ & 100 & $\mathbf{4 . 3 9 . 2 9}-86$ & $\mathbf{4 . 3 9 . 3 0 - 7}$ & $>95: 5$ \\
13 & $\mathbf{4 . 3 9 . 1 1}$ & $\mathrm{CN}^{2}$ & 15 & - & - & - \\
14 & $\mathbf{4 . 3 9 . 1 2}$ & $\mathrm{NO}_{2}$ & 19 & - & - & - \\
\hline
\end{tabular}

[a] Purification problems, inseparable 4.39.25 and 4.39.26. [b]Yields are based on ${ }^{1} \mathrm{H}$ NMR ratio of 4.39.25/4.39.26 and mass of the isolated mixture.

Table 4.5. Para-substituted aryl compounds employed in Heck coupling with 4.25.1.

The Heck reaction with $p$-substituted aryl iodides delivered high yields when using electron-rich aryl iodides but low to moderate yields with electron-deficient aryl iodides (Scheme 4.40). For this reason, the attempt to improve the yield of major regioisomer product, tri-substituted ethylenes bearing an electron deficient aryl group, was realized by performing the reaction in the presence of an additive such as $\mathrm{PPh}_{3}$. However, when the optimized Heck reaction involving ethyl 4-iodobenzoate 4.39.8 (Scheme 4.40, eq 1) was repeated under condition I $\left(\mathrm{PPh}_{3}(12 \mathrm{~mol} \%)\right.$ (Scheme 4.40, eq 2), the stereoselectivity of the major product 4.39.27 decreased from $(E / Z)=50: 1$ to $(E / Z)=2.9: 1$. In addition, the marginal decrease in the isolated yield of $(E)-\mathbf{4 . 3 9 . 2 7}$ (from 73\% to 67\%) led to the halt of further efforts for yieldimprovement (Scheme 4.40). 


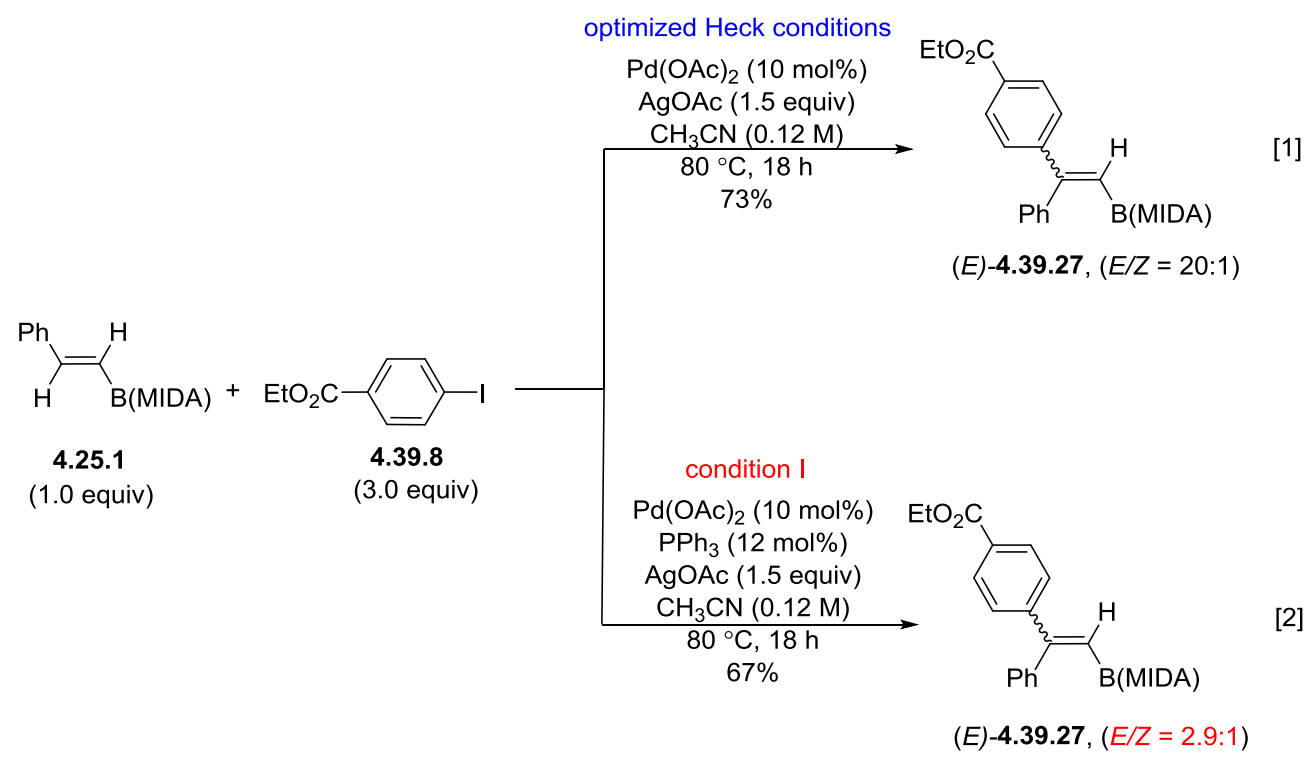

Scheme 4.40 The influence of $\mathrm{PPh}_{3}$ on the Heck reaction with 4.39.8.

\section{$\underline{\text { Heck Reaction with Meta-Substituted Aryl Iodides }}$}

The use of meta-substituted aryl iodide was then explored to understand the influence of the position of the substituent on the phenyl ring on the reaction (Scheme 4.41, Table 4.6, entries $1-4)$. The investigation gave encouraging results with a similar trend as previously observed with the para-substituted aryl compounds. The electron-donating substituents 1-iodo3-methylbenzene 4.41.1 and 1-iodo-3-methoxybenzene 4.41.2 gave the major regioisomers 4.41.5 and 4.41.7 respectively in high yield and excellent stereoselectivity as a single $(E)$-isomer was formed (Table 4.6, entries $1-2$ ). When using electron-deficient substrates such as bromo3-iodobenzene 4.41.3, major regioisomer 4.41.9 was isolated in high 73\%. However, there was a decrease in the stereoselectivity as a mixture of $E / Z$ isomers (15:1) was obtained. Similarly, a lower regioselectivity was observed as the minor regioisomer, 4.41 .10 (26\%), was afforded in substantial amount (Table 4.6, entry 3). Nonetheless, the method showed tolerance of the bromine atom. A similar trend was observed in the use of methyl 3-iodobenzoate 4.41.4 (Table 4.6, entry 4). 


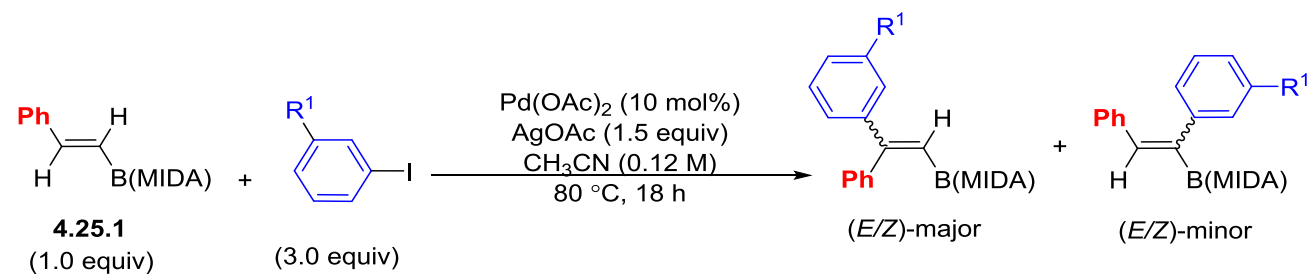

Scheme 4.41. Heck arylation of MIDA boronate 4.25.1 with $m$-substituted aryl iodides.

\begin{tabular}{|c|c|c|c|c|c|c|}
\hline entry & $\begin{array}{l}\text { aryl } \\
\text { iodide }\end{array}$ & $\mathrm{R}^{1}$ & $\begin{array}{l}\text { conversion } \\
(\%) \text { of } \mathbf{4 . 2 5 . 1}\end{array}$ & $\begin{array}{l}\text { yield }(\%) \text { of } \\
\text { major } \\
\text { regioisomer }\end{array}$ & $\begin{array}{l}\text { yield }(\%) \text { of } \\
\text { minor } \\
\text { regioisomer }\end{array}$ & $\begin{array}{l}(E / Z)- \\
\text { selectivity of } \\
\text { major } \\
\text { regioisomer }\end{array}$ \\
\hline 1 & 4.41.1 & $\mathrm{Me}$ & 100 & 4.41.5-85 & 4.41.6-15 & $>95: 5$ \\
\hline 2 & 4.41.2 & $\mathrm{OMe}$ & 100 & 4.41.7-90 & 4.41.8-9 & $>95: 5$ \\
\hline 3 & 4.41 .3 & $\mathrm{Br}$ & 100 & 4.41.9-73 & 4.41.10-26 & $15: 1$ \\
\hline 4 & 4.41.4 & $\mathrm{CO}_{2} \mathrm{Me}$ & 100 & 4.41.11-72 & 4.41.12-25 & $>95: 5$ \\
\hline
\end{tabular}

Table 4.6. Meta-substituted aryl compounds employed in Heck coupling with 4.25.1.

We have shown that the MIDA boronate 4.25.1 was successfully employed in the Heck reaction with aryl iodides with various substitution patterns (ortho-, meta-, and para-) to give the tri-substituted aryl olefins in high yield and good stereoselectivity in favour of the (E)-isomer. However, the limitation of the reaction is in the steric sensitivity towards orthosubstituted aryl iodides. Nonetheless, the reaction conditions showed tolerance for a panel of para-substituents, both electron-donating and withdrawing, and hence demonstrates flexibility that could potentially allow for a general application.

\subsection{Heteroaromatics}

The next stage in the scope investigation involved the incorporation of nitrogen heterocycles (Table 4.7, entries 1 - 9, 4.42.1-4.42.8). The bromo-pyrimidine 4.42.1 and pyridine 4.42.2 substrates were specifically employed to understand whether the activating 
properties from the nitrogen could enhance the activity of the bromide, making it a potential coupling partner (Table 4.7, entries 1 and 2). Unfortunately, the bromo-substrates remained inactive. This is also true for 2-iodopyridine 4.42.3 and 1-benzyl-4-iodopyrazole $\mathbf{4 . 4 2 . 4}$ (Table 4, entries 3 and 4). There was also no conversion observed with $t$-Boc-protected 3-iodo indole 4.42.5 (Table 4.7, entry 5). As previously suspected, the presence of the nitrogen with its lone pair of electrons could account for the unsuccessful Heck coupling with nitrogen containing heterocycles.

The Lewis basicity of the pyridine nitrogen was reduced by introducing a chlorine atom at the $\mathrm{C} 2$ position through the use of 2-chloro-5-iodo-pyridine $\mathbf{4 . 4 2 . 6}$ (Table 4.7, entry 6). Under the optimized reaction condition, a low $15 \%$ conversion of 4.25 .1 into desired olefin 4.42.13 was estimated by ${ }^{1} \mathrm{H}$ NMR analysis (Table 4.7, entry 6). When the reaction with hetero-iodide 4.42.6 was repeated in the presence of catalytic amount of $\mathrm{PPh}_{3}(12 \mathrm{~mol} \%)$ as a ligand, there was an improvement in the conversion from previously 15\% to 54\% (Table 4.7, entries 6 and 7). More interestingly, in the analysis of the ${ }^{1} \mathrm{H}$ NMR spectrum, there was only a single product observed from the coupling with 5-iodo-2-chloropyridine 4.42.6 as peaks correlating to the expected accompanying minor regioisomeric product in the Heck reaction were absent. It was to our delight that there was complete regioselectivity as only one regioisomer $\mathbf{4 . 4 2 . 1 3}$ was formed, which was separable from the starting phenyl vinyl boronate 4.25.1 and was isolated in $53 \%$ yield as a mixture of stereoisomers $(E / Z=3: 1)$ (Table 4.7, entry 7). Additionally, it was then believed that a bulkier ligand could give a further improvement, however, there was no enhancement in the conversion of $\mathbf{4 . 2 5 . 1}$ with the use of $\mathrm{P}(o \text {-Tolyl })_{3}$.

The use of a sulfur-containing heteroaromatic iodide was then studied in the Heck reaction between 3-iodothiophene 4.41.6 and 4.25.1. The low conversion of $15 \%$ was inadequate for product isolation from the starting material 4.25.1 (Table 4.7, entry 8). Therefore, 
the Heck reaction was repeated with the addition of catalytic amount of $\mathrm{PPh}_{3}\left(12 \mathrm{~mol}^{\%}\right)$. Unfortunately, there was no improvement in spite of the ligand addition.

The use of 5-iododihydrofuran $\mathbf{4 . 4 2 . 8}$, on the other hand, provided encouraging results. The incomplete conversion (86\%) of phenyl vinyl boronate 4.25.1 did not hamper the product isolation because the desired Heck product 4.42.15 was separable from the mixture of starting material and minor regioisomer with a yield of $74 \%$ as a mixture of stereoisomers $(E / Z=13: 1)$ (Table 4.7, entry 9) The moderate stereoselectivity 13:1 is a marked improvement from the results obtained with the other heteroaromatic iodides examined.

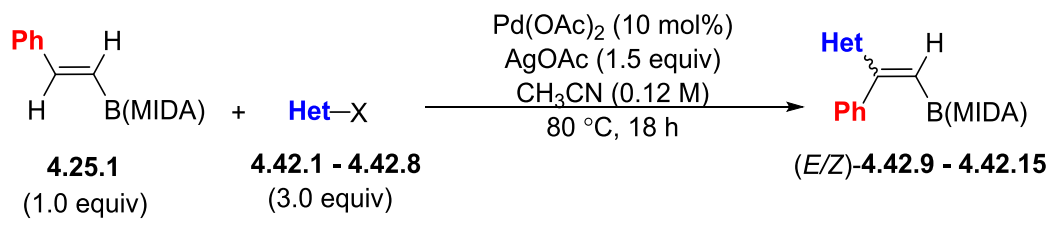

Scheme 4.42. Extension of the scope on heterocycles.

\begin{tabular}{lccc}
\hline entry & Heck product & $\begin{array}{c}\text { conversion } \\
(\%) \text { of } \mathbf{4 . 2 5 . 1}\end{array}$ & $\begin{array}{c}\text { Yield (\%) } \\
(E / Z- \\
\text { stereoselectivity })\end{array}$ \\
\hline & & & 0 \\
\hline
\end{tabular}




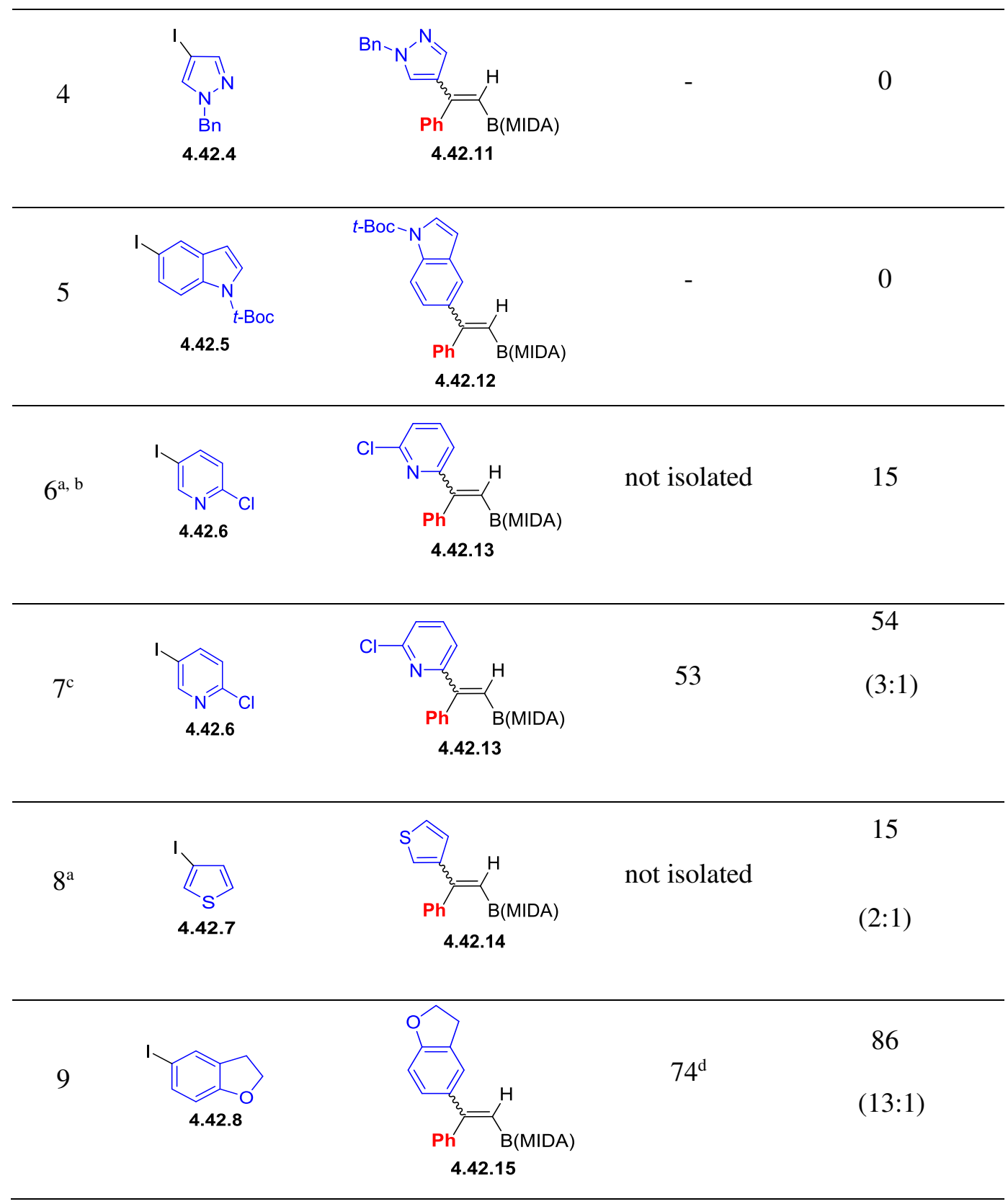

[a] No improvement with $\mathrm{PPh}_{3}(12 \mathrm{~mol} \%)$. [b] No improvement with $\mathrm{P}(o \text {-toly })_{3}(12 \mathrm{~mol} \%)$. [c] No improvement with $\mathrm{P}(o \text {-toly })_{3}(12 \mathrm{~mol} \%)$ in DMF at $140{ }^{\circ} \mathrm{C}$. [c] Addition of $\mathrm{PPh}_{3}(12 \mathrm{~mol} \%)$. [d] Major product isolated as a mixture with starting material and the minor regioisomeric products.

Table 4.7. Heck coupling attempted on heterocycles.

We have shown that the scope of Heck reaction between phenyl vinyl boronate 4.25.1 and heterocycles is narrow. Although some improvements in the conversion of $\mathbf{4 . 2 5 . 1}$ can be achieved by the presence of an additive like $\mathrm{PPh}_{3}$, the Heck reaction under our optimized 
reaction condition showed little tolerance with heteroaromatic aryl iodides as the coupling partner.

\subsection{Heck Cross-Coupling Between $s p^{2}-s p$}

The Heck reaction was also attempted between $s p^{2}-s p$ carbon that is $\mathbf{4 . 2 5 . 1}$ and alkyne 4.43.1 (Scheme 4.43). However, no conversion of the starting reagent 4.25.1 to the desired Heck product was observed.

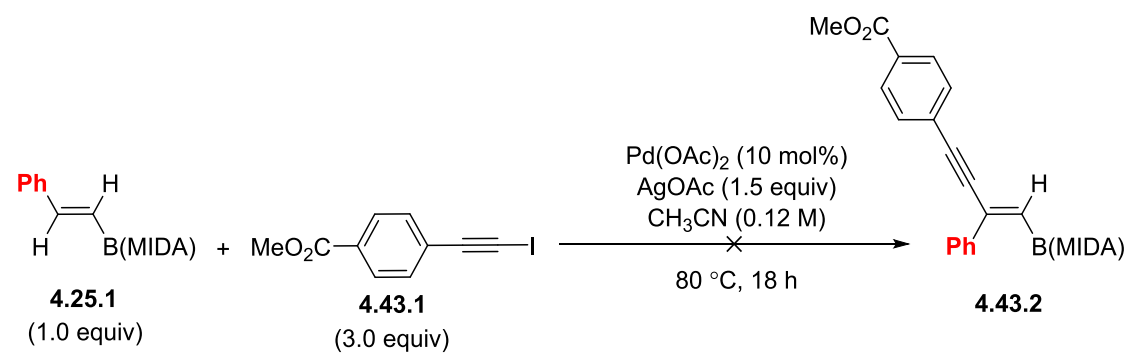

Scheme 4.43. Heck coupling attempted on sp-hybridized carbon.

\subsection{Heck Cross-Coupling Between $s p^{2}-s p^{3}$}

Similarly, the methodology was also extended onto $s p^{2}-s p^{3}$ Heck cross-coupling reaction (Table 4.8, entries 1 and 2). There was no conversion observed when the Heck reaction was performed using alkyl iodide 4.44.1 and allyl iodide 4.44.2 under our optimized reaction condition.

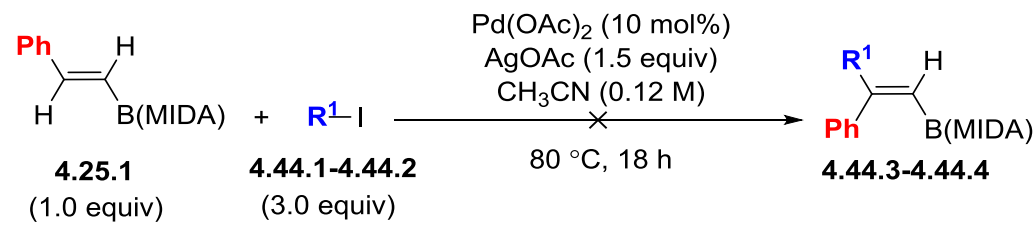

Scheme 4.44. Heck coupling between $s p^{2}-s p^{3}$ substrates. 


entry

Table 4.8. Heck coupling attempted on alkyl iodides.

$\underline{\text { Summary }}$

We have shown that the MIDA boronate $\mathbf{4}$.25.1 could be successfully used in the Heck coupling reaction with a series of substituted aryl iodides with various substitution patterns (ortho-, meta-and para-) to give tri-substituted aryl olefins in high yield and good stereoselectivity. However, the limitation of the reaction is in the steric sensitivity towards orthosubstituted aryl iodides.

Overall, the scope of the reaction involves the coupling of boron MIDA ester 4.25.1 with different types of iodide (aryl-, alkyl-, alkynyl-, heteroatomatics) where the Heck optimized reaction condition has demonstrated versatility but are limited by reactions between $s p-s p^{2}$ and $s p^{3}-s p^{2}$ coupling.

\subsubsection{Proposed Mechanism and Structure Determination}

The Heck cross-coupling reaction between 4.25.1 and aryl iodides 4.25.2 gave two separable regioisomers 4.25.3 and 4.25.4 (Scheme 4.45). 


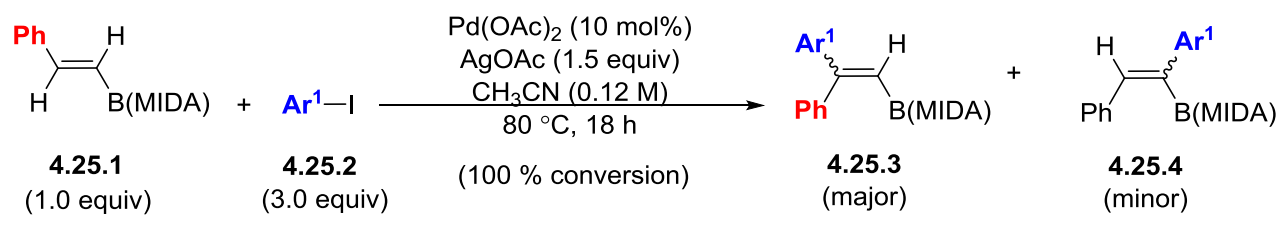

Scheme 4.45. Optimized reaction condition for the Heck coupling with MIDA boronate 4.25.1.

The catalytic cycle detailed in Scheme 4.46 is proposed in accordance to the data gathered from the rigorous ${ }^{1} \mathrm{H}$ NMR analysis of the regioisomers obtained from the Heck reaction and the X-ray crystallography. The simplest $\mathrm{Pd}(\mathrm{II}) / \mathrm{Pd}(\mathrm{IV})$ catalytic cycle is proposed where firstly, the oxidative addition of $\operatorname{Pd}(0)$ occurs with aryl iodide in the presence of the halophilic silver(I) (from AgOAc), which sequesters the iodide to form silver(I)iodide salts ${ }^{264}$ and the arylpalladium acetate $\mathbf{A}$. The subsequent olefin-coordination step gives complex $\mathbf{B}$ which was followed by the critical olefin insertion step to give C. It is during the carbometallation step that the regioselectivity of the eventual Heck products is decided. The proposed catalytic cycle shows the palladium(II) atom selectively inserts at the carbon $\alpha$-to the boron atom to be consistent with the outcome of the Heck reaction. The complex $\mathbf{C}$ then undergoes a $\mathrm{C}-\mathrm{C}$ bond rotation to give $\mathbf{D}$. The bond rotation is required to achieve a syn-coplanar conformation between the $s p^{3}$-bonded $\beta$-hydrogen and the palladium atom for the syn- $\beta$-hydride elimination to occur and give $\mathbf{E}$. As proposed by Heck, the $\beta$-hydride elimination can be a reversible process, ${ }^{263,265}$ and thus accounts for the isomerization in the final Heck products. ${ }^{263}$ Alkene dissociation then releases the Heck product while the base promoted reductive elimination of the the complex $\mathbf{F}$ regenerates the active catalyst $\operatorname{Pd}(0)$, which is ready to re-enter the catalytic cycle (Scheme 4.46). It is worth noting that in the absence of a phosphine ligands or an amine base during the Heck reaction, the formation of the active catalyst $\operatorname{Pd}(0)$ from the catalyst precursor Pd(II) is inspired by Jeffery's conditions ${ }^{263,266}$ where clusters of $\operatorname{Pd}(0)$ nanoparticles could be generated upon mixing $\mathrm{Pd}(\mathrm{OAc})$ and AgOAc. ${ }^{266 \mathrm{~b}}$ The nanoparticles would be stabilized by cations $\left(\mathrm{Ag}^{+}\right)$upon the $\mathrm{Pd}-\mathrm{OAc}$ bond cleavage. ${ }^{263}$ 


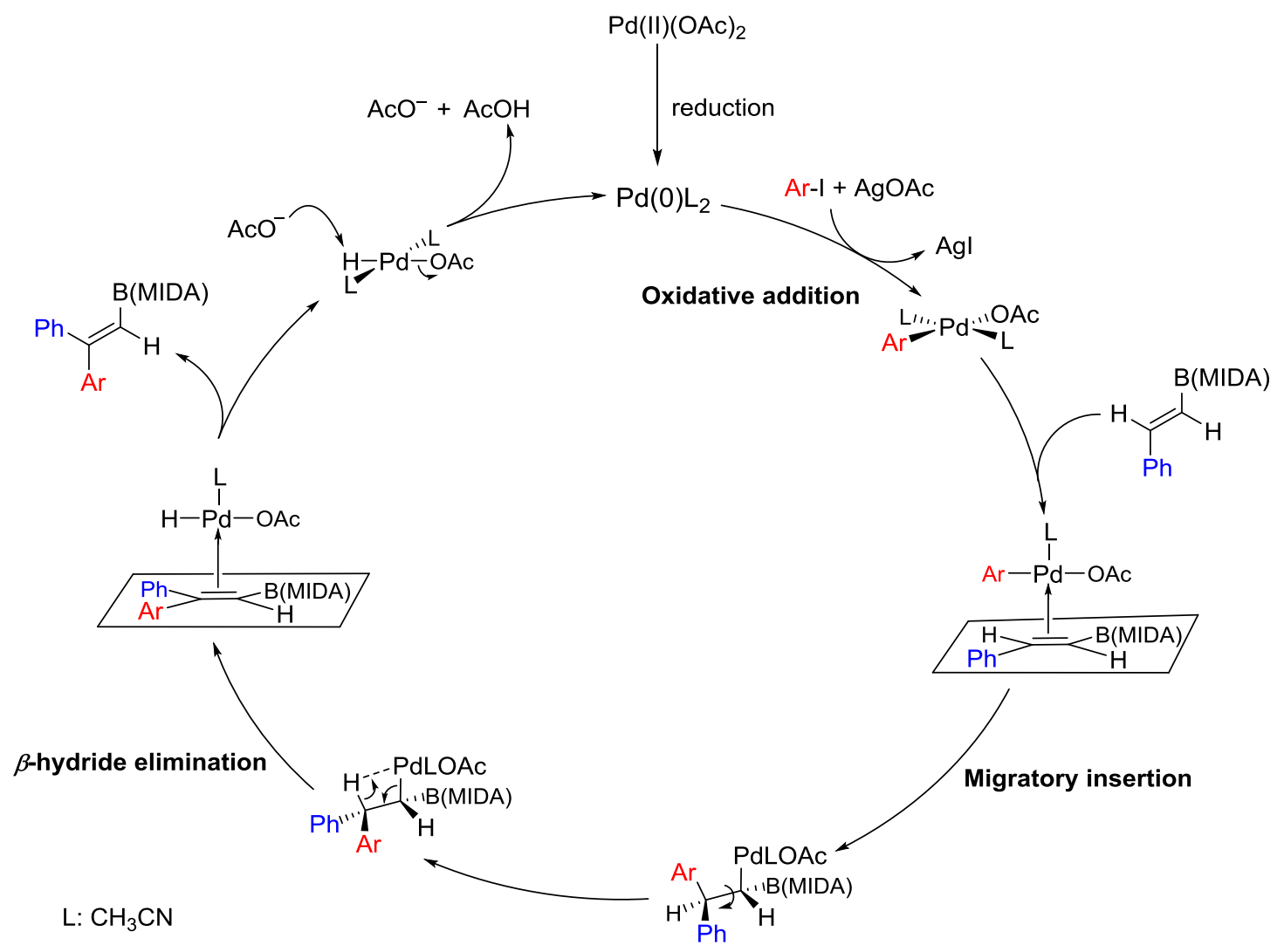

Scheme 4.46. Proposed catalytic cycle of the Heck cross-coupling reaction of boronate MIDA ester 4.25.1.

$\underline{\text { Regioselectivity of Major Product }}$

An example to illustrate the way in which the regioselectivity of the major Heck products were derived and determined is through analysis of the spectroscopic data of (E)-4.39.23 (Figures 4.6-4.8). Firstly, the ${ }^{1} \mathrm{H}$ NMR of substrate $(E)-\mathbf{4 . 3 9 . 2 3}$ reflects the characteristic singlet peak of $\mathrm{H} 3$ alkenyl proton (found at $6.35 \mathrm{ppm}$ ) (Figure X). Secondly, in the case of $(E)-4.39 .23$, the protons from the simple phenyl ring $(\mathrm{H} 13-\mathrm{H} 15)$ are distinguishable from the protons ( $\mathrm{H} 6$ and $\mathrm{H} 7$ ) attached to a phenyl ring bearing the methyl ketone (Figure 4.6). 


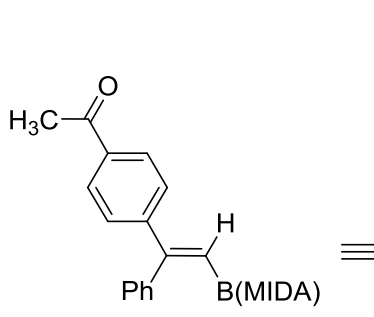

$(E)-4.39 .6$

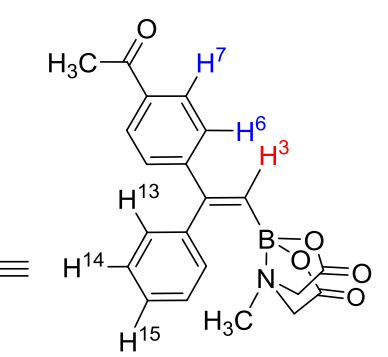

$(E)-4.39 .23$

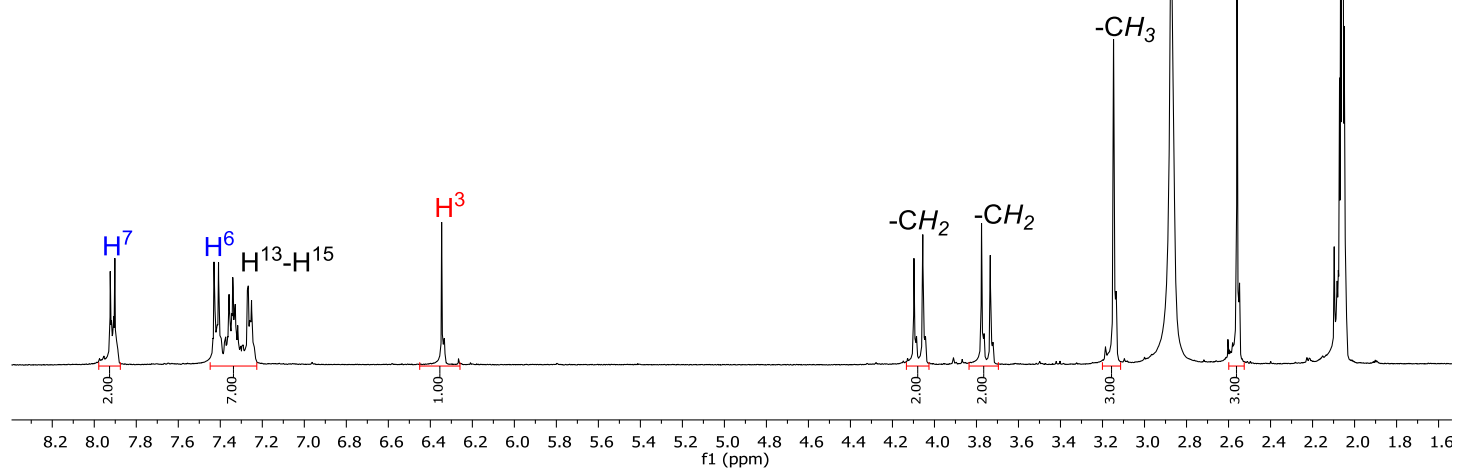

Figure 4.6. HMQC of Heck product (E)-4.39.23.

The proton $\mathrm{H} 3$ is positioned at $\mathrm{C} 3, \alpha$-to the boron atom, making it key in defining the regioselectivity of the major product (Figure 4.7). This is as the carbon atom directly linked to the boron does not show a coupling of ${ }^{13} \mathrm{C}$ with ${ }^{11} \mathrm{~B}$ due to the rapid quadrupolar relaxation rate which results in a loss of signal intensity. ${ }^{267}$ Consequently, the protons $\alpha$-to the boron atom will not show a correlation with its connecting carbon on the Heteronuclear Multiple Quantum Coherence (HMQC) spectrum. This is evident in the HMQC of substrate $(E)-\mathbf{4 . 3 9 . 2 3}$, where the $\mathrm{C}-\mathrm{H} 3$ coupling is absent (Figure 4.7), thereby confirming the proposed structure of the major regioisomer of the Heck products. 


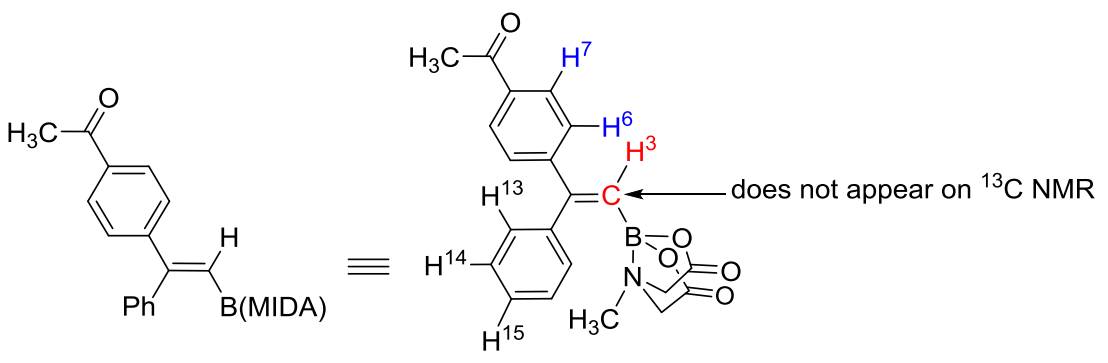

(E)-4.39.23

$(E)-4.39 .23$

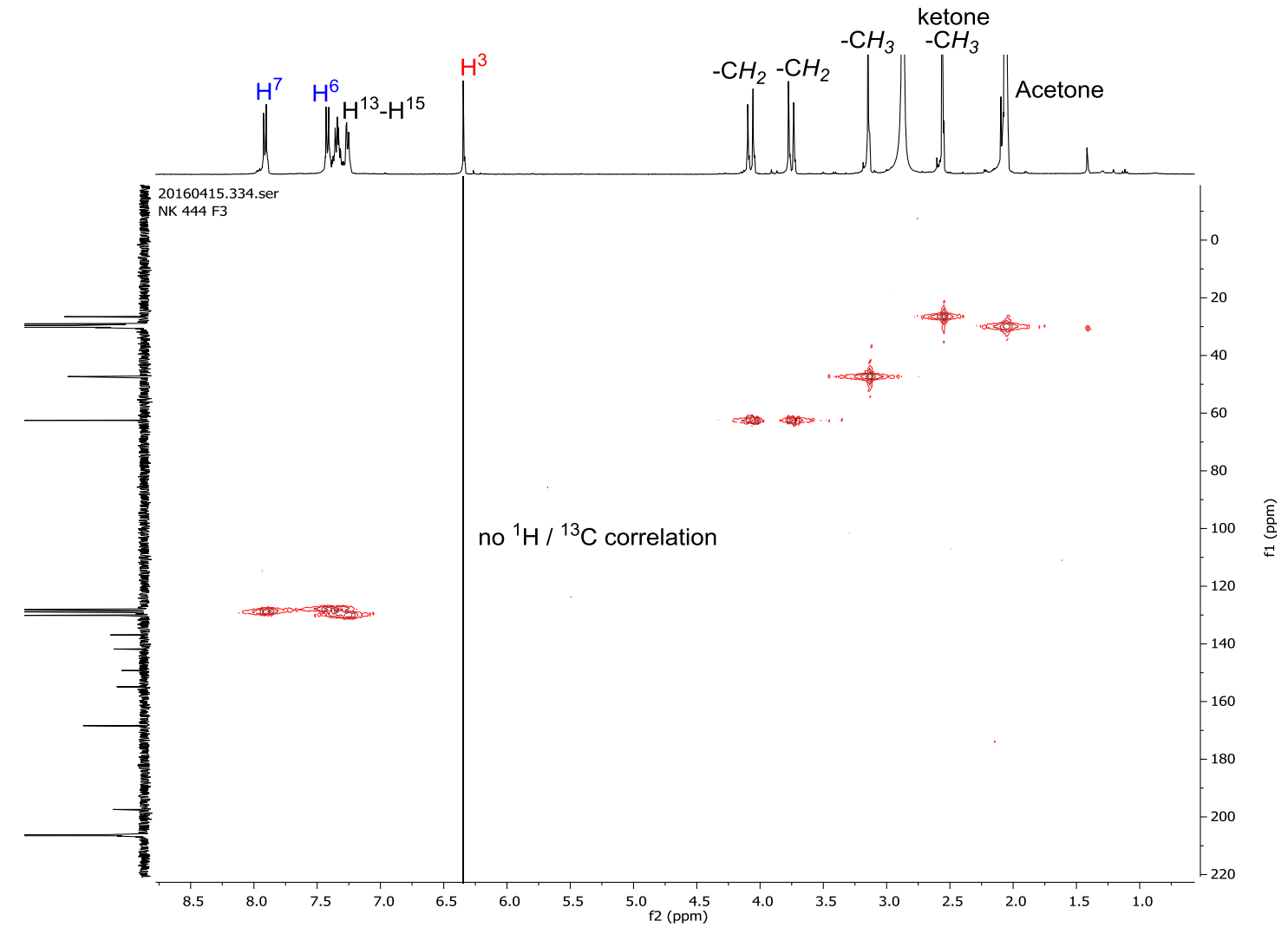

Figure 4.7. HMQC of Heck product (E)-4.39.23.

Stereoselectivity of Major Product

The stereoselectivity ( $E$ or $Z$ ), on the other hand, was validated by Nuclear Overhauser Effect Spectroscopy (NOESY) (Figure 4.8). It is worth pointing that this was only possible for olefins with electron-withdrawing aryl substituents because it allows for distinguishable aromatic protons. The correlation between $\mathrm{H} 3$ and $\mathrm{H} 6$ was clearly reflected in the NOESY experiment of $(E)-\mathbf{4 . 3 9 . 2 3}$, thereby confirming the proposed $(E)$-stereoselectivity for the Heck products (Figure 4.8). 


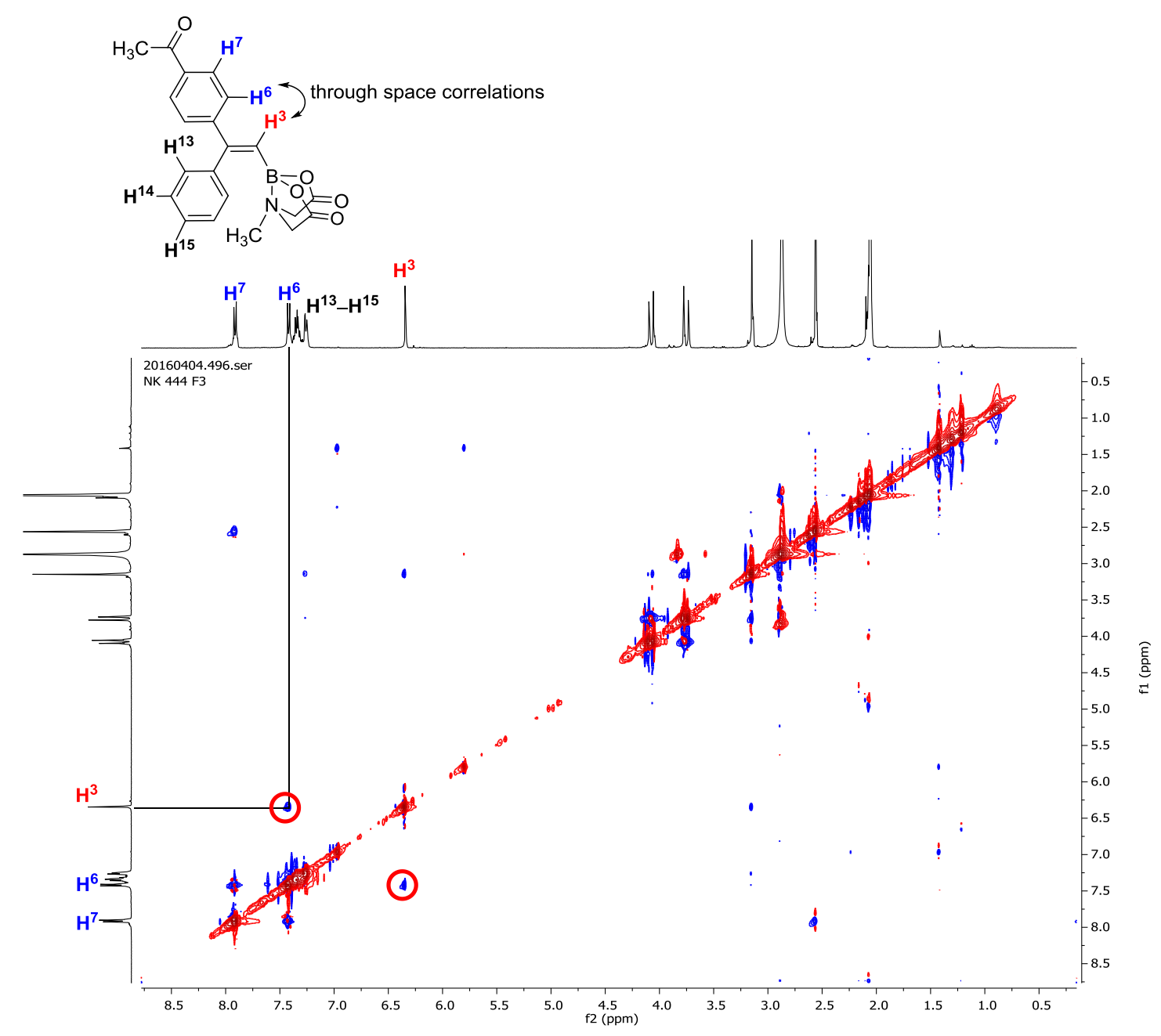

Figure 4.8. NOESY of Heck product (E)-4.39.23.

In cases where the aromatic protons are indistinguishable, the use of NMR techniques such as NOESY to determine the type of geometric isomerism was insufficient (Figure 4.9). This is especially applicable for the Heck tri-substituted ethylene products with electrondonating aryl substituents such as (E)-4.29.1. A validation in the form of an X-ray crystal structure of the single diastereomer $(E)$-4.29.1, obtained in the Heck reaction, was thus acquired (Figure 4.9). 


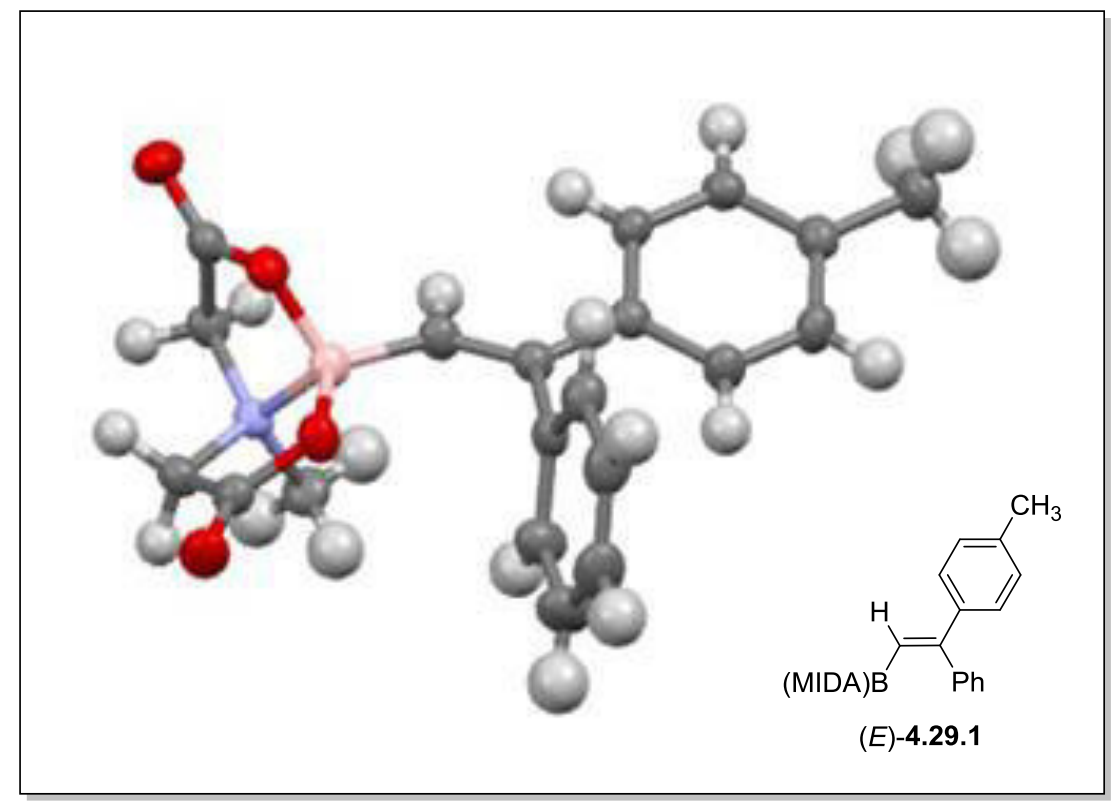

Figure 4.9. X-ray crystal structure of $(E)$-4.29.1.

\section{Hypothesis for the Observed Regioselectivity}

The yield of the minor regioisomer from the Heck reaction increased when aryl iodides bearing electron withdrawing groups were used $\left(\mathrm{R}=-\mathrm{CO}_{2} \mathrm{Et}\right.$, $\left.-\mathrm{COOH},-\mathrm{C}(\mathrm{O}) \mathrm{Me}\right)$ (refer to Section 4.2.3.3.1.1, Table 4.5). This observation led us to believe that the factors governing the regioselectivity was not solely based on steric interactions, but exists in combination with other factors such as the electronic factor.

We propose two possible transition state structures that may emerge from the 1,2-migratory insertion step of the $\mathrm{C}=\mathrm{C} \pi$-system into the $\mathrm{Pd}-\mathrm{Ar}^{1}$ bond (Scheme 4.47). There are two available carbon atoms for insertion, $\mathrm{C} \alpha$ and $\mathrm{C} \beta$ to the boron of MIDA boronate. The palladium insertion at $\mathrm{C} \alpha$ would give rise to transition state I (TS-I, Scheme 4.47), while the insertion at $\mathrm{C} \beta$ would give rise to transition state II (TS-II, Scheme 4.47). The reaction pathway through TS-I would subsequently deliver the major regioisomer while the minor regioisomer would be obtained through TS-II. 
In the event of TS-I, there would be a reduced steric interactions between the palladium atom and the boronate MIDA ester group potentially due to the slightly longer metal-vinyl (palladium-carbon) bond length of approximately $2.04 \AA^{268}$ compared to a vinyl $\mathrm{C}-\mathrm{C}$ bond length (such as styrene) of approximately $1.47 \AA$ (Scheme 4.47 ) ${ }^{269}$ On the contrary, TS-II suffers from destabilization due to steric hindrance between the aryl ring and the boronate MIDA ester group (TS-II, Scheme 4.47). Other factors such as electronic effects may play an equally significant role in the regioselectivity but these factors have not been systematically addressed. Overall, based on the results obtained from the Heck reaction, steric factor predominates as the major product ultimately derives from the proposed TS-I.

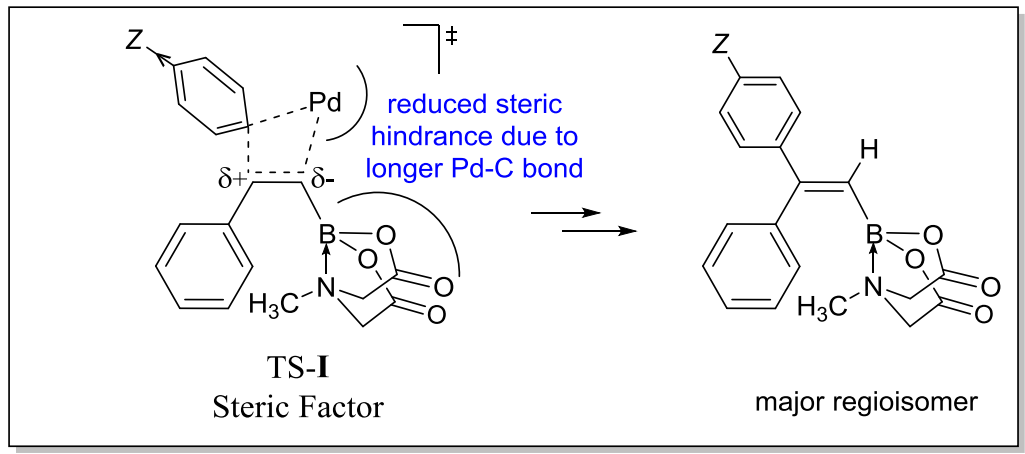

$Z$ = electron withdrawing substituents

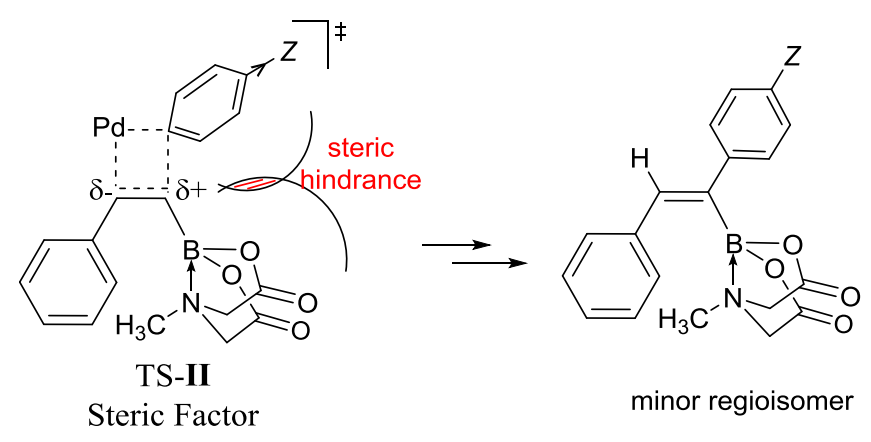

Note: Ligands coordinated to Pd atom have been omitted for clarity.

Scheme 4.47. Hypothetical transition states of 1,2-migratory insertion step. 


\subsubsection{Late-Stage Functionalization}

\subsection{Suzuki-Miyaura Cross-Coupling}

The presence of the MIDA boronate group in the final olefin product proves advantageous as it allows for a late-stage functionalization through coupling reactions (Scheme 4.48). An example was carried out to illustrate this point. The olefin $(E)-\mathbf{4 . 2 9 . 1}$ was employed in the Suzuki-Miyaura cross-coupling with a simple aromatic substrate 3-bromoanisole 4.48.1 to give the desired tri-aryl substituted olefin $\mathbf{4 . 4 8 . 2}$ in good yield of $80 \%$ to afford a single diastereomer (Scheme 4.48). This demonstrates the attachment of three distinctive aryl groups around the $\mathrm{C}=\mathrm{C}$ double bond through the Heck/Suzuki combination strategy.

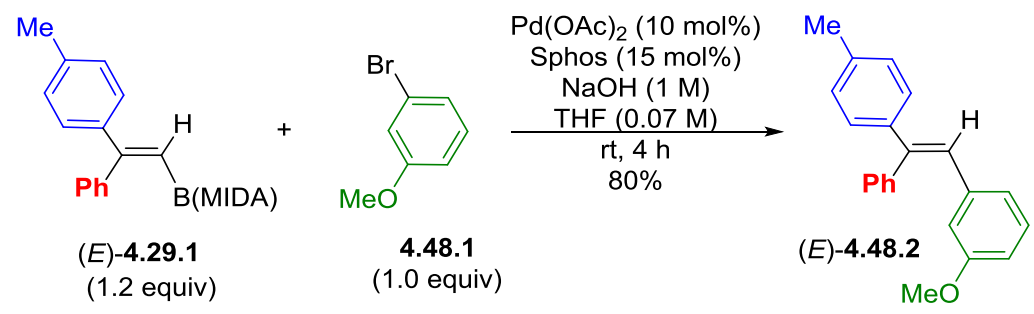

Scheme 4.48. Suzuki coupling between $(E)$-4.29.1 and 4.48.1 to give tri-aryl substituted olefin $(E)$-4.48.2

\subsection{Conditions towards Tetra-Aryl Substituted Olefins}

\section{Method I: Heck Reaction of Tri-Substituted Ethylenes}

The success in obtaining a tri-aryl substituted olefins has sparked an interest in the formation of tetra- substituted olefins 4.49.1-4.49.2 by using the Heck coupling reaction under forcing conditions (Scheme 4.49). Therefore, several conditions were tested including a change in temperature and solvent (Table 4.9, entries 1-3). Unfortunately, neither the increase of silver(II) acetate equivalent nor the reaction temperature led to any conversion of the trisubstituted starting materials (4.29.1 - 4.29.2) (Table 4.9, entries 1-3). 


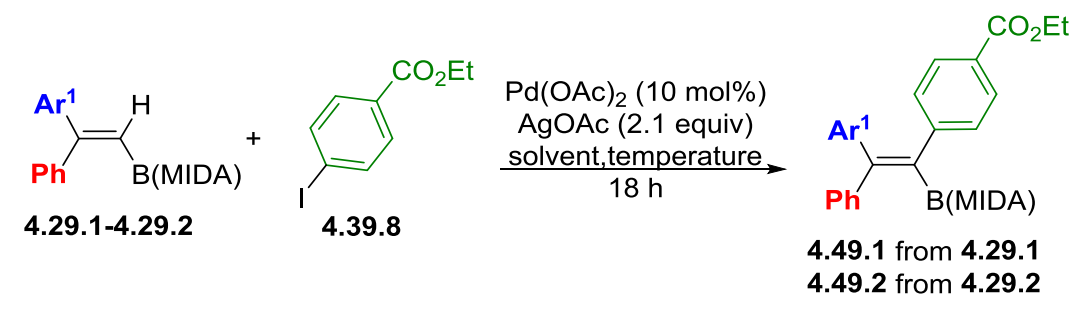

Scheme 4.49. Efforts towards tetra-substituted olefins 4.49.1 - 4.49.2.

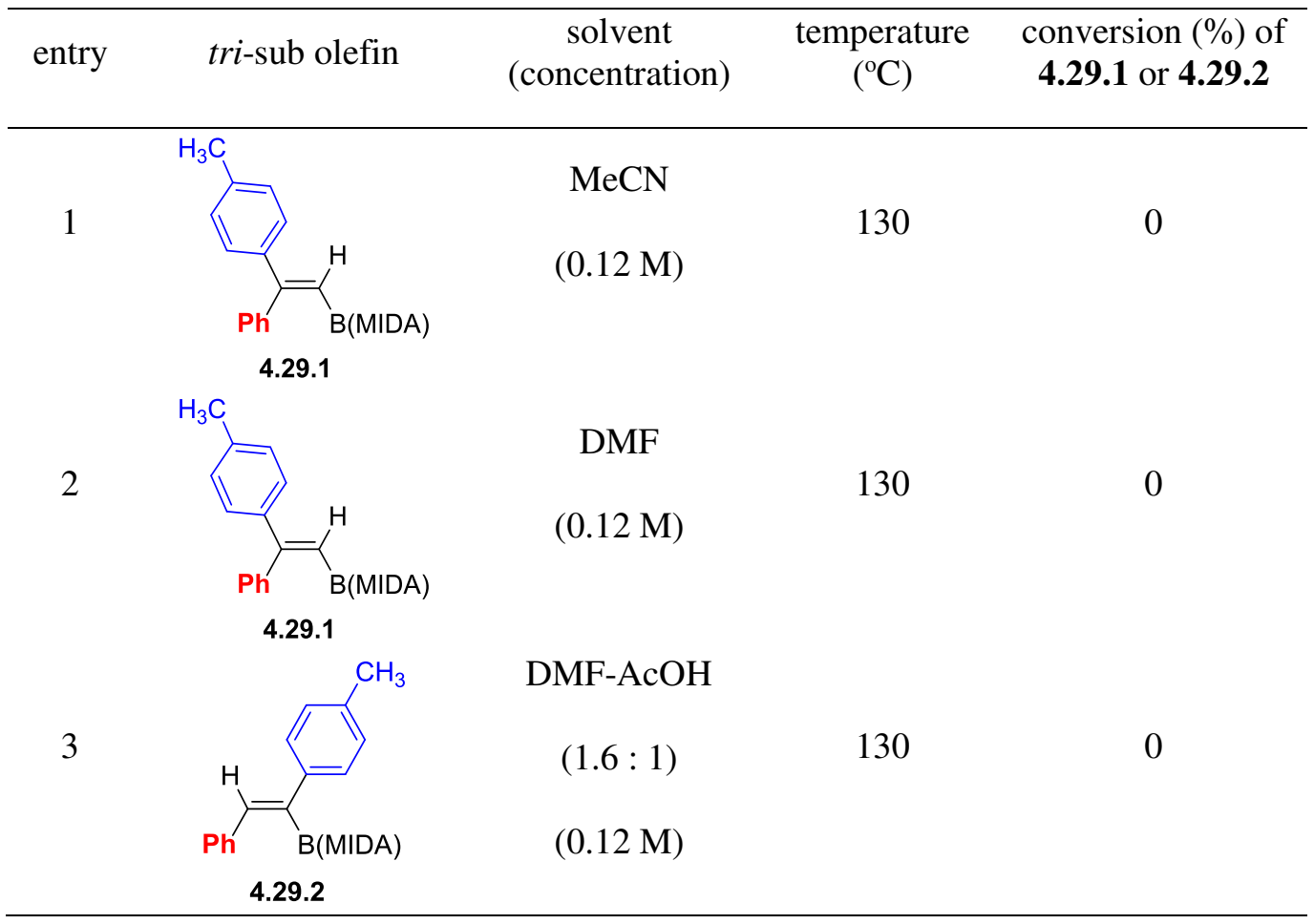

Table 4.9. Efforts towards tetra-substituted olefins 4.49.1-4.49.2.

Method II: Bromination of Tri-Substituted Ethylenes

The unsuccessful attempts shifted our focus to other synthetic alternatives such as bromination. According to the procedure described by Nunez et al. for the synthesis of tamoxifen in 2009, which was later reproduced by Cahiez et al. in 2014, ${ }^{239}$ the $\mathrm{C}-\mathrm{H}$ bond of the tri-aryl ethylene 4.25.3 can be converted into a $\mathrm{C}-\mathrm{Br}$ bond 4.50.1 through the use of $\mathrm{Br}_{2}$ solution in the presence of excess $\mathrm{Et}_{3} \mathrm{~N}$ (Scheme 4.50). ${ }^{239,270}$ 


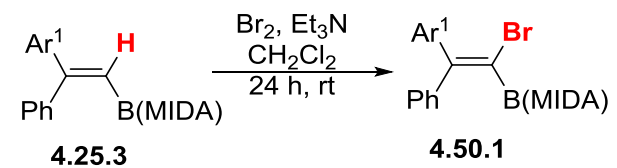

Scheme 4.50. Bromination of tri-aryl ethylene 4.50.1.

We had anticipated the dibrominated compound 4.51.1 which could be isolated and characterized prior to a $\mathrm{H}-\mathrm{Br}$ elimination with a suitable base $\left(\mathrm{Et}_{3} \mathrm{~N}\right)$ to give the desired tetrasubstituted olefin $(E)$-4.51.2, which could then be submitted to a Negishi or Suzuki-Miyaura cross-coupling to give the desired tetra-aryl ethylene (E)-4.51.3 (Scheme 4.51). However, when these conditions were applied on $(E)-4.48 .2$, the desired dibrominated 4.51.1 was not observed (Scheme 4.51). The reaction conditions resulted in an olefin isomerization to give (E/Z)-4.48.2. The isomers $(E / Z)-\mathbf{4 . 4 8 . 2}$ in a ratio of 4:1 were recovered exclusively with no traces of the desired product. The GC-MS confirmed the absence of any bromine atom present in the recovered mixture of stereoisomers (Scheme 4.51). 


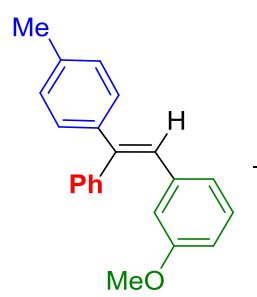

(E)-4.48.2

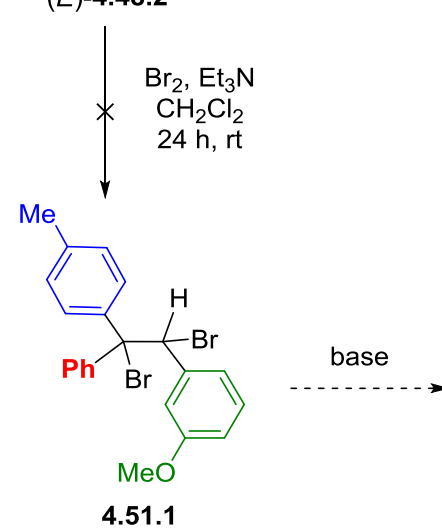

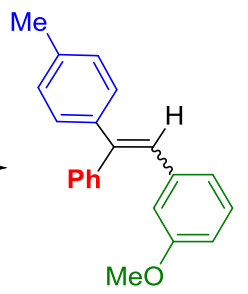

(E/Z)-4.48.2

(4:1)

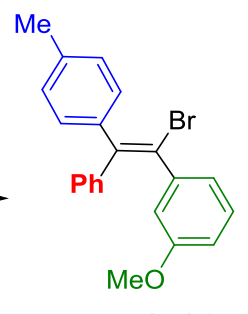

(E)-4.51.2

Negishi or Suzuki coupling

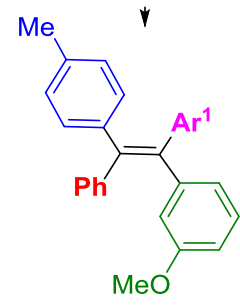

(E)-4.51.3

Scheme 4.51. Bromination of (E)-4.48.2 led to isomerization to give mixture of isomers $(E / Z)-4.48 .2$.

Therefore, the attempt to install the bromine functionality was re-attempted in two separate steps and applied on tri-aryl ethylene (E/Z)-4.48.2 following the procedure described by Okamura et al. (Scheme 4.52). ${ }^{271}$ Unfortunately 4.51.1 was not isolated in the dibromination of substrate (E/Z)-4.48.2, which gave a myriad of products (Scheme 4.52) that were inseparable by chromatography on silica gel, and led to a mixture of compounds. These products could not be identified by the analysis of ${ }^{1} \mathrm{H}$ NMR spectrum of the crude mixture because of the lack of characteristic peaks. This outcome could be the result of a simultaneous partial bromination of the electron-rich aryl rings and/or double bond (compounds 4.52.1-4.52.4). The lack of specificity in the bromination reaction was reflected in the ${ }^{1} \mathrm{H}$ NMR spectrum. The absence of 
the characteristic singlet corresponding to the dibrominated desired compound 4.51 .1 on the ${ }^{1} \mathrm{H}$ NMR confirmed that the desired product was not formed as part of the cocktail of products that was obtained.
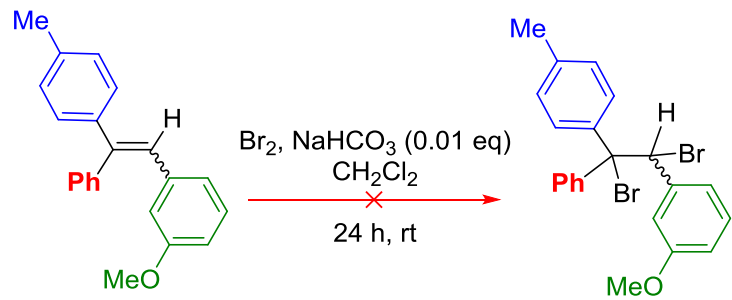

(E/Z)-4.48.2

4.51.1
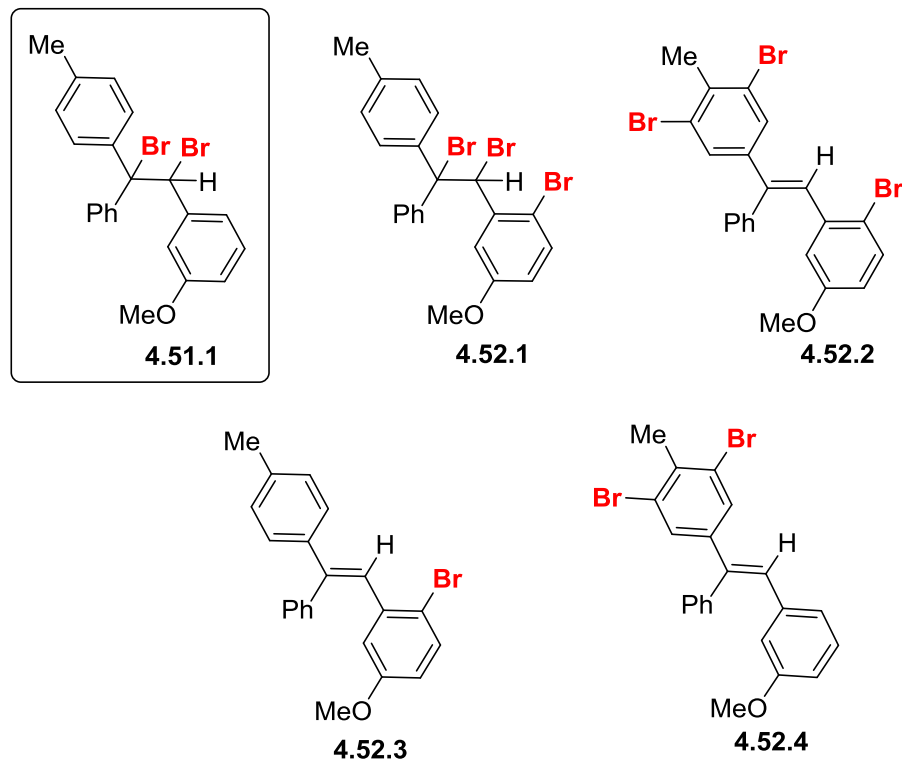

Scheme 4.52. Failed bromination of $(E / Z)-4.48 .2$ and possibilities of brominated products 4.52.1 - 4.52.4

\section{Conclusion}

In summary, a Heck cross-coupling between 1,2-phenyl vinyl boronate MIDA 4.25.1 ester and a range of aryl iodides $\mathbf{4 . 2 5 . 2}$ has been developed (Scheme 4.53). The reaction is efficient, high-yielding, regio- and stereoselective. The broad variety of tolerated aryl substituents including functionalizable groups such as a bromine atom reflects the versatility of the methodology. Moreover the late-stage functionalization of the remainder boronate MIDA ester present in the Heck products through the Suzuki-Miyaura cross-coupling, afforded an easy 
and modular access to tri-arylethylenes. Therefore, the flexible palladium-catalyzed Heck/Suzuki combination could emerge as a straightforward approach towards the construction of libraries of tri-arylethylene derivatives.

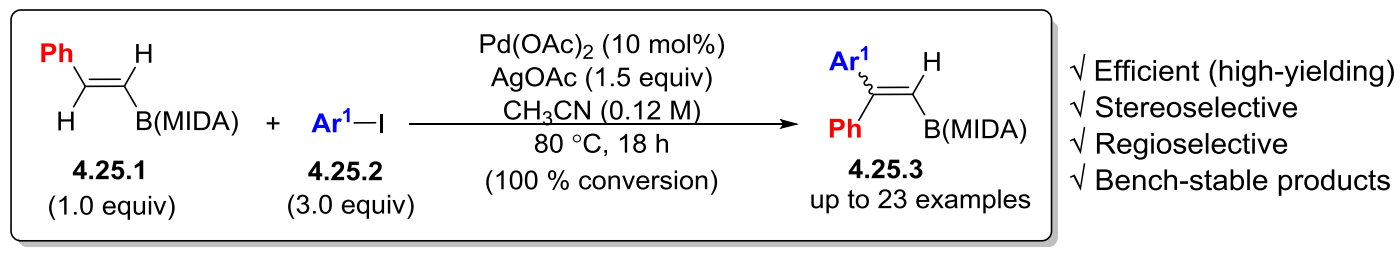

Scheme 4.53. Heck cross-coupling between 4.25.1 ester and a range of aryl iodides 4.25.2

\subsubsection{Perspectives}

The scope of the Heck reaction could benefit from a detailed catalyst screening where reaction involving electron deficient bromides could then be repeated. The obvious limitation of the developed method is the choice of aryl group at the $\mathrm{C} 2$ position of the organoboron coupling olefin 4.25.1, which is currently limited to a simple phenyl ring (Scheme 4.54). Recently, Watson et al. has developed a palladium-catalyzed boryl-Heck reaction which transforms terminal olefins into trans-alkenyl boronic esters. ${ }^{260}$ Although a library of diverse alkenyl boronate derivatives were synthesized, no examples of alkenyl boronate MIDA esters were reported. Therefore, it is worth evaluating the outcome of the boryl-Heck alkenylation of substituted styrenes with boronate MIDA ester under the optimized conditions established by Watsons et al.

Alternatively the originally adopted cross-metathesis strategy reported by Burke et al..$^{257}$ to access 4.25.1, as reflected in Scheme 4.54, could be extended to a panel of substituted styrenes with vinyl boron MIDA ester 4.26.1 to create a library of 1,2-aryl vinyl boron MIDA esters as substrates for the Heck arylation of aryl iodides. These 1,2-aryl vinyl boron MIDA derivatives, have also been reported by Burke et $a .^{257}$ 
In contrast to the developed methodology which proceeds in a stepwise manner by first obtaining the $d i$-substituted olefin 4.25.1 followed by the Heck coupling (Scheme 4.54), the access to tri-substituted olefins with two different types of aromatic groups attached can be carried out under suitable coupling conditions employing two different aromatic halides to give gem-arylated olefins 4.55.1 in a one-pot synthesis (Scheme 4.55). Although the Heck diarylation of vinyl boron MIDA ester 4.26.1 is a more attractive option, it is also more challenging as the optimal conditions for the controlled mono Heck cross-coupling would need to be established. This requires screening of a series of catalytic systems including, but not limited to, the effects of different catalysts, temperatures, types of ligands and solvents. Moreover, the potential of attaching distinctive aryl groups around the $\mathrm{C}=\mathrm{C}$ by the controlled double Heck arylation removes the limitation of having a phenyl ring on $\mathrm{C} 2\left(\mathrm{Ar}^{1} \neq \mathrm{Ph}\right)$, thereby creating a diversified library of tri-substituted ethylenes. (Scheme 4.55)

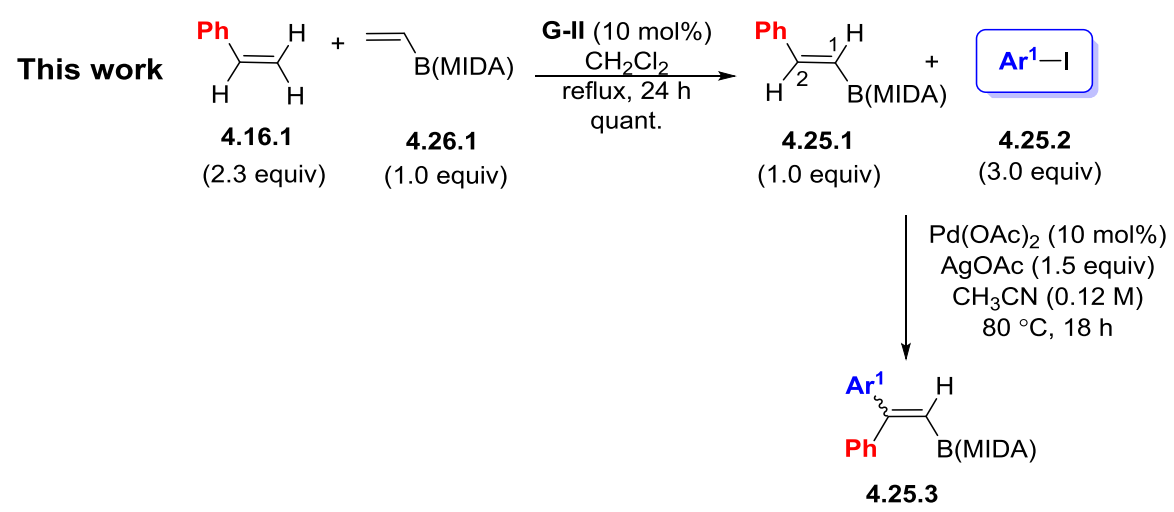

Scheme 4.54. Developed stepwise access to tri-substituted olefins 4.25.3 from styrene 4.16.1.

Future work

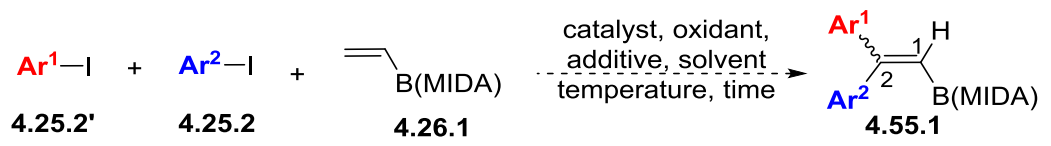

Scheme 4.55. One-pot double Heck arylation of vinyl boron MIDA ester 4.26.1. 
This page has been intentionally left blank. 
This page has been intentionally left blank. 


\section{Chapter 5}

\subsection{Experimental}

5.1.1 Experimental Procedure for Chapter I

5.1.2 Experimental Procedure for Chapter II

5.1.3 Experimental Procedure for Chapter III

5.1.4 Experimental Procedure for Chapter IV 
This page has been intentionally left blank. 


\subsection{Experimental}

\section{General Information}

All anhydrous and oxygen sensitive reactions were performed either under argon or nitrogen atmosphere using oven-dried glassware $\left(120^{\circ} \mathrm{C}\right)$, that were cooled under vacuum. Anhydrous THF and $\mathrm{E}_{\mathrm{t} 2} \mathrm{O}$ were obtained by distillation from sodium metal and benzophenone prior to use under nitrogen. Anhydrous $\mathrm{CH}_{2} \mathrm{Cl}_{2}$ was obtained by distillation from $\mathrm{CaH}_{2}$ prior to use under nitrogen atmosphere. Anhydrous methanol was obtained by distillation from activated magnesium under nitrogen. All other anhydrous solvents and reagents, that were commercially available, were used as received.

Proton nuclear magnetic resonance $\left({ }^{1} \mathrm{H}\right.$ NMR) spectra were recorded using the Bruker Advance DPX at 300,400 , or $500 \mathrm{MHz}$ or JEOL ECA $400 \mathrm{MHz}$ in deuterated solvents (Chloroform- $d$, acetone- $d_{6}$ or deuterium oxide) and the same instruments recorded the ${ }^{13} \mathrm{C}$ NMR spectra at 75, 100 or $125 \mathrm{MHz}$. The peak positions were recorded in ppm relative to an internal standard and coupling constants $J$ were recorded in $\mathrm{Hz}$.

Flash chromatography on silica gel was performed with silica gel 230 - 400 mesh. Mass spectra were recorded on Finnigan LCQ DECA XP MAX Ultra instrument or a Finnigan PolarisQ, GCMS XP mass spectrometer. The ionization techniques used were either electron impact (EI) or electrospray (ESI). High Resolution Mass Spectroscopy (HRMS) was performed using Waters Q-Tof premier Mass Spectrometer. Infrared spectra were recorded neat using Bruker Optics TESOR 27 FT-IR Spectrometer, or with nujol on NaCl plate using Shimadzu IR Prestige-21 FT-IR or Bruker Alpha-E FTIR.

Specific optical rotations were recorded on Jasco-1030 polarimeter. The units provided were $10^{-1} \mathrm{deg} \cdot \mathrm{cm}^{2} \cdot \mathrm{g}^{-1}$. The wavelength at which angles of rotations were measured was $589 \mathrm{~nm}$. 
This page has been intentionally left blank. 


\subsubsection{Experimental Procedure for Chapter I}

(S)-1-chloropent-4-en-2-ol, (1.36.5):

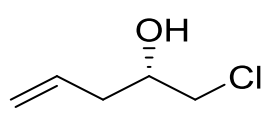

Vinyl magnesium bromide was prepared by dropwise addition of vinyl bromide (10.0 $\mathrm{mL})$ to a stirring suspension of magnesium turnings $(0.73 \mathrm{~g}, 30.00 \mathrm{mmol})$ in anhydrous tetrahydrofuran $(35 \mathrm{~mL})$. The mixture was heated at reflux for 1 hour. The vinyl magnesium bromide was added dropwise to a cooled $\left(-68{ }^{\circ} \mathrm{C}\right)$ solution of $(S)$-epichlorohydrin $\mathbf{1 . 3 6 . 6}(0.85 \mathrm{~mL}, 10.81 \mathrm{mmol})$ containing copper(I)iodide $(0.21 \mathrm{~g}, 1.08 \mathrm{mmol})$ in anhydrous diethyl ether $(45 \mathrm{~mL})$. The mixture was stirred at $-68{ }^{\circ} \mathrm{C}$ for 1 hour. The reaction mixture was quenched with saturated aqueous ammonium chloride and allowed to warm to room temperature. The layers were separated and the aqueous layer was extracted with diethyl ether $(3 \times 10 \mathrm{~mL})$. The combined organic extracts were washed with brine, dried over anhydrous magnesium sulphate, filtered through celite and concentrated in vacuo to give $\mathbf{1 . 3 6 . 5}(1.17 \mathrm{~g}, 90 \%)$ as a colourless clear oil.

Formula: $\mathrm{C}_{5} \mathrm{H}_{9} \mathrm{ClO}$

Mass: $120.6 \mathrm{~g} / \mathrm{mol}$

${ }^{1} \mathrm{H}$ NMR $\left(400 \mathrm{MHz}, \mathrm{CDCl}_{3}\right): \delta 5.82\left(1 \mathrm{H}, \mathrm{ddt}, J=17.1,7.1,3.2 \mathrm{~Hz}, \mathrm{R}-\mathrm{CH}=\mathrm{CH}_{2}\right), 5.17(2 \mathrm{H}, \mathrm{dd}$, $\left.J=13.1,1.4 \mathrm{~Hz}, \mathrm{R}-\mathrm{CH}=\mathrm{CH}_{2}\right), 3.88\left(1 \mathrm{H}, \mathrm{m}, \mathrm{Cl}-\mathrm{CH}_{2}-\mathrm{CH}-\mathrm{R}\right) 3.63(1 \mathrm{H}, \mathrm{dd}, J=11.2,4.1 \mathrm{~Hz}, \mathrm{Cl}-$ $\mathrm{CHH}-\mathrm{R}), 3.51(1 \mathrm{H}, \mathrm{dd}, J=11.2,6.8 \mathrm{~Hz}, \mathrm{Cl}-\mathrm{CH} H-\mathrm{R}), 2.35\left(2 \mathrm{H}, \mathrm{m}, \mathrm{R}-\mathrm{CH}_{2}-\mathrm{CH}=\mathrm{CH}_{2}\right)$.

${ }^{13}$ C NMR (100 MHz, $\left.\mathrm{CDCl}_{3}\right): \delta 133.2,118.6,70.5,49.3,38.6$.

MS (ESI) $m / z$ (rel intensity): $121[\mathrm{M}+\mathrm{H}]^{+}$

The obtained data are consistent with literature values ${ }^{1}$ 


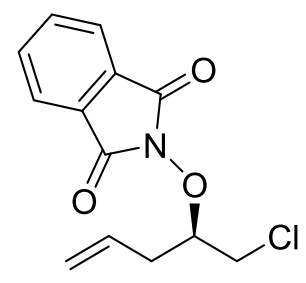

(S)-1-Chloropent-4-en-2-ol, 1.36.5 (2.00 g, $16.60 \mathrm{mmol})$ in anhydrous tetrahydrofuran $(50 \mathrm{~mL})$ was added to a mixture of $N$-hydroxyphthalimide $(3.25 \mathrm{~g}, 19.90 \mathrm{mmol})$ and triphenylphosphine $(6.53 \mathrm{~g}, 24.70 \mathrm{mmol})$. The mixture was then cooled to $0{ }^{\circ} \mathrm{C}$ and diisopropyl azodicarboxylate (5.80 $\mathrm{mL}, 29.70 \mathrm{mmol})$ in anhydrous tetrahydrofuran $(5 \mathrm{~mL})$ was added dropwise with the temperature maintained at $0{ }^{\circ} \mathrm{C}$ until complete addition. The reaction mixture was warmed to room temperature and left to stir overnight. The solvent was removed in vacuo. The residue was purified by dry-loading flash chromatography on silica gel eluting with $($ Hexane/EtOAc $=100: 0$ to $85: 15)$ to give the hydroxylamine $\mathbf{1 . 3 7 . 1}(4.30 \mathrm{~g}, 98 \%)$ as a colourless solid.

Formula: $\mathrm{C}_{13} \mathrm{H}_{12} \mathrm{ClNO}_{3}$

Mass: $265.7 \mathrm{~g} / \mathrm{mol}$

$\mathbf{M p}=60-71^{\circ} \mathrm{C}$

IR $v_{\max }$ (neat): $1790,1732,1612,1188 \mathrm{~cm}^{-1}$

${ }^{1} \mathbf{H}$ NMR (400 MHz, $\left.\mathrm{CDCl}_{3}\right): \delta$ 7.90-7.76 (4H, m, Ar- $\left.H\right), 5.96(1 \mathrm{H}, \mathrm{ddt}, J=17.4,7.3,3.2 \mathrm{~Hz}$ $\left.\mathrm{R}-\mathrm{C} H=\mathrm{CH}_{2}\right), 5.23(1 \mathrm{H}, \mathrm{dd}, J=16.7,1.4 \mathrm{~Hz}, \mathrm{R}-\mathrm{CH}=\mathrm{C} H \mathrm{H}), 5.21(1 \mathrm{H}, \mathrm{dd}, J=11.7,1.8 \mathrm{~Hz}, \mathrm{R}-$ $\mathrm{CH}=\mathrm{CH} H), 4.45\left(1 \mathrm{H}, \mathrm{m}, \mathrm{Cl}-\mathrm{CH}_{2}-\mathrm{CH}-\mathrm{R}\right), 3.78\left(2 \mathrm{H}, \mathrm{dd}, J=11.9,4.6 \mathrm{~Hz}, \mathrm{Cl}-\mathrm{CH}_{2}-\mathrm{R}\right), 2.73(1 \mathrm{H}$, ddd, $\left.J=14.7,6.4,6.0 \mathrm{~Hz}, \mathrm{R}-\mathrm{CHH}-\mathrm{CH}=\mathrm{CH}_{2}\right), 2.63(1 \mathrm{H}, \mathrm{ddd}, J=16.1,11.2,6.8 \mathrm{~Hz}, \mathrm{R}-\mathrm{CH} H-$ $\left.\mathrm{CH}=\mathrm{CH}_{2}\right)$.

${ }^{13} \mathrm{C}$ NMR (100 MHz, $\left.\mathrm{CDCl}_{3}\right): \delta 163.7,134.6,131.8,128.8,123.6,118.9,86.0,43.4,34.9$.

GCMS (ESI) $m / z$ (rel intensity): $289[\mathrm{M}+\mathrm{Na}]^{+}$ 
HRMS (ESI-TOF): Calculated for $\mathrm{C}_{13} \mathrm{H}_{13}{ }^{35} \mathrm{ClNO}_{3}[\mathrm{M}+\mathrm{H}]^{+}:$266.0584. Found: 266.0580 .

$[\alpha]_{\mathbf{D}}{ }^{23}:+8.9\left(c=0.2, \mathrm{CH}_{2} \mathrm{Cl}_{2}\right)$.

$(R)$ - $O$-(1-chloropent-4-en-2-yl)hydroxylamine, (1.37.1a):<smiles>C=CCC(CCl)ON</smiles>

Hydrazine monohydrate $(0.45 \mathrm{~mL}, 14.2 \mathrm{mmol})$ was added to a solution of compound $\mathbf{1 . 3 7 . 1}$ $(0.59 \mathrm{~g}, 2.22 \mathrm{mmol})$ in dichloromethane $(20 \mathrm{~mL})$ at $0{ }^{\circ} \mathrm{C}$. The mixture was warmed to room temperature, stirred for 15 minutes, filtered through celite, and concentrated in vacuo to give the hydroxylamine 1.37.1a $(0.27 \mathrm{~g}, 90 \%)$ as a colourless oil which was used without further purification.

Formula: $\mathrm{C}_{5} \mathrm{H}_{10} \mathrm{ClNO}$

Mass: $135.6 \mathrm{~g} / \mathrm{mol}$

IR $v_{\max }$ (neat): $3306,2982,1043 \mathrm{~cm}^{-1}$

${ }^{1} \mathbf{H}$ NMR $\left(400 \mathrm{MHz}, \mathrm{CDCl}_{3}\right): \delta 5.80\left(1 \mathrm{H}, \mathrm{ddt}, J=17.4,7.3,3.2 \mathrm{~Hz}, \mathrm{R}-\mathrm{CH}=\mathrm{CH}_{2}\right), 5.17(1 \mathrm{H}, \mathrm{dd}$, $J=3.2,1.4 \mathrm{~Hz}, \mathrm{C} H \mathrm{H}=\mathrm{CHR}), 5.13(1 \mathrm{H}, \mathrm{dd}, J=3.9,1.4 \mathrm{~Hz}, \mathrm{CH} H=\mathrm{CH}-\mathrm{R}), 3.77\left(1 \mathrm{H}, \mathrm{m}, \mathrm{Cl}_{-} \mathrm{CH}_{2}-\right.$ $\mathrm{CH}-\mathrm{R}), 3.64\left(2 \mathrm{H}, \mathrm{dd}, J=11.4,4.6 \mathrm{~Hz}, \mathrm{R}-\mathrm{CH}_{2}-\mathrm{Cl}\right), 2.42(1 \mathrm{H}, \mathrm{ddd}, J=11.5,7.6,1.4 \mathrm{~Hz}, \mathrm{R}-\mathrm{C} H \mathrm{H}-$ Cl), $2.36(1 \mathrm{H}, \mathrm{ddd}, J=11.8,7.1,1.4 \mathrm{~Hz}, \mathrm{R}-\mathrm{CH} H-\mathrm{Cl})$.

${ }^{13}$ C NMR (100 MHz, $\left.\mathrm{CDCl}_{3}\right): \delta 134.1,117.6,80.4,48.9,38.6$.

MS (ESI) $m / z$ (rel intensity): $158(\mathrm{M}+\mathrm{Na})^{+}$

HRMS (ESI-TOF): Calculated for $\mathrm{C}_{5} \mathrm{H}_{11}{ }^{35} \mathrm{CINO}[\mathrm{M}+\mathrm{H}]^{+}$: 136.0529; found: 136.0528 .

$[\alpha]_{\mathbf{D}}{ }^{21}:+3.0\left(c=1.0, \mathrm{CH}_{2} \mathrm{Cl}_{2}\right)$. 


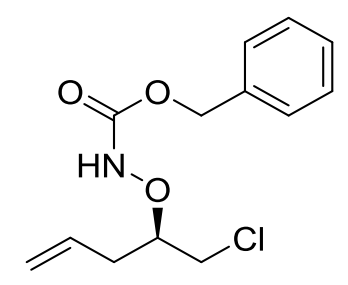

Benzyl chloroformate $(1.67 \mathrm{~mL}, 12.30 \mathrm{mmol})$ was added to a solution of hydroxylamine 1.37.1a (1.25 g, $10.30 \mathrm{mmol})$ in $\mathrm{CH}_{2} \mathrm{Cl}_{2} / \mathrm{H}_{2} \mathrm{O}(1: 1)(78 \mathrm{~mL})$ containing anhydrous sodium carbonate $(1.31 \mathrm{~g}, 12.30 \mathrm{mmol})$ at $0{ }^{\circ} \mathrm{C}$. The mixture was stirred at room temperature for 3 hours. The organic layer was separated, and the aqueous layer was extracted with $\mathrm{CH}_{2} \mathrm{Cl}_{2}(3 \times 20 \mathrm{~mL})$. The combined organic layers were dried over anhydrous magnesium sulphate, filtered and concentrated in vacuo. The residue was purified by flash chromatography on silica gel eluting with $($ Hexane/EtOAc $=75: 25)$ to give hydroxylamine 1.36.4 $(2.39 \mathrm{~g}, 86 \%)$ as a colourless oil.

Formula: $\mathrm{C}_{13} \mathrm{H}_{16} \mathrm{ClNO}_{3}$

Mass: $369.7 \mathrm{~g} / \mathrm{mol}$

IR $v_{\max }$ (neat): 3083, 2982, 1707, 1647, 754, $698 \mathrm{~cm}^{-1}$.

${ }^{1} \mathbf{H}$ NMR $\left(400 \mathrm{MHz}, \mathrm{CDCl}_{3}\right): \delta$ 7.40-7.34 (5H, m, Ar-H), $5.81(1 \mathrm{H}, \mathrm{ddt}, J=17.4,7.3,3.2 \mathrm{~Hz}$, $\left.\mathrm{CH}_{2}=\mathrm{CH}-\mathrm{R}\right), 5.18\left(2 \mathrm{H}, \mathrm{s}, \mathrm{Ph}-\mathrm{CH}_{2}-\mathrm{R}\right), 5.15\left(2 \mathrm{H}, \mathrm{dd}, J=5.0,1.8 \mathrm{~Hz}, \mathrm{CH}_{2}=\mathrm{CH}-\mathrm{R}\right), 4.10(1 \mathrm{H}, \mathrm{m}$, Cl-CH $2-\mathrm{CH}-\mathrm{R}), 3.72(1 \mathrm{H}, \mathrm{dd}, J=11.9,5.0 \mathrm{~Hz}, \mathrm{Cl}-\mathrm{C} H \mathrm{H}-\mathrm{R}), 3.63(1 \mathrm{H}, \mathrm{dd}, J=7.5,4.6 \mathrm{~Hz}, \mathrm{Cl}-$ CHH-R), 2.49 (2H, ddd, $J=7.5,3.4,1.4 \mathrm{~Hz}, \mathrm{R}-\mathrm{CH}_{2}-\mathrm{CH}=\mathrm{CH}_{2}$ ).

${ }^{13}$ C NMR $\left(100 \mathrm{MHz}, \mathrm{CDCl}_{3}\right): \delta 157.8,135.4,132.9,128.7,128.6,128.5,118.6,83.9,67.9$, $43.9,34.8$

MS (ESI) $m / z$ (rel intensity): $m / z: 270[\mathrm{M}+\mathrm{H}]^{+}$

HRMS (ESI-TOF) $m / z$ : Calculated for $\mathrm{C}_{13} \mathrm{H}_{16}{ }^{35} \mathrm{ClNO}_{3} \mathrm{Na}[\mathrm{M}+\mathrm{Na}]^{+}:$292.0716; found: 292.0713. 
HRMS (ESI-TOF) $m / z$ : Calculated for $\mathrm{C}_{13} \mathrm{H}_{16}{ }^{37} \mathrm{ClNO}_{3} \mathrm{Na}[\mathrm{M}+\mathrm{Na}]^{+}$: 294.0687; found: 294.0680.

$[\alpha]_{\mathbf{D}}{ }^{22}:+27.8\left(c=\mathrm{m} 0.3, \mathrm{CH}_{2} \mathrm{Cl}_{2}\right)$.

(5R)-benzyl 5-(chloromethyl)-3-methoxyisoxazolidine-2-carboxylate, (1.36.3):<smiles>CO[C@H]1C[C@H](CCl)ON1C(=O)OCc1ccccc1</smiles>

Amberlyst-15 (66.0 mg, $0.48 \mathrm{mmol})$ was added to a solution of $\mathbf{1 . 3 6 . 4}(2.56 \mathrm{~g}, 9.50 \mathrm{mmol})$ in methanol $(52 \mathrm{~mL})$ at $-78{ }^{\circ} \mathrm{C}$. The temperature was maintained at $-78^{\circ} \mathrm{C}$ while a mixture of ozone and oxygen was bubbled through the solution until a blue colour was observed. Dimethyl sulfide $(8.40 \mathrm{~mL})$ was added to the solution and it was stirred overnight, then filtered and concentrated in vacuo. Water was added, and the mixture was extracted with ethyl acetate (3 X $30 \mathrm{~mL})$. The combined organic extracts were washed with brine, dried over anhydrous magnesium sulphate, filtered through celite and concentrated in vacuo to give isoxazolidine $\mathbf{1 . 3 6 . 3}(1.95 \mathrm{~g}, 72 \%)$ as a colourless clear oil and as an inconsequential 1:1 mixture of diastereomers.

Formula: $\mathrm{C}_{13} \mathrm{H}_{16} \mathrm{ClNO}_{4}$

Mass: $285.7 \mathrm{~g} / \mathrm{mol}$

IR $v_{\max }$ (neat): 3034, 2955, 1454, 1741, 7752, $698 \mathrm{~cm}^{-1}$.

${ }^{1} \mathrm{H}$ NMR $\left(400 \mathrm{MHz}, \mathrm{CDCl}_{3}\right): \delta$ 7.40-7.31 $(5 \mathrm{H}, \mathrm{m}, \mathrm{Ar}-H), 5.47(1 \mathrm{H}, \mathrm{dd}, J=6.3,1.6 \mathrm{~Hz}, \mathrm{MeO}-$ $\mathrm{CH}-\mathrm{R})$, 5.27-5.17 (3H, m, MeO-CH'-R and $\left.\mathrm{Ph}_{-} \mathrm{CH}_{2}-\mathrm{R}\right), 4.63\left(1 \mathrm{H}, \mathrm{m}, \mathrm{ClCH}_{2}-\mathrm{CH}-\mathrm{R}\right), 4.35(1 \mathrm{H}$, m, $\left.\mathrm{ClCH}_{2}-\mathrm{C} H^{\prime}-\mathrm{R}\right), 3.71(1 \mathrm{H}, \mathrm{dd}, J=11.4,5.5 \mathrm{~Hz}, \mathrm{R}-\mathrm{C} H \mathrm{H}-\mathrm{Cl}), 3.64(1 \mathrm{H}, \mathrm{dd}, J=11.4,6.8 \mathrm{~Hz}$, R-CHH-Cl), 2.58 (1H, ddd, $J=14.8,6.4,1.4 \mathrm{~Hz}, \mathrm{MeO}-\mathrm{CH}-\mathrm{C} H \mathrm{H}-\mathrm{R}), 2.46$ (1H, ddd, $J=13.6$, 7.3, $1.8 \mathrm{~Hz}, \mathrm{MeO}-\mathrm{CH}-\mathrm{CH} H-\mathrm{R}), 2.36$ (1H, ddd, $\left.J=14.7,7.1,1.4 \mathrm{~Hz}, \mathrm{MeO}-\mathrm{CH}-\mathrm{CH}{ }^{\prime} \mathrm{H}-\mathrm{R}\right), 2.22$ $\left(1 \mathrm{H}, \mathrm{ddd}, J=13.6,6.7,1.8 \mathrm{~Hz}, \mathrm{MeO}-\mathrm{CH}-\mathrm{CH} H^{\prime}-\mathrm{R}\right)$. 
${ }^{13} \mathbf{C}$ NMR (100 MHz, $\left.\mathrm{CDCl}_{3}\right): \delta 157.0,145.7,135.6,128.8,128.7,128.5,128.3,91.2,90.3$, $80.8,56.2,56.0,44.8,44.7,43.6,39.3,39.1$.

MS (ESI) $m / z$ (rel intensity): $309(\mathrm{M}+\mathrm{Na})^{+}$

MS (ESI-TOF, HR) m/z (M+Na) cald for $\mathrm{C}_{13} \mathrm{H}_{16}{ }^{35} \mathrm{ClNO}_{4} \mathrm{Na}$ : 308.0666; found: 308.0667 .

MS (ESI-TOF, HR) $m / z(\mathrm{M}+\mathrm{H})^{+}$Calculated for $\mathrm{C}_{13} \mathrm{H}_{17}{ }^{37} \mathrm{ClNO}_{4}$ : 310.0636 ; found: 310.0645 .

$[\alpha]_{\mathbf{D}}{ }^{22}:-10.4\left(c=8.8, \mathrm{CH}_{2} \mathrm{Cl}_{2}\right)$.

(3S,5R)-benzyl 3-allyl-5-(chloromethyl)isoxazolidine-2-carboxylate, (1.36.2):<smiles>C=CCC1CC(CCl)ON1C(=O)OCc1ccccc1</smiles>

Boron trifluoride diethyl etherate $\left(46 \% \mathrm{BF}_{3}\right.$ basis $)\left(\mathrm{BF}_{3} . \mathrm{OEt}_{2}\right)(1.78 \mathrm{~mL}, 14.2 \mathrm{mmol})$ was added dropwise to a solution of $\mathbf{1 . 3 6 . 3}(1.56 \mathrm{~g}, 5.46 \mathrm{mmol})$ and allyltrimethylsilane (2.61 $\mathrm{mL}, 16.4$ mmol) in anhydrous dichloromethane $(80 \mathrm{~mL})$ at $-70{ }^{\circ} \mathrm{C}$. The mixture was stirred at $-40{ }^{\circ} \mathrm{C}$ for $24 \mathrm{~h}$. The mixture was quenched with triethylamine $(2.0 \mathrm{~mL})$ at $-40{ }^{\circ} \mathrm{C}$, then allowed to warm to room temperature. Water was added, and the mixture was extracted with dichloromethane (3 X $20 \mathrm{~mL}$ ). The combined organic extracts were washed with brine, dried over anhydrous sodium sulphate, filtered and concentrated in vасио. The residue was purified by flash chromatography on silica gel eluting with (Hexane/EtOAc $=97: 3$ to $96: 4)$ to give isoxazolidine 1.36.2 $(1.42 \mathrm{~g}, 88 \%)$ as a clear yellow oil $(\mathrm{dr}=>95: 5)$.

Formula: $\mathrm{C}_{15} \mathrm{H}_{18} \mathrm{ClNO}_{3}$ Mass: $295.8 \mathrm{~g} / \mathrm{mol}$

IR $v_{\max }$ (neat): 3066, 3032, 2954, 1703, 1641, 993, 918, 754, $698 \mathrm{~cm}^{-1}$.

${ }^{1} \mathbf{H}$ NMR $\left(400 \mathrm{MHz}, \mathrm{CDCl}_{3}\right): \delta 7.41-7.31(5 \mathrm{H}, \mathrm{m}, \mathrm{Ar}-H), 5.78(1 \mathrm{H}, \mathrm{ddt}, J=12.1,6.9,3.7 \mathrm{~Hz}$, $\left.\mathrm{CH}_{2}=\mathrm{C} H-\mathrm{R}\right), 5.23(1 \mathrm{H}, \mathrm{d}, J=12.4 \mathrm{~Hz}, \mathrm{Ph}-\mathrm{CH} H-\mathrm{R}), 5.16(1 \mathrm{H}, \mathrm{d}, J=12.4 \mathrm{~Hz}, \mathrm{Ph}-\mathrm{CH} H-\mathrm{R}), 4.47$ 
$\left(1 \mathrm{H}, \mathrm{m}, \mathrm{CH}_{2}=\mathrm{CHCH}_{2}-\mathrm{CH}-\mathrm{R}\right), 4.38\left(1 \mathrm{H}, \mathrm{m}, \mathrm{ClCH}_{2}-\mathrm{CH}-\mathrm{R}\right), 3.54(1 \mathrm{H}, \mathrm{dd}, J=11.2,5.5 \mathrm{~Hz}, \mathrm{Cl}-$

CHH-R), 3.29 (1H, dd, $J=11.4,7.8 \mathrm{~Hz}, \mathrm{Cl}-\mathrm{CH} H-\mathrm{R}), 2.47$ (2H, ddd, $J=16.9,14.2,6.8 \mathrm{~Hz}$, $\left.\mathrm{CH}_{2}=\mathrm{CH}-\mathrm{CH}_{2}-\mathrm{R}\right), 2.30\left(2 \mathrm{H}, \mathrm{m}, \mathrm{ClCH}_{2}-\mathrm{CH}-\mathrm{CH}_{2}-\mathrm{R}\right)$.

${ }^{13} \mathrm{C}$ NMR $\left(100 \mathrm{MHz}, \mathrm{CDCl}_{3}\right): \delta 157.7,135.9,133.8,128.7,128.6,128.5,118.4,80.2,68.2$, $58.7,44.5,39.1,36.7$.

MS (ESI) $m / z$ (rel intensity): $297(\mathbf{M}+\mathrm{H})^{+}$

MS (ESI-TOF, HR) $m / z(\mathrm{M}+\mathrm{Na})^{+}$Calculated for $\mathrm{C}_{15} \mathrm{H}_{18}{ }^{35} \mathrm{ClNO}_{3} \mathrm{Na}$ : 318.0873; found: 318.0878.

MS (ESI-TOF, HR) $\mathrm{m} / z(\mathrm{M}+\mathrm{Na})^{+}$Calculated for $\mathrm{C}_{15} \mathrm{H}_{18}{ }^{37} \mathrm{ClNO}_{3} \mathrm{Na}$ : 320.0843; found: 320.0849 .

$[\alpha]_{\mathrm{D}}{ }^{22}:+93.5\left(c=3.5, \mathrm{CH}_{2} \mathrm{Cl}_{2}\right)$.

\section{2-((3R,5R)-2-((benzyloxy)carbonyl)-5-(chloromethyl)isoxazolidin-3-yl)acetic acid,}

(1.36.1):<smiles>O=C(O)C[C@@H]1C[C@H](CCl)ON1C(=O)O</smiles>

Ruthenium trichloride $(0.91 \mathrm{mg}, 0.00440 \mathrm{mmol}$ ) was added to a solution of isoxazolidine $\mathbf{1 . 3 6 . 2}$ $(0.060 \mathrm{~g}, 0.20 \mathrm{mmol})$, and sodium metaperiodate $(0.175 \mathrm{~g}, 0.82 \mathrm{mmol})$ in chloroform $(0.40 \mathrm{~mL})$, acetonitrile $(0.40 \mathrm{~mL})$, water $(0.60 \mathrm{~mL})$. The mixture was stirred vigorously at room temperature for $2 \mathrm{~h}$. Dichloromethane ( $2 \mathrm{~mL})$ was added the phases were separated. The aqueous layer was extracted with dichloromethane $(3 \times 10 \mathrm{~mL})$. The combined organic extracts were washed with brine, dried over anhydrous magnesium sulphate, filtered and concentrated in vacuo to give 1.36.1 $(0.06 \mathrm{~g}, 96 \%)$ as a bright orange-yellow oil.

Formula: $\mathrm{C}_{14} \mathrm{H}_{16} \mathrm{ClNO}_{5}$

Mass: $313.7 \mathrm{~g} / \mathrm{mol}$

IR $v_{\max }$ (neat): 2961, 1732, 1714, 1454, 1423, 752, $698 \mathrm{~cm}^{-1}$. 
${ }^{1} \mathbf{H}$ NMR $\left(400 \mathrm{MHz}, \mathrm{CDCl}_{3}\right): \delta$ 7.32-7.41 $(5 \mathrm{H}, \mathrm{m}, \mathrm{Ar}-H), 5.26(1 \mathrm{H}, \mathrm{d}, J=12.1 \mathrm{~Hz}, \mathrm{Ph}-\mathrm{CHH}-$ R), $5.20(1 \mathrm{H}, \mathrm{d}, J=12.1 \mathrm{~Hz}, \mathrm{Ph}-\mathrm{CH} H-\mathrm{R}), 4.73\left(1 \mathrm{H}, \mathrm{m}, \mathrm{CO}_{2} \mathrm{H}-\mathrm{CH}_{2}-\mathrm{CH}-\mathrm{R}\right), 4.50(1 \mathrm{H}, \mathrm{m}, \mathrm{Cl}-$ $\left.\mathrm{CH}_{2}-\mathrm{CH}-\mathrm{R}\right), 3.56(1 \mathrm{H}, \mathrm{dd}, J=11.7,5.3 \mathrm{~Hz}, \mathrm{Cl}-\mathrm{CHH}-\mathrm{R}), 3.33(1 \mathrm{H}, \mathrm{dd}, J=11.2,7.5 \mathrm{~Hz}, \mathrm{Cl}-$ CHH-R) $2.90\left(1 \mathrm{H}, \mathrm{dd}, J=16.9,5.5 \mathrm{~Hz}, \mathrm{CO}_{2} \mathrm{H}-\mathrm{C} H \mathrm{H}-\mathrm{R}\right), 2.60\left(1 \mathrm{H}, \mathrm{dd}, J=16.7,8.5 \mathrm{~Hz}, \mathrm{CO}_{2} \mathrm{H}-\right.$ CHH-R), 2.48 (1H, ddd, $\left.J=17.6,8.2,4.6 \mathrm{~Hz}, \mathrm{CO}_{2} \mathrm{H}-\mathrm{CH}_{2} \mathrm{CH}-\mathrm{C} H \mathrm{H}-\mathrm{R}\right), 2.33$ (1H, ddd, $J=17.6$, 8.5, $\left.4.1 \mathrm{~Hz}, \mathrm{CO}_{2} \mathrm{H}-\mathrm{CH}_{2} \mathrm{CH}-\mathrm{CH} H-\mathrm{R}\right)$.

${ }^{13} \mathrm{C}$ NMR (100 MHz, $\left.\mathrm{CDCl}_{3}\right): \delta 174.7,157.4,135.5,128.7,128.6,128.4,79.9,68.5,55.7,44.3$, 38.9, 37.6.

MS (ESI) $m / z$ (rel intensity): $336(\mathrm{M}+\mathrm{Na})^{+}$

MS (ESI-TOF, HR) $m / z(\mathrm{M}+\mathrm{Na})^{+}$Calculated for $\mathrm{C}_{14} \mathrm{H}_{16}{ }^{35} \mathrm{ClNO}_{5} \mathrm{Na}$ : 336.0615; found: 336.0612. $[\alpha]_{\mathrm{D}}{ }^{22}:+13.1\left(c=2.0, \mathrm{CH}_{2} \mathrm{Cl}_{2}\right)$.

benzyl (3R,5R)-3-(2-(2-(2-(benzyloxy)-2-oxoethyl)-2-methylhydrazinyl)-2-oxoethyl)-5(chloromethyl)isoxazolidine-2-carboxylate, (1.37.2):<smiles>CN(CC(=O)OCc1ccccc1)NC(=O)C[C@@H]1C[C@H](CCl)ON1C(=O)OCc1ccccc1</smiles>

Dimethylformamide $(0.50 \mathrm{~mL})$ was added to a solution of acid, $\mathbf{1 . 3 6 . 1}(1.52 \mathrm{~g}, 4.84 \mathrm{mmol})$ and oxalyl chloride $(0.741 \mathrm{~mL}, 8.65 \mathrm{mmol})$ in dichloromethane $(15 \mathrm{~mL})$. The mixture was stirred for $0.5 \mathrm{~h}$. The volatiles were removed in vacuo and the residue was taken up in dichloromethane (83 mL). Hydrazine $\mathbf{1 . 3 7 . 4}(1.12 \mathrm{~g}, 5.60 \mathrm{mmol})$ followed by pyridine $(0.700 \mathrm{~mL}, 8.65 \mathrm{mmol})$ were added and the mixture was stirred for $24 \mathrm{~h}$. The volatiles were removed in vacuo and the residue was purified by flash chromatography on silica gel eluting with $(\mathrm{EtOAc} / \mathrm{MeOH}=70: 30)$ to give the hydrazide $\mathbf{1 . 3 7 . 2}(1.65 \mathrm{~g}, 70 \%)$ as a dark brown oil. 
IR $v_{\max }$ (neat): $2955,1714,1668,1385 \mathrm{~cm}^{-1}$.

${ }^{1}$ H NMR (400 MHz, $\left.\mathrm{CDCl}_{3}\right): \delta$ 7.38-7.35 (10H, m, Ar- $\left.H\right)$, 5.20-5.14 (4H, m, Ph-CH $\left.-\mathrm{R}\right), 4.72$ (1H, m, R-CH-CH $2{ }_{2} \mathrm{CONHR}$ '), 4.48 (1H, m, R-CH-CH $\left.2 \mathrm{Cl}\right), 3.73(1 \mathrm{H}, \mathrm{d}, J=18.0 \mathrm{~Hz}, \mathrm{R}-\mathrm{NCHH}-$ $\left.\mathrm{CO}_{2} \mathrm{Bn}\right), 3.68\left(1 \mathrm{H}, \mathrm{d}, J=18.0 \mathrm{~Hz}, \mathrm{R}-\mathrm{NCH} H-\mathrm{CO}_{2} \mathrm{Bn}\right), 3.52(1 \mathrm{H}, \mathrm{dd}, J=12.1,5.7 \mathrm{~Hz}, \mathrm{Cl}-\mathrm{CHH}-$ R), $3.32(1 \mathrm{H}, \mathrm{dd}, J=11.4,8.2 \mathrm{~Hz}, \mathrm{Cl}-\mathrm{CH} H-\mathrm{R}), 2.74\left(3 \mathrm{H}, \mathrm{s}, \mathrm{R}-\mathrm{NCH}_{3}\right), 2.47$ (1H, dd, $J=14.3$, $6.4 \mathrm{~Hz}, \mathrm{R}-\mathrm{C} H \mathrm{H}-\mathrm{CONH}), 2.32(1 \mathrm{H}, \mathrm{dd}, J=14.3,6.4 \mathrm{~Hz}, \mathrm{R}-\mathrm{CH} H-\mathrm{CONH}), 2.39$ (2H, m, Cl$\left.\mathrm{CH}_{2} \mathrm{CH}-\mathrm{CH}_{2}-\mathrm{R}\right)$.

${ }^{13} \mathrm{C}$ NMR $\left(100 \mathrm{MHz}, \mathrm{CDCl}_{3}\right): \delta 170.7,167.9,157.8,135.8,135.6,135.4,135.2,128.9,128.8$, $128.7,128.6,80.4,68.6,66.8,60.6,57.8,44.6,44.2,39.9,37.0$.

MS (ESI) $m / z$ (rel intensity): $512(\mathrm{M}+\mathrm{Na})^{+}$

HRMS (ESI-TOF): Calculated for $\mathrm{C}_{24} \mathrm{H}_{28}{ }^{35} \mathrm{ClN}_{3} \mathrm{O}_{6} \mathrm{Na}[\mathrm{M}+\mathrm{Na}]^{+}:$512.1564; found: 512.1560.

HRMS (ESI-TOF): Calculated for $\mathrm{C}_{24} \mathrm{H}_{28}{ }^{37} \mathrm{ClN}_{3} \mathrm{O}_{6} \mathrm{Na}[\mathrm{M}+\mathrm{Na}]^{+}:$514.1535; found: 514.1539.

$[\alpha]_{\mathrm{D}}{ }^{22}:+42.5\left(c=11.3, \mathrm{CH}_{2} \mathrm{Cl}_{2}\right)$.

(3R,5R)-benzyl 5-(azidomethyl)-3-(2-(2-(2-(benzyloxy)-2-oxoethyl)-2-methylhydrazinyl)2-oxoethyl)isoxazolidine-2-carboxylate, (1.37.3):<smiles>CN(CC(=O)OCc1ccccc1)NC(=O)C[C@@H]1CC(CN)ON1C(=O)O</smiles>

$n-\mathrm{Bu}_{4} \mathrm{~N}^{+} \mathrm{N}_{3}{ }^{-}(0.0820 \mathrm{~g}, 0.330 \mathrm{mmol})$ was added to a stirred solution of $\mathbf{1 . 3 7 . 2}(0.080 \mathrm{~g}, 0.163$ mmol $)$ in tetrahydrofuran $(5 \mathrm{~mL})$ at $25^{\circ} \mathrm{C}$. The mixture was heated at reflux for $24 \mathrm{~h}$. The solvent 
was removed in vacuo and the residue was purified by flash chromatography on silica gel eluting with $(\mathrm{EtOAc} / \mathrm{MeOH}=90: 10)$ to give azide $\mathbf{1 . 3 7 . 3}(0.067 \mathrm{~g}, 83 \%)$ as a dark brown oil.

Formula: $\mathrm{C}_{24} \mathrm{H}_{28} \mathrm{~N}_{6} \mathrm{O}_{6}$ Mass: $496.5 \mathrm{~g} / \mathrm{mol}$

IR $v_{\max }$ (neat): 3242, 3065, 3034, 2957, 2104, 1745, 1693, 1497, 745, 698.

${ }^{1} \mathrm{H}$ NMR $\left(400 \mathrm{MHz}, \mathrm{CDCl}_{3}\right): \delta$ 7.39-7.33 (10H, m, Ar-H), 5.26-5.14 (4H, m, Ph-CH $\left.-\mathrm{R}\right), 4.68$ $\left(1 \mathrm{H}, \mathrm{m}, \mathrm{R}-\mathrm{CH}-\mathrm{CH}_{2} \mathrm{CON} H \mathrm{R}\right.$ '), $4.29\left(1 \mathrm{H}, \mathrm{m}, \mathrm{R}-\mathrm{C} H-\mathrm{CH}_{2} \mathrm{~N}_{3}\right), 3.73(1 \mathrm{H}, \mathrm{d}, J=18.0 \mathrm{~Hz}, \mathrm{R}-\mathrm{NC} H \mathrm{H}-$ $\left.\mathrm{CO}_{2} \mathrm{Bn}\right), 3.68\left(1 \mathrm{H}, \mathrm{d}, J=18.0 \mathrm{~Hz}, \mathrm{R}-\mathrm{NCH} H-\mathrm{CO}_{2} \mathrm{Bn}\right), 3.32\left(1 \mathrm{H}, \mathrm{dd}, J=7.1,4.6 \mathrm{~Hz}, \mathrm{~N}_{3}-\mathrm{CHH}-\right.$ R), $3.15\left(1 \mathrm{H}, \mathrm{dd}, J=8.7,4.6 \mathrm{~Hz}, \mathrm{~N}_{3}-\mathrm{CH} H-\mathrm{R}\right), 2.75\left(3 \mathrm{H}, \mathrm{s},-\mathrm{NCH}_{3}\right), 2.45(1 \mathrm{H}, \mathrm{dd}, J=14.2,6.4$ $\mathrm{Hz}, \mathrm{R}-\mathrm{C} H \mathrm{H}-\mathrm{CONH}), 2.32(1 \mathrm{H}, \mathrm{dd}, J=14.4,6.6 \mathrm{~Hz}, \mathrm{R}-\mathrm{CH} H-\mathrm{CONH}), 1.64\left(2 \mathrm{H}, \mathrm{m}, \mathrm{N}_{3}-\mathrm{CH}_{2} \mathrm{CH}-\right.$ $\left.\mathrm{CH}_{2}-\mathrm{R}\right)$

${ }^{13}$ C NMR $\left(100 \mathrm{MHz}, \mathrm{CDCl}_{3}\right): \delta$ 170.6, 167.9, 157.5, 135.7, 135.6, 135.4, 135.2, 128.9, 128.8, $128.7,128.6,80.4,68.5,66.9,57.8,56.9,44.6,44.2,39.9,37.0$.

MS (ESI) $m / z$ (rel intensity): $519(\mathrm{M}+\mathrm{Na})^{+}$

HRMS (ESI-TOF): Calculated for $\mathrm{C}_{24} \mathrm{H}_{28}{ }^{35} \mathrm{ClN}_{6} \mathrm{O}_{6} \mathrm{Na}[\mathrm{M}+\mathrm{Na}]^{+}:$519.1968. Found: 519.1968.

$[\alpha]_{\mathbf{D}}{ }^{22}:+23.3\left(c=2.5, \mathrm{CH}_{2} \mathrm{Cl}_{2}\right)$.

$N$-((3R,5R)-3,6-diamino-5-hydroxyhexanamido)- $N$-methylglycine, (1.1.1):<smiles>CN(CC(=O)O)NC(=O)C[C@@H](N)C[C@H](O)CN</smiles>

A solution of $\mathbf{1 . 3 7 . 3}(24.1 \mathrm{mg}, 0.0485 \mathrm{mmol})$ in a 2:1 mixture of methanol and 5\% acetic acid (2.28 $\mathrm{mL})$ containing $10 \%$ palladium on charcoal $(4 \mathrm{mg})$ in a Fisher-Porter tube was placed under $\mathrm{H}_{2}$ at $40 \mathrm{psi}$ and stirred at room temperature for $24 \mathrm{~h}$. The mixture was then filtered through a wet celite pad washing with water. The volatiles were in vacuo and the residue was 
purified by ion-exchange chromatography on amberlite CG-50 $\left(\mathrm{NH}_{4}{ }^{+}\right.$resin), eluting with water followed by $0.5 \% \mathrm{NH}_{4} \mathrm{OH}(\mathrm{aq})$. Ninhydrin-active fractions were collected and concentrated in vacuo to give (+)-Negamycin 1.1.1 (11.3 mg, 94\%) as white powder.

Formula: $\mathrm{C}_{9} \mathrm{H}_{20} \mathrm{~N}_{4} \mathrm{O}_{4}$

Mass: $248.3 \mathrm{~g} / \mathrm{mol}$

$\mathbf{M p}=114-117^{\circ} \mathrm{C}\left(\text { lit mp: } 110-120^{\circ} \mathrm{C}\right)^{2}$

${ }^{1} \mathbf{H}$ NMR $\left(400 \mathrm{MHz}, \mathrm{CDCl}_{3}\right): \delta 3.84\left(1 \mathrm{H}, \mathrm{m}, \mathrm{R}-\mathrm{CH}-\mathrm{CH}_{2} \mathrm{CONHR}\right.$ '), $3.29(1 \mathrm{H}, \mathrm{m}, \mathrm{R}-\mathrm{CH}-$ $\left.\mathrm{CH}_{2} \mathrm{NH}_{2}\right), 3.23\left(2 \mathrm{H}, \mathrm{s}, \mathrm{R}-\mathrm{CH}_{2} \mathrm{CO}_{2} \mathrm{H}\right), 2.88\left(1 \mathrm{H}, \mathrm{dd}, J=13.2,3.5 \mathrm{~Hz}, \mathrm{NH}_{2}-\mathrm{CHH}-\mathrm{R}\right), 2.71(1 \mathrm{H}$, dd, $\left.J=13.2,9.0 \mathrm{~Hz}, \mathrm{NH}_{2}-\mathrm{CH} H-\mathrm{R}\right), 2.46\left(3 \mathrm{H}, \mathrm{s}, \mathrm{R}-\mathrm{NCH}_{3}\right), 2.25(1 \mathrm{H}, \mathrm{dd}, J=14.6,6.8 \mathrm{~Hz}, \mathrm{R}-$ CHH-CONH-R'), 2.20 (1H, dd, $J=14.5,6.8 \mathrm{~Hz}, \mathrm{R}-\mathrm{CH} H-\mathrm{CONH}-\mathrm{R}$ '), $1.44\left(2 \mathrm{H}, \mathrm{m}, \mathrm{H}_{2} \mathrm{~N}-\right.$ $\left.\mathrm{CH}_{2} \mathrm{CH}-\mathrm{CH}_{2}-\mathrm{R}\right)$.

${ }^{13} \mathrm{C}$ NMR $\left(100 \mathrm{MHz}, \mathrm{CDCl}_{3}\right): \delta 177.1,170.9,65.6,61.0,45.2,44.9,43.8,41.2,39.6$.

$[\alpha]_{\mathrm{D}}{ }^{23}:+2.5\left(c=2.0, \mathrm{H}_{2} \mathrm{O}\right)$ lit: $[\alpha]_{\mathrm{D}}^{29}:+2.5\left(c=2.0, \mathrm{H}_{2} \mathrm{O}\right)^{2}$

benzyl 2-(1-methylhydrazinyl)acetate, (11):<smiles>CN(N)CC(=O)OCc1ccccc1</smiles>

Suspension of N-methylhydrazine sulfate $(0.200 \mathrm{~g}, 1.39 \mathrm{mmol})$ and triethylamine $(0.66 \mathrm{~mL}$, $4.73 \mathrm{mmol})$ was stirred in dichloromethane $(5 \mathrm{~mL})$ at room temperature for $24 \mathrm{~h}$. Benzyl bromoacetate $(0.22 \mathrm{~mL}, 1.39 \mathrm{mmol})$ in dichloromethane $(1.40 \mathrm{~mL})$ was added dropwise to the homogenous solution at $0{ }^{\circ} \mathrm{C}$. The mixture was stirred at $0{ }^{\circ} \mathrm{C}$ for $0.5 \mathrm{~h}$ and at $25{ }^{\circ} \mathrm{C}$ for $24 \mathrm{~h}$. The solvent was removed in vacuo and the residue was purified by on silica gel eluting with $(\mathrm{EtOAc} / \mathrm{MeOH}=95: 5)$ to give the hydrazine $(\mathbf{1 1}),(0.087 \mathrm{~g}, 31 \%)$ as a clear colourless oil.

Formula: $\mathrm{C}_{19} \mathrm{H}_{17} \mathrm{BFNO}_{4}$

Mass: $353.2 \mathrm{~g} / \mathrm{mol}$ 
${ }^{1} \mathrm{H}$ NMR (400 MHz, $\left.\mathrm{CDCl}_{3}\right): \delta 7.25(5 \mathrm{H}, \mathrm{m}, \mathrm{Ar}-\mathrm{H}), 5.15\left(2 \mathrm{H}, \mathrm{s}, \mathrm{Ph}-\mathrm{CH}_{2}-\mathrm{R}\right), 3.43\left(2 \mathrm{H}, \mathrm{s}, \mathrm{NCH}_{2}\right)$, $3.21\left(2 \mathrm{H}\right.$, br s, $\left.\mathrm{NH}_{2}\right), 2.61\left(3 \mathrm{H}, \mathrm{s}, \mathrm{NCH}_{3}\right)$.

${ }^{13}$ C NMR (100 MHz, $\left.\mathrm{CDCl}_{3}\right): \delta 170.2,135.6,128.6,128.4,128.3,66.4,62.4,48.7$.

\section{Tetrabutylammonium Azide, ${ }^{60}(12)$ :}

A solution of $\mathrm{NaN}_{3}(1.00 \mathrm{~g}, 15.4 \mathrm{mmol})$ in water $(2.31 \mathrm{~mL})$ was added to tetrabutylammonium hydroxide (2.00 g, $7.69 \mathrm{mmol})$. Dichloromethane $(12 \mathrm{~mL})$ was added, and the organic layer was extracted. Extractions of aqueous layer with dichloromethane ( $3 \mathrm{X} 4 \mathrm{~mL})$. The combined organic layers were dried over anhydrous magnesium sulphate, filtered and concentrated to give the salt as a white crystalline solid $(2.10 \mathrm{~g}, 96 \%)$.

Formula: $\mathrm{C}_{19} \mathrm{H}_{17} \mathrm{BFNO}_{4}$

Mass: $353.2 \mathrm{~g} / \mathrm{mol}$

IR $v_{\max }$ (neat): $2014 \mathrm{~cm}^{-1}$

\subsubsection{Experimental Procedure for Chapter II}

Hex-5-enoic Acid, ${ }^{1}$ (2.14.7):<smiles>C=CCCCC(=O)O</smiles>

Aqueous $\mathrm{H}_{2} \mathrm{O}_{2}(30 \%, 22.7 \mathrm{~mL}, 0.20 \mathrm{~mol})$ was added dropwise to a stirred solution of cyclohexanone $(9.80 \mathrm{~g}, 0.10 \mathrm{~mol})$ in methanol $(10 \mathrm{~mL})$ at $25^{\circ} \mathrm{C}$. The mixture was then added dropwise to a mixture of $\mathrm{FeSO}_{4} \cdot 6 \mathrm{H}_{2} \mathrm{O}(27.8 \mathrm{~g}, 0.10 \mathrm{~mol})$ and $\mathrm{CuSO}_{4} \cdot 5 \mathrm{H}_{2} \mathrm{O}(25 \mathrm{~g}, 0.10 \mathrm{~mol})$ in water $(180 \mathrm{~mL})$. The mixture was extracted with ether $(3 \mathrm{X} 20 \mathrm{~mL})$. The combined organic extracts were washed with aqueous sodium hydroxide $(20 \%, 2$ X $10 \mathrm{~mL})$. The alkaline extract was then brought to $\mathrm{pH} 2$ with aqueous sulfuric acid $(20 \%, 25 \mathrm{~mL})$ and the solution was extracted with ether ( 3 X $20 \mathrm{~mL})$. The combined organic extracts were washed with water, brine, 
dried over anhydrous magnesium sulphate, filtered and concentrated in vacuo. The residue was distilled in vacuo collecting the fraction at $87-90{ }^{\circ} \mathrm{C} / 10 \mathrm{mmHg}$, to give the acid $\mathbf{2 . 1 4 . 7}$ as colourless liquid (3.36 g, 30\%).

Formula: $\mathrm{C}_{6} \mathrm{H}_{10} \mathrm{O}_{2}$

Mass: $114.1 \mathrm{~g} / \mathrm{mol}$

${ }^{1} \mathbf{H}$ NMR $\left(400 \mathrm{MHz}, \mathrm{CDCl}_{3}\right): \delta 5.78\left(1 \mathrm{H}, \mathrm{ddt}, J=17.0,10.2,6.7 \mathrm{~Hz}, \mathrm{R}-\mathrm{CH}=\mathrm{CH}_{2}\right), 5.08-4.97$ $\left(2 \mathrm{H}, \mathrm{m}, \mathrm{R}-\mathrm{CH}=\mathrm{CH} \mathrm{H}_{2}\right), 2.37\left(2 \mathrm{H}, \mathrm{t}, J=7.5 \mathrm{~Hz}, \mathrm{R}-\mathrm{CH}_{2} \mathrm{COOH}\right), 2.12(2 \mathrm{H}, \mathrm{dt}, J=6.8,1.3 \mathrm{~Hz}, \mathrm{R}-$ $\left.\mathrm{CH}_{2} \mathrm{CH}=\mathrm{CH}_{2}\right), 1.75\left(2 \mathrm{H}\right.$, quint, $\left.J=7.8 \mathrm{~Hz}, \mathrm{R}-\mathrm{CH}_{2}-\mathrm{R}^{\prime}\right)$.

${ }^{13} \mathrm{C}$ NMR $\left(100 \mathrm{MHz}, \mathrm{CDCl}_{3}\right): \delta 180.5,137.5,115.6,100.0,33.1,23.8$.

2-aminoisoindoline-1,3-dione, (2.14.6):<smiles>NN1C(=O)c2ccccc2C1=O</smiles>

Hydrazine monohydrate $(0.36 \mathrm{~mL}, 7.48 \mathrm{mmol})$ was added dropwise to a suspension of phthalimide $(1.00 \mathrm{~g}, 6.80 \mathrm{mmol})$ in aqueous ethanol $(95 \%, 7.0 \mathrm{~mL})$ at $5{ }^{\circ} \mathrm{C}$ for $2 \mathrm{~h}$. The mixture was then diluted with ice water $(14 \mathrm{~mL})$, stirred, filtered, washed with water, and dried in air to give the product 2.14.6 as white powder $(0.83 \mathrm{~g}, 75 \%)$.

Formula: $\mathrm{C}_{8} \mathrm{H}_{6} \mathrm{~N}_{2} \mathrm{O}_{2}$

Mass: $162.1 \mathrm{~g} / \mathrm{mol}$

Mp: $199-202{ }^{\circ} \mathrm{C}^{2}$

${ }^{1} \mathbf{H}$ NMR $\left(400 \mathrm{MHz}, \mathrm{CDCl}_{3}\right): \delta 7.89-7.84(2 \mathrm{H}, \mathrm{m}, \mathrm{Ar}-\mathrm{H}), 7.76-7.72(2 \mathrm{H}, \mathrm{m}, \mathrm{Ar}-\mathrm{H}), 4.72(2 \mathrm{H}$, brs, $\mathrm{R}-\mathrm{N} H_{2}$ ).

${ }^{13}$ C NMR (100 MHz, $\left.\mathrm{CDCl}_{3}\right): \delta 166.8,134.4,130.3,123.6$. 


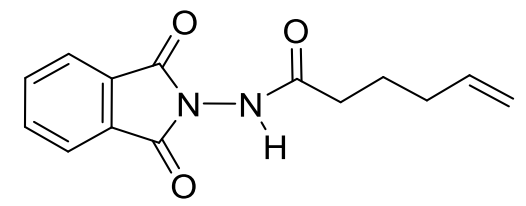

Dimethylformamide $(0.05 \mathrm{~mL})$ was added to a solution of hex-5-enoic acid, 2.14.7 (0.48 g, 4.21 mmol) and oxalyl chloride $(0.55 \mathrm{~mL}, 6.31 \mathrm{mmol})$ in dichloromethane $(15 \mathrm{~mL})$. The mixture was stirred for $0.5 \mathrm{~h}$. The volatiles were removed in vacuo and the residue was taken up in dichloromethane (5 mL). A solution of 2.14.6 (1.24 g, $7.65 \mathrm{mmol})$ in dichloromethane $(25 \mathrm{~mL})$ followed by sodium bicarbonate $(0.64 \mathrm{~g}, 7.65 \mathrm{mmol})$ were added and the mixture was stirred for $24 \mathrm{~h}$. The volatiles were removed in vacuo and the residue was purified by flash chromatography on silica gel eluting with $($ Hexane/EtOAc $=70: 30)$ to give the hydrazide 2.14.5 $(0.82 \mathrm{~g}, 72 \%)$ as light brown powder.

Formula: $\mathrm{C}_{14} \mathrm{H}_{14} \mathrm{~N}_{2} \mathrm{O}_{3}$

Mass: $258.3 \mathrm{~g} / \mathrm{mol}$

Mp: $105-108^{\circ} \mathrm{C}$

IR $v_{\max }$ (neat): $3267,1751,1668,984,928,885,760$.

${ }^{1} \mathbf{H}$ NMR $\left(400 \mathrm{MHz}, \mathrm{CDCl}_{3}\right): \delta 7.96(2 \mathrm{H}, \mathrm{dd}, J=5.4,3.0 \mathrm{~Hz}, \mathrm{Ar}-H), 7.86(2 \mathrm{H}, \mathrm{dd}, J=5.4,3.2$ $\mathrm{Hz}, \mathrm{Ar}-H), 5.75\left(1 \mathrm{H}, \mathrm{ddt}, J=17.0,10.2,6.7 \mathrm{~Hz}, \mathrm{R}-\mathrm{CH}=\mathrm{CH}_{2}\right), 5.07-4.94\left(2 \mathrm{H}, \mathrm{m}, \mathrm{R}-\mathrm{CH}=\mathrm{CH}_{2}\right)$, $2.75\left(2 \mathrm{H}, \mathrm{t}, J=7.2 \mathrm{~Hz}, \mathrm{R}-\mathrm{CH}_{2}-\mathrm{CONH}-\mathrm{R}\right.$ '), $2.09\left(2 \mathrm{H}, \mathrm{q}, J=7.2 \mathrm{~Hz}, \mathrm{R}-\mathrm{CH}_{2}-\mathrm{CH}=\mathrm{CH}_{2}\right), 1.76(2 \mathrm{H}$, quint, $J=7.3 \mathrm{~Hz}$, R-CONH-CH $\left.\mathrm{CH}_{2}-\mathrm{R}^{\prime}\right)$.

${ }^{13}$ C NMR $\left(100 \mathrm{MHz}, \mathrm{CDCl}_{3}\right): \delta$ 172.6, 165.0, 137.6, 135.3, 130.0, 124.5, 115.6, 100.0, 32.7, 23.5.

MS (ESI) $m / z$ (rel intensity): $259(\mathbf{M}+\mathrm{H})^{+}$

HRMS (ESI-TOF) $m / z(\mathrm{M}+\mathrm{H})^{+}$Calculated for $\mathrm{C}_{14} \mathrm{H}_{14} \mathrm{~N}_{2} \mathrm{O}_{3}$ : 259.1083; found: 259.1085 . 


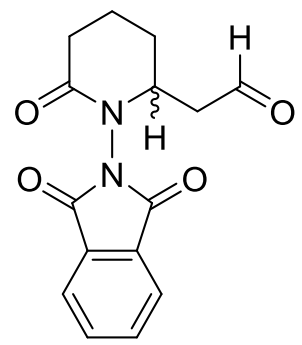

Croton aldehyde $(0.26 \mathrm{~mL}, 3.17 \mathrm{mmol})$ was added to a solution of hydrazide $\mathbf{2 . 1 4 . 5}(0.16 \mathrm{~g}$, $0.63 \mathrm{mmol})$ in dichloroethane $(5 \mathrm{~mL})$ under nitrogen atmosphere. A solution of Grubbs-II catalyst $(27 \mathrm{mg})$ in dichloroethane $(2 \mathrm{~mL})$ was then added sequentially over $4 \mathrm{~h}$. After the first addition of the catalyst, the mixture was heated at reflux until addition completes and a further hour thereafter. The reaction mixture was cooled and the solvent was removed in vacuo.The residue was purified by flash chromatography on silica gel eluting with $($ Hexane/EtOAc $=$ 70:30) to give aldehyde $\mathbf{2 . 1 4 . 3},(0.174 \mathrm{~g}, 96 \%)$ as a dark brown solid.

Formula: $\mathrm{C}_{15} \mathrm{H}_{14} \mathrm{~N}_{2} \mathrm{O}_{4}$

Mass: $286.3 \mathrm{~g} / \mathrm{mol}$

Mp: $126-129^{\circ} \mathrm{C}$

IR $v_{\max }$ (neat): 1790, 1732, 1666, 1371

${ }^{1} \mathbf{H}$ NMR $\left(400 \mathrm{MHz}, \mathrm{CDCl}_{3}\right): \delta 9.72(1 \mathrm{H}, \mathrm{s}, \mathrm{R}-\mathrm{CHO}), 7.95-7.87(2 \mathrm{H}, \mathrm{m}, \mathrm{Ar}-H), 7.84-7.77$ $(2 \mathrm{H}, \mathrm{m}, \mathrm{Ar}-H), 4.51(1 \mathrm{H}, \mathrm{tt}, J=7.0,5.3 \mathrm{~Hz}, \beta$ to $-\mathrm{CHO}), 3.05(1 \mathrm{H}, \mathrm{dd}, J=5.4,1.4 \mathrm{~Hz}, \mathrm{R}-\mathrm{CHH}-$ CHO), $3.00(1 \mathrm{H}, \mathrm{dd}, J=5.4,1.4 \mathrm{~Hz}, \mathrm{R}-\mathrm{CH} H-\mathrm{CHO}), 2.70-2.57$ (2H, m, $\alpha$ to $-\mathrm{C}(\mathrm{O}) \mathrm{N}-\mathrm{NPhth})$, $2.32(1 \mathrm{H}, \mathrm{m}, \gamma$ to $-\mathrm{C}(\mathrm{O}) \mathrm{N}-\mathrm{NPhth}), 2.05-1.95(2 \mathrm{H}, \mathrm{m}, \beta$ to $-\mathrm{C}(\mathrm{O}) \mathrm{N}-\mathrm{NPhth}), 1.89(1 \mathrm{H}, \mathrm{m}, \gamma$ to $\mathrm{C}(\mathrm{O}) \mathrm{N}-\mathrm{NPhth})$.

${ }^{13} \mathrm{C}$ NMR (100 MHz, $\left.\mathrm{CDCl}_{3}\right): \delta$ 199.1, 168.5, 165.9, 165.5, 134.9, 130.0, 124.2, 124.1, 100.0, $56.1,48.2,32.4,29.4,18.6$

MS (ESI) $m / z$ (rel intensity): $287(\mathbf{M}+\mathrm{H})^{+}$ 
HRMS (ESI-TOF): Calculated for $\mathrm{C}_{15} \mathrm{H}_{14} \mathrm{~N}_{2} \mathrm{O}_{4}[\mathrm{M}+\mathrm{H}]^{+}:$: 287.1032. Found: 287.1034.

Methyl 4-(1-(1,3-dioxoisoindolin-2-yl)-6-oxopiperidin-2-yl)but-2-enoate, (E/Z-2.16.12):

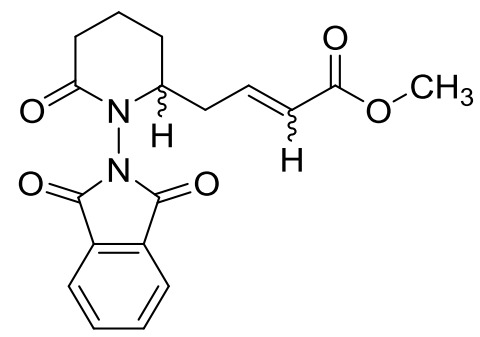

The aldehyde $\mathbf{2 . 1 4 . 3}$ ( $0.370 \mathrm{~g}, 1.29 \mathrm{mmol})$ in dichloromethane $(7 \mathrm{~mL})$ was added to a solution of methyl ester phosphonate 2.16.11 ( $0.500 \mathrm{~g}, 1.42 \mathrm{mmol})$ in dichloromethane (5 mL) and stirred for $4 \mathrm{~h}$ at room temperature. The solvent was removed in vacuo and the residue was purified by flash chromatography on silica gel eluting with $($ Hexane/EtOAc $=60: 40)$ to give an inseparable mixture of isomers $(E / Z)-\mathbf{2 . 1 6 . 1 2},(0.310 \mathrm{~g}, 70 \%)$ as an off-white pellet, $(E / Z=7.3: 1)$.

Formula: $\mathrm{C}_{18} \mathrm{H}_{18} \mathrm{~N}_{2} \mathrm{O}_{5}$

Mass: $356.2 \mathrm{~g} / \mathrm{mol}$

Mp: $113-116^{\circ} \mathrm{C}$

IR $v_{\max }$ (neat): 1790, 1732, 1462, 1377, 1190, 995, 881, 752.

${ }^{1} \mathbf{H}$ NMR $\left(400 \mathrm{MHz}, \mathrm{CDCl}_{3}\right): \delta 7.90-7.85(2 \mathrm{H}, \mathrm{m}, \mathrm{Ar}-H), 7.80-7.74(2 \mathrm{H}, \mathrm{m}, \mathrm{Ar}-H), 6.80(1 \mathrm{H}$, m, $\left.\mathrm{R}_{\mathrm{E}}-\mathrm{CH}=\mathrm{CHCO}_{2} \mathrm{Me}\right), 6.28\left(1 \mathrm{H}, \mathrm{m}, \mathrm{R}_{\mathrm{Z}}-\mathrm{C} H=\mathrm{CHCO}_{2} \mathrm{Me}\right), 5.83\left(1 \mathrm{H}, \mathrm{d}, J=15.6 \mathrm{~Hz}, \mathrm{R}_{\mathrm{E}}-\right.$ $\left.\mathrm{CH}=\mathrm{CHCO}_{2} \mathrm{Me}\right), 4.02(1 \mathrm{H}, \mathrm{m}, \delta$ to $-\mathrm{C}(\mathrm{O}) \mathrm{N}-\mathrm{NPhth}), 3.62\left(1 \mathrm{H}, \mathrm{s}, \mathrm{R}_{\mathrm{E}}-\mathrm{C}(\mathrm{O}) \mathrm{OCH}_{3}\right), 3.14(1 \mathrm{H}, \mathrm{m}$,

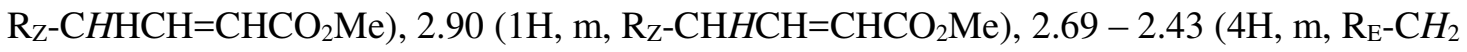
$-\mathrm{CH}=\mathrm{CHCO}_{2} \mathrm{Me}$ and $\alpha$ to $\left.-\mathrm{C}(\mathrm{O}) \mathrm{N}-\mathrm{NPhth}\right), 2.15(1 \mathrm{H}, \mathrm{m}, \gamma$ to $-\mathrm{C}(\mathrm{O}) \mathrm{N}-\mathrm{NPhth}), 2.07-1.75(3 \mathrm{H}$, $\mathrm{m}, \beta$ and $\gamma$ to $-\mathrm{C}(\mathrm{O}) \mathrm{N}-\mathrm{NPhth})$.

${ }^{13} \mathrm{C}$ NMR (100 MHz, $\left.\mathrm{CDCl}_{3}\right): \delta$ 168.7, 166.3, 165.7, 143.6, 134.9, 134.8, 132.0, 128.6, 128.5, $124.2,124.0,123.9,60.5,51.6,36.8,32.5,28.7,18.6$. 
HRMS (ESI-TOF): Calculated for $\mathrm{C}_{18} \mathrm{H}_{18} \mathrm{~N}_{2} \mathrm{O}_{5}[\mathrm{M}+\mathrm{H}]^{+}:$343.1294. Found: 343.1291.

Methyl 2-((2S,3aS)-2-(2-methoxy-2-oxoethyl)-7-oxooctahydropyrazolo[1,5-a]pyridine-1carbonyl)benzoate, $(2.16 .13)$ :<smiles>COC(=O)CC1CC2CCCC(=O)N2N1C(=O)c1ccccc1C(=O)OC</smiles>

Potassium carbonate $(0.22 \mathrm{~g}, 1.61 \mathrm{mmol})$ was added to a solution of the Wittig products (E/Z)-2.16.12 (0.500 g, $1.46 \mathrm{mmol})$ in a 1:1 mixture of methanol and dichoromethane $(10 \mathrm{~mL})$. The reaction mixture was stirred for $1 \mathrm{~min}$. The solvents were removed in vacuo and the residue was purified by flash chromatography on silica gel eluting with $($ Hexane/EtOAc $=50: 50$ to 0:100) to give tetrahydropyrazolidine $\mathbf{2 . 1 6 . 1 3},(0.449 \mathrm{~g}, 82 \%)$ as white crystals $(\mathrm{dr}>95: 5)$.

Formula: $\mathrm{C}_{19} \mathrm{H}_{22} \mathrm{~N}_{2} \mathrm{O}_{6}$

Mass: $374.4 \mathrm{~g} / \mathrm{mol}$

Mp: $159-162^{\circ} \mathrm{C}$

IR $v_{\max }$ (neat): 1728, 1715, 1645, 1445, 1377, 775.

${ }^{1}$ H NMR (400 MHz, CDCl $): \delta 7.81(1 \mathrm{H}, \mathrm{d}, J=7.4 \mathrm{~Hz}, \mathrm{Ar}-H), 7.54(1 \mathrm{H}, \mathrm{d}, J=7.4 \mathrm{~Hz}, \mathrm{Ar}-H)$, $7.43(1 \mathrm{H}, \mathrm{t}, J=6.8 \mathrm{~Hz}, \mathrm{Ar}-H), 7.31(1 \mathrm{H}, \mathrm{t}, J=7.1 \mathrm{~Hz}, \mathrm{Ar}-H), 4.79\left(1 \mathrm{H}, \mathrm{s}, \mathrm{R}-\mathrm{CHCH}_{2} \mathrm{C}(\mathrm{O})-\right.$ $\left.\mathrm{OCH}_{3}\right), 3.80\left(4 \mathrm{H}, \mathrm{s}, \mathrm{R}-\mathrm{C}(\mathrm{O}) \mathrm{OCH}_{3}\right.$ and $\delta$ to $\left.-\mathrm{C}(\mathrm{O}) \mathrm{OCH}_{3}\right), 3.61\left(3 \mathrm{H}, \mathrm{s}, \mathrm{Ar}-\mathrm{C}(\mathrm{O}) \mathrm{OCH}_{3}\right), 3.12(1 \mathrm{H}$, d, $\left.J=15.1 \mathrm{~Hz}, \mathrm{R}-\mathrm{C} H \mathrm{H}-\mathrm{CHCH}_{2} \mathrm{C}(\mathrm{O}) \mathrm{OCH}_{3}\right), 2.69\left(2 \mathrm{H}, \mathrm{m}, \alpha\right.$ to $\left.-\mathrm{C}(\mathrm{O}) \mathrm{OCH}_{3}\right), 2.47(1 \mathrm{H}, \mathrm{dd}, J=$ 15.5, 9.9 Hz, R-CHH-CHCH $\left.2 \mathrm{C}(\mathrm{O}) \mathrm{OCH}_{3}\right), 2.05$ (2H, brs), 1.60 (2H, ddd, $J=12.5,10.1,7.1 \mathrm{~Hz}$, R-CH $\left.H_{2}-\mathrm{C}(\mathrm{O}) \mathrm{NR}_{2}\right), 1.45$ (2H, s, brs). 
${ }^{13} \mathrm{C}$ NMR (100 MHz, $\left.\mathrm{CDCl}_{3}\right): \delta$ 172.9, 171.2, 170.5, 166.4, 137.7, 132.3, 129.1, 128.8, 128.5, $126.260 .0,54.2,52.4,51.8,39.5,39.3,32.7,27.8,18.9$.

MS (ESI) $m / z$ (rel intensity): $397[\mathrm{M}+\mathrm{Na}]^{+}$

HRMS (ESI-TOF): Calculated for $\mathrm{C}_{19} \mathrm{H}_{22} \mathrm{~N}_{2} \mathrm{O}_{6}[\mathrm{M}+\mathrm{H}]^{+}: 375.1556$. Found: 375.1554.

\subsubsection{Experimental Procedure for Chapter III}

methyl (S)-3-((tert-butyldiphenylsilyl)oxy)-2-methylpropanoate, (3.42.1):

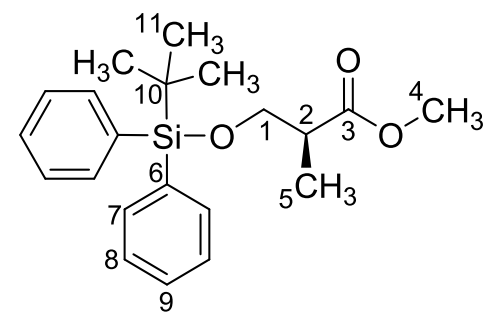

Tert-Butyldiphenylsilylchloride $(2.30 \mathrm{~mL}, 8.89 \mathrm{mmol})$ was added to a solution of commercially available (+)-(S)-methyl 3-hydroxy-2-methylpropionate $3.40 .5(0.93 \mathrm{~mL}, 8.46 \mathrm{mmol})$ and imidazole $(1.44 \mathrm{~g}, 21.1 \mathrm{mmol})$ in DMF $(2 \mathrm{~mL})$ at room temperature under argon. The reaction mixture was stirred at room temperature for $3 \mathrm{~h}$. Water was added, and the mixture was extracted with $\mathrm{Et}_{2} \mathrm{O}(3 \times 20 \mathrm{~mL})$. The combined organic extracts were washed sequentially with $1 \mathrm{M} \mathrm{HCl}$, saturated aqueous hydrogencarbonate solution and brine, then dried over anhydrous magnesium sulphate, filtered and concentrated in vасио. The residue was purified by flash chromatography on silica gel eluting with $(\mathrm{PET} / \mathrm{EtOAc}=100: 0$ to $95: 5)$ to give $\mathbf{3 . 4 2 . 1}(2.99 \mathrm{~g}, 99 \%)$ as a colorless oil.

Formula: $\mathrm{C}_{21} \mathrm{H}_{28} \mathrm{O}_{3} \mathrm{Si}$

Mass: $356.2 \mathrm{~g} / \mathrm{mol}$ 
${ }^{1}$ H NMR (400 MHz, Chloroform- $d$ ): $\delta 7.73-7.65\left(4 \mathrm{H}, \mathrm{m}, 4 \mathrm{H}_{9}\right), 7.55-7.34\left(6 \mathrm{H}, \mathrm{m}, 4 \mathrm{H}_{10}+\right.$ $\left.2 \mathrm{H}_{11}\right), 3.86\left(1 \mathrm{H}, \mathrm{dd}, J=9.8,6.9 \mathrm{~Hz}, \mathrm{H}_{1}\right), 3.76\left(1 \mathrm{H}, \mathrm{dd}, J=9.8,5.7 \mathrm{~Hz}, \mathrm{H}_{1^{\prime}}\right), 3.71\left(3 \mathrm{H}, \mathrm{s}, \mathrm{H}_{4}\right)$, $2.75\left(1 \mathrm{H}, \mathrm{q}, J=7.0 \mathrm{~Hz}, \mathrm{H}_{2}\right), 1.18\left(3 \mathrm{H}, \mathrm{d}, J=7.0 \mathrm{~Hz}, \mathrm{H}_{5}\right), 1.06\left(9 \mathrm{H}, \mathrm{s}, \mathrm{H}_{11}\right)$.

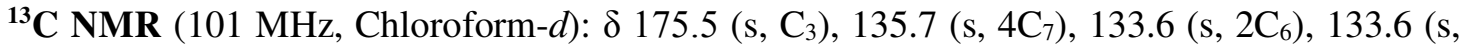
$\left.4 \mathrm{C}_{8}\right), 129.8\left(\mathrm{~s}, 2 \mathrm{C}_{9}\right), 127.8\left(\mathrm{~s}, 4 \mathrm{C}_{8}\right), 66.0\left(\mathrm{~s}, \mathrm{C}_{1}\right), 51.7\left(\mathrm{~s}, \mathrm{C}_{4}\right), 42.5\left(\mathrm{~s}, \mathrm{C}_{2}\right), 26.8\left(\mathrm{~s}, 3 \mathrm{C}_{11}\right), 19.4(\mathrm{~s}$, $\left.\mathrm{C}_{10}\right), 13.6\left(\mathrm{~s}, \mathrm{C}_{5}\right)$.

GCMS (EI) $m / z$ (rel intensity): 51 (22), 52 (20), 70 (88), 77 (50), 78 (79), 105 (24), 117 (24), 183 (73), 191 (100), 199 (50), 239 (52), 269 (41).

The data is consistent with reported literatures ${ }^{272}$

(S)-3-((tert-butyldiphenylsilyl)oxy)-N-methoxy-N,2-dimethylpropanamide, (3.42.2):

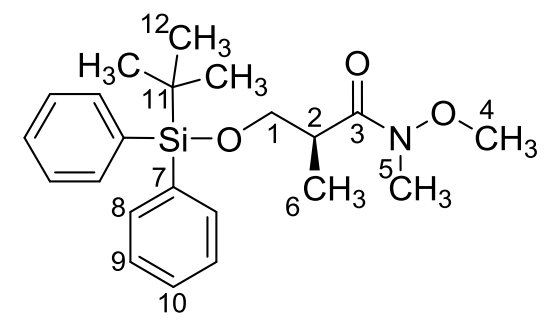

A solution of $i$-PrMgCl in THF (20 mL, $2.0 \mathrm{M}$ ) was added dropwise to a slurried mixture of 3.42.1 (4.75 g, $13.3 \mathrm{mmol})$ and $\mathrm{N}, \mathrm{O}$-dimethylhydroxylamine (2.02 g, $20.7 \mathrm{mmol})$ in THF (31 $\mathrm{mL}$ ) at $-40{ }^{\circ} \mathrm{C}$ over a period of $1 \mathrm{~h}$ under argon. The reaction mixture was stirred at $-10{ }^{\circ} \mathrm{C}$ for 2 h. The reaction mixture was quenched with saturated aqueous ammonium chloride at $-10{ }^{\circ} \mathrm{C}$, then allowed to warm to room temperature. The layers were separated and the aqueous layer was extracted with $\mathrm{Et}_{2} \mathrm{O}(3 \times 15 \mathrm{~mL})$. The combined organic extracts were washed with brine, dried over anhydrous magnesium sulphate, filtered and concentrated in vacuo. The residue was purified by flash chromatography on silica gel eluting with $(\mathrm{PET} / \mathrm{EtOAc}=80: 20)$ to give 3.42.2 $(5.09 \mathrm{~g}, 99 \%)$ as white crystals. 
${ }^{1}$ H NMR (400 MHz, Chloroform- $d$ ): $\delta 7.56-7.51\left(4 \mathrm{H}, \mathrm{m}, 4 \mathrm{H}_{9}\right), 7.30-7.21\left(6 \mathrm{H}, \mathrm{m}, 4 \mathrm{H}_{10}+\right.$ $\left.2 \mathrm{H}_{11}\right), 3.80\left(1 \mathrm{H}, \mathrm{dd}, J=9.4,8.0 \mathrm{~Hz}, \mathrm{H}_{1}\right), 3.52\left(3 \mathrm{H}, \mathrm{s}, \mathrm{H}_{4}\right), 3.47\left(1 \mathrm{H}, \mathrm{dd}, J=9.6,6.3 \mathrm{~Hz}, \mathrm{H}_{1^{\prime}}\right)$, $3.06\left(4 \mathrm{H}\right.$, br s, $\left.\mathrm{H}_{2}+\mathrm{H}_{5}\right), 0.95\left(3 \mathrm{H}, \mathrm{d}, J=6.8 \mathrm{~Hz}, \mathrm{H}_{6}\right), 0.90\left(9 \mathrm{H}, \mathrm{s}, \mathrm{H}_{12}\right)$.

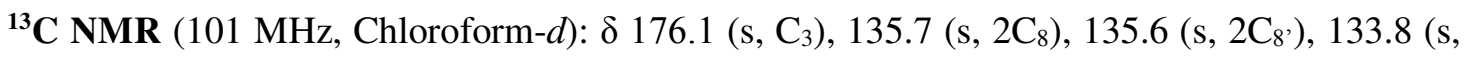
$\left.\mathrm{C}_{7}\right), 133.6\left(\mathrm{~s}, \mathrm{C}_{7}\right), 129.7\left(\mathrm{~s}, 2 \mathrm{C}_{10}\right), 127.7\left(\mathrm{~s}, 4 \mathrm{C}_{9}\right), 66.3\left(\mathrm{~s}, \mathrm{C}_{1}\right), 61.6\left(\mathrm{~s}, \mathrm{C}_{4}\right), 38.1\left(\mathrm{~s}, \mathrm{C}_{2}\right), 32.2(\mathrm{~s}$, $\left.3 \mathrm{C}_{5}\right), 26.9\left(\mathrm{~s}, 3 \mathrm{C}_{12}\right), 19.3\left(\mathrm{~s}, \mathrm{C}_{11}\right), 13.9\left(\mathrm{~s}, \mathrm{C}_{6}\right)$.

The data is consistent with reported literatures. ${ }^{273}$

(S)-3-((tert-butyldiphenylsilyl)oxy)-2-methylpropanal, (3.42.3):

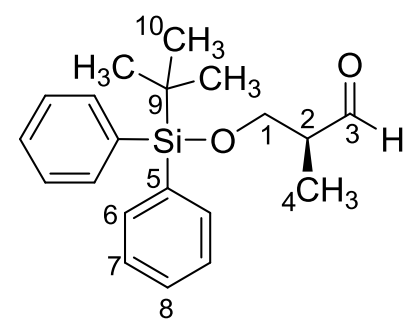

A solution of DIBAL-H in THF (3.90 mL, 1.0 M) was added dropwise to a solution of Weinreb amide 3.42.2 (1.0 g, $2.59 \mathrm{mmol})$ in THF $(9 \mathrm{~mL})$ at $-78{ }^{\circ} \mathrm{C}$ over a period of 15 minutes under argon. The reaction mixture was stirred at $-78^{\circ} \mathrm{C}$ for $1 \mathrm{~h}$ and at $-40{ }^{\circ} \mathrm{C}$ for a further $0.5 \mathrm{~h}$. The reaction mixture was slowly quenched with an excess amount of saturated aqueous sodium potassium tartarate solution (Rochelle), then allowed to warm to room temperature. Diethyl ether was added and the mixture was vigorously stirred at room temperature for $0.5 \mathrm{~h}$. The layers were separated and the aqueous layer was extracted with $\mathrm{Et}_{2} \mathrm{O}(3 \times 15 \mathrm{~mL})$. The combined organic extracts were washed with brine, dried over anhydrous sodium sulphate, filtered and concentrated in vacuo. The residue was azeotropically dried with toluene twice to give the crude aldehyde 3.42.3 (0.70 g, 82\%) as a colorless oil which was used in the proceeding allylation step without further purification. 
${ }^{1}$ H NMR (400 MHz, Chloroform- $d$ ): $\delta 9.77\left(1 \mathrm{H}, \mathrm{d}, J=1.6 \mathrm{~Hz}, \mathrm{H}_{3}\right), 7.67-7.63\left(4 \mathrm{H}, \mathrm{m}, 4 \mathrm{H}_{9}\right)$, $7.47-7.36\left(6 \mathrm{H}, \mathrm{m}, 4 \mathrm{H}_{10}+2 \mathrm{H}_{11}\right), 3.91\left(1 \mathrm{H}, \mathrm{dd}, J=10.3,5.0 \mathrm{~Hz}, \mathrm{H}_{1}\right), 3.85(1 \mathrm{H}, \mathrm{dd}, J=10.3$, $\left.6.4 \mathrm{~Hz}, \mathrm{H}_{1}\right), 2.61-2.54\left(1 \mathrm{H}, \mathrm{m}, \mathrm{H}_{2}\right), 1.10\left(3 \mathrm{H}, \mathrm{d}, J=7.0 \mathrm{~Hz}, \mathrm{H}_{4}\right), 1.04\left(9 \mathrm{H}, \mathrm{s}, \mathrm{H}_{10}\right)$

${ }^{13}$ C NMR (101 MHz, Chloroform- $d$ ): $\delta 204.6$ (s, C 3 ), 135.7 (s, 4C 6 ), 133.3 (s, 2C ), 130.0 (s, $\left.2 \mathrm{C}_{8}\right), 127.9\left(\mathrm{~s}, 4 \mathrm{C}_{7}\right), 64.3\left(\mathrm{~s}, \mathrm{C}_{1}\right), 49.0\left(\mathrm{~s}, \mathrm{C}_{2}\right), 26.9\left(\mathrm{~s}, \mathrm{C}_{10}\right), 19.4\left(\mathrm{~s}, \mathrm{C}_{9}\right), 10.5\left(\mathrm{~s}, \mathrm{C}_{4}\right)$.

GCMS (EI) m/z (rel intensity): 77 (22), 91 (25), 105 (55), 153 (27), 183 (57), 213 (100), 214 (21), 299 (81).

The data is consistent with reported literatures. ${ }^{272 a}$

(2S,3S)-1-((tert-butyldiphenylsilyl)oxy)-2-methylhex-5-en-3-ol, (3.42.4):

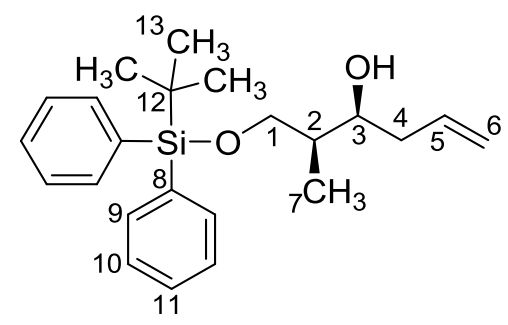

A solution of the aldehyde $\mathbf{3 . 4 2 . 3}(4.28 \mathrm{~g}, 13.1 \mathrm{mmol})$ in $\mathrm{Et}_{2} \mathrm{O}(26 \mathrm{~mL})$ was added to a suspension of $(R, R)-\mathbf{T i}(9.73 \mathrm{~g}, 15.7 \mathrm{mmol})$ in $\mathrm{Et}_{2} \mathrm{O}(105 \mathrm{~mL})$ at $-78{ }^{\circ} \mathrm{C}$ over a period of 10 minutes under argon. The reaction mixture was stirred at $-78{ }^{\circ} \mathrm{C}$ for $24 \mathrm{~h}$. The reaction mixture was quenched with deionized water at $-78{ }^{\circ} \mathrm{C}$, then allowed to warm to room temperature. The mixture was stirred at room temperature for $24 \mathrm{~h}$. The reaction media was filtered on a pad of Celite ${ }^{\circledR}$. The layers were separated and the aqueous layer was extracted with $\mathrm{Et}_{2} \mathrm{O}(3 \times 15 \mathrm{~mL})$. The combined organic extracts were washed with brine, dried over anhydrous magnesium sulphate, filtered and concentrated in vacuo. Pentane $(200 \mathrm{~mL})$ was added, the mixture was stirred vigorously at room temperature for $4 \mathrm{~h}$, filtered and concentrated in vacuo. The residue 
was purified by flash chromatography on silica gel eluting with (PET/EtOAc $=90: 10$ to $85: 15)$ to give homoallylic alcohol 3.42.4 (3.34 g, 69\%) as a colorless oil $(\mathrm{dr}=19: 1)$.

Formula: $\mathrm{C}_{23} \mathrm{H}_{32} \mathrm{O}_{2} \mathrm{Si}$

Mass: $368.2 \mathrm{~g} / \mathrm{mol}$

IR $v_{\max }$ (neat): 3455, 2931, 1641, 1589, 1472, 1427, 1391, 1362, 1109, 822, 739, 700, 612 $\mathrm{cm}^{-1}$.

${ }^{1} \mathbf{H}$ NMR (400 MHz, Chloroform- $d$ ): $\delta 7.75-7.68\left(4 \mathrm{H}, \mathrm{m}, 4 \mathrm{H}_{9}\right), 7.55-7.35\left(6 \mathrm{H}, \mathrm{m}, 4 \mathrm{H}_{10}+\right.$ $\left.2 \mathrm{H}_{11}\right), 5.88\left(1 \mathrm{H}, \mathrm{ddt}, J=17.2,10.2,7.1,1.0 \mathrm{~Hz}, \mathrm{H}_{5}\right), 5.21-5.06\left(2 \mathrm{H}, \mathrm{m}, \mathrm{H}_{6}\right), 4.00-3.92(1 \mathrm{H}$, m, $\left.\mathrm{H}_{3}\right), 3.78\left(1 \mathrm{H}, \mathrm{dd}, J=10.1,4.4 \mathrm{~Hz}, \mathrm{H}_{1}\right), 3.72\left(1 \mathrm{H}, \mathrm{dd}, J=10.1,5.6 \mathrm{~Hz}, \mathrm{H}_{1}\right), 2.84(1 \mathrm{H}, \mathrm{d}, J$ $=3.5 \mathrm{~Hz},-\mathrm{OH}), 2.40-2.15\left(2 \mathrm{H}, \mathrm{m}, \mathrm{H}_{4}\right), 1.88-1.71\left(1 \mathrm{H}, \mathrm{m}, \mathrm{H}_{2}\right), 1.10\left(9 \mathrm{H}, \mathrm{s}, \mathrm{H}_{13}\right), 0.99(3 \mathrm{H}$, $\left.\mathrm{d}, J=7.1,1.1 \mathrm{~Hz}, \mathrm{H}_{7}\right)$.

${ }^{13}$ C NMR (101 MHz, Chloroform- $d$ ): $\delta 135.8$ (s, $\left.\mathrm{C}_{6}\right), 135.7$ (s, 4C $\left.\mathrm{C}_{9}\right), 133.2\left(\mathrm{~s}, \mathrm{C}_{8}\right), 133.1\left(\mathrm{~s}, \mathrm{C}_{8}\right)$, $129.9\left(\mathrm{~s}, 2 \mathrm{C}_{11}\right), 127.9\left(\mathrm{~s}, 4 \mathrm{C}_{10}\right), 117.2\left(\mathrm{~s}, \mathrm{C}_{5}\right), 73.4\left(\mathrm{~s}, \mathrm{C}_{3}\right), 68.6\left(\mathrm{~s}, \mathrm{C}_{1}\right), 39.1\left(\mathrm{~s}, \mathrm{C}_{4}\right), 38.9\left(\mathrm{~s}, \mathrm{C}_{2}\right)$ $27.0\left(\mathrm{~s}, 3 \mathrm{C}_{13}\right), 19.3\left(\mathrm{~s}, \mathrm{C}_{12}\right), 10.4\left(\mathrm{~s}, \mathrm{C}_{7}\right)$.

GCMS (EI) m/z (rel intensity): 95 (83), 135 (16), 139 (16), 181 (12), 199 (100), 200 (19), 229 (16), $230(4)$.

HRMS (ESI-TOF): Calculated for $\mathrm{C}_{23} \mathrm{H}_{32} \mathrm{O}_{2} \mathrm{SiNa}$ [M + Na] $]^{+}:$391.2069. Found: 391.2064.

The data is consistent with reported literatures. ${ }^{274}$ 
(3.40.4):

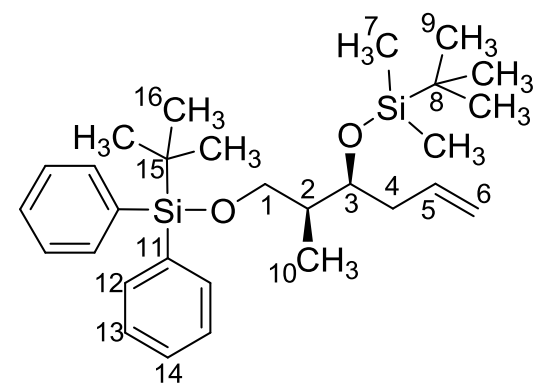

The base 2,6-lutidine $(0.61 \mathrm{~mL}, 5.22 \mathrm{mmol})$ and TBSOTf $(0.60 \mathrm{~mL}, 2.61 \mathrm{mmol})$ were successively added to a solution of homoallylic alcohol $\mathbf{3 . 4 2 . 4}(0.64 \mathrm{~g}, 1.74 \mathrm{mmol})$ in $\mathrm{CH}_{2} \mathrm{Cl}_{2}$ $(17 \mathrm{~mL})$ at $-78^{\circ} \mathrm{C}$. The reaction mixture was allowed to warm to room temperature and stirred overnight. A saturated aqueous solution of $\mathrm{NaHCO}_{3}(14 \mathrm{~mL})$ was added, and the two phases were separated. The aqueous layer was extracted with $\mathrm{CH}_{2} \mathrm{Cl}_{2}(3 \times 15 \mathrm{~mL})$. The combined organic extracts were washed with brine, dried over anhydrous magnesium sulphate, filtered and concentrated in vacuo. The residue was purified by flash chromatography on silica gel eluting with $(\mathrm{PET} / \mathrm{EtOAc}=95: 5)$ to give alkene 3.40.4 $(0.84 \mathrm{~g}, 99 \%)$ as a colorless oil.

Formula: $\mathrm{C}_{29} \mathrm{H}_{46} \mathrm{O}_{2} \mathrm{Si}_{2}$

Mass: $482.3 \mathrm{~g} / \mathrm{mol}$

${ }^{1}$ H NMR (400 MHz, Chloroform- $d$ ): $\delta 7.68-7.63\left(4 \mathrm{H}, \mathrm{m}, 4 \mathrm{H}_{12}\right), 7.42-7.34\left(6 \mathrm{H}, \mathrm{m}, 4 \mathrm{H}_{13}+\right.$ $\left.2 \mathrm{H}_{14}\right), 5.79-5.66\left(1 \mathrm{H}, \mathrm{m}, \mathrm{H}_{5}\right), 5.05-4.96\left(2 \mathrm{H}, \mathrm{m}, \mathrm{H}_{6}\right), 3.90\left(1 \mathrm{H}, \mathrm{dt}, J=6.5,3.1 \mathrm{~Hz}, \mathrm{H}_{3}\right), 3.61$ $\left(1 \mathrm{H}, \mathrm{dd}, J=9.9,6.9 \mathrm{~Hz}, \mathrm{H}_{1}\right), 3.46\left(1 \mathrm{H}, \mathrm{dd}, J=9.9,6.5 \mathrm{~Hz}, \mathrm{H}_{1^{\prime}}\right), 2.28-2.12\left(2 \mathrm{H}, \mathrm{m}, \mathrm{H}_{4}\right), 1.80$ $-1.69\left(1 \mathrm{H}, \mathrm{m}, \mathrm{H}_{2}\right), 1.05\left(9 \mathrm{H}, \mathrm{s}, \mathrm{H}_{13}\right), 0.85-0.83\left(12 \mathrm{H}, \mathrm{m}, \mathrm{H}_{9}+\mathrm{H}_{10}\right), 0.03\left(3 \mathrm{H}, \mathrm{s}, \mathrm{H}_{7}\right),-0.01$ $\left(3 \mathrm{H}, \mathrm{s}, \mathrm{H}_{7}\right)$.

${ }^{13}$ C NMR (101 MHz, Chloroform- $d$ ): $\delta 135.5$ (s, 4C $\mathrm{C}_{12}$ ), 135.3 (s, $\mathrm{C}_{5}$ ), 133.9 (s, 2C $\mathrm{C}_{11}$ ), 129.4 (s, $\left.\mathrm{C}_{14}\right), 129.3\left(\mathrm{~s}, \mathrm{C}_{14}\right), 127.4\left(\mathrm{~s}, 4 \mathrm{C}_{13}\right), 116.4\left(\mathrm{~s}, \mathrm{C}_{6}\right), 71.4\left(\mathrm{~s}, \mathrm{C}_{3}\right), 66.1\left(\mathrm{~s}, \mathrm{C}_{1}\right), 39.6\left(\mathrm{~s}, \mathrm{C}_{2}\right), 39.5(\mathrm{~s}$, $\left.\mathrm{C}_{4}\right), 26.8\left(\mathrm{~s}, 3 \mathrm{C}_{16}\right), 25.8\left(\mathrm{~s}, 3 \mathrm{C}_{9}\right), 19.1\left(\mathrm{~s}, \mathrm{C}_{15}\right), 18.0\left(\mathrm{~s}, \mathrm{C}_{8}\right), 10.3\left(\mathrm{~s}, \mathrm{C}_{10}\right),-4.2\left(\mathrm{~s}, \mathrm{C}_{7}\right),-4.9\left(\mathrm{~s}, \mathrm{C}_{7}\right)$. 
GCMS (EI) m/z (rel intensity): 73 (42), 91 (44), 95 (100), 131 (27), 135 (51), 175 (22), 193 (25), 197 (21), 209(59), 235 (19), 271 (33), 313 (24).

The data is consistent with reported literatures. ${ }^{275}$

\section{(5S,3S,2S)-3-((tert-butyldimethylsilyl)oxy)-1-((tert-butyldiphenylsilyl)oxy)-5-hydroxy-2-}

methyloctan-7-one, (3.42.5):

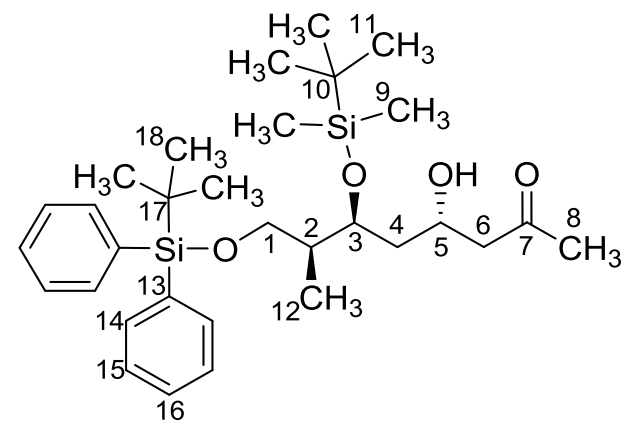

Mukaiyama Aldol:

Dimethylaluminium chloride $(82.5 \mu \mathrm{L}, 0.083 \mathrm{mmol})$ was added dropwise to a stirring solution of aldehyde $\mathbf{3 . 4 0 . 3}$ (40.0 mg, $0.083 \mathrm{mmol})$ and (isopropenyloxy)trimethylsilane (11.8 mg, 0.091 mmol) in dichloromethane $(3 \mathrm{~mL})$ at $-78{ }^{\circ} \mathrm{C}$. The reaction mixture was stirred for $0.5 \mathrm{~h}$ then quenched by $\mathrm{NaHCO}_{3}(1 \mathrm{~mL})$ at the $-78{ }^{\circ} \mathrm{C}$ temperature and allowed to warm to room temperature. The phases were separated and extracted with EtOAc $(3 \times 5 \mathrm{~mL})$ and the combined organic extracts were dried over anhydrous magnesium sulphate, filtered and concentrated in vacuo to give methyl ketone $\mathbf{3 . 4 5 . 5}(0.025 \mathrm{~g}, 56 \%)$ as a dark viscous oil, $(\mathrm{dr}=3: 1)$.

\section{Wacker Oxidation:}

Tetrahydrofuran $(1.30 \mathrm{~mL})$ and water $(0.12 \mathrm{~mL})$ were added to a mixture of $\mathrm{PdCl}_{2}(3.37 \mathrm{mg}$, $19.0 \mu \mathrm{mol})$ and $\mathrm{CuCl}(18.8 \mathrm{mg}, 0.19 \mathrm{mmol})$. Oxygen was bubbled through the reaction mixture for $1.5 \mathrm{~h}$ before the alkene $3.42 .6(0.10 \mathrm{~g}, 0.19 \mathrm{mmol})$ was added. The reaction mixture was stirred at room temperature for $18 \mathrm{~h}$ under continuous oxygen bubbling. Water was added, and 
mixture was extracted with $\mathrm{CH}_{2} \mathrm{Cl}_{2}(3 \times 5 \mathrm{~mL})$. The combined organic extracts were washed with a solution of $\mathrm{LiCl}$, dried over anhydrous magnesium sulphate, filtered and concentrated in vacuo. The residue was purified by flash chromatography on silica gel eluting with (PET/EtOAc $=70: 30)$ to give methyl ketone $\mathbf{3 . 4 2 . 5}(0.075 \mathrm{~g}, 73 \%)$ as a yellow oil.

Formula: $\mathrm{C}_{31} \mathrm{H}_{50} \mathrm{O}_{4} \mathrm{Si}_{2}$

Mass: $542.32 \mathrm{~g} / \mathrm{mol}$

${ }^{1}$ H NMR (400 MHz, Chloroform- $d$ ): $\delta 7.69-7.61\left(4 \mathrm{H}, \mathrm{m}, \mathrm{H}_{14}\right), 7.45-7.34\left(6 \mathrm{H}, \mathrm{m}, 2 \mathrm{H}_{16}+4 \mathrm{H}_{15}\right)$, $4.12\left(1 \mathrm{H}, \mathrm{m}, \mathrm{H}_{5}\right), 4.00\left(1 \mathrm{H}, \mathrm{m}, \mathrm{H}_{3}\right), 3.64\left(1 \mathrm{H}, \mathrm{dd}, J=9.9,5.4 \mathrm{~Hz}, \mathrm{H}_{1}\right), 3.51\left(1 \mathrm{H}, \mathrm{dd}, J=9.9,6.4 \mathrm{~Hz}, \mathrm{H}_{1^{\prime}}\right)$, $3.24(1 \mathrm{H}, \mathrm{s},-\mathrm{OH}), 2.10\left(3 \mathrm{H}, \mathrm{s}, \mathrm{H}_{8}\right), 1.91-1.73\left(1 \mathrm{H}, \mathrm{m}, \mathrm{H}_{2}\right), 1.61-1.51\left(2 \mathrm{H}, \mathrm{m}, \mathrm{H}_{4}\right), 1.42\left(2 \mathrm{H}, \mathrm{m}, \mathrm{H}_{6}\right)$, $1.02\left(9 \mathrm{H}, \mathrm{s}, \mathrm{H}_{18}\right), 0.92\left(3 \mathrm{H}, \mathrm{dd}, J=7.1,2.1 \mathrm{~Hz}, \mathrm{H}_{12}\right), 0.82\left(9 \mathrm{H}, \mathrm{s}, \mathrm{H}_{11}\right), 0.06\left(3 \mathrm{H}, \mathrm{s}, \mathrm{H}_{9}\right), 0.00\left(3 \mathrm{H}, \mathrm{s}, \mathrm{H}_{9}\right.$ )

${ }^{13}$ C NMR (101 MHz, Chloroform- $d$ ): $\delta 209.4\left(\mathrm{~s}, \mathrm{C}_{7}\right), 135.6$ (s, 4C $\mathrm{C}_{14}$ ), 133.9 (s, $\left.\mathrm{C}_{13}\right), 133.8$ (s, $\left.\mathrm{C}_{13}{ }^{3}\right), 129.6\left(\mathrm{~s}, 2 \mathrm{C}_{16}\right), 127.6\left(\mathrm{~s}, 4 \mathrm{C}_{15}\right), 70.5\left(\mathrm{~s}, \mathrm{C}_{3}\right), 65.3\left(\mathrm{~s}, \mathrm{C}_{1}\right), 64.5\left(\mathrm{~s}, \mathrm{C}_{5}\right), 50.6\left(\mathrm{~s}, \mathrm{C}_{6}\right), 40.9$

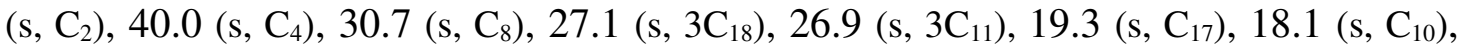
$12.5\left(\mathrm{~s}, \mathrm{C}_{12}\right),-4.4\left(\mathrm{~s}, \mathrm{C}_{9}\right),-4.5\left(\mathrm{~s}, \mathrm{C}_{9}{ }^{\prime}\right)$.

(2S,3S,5R)-3-((tert-butyldimethylsilyl)oxy)-1-((tert-butyldiphenylsilyl)oxy)-2-methyloct8-en-5-ol, (3.42.6):

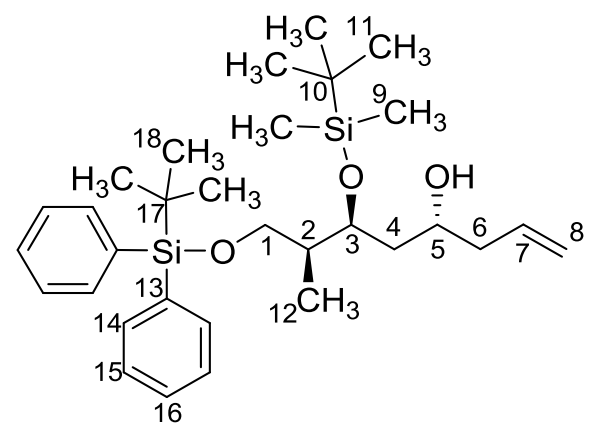

A solution of osmium tetroxide in tert-butanol $(0.44 \mathrm{~mL}, 0.0344 \mathrm{mmol}, 0.079 \mathrm{M})$ followed by NMO (0.22 g, $1.89 \mathrm{mmol})$ were added to a mixture of alkene $\mathbf{3 . 4 0 . 4}(0.83 \mathrm{~g}, 1.72 \mathrm{mmol})$, water $(4.50 \mathrm{~mL})$ and tert-butanol $(4.50 \mathrm{~mL})$ at room temperature. The reaction mixture was stirred at 
room temperature for $8 \mathrm{~h}$, and additional portions of osmium tetroxide $(0.44 \mathrm{~mL}, 0.0344 \mathrm{mmol}$, $0.079 \mathrm{M})$ and NMO (0.22 $\mathrm{g}, 1.89 \mathrm{mmol})$ were added. The mixture was stirred at room temperature for $20 \mathrm{~h}$. Solid $\mathrm{Na}_{2} \mathrm{~S}_{2} \mathrm{O}_{3}(1.80 \mathrm{~g})$, Celite (3.50 g), and EtOAc (7.0 mL) were added, and the mixture was filtered through a pad of Celite ${ }^{\circledR}$ which was thoroughly washed with EtOAc. The filtrate was concentrated in vacuo, and the residue was dissolved in (1:1) mixture of $\mathrm{H}_{2} \mathrm{O}$ :THF $(20 \mathrm{~mL})$. Sodium metaperiodate $(0.92 \mathrm{~g}, 4.30 \mathrm{mmol})$ was then added to the mixture and stirred for $3 \mathrm{~h}$ at room temperature. The biphasic mixture was filtered through a pad of Celite ${ }^{\circledR}$, the layers were separated, and the aqueous layer was extracted with EtOAc ( 3 x 15 $\mathrm{mL}$ ). The combined organic extracts were washed with brine, dried over anhydrous magnesium sulphate, filtered and concentrated in vacuo. The residue was azeotropically dried with toluene twice to give the crude aldehyde $\mathbf{3 . 4 0 . 3}$ as a yellow oil which was used in the proceeding allylation step without further purification.

A solution of the aldehyde $\mathbf{3 . 4 0 . 3}\left(1.72 \mathrm{mmol}, 1.0\right.$ equiv) in $\mathrm{Et}_{2} \mathrm{O}(5 \mathrm{~mL})$ was added to a suspension of $(S, S)-\mathbf{T i}(1.28 \mathrm{~g}, 2.06 \mathrm{mmol})$ in $\mathrm{Et}_{2} \mathrm{O}(14 \mathrm{~mL})$ at $-78^{\circ} \mathrm{C}$ over a period of 10 minutes under argon. The mixture was stirred at $-78{ }^{\circ} \mathrm{C}$ for $24 \mathrm{~h}$. The reaction mixture was quenched with deionized water at $-78{ }^{\circ} \mathrm{C}$, then allowed to warm to room temperature. The mixture was stirred at room temperature for $24 \mathrm{~h}$. The reaction media was filtered through a pad of Celite®. The layers were separated and the aqueous layer was extracted with $\mathrm{Et}_{2} \mathrm{O}(3 \times 15 \mathrm{~mL})$. The combined organic extracts were washed with brine, dried over anhydrous magnesium sulphate, filtered and concentrated in vacuo. Pentane $(200 \mathrm{~mL})$ was added, the mixture was stirred vigorously at room temperature for $4 \mathrm{~h}$, filtered and concentrated in vacuo. The residue was purified by flash chromatography on silica gel eluting with (PET/EtOAc $=85: 15$ to $80: 20)$ to give homoallylic alcohol 3.42.6 (0.48 g, 53\%) as a yellow oil.

Formula: $\mathrm{C}_{31} \mathrm{H}_{50} \mathrm{O}_{3} \mathrm{Si}_{2}$

Mass: $526.3 \mathrm{~g} / \mathrm{mol}$ 
${ }^{1}$ H NMR (400 MHz, Chloroform- $d$ ): $\delta 7.69-7.62\left(4 \mathrm{H}, \mathrm{m}, 4 \mathrm{H}_{14}\right), 7.45-7.33\left(6 \mathrm{H}, \mathrm{m}, 4 \mathrm{H}_{15}+\right.$ $\left.2 \mathrm{H}_{16}\right), 5.77-5.67\left(1 \mathrm{H}, \mathrm{m}, \mathrm{H}_{7}\right), 5.14-5.04\left(2 \mathrm{H}, \mathrm{m}, \mathrm{H}_{8}\right), 4.01\left(1 \mathrm{H}, \mathrm{q}, J=5.2 \mathrm{~Hz}, \mathrm{H}_{5}\right), 3.86-$ $3.77\left(1 \mathrm{H}, \mathrm{m}, \mathrm{H}_{3}\right), 3.62\left(1 \mathrm{H}, \mathrm{dd}, J=10.0,5.1 \mathrm{~Hz}, \mathrm{H}_{1}\right), 3.50\left(1 \mathrm{H}, \mathrm{dd}, J=10.0,6.1 \mathrm{~Hz}, \mathrm{H}_{1}{ }^{\prime}\right), 2.18$ $-2.11\left(2 \mathrm{H}, \mathrm{m}, \mathrm{H}_{6}\right), 1.93-1.82\left(1 \mathrm{H}, \mathrm{m}, \mathrm{H}_{2}\right), 1.59-1.47\left(2 \mathrm{H}, \mathrm{m}, \mathrm{H}_{4}\right), 0.98(3 \mathrm{H}, \mathrm{d}, J=6.9 \mathrm{~Hz}$ $\left.\mathrm{H}_{12}\right), 1.05$ (9H, s, $\left.\mathrm{H}_{18}\right), 0.87$ (9H, s, $\left.\mathrm{H}_{11}\right), 0.09$ (3H, s, $\left.\mathrm{H}_{9}\right), 0.04$ (3H, s, $\mathrm{H}_{9}$ )

${ }^{13}$ C NMR (101 MHz, Chloroform- $d$ ): $\delta 135.7$ (s, 4C 14 ), 134.9 (s, $\mathrm{C}_{7}$ ), 133.9 (s, 2C 13 ), 129.7 (s, 2C $\left.\mathrm{C}_{16}\right), 127.8\left(\mathrm{~s}, 4 \mathrm{C}_{15}\right), 117.8\left(\mathrm{~s}, \mathrm{C}_{8}\right), 71.9\left(\mathrm{~s}, \mathrm{C}_{5}\right), 67.9\left(\mathrm{~s}, \mathrm{C}_{3}\right), 65.7\left(\mathrm{~s}, \mathrm{C}_{1}\right), 42.6\left(\mathrm{~s}, \mathrm{C}_{6}\right), 40.7(\mathrm{~s}$, $\left.\mathrm{C}_{2}\right), 40.0\left(\mathrm{~s}, \mathrm{C}_{4}\right), 27.0\left(\mathrm{~s}, 3 \mathrm{C}_{18}\right), 26.1\left(\mathrm{~s}, 3 \mathrm{C}_{11}\right), 19.4\left(\mathrm{~s}, \mathrm{C}_{17}\right), 18.2\left(\mathrm{~s}, \mathrm{C}_{10}\right), 13.3\left(\mathrm{~s}, \mathrm{C}_{12}\right),-4.2(\mathrm{~s}$, $\left.\mathrm{C}_{9}\right),-4.5\left(\mathrm{~s}, \mathrm{C}_{9}\right)^{\prime}$.

GCMS (EI) $m / z$ (rel intensity): 73 (58), 75 (100), 79 (22), 91 (53), 121 (53), 135 (78), 195 (25), 197 (30), 199 (71), 209 (22), 267 (20), 337 (14).

The data is consistent with reported literatures. ${ }^{275}$

(5R,3S,2S)-3-((tert-butyldimethylsilyl)oxy)-1-((tert-butyldiphenylsilyl)oxy)-2-methyloct-1en-5-yl acetate, (3.53.1):

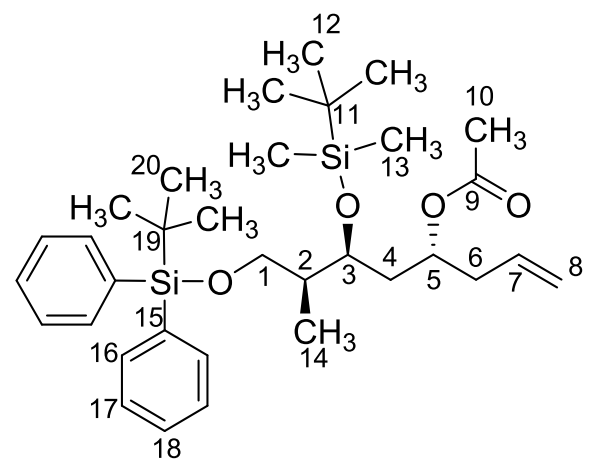

Alcohol Protection ${ }^{276}$ :

Pyridine $(0.23 \mathrm{~mL}, 2.87 \mathrm{mmol})$ and DMAP $(1 \mathrm{mg}, 8.19 \mu \mathrm{mol})$ were added to a cooled solution of alcohol 3.42.6 (0.167 $\mathrm{g}, 0.316 \mathrm{mmol})$ and acetic anhydride $(0.1 \mathrm{~mL}, 0.475 \mathrm{mmol})$ in dichloromethane $(0.5 \mathrm{~mL})$ at $0{ }^{\circ} \mathrm{C}$ under argon atmosphere. The reaction mixture was stirred at 
room temperature for $2 \mathrm{~h}$. Saturated bicarbonate solution was then added $(1 \mathrm{~mL})$ and stirred for an additional $2 \mathrm{~h}$. The mixture was extracted with $\mathrm{Et}_{2} \mathrm{O}(3 \mathrm{X} 5 \mathrm{~mL})$, and the combined organic extracts were washed with copper sulphate solution, dried over anhydrous magnesium sulphate, filtered and concentrated in vacuo. The residue was purified by flash chromatography on silica gel eluting with $(\mathrm{PET} / \mathrm{EtOAc}=95: 5)$ to give allyl acetate 3.54.1 $(0.14 \mathrm{~g}, 78 \%)$ as a colorless oil.

Formula: $\mathrm{C}_{33} \mathrm{H}_{52} \mathrm{O}_{4} \mathrm{Si}_{2}$

Mass: $568.3 \mathrm{~g} / \mathrm{mol}$

${ }^{1} \mathbf{H}$ NMR (400 MHz, Chloroform- $d$ ): $\delta 7.69-7.63\left(4 \mathrm{H}, \mathrm{m}, 4 \mathrm{H}_{16}\right), 7.46-7.34\left(6 \mathrm{H}, \mathrm{m}, 4 \mathrm{H}_{17}+\right.$ $\left.2 \mathrm{H}_{18}\right), 5.81-5.65\left(1 \mathrm{H}, \mathrm{m}, \mathrm{H}_{7}\right), 5.12-5.01\left(2 \mathrm{H}, \mathrm{m}, \mathrm{H}_{8}\right), 4.95-4.85\left(1 \mathrm{H}, \mathrm{m}, \mathrm{H}_{2}\right), 3.94(1 \mathrm{H}, \mathrm{ddd}, J=$ 7.8, 5.1, $\left.2.7 \mathrm{~Hz}, \mathrm{H}_{3}\right), 3.62\left(1 \mathrm{H}, \mathrm{dd}, J=9.9,6.9 \mathrm{~Hz}, \mathrm{H}_{1}\right), 3.51\left(1 \mathrm{H}, \mathrm{dd}, J=9.9,6.1 \mathrm{~Hz}, \mathrm{H}_{1^{\prime}}\right), 2.41-2.25$ $\left(2 \mathrm{H}, \mathrm{m}, \mathrm{H}_{6}\right), 2.02\left(3 \mathrm{H}, \mathrm{s}, \mathrm{H}_{10}\right), 1.81-1.69\left(2 \mathrm{H}, \mathrm{m}, \mathrm{H}_{4}\right), 1.63\left(1 \mathrm{H}, \mathrm{m}, \mathrm{H}_{2}\right), 1.05\left(9 \mathrm{H}, \mathrm{s}, \mathrm{H}_{20}\right), 0.83(9 \mathrm{H}$, s, $\left.\mathrm{H}_{12}\right), 0.76\left(3 \mathrm{H}, \mathrm{d}, J=6.8 \mathrm{~Hz}, \mathrm{H}_{14}\right), 0.02\left(3 \mathrm{H}, \mathrm{s}, \mathrm{H}_{13}\right),-0.02\left(3 \mathrm{H}, \mathrm{s}, \mathrm{H}_{13}\right)$.

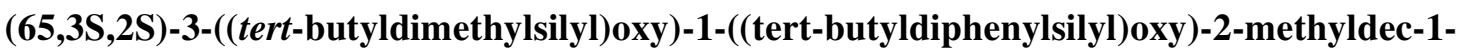
ene-5,7-diol, (3.51.1):

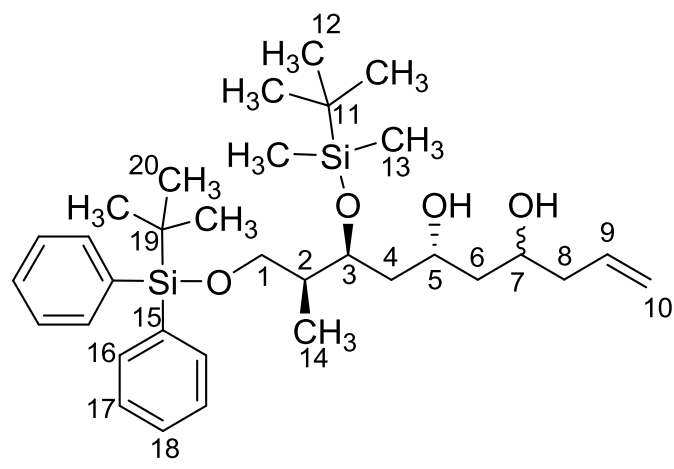

A solution of osmium tetroxide in tert-butanol $(65.0 \mu \mathrm{L}, 5.13 \mu \mathrm{mol}, 0.079 \mathrm{M})$ followed by NMO $(0.33 \mathrm{~g}, 0.28 \mathrm{mmol})$ were added to a mixture of alkene $\mathbf{3 . 4 2 . 6}(0.13 \mathrm{~g}, 0.26 \mathrm{mmol})$, water $(4.5$ $\mathrm{mL})$ and tert-butanol $(4.5 \mathrm{~mL})$ at room temperature. The reaction mixture was stirred at room temperature for $8 \mathrm{~h}$, and additional portions of osmium tetroxide $(65.0 \mu \mathrm{L}, 5.13 \mu \mathrm{mol}, 0.079 \mathrm{M})$ 
and NMO (0.33 g, $0.28 \mathrm{mmol})$ were added. The mixture was stirred at room temperature for 20 h. Solid $\mathrm{Na}_{2} \mathrm{~S}_{2} \mathrm{O}_{3}(0.30 \mathrm{~g})$, Celite $(0.53 \mathrm{~g})$, and EtOAc $(1 \mathrm{~mL})$ were added, and the mixture was filtered through a pad of Celite® which was thoroughly washed with EtOAc. The filtrate was concentrated in vacuo, and the residue was dissolved in (1:1) mixture of $\mathrm{H}_{2} \mathrm{O}$ :THF $(3 \mathrm{~mL})$. Sodium metaperiodate $(0.14 \mathrm{~g}, 0.64 \mathrm{mmol})$ was then added to the mixture and stirred for $3 \mathrm{~h}$ at room temperature. The biphasic mixture was filtered through a pad of Celite ${ }^{\circledR}$, the layers were separated, and the aqueous layer was extracted with EtOAc $(3 \times 5 \mathrm{~mL})$. The combined organic extracts were washed with brine, dried over anhydrous magnesium sulphate, filtered and concentrated in vacuo. The residue was azeotropically dried with toluene twice to give the crude aldehyde 3.57.1 as a yellow oil which was used in the proceeding allylation step without further purification.

\section{Barbier Allylation: ${ }^{277}$}

Allyl bromide ( $31.2 \mu \mathrm{L}, 0.359 \mathrm{mmol})$ was added to a mixture of aldehyde $\mathbf{3 . 5 7 . 1}(0.136 \mathrm{~g}, 0.256$ $\mathrm{mmol})$ and indium powder $(0.0324 \mathrm{~g}, 0.282 \mathrm{mmol})$ in water $(1.5 \mathrm{~mL})$ and $\mathrm{THF}(1.5 \mathrm{~mL})$ at room temperature. The reaction was stirred vigorously at room temeperature until the metal had dissolved ( $2 \mathrm{~h}$ to $3 \mathrm{~h})$ at which time the TLC indicated a complete reaction. Aqueous $\mathrm{NaHCO}_{3}$ (10\%) was added and the mixture was extracted with EtOAc $(3 \times 5 \mathrm{~mL})$, and the combined organic extracts were dried over anhydrous magnesium sulphate, filtered and concentrated in vacuo. The residue was purified by flash chromatography on silica gel eluting with (PET/EtOAc $=60: 40)$ to give homoallylic alcohol 3.51.1 $(0.0677 \mathrm{~g}, 46 \%)$ as a yellow oil, $(\mathrm{dr}=1: 1)$.

Formula: $\mathrm{C}_{33} \mathrm{H}_{54} \mathrm{O}_{4} \mathrm{Si}_{2}$

Mass: $570.1 \mathrm{~g} / \mathrm{mol}$

${ }^{1} \mathbf{H}$ NMR (400 MHz, Chloroform- $d$ ): $\delta 7.66-7.57\left(4 \mathrm{H}, \mathrm{m}, 4 \mathrm{H}_{16}\right), 7.44-7.30\left(6 \mathrm{H}, \mathrm{m}, 4 \mathrm{H}_{17}+2 \mathrm{H}_{18}\right)$, $5.77\left(1 \mathrm{H}, \mathrm{m}, \mathrm{H}_{9}\right), 5.16-4.85\left(2 \mathrm{H}, \mathrm{m}, \mathrm{H}_{10}\right), 4.16-3.75\left(2 \mathrm{H}, \mathrm{m}, \mathrm{H}_{7}+\mathrm{H}_{3}\right), 3.68-3.54\left(2 \mathrm{H}, \mathrm{m}, \mathrm{H}_{1}+\mathrm{H}_{5}\right)$, $3.46(1 \mathrm{H}, \mathrm{dd}, J=9.5,5.0 \mathrm{~Hz}), 2.25-2.09\left(2 \mathrm{H}, \mathrm{m}, \mathrm{H}_{8}\right), 2.01\left(1 \mathrm{H}, \mathrm{m}, \mathrm{H}_{2}\right), 1.84-1.64\left(2 \mathrm{H}, \mathrm{m}, \mathrm{H}_{4}\right), 1.48$ 
$\left(1 \mathrm{H}, \mathrm{m}, \mathrm{H}_{6}\right), 1.38\left(1 \mathrm{H}, \mathrm{m}, \mathrm{H}_{6}\right), 1.02\left(9 \mathrm{H}, \mathrm{s}, \mathrm{H}_{20}\right), 0.89-0.77\left(12 \mathrm{H}, \mathrm{m}, 9 \mathrm{H}_{12}+3 \mathrm{H}_{14}\right), 0.09-0.01(3 \mathrm{H}, \mathrm{s}$, $\left.\mathrm{H}_{13}\right), 0.01--0.06\left(3 \mathrm{H}, \mathrm{s}, \mathrm{H}_{13^{\prime}}\right)$.

tert-butyl(((2S,3S)-3-((4-methoxybenzyl)oxy)-2-methylhex-5-en-1-yl)oxy)diphenylsilane, (3.59.1):

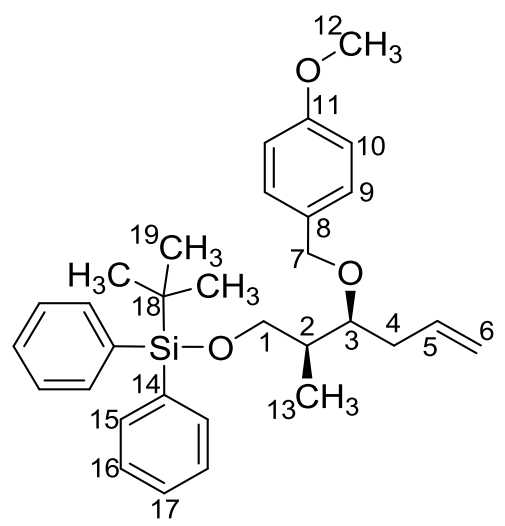

4-Methoxybenzyl-2,2,2-trichloroacetimidate $(0.50 \mathrm{~mL}, 2.41 \mathrm{mmol})$ and camphor sulfonic acid (0.0559 $\mathrm{g}, 0.241 \mathrm{mmol})$ was added to a solution of homoallylic alcohol $\mathbf{3 . 4 2 . 4}(0.443 \mathrm{~g}, 1.20$ $\mathrm{mmol})$ in $\mathrm{CH}_{2} \mathrm{Cl}_{2}(12 \mathrm{~mL})$ at $0{ }^{\circ} \mathrm{C}$. The reaction mixture was allowed to warm to room temperature and stirred overnight. A saturated aqueous solution of $\mathrm{NaHCO}_{3}(14 \mathrm{~mL})$ was added, and the two phases were separated. The aqueous layer was extracted with $\mathrm{CH}_{2} \mathrm{Cl}_{2}(3 \times 15 \mathrm{~mL})$. The combined organic extracts were washed with brine, dried over anhydrous magnesium sulphate, filtered and concentrated in vacuo. The residue was purified by flash chromatography on silica gel eluting with $(\mathrm{PET} / \mathrm{EtOAc}=95: 5)$ to give alkene 3.59.1 $(0.370 \mathrm{~g}, 63 \%)$ as a colorless oil.

Formula: $\mathrm{C}_{31} \mathrm{H}_{40} \mathrm{O}_{3} \mathrm{Si}$

Mass: $488.3 \mathrm{~g} / \mathrm{mol}$

IR $v_{\max }$ (neat): $1613,1511,1463,1441,1246,1173,1110,1073,1037,821,740,701,612 \mathrm{~cm}^{-}$ 1 . 
${ }^{1}$ H NMR (400 MHz, Chloroform- $d$ ): $\delta 7.71-7.62\left(4 \mathrm{H}, \mathrm{m}, 4 \mathrm{H}_{15}\right), 7.48-7.32\left(6 \mathrm{H}, \mathrm{m}, 4 \mathrm{H}_{16}+\right.$ $\left.2 \mathrm{H}_{17}\right), 6.84\left(4 \mathrm{H}, \mathrm{m}, 2 \mathrm{H}_{9}+2 \mathrm{H}_{10}\right), 5.90-5.74\left(1 \mathrm{H}, \mathrm{m}, \mathrm{H}_{5}\right), 5.13-5.00\left(2 \mathrm{H}, \mathrm{m}, \mathrm{H}_{6}\right), 4.53(1 \mathrm{H}, \mathrm{d}$, $\left.J=11.1 \mathrm{~Hz}, \mathrm{H}_{7}\right), 4.39\left(1 \mathrm{H}, \mathrm{d}, J=11.1 \mathrm{~Hz}, \mathrm{H}_{7^{\prime}}\right), 3.80\left(3 \mathrm{H}, \mathrm{s}, \mathrm{H}_{12}\right), 3.74-3.63\left(2 \mathrm{H}, \mathrm{m}, \mathrm{H}_{1}+\mathrm{H}_{3}\right)$, $3.55\left(1 \mathrm{H}, \mathrm{dd}, J=9.8,6.1 \mathrm{~Hz}, \mathrm{H}_{1}{ }^{\prime}\right), 2.42-2.34\left(1 \mathrm{H}, \mathrm{m}, \mathrm{H}_{4}\right), 2.32-2.23\left(1 \mathrm{H}, \mathrm{m}, \mathrm{H}_{4}{ }^{\prime}\right), 1.89(1 \mathrm{H}$, $\left.\mathrm{qd}, J=7.1,3.6 \mathrm{~Hz}, \mathrm{H}_{2}\right), 1.07\left(9 \mathrm{H}, \mathrm{s}, \mathrm{H}_{19}\right), 0.92\left(3 \mathrm{H}, \mathrm{d}, J=6.8 \mathrm{~Hz}, \mathrm{H}_{13}\right)$

${ }^{13}$ C NMR (101 MHz, Chloroform- $d$ ): $\delta 159.1$ (s, C 11 ), 135.7 (s, 4C 15$), 135.3$ (s, $\left.\mathrm{C}_{5}\right), 134.0$ (s, $\left.2 \mathrm{C}_{14}\right), 131.4\left(\mathrm{~s}, \mathrm{C}_{8}\right), 129.7\left(\mathrm{~s}, 2 \mathrm{C}_{17}\right), 129.3\left(\mathrm{~s}, 2 \mathrm{C}_{9}\right), 127.7\left(\mathrm{~s}, 4 \mathrm{C}_{16}\right), 116.7\left(\mathrm{~s}, \mathrm{C}_{6}\right), 113.8\left(\mathrm{~s}, 2 \mathrm{C}_{10}\right)$, $78.6\left(\mathrm{~s}, \mathrm{C}_{3}\right), 71.9\left(\mathrm{~s}, \mathrm{C}_{7}\right), 66.1\left(\mathrm{~s}, \mathrm{C}_{1}\right), 55.4\left(\mathrm{~s}, \mathrm{C}_{12}\right), 39.0\left(\mathrm{~s}, \mathrm{C}_{2}\right), 36.3\left(\mathrm{~s}, \mathrm{C}_{4}\right), 27.0\left(\mathrm{~s}, 3 \mathrm{C}_{19}\right), 19.4$ $\left(\mathrm{s}, \mathrm{C}_{18}\right), 11.3\left(\mathrm{~s}, \mathrm{C}_{13}\right)$.

GCMS (EI) m/z (rel intensity): 67 (11), 77 (13), 95 (91), 121 (7), 135 (17), 139 (18), 181 (10), 199 (100), 200 (17), 211 (7), 229 (19), 269 (8).

HRMS (ESI-TOF): Calculated for $\mathrm{C}_{31} \mathrm{H}_{40} \mathrm{O}_{3} \mathrm{SiNa}[\mathrm{M}+\mathrm{Na}]^{+}:$511.2636. Found: 511.2639.

$[\alpha]_{\mathbf{D}}{ }^{20}:+4.4\left(c=4.0, \mathrm{CH}_{3} \mathrm{Cl}\right)$

$(5 R, 3 S, 2 S)-1-((t e r t-b u t y l d i p h e n y l s i l y l) o x y)-3-((4-m e t h o x y b e n z y l) o x y)-2-m e t h y l o c t-7-e n-5-$ ol, (3.59.3):

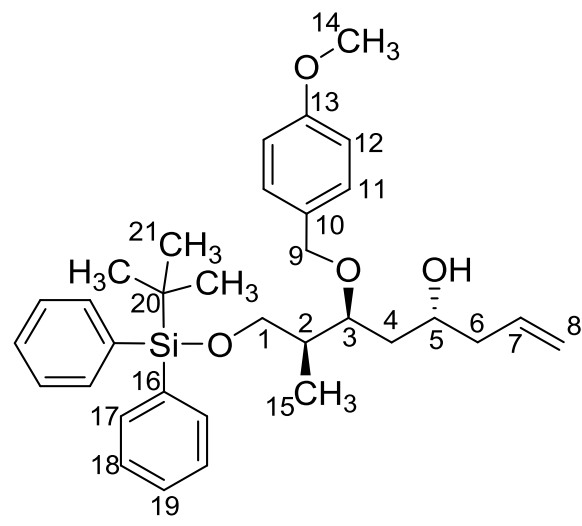

A solution of osmium tetroxide in tert-butanol $(0.50 \mathrm{~mL}, 0.0392 \mathrm{mmol}, 0.079 \mathrm{M})$ followed by NMO (0.252 g, $2.16 \mathrm{mmol})$ were added to a mixture of alkene $\mathbf{3 . 5 9 . 1}(0.957 \mathrm{~g}, 1.96 \mathrm{mmol})$, 
water $(2 \mathrm{~mL})$ and tert-butanol $(9 \mathrm{~mL})$ and THF $(9 \mathrm{~mL})$ at room temperature. The reaction mixture was stirred at room temperature for $8 \mathrm{~h}$, and additional portions of osmium tetroxide $(0.50 \mathrm{~mL}, 0.0392 \mathrm{mmol}, 0.079 \mathrm{M})$ and NMO $(0.252 \mathrm{~g}, 2.16 \mathrm{mmol})$ were added. The mixture was stirred at room temperature for $20 \mathrm{~h}$. Solid $\mathrm{Na}_{2} \mathrm{~S}_{2} \mathrm{O}_{3}(2.10 \mathrm{~g})$, Celite (4.10 g), and EtOAc (8 $\mathrm{mL}$ ) were added, and the mixture was filtered through a pad of Celite ${ }^{\circledR}$ which was thoroughly washed with EtOAc. The filtrate was concentrated in vacuo, and the residue was dissolved in (1:2) mixture of $\mathrm{H}_{2} \mathrm{O}: \mathrm{MeOH}(33 \mathrm{~mL})$. Sodium metaperiodate $(2.51 \mathrm{~g}, 11.8 \mathrm{mmol})$ was then added to the mixture and stirred for $3 \mathrm{~h}$ at room temperature. The biphasic mixture was filtered through a pad of Celite ${ }^{\circledR}$, the layers were separated, and the aqueous layer was extracted with EtOAc $(3 \times 15 \mathrm{~mL})$. The combined organic extracts were washed with brine, dried over anhydrous magnesium sulphate, filtered and concentrated in vacuo. The residue was azeotropically dried with toluene twice to give the crude aldehyde as a yellow oil which was used in the proceeding allylation step without further purification.

A solution of the aldehyde $\mathbf{3 . 5 9 . 2}\left(1.96 \mathrm{mmol}, 1.0\right.$ equiv) in $\mathrm{Et}_{2} \mathrm{O}(5 \mathrm{~mL})$ was added to a suspension of $(S, S)-\mathbf{T i}(1.45 \mathrm{~g}, 2.35 \mathrm{mmol})$ in $\mathrm{Et}_{2} \mathrm{O}(16 \mathrm{~mL})$ at $-78^{\circ} \mathrm{C}$ over a period of 10 minutes under argon. The mixture was stirred at $-78{ }^{\circ} \mathrm{C}$ for $24 \mathrm{~h}$. The reaction mixture was quenched with deionized water at $-78{ }^{\circ} \mathrm{C}$, then allowed to warm to room temperature. The mixture was stirred at room temperature for $24 \mathrm{~h}$. The reaction media was filtered through a pad of Celite ${ }^{\circledR}$. The layers were separated and the aqueous layer was extracted with $\mathrm{Et}_{2} \mathrm{O}(3 \times 15 \mathrm{~mL})$. The combined organic extracts were washed with brine, dried over anhydrous magnesium sulphate, filtered and concentrated in vacuo. Pentane $(250 \mathrm{~mL})$ was added, the mixture was stirred vigorously at room temperature for $4 \mathrm{~h}$, filtered and concentrated in vacuo. The residue was purified by flash chromatography on silica gel eluting with (PET/EtOAc $=75: 25$ to $70: 30)$ to give homoallylic alcohol $\mathbf{3 . 5 9 . 3}(0.313 \mathrm{~g}, 30 \%)$ as a yellow oil, $(\mathrm{dr}=19: 1)$.

Formula: $\mathrm{C}_{33} \mathrm{H}_{44} \mathrm{O}_{4} \mathrm{Si}$

Mass: $532.3 \mathrm{~g} / \mathrm{mol}$ 
IR $v_{\max }$ (neat): 2931, 1612, 1513, 1463, 1427, 1247, 1171, 1109, 1034, 822, 740, 700, 612 $\mathrm{cm}^{-1}$.

${ }^{1}$ H NMR (400 MHz, Chloroform- $d$ ): $\delta 7.70-7.57$ (4H, m, 4H $\left.\mathrm{H}_{12}\right), 7.58-7.47\left(2 \mathrm{H}, \mathrm{m}, \mathrm{H}_{11}\right), 7.45$ $-7.33\left(6 \mathrm{H}, \mathrm{m}, 4 \mathrm{H}_{13}+2 \mathrm{H}_{14}\right), 6.91-6.76\left(2 \mathrm{H}, \mathrm{m}, \mathrm{H}_{12}\right), 5.73\left(1 \mathrm{H}, \mathrm{ddt}, J=19.4,9.5,7.1 \mathrm{~Hz}, \mathrm{H}_{7}\right)$, $5.12-5.01\left(2 \mathrm{H}, \mathrm{m}, \mathrm{H}_{8}\right), 4.56\left(1 \mathrm{H}, \mathrm{s}, \mathrm{H}_{9}\right), 4.51\left(1 \mathrm{H}, \mathrm{s}, \mathrm{H}_{9}\right), 3.82-3.77\left(2 \mathrm{H}, \mathrm{m}, \mathrm{H}_{3}+\mathrm{H}_{5}\right), 3.76$ $\left(3 \mathrm{H}, \mathrm{s}, \mathrm{H}_{14}\right), 3.70\left(1 \mathrm{H}, \mathrm{dd}, J=10.0,5.6 \mathrm{~Hz}, \mathrm{H}_{1}\right), 3.54\left(1 \mathrm{H}, \mathrm{dd}, J=10.0,6.4 \mathrm{~Hz}, \mathrm{H}_{1}{ }^{\prime}\right), 2.44(1 \mathrm{H}$, d, $J=3.9 \mathrm{~Hz},-\mathrm{OH}), 2.17-2.11\left(2 \mathrm{H}, \mathrm{m}, \mathrm{H}_{6}\right), 2.03-1.91\left(1 \mathrm{H}, \mathrm{m}, \mathrm{H}_{2}\right), 1.67-1.49\left(2 \mathrm{H}, \mathrm{m}, \mathrm{H}_{4}\right)$, $1.06\left(9 \mathrm{H}, \mathrm{s}, \mathrm{H}_{21}\right), 0.99\left(3 \mathrm{H}, \mathrm{d}, J=6.9 \mathrm{~Hz}, \mathrm{H}_{15}\right)$.

${ }^{13}$ C NMR (101 MHz, Chloroform- $d$ ): $\delta 159.2\left(\mathrm{~s}, \mathrm{C}_{13}\right), 135.7$ (s, 4C 17 ), 134.9 (s, $\left.\mathrm{C}_{7}\right), 133.8$ (s, $\left.2 \mathrm{C}_{16}\right), 130.7\left(\mathrm{~s}, \mathrm{C}_{10}\right), 129.7\left(\mathrm{~s}, \mathrm{C}_{19}\right), 129.6\left(\mathrm{~s}, \mathrm{C}_{19}\right), 128.8\left(\mathrm{~s}, 2 \mathrm{C}_{11}\right), 127.8\left(\mathrm{~s}, 4 \mathrm{C}_{18}\right), 117.8\left(\mathrm{~s}, \mathrm{C}_{8}\right)$, $113.9\left(\mathrm{~s}, 2 \mathrm{C}_{12}\right), 78.1\left(\mathrm{~s}, \mathrm{C}_{3}\right), 72.1\left(\mathrm{~s}, \mathrm{C}_{9}\right), 68.0\left(\mathrm{~s}, \mathrm{C}_{5}\right), 65.6\left(\mathrm{~s}, \mathrm{C}_{1}\right), 55.3\left(\mathrm{~s}, \mathrm{C}_{14}\right), 42.3\left(\mathrm{~s}, \mathrm{C}_{6}\right), 39.0$ $\left(\mathrm{s}, \mathrm{C}_{2}\right), 37.4\left(\mathrm{~s}, \mathrm{C}_{4}\right), 27.0\left(\mathrm{~s}, 3 \mathrm{C}_{21}\right), 19.4\left(\mathrm{~s}, \mathrm{C}_{20}\right), 13.0\left(\mathrm{~s}, \mathrm{C}_{15}\right)$.

GCMS (EI) m/z (rel intensity): 76 (9), 77 (8), 96 (13), 97 (13), 115 (12), 178 (64), 179 (100), 180 (14), 193 (12), 194 (82), 195 (14).

HRMS (ESI-TOF): Calculated for $\mathrm{C}_{33} \mathrm{H}_{44} \mathrm{O}_{4} \mathrm{SiNa}[\mathrm{M}+\mathrm{Na}]^{+}:$555.2887. Found: 555.2901.

$[\alpha]_{\mathbf{D}}{ }^{\mathbf{2 0}}:+11.4\left(c=5.0, \mathrm{CH}_{3} \mathrm{Cl}\right)$ 


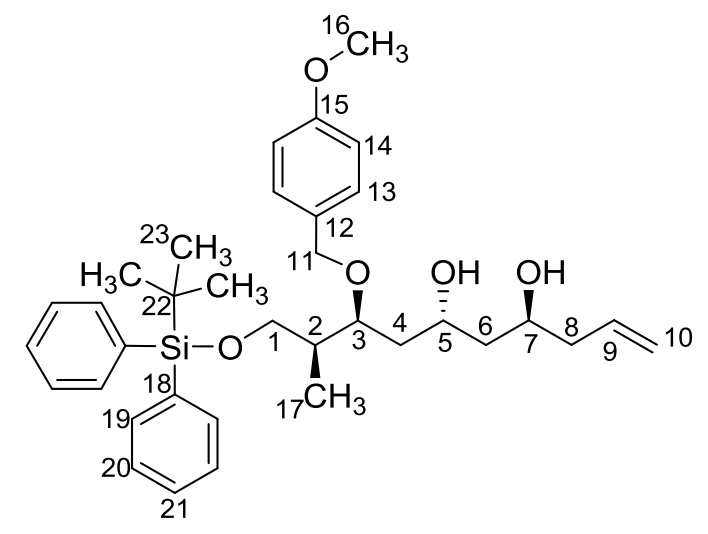

A solution of osmium tetroxide in tert-butanol $(0.41 \mathrm{~mL}, 0.0325 \mathrm{mmol}, 0.079 \mathrm{M})$ followed by NMO $(0.210 \mathrm{~g}, 1.79 \mathrm{mmol})$ were added to a mixture of homoallylic alcohol $\mathbf{3 . 5 9 . 3}(0.867 \mathrm{~g}$, $1.63 \mathrm{mmol})$, water $(1.50 \mathrm{~mL})$ and tert-butanol $(7.50 \mathrm{~mL})$ and $\mathrm{THF}(7.50 \mathrm{~mL})$ at room temperature. The reaction mixture was stirred at room temperature for $8 \mathrm{~h}$, and additional portions of osmium tetroxide $(0.41 \mathrm{~mL}, 0.0325 \mathrm{mmol}, 0.079 \mathrm{M})$ and NMO $(0.210 \mathrm{~g}, 1.79 \mathrm{mmol})$ were added. The mixture was stirred at room temperature for $20 \mathrm{~h}$. Solid $\mathrm{Na}_{2} \mathrm{~S}_{2} \mathrm{O}_{3}(1.75 \mathrm{~g})$, Celite $(3.40 \mathrm{~g})$, and EtOAc $(7 \mathrm{~mL})$ were added, and the mixture was filtered through a pad of Celite ${ }^{\circledR}$ which was thoroughly washed with EtOAc. The filtrate was concentrated in vacuo, and the residue was dissolved in (1:2) mixture of $\mathrm{H}_{2} \mathrm{O}: \mathrm{MeOH}(27 \mathrm{~mL})$. Sodium metaperiodate (2.09 $\mathrm{g}, 9.76 \mathrm{mmol}$ ) was then added to the mixture and stirred for $3 \mathrm{~h}$ at room temperature. The biphasic mixture was filtered through a pad of Celite®, the layers were separated, and the aqueous layer was extracted with EtOAc $(3 \times 15 \mathrm{~mL})$. The combined organic extracts were washed with brine, dried over anhydrous magnesium sulphate, filtered and concentrated in vасио. The residue was azeotropically dried with toluene twice to give the crude aldehyde as a yellow oil which was used in the proceeding allylation step without further purification. 
A solution of the aldehyde $\mathbf{3 . 5 9 . 4}\left(1.63 \mathrm{mmol}, 1.0\right.$ equiv) in $\mathrm{Et}_{2} \mathrm{O}(4 \mathrm{~mL})$ was added to a suspension of $(R, R)$ - $\mathbf{T i}(1.21 \mathrm{~g}, 1.96 \mathrm{mmol})$ in $\mathrm{Et}_{2} \mathrm{O}(13 \mathrm{~mL})$ at $-78{ }^{\circ} \mathrm{C}$ over a period of 10 minutes under argon. The mixture was stirred at $-78{ }^{\circ} \mathrm{C}$ for $24 \mathrm{~h}$. The reaction mixture was quenched with deionized water at $-78{ }^{\circ} \mathrm{C}$, then allowed to warm to room temperature. The mixture was stirred at room temperature for $24 \mathrm{~h}$. The reaction media was filtered through a pad of Celite®. The layers were separated and the aqueous layer was extracted with $\mathrm{Et}_{2} \mathrm{O}(3 \times 15$ $\mathrm{mL}$ ). The combined organic extracts were washed with brine, dried over anhydrous magnesium sulphate, filtered and concentrated in vacuo. Pentane $(210 \mathrm{~mL})$ was added, the mixture was stirred vigorously at room temperature for $4 \mathrm{~h}$, filtered and concentrated in vacuo. The residue was purified by flash chromatography on silica gel eluting with (PET/EtOAc $=60: 40$ to 55:45) to give diol 3.59.5 $(0.197 \mathrm{~g}, 21 \%)$ as a yellow oil $(\mathrm{dr}=.19: 1)$

Formula: $\mathrm{C}_{35} \mathrm{H}_{48} \mathrm{O}_{5} \mathrm{Si}$

Mass: $576.3 \mathrm{~g} / \mathrm{mol}$

IR $v_{\max }$ (neat): 2930, 2857, 1609, 1513, 1463, 1427, 1248, 1169, 1108, 1034, 822, 740, 701, 611 $\mathrm{cm}^{-1}$.

${ }^{1} \mathbf{H}$ NMR $(400 \mathrm{MHz}$, Chloroform- $d): \delta 7.68-7.62\left(4 \mathrm{H}, \mathrm{m}, 4 \mathrm{H}_{20}\right), 7.45-7.33\left(6 \mathrm{H}, \mathrm{m}, 4 \mathrm{H}_{19}+\right.$ $\left.2 \mathrm{H}_{21}\right), 7.22-7.18\left(2 \mathrm{H}, \mathrm{m}, \mathrm{H}_{13}\right), 6.87-6.82\left(2 \mathrm{H}, \mathrm{m}, \mathrm{H}_{14}\right), 5.79(1 \mathrm{H}, \mathrm{ddt}, J=16.5,11.0,7.1 \mathrm{~Hz}$, $\left.\mathrm{H}_{9}\right), 5.14-5.07\left(2 \mathrm{H}, \mathrm{m}, \mathrm{H}_{10}\right), 4.45\left(2 \mathrm{H}, \mathrm{s}, \mathrm{H}_{11}\right), 4.13-4.04\left(1 \mathrm{H}, \mathrm{m}, \mathrm{H}_{7}\right), 3.92(1 \mathrm{H}, \mathrm{p}, J=6.3$ $\left.\mathrm{Hz}, \mathrm{H}_{5}\right), 3.79\left(2 \mathrm{H}\right.$, br s, $\left.\mathrm{H}_{3}+\mathrm{H}_{16}\right), 3.70\left(1 \mathrm{H}, \mathrm{dd}, J=10.1,5.5 \mathrm{~Hz}, \mathrm{H}_{1}\right), 3.54(1 \mathrm{H}, \mathrm{dd}, J=10.1$, $\left.6.2 \mathrm{~Hz}, \mathrm{H}_{1^{\prime}}\right), 2.25-2.18\left(2 \mathrm{H}, \mathrm{m}, \mathrm{H}_{8}\right), 1.99\left(1 \mathrm{H}, \mathrm{p}, J=6.3 \mathrm{~Hz}, \mathrm{H}_{2}\right), 1.71(1 \mathrm{H}, \mathrm{ddd}, J=14.4,9.4$, $\left.3.5 \mathrm{~Hz}, \mathrm{H}_{4}\right), 1.59-1.50\left(3 \mathrm{H}, \mathrm{m} . \mathrm{H}_{4},+2 \mathrm{H}_{6}\right), 1.06\left(9 \mathrm{H}, \mathrm{s}, \mathrm{H}_{23}\right), 1.01\left(3 \mathrm{H}, \mathrm{d}, J=6.8 \mathrm{~Hz}, \mathrm{H}_{17}\right)$

${ }^{13}$ C NMR (101 MHz, Chloroform-d): $\delta 159.3$ (s, $\mathrm{C}_{15}$ ), 135.7 (s, 4C 19 ), 135.0 (s, $\mathrm{C}_{9}$ ), 133.8 (s, 2 $\left.\mathrm{C}_{18}\right), 130.6\left(\mathrm{~s}, \mathrm{C}_{12}\right), 129.7\left(\mathrm{~s}, 2 \mathrm{C}_{13}\right), 127.8\left(\mathrm{~s}, 4 \mathrm{C}_{20}\right), 117.9\left(\mathrm{~s}, \mathrm{C}_{10}\right), 113.9\left(\mathrm{~s}, 2 \mathrm{C}_{14}\right), 77.7\left(\mathrm{~s}, \mathrm{C}_{3}\right)$, 72.1 (s, $\left.\mathrm{C}_{11}\right), 68.4\left(\mathrm{~s}, \mathrm{C}_{5}\right), 66.7\left(\mathrm{~s}, \mathrm{C}_{7}\right), 65.7$ (s, $\left.\mathrm{C}_{1}\right), 55.4$ (s, $\left.\mathrm{C}_{16}\right), 42.2\left(\mathrm{~s}, \mathrm{C}_{8}\right), 42.1$ (s, $\left.\mathrm{C}_{6}\right), 39.0$ (s, $\left.\mathrm{C}_{2}\right), 37.6\left(\mathrm{~s}, \mathrm{C}_{4}\right), 27.0\left(\mathrm{~s}, 3 \mathrm{C}_{23}\right), 19.4\left(\mathrm{~s}, \mathrm{C}_{22}\right), 13.1\left(\mathrm{~s}, \mathrm{C}_{17}\right)$. 
GCMS (EI) m/z (rel intensity): 89 (19), 96 (16), 115 (14), 178 (61), 179 (100), 180 (17), 193 (14), 194 (80), 195 (17).

HRMS (ESI-TOF): Calculated for $\mathrm{C}_{35} \mathrm{H}_{48} \mathrm{O}_{5} \mathrm{SiNa}[\mathrm{M}+\mathrm{Na}]^{+}:$599.3150. Found: 599.3163.

$[\alpha]_{\mathbf{D}}{ }^{20}:-5.7\left(c=2.4, \mathrm{CH}_{3} \mathrm{Cl}\right)$

((2S,3S)-4-((4R,6S)-6-allyl-2,2-dimethyl-1,3-dioxan-4-yl)-3-((4-methoxybenzyl)oxy)-2methylbutoxy)(tert-butyl)diphenylsilane, (3.59.6):

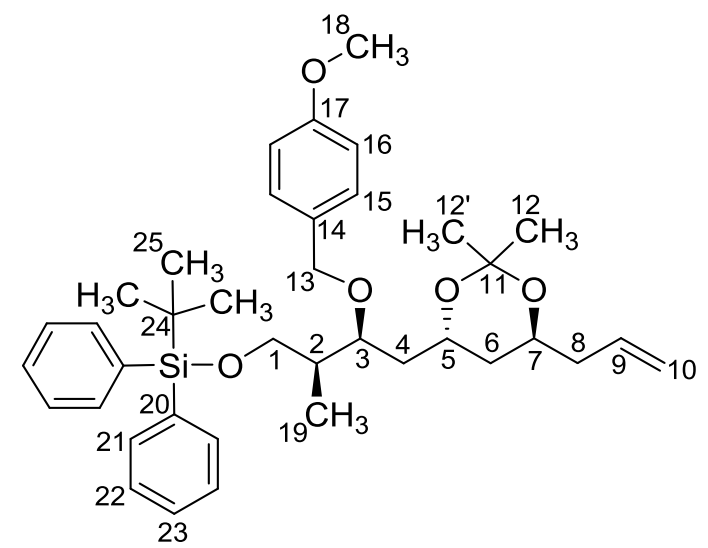

2,2-Dimethoxypropane $(1.30 \mathrm{~mL}, 10.2 \mathrm{mmol})$ followed by a catalytic amount of PPTS $(1 \mathrm{mg}$, $5.1 \mu \mathrm{mol})$ were added to a solution of diol $\mathbf{3 . 5 9 . 5}(0.29 \mathrm{~g}, 0.511 \mathrm{mmol})$ in $\mathrm{CH}_{2} \mathrm{Cl}_{2}(3 \mathrm{~mL})$ at room temperature. The reaction mixture was stirred at room temperature for $5 \mathrm{~h}$. Water was added, and the mixture was extracted with $\mathrm{CH}_{2} \mathrm{Cl}_{2}(3 \mathrm{X} 20 \mathrm{~mL})$. The combined organic extracts were washed with brine, then dried over anhydrous magnesium sulphate, filtered and concentrated in vacuo. The residue was purified by flash chromatography on silica gel eluting with $(\mathrm{PET} / \mathrm{EtOAc}=97: 3$ to $95: 5)$ to give trans-acetonide $\mathbf{3 . 5 9 . 6}(0.227 \mathrm{~g}, 72 \%)$ as a colorless oil.

Formula: $\mathrm{C}_{38} \mathrm{H}_{52} \mathrm{O}_{5} \mathrm{Si}$

Mass: $616.4 \mathrm{~g} / \mathrm{mol}$

IR $v_{\max }$ (neat): 1613, 1588, 1514, 1463, 1378, 1247, 1224, 1171, 1111, 1075, 1037, 942, 914 , $823 \mathrm{~cm}^{-1}$. 
${ }^{1}$ H NMR (400 MHz, Acetone- $\left.d_{6}\right) \delta 7.72-7.56\left(4 \mathrm{H}, \mathrm{m}, 4 \mathrm{H}_{21}\right), 7.43-7.31\left(6 \mathrm{H}, \mathrm{m}, 4 \mathrm{H}_{22}+2 \mathrm{H}_{23}\right.$ ), $7.19-7.13\left(2 \mathrm{H}, \mathrm{m}, \mathrm{H}_{15}\right), 6.85-6.80\left(2 \mathrm{H}, \mathrm{m}, \mathrm{H}_{16}\right), 5.79\left(1 \mathrm{H}, \mathrm{ddt}, J=17.0,10.2,6.8 \mathrm{~Hz}, \mathrm{H}_{9}\right)$, $5.14-4.99\left(2 \mathrm{H}, \mathrm{m}, \mathrm{H}_{10}\right), 4.48\left(1 \mathrm{H}, \mathrm{d}, J=10.7 \mathrm{~Hz}, \mathrm{H}_{13}\right), 4.43\left(1 \mathrm{H}, \mathrm{d}, J=10.8 \mathrm{~Hz}, \mathrm{H}_{13^{\prime}}\right), 4.01$ $\left(1 \mathrm{H}, \mathrm{tdd}, J=9.6,6.1,3.9 \mathrm{~Hz}, \mathrm{H}_{7}\right), 3.93-3.82\left(2 \mathrm{H}, \mathrm{m}, \mathrm{H}_{3}+\mathrm{H}_{5}\right), 3.80-3.71\left(4 \mathrm{H}, \mathrm{m}, \mathrm{H}_{1}+\mathrm{H}_{18}\right)$, $3.56\left(1 \mathrm{H}, \mathrm{dd}, J=10.0,6.7 \mathrm{~Hz}, \mathrm{H}_{1^{\prime}}\right), 2.37-2.25\left(1 \mathrm{H}, \mathrm{m}, \mathrm{H}_{8}\right), 2.24-2.12\left(1 \mathrm{H}, \mathrm{m}, \mathrm{H}_{8^{\prime}}\right), 1.93(1 \mathrm{H}$, dtq, $\left.J=10.3,7.0,3.4 \mathrm{~Hz}, \mathrm{H}_{2}\right), 1.69-1.49\left(4 \mathrm{H}, \mathrm{m}, \mathrm{H}_{4}+\mathrm{H}_{6}\right), 1.44\left(3 \mathrm{H}, \mathrm{s}, \mathrm{H}_{12}\right), 1.41\left(3 \mathrm{H}, \mathrm{s}, \mathrm{H}_{12}\right)$, $1.07\left(9 \mathrm{H}, \mathrm{s}, \mathrm{H}_{25}\right), 0.93\left(3 \mathrm{H}, \mathrm{d}, J=6.9,1.0 \mathrm{~Hz} \mathrm{H} \mathrm{H}_{19}\right)$.

${ }^{13}$ C NMR (101 MHz, Acetone- $\left.d_{6}\right) \delta 159.1\left(\mathrm{~s}, \mathrm{C}_{17}\right), 135.7$ (s, 4C 21$), 134.6$ (s, C9), $134.0\left(\mathrm{~s}, \mathrm{C}_{20}\right)$, $133.9\left(\mathrm{~s}, \mathrm{C}_{20^{\circ}}\right), 131.3\left(\mathrm{~s}, \mathrm{C}_{14}\right), 129.6\left(\mathrm{~s}, 2 \mathrm{C}_{23}\right), 129.2\left(\mathrm{~s}, 2 \mathrm{C}_{15}\right), 127.7\left(\mathrm{~s}, 4 \mathrm{C}_{22}\right), 116.9\left(\mathrm{~s}, \mathrm{C}_{10}\right)$, $113.8\left(\mathrm{~s}, 2 \mathrm{C}_{16}\right), 100.3\left(\mathrm{~s}, \mathrm{C}_{11}\right), 75.9\left(\mathrm{~s}, \mathrm{C}_{3}\right), 72.6\left(\mathrm{~s}, \mathrm{C}_{13}\right), 66.3\left(\mathrm{~s}, \mathrm{C}_{5}\right), 65.7\left(\mathrm{~s}, \mathrm{C}_{1}\right), 63.8\left(\mathrm{~s}, \mathrm{C}_{7}\right)$, $55.3\left(\mathrm{~s}, \mathrm{C}_{18}\right), 40.3\left(\mathrm{~s}, \mathrm{C}_{2}\right), 39.8\left(\mathrm{~s}, \mathrm{C}_{8}\right), 39.2\left(\mathrm{~s}, \mathrm{C}_{6}\right), 38.4\left(\mathrm{~s}, \mathrm{C}_{4}\right), 27.0\left(\mathrm{~s}, 3 \mathrm{C}_{25}\right), 25.2\left(\mathrm{~s}, \mathrm{C}_{12}\right), 25.1$ $\left(\mathrm{s}, \mathrm{C}_{12}\right), 19.4\left(\mathrm{~s}, \mathrm{C}_{24}\right), 11.7\left(\mathrm{~s}, \mathrm{C}_{19}\right)$.

GCMS (EI) m/z (rel intensity): 55 (7), 57 (37), 77 (4), 79 (5), 95 (6), 145 (13), 161 (4), 177 (12), 205 (100), 206 (14), 220 (28), 221 (5).

HRMS (ESI-TOF): Calculated for $\mathrm{C}_{38} \mathrm{H}_{52} \mathrm{O}_{5} \mathrm{SiNa}[\mathrm{M}+\mathrm{Na}]^{+}:$639.3479. Found: 639.3476. 
(E)-5-((4S,6R)-6-((2S,3S)-4-((tert-butyldiphenylsilyl)oxy)-2-((4-methoxybenzyl)oxy)-3methylbutyl)-2,2-dimethyl-1,3-dioxan-4-yl)pent-3-en-2-one, (3.59.7):

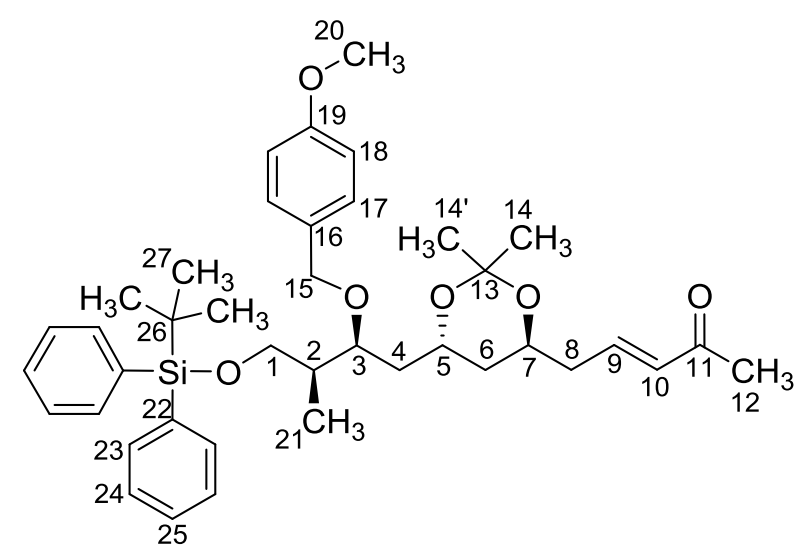

A solution of osmium tetroxide in tert-butanol $(23.0 \mu \mathrm{L}, 1.82 \mu \mathrm{mol}, 0.079 \mathrm{M})$ followed by NMO $(11.7 \mathrm{mg}, 100 \mu \mathrm{mol})$ were added to a mixture of alkene $\mathbf{3 . 5 9 . 6}(0.056 \mathrm{~g}, 0.0908 \mathrm{mmol})$, water $(0.1 \mathrm{~mL}), \mathrm{THF}(0.4 \mathrm{~mL})$ and tert-butanol $(0.4 \mathrm{~mL})$ at room temperature. The reaction mixture was stirred at room temperature for $8 \mathrm{~h}$, and additional portions of osmium tetroxide $(23.0 \mu \mathrm{L}$, $1.82 \mu \mathrm{mol}, 0.079 \mathrm{M})$ and NMO $(11.7 \mathrm{mg}, 100 \mu \mathrm{mol})$ were added. The mixture was stirred at room temperature for $20 \mathrm{~h}$. Solid $\mathrm{Na}_{2} \mathrm{~S}_{2} \mathrm{O}_{3}(1.0 \mathrm{~g})$, Celite $(0.20 \mathrm{~g})$, and EtOAc $(0.5 \mathrm{~mL})$ were added, and the mixture was filtered through a pad of Celite ${ }^{\circledR}$ which was thoroughly washed with EtOAc. The filtrate was concentrated in vacuo, and the residue was dissolved in (1:1) mixture of $\mathrm{H}_{2} \mathrm{O}$ :THF $(1 \mathrm{~mL})$. Sodium metaperiodate $(0.117 \mathrm{~g}, 0.545 \mathrm{mmol})$ was then added to the mixture and stirred for $3 \mathrm{~h}$ at room temperature. The biphasic mixture was filtered through a pad of Celite ${ }^{\circledR}$, the layers were separated, and the aqueous layer was extracted with EtOAc (3 x $5 \mathrm{~mL}$ ). The combined organic extracts were washed with brine, dried over anhydrous magnesium sulphate, filtered and concentrated in vасио. The residue was azeotropically dried with toluene twice to give the crude aldehyde as a yellow oil which was used in the proceeding Wittig olefination step without further purification. 
1-(Triphenylphosphoranylidene)acetone $\mathbf{3 . 5 1 . 4}(0.261 \mathrm{~g}, 0.820 \mathrm{mmol})$ was added to a solution of aldehyde $3.51 .3(0.338 \mathrm{~g}, 0.547 \mathrm{mmol})$ in THF $(2 \mathrm{~mL})$ at room temperature. The reaction mixture was brought to reflux for $24 \mathrm{~h}$ then cooled and concentrated in vacuo. The residue was purified by flash chromatography on silica gel eluting with $(\mathrm{PET} / \mathrm{EtOAc}=60: 40)$ to give $\alpha, \beta$ unsaturated methyl ketone 3.59.7 (0.13 g, 36\%) as a yellow oil.

Formula: $\mathrm{C}_{40} \mathrm{H}_{54} \mathrm{O}_{6} \mathrm{Si}$

Mass: $658.4 \mathrm{~g} / \mathrm{mol}$

IR $v_{\max }$ (neat): 1724, 1659, 1612, 1588, 1513, 1463, 1428, 1379, 1247, 1223, 1170, 1110, 1073, $1035,938,908,822 \mathrm{~cm}^{-1}$.

${ }^{1} \mathbf{H}$ NMR $\left(400 \mathrm{MHz}\right.$, Acetone- $\left.d_{6}\right) \delta 7.68-7.64\left(4 \mathrm{H}, \mathrm{m}, \mathrm{H}_{23}\right), 7.44-7.32\left(6 \mathrm{H}, \mathrm{m}, 4 \mathrm{H}_{24}+2 \mathrm{H}_{25}\right)$, $7.20-7.13\left(2 \mathrm{H}, \mathrm{m}, \mathrm{H}_{17}\right), 6.85-6.82\left(2 \mathrm{H}, \mathrm{m}, \mathrm{H}_{18}\right), 6.78\left(1 \mathrm{H}, \mathrm{dt}, J=15.9,6.9 \mathrm{~Hz}, \mathrm{H}_{9}\right), 6.12(1 \mathrm{H}$, ddt, $\left.J=16.0,5.3,1.3 \mathrm{~Hz}, \mathrm{H}_{10}\right), 4.48\left(1 \mathrm{H}, \mathrm{d}, J=10.7 \mathrm{~Hz}, \mathrm{H}_{15}\right), 4.42\left(1 \mathrm{H}, \mathrm{d}, J=10.8 \mathrm{~Hz}, \mathrm{H}_{15}\right)$, $4.02\left(1 \mathrm{H}, \mathrm{tdd}, J=9.0,6.6,3.8 \mathrm{~Hz}, \mathrm{H}_{5}\right), 3.98-3.92\left(1 \mathrm{H}, \mathrm{m}, \mathrm{H}_{7}\right), 3.89(1 \mathrm{H}, \mathrm{dt}, J=9.3,4.0 \mathrm{~Hz}$, $\left.\mathrm{H}_{3}\right), 3.78\left(3 \mathrm{H}, \mathrm{s}, \mathrm{H}_{20}\right), 3.74\left(1 \mathrm{H}, \mathrm{dd}, J=9.9,6.9 \mathrm{~Hz}, \mathrm{H}_{1}\right), 3.57\left(1 \mathrm{H}, \mathrm{dd}, J=10.0,6.6 \mathrm{~Hz}, \mathrm{H}_{1^{\prime}}\right)$, $2.47-2.30\left(2 \mathrm{H}, \mathrm{m}, \mathrm{H}_{8}\right), 2.24\left(3 \mathrm{H}, \mathrm{s}, \mathrm{H}_{12}\right), 1.94\left(1 \mathrm{H}, \mathrm{qd}, J=6.8,3.3 \mathrm{~Hz}, \mathrm{H}_{2}\right), 1.68-1.48(4 \mathrm{H}$, m, $\left.\mathrm{H}_{4}+\mathrm{H}_{6}\right), 1.37\left(3 \mathrm{H}, \mathrm{s}, \mathrm{H}_{14}\right), 1.36\left(3 \mathrm{H}, \mathrm{s}, \mathrm{H}_{14}\right), 1.07\left(9 \mathrm{H}, \mathrm{s}, \mathrm{H}_{27}\right), 0.93\left(3 \mathrm{H}, \mathrm{d}, J=7.0 \mathrm{~Hz}, \mathrm{H}_{21}\right)$.

${ }^{13}$ C NMR (101 MHz, Acetone-d $\left.d_{6}\right) \delta 198.5\left(\mathrm{~s}, \mathrm{C}_{11}\right), 159.1\left(\mathrm{~s}, \mathrm{C}_{19}\right), 144.1\left(\mathrm{~s}, \mathrm{C}_{9}\right), 135.7\left(\mathrm{~s}, 4 \mathrm{C}_{23}\right)$, $133.9\left(\mathrm{~s}, 2 \mathrm{C}_{22}\right), 133.1\left(\mathrm{~s}, \mathrm{C}_{10}\right), 131.2\left(\mathrm{~s}, \mathrm{C}_{16}\right), 129.6\left(\mathrm{~s}, 2 \mathrm{C}_{25}\right), 129.2\left(\mathrm{~s}, 2 \mathrm{C}_{17}\right), 127.7\left(\mathrm{~s}, 4 \mathrm{C}_{24}\right)$, $113.8\left(\mathrm{~s}, 2 \mathrm{C}_{18}\right), 100.6\left(\mathrm{~s}, \mathrm{C}_{13}\right), 75.9\left(\mathrm{~s}, \mathrm{C}_{3}\right), 72.6\left(\mathrm{~s}, \mathrm{C}_{15}\right), 65.7\left(\mathrm{~s}, \mathrm{C}_{7}\right), 65.6\left(\mathrm{~s}, \mathrm{C}_{1}\right), 63.7\left(\mathrm{~s}, \mathrm{C}_{5}\right)$, $55.3\left(\mathrm{~s}, \mathrm{C}_{20}\right), 39.7\left(\mathrm{~s}, \mathrm{C}_{2}\right), 39.2\left(\mathrm{~s}, \mathrm{C}_{6}\right), 38.6\left(\mathrm{~s}, \mathrm{C}_{8}\right), 38.6\left(\mathrm{~s}, \mathrm{C}_{4}\right), 27.0\left(\mathrm{~s}, 3 \mathrm{C}_{27}\right), 26.9\left(\mathrm{~s}, \mathrm{C}_{12}\right) 25.1$ (s, 2C 14$), 19.4\left(\mathrm{~s}, \mathrm{C}_{26}\right), 11.8\left(\mathrm{~s}, \mathrm{C}_{21}\right)$.

GCMS (EI) $m / z$ (rel intensity): 57 (41), 95 (6), 161 (2), 177 (12), 205 (100), 206 (16), 220 (26), $221(4)$

HRMS (ESI-TOF): Calculated for $\mathrm{C}_{40} \mathrm{H}_{54} \mathrm{O}_{6} \mathrm{SiNa}[\mathrm{M}+\mathrm{Na}]^{+}$: 681.3582. Found: 681.3582. 
$[\alpha]_{\mathrm{D}}{ }^{23}:-4.6\left(c=5.2, \mathrm{CH}_{3} \mathrm{Cl}\right)$

$(E)-5-((4 S, 6 R)-6-((2 S, 3 S)-4-((t e r t-b u t y l d i p h e n y l s i l y l) o x y)-2-((4-m e t h o x y b e n z y l) o x y)-3-$ methylbutyl)-2,2-dimethyl-1,3-dioxan-4-yl)pent-3-en-2-ol, (3.59.8):

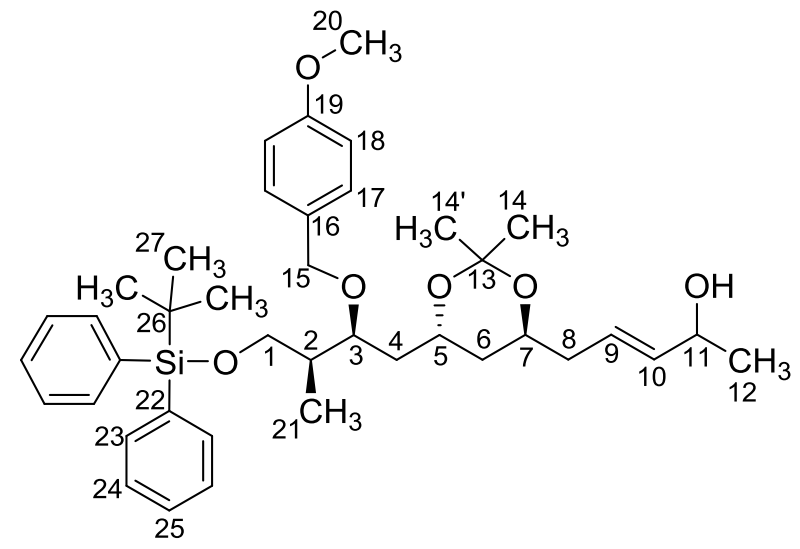

Cerium trichloride heptahydrate $\left(\mathrm{CsCl}_{3} \bullet 7 \mathrm{H} 2 \mathrm{O}\right)(0.147 \mathrm{~g}, 0.395 \mathrm{mmol})$ was added to a solution of $\alpha, \beta$-unsaturated methyl ketone $3.59 .7(0.13 \mathrm{~g}, 0.197 \mathrm{mmol})$ in methanol $(3 \mathrm{~mL})$ at room temperature and stirred for $10 \mathrm{~min}$. The reaction mixture was then cooled to $0{ }^{\circ} \mathrm{C}$ and sodium borohydride $(0.0100 \mathrm{~g}, 0.237 \mathrm{mmol})$ was added portion wise. The mixture was stirred at $0{ }^{\circ} \mathrm{C}$ for $15 \mathrm{~min}$ and at room temperature for $4 \mathrm{~h}$. Saturated aqueous ammonium chloride solution and sodium potassium tartarate (Rochelle) were added, and the mixture was extracted with EtOAc (3 X $20 \mathrm{~mL}$ ). The combined organic extracts were dried over anhydrous magnesium sulphate, filtered and concentrated in vacuo to give the crude alcohol $\mathbf{3 . 5 9 . 8}(0.100 \mathrm{~g}, 77 \%)$ as a yellow oil which was used in the proceeding acetonide deprotection step without further purification.

Formula: $\mathrm{C}_{40} \mathrm{H}_{56} \mathrm{O}_{6} \mathrm{Si}$

Mass: $660.4 \mathrm{~g} / \mathrm{mol}$

IR $v_{\max }$ (neat): 1613, 1513, 1463, 1428, 1378, 1247, 1171, 1110, 1068, 1036, 822, 740, 702, 669, $613 \mathrm{~cm}^{-1}$.

${ }^{1} \mathbf{H}$ NMR (400 MHz, Acetone- $\left.d_{6}\right) \delta 7.59-7.55\left(4 \mathrm{H}, \mathrm{m}, 4 \mathrm{H}_{23}\right), 7.34-7.22\left(6 \mathrm{H}, \mathrm{m}, 4 \mathrm{H}_{24}+2 \mathrm{H}_{25}\right.$ ), $7.12-7.01\left(2 \mathrm{H}, \mathrm{m}, \mathrm{H}_{17}\right), 6.76-6.71\left(2 \mathrm{H}, \mathrm{m}, \mathrm{H}_{18}\right), 5.54-5.46\left(2 \mathrm{H}, \mathrm{m}, \mathrm{H}_{9}+\mathrm{H}_{10}\right), 4.39(2 \mathrm{H}$, 
d, $\left.J=10.7 \mathrm{~Hz}, \mathrm{H}_{15}\right), 4.34\left(2 \mathrm{H}, \mathrm{d}, J=11.2 \mathrm{~Hz}, \mathrm{H}_{15}{ }^{\circ}\right), 4.19-4.10\left(1 \mathrm{H}, \mathrm{m}, \mathrm{H}_{11}\right), 4.04-3.89(1 \mathrm{H}$, $\left.\mathrm{m}, \mathrm{H}_{7}\right), 3.84-3.70\left(1 \mathrm{H}, \mathrm{m}, \mathrm{H}_{3}\right), 3.67\left(3 \mathrm{H}, \mathrm{s}, \mathrm{H}_{20}\right), 3.66-3.60\left(1 \mathrm{H}, \mathrm{m}, \mathrm{H}_{1}\right), 3.50-3.43(1 \mathrm{H}, \mathrm{m}$, $\left.\mathrm{H}_{1^{\prime}}\right), 3.41-3.31\left(1 \mathrm{H}, \mathrm{m}, \mathrm{H}_{5}\right), 2.12-1.99\left(2 \mathrm{H}, \mathrm{m}, \mathrm{H}_{8}\right), 1.85\left(1 \mathrm{H}, \mathrm{tqd}, J=9.8,6.5,3.4 \mathrm{~Hz}, \mathrm{H}_{2}\right)$, $1.59-1.39\left(4 \mathrm{H}, \mathrm{m}, \mathrm{H}_{4}+\mathrm{H}_{8}\right), 1.27\left(1 \mathrm{H}, \mathrm{d}, J=4.3 \mathrm{~Hz}, \mathrm{H}_{12}\right), 1.17\left(3 \mathrm{H}, \mathrm{s}, \mathrm{H}_{14}\right), 1.13\left(3 \mathrm{H}, \mathrm{s}, \mathrm{H}_{14}\right)$, $0.98\left(9 \mathrm{H}, \mathrm{s}, \mathrm{H}_{27}\right), 0.83\left(2 \mathrm{H}, \mathrm{d}, J=6.9 \mathrm{~Hz}, \mathrm{H}_{21}\right)$.

${ }^{13}$ C NMR (101 MHz, Acetone- $\left.d_{6}\right) \delta 159.0\left(\mathrm{~s}, \mathrm{C}_{19}\right), 136.8\left(\mathrm{~s}, \mathrm{C}_{10}\right), 135.7\left(\mathrm{~s}, 4 \mathrm{C}_{23}\right), 133.9\left(\mathrm{~s}, 2 \mathrm{C}_{22}\right)$, $131.3\left(\mathrm{~s}, \mathrm{C}_{16}\right), 129.6\left(\mathrm{~s}, 2 \mathrm{C}_{25}\right), 129.2\left(\mathrm{~s}, 2 \mathrm{C}_{17}\right), 127.7\left(\mathrm{~s}, 4 \mathrm{C}_{24}\right), 126.1\left(\mathrm{~s}, \mathrm{C}_{9}\right), 113.8\left(\mathrm{~s}, 2 \mathrm{C}_{18}\right)$, $100.3\left(\mathrm{~s}, \mathrm{C}_{13}\right), 75.9\left(\mathrm{~s}, \mathrm{C}_{3}\right), 72.6\left(\mathrm{~s}, \mathrm{C}_{15}\right), 72.4\left(\mathrm{~s}, \mathrm{C}_{5}\right), 68.7\left(\mathrm{~s}, \mathrm{C}_{11}\right), 65.7\left(\mathrm{~s}, \mathrm{C}_{1}\right), 64.6\left(\mathrm{~s}, \mathrm{C}_{7}\right), 55.3$ (s, $\left.\mathrm{C}_{20}\right), 39.7\left(\mathrm{~s}, \mathrm{C}_{2}\right), 39.2\left(\mathrm{~s}, \mathrm{C}_{8}\right), 39.0\left(\mathrm{~s}, \mathrm{C}_{6}\right), 37.9\left(\mathrm{~s}, \mathrm{C}_{4}\right), 29.7\left(\mathrm{~s}, \mathrm{C}_{12}\right), 27.0\left(\mathrm{~s}, 3 \mathrm{C}_{27}\right), 25.1(\mathrm{~s}$, $\left.2 \mathrm{C}_{14}\right), 19.3\left(\mathrm{~s}, \mathrm{C}_{26}\right), 11.7\left(\mathrm{~s}, \mathrm{C}_{21}\right)$.

GCMS (EI) m/z (rel intensity): 57 (37), 77 (4), 79 (2), 95 (7), 161 (2), 177 (14), 205 (100), 206 (17), $220(30), 221(2)$.

HRMS (ESI-TOF): Calculated for $\mathrm{C}_{40} \mathrm{H}_{56} \mathrm{O}_{6} \mathrm{SiNa}[\mathrm{M}+\mathrm{Na}]^{+}$: 683.3730. Found: 683.3738.

$(6 S, 8 R, 10 S, 11 S, E)-12-((t e r t-b u t y l d i p h e n y l s i l y l) o x y)-10-((4-m e t h o x y b e n z y l) o x y)-11-$ methyldodec-3-ene-2,6,8-triol, (3.50.1b):

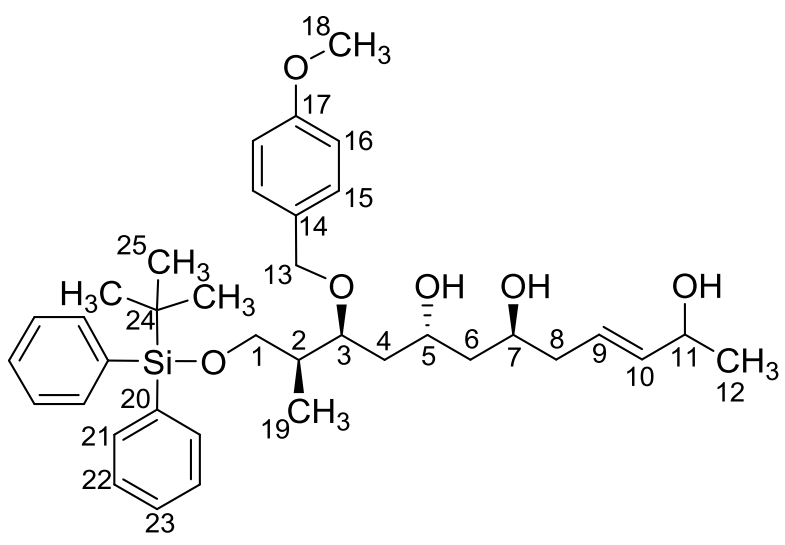

Aqueous $\mathrm{HCl}(0.015 \mathrm{~mL}, 0.0151 \mathrm{mmol}, 2 \mathrm{M})$ was added to a solution of alcohol $\mathbf{3 . 5 9 . 8}(0.100$ g, $0.151 \mathrm{mmol})$ in $\mathrm{CH}_{2} \mathrm{Cl}_{2}(3 \mathrm{~mL})$ at $0{ }^{\circ} \mathrm{C}$ and stirred at room temperature for $72 \mathrm{~h}$. Saturated 
aqueous sodium carbonate $(5 \mathrm{~mL})$ and water $(5 \mathrm{~mL})$ were added. The phases were separated and the aqueous layer was extracted with $\mathrm{CH}_{2} \mathrm{Cl}_{2}(3 \mathrm{X} 20 \mathrm{~mL})$. The combined organic extracts were dried over anhydrous magnesium sulphate, filtered and concentrated in vacuo to give the crude triol 3.50.1b $(0.0740 \mathrm{~g}, 79 \%)$ as a yellow oil which was used in the proceeding iron-mediated cyclization step without further purification.

Formula: $\mathrm{C}_{37} \mathrm{H}_{52} \mathrm{O}_{6} \mathrm{Si}$

Mass: $620.3 \mathrm{~g} / \mathrm{mol}$

IR $v_{\max }$ (neat): 3422, 1612, 1514, 1463, 1428, 1362, 1249, 1171, 1111, 1074, 1037, 823, 741, $703,611 \mathrm{~cm}^{-1}$.

${ }^{1} \mathbf{H}$ NMR $\left(400 \mathrm{MHz}\right.$, Acetone- $\left.d_{6}\right) \delta 7.69-7.63\left(4 \mathrm{H}, \mathrm{m}, 4 \mathrm{H}_{21}\right), 7.43-7.34\left(6 \mathrm{H}, \mathrm{m}, 4 \mathrm{H}_{22}+2 \mathrm{H}_{23}\right.$ ), $7.21-7.16\left(2 \mathrm{H}, \mathrm{m}, \mathrm{H}_{15}\right), 6.88-6.80\left(2 \mathrm{H}, \mathrm{m}, \mathrm{H}_{16}\right), 5.66-5.52\left(2 \mathrm{H}, \mathrm{m}, \mathrm{H}_{9}+\mathrm{H}_{10}\right), 4.43(2 \mathrm{H}$, s, $\left.\mathrm{H}_{13}\right), 4.22\left(1 \mathrm{H}, \mathrm{dqd}, J=13.3,6.9,3.7 \mathrm{~Hz}, \mathrm{H}_{11}\right), 4.10-4.03\left(1 \mathrm{H}, \mathrm{m}, \mathrm{H}_{7}\right), 3.93-3.82(1 \mathrm{H}, \mathrm{m}$, $\left.\mathrm{H}_{3}\right), 3.79-3.69\left(4 \mathrm{H}, \mathrm{m}, \mathrm{H}_{1}+3 \mathrm{H}_{18}\right), 3.54\left(1 \mathrm{H}, \mathrm{dd}, J=9.8,6.5 \mathrm{~Hz}, \mathrm{H}_{1}\right), 2.18-2.08\left(2 \mathrm{H}, \mathrm{m}, \mathrm{H}_{8}\right)$, $2.04-1.93\left(1 \mathrm{H}, \mathrm{m}, \mathrm{H}_{2}\right), 1.75-1.46\left(4 \mathrm{H}, \mathrm{m}, \mathrm{H}_{4}+\mathrm{H}_{6}\right), 1.25\left(3 \mathrm{H}, \mathrm{d}, J=10.9 \mathrm{~Hz}, \mathrm{H}_{12}\right), 1.06(9 \mathrm{H}$, s, $\left.\mathrm{H}_{25}\right), 0.99\left(3 \mathrm{H}, \mathrm{d}, J=6.6 \mathrm{~Hz}, \mathrm{H}_{19}\right)$.

${ }^{13}$ C NMR (101 MHz, Acetone- $\left.d_{6}\right) \delta 159.3\left(\mathrm{~s}, \mathrm{C}_{17}\right), 137.5\left(\mathrm{~s}, \mathrm{C}_{10}\right), 135.7\left(\mathrm{~s}, 4 \mathrm{C}_{21}\right), 133.8\left(\mathrm{~s}, 2 \mathrm{C}_{20}\right)$,

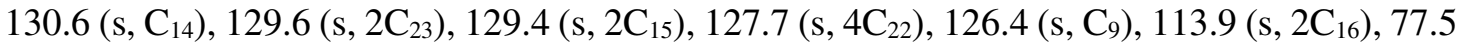
$\left(\mathrm{s}, \mathrm{C}_{3}\right), 72.1\left(\mathrm{~s}, \mathrm{C}_{13}\right), 68.5\left(\mathrm{~s}, \mathrm{C}_{5}\right), 66.5\left(\mathrm{~s}, \mathrm{C}_{11}\right), 65.6\left(\mathrm{~s}, \mathrm{C}_{1}\right), 64.6\left(\mathrm{~s}, \mathrm{C}_{7}\right), 55.3\left(\mathrm{~s}, \mathrm{C}_{18}\right), 44.5(\mathrm{~s}$, $\left.\mathrm{C}_{4}\right), 41.4\left(\mathrm{~s}, \mathrm{C}_{8}\right), 38.9\left(\mathrm{~s}, \mathrm{C}_{2}\right), 37.8\left(\mathrm{~s}, \mathrm{C}_{6}\right), 27.0\left(\mathrm{~s}, 3 \mathrm{C}_{25}\right), 23.3\left(\mathrm{~s}, \mathrm{C}_{12}\right), 19.4\left(\mathrm{~s}, \mathrm{C}_{24}\right), 13.0\left(\mathrm{~s}, \mathrm{C}_{19}\right)$.

GCMS (EI) $m / z$ (rel intensity): 77 (11), 121 (4), 199 (100), 200 (19), 201 (5).

HRMS (ESI-TOF): Calculated for $\mathrm{C}_{37} \mathrm{H}_{52} \mathrm{O}_{6} \mathrm{SiNa}[\mathrm{M}+\mathrm{Na}]^{+}$: 643.3422. Found: 643.3425. 


\section{(R)-1-((4S,6R)-6-((2S,3S)-4-((tert-butyldiphenylsilyl)oxy)-2-((4-methoxybenzyl)oxy)-3-}

methylbutyl)-2,2-dimethyl-1,3-dioxan-4-yl)pent-4-en-2-ol, (3.63.1):

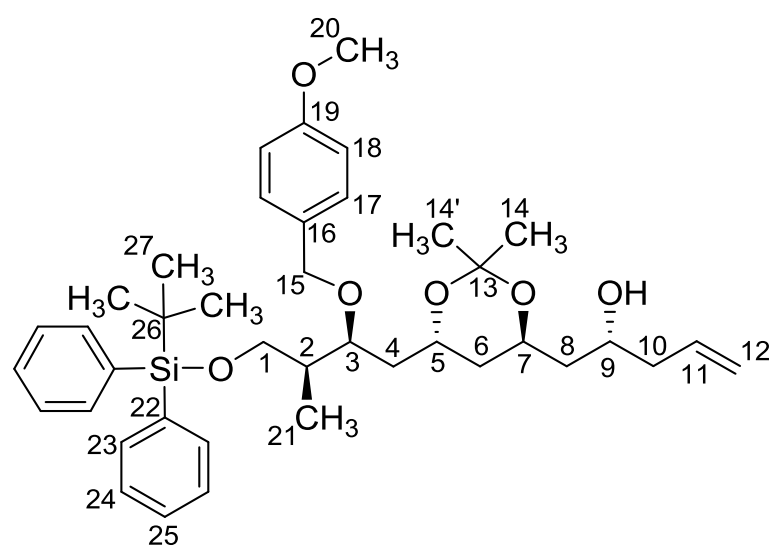

A solution of osmium tetroxide in tert-butanol $(44.4 \mu \mathrm{L}, 3.50 \mu \mathrm{mol}, 0.079 \mathrm{M})$ followed by NMO (22.6 mg, $193 \mu \mathrm{mol})$ were added to a mixture of acetonide 3.59 .6 (0.108 g, $0.175 \mathrm{mmol})$, water $(0.20 \mathrm{~mL})$ and tert-butanol $(0.80 \mathrm{~mL})$ and THF $(0.80 \mathrm{~mL})$ at room temperature. The reaction mixture was stirred at room temperature for $8 \mathrm{~h}$, and additional portions of osmium tetroxide (44.4 $\mu \mathrm{L}, 3.50 \mu \mathrm{mol}, 0.079 \mathrm{M})$ and NMO (22.6 mg, $193 \mu \mathrm{mol})$ were added. The mixture was stirred at room temperature for $20 \mathrm{~h}$. Solid $\mathrm{Na}_{2} \mathrm{~S}_{2} \mathrm{O}_{3}(0.20 \mathrm{~g})$, Celite $(0.40 \mathrm{~g})$, and EtOAc $(1 \mathrm{~mL})$ were added, and the mixture was filtered through a pad of Celite ${ }^{\circledR}$ which was thoroughly washed with EtOAc. The filtrate was concentrated in vacuo, and the residue was dissolved in (1:2) mixture of $\mathrm{H}_{2} \mathrm{O}: \mathrm{MeOH}(3 \mathrm{~mL})$. Sodium metaperiodate $(0.225 \mathrm{~g}, 1.05 \mathrm{mmol})$ was then added to the mixture and stirred for $3 \mathrm{~h}$ at room temperature. The biphasic mixture was filtered through a pad of Celite ${ }^{\circledR}$, the layers were separated, and the aqueous layer was extracted with EtOAc (3 x $15 \mathrm{~mL}$ ). The combined organic extracts were washed with brine, dried over anhydrous magnesium sulphate, filtered and concentrated in vасио. The residue was azeotropically dried with toluene twice to give the crude aldehyde as a yellow oil which was used in the proceeding allylation step without further purification. 
A solution of the aldehyde $\mathbf{3 . 5 1 . 3}\left(0.175 \mathrm{mmol}, 1.0\right.$ equiv) in $\mathrm{Et}_{2} \mathrm{O}(0.5 \mathrm{~mL})$ was added to a suspension of $(S, S)-\mathbf{T i}(0.130 \mathrm{~g}, 0.210 \mathrm{mmol})$ in $\mathrm{Et}_{2} \mathrm{O}(1.5 \mathrm{~mL})$ at $-78{ }^{\circ} \mathrm{C}$ over a period of 10 minutes under argon. The mixture was stirred at $-78{ }^{\circ} \mathrm{C}$ for $24 \mathrm{~h}$. The reaction mixture was quenched with deionized water at $-78{ }^{\circ} \mathrm{C}$, then allowed to warm to room temperature. The mixture was stirred at room temperature for $24 \mathrm{~h}$. The reaction media was filtered through a pad of Celite ${ }^{\circledR}$. The layers were separated and the aqueous layer was extracted with $\mathrm{Et}_{2} \mathrm{O}(3 \times 15$ $\mathrm{mL}$ ). The combined organic extracts were washed with brine, dried over anhydrous magnesium sulphate, filtered and concentrated in vacuo. Pentane $(23 \mathrm{~mL})$ was added, the mixture was stirred vigorously at room temperature for $4 \mathrm{~h}$, filtered and concentrated in vacuo. The residue was purified by flash chromatography on silica gel eluting with $(\mathrm{PET} / \mathrm{EtOAc}=65: 35)$ to give homoallylic alcohol 3.63.1 (69.6 mg, 60\%) as a yellow oil.

Formula: $\mathrm{C}_{40} \mathrm{H}_{56} \mathrm{O}_{6} \mathrm{Si}$

Mass: $660.4 \mathrm{~g} / \mathrm{mol}$

${ }^{1}$ H NMR (400 MHz, Acetone- $\left.d_{6}\right) \delta 7.68-7.65\left(4 \mathrm{H}, \mathrm{m}, \mathrm{H}_{23}\right), 7.43-7.32\left(6 \mathrm{H}, \mathrm{m}, 4 \mathrm{H}_{24}+2 \mathrm{H}_{25}\right)$, $7.16\left(2 \mathrm{H}, \mathrm{d}, J=8.7 \mathrm{~Hz}, \mathrm{H}_{17}\right), 6.83\left(2 \mathrm{H}, \mathrm{d}, J=8.7 \mathrm{~Hz}, \mathrm{H}_{18}\right), 5.83(1 \mathrm{H}, \mathrm{ddt}, J=17.5,10.4,7.1$ $\left.\mathrm{Hz}, \mathrm{H}_{11}\right), 5.14-5.07\left(2 \mathrm{H}, \mathrm{m}, \mathrm{H}_{12}\right), 4.47\left(1 \mathrm{H}, \mathrm{d}, J=10.8 \mathrm{~Hz}, \mathrm{H}_{15}\right), 4.42(1 \mathrm{H}, \mathrm{d}, J=10.7 \mathrm{~Hz}$, $\left.\mathrm{H}_{15^{\circ}}\right), 4.21-4.11\left(1 \mathrm{H}, \mathrm{m}, \mathrm{H}_{5}\right), 4.07-3.97\left(1 \mathrm{H}, \mathrm{m}, \mathrm{H}_{7}\right), 3.97-3.84\left(2 \mathrm{H}, \mathrm{m}, \mathrm{H}_{3}+\mathrm{H}_{9}\right), 3.78(3 \mathrm{H}$, s, $\left.\mathrm{H}_{20}\right), 3.74\left(1 \mathrm{H}, \mathrm{dd}, J=10.0,7.0 \mathrm{~Hz}, \mathrm{H}_{1}\right), 3.56\left(1 \mathrm{H}, \mathrm{dd}, J=9.9,6.6 \mathrm{~Hz}, \mathrm{H}_{1}{ }^{\prime}\right), 2.28-2.21(2 \mathrm{H}$, m, $\left.\mathrm{H}_{10}\right), 1.94\left(1 \mathrm{H}, \mathrm{dtq}, J=10.2,6.8,3.9 \mathrm{~Hz}, \mathrm{H}_{2}\right), 1.72-1.50\left(6 \mathrm{H}, \mathrm{m}, 2 \mathrm{H}_{4}+2 \mathrm{H}_{6}+2 \mathrm{H}_{8}\right), 1.38$ $\left(3 \mathrm{H}, \mathrm{s}, \mathrm{H}_{14}\right), 1.35\left(3 \mathrm{H}, \mathrm{s}, \mathrm{H}_{14^{\prime}}\right), 1.07\left(9 \mathrm{H}, \mathrm{s}, \mathrm{H}_{25}\right), 0.92\left(3 \mathrm{H}, \mathrm{d}, J=6.9 \mathrm{~Hz}, \mathrm{H}_{21}\right)$.

${ }^{13}$ C NMR (101 MHz, Acetone- $\left.d_{6}\right) \delta 159.0\left(\mathrm{~s}, \mathrm{C}_{19}\right), 135.6\left(\mathrm{~s}, 4 \mathrm{C}_{23}\right), 134.9\left(\mathrm{~s}, \mathrm{C}_{11}\right), 133.9\left(\mathrm{~s}, 2 \mathrm{C}_{22}\right)$, $131.2\left(\mathrm{~s}, \mathrm{C}_{16}\right), 129.6\left(\mathrm{~s}, 2 \mathrm{C}_{25}\right), 129.2\left(\mathrm{~s}, 2 \mathrm{C}_{17}\right), 127.6\left(\mathrm{~s}, 4 \mathrm{C}_{24}\right), 117.7\left(\mathrm{~s}, \mathrm{C}_{12}\right), 113.8\left(\mathrm{~s}, 2 \mathrm{C}_{18}\right)$ $100.5\left(\mathrm{~s}, \mathrm{C}_{13}\right), 75.9\left(\mathrm{~s}, \mathrm{C}_{3}\right), 72.5\left(\mathrm{~s}, \mathrm{C}_{15}\right), 67.9\left(\mathrm{~s}, \mathrm{C}_{9}\right), 65.6\left(\mathrm{~s}, \mathrm{C}_{1}\right), 64.3\left(\mathrm{~s}, \mathrm{C}_{5}\right), 63.9\left(\mathrm{~s}, \mathrm{C}_{7}\right), 55.3$ (s, $\left.\mathrm{C}_{20}\right), 42.0\left(\mathrm{~s}, \mathrm{C}_{10}\right), 41.2\left(\mathrm{~s}, \mathrm{C}_{6}\right), 39.7\left(\mathrm{~s}, \mathrm{C}_{2}\right), 39.1\left(\mathrm{~s}, \mathrm{C}_{8}\right), 38.4\left(\mathrm{~s}, \mathrm{C}_{4}\right), 26.9\left(\mathrm{~s}, \mathrm{C}_{27}\right), 25.0(\mathrm{~s}$, $\left.2 \mathrm{C}_{14}\right), 19.3\left(\mathrm{~s}, \mathrm{C}_{26}\right), 11.7\left(\mathrm{~s}, \mathrm{C}_{21}\right)$. 


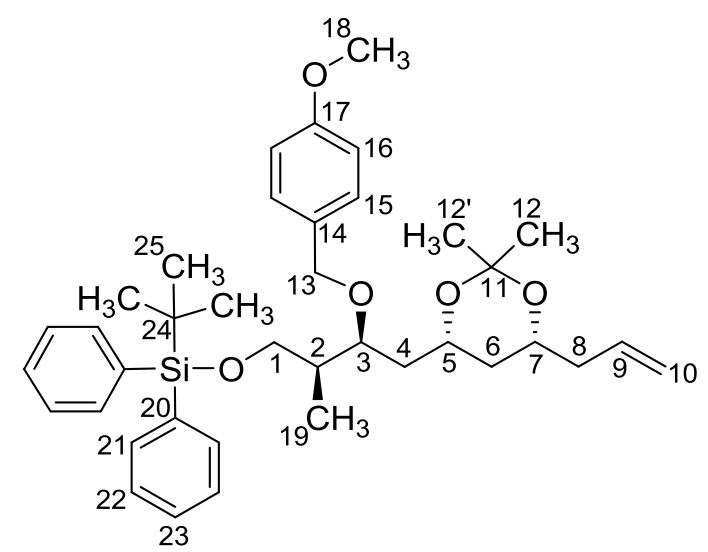

2,2-Dimethoxypropane $(0.18 \mathrm{~mL}, 1.50 \mathrm{mmol})$ followed by a catalytic amount of PPTS $(0.4 \mathrm{mg}$, $1.5 \mu \mathrm{mol})$ were added to a solution of diol $\mathbf{3 . 6 0 . 1}(0.086 \mathrm{~g}, 0.149 \mathrm{mmol})$ in $\mathrm{CH}_{2} \mathrm{Cl}_{2}(1 \mathrm{~mL})$ at room temperature. The reaction mixture was stirred at room temperature for $5 \mathrm{~h}$. Water was added, and the mixture was extracted with $\mathrm{CH}_{2} \mathrm{Cl}_{2}(3 \mathrm{X} 20 \mathrm{~mL})$. The combined organic extracts were washed with brine, then dried over anhydrous magnesium sulphate, filtered and concentrated in vacuo. The residue was purified by flash chromatography on silica gel eluting with $(\mathrm{PET} / \mathrm{EtOAc}=97: 3$ to $95: 5)$ to give trans-acetonide $\mathbf{3 . 6 0 . 2}(0.050 \mathrm{~g}, 54 \%)$ as a colorless oil.

Formula: $\mathrm{C}_{38} \mathrm{H}_{52} \mathrm{O}_{5} \mathrm{Si}$

Mass: $616.4 \mathrm{~g} / \mathrm{mol}$

${ }^{1}$ H NMR (400 MHz, Acetone- $\left.d_{6}\right) \delta 7.68-7.64\left(4 \mathrm{H}, \mathrm{m}, 4 \mathrm{H}_{21}\right), 7.45-7.32\left(6 \mathrm{H}, \mathrm{m}, 4 \mathrm{H}_{22}+2 \mathrm{H}_{23}\right)$, $7.18\left(2 \mathrm{H}, \mathrm{m}, \mathrm{H}_{15}\right), 6.86-6.80\left(2 \mathrm{H}, \mathrm{m}, \mathrm{H}_{16}\right), 5.73\left(1 \mathrm{H}, \mathrm{m}, \mathrm{H}_{9}\right), 5.12-5.01\left(2 \mathrm{H}, \mathrm{m}, \mathrm{H}_{10}\right), 4.44$ $\left(2 \mathrm{H}, \mathrm{d}, J=10.8 \mathrm{~Hz}, \mathrm{H}_{13}\right), 4.02\left(1 \mathrm{H}, \mathrm{ddt}, J=11.7,9.0,3.1 \mathrm{~Hz}, \mathrm{H}_{7}\right), 3.95(1 \mathrm{H}, \mathrm{dt}, J=9.3,3.5,3.1$ $\left.\mathrm{Hz}, \mathrm{H}_{3}\right), 3.87\left(1 \mathrm{H}, \mathrm{dtd}, J=12.1,6.2,2.3 \mathrm{~Hz}, \mathrm{H}_{5}\right), 3.78\left(3 \mathrm{H}, \mathrm{s}, \mathrm{H}_{18}\right), 3.72(1 \mathrm{H}, \mathrm{dd}, J=10.0,6.9$ $\left.\mathrm{Hz}, \mathrm{H}_{1}\right), 3.54\left(1 \mathrm{H}, \mathrm{dd}, J=10.1,6.5 \mathrm{~Hz}, \mathrm{H}_{1^{\prime}}\right), 2.28\left(1 \mathrm{H}, \mathrm{m}, \mathrm{H}_{8}\right), 2.14\left(1 \mathrm{H}, \mathrm{m}, \mathrm{H}_{8^{\prime}}\right), 1.85(1 \mathrm{H}, \mathrm{qt}$, $\left.\left.J=10.2,5.2 \mathrm{~Hz}, \mathrm{H}_{2}\right), 1.64-1.50\left(4 \mathrm{H}, \mathrm{m}, \mathrm{H}_{4}+\mathrm{H}_{6}\right), 1.44\left(3 \mathrm{H}, \mathrm{s}, \mathrm{H}_{12}\right), 1.41\left(3 \mathrm{H}, \mathrm{s}, \mathrm{H}_{12}\right)^{\prime}\right), 1.06$ (9H, s, $\left.\mathrm{H}_{25}\right), 0.91\left(3 \mathrm{H}, \mathrm{d}, J=6.9,1.0 \mathrm{~Hz}, \mathrm{H}_{19}\right)$. 
${ }^{13}$ C NMR (101 MHz, Acetone- $\left.d_{6}\right) \delta 159.0\left(\mathrm{~s}, \mathrm{C}_{17}\right), 135.7\left(\mathrm{~s}, 4 \mathrm{C}_{21}\right), 134.3\left(\mathrm{~s}, \mathrm{C}_{9}\right), 134.0\left(\mathrm{~s}, \mathrm{C}_{20}\right)$,

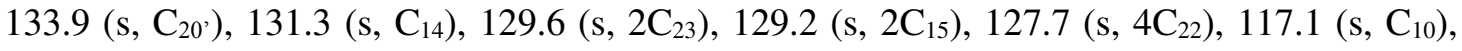
$113.7\left(\mathrm{~s}, 2 \mathrm{C}_{16}\right), 98.5\left(\mathrm{~s}, \mathrm{C}_{11}\right), 75.1\left(\mathrm{~s}, \mathrm{C}_{3}\right), 72.7\left(\mathrm{~s}, \mathrm{C}_{13}\right), 68.7\left(\mathrm{~s}, \mathrm{C}_{5}\right), 65.8\left(\mathrm{~s}, \mathrm{C}_{1}\right), 55.3\left(\mathrm{~s}, \mathrm{C}_{18}\right)$, $40.9\left(\mathrm{~s}, \mathrm{C}_{2}\right), 40.0\left(\mathrm{~s}, \mathrm{C}_{8}\right), 39.9\left(\mathrm{~s}, \mathrm{C}_{6}\right), 37.1\left(\mathrm{~s}, \mathrm{C}_{4}\right), 29.7\left(\mathrm{~s}, \mathrm{C}_{12}\right), 27.0\left(\mathrm{~s}, 3 \mathrm{C}_{25}\right), 20.0\left(\mathrm{~s}, \mathrm{C}_{12}\right), 19.3$ $\left(\mathrm{s}, \mathrm{C}_{24}\right), 11.7\left(\mathrm{~s}, \mathrm{C}_{19}\right)$.

\section{(7S,5R,3S,2S,E)-1-((tert-butyldiphenylsilyl)oxy)-5,7-dihydroxy-9-((4-} methoxybenzyl)oxy)-2-methyl-11-phenylundec-9-en-11-one (3.64.2)

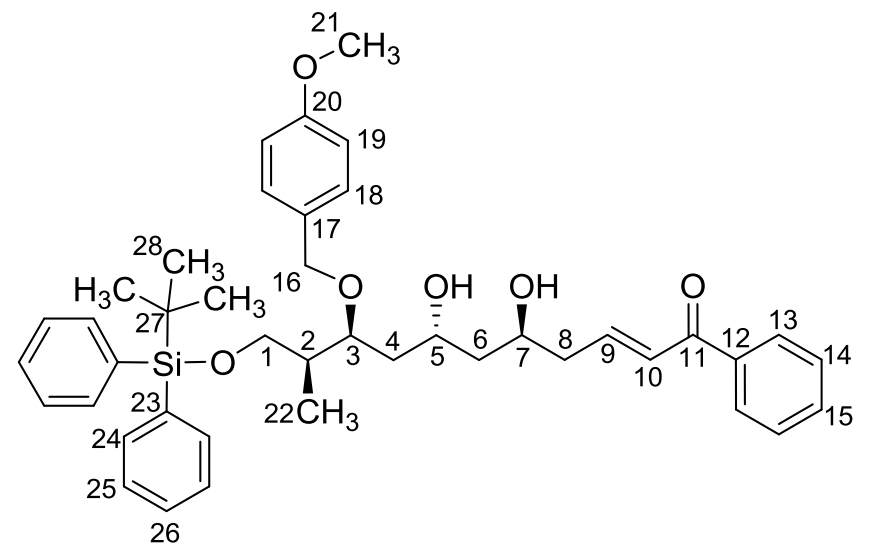

A solution of the Grubbs $2^{\text {nd }}$ generation $(0.0150 \mathrm{~g}, 0.0175 \mathrm{mmol})$ in $\mathrm{CH}_{2} \mathrm{Cl}_{2}(5 \mathrm{~mL})$ was added portion wise $(1.5 \mathrm{~mL} / \mathrm{h})$ to a solution of diol $\mathbf{3 . 5 9 . 5}(0.101 \mathrm{~g}, 0.175 \mathrm{mmol})$ and phenyl vinyl ketone 3.64.1 ( $0.13 \mathrm{~g}, 0.984 \mathrm{mmol})$ in $\mathrm{CH}_{2} \mathrm{Cl}_{2}(6 \mathrm{~mL})$ at $40{ }^{\circ} \mathrm{C}$ under a stream of argon passing through the system. The reaction mixture was stirred at reflux for a further hour, then cooled and concentrated in vacuo. The residue was purified by flash chromatography on silica gel eluting with $(\mathrm{PET} / \mathrm{EtOAc}=40: 60)$ to give $\alpha, \beta$-unsaturated phenyl ketone 3.64.2 $(0.035 \mathrm{~g}, 29 \%)$ as a yellow oil.

Formula: $\mathrm{C}_{42} \mathrm{H}_{52} \mathrm{O}_{6} \mathrm{Si}$

Mass: $680.3 \mathrm{~g} / \mathrm{mol}$

IR (neat): 3424, 1671, 1613, 1579, 1513, 1463, 1448, 1427, 1390, 1247, 1173, 1109, 1070 , $1034,938,822 \mathrm{~cm}^{-1}$. 
${ }^{1}$ H NMR $(400 \mathrm{MHz}$, Acetone-d $\left.)\right) \delta 7.92\left(2 \mathrm{H}, \mathrm{d}, J=7.8 \mathrm{~Hz}, \mathrm{H}_{13}\right), 7.64\left(4 \mathrm{H}, \mathrm{d}, J=7.2 \mathrm{~Hz}, \mathrm{H}_{24}\right)$, $7.57-7.51\left(1 \mathrm{H}, \mathrm{m}, \mathrm{H}_{10}\right), 7.49-7.32\left(8 \mathrm{H}, \mathrm{m}, 2 \mathrm{H}_{14}+4 \mathrm{H}_{25}+2 \mathrm{H}_{26}\right), 7.20\left(2 \mathrm{H}, \mathrm{d}, J=8.0 \mathrm{~Hz}, \mathrm{H}_{18}\right)$, $7.04\left(1 \mathrm{H}, \mathrm{dt}, J=14.8,7.3 \mathrm{~Hz}, \mathrm{H}_{9}\right), 6.85\left(2 \mathrm{H}, \mathrm{d}, J=8.3 \mathrm{~Hz}, \mathrm{H}_{19}\right), 4.45\left(2 \mathrm{H}, \mathrm{s}, \mathrm{H}_{16}\right), 4.15-4.02$ $\left(2 \mathrm{H}, \mathrm{m}, \mathrm{H}_{5}+\mathrm{H}_{7}\right), 3.82-3.71\left(4 \mathrm{H}, \mathrm{m}, \mathrm{H}_{3}+3 \mathrm{H}_{21}\right), 3.69\left(2 \mathrm{H}, \mathrm{dd}, J=10.1,5.5 \mathrm{~Hz}, \mathrm{H}_{1}\right), 3.54(2 \mathrm{H}$, dd, $\left.J=10.1,5.9 \mathrm{~Hz}, \mathrm{H}_{1}{ }^{\prime}\right), 2.54-2.37\left(2 \mathrm{H}, \mathrm{m}, \mathrm{H}_{8}\right), 1.99\left(1 \mathrm{H}, \mathrm{dt}, J=12.7,6.4 \mathrm{~Hz}, \mathrm{H}_{2}\right), 1.72(1 \mathrm{H}$, ddd, $\left.J=13.6,9.7,3.4 \mathrm{~Hz}, \mathrm{H}_{6}\right), 1.62-1.47\left(2 \mathrm{H}, \mathrm{m}, \mathrm{H}_{4}+\mathrm{H}_{6}\right)$.

${ }^{13}$ C NMR (101 MHz, Acetone- $\left.d_{6}\right) \delta 190.7$ (s, C 11 ), $159.4\left(\mathrm{~s}, \mathrm{C}_{20}\right), 145.9$ (s, C 9 ), 137.9 (s, C 12 ), $135.7\left(\mathrm{~s}, 4 \mathrm{C}_{24}\right), 133.7\left(\mathrm{~s}, 2 \mathrm{C}_{23}\right), 132.9\left(\mathrm{~s}, \mathrm{C}_{10}\right), 129.8\left(\mathrm{~s}, \mathrm{C}_{17}\right), 128.7\left(\mathrm{~s}, 2 \mathrm{C}_{14}+2 \mathrm{C}_{18}\right), 128.2(\mathrm{~s}$, $\left.2 \mathrm{C}_{13}+\mathrm{C}_{26}\right), 127.8\left(\mathrm{~s}, \mathrm{C}_{15}\right), 114.0\left(\mathrm{~s}, 4 \mathrm{C}_{25}\right), 77.9\left(\mathrm{~s}, 2 \mathrm{C}_{19}\right), 72.1\left(\mathrm{~s}, \mathrm{C}_{16}\right), 68.2\left(\mathrm{~s}, \mathrm{C}_{7}\right), 66.8\left(\mathrm{~s}, \mathrm{C}_{5}\right)$, $65.6\left(\mathrm{~s}, \mathrm{C}_{1}\right), 55.4\left(\mathrm{~s}, \mathrm{C}_{21}\right), 42.5\left(\mathrm{~s}, \mathrm{C}_{8}\right), 41.0\left(\mathrm{~s}, \mathrm{C}_{6}\right), 38.9\left(\mathrm{~s}, \mathrm{C}_{2}\right), 37.2\left(\mathrm{~s}, \mathrm{C}_{4}\right), 27.0\left(\mathrm{~s}, \mathrm{C}_{28}\right), 19.4$ $\left(\mathrm{s}, \mathrm{C}_{27}\right), 13.4\left(\mathrm{~s}, \mathrm{C}_{22}\right)$.

GCMS (EI) m/z (rel intensity): 55 (100), 56 (82), 57 (63), 69 (80), 70 (77), 83 (66), 84 (29), 97 (45).

HRMS (ESI-TOF): Calculated for $\mathrm{C}_{42} \mathrm{H}_{52} \mathrm{O}_{6} \mathrm{SiNa}[\mathrm{M}+\mathrm{Na}]^{+}$: 703.3425. Found: 703.3425.

$[\alpha]_{\mathbf{D}}{ }^{23}:-13.7\left(c=1.1, \mathrm{CH}_{3} \mathrm{Cl}\right)$ 


\subsubsection{Experimental Procedure for Chapter IV}

\section{General procedure for Heck coupling reactions}

\section{Method A (Sealed vial)}

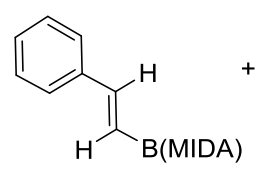

2-phenylvinyl boronic acid MIDA ester

A
$\mathrm{Pd}(\mathrm{OAc})_{2}(10 \mathrm{~mol} \%)$<smiles>Ic1c[R1]ccc1</smiles>

AgOAc (1.5 equiv) $\mathrm{CH}_{3} \mathrm{CN}(0.12 \mathrm{M})$ $80^{\circ} \mathrm{C}, 18 \mathrm{~h}$

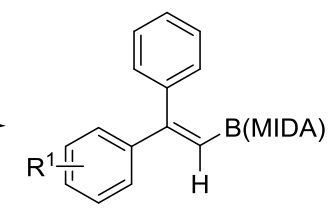

lodo-aryls

B

To an oven-dried vial was added 2-phenylvinyl boronic acid MIDA ester (1.0 equiv) and B (3.0 equiv) (if solid), followed by the oxidant $\mathrm{AgOAc}$ (1.5 equiv) and the catalyst $\mathrm{Pd}(\mathrm{OAc})_{2}(10$ mol\%) under an argon atmosphere. B (if liquid) was added after the catalyst. To this mixture was added anhydrous $\mathrm{CH}_{3} \mathrm{CN}(0.12 \mathrm{M})$. The vial was sealed with a PTFE-lined septum screw cap and heated at $80^{\circ} \mathrm{C}$ for 18 hours. The reaction mixture was left to cool to room temperature and filtered over a pad of Celite $\odot$. The filtrate was concentrated in vacuo. The crude material was dry loaded onto Celite $\odot$ from an acetone solution and was purified by flash chromatography on silica $\left(\mathrm{Et}_{2} \mathrm{O} /\right.$ acetone $=100: 0$ to $\left.70: 30\right)$ to afford the respective Heck coupling products.

\section{Method B}

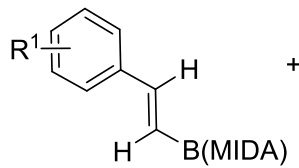

C

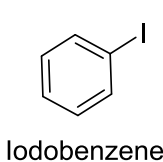

D

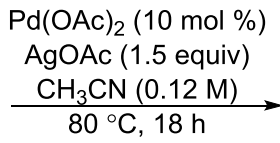

$80^{\circ} \mathrm{C}, 18 \mathrm{~h}$

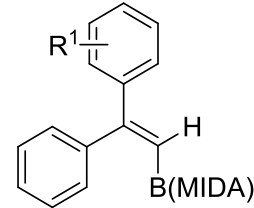

To an oven-dried round bottom flask was added $\mathbf{C}$ (1.0 equiv) (if solid), the oxidant AgOAc (1.5 equiv) and the catalyst $\mathrm{Pd}(\mathrm{OAc})_{2}(10 \mathrm{~mol} \%)$ followed by iodobenzene (3.0 equiv) under nitrogen atmosphere. $\mathbf{C}$ (if liquid) was added after the catalyst. To this mixture was added anhydrous $\mathrm{CH}_{3} \mathrm{CN}(0.12 \mathrm{M})$. The flask was fitted with a reflux condenser and heated at $80^{\circ} \mathrm{C}$ 
for 18 hours under nitrogen atmosphere. The reaction mixture was left to cool to room temperature and filtered over a pad of Celite 0 . The filtrate was concentrated in vacuo. The crude material was dry loaded onto Celite $\odot$ from an acetone solution and was purified by flash chromatography on silica $\left(\mathrm{Et}_{2} \mathrm{O} /\right.$ acetone $=100: 0$ to $\left.50: 50\right)$ to afford the respective Heck coupling products. 
This page has been intentionally left blank. 


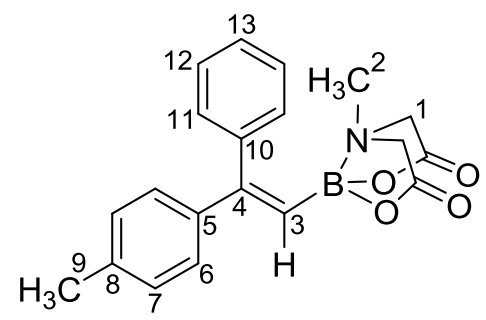

4.29.1 was prepared according to the general procedure A using 4-iodotoluene 4.12.2 (252 $\mathrm{mg}$, $116 \mathrm{mmol})$. Purification by flash chromatography on silica gel $\left(\mathrm{Et}_{2} \mathrm{O} /\right.$ Acetone $=83: 17$ to $\left.80: 20\right)$ to give 4.29.1 $(115 \mathrm{mg}, 85 \%)$ as a colorless oil $(E / Z=>50: 1)$.

Formula: $\mathrm{C}_{20} \mathrm{H}_{20} \mathrm{BNO}_{4}$

Mass: $349.2 \mathrm{~g} / \mathrm{mol}$

IR $v_{\max }$ (neat): 1761, 1608, 1595, 1509, 1455, 1336, 1290, 1447, 1229, 1191, 1157, 1108, 1083, $1025 \mathrm{~cm}^{-1}$.

${ }^{1}$ H NMR (400 MHz, Acetone- $\left.d_{6}\right) \delta 7.35-7.25\left(3 \mathrm{H}, \mathrm{m}, 2 \mathrm{H}_{7}+\mathrm{H}_{13}\right), 7.25-7.20\left(2 \mathrm{H}, \mathrm{m}, 2 \mathrm{H}_{11}\right)$, $7.20-7.14\left(2 \mathrm{H}, \mathrm{m}, 2 \mathrm{H}_{6}\right), 7.14-7.07\left(2 \mathrm{H}, \mathrm{m}, 2 \mathrm{H}_{12}\right), 6.15\left(1 \mathrm{H}, \mathrm{s}, \mathrm{H}_{3}\right), 4.03(2 \mathrm{H}, \mathrm{d}, J=16.7 \mathrm{~Hz}$, $\left.\mathrm{H}_{1}\right), 3.73\left(2 \mathrm{H}, \mathrm{d}, J=16.7 \mathrm{~Hz}, \mathrm{H}_{1}{ }^{\prime}\right), 3.09\left(3 \mathrm{H}, \mathrm{s}, \mathrm{H}_{2}\right), 2.29\left(3 \mathrm{H}, \mathrm{s}, \mathrm{H}_{9}\right)$.

${ }^{13}$ C NMR (101 MHz, Acetone- $\left.d_{6}\right) \delta 168.5$ (s, 2으), $155.9\left(\mathrm{~s}, \mathrm{C}_{4}\right), 142.7\left(\mathrm{~s}, \mathrm{C}_{5}\right), 142.3\left(\mathrm{~s}, \mathrm{C}_{10}\right)$, $138.0\left(\mathrm{~s}, \mathrm{C}_{8}\right), 130.3\left(\mathrm{~s}, 2 \mathrm{C}_{6}\right), 129.5\left(\mathrm{~s}, 2 \mathrm{C}_{7}\right), 128.8\left(\mathrm{~s}, 2 \mathrm{C}_{12}\right), 128.0\left(\mathrm{~s}, 2 \mathrm{C}_{11}\right), 127.8\left(\mathrm{~s}, \mathrm{C}_{13}\right), 62.6$ $\left(\mathrm{s}, 2 \mathrm{C}_{1}\right), 47.4\left(\mathrm{~s}, \mathrm{C}_{2}\right) 21.0\left(\mathrm{~s}, \mathrm{C}_{9}\right)$.

GCMS (EI) m/z (rel intensity): 179 (100), 180 (35), 193 (32), 194 (88), 195 (28), 220 (28), 221 (4).

HRMS (ESI-TOF): Calculated for $\mathrm{C}_{20} \mathrm{H}_{20} \mathrm{BNO}_{4} \mathrm{Na}[\mathrm{M}+\mathrm{Na}]^{+}: 372.1378$. Found: 372.1379 . 


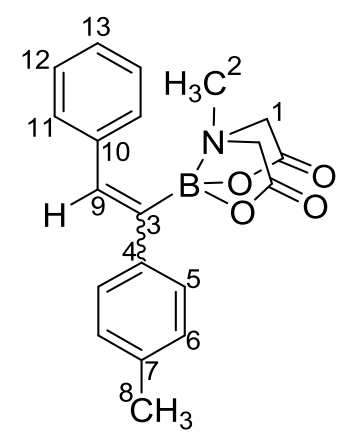

4.29.2 was prepared according to the general procedure A using 4-iodotoluene 4.12.2 (252 mg, $116 \mathrm{mmol})$. Purification by flash chromatography on silica gel $\left(\mathrm{Et}_{2} \mathrm{O} /\right.$ Acetone $\left.=85: 15\right)$ to give 4.29.2 $(23 \mathrm{mg}, 14 \%)$ as a colorless oil $(E / Z=1: 1)$.

Formula: $\mathrm{C}_{20} \mathrm{H}_{20} \mathrm{BNO}_{4}$

Mass: $349.2 \mathrm{~g} / \mathrm{mol}$

IR $v_{\max }$ (neat): $1763,1708,1606,1509,1448,1335,1287,1102,1071,1027 \mathrm{~cm}^{-1}$.

${ }^{1} \mathbf{H}$ NMR $\left(400 \mathrm{MHz}\right.$, Acetone- $\left.d_{6}\right) \delta 7.60-6.38\left(20 \mathrm{H}, \mathrm{m}, 2 \mathrm{H}_{5 E}+2 \mathrm{H}_{5 Z}+2 \mathrm{H}_{6 E}+2 \mathrm{H}_{6 \mathrm{Z}}+\mathrm{H}_{9 E}+\mathrm{H}_{9 Z}\right.$ $\left.+2 \mathrm{H}_{11 E}+2 \mathrm{H}_{11 Z}+2 \mathrm{H}_{12 E}+2 \mathrm{H}_{12 Z}+\mathrm{H}_{13 E}+\mathrm{H}_{13 Z}\right), 4.00\left(2 \mathrm{H}, \mathrm{d}, J=16.8 \mathrm{~Hz}, \mathrm{H}_{1 E}\right), 3.95(2 \mathrm{H}, \mathrm{d}, J=$ $\left.16.4 \mathrm{~Hz}, \mathrm{H}_{1 Z}\right), 3.45\left(2 \mathrm{H}, \mathrm{d}, J=16.4 \mathrm{~Hz}, \mathrm{H}_{1 Z}\right), 3.45\left(2 \mathrm{H}, \mathrm{d}, J=16.4 \mathrm{~Hz}, \mathrm{H}_{1 Z}\right), 3.21\left(3 \mathrm{H}, \mathrm{s}, \mathrm{H}_{2 E}\right)$, $2.95\left(3 \mathrm{H}, \mathrm{s}, \mathrm{H}_{2 \mathrm{Z}}\right), 2.32\left(3 \mathrm{H}, \mathrm{s}, \mathrm{H}_{8 E}\right), 2.26\left(3 \mathrm{H}, \mathrm{s}, \mathrm{H}_{9 \mathrm{Z}}\right)$.

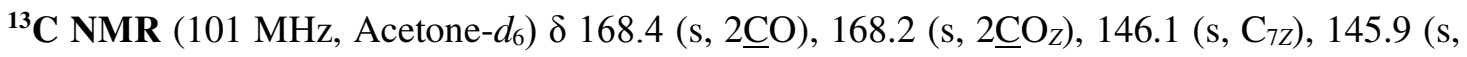

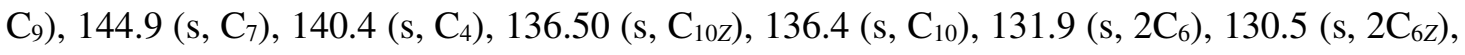
$129.8-129.3\left(\mathrm{~m}, 2 \mathrm{C}_{12}, 2 \mathrm{C}_{12 Z}\right), 128.9\left(\mathrm{~s}, 2 \mathrm{C}_{11 Z}\right), 128.7\left(\mathrm{~s}, 2 \mathrm{C}_{11}\right), 128.1\left(\mathrm{~s}, \mathrm{C}_{13}\right), 127.5\left(\mathrm{~s}, 2 \mathrm{C}_{5}\right)$, $126.4\left(\mathrm{~s}, 2 \mathrm{C}_{5 z}\right), 63.6\left(\mathrm{~s}, 2 \mathrm{C}_{1 z}\right), 63.3\left(\mathrm{~s}, 2 \mathrm{C}_{1}\right), 47.9\left(\mathrm{~s}, \mathrm{C}_{2 z}\right), 47.8\left(\mathrm{~s}, \mathrm{C}_{2}\right), 21.3\left(\mathrm{~s}, \mathrm{C}_{8 z}\right), 21.1\left(\mathrm{~s}, \mathrm{C}_{8}\right)$. GCMS (EI) m/z (rel intensity): 74 (2), 77 (4), 155 (23), 156 (100), 157 (8), 158 (6), 283 (5), 284 (100), 285 (23).

HRMS (ESI-TOF): Calculated for $\mathrm{C}_{20} \mathrm{H}_{20} \mathrm{BNO}_{4} \mathrm{Na}[\mathrm{M}+\mathrm{Na}]^{+}: 372.1378$. Found: 372.1378 . 


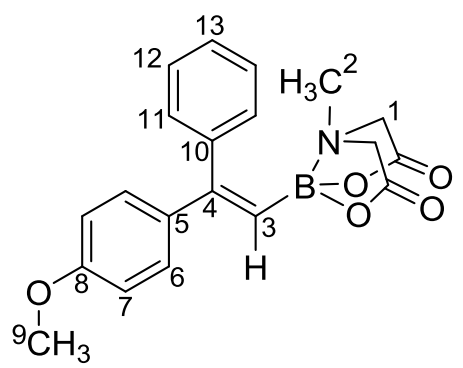

4.39.13 was prepared according to the general procedure A using 4-iodoanisole 4.39.1 (231 mg, $0.99 \mathrm{mmol})$. Purification by flash chromatography on silica gel $\left(\mathrm{Et}_{2} \mathrm{O} /\right.$ Acetone $=83: 17$ to 80:20) to give 4.39.13 $(112 \mathrm{mg}, 93 \%)$ as a colorless oil $(E / Z=19: 1)$.

Formula: $\mathrm{C}_{20} \mathrm{H}_{20} \mathrm{BNO}_{5}$

Mass: $365.2 \mathrm{~g} / \mathrm{mol}$

IR $v_{\max }$ (neat): 1769, 1751, 1604, 1509, 1460, 1356, 1336, 1285, 1248, 1178, 1108, 1082, 1024 , $1004,986 \mathrm{~cm}^{-1}$.

${ }^{1}$ H NMR (400 MHz, Acetone- $\left.d_{6}\right) \delta 7.35-7.25\left(3 \mathrm{H}, \mathrm{m}, 2 \mathrm{H}_{6}+\mathrm{H}_{13}\right), 7.25-7.19\left(4 \mathrm{H}, \mathrm{m}, 2 \mathrm{H}_{11}+\right.$ $\left.2 \mathrm{H}_{12}\right), 6.90-6.66\left(2 \mathrm{H}, \mathrm{m}, \mathrm{H}_{7}\right), 6.09\left(1 \mathrm{H}, \mathrm{s}, \mathrm{H}_{3}\right), 4.02\left(2 \mathrm{H}, \mathrm{d}, J=16.7 \mathrm{~Hz}, \mathrm{H}_{1}\right), 3.77\left(3 \mathrm{H}, \mathrm{s}, \mathrm{H}_{9}\right)$, $3.66\left(2 \mathrm{H}, \mathrm{d}, J=16.7 \mathrm{~Hz}, \mathrm{H}_{1}{ }^{\prime}\right), 3.08\left(3 \mathrm{H}, \mathrm{s}, \mathrm{H}_{2}\right)$.

${ }^{13}$ C NMR (101 MHz, Acetone- $\left.d_{6}\right) \delta 168.6$ (s, 2CO), $160.4\left(\mathrm{~s}, \mathrm{C}_{8}\right), 155.6\left(\mathrm{~s}, \mathrm{C}_{4}\right), 142.9\left(\mathrm{~s}, \mathrm{C}_{5}\right)$,

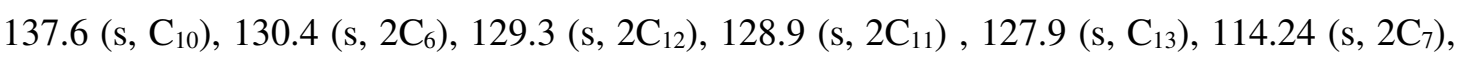
$62.7\left(\mathrm{~s}, 2 \mathrm{C}_{1}\right), 55.6\left(\mathrm{~s}, \mathrm{C}_{9}\right), 47.4\left(\mathrm{~s}, \mathrm{C}_{2}\right)$.

GCMS (EI) $m / z$ (rel intensity): 194 (8), 195 (56), 196 (9), 209 (9), 210 (100), 211 (15).

HRMS (ESI-TOF): Calculated for $\mathrm{C}_{20} \mathrm{H}_{20} \mathrm{BNO}_{5} \mathrm{Na}[\mathrm{M}+\mathrm{Na}]^{+}: 388.1327$. Found: 388.1328 . 
(E)-(1-(4-methoxyphenyl)-2-phenylvinyl)boronic acid MIDA ester, (4.39.14):

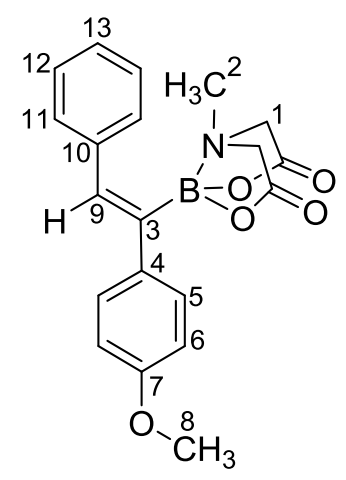

4.39.14 was prepared according to the general procedure A using 4-iodoanisole 4.39.1 (231 mg, $0.99 \mathrm{mmol})$. Purification by flash chromatography on silica gel $\left(\mathrm{Et}_{2} \mathrm{O} /\right.$ Acetone $\left.=85: 15\right)$ to give 4.39.14 $(10 \mathrm{mg}, 7 \%)$ as a colorless oil $(E / Z=3: 1)$.

Formula: $\mathrm{C}_{20} \mathrm{H}_{20} \mathrm{BNO}_{5}$ Mass: $365.2 \mathrm{~g} / \mathrm{mol}$

IR $v_{\max }$ (neat): $1770,1672,1599,1509,1456,1335,1285,1245,1176,1106,1074,1027 \mathrm{~cm}^{-1}$. ${ }^{1}$ H NMR $\left(400 \mathrm{MHz}\right.$, Acetone- $\left.d_{6}\right) \delta 7.42-7.23\left(6 \mathrm{H}, \mathrm{m}, 2 \mathrm{H}_{5}+2 \mathrm{H}_{11}+2 \mathrm{H}_{12}\right), 7.24-7.16(3 \mathrm{H}, \mathrm{m}$, $\left.\mathrm{H}_{12}+\mathrm{H}_{9}\right), 6.89\left(2 \mathrm{H}, \mathrm{d}, J=8.8 \mathrm{~Hz}, \mathrm{H}_{6}\right), 4.01\left(2 \mathrm{H}, \mathrm{d}, J=16.7 \mathrm{~Hz}, \mathrm{H}_{1}\right), 3.79\left(3 \mathrm{H}, \mathrm{s}, \mathrm{H}_{8}\right), 3.47(2 \mathrm{H}$, $\left.\mathrm{d}, J=16.8 \mathrm{~Hz}, \mathrm{H}_{1}\right), 2.95\left(3 \mathrm{H}, \mathrm{s}, \mathrm{H}_{2}\right)$.

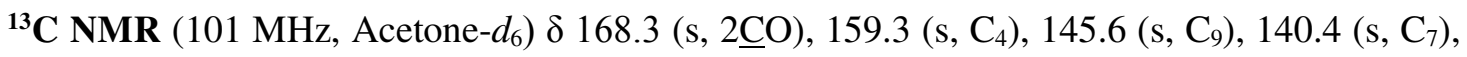
$139.9\left(\mathrm{~s}, \mathrm{C}_{10}\right), 130.7,\left(\mathrm{~s}, 2 \mathrm{C}_{12}\right) 129.4\left(\mathrm{~s}, 2 \mathrm{C}_{11}\right), 128.7\left(\mathrm{~s}, 2 \mathrm{C}_{5}\right), 127.4\left(\mathrm{~s}, \mathrm{C}_{13}\right), 114.2\left(\mathrm{~s}, 2 \mathrm{C}_{6}\right), 63.2$ $\left(\mathrm{s}, 2 \mathrm{C}_{1}\right), 55.5\left(\mathrm{~s}, \mathrm{C}_{8}\right), 47.7\left(\mathrm{~s}, \mathrm{C}_{2}\right)$.

HRMS (EI-TOF): Calculated for $\mathrm{C}_{20} \mathrm{H}_{20} \mathrm{BNO}_{5} \mathrm{Na}[\mathrm{M}+\mathrm{Na}]^{+}:$388.1327. Found: 388.1328 . 


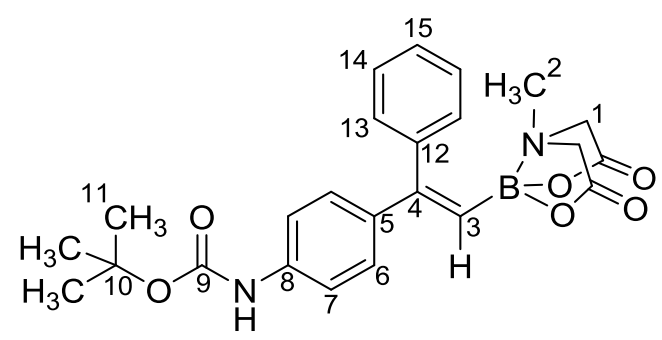

4.39.15 was prepared according to the general procedure A using tert-butyl N-(4iodophenyl)carbamate 4.39 .3 (156 $\mathrm{mg}, 0.49 \mathrm{mmol})$. Purification by flash chromatography on silica gel $\left(\mathrm{Et}_{2} \mathrm{O} /\right.$ Acetone $=83: 17$ to $\left.80: 20\right)$ to give $\mathbf{4 . 3 9 . 1 5}(54 \mathrm{mg}, 73 \%)$ as a colorless oil $(E / Z$ $=>50: 1)$.

Formula: $\mathrm{C}_{24} \mathrm{H}_{27} \mathrm{BN}_{2} \mathrm{O}_{6}$

Mass: $450.3 \mathrm{~g} / \mathrm{mol}$

IR $v_{\max }$ (neat): 1760, 1706, 1606, 1593, 1520, 1455, 1408, 1365, 1337, 1315, 1290, 1231, 1156, $1108,1084,1052,1025 \mathrm{~cm}^{-1}$.

${ }^{1}$ H NMR (400 MHz, Acetone- $\left.d_{6}\right) \delta 8.43\left(1 \mathrm{H}\right.$, brs, NH), $7.47\left(2 \mathrm{H}\right.$, brd, $\left.J=8.7 \mathrm{~Hz}, \mathrm{H}_{7}\right), 7.35-$ $7.26\left(3 \mathrm{H}, \mathrm{m}, 2 \mathrm{H}_{14}+\mathrm{H}_{15}\right), 7.26-7.17\left(4 \mathrm{H}, \mathrm{m}, 2 \mathrm{H}_{6}+2 \mathrm{H}_{13}\right), 6.13\left(1 \mathrm{H}, \mathrm{s}, \mathrm{H}_{3}\right), 4.01(2 \mathrm{H}, \mathrm{d}, J=$ 16.7 Hz, $\left.\mathrm{H}_{1}\right), 3.67\left(2 \mathrm{H}, \mathrm{d}, J=16.7 \mathrm{~Hz}, \mathrm{H}_{1}{ }^{\prime}\right), 3.07\left(3 \mathrm{H}, \mathrm{s}, \mathrm{H}_{2}\right), 1.47\left(9 \mathrm{H}, \mathrm{s}, \mathrm{H}_{11}\right)$.

${ }^{13}$ C NMR (101 MHz, Acetone- $\left.d_{6}\right) \delta 168.5$ (s, 2CO), 155.6 (s, C9), $153.6\left(\mathrm{~s}, \mathrm{C}_{4}\right), 142.7$ (s, C 8 ), $140.2\left(\mathrm{~s}, \mathrm{C}_{5}\right), 139.0\left(\mathrm{~s}, \mathrm{C}_{12}\right), 130.3\left(\mathrm{~s}, 2 \mathrm{C}_{14}\right), 128.8\left(\mathrm{~s}, 2 \mathrm{C}_{13}\right), 128.5\left(\mathrm{~s}, 2 \mathrm{C}_{6}\right), 127.8\left(\mathrm{~s}, \mathrm{C}_{15}\right), 118.5$ $\left(\mathrm{s}, 2 \mathrm{C}_{7}\right), 80.0\left(\mathrm{~s}, \mathrm{C}_{10}\right), 62.6\left(\mathrm{~s}, 2 \mathrm{C}_{1}\right), 47.4\left(\mathrm{~s}, \mathrm{C}_{2}\right), 28.5\left(\mathrm{~s}, \mathrm{C}_{11}\right)$.

GCMS (EI) $m / z$ (rel intensity): 207 (100), 208 (18), 222 (53), 223 (4).

HRMS (ESI-TOF): Calculated for $\mathrm{C}_{24} \mathrm{H}_{27} \mathrm{BN}_{2} \mathrm{O}_{6} \mathrm{Na}[\mathrm{M}+\mathrm{Na}]^{+}:$473.1854. Found: 473.1854 . 
(E)-(2-phenyl-2-(4-(trifluoromethyl)phenyl)vinyl)boronic acid MIDA ester, (4.39.17):

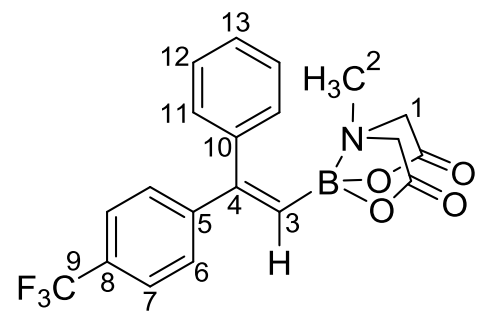

4.39.17 was prepared according to the general procedure A using 4-iodobenzotrifluoride 4.39.4 (90 mg, $0.33 \mathrm{mmol})$. Purification by flash chromatography on silica gel $\left(\mathrm{Et}_{2} \mathrm{O} /\right.$ Acetone $\left.=85: 15\right)$ to give 4.39.17 (34 $\mathrm{mg}, 78 \%)$ as a colorless oil $(E / Z=20: 1)$.

Formula: $\mathrm{C}_{20} \mathrm{H}_{17} \mathrm{BF}_{3} \mathrm{NO}_{4}$

Mass: $403.2 \mathrm{~g} / \mathrm{mol}$

IR $v_{\max }$ (neat): 1759, 1710, 1615, 1452, 1411, 1322, 1289, 1225, 1160, 1110, 1065, 1025, 1015, $960 \mathrm{~cm}^{-1}$.

${ }^{1} \mathrm{H}$ NMR $\left(400 \mathrm{MHz}\right.$, Acetone- $\left.d_{6}\right) \delta 7.68\left(2 \mathrm{H}, \mathrm{d}, J=8.4 \mathrm{~Hz}, \mathrm{H}_{7}\right), 7.50\left(2 \mathrm{H}, \mathrm{d}, J=8.6 \mathrm{~Hz}, \mathrm{H}_{6}\right)$, $7.41-7.21\left(5 \mathrm{H}, \mathrm{m}, \mathrm{H}_{11}+\mathrm{H}_{12}+\mathrm{H}_{13}\right), 6.34\left(1 \mathrm{H}, \mathrm{s}, \mathrm{H}_{3}\right), 4.11\left(2 \mathrm{H}, \mathrm{d}, J=16.8 \mathrm{~Hz}, \mathrm{H}_{1}\right), 3.80(2 \mathrm{H}$, $\left.\mathrm{d}, J=16.8 \mathrm{~Hz}, \mathrm{H}_{1}\right), 3.15\left(3 \mathrm{H}, \mathrm{s}, \mathrm{H}_{1}\right)$.

${ }^{13}$ C NMR (101 MHz, Acetone- $\left.d_{6}\right) \delta 168.6(\mathrm{~s}, 2 \mathrm{CO}), 154.6\left(\mathrm{~s}, \mathrm{C}_{4}\right), 149.0\left(\mathrm{~s}, \mathrm{C}_{5}\right), 141.8\left(\mathrm{~s}, \mathrm{C}_{10}\right)$, $130.3\left(\mathrm{~s}, 2 \mathrm{C}_{12}\right), 129.6\left(\mathrm{q}, J=36.5 \mathrm{~Hz}, \mathrm{C}_{8}\right), 129.1\left(\mathrm{~s}, 2 \mathrm{C}_{6}\right), 128.7\left(\mathrm{~s}, 2 \mathrm{C}_{11}\right), 128.3\left(\mathrm{~s}, \mathrm{C}_{13}\right), 125.8$ $\left(\mathrm{q}, J=3.8 \mathrm{~Hz}, 2 \mathrm{C}_{7}\right), 125.4\left(\mathrm{q}, J=269.5 \mathrm{~Hz}, \mathrm{C}_{9}\right), 62.5\left(\mathrm{~s}, 2 \mathrm{C}_{1}\right), 47.3\left(\mathrm{~s}, \mathrm{C}_{2}\right)$.

GCMS (EI) m/z (rel intensity): 248 (100), 249 (16), 250 (1), 412 (48), 413 (14), 414 (86), 415 (22), $416(52)$.

HRMS (ESI-TOF): Calculated for $\mathrm{C}_{20} \mathrm{H}_{17} \mathrm{BF}_{3} \mathrm{NO}_{4} \mathrm{Na}[\mathrm{M}+\mathrm{Na}]^{+}: 426.1095$. Found: 426.1095. 


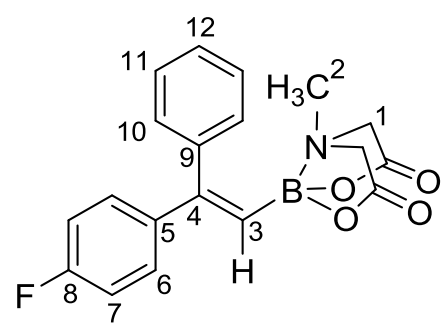

4.39.19 was prepared according to the general procedure A using 1-fluoro-4-iodobenzene 4.22.2 $(102 \mathrm{mg}, 0.46 \mathrm{mmol})$. Purification by flash chromatography on silica gel $\left(\mathrm{Et}_{2} \mathrm{O} /\right.$ Acetone $=83: 17$ to $80: 20)$ to give $\mathbf{4 . 3 9 . 1 9}(39 \mathrm{mg}, 73 \%)$ as a colorless oil $(E / Z=11: 1)$.

Formula: $\mathrm{C}_{19} \mathrm{H}_{17} \mathrm{BFNO}_{4}$

Mass: $353.2 \mathrm{~g} / \mathrm{mol}$

IR $v_{\max }$ (neat): 1772, 1752, 1625, 1598, 1504, 1461, 1337, 1283, 1221, 1158, 1126, 1112, 1083 $\mathrm{cm}^{-1}$.

${ }^{1} \mathbf{H}$ NMR $\left(400 \mathrm{MHz}\right.$, Acetone- $\left.d_{6}\right) \delta 7.38-7.19\left(7 \mathrm{H}, \mathrm{m}, 2 \mathrm{H}_{6}+2 \mathrm{H}_{10}+2 \mathrm{H}_{11}+\mathrm{H}_{12}\right), 7.12-6.89$ $\left(2 \mathrm{H}, \mathrm{m}, \mathrm{H}_{7}\right), 6.16\left(1 \mathrm{H}, \mathrm{s}, \mathrm{H}_{3}\right), 4.05\left(2 \mathrm{H}, \mathrm{d}, J=16.8 \mathrm{~Hz}, \mathrm{H}_{1}\right), 3.70\left(2 \mathrm{H}, \mathrm{d}, J=16.8 \mathrm{~Hz}, \mathrm{H}_{1}\right), 3.10$ $\left(3 \mathrm{H}, \mathrm{s}, \mathrm{H}_{2}\right)$.

${ }^{13}$ C NMR (101 MHz, Acetone- $\left.d_{6}\right) \delta 168.5(2 \underline{\mathrm{CO}}), 163.2\left(\mathrm{~d}, J=48.7 \mathrm{~Hz} \mathrm{C}_{8}\right), 154.8\left(\mathrm{~s}, \mathrm{C}_{4}\right), 142.3$ (s, $\left.\mathrm{C}_{5}\right), 141.6\left(\mathrm{~s}, \mathrm{C}_{9}\right), 130.3\left(\mathrm{~s}, 2 \mathrm{C}_{11}\right), 130.0\left(\mathrm{~d}, J=8.2 \mathrm{~Hz}, 2 \mathrm{C}_{6},{ }^{19} \mathrm{~F}-\mathrm{C}-\mathrm{C}-H\right)^{[\mathrm{a}]}, 128.9\left(\mathrm{~s}, 2 \mathrm{C}_{10}\right)$, $128.0\left(\mathrm{~s}, \mathrm{C}_{12}\right), 115.5\left(\mathrm{~d}, J=21.5 \mathrm{~Hz}, 2 \mathrm{C}_{7}\right), 62.6\left(\mathrm{~s}, 2 \mathrm{C}_{1}\right), 47.4\left(\mathrm{~s}, \mathrm{C}_{2}\right)$.

GCMS (EI) m/z (rel intensity): 165 (32), 166 (12), 167 (19), 195 (10), 196 (57), 197 (11), 209 (12), 210 (100), $211(16)$.

HRMS (ESI-TOF): Calculated for $\mathrm{C}_{19} \mathrm{H}_{17} \mathrm{BFNO}_{4} \mathrm{Na}[\mathrm{M}+\mathrm{Na}]^{+}$: 376.1127 . Found: 376.1128. 


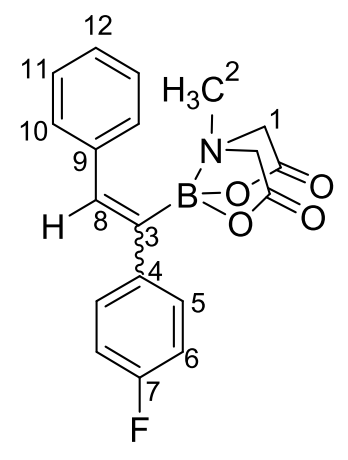

4.39.20 was prepared according to the general procedure A using 1-fluoro-4-iodobenzene 4.22.2 $(102 \mathrm{mg}, 0.46 \mathrm{mmol})$. Purification by flash chromatography on silica gel $\left(\mathrm{Et}_{2} \mathrm{O} /\right.$ Acetone $=$ 85:15) to give 4.39.20 (6 mg, $8 \%)$ as a colorless oil $(E / Z=1: 1)$.

Formula: $\mathrm{C}_{19} \mathrm{H}_{17} \mathrm{BFNO}_{4}$ Mass: $353.2 \mathrm{~g} / \mathrm{mol}$ IR $v_{\max }$ (neat): 3059, 1768, 1745, 1597, 1572, 1506, 1362, 1279, 1231, 1160, 1099, 1072, 1030 $\mathrm{cm}^{-1}$

${ }^{1}$ H NMR $\left(400 \mathrm{MHz}\right.$, Acetone- $\left.d_{6}\right) \delta 7.49-7.41\left(2 \mathrm{H}, \mathrm{m}, \mathrm{H}_{10}\right), 7.40-7.33\left(2 \mathrm{H}, \mathrm{m}, \mathrm{H}_{11}\right), 7.32-$ $7.19\left(4 \mathrm{H}, \mathrm{m}, 2 \mathrm{H}_{5}+\mathrm{H}_{8}+\mathrm{H}_{12}\right), 7.13-6.99\left(2 \mathrm{H}, \mathrm{m}, \mathrm{H}_{6}\right), 4.02\left(2 \mathrm{H}, \mathrm{d}, J=16.7 \mathrm{~Hz}, \mathrm{H}_{1}\right), 3.50(2 \mathrm{H}$, $\left.\mathrm{d}, J=16.9 \mathrm{~Hz}, \mathrm{H}_{1^{\prime}}\right), 2.98\left(3 \mathrm{H}, \mathrm{s}, \mathrm{H}_{2}\right)$.

(E/Z)-mixture obtained, only $(E)$-isomer is described.

${ }^{13}$ C NMR (101 MHz, Acetone- $\left.d_{6}\right) \delta 168.3$ (s, 2CO), 146.6 (s, C 8 ), 140.2 (s, C 7 ), 135.6 (s, C 4 ), 133.7 (s, $\left.\mathrm{C}_{9}\right), 131.5$ (d, $\left.J=7.8 \mathrm{~Hz}, 2 \mathrm{C}_{5}\right), 129.4\left(\mathrm{~s}, 2 \mathrm{C}_{11}\right), 128.9$ (s, 2C 10$), 128.4,127.7\left(\mathrm{~s}, \mathrm{C}_{12}\right)$, $116.0(\mathrm{~d}, J=21.6 \mathrm{~Hz}), 115.4\left(\mathrm{~d}, J=21.4 \mathrm{~Hz}, 2 \mathrm{C}_{6}\right), 63.4\left(\mathrm{~s}, 2 \mathrm{C}_{1}\right), 47.9\left(\mathrm{~s}, \mathrm{C}_{2}\right)$.

(E/Z)-mixture obtained, only $(E)$-isomer is described.

GCMS (EI) m/z (rel intensity): 164 (3), 176 (17), 177 (12), 178 (100), 179 (99), 180 (15), 258 (59), 259 (10), 260 (53), 261 (8). 
HRMS (ESI-TOF): Calculated for $\mathrm{C}_{19} \mathrm{H}_{17} \mathrm{BFNO}_{4} \mathrm{Na}[\mathrm{M}+\mathrm{Na}]^{+}:$376.1127. Found: 376.1129.

(E)-(2-(4-bromophenyl)-2-phenylvinyl)boronic acid MIDA ester, (4.39.21):

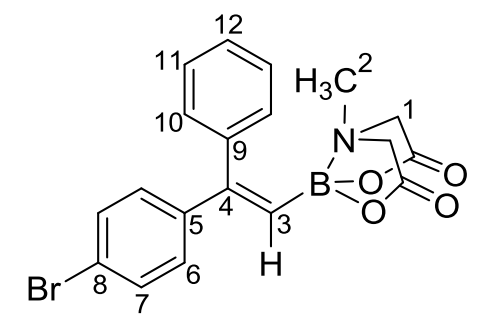

4.39.21 was prepared according to the general procedure A using 1-bromo-4-iodobenzene 4.39.5 (128 mg, $0.45 \mathrm{mmol})$. Purification by flash chromatography on silica gel ( $\mathrm{Et}_{2} \mathrm{O} /$ Acetone $=83: 17$ to $80: 20)$ to give $\mathbf{4 . 3 9 . 2 1}(44 \mathrm{mg}, 70 \%)$ as a colorless oil $(E / Z=17: 1)$.

Formula: $\mathrm{C}_{19} \mathrm{H}_{17} \mathrm{BBrNO}_{4}$

Mass: $414.1 \mathrm{~g} / \mathrm{mol}$

IR $v_{\max }$ (neat): 1770, 1752, 1709, 1597, 1485, 1461, 1356, 1337, 1283, 1223, 1161, 1126, 1112 , $1071,1024,1006,988 \mathrm{~cm}^{-1}$.

${ }^{1} \mathbf{H}$ NMR $\left(400 \mathrm{MHz}\right.$, Acetone- $\left.d_{6}\right) \delta 7.61-7.41\left(2 \mathrm{H}, \mathrm{m}, \mathrm{H}_{7}\right), 7.37-7.20\left(7 \mathrm{H}, \mathrm{m}, 2 \mathrm{H}_{6}+2 \mathrm{H}_{10}+\right.$ $\left.2 \mathrm{H}_{11}+\mathrm{H}_{12}\right), 6.23\left(1 \mathrm{H}, \mathrm{s}, \mathrm{H}_{3}\right), 4.10\left(2 \mathrm{H}, \mathrm{d}, J=16.8 \mathrm{~Hz}, \mathrm{H}_{1}\right), 3.72\left(2 \mathrm{H}, \mathrm{d}, J=16.8 \mathrm{~Hz}, \mathrm{H}_{1^{\prime}}\right), 3.11$ $\left(3 \mathrm{H}, \mathrm{s}, \mathrm{H}_{2}\right)$.

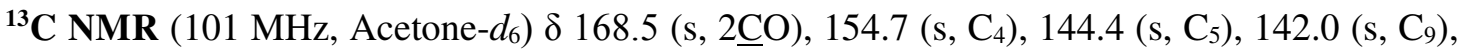
$131.9\left(\mathrm{~s}, 2 \mathrm{C}_{7}\right), 130.3\left(\mathrm{~s}, 2 \mathrm{C}_{11}\right), 130.1\left(\mathrm{~s}, 2 \mathrm{C}_{10}\right), 129.0\left(\mathrm{~s}, 2 \mathrm{C}_{6}\right), 128.1\left(\mathrm{~s}, \mathrm{C}_{12}\right), 122.0\left(\mathrm{~s}, \mathrm{C}_{8}\right), 62.7$ $\left(\mathrm{s}, 2 \mathrm{C}_{1}\right), 47.5\left(\mathrm{~s}, \mathrm{C}_{2}\right)$.

GCMS (EI) m/z (rel intensity): 176 (17), 177 (12), 178 (100), 179 (95), 258 (55), 259 (10), 260 (53), $261(9)$.

HRMS (ESI-TOF): Calculated for $\mathrm{C}_{19} \mathrm{H}_{17} \mathrm{~B}^{79} \mathrm{BrNO}_{4} \mathrm{Na}[\mathrm{M}+\mathrm{Na}]^{+}$: 436.0326. Found: 436.0325.

HRMS (ESI-TOF): Calculated for $\mathrm{C}_{19} \mathrm{H}_{17} \mathrm{~B}^{81} \mathrm{BrNO}_{4} \mathrm{Na}[\mathrm{M}+\mathrm{Na}]^{+}$: 438.0305. Found: 438.0306. 


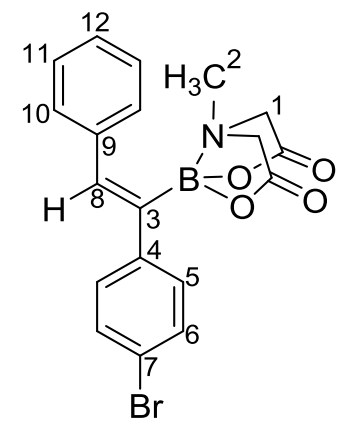

4.39.22 was prepared according to the general procedure A using 1-bromo-4-iodobenzene 4.39.5 (128 mg, $0.45 \mathrm{mmol})$. Purification by flash chromatography on silica gel $\left(\mathrm{Et}_{2} \mathrm{O} /\right.$ Acetone $=85: 15$ to $80: 20)$ to give $\mathbf{4 . 3 9 . 2 2}(15 \mathrm{mg}, 17 \%)$ as a colorless oil $(E / Z=5: 1)$.

Formula: $\mathrm{C}_{19} \mathrm{H}_{17} \mathrm{BBrNO}_{4}$ Mass: $414.1 \mathrm{~g} / \mathrm{mol}$

IR $v_{\max }$ (neat): 1766, 1708, 1590, 1485, 1455, 1336, 1287, 1224, 1070, 1029, 1008, $817 \mathrm{~cm}^{-1}$.

${ }^{1}$ H NMR $\left(400 \mathrm{MHz}\right.$, Acetone- $\left.d_{6}\right) \delta 7.38-7.34\left(2 \mathrm{H}, \mathrm{m}, \mathrm{H}_{12(E)}+\mathrm{H}_{12(Z)}\right), 7.25-7.21\left(2 \mathrm{H}, \mathrm{m}, \mathrm{H}_{6(E)}\right.$ $\left.+\mathrm{H}_{6(Z)}\right), 7.16-7.13\left(2 \mathrm{H}, \mathrm{m}, \mathrm{H}_{5(E)}+\mathrm{H}_{5(Z)}\right), 7.06\left(2 \mathrm{H}, \mathrm{m}, \mathrm{H}_{8(E)}+\mathrm{H}_{8(Z)}\right), 6.96-6.85\left(4 \mathrm{H}, \mathrm{m}, 2 \mathrm{H}_{P h}\right.$ $\left.(E)+2 \mathrm{H}_{\mathrm{Ph}(Z)}\right), 3.96\left(2.37 \mathrm{H}, \mathrm{d}, J=17.0 \mathrm{~Hz}, 2 \mathrm{H}_{1(E)}+0.37 \mathrm{H}_{1(\mathrm{Z})}\right), 3.53\left(2 \mathrm{H}, \mathrm{d}, J=17.0 \mathrm{~Hz}, \mathrm{H}_{1^{\prime}}\right)$, $3.40\left(0.38 \mathrm{H}, \mathrm{d}, J=17.0 \mathrm{~Hz}, \mathrm{H}_{1(\mathrm{Z})}\right), 3.13\left(3 \mathrm{H}, \mathrm{s}, \mathrm{H}_{2}\right), 2.88\left(0.65 \mathrm{H}, \mathrm{s}, \mathrm{H}_{2(\mathrm{Z})}\right)$

(E/Z)-mixture obtained.

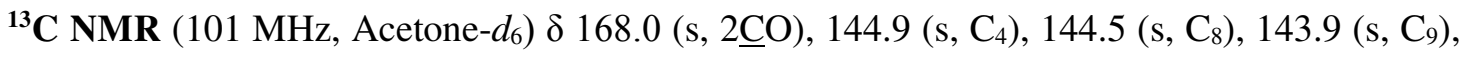
$133.9\left(\mathrm{~s}, 2 \mathrm{C}_{6}\right), 131.9\left(\mathrm{~s}, 2 \mathrm{C}_{5}\right), 131.2\left(\mathrm{~s}, 2 \mathrm{C}_{11}\right), 128.4\left(\mathrm{~s}, 2 \mathrm{C}_{10}\right), 126.9\left(\mathrm{~s}, \mathrm{C}_{12}\right), 120.9\left(\mathrm{~s}, \mathrm{C}_{7}\right), 63.5$ $\left(\mathrm{s}, 2 \mathrm{C}_{1}\right), 48.1\left(\mathrm{~s}, \mathrm{C}_{2}\right)$.

(E/Z)-mixture obtained, only $(E)$-isomer is described.

GCMS (EI) m/z (rel intensity): 177 (11), 178 (100), 179 (89), 180 (14), 258 (45), 259 (9), 260 (48), $261(7)$. 
HRMS (ESI-TOF): Calculated for $\mathrm{C}_{19} \mathrm{H}_{17} \mathrm{~B}^{79} \mathrm{BrNO}_{4} \mathrm{Na}[\mathrm{M}+\mathrm{Na}]^{+}$: 436.0326. Found: 436.0326 .

HRMS (ESI-TOF): Calculated for $\mathrm{C}_{19} \mathrm{H}_{17} \mathrm{~B}^{81} \mathrm{BrNO}_{4} \mathrm{Na}[\mathrm{M}+\mathrm{Na}]^{+}$: 438.0305. Found: 438.0306.

(E)-(2-(4-acetylphenyl)-2-phenylvinyl)boronic acid MIDA ester, (4.39.23):

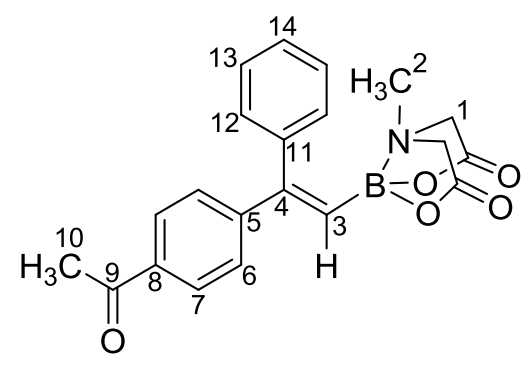

4.39.23 was prepared according to the general procedure A using 4-iodoacetophenone 4.39.6 $(61 \mathrm{mg}, 0.25 \mathrm{mmol})$. Purification by flash chromatography on silica gel $\left(\mathrm{Et}_{2} \mathrm{O} /\right.$ Acetone $=83: 17$ to $80: 20)$ to give $\mathbf{4 . 3 9 . 2 3 ~ ( 2 2 ~} \mathrm{mg}, 72 \%)$ as a colorless oil $(E / Z=20: 1)$.

Formula: $\mathrm{C}_{21} \mathrm{H}_{20} \mathrm{BNO}_{5}$

Mass: $377.2 \mathrm{~g} / \mathrm{mol}$

IR $v_{\max }$ (neat): 1760, 1708, 1677, 1600, 1404, 1357, 1267, 1156, 1108, 1083, $1025 \mathrm{~cm}^{-1}$.

${ }^{1} \mathbf{H}$ NMR $\left(400 \mathrm{MHz}\right.$, Acetone- $\left.d_{6}\right) \delta 8.05-7.86\left(2 \mathrm{H}, \mathrm{m}, \mathrm{H}_{7}\right), 7.46-7.38\left(2 \mathrm{H}, \mathrm{m}, 2 \mathrm{H}_{6}\right), 7.38-$ $7.30\left(3 \mathrm{H}, \mathrm{m}, 2 \mathrm{H}_{12}+\mathrm{H}_{14}\right), 7.30-7.23\left(2 \mathrm{H}, \mathrm{m}, 2 \mathrm{H}_{13}\right), 6.35\left(1 \mathrm{H}, \mathrm{s}, \mathrm{H}_{3}\right), 4.09(2 \mathrm{H}, \mathrm{d}, J=16.5 \mathrm{~Hz}$ $\left.\mathrm{H}_{1}\right), 3.75\left(2 \mathrm{H}, \mathrm{d}, J=16.5 \mathrm{~Hz}, \mathrm{H}_{1^{\prime}}\right), 3.14\left(3 \mathrm{H}, \mathrm{s}, \mathrm{H}_{2}\right), 2.56\left(3 \mathrm{H}, \mathrm{s}, \mathrm{H}_{10}\right)$.

${ }^{13}$ C NMR (101 MHz, Acetone- $\left.d_{6}\right) \delta 197.4\left(\mathrm{~s}, \mathrm{C}_{9}\right), 168.4(\mathrm{~s}, 2 \underline{\mathrm{CO}}), 154.9\left(\mathrm{~s}, \mathrm{C}_{4}\right), 149.2\left(\mathrm{~s}, \mathrm{C}_{5}\right)$, $141.8\left(\mathrm{~s}, \mathrm{C}_{11}\right), 136.9\left(\mathrm{~s}, \mathrm{C}_{8}\right), 130.2\left(\mathrm{~s}, 2 \mathrm{C}_{13}\right), 129.0\left(\mathrm{~s}, 2 \mathrm{C}_{12}\right) 128.9\left(\mathrm{~s}, 2 \mathrm{C}_{7}\right), 128.3\left(\mathrm{~s}, 2 \mathrm{C}_{6}\right), 128.2$ $\left(\mathrm{s}, \mathrm{C}_{14}\right), 62.5\left(\mathrm{~s}, 2 \mathrm{C}_{1}\right), 47.3\left(\mathrm{~s}, \mathrm{C}_{2}\right), 26.6\left(\mathrm{~s}, \mathrm{C}_{10}\right)$.

GCMS (EI) $m / z$ (rel intensity): 205 (100), 206 (16), 207 (2), 220 (27).

HRMS (ESI-TOF): Calculated for $\mathrm{C}_{21} \mathrm{H}_{21} \mathrm{BNO}_{5} \mathrm{H}[\mathrm{M}+\mathrm{H}]^{+}:$378.1507. Found: 378.1508. 


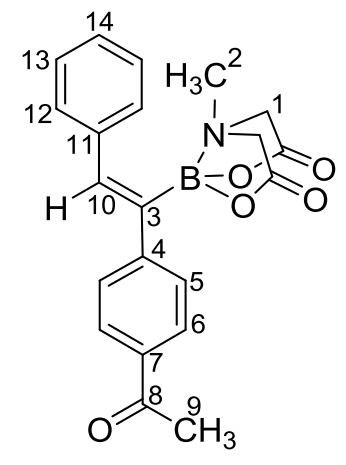

4.39.24 was prepared according to the general procedure A using 4-iodoacetophenone 4.39.6 $(61 \mathrm{mg}, 0.25 \mathrm{mmol})$. Purification by flash chromatography on silica gel $\left(\mathrm{Et}_{2} \mathrm{O} /\right.$ Acetone $\left.=85: 15\right)$ to give 4.39.24 (10 $\mathrm{mg}, 26 \%)$ as a colorless oil $(E / Z=22: 1)$.

Formula: $\mathrm{C}_{21} \mathrm{H}_{20} \mathrm{BNO}_{5}$ Mass: $377.2 \mathrm{~g} / \mathrm{mol}$

IR $v_{\max }$ (neat): 1764, 1708, 1677, 1600, 1451, 1359, 1336, 1269, 1184, 1150, 1063, $1030 \mathrm{~cm}^{-1}$. ${ }^{1}$ H NMR (400 MHz, Acetone- $\left.d_{6}\right) \delta 8.05-7.69\left(2 \mathrm{H}, \mathrm{m}, \mathrm{H}_{6}\right), 7.62-7.41\left(2 \mathrm{H}, \mathrm{m}, \mathrm{H}_{5}\right), 7.44-$ $7.36\left(2 \mathrm{H}, \mathrm{m}, 2 \mathrm{H}_{13}\right), 7.35-7.19\left(4 \mathrm{H}, \mathrm{m}, \mathrm{H}_{10}+2 \mathrm{H}_{12}+\mathrm{H}_{14}\right), 3.98\left(2 \mathrm{H}, \mathrm{d}, J=16.9 \mathrm{~Hz}, \mathrm{H}_{1}\right), 3.39$ $\left(2 \mathrm{H}, \mathrm{d}, J=16.9 \mathrm{~Hz}, \mathrm{H}_{1}\right), 2.89\left(3 \mathrm{H}, \mathrm{s}, \mathrm{H}_{2}\right), 2.49\left(3 \mathrm{H}, \mathrm{s}, \mathrm{H}_{9}\right)$.

${ }^{13}$ C NMR (101 MHz, Acetone- $d_{6}$ ) $\delta 197.66$ (s, C 8 ), 168.24 (s, 2ㄷ), 152.92 (s, C4), 147.00 (s, $\left.\mathrm{C}_{10}\right), 139.93\left(\mathrm{~s}, \mathrm{C}_{11}\right), 136.10\left(\mathrm{~s}, \mathrm{C}_{7}\right), 129.87\left(\mathrm{~s}, 2 \mathrm{C}_{5}\right), 129.38\left(\mathrm{~s}, 2 \mathrm{C}_{13}\right), 128.93\left(\mathrm{~s}, 2 \mathrm{C}_{12}\right), 128.81$ $\left(\mathrm{s}, 2 \mathrm{C}_{6}\right), 127.81\left(\mathrm{~s}, \mathrm{C}_{14}\right), 63.36\left(\mathrm{~s}, 2 \mathrm{C}_{1}\right), 47.94\left(\mathrm{~s}, \mathrm{C}_{2}\right), 26.72\left(\mathrm{~s}, \mathrm{C}_{9}\right)$.

GCMS (EI) m/z (rel intensity): 207 (100), 208 (19), 222 (70), 223 (14).

HRMS (ESI-TOF): Calculated for $\mathrm{C}_{21} \mathrm{H}_{20} \mathrm{BNO}_{5} \mathrm{Na}[\mathrm{M}+\mathrm{Na}]^{+}: 400.1327$. Found: 400.1330 . 


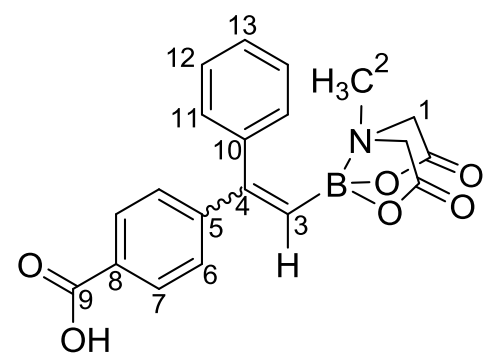

4.39.25/4.39.26 was prepared according to the general procedure A using 4-iodobenzoic acid 4.39.7 (113 mg, $0.46 \mathrm{mmol}$ ). Purification by flash chromatography on silica gel (Acetone/TFA $=90: 10)$ to give a mixture of regioisomers $4.39 .25(40 \mathrm{mg}, 70 \%)$ and $\mathbf{4 . 3 9 . 2 6}(21 \mathrm{mg}, 28 \%)$ as a colorless oil $(E / Z=11: 1)$

Formula: $\mathrm{C}_{20} \mathrm{H}_{18} \mathrm{BNO}_{6}$ Mass: $379.2 \mathrm{~g} / \mathrm{mol}$

IR $v_{\max }$ (neat): $3433,1748,1701,1607,1361,1228,1027,864,776,703 \mathrm{~cm}^{-1}$.

${ }^{1} \mathbf{H}$ NMR $\left(400 \mathrm{MHz}\right.$, Acetone- $\left.d_{6}\right) \delta 8.13-7.21\left(9 \mathrm{H}, \mathrm{m}, 2 \mathrm{H}_{6}+2 \mathrm{H}_{7}+2 \mathrm{H}_{11}+2 \mathrm{H}_{12}+\mathrm{H}_{13}\right), 6.34$ $\left(1 \mathrm{H}, \mathrm{s}, \mathrm{H}_{3}\right), 4.07\left(2 \mathrm{H}, \mathrm{d}, J=16.7 \mathrm{~Hz}, \mathrm{H}_{1}\right), 3.76\left(2 \mathrm{H}, \mathrm{d}, J=16.7 \mathrm{~Hz}, \mathrm{H}_{1}\right), 3.14\left(3 \mathrm{H}, \mathrm{s}, \mathrm{H}_{2}\right)$.

${ }^{13}$ C NMR (101 MHz, Acetone- $\left.d_{6}\right) \delta 172.2(\mathrm{~s}, 2 \underline{\mathrm{CO}}) 168.6\left(\mathrm{~s}, \mathrm{C}_{9}\right), 155.1\left(\mathrm{~s}, \mathrm{C}_{4}\right), 149.5\left(\mathrm{~s}, \mathrm{C}_{5}\right)$, $142.0\left(\mathrm{~s}, \mathrm{C}_{10}\right), 132.1\left(\mathrm{~s}, \mathrm{C}_{8}\right), 130.3\left(\mathrm{~s}, 2 \mathrm{C}_{6}+2 \mathrm{C}_{7}\right), 129.0\left(\mathrm{~s}, 2 \mathrm{C}_{12}\right), 128.1\left(\mathrm{~s}, 2 \mathrm{C}_{11}+\mathrm{C}_{13}\right), 62.7(\mathrm{~s}$, $\left.2 \mathrm{C}_{1}\right), 47.5\left(\mathrm{~s}, \mathrm{C}_{2}\right)$.

HRMS (ESI-TOF): Calculated for $\mathrm{C}_{20} \mathrm{H}_{18} \mathrm{BNO}_{6} \mathrm{Na}[\mathrm{M}+\mathrm{Na}]^{+}:$402.1119. Found: 402.1121 . 
(E)-(2-(4-(ethoxycarbonyl)phenyl)-2-phenylvinyl)boronic acid MIDA ester, (4.39.27):

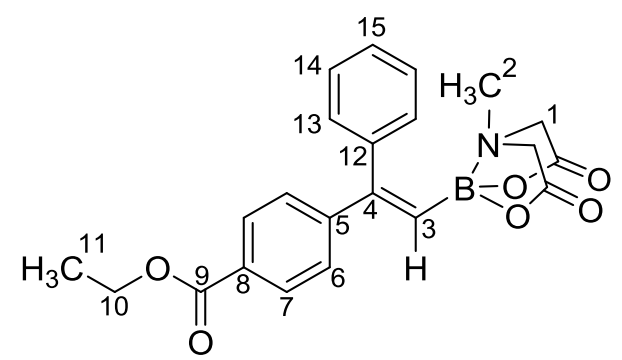

4.39.27 was prepared according to the general procedure A using ethyl 4-iodobenzoate 4.39.8 (297 mg, $108 \mathrm{mmol})$. Purification by flash chromatography on silica gel $\left(\mathrm{Et}_{2} \mathrm{O} /\right.$ Acetone $=83: 17$ to $80: 20)$ to give $\mathbf{4 . 3 9 . 2 7}(106 \mathrm{mg}, 73 \%)$ as a colorless oil $(E / Z=50: 1)$.

Formula: $\mathrm{C}_{22} \mathrm{H}_{22} \mathrm{BNO}_{6}$ Mass: $407.2 \mathrm{~g} / \mathrm{mol}$

IR $v_{\max }$ (neat): $1762,1707,1606,1450,1364,1136,1274,1224,1156,1102,1082,1022 \mathrm{~cm}^{-1}$.

${ }^{1} \mathbf{H}$ NMR $\left(400 \mathrm{MHz}\right.$, Acetone- $\left.d_{6}\right) \delta 7.93\left(2 \mathrm{H}, \mathrm{d}, J=8.6 \mathrm{~Hz}, \mathrm{H}_{7}\right), 7.40\left(2 \mathrm{H}, \mathrm{d}, J=8.6 \mathrm{~Hz}, \mathrm{H}_{6}\right)$, $7.37-7.18\left(5 \mathrm{H}, \mathrm{m}, \mathrm{H}_{\mathrm{Ph}}\right), 6.33\left(1 \mathrm{H}, \mathrm{s}, \mathrm{H}_{3}\right), 4.35\left(2 \mathrm{H}, \mathrm{q}, J=7.0 \mathrm{~Hz}, \mathrm{H}_{10}\right), 4.10(2 \mathrm{H}, \mathrm{d}, J=16.8$ $\left.\mathrm{Hz}, \mathrm{H}_{1}\right), 3.79\left(2 \mathrm{H}, \mathrm{d}, J=16.8 \mathrm{~Hz}, \mathrm{H}_{1}\right), 3.13\left(3 \mathrm{H}, \mathrm{s}, \mathrm{H}_{2}\right), 1.35\left(3 \mathrm{H}, \mathrm{t}, J=7.2 \mathrm{~Hz}, \mathrm{H}_{11}\right)$.

${ }^{13}$ C NMR (101 MHz, Acetone- $\left.d_{6}\right) \delta 168.5$ (s, 2므), $166.5\left(\mathrm{~s}, \mathrm{C}_{9}\right), 155.1\left(\mathrm{~s}, \mathrm{C}_{4}\right), 149.6\left(\mathrm{~s}, \mathrm{C}_{5}\right)$, $142.0\left(\mathrm{~s}, \mathrm{C}_{12}\right), 130.4\left(\mathrm{~s}, 2 \mathrm{C}_{14}+\mathrm{C}_{8}\right), 130.0\left(\mathrm{~s}, 2 \mathrm{C}_{7}\right), 129.0\left(\mathrm{~s}, 2 \mathrm{C}_{13}\right), 128.2\left(\mathrm{~s}, 2 \mathrm{C}_{6}+\mathrm{C}_{15}\right), 62.7(\mathrm{~s}$, $\left.2 \mathrm{C}_{1}\right), 61.5\left(\mathrm{~s}, \mathrm{C}_{10}\right), 47.5\left(\mathrm{~s}, \mathrm{C}_{2}\right), 14.7\left(\mathrm{~s}, \mathrm{C}_{11}\right)$.

GCMS (EI) m/z (rel intensity): 227 (1), 258 (53), 259 (37), 260 (46), 272 (27), 273 (53), 274 (35), 275 (12), 289 (31), 304 (100), 305 (24), 306 (3), 394 (100), 395 (26).

HRMS (ESI-TOF): Calculated for $\mathrm{C}_{22} \mathrm{H}_{22} \mathrm{BNO}_{6} \mathrm{Na}[\mathrm{M}+\mathrm{Na}]^{+}$: 430.1432. Found: 430.1434. 


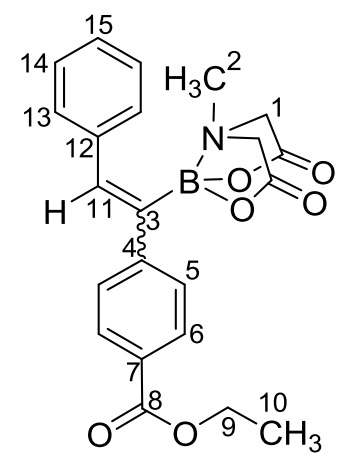

4.39.2 was prepared according to the general procedure A using ethyl 4-iodobenzoate 4.39.8 $(297 \mathrm{mg}, 108 \mathrm{mmol})$. Purification by flash chromatography on silica gel $\left(\mathrm{Et}_{2} \mathrm{O} /\right.$ Acetone $\left.=83: 15\right)$ to give 4.39.2 (33 $\mathrm{mg}, 16 \%)$ as a colorless oil $(E / Z=13: 1)$.

Formula: $\mathrm{C}_{22} \mathrm{H}_{22} \mathrm{BNO}_{6}$ Mass: $407.2 \mathrm{~g} / \mathrm{mol}$

IR $v_{\max }$ (neat): $1768,1699,1605,1572,1492,1365,1276,1230,1182,1103,1019 \mathrm{~cm}^{-1}$.

${ }^{1} \mathbf{H}$ NMR (400 MHz, Acetone- $\left.d_{6}\right) \delta 7.97\left(2 \mathrm{H}, \mathrm{d}, J=8.4 \mathrm{~Hz}, \mathrm{H}_{6}\right), 7.55\left(2 \mathrm{H}, \mathrm{d}, J=8.4 \mathrm{~Hz}, \mathrm{H}_{5}\right)$, $7.47-6.84\left(6 \mathrm{H}, \mathrm{m}, \mathrm{H}_{11}+2 \mathrm{H}_{13}+2 \mathrm{H}_{14}+\mathrm{H}_{15}\right), 4.35\left(2 \mathrm{H}, \mathrm{q}, J=7.1 \mathrm{~Hz}, \mathrm{H}_{9}\right), 4.03(2 \mathrm{H}, \mathrm{d}, J=16.9$ $\left.\mathrm{Hz}, \mathrm{H}_{1}\right), 3.48\left(2 \mathrm{H}, \mathrm{d}, J=16.9 \mathrm{~Hz}, \mathrm{H}_{1}\right), 2.99\left(3 \mathrm{H}, \mathrm{s}, \mathrm{H}_{2}\right), 1.37\left(3 \mathrm{H}, \mathrm{t}, J=7.1 \mathrm{~Hz}, \mathrm{H}_{10}\right)$.

${ }^{13}$ C NMR (101 MHz, Acetone- $\left.d_{6}\right) \delta 168.2(\mathrm{~s}, 2 \underline{\mathrm{CO}}), 166.7$ (s, $\left.\mathrm{C}_{8}\right), 152.8\left(\mathrm{~s}, \mathrm{C}_{4}\right), 147.0\left(\mathrm{~s}, \mathrm{C}_{11}\right)$, $139.9\left(\mathrm{~s}, \mathrm{C}_{12}\right), 129.8\left(2 \mathrm{C}_{6}+\mathrm{C}_{7}\right), 129.39\left(\mathrm{~s}, 2 \mathrm{C}_{14}\right), 128.8\left(\mathrm{~s}, 2 \mathrm{C}_{13}+2 \mathrm{C}_{5}\right), 127.7\left(\mathrm{~s}, \mathrm{C}_{15}\right), 63.3(\mathrm{~s}$, $\left.2 \mathrm{C}_{1}\right), 61.3\left(\mathrm{~s}, \mathrm{C}_{9}\right), 47.9\left(\mathrm{~s}, \mathrm{C}_{2}\right), 14.6\left(\mathrm{~s}, 2 \mathrm{C}_{10}\right)$.

HRMS (ESI-TOF): Calculated for $\mathrm{C}_{22} \mathrm{H}_{22} \mathrm{BNO}_{6} \mathrm{Na}[\mathrm{M}+\mathrm{Na}]^{+}: 430.1432$. Found: 430.1430 . 


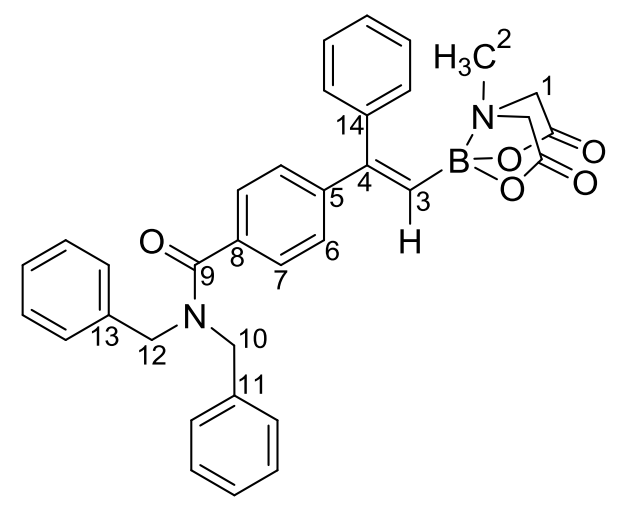

4.39.29 was prepared according to the general procedure A using $N, N$-dibenzyl-4iodobenzamide 4.39.9 (223 mg, $0.49 \mathrm{mmol})$. Purification by flash chromatography on silica gel $\left(\mathrm{Et}_{2} \mathrm{O} /\right.$ Acetone $=83: 17$ to $\left.80: 20\right)$ to give $\mathbf{4 . 3 9 . 2 9}(83 \mathrm{mg}, 86 \%)$ as a colorless oil $(E / Z=>50: 1)$.

Formula: $\mathrm{C}_{34} \mathrm{H}_{31} \mathrm{BN}_{2} \mathrm{O}_{5}$ Mass: $558.4 \mathrm{~g} / \mathrm{mol}$

IR $v_{\max }$ (neat): 1760, 1709, 1627, 1494, 1451, 1421, 1356, 1335, 1287, 1249, 1027, $855 \mathrm{~cm}^{-1}$.

${ }^{1}$ H NMR (400 MHz, Acetone- $\left.d_{6}\right) \delta 7.44-7.05\left(19 \mathrm{H}, \mathrm{m}, \mathrm{H}_{\mathrm{Ph}}\right), 6.15\left(1 \mathrm{H}, \mathrm{s}, \mathrm{H}_{3}\right), 4.54(2 \mathrm{H}, \mathrm{brs}$, $\left.\mathrm{H}_{10}\right), 4.39\left(2 \mathrm{H}\right.$, brs, $\left.\mathrm{H}_{12}\right), 3.94\left(2 \mathrm{H}, \mathrm{d}, J=16.7 \mathrm{~Hz}, \mathrm{H}_{1}\right), 3.62\left(2 \mathrm{H}, \mathrm{d}, J=16.7 \mathrm{~Hz}, \mathrm{H}_{1}\right), 3.01(3 \mathrm{H}$, $\left.\mathrm{s}, \mathrm{H}_{2}\right)$.

${ }^{13}$ C NMR (101 MHz, Acetone- $\left.d_{6}\right) \delta 172.1\left(\mathrm{~s}, \mathrm{C}_{9}\right), 168.5$ (s, 2으), $155.1\left(\mathrm{~s}, \mathrm{C}_{4}\right), 146.2\left(\mathrm{~s}, \mathrm{C}_{13}+\right.$ $\left.\mathrm{C}_{11}\right), 142.1\left(\mathrm{~s}, \mathrm{C}_{5}\right), 136.7\left(\mathrm{~s}, \mathrm{C}_{14}\right), 130.4\left(\mathrm{~s}, 2 \mathrm{C}_{6}\right), 130.2\left(\mathrm{~s}, 4 \mathrm{C}_{\mathrm{Ph}}\right), 129.6\left(\mathrm{~s}, 2 \mathrm{C}_{\mathrm{Ph}}\right), 128.9\left(\mathrm{~s}, 2 \mathrm{C}_{\mathrm{Ph}}\right)$, $128.1\left(\mathrm{~s}, 5 \mathrm{C}_{\mathrm{Ph}}\right), 127.5\left(\mathrm{~s}, 2 \mathrm{C}_{\mathrm{Ph}}+2 \mathrm{C}_{7}\right), 62.6\left(\mathrm{~s}, 2 \mathrm{C}_{1}\right), 47.5\left(\mathrm{~s}, \mathrm{C}_{2}\right)$.

.HRMS (ESI-TOF): Calculated for $\mathrm{C}_{34} \mathrm{H}_{31} \mathrm{BN}_{2} \mathrm{O}_{5} \mathrm{Na}[\mathrm{M}+\mathrm{Na}]^{+}:$581.2218. Found: 581.2223 


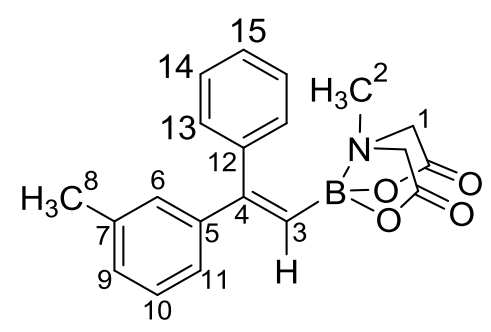

4.41.5 was prepared according to the general procedure A using 1-iodo-3-methylbenzene 4.41.1 $(102 \mathrm{mg}, 0.47 \mathrm{mmol})$. Purification by flash chromatography on silica gel $\left(\mathrm{Et}_{2} \mathrm{O} /\right.$ Acetone $=83: 17$ to $80: 20)$ to give $4.41 .5(46 \mathrm{mg}, 85 \%)$ as a colorless oil $(E / Z=>50: 1)$.

Formula: $\mathrm{C}_{20} \mathrm{H}_{20} \mathrm{BNO}_{4}$

Mass: $349.2 \mathrm{~g} / \mathrm{mol}$

IR $v_{\max }$ (neat): 1759, 1709, 1596, 1453, 1355, 1331, 1291, 1223, 1153, 1110, 1084, 1023, 991 $\mathrm{cm}^{-1}$.

${ }^{1}$ H NMR (400 MHz, Acetone- $\left.d_{6}\right) \delta 7.49-6.97\left(9 \mathrm{H}, \mathrm{m}, 5 \mathrm{H}_{\mathrm{Ph}}+\mathrm{H}_{6}+\mathrm{H}_{9}+\mathrm{H}_{10}+\mathrm{H}_{11}\right), 6.17(1 \mathrm{H}$, s, $\left.\mathrm{H}_{3}\right), 4.05\left(2 \mathrm{H}, \mathrm{d}, J=16.8 \mathrm{~Hz}, \mathrm{H}_{1}\right), 3.71\left(2 \mathrm{H}, \mathrm{d}, J=16.8 \mathrm{~Hz}, \mathrm{H}_{1^{\prime}}\right), 3.10\left(3 \mathrm{H}, \mathrm{s}, \mathrm{H}_{2}\right), 2.27(3 \mathrm{H}$, s, $\left.\mathrm{H}_{8}\right)$.

${ }^{13}$ C NMR (101 MHz, Acetone- $\left.d_{6}\right) \delta 168.5(\mathrm{~s}, 2 \underline{\mathrm{CO}}), 156.2\left(\mathrm{~s}, \mathrm{C}_{4}\right), 145.2\left(\mathrm{~s}, \mathrm{C}_{5}\right), 142.6\left(\mathrm{~s}, \mathrm{C}_{12}\right)$, $138.2\left(\mathrm{~s}, \mathrm{C}_{7}\right), 130.3\left(\mathrm{~s}, 2 \mathrm{C}_{14}\right), 129.0\left(\mathrm{~s}, \mathrm{C}_{6}\right), \delta 128.8\left(\mathrm{~s}, 2 \mathrm{C}_{13}+\mathrm{C}_{15}\right), 128.6\left(\mathrm{~s}, \mathrm{C}_{9}\right), 127.8\left(\mathrm{~s}, \mathrm{C}_{11}\right)$ , $125.4\left(\mathrm{~s}, \mathrm{C}_{10}\right), 62.6\left(\mathrm{~s}, 2 \mathrm{C}_{1}\right), 47.4\left(\mathrm{~s}, \mathrm{C}_{2}\right), 21.5\left(\mathrm{~s}, \mathrm{C}_{8}\right)$.

GCMS (EI) m/z (rel intensity): 93 (1), 105 (9), 115 (5), 116 (6), 116 (2), 117 (3), 144 (1), 145 (12), 205 (100), 206 (16), 220 (28).

HRMS (ESI-TOF): Calculated for $\mathrm{C}_{20} \mathrm{H}_{20} \mathrm{BNO}_{4} \mathrm{Na}[\mathrm{M}+\mathrm{Na}]^{+}:$372.1378. Found: 372.1379 . 


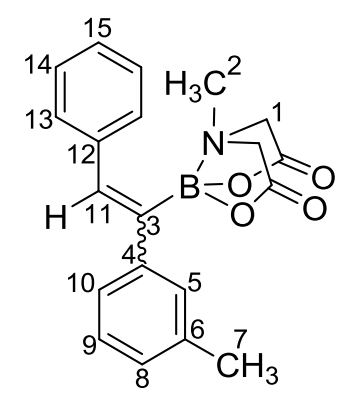

4.41.6 was prepared according to the general procedure A using 1-iodo-3-methylbenzene 4.41.1 $(102 \mathrm{mg}, 0.47 \mathrm{mmol})$. Purification by flash chromatography on silica gel $\left(\mathrm{Et}_{2} \mathrm{O} /\right.$ Acetone $=$ 85:15) to give 4.41.6 $(10 \mathrm{mg}, 15 \%)$ as a colorless oil $(E / Z=1: 1)$.

Formula: $\mathrm{C}_{20} \mathrm{H}_{20} \mathrm{BNO}_{4}$ Mass: $349.2 \mathrm{~g} / \mathrm{mol}$

IR $v_{\max }$ (neat): $1763,1709,1598,1450,1335,1288,1245,1178,1155,1104,1073 \mathrm{~cm}^{-1}$.

${ }^{1}$ H NMR (400 MHz, Acetone- $\left.d_{6}\right) \delta 7.20-6.99\left(3 \mathrm{H}, \mathrm{m}, 2 \mathrm{H}_{13}+\mathrm{H}_{15}\right), 7.01-6.75\left(6 \mathrm{H}, \mathrm{m}, \mathrm{H}_{5}+\right.$ $\left.\mathrm{H}_{9}+\mathrm{H}_{10}+\mathrm{H}_{11}+2 \mathrm{H}_{14}\right), 6.69\left(1 \mathrm{H}, \mathrm{m}, \mathrm{H}_{8}\right), 3.87\left(2 \mathrm{H}, \mathrm{d}, J=16.7 \mathrm{~Hz}, \mathrm{H}_{1}\right), 3.30(2 \mathrm{H}, \mathrm{d}, J=16.8$ $\left.\mathrm{Hz}, \mathrm{H}_{1}{ }^{\prime}\right), 3.14\left(3 \mathrm{H}, \mathrm{s}, \mathrm{H}_{2}\right), 2.20\left(3 \mathrm{H}, \mathrm{s}, \mathrm{H}_{7}\right)$.

${ }^{13}$ C NMR (101 MHz, Acetone- $\left.d_{6}\right) \delta 168.2(\mathrm{~s}, 2 \underline{\mathrm{CO}}), 155.0\left(\mathrm{~s}, \mathrm{C}_{4}\right), 145.9\left(\mathrm{~s}, \mathrm{C}_{11}\right), 137.2\left(\mathrm{~s}, \mathrm{C}_{6}\right)$, $132.6\left(\mathrm{~s}, \mathrm{C}_{12}\right), 130.3\left(\mathrm{~s}, 2 \mathrm{C}_{14}\right), 129.4\left(\mathrm{~s}, \mathrm{C}_{9}\right), 128.7\left(\mathrm{~s}, \mathrm{C}_{8}\right), 128.0\left(\mathrm{~s}, 2 \mathrm{C}_{13}\right), 127.6\left(\mathrm{~s}, \mathrm{C}_{15}\right), 126.5$ $\left(\mathrm{s}, \mathrm{C}_{5}\right), 126.4\left(\mathrm{~s}, \mathrm{C}_{10}\right), 63.2\left(\mathrm{~s}, 2 \mathrm{C}_{1}\right), 47.7\left(\mathrm{~s}, \mathrm{C}_{2}\right), 21.5\left(\mathrm{~s}, \mathrm{C}_{7}\right)$

GCMS (EI) m/z (rel intensity): 193 (13), 194 (84), 195 (13), 205 (100), 206 (17), 268 (13), 269 (45), 270 (10), 284 (100).

HRMS (ESI-TOF): Calculated for $\mathrm{C}_{20} \mathrm{H}_{20} \mathrm{BNO}_{4} \mathrm{Na}[\mathrm{M}+\mathrm{Na}]^{+}:$372.1378. Found: 372.1378 . 


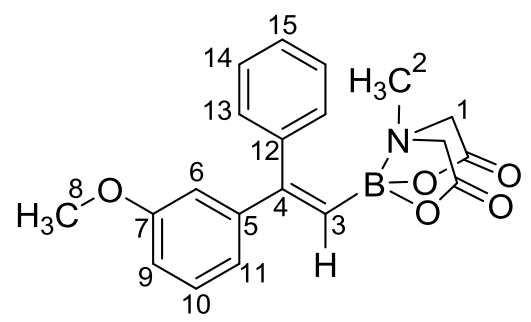

4.41.7 was prepared according to the general procedure A using 3-iodoanisole 4.41.2 (107 $\mathrm{mg}$, $0.46 \mathrm{mmol})$. Purification by flash chromatography on silica gel $\left(\mathrm{Et}_{2} \mathrm{O} /\right.$ Acetone $=83: 17$ to $\left.80: 20\right)$ to give 4.41.7 (50 $\mathrm{mg}, 90 \%)$ as a colorless oil $(E / Z=>50: 1)$.

Formula: $\mathrm{C}_{20} \mathrm{H}_{20} \mathrm{BNO}_{5}$

Mass: $365.2 \mathrm{~g} / \mathrm{mol}$

IR $v_{\max }$ (neat): 1760, 1708, 1595, 1577, 1485, 1451, 1430, 1353, 1335, 1285, 1223, 1202, 1152 , $1107,1084,1007,992,891,876,777,705 \mathrm{~cm}^{-1}$.

${ }^{1}$ H NMR (400 MHz, Acetone- $\left.d_{6}\right) \delta 7.49-6.99\left(6 \mathrm{H}, \mathrm{m}, \mathrm{H}_{10}+2 \mathrm{H}_{13}+2 \mathrm{H}_{14}+\mathrm{H}_{15}\right), 6.82-6.51$ (3H, m, $\left.\mathrm{H}_{6}+\mathrm{H}_{9}+\mathrm{H}_{11}\right), 6.10\left(1 \mathrm{H}, \mathrm{s}, \mathrm{H}_{3}\right), 3.91\left(2 \mathrm{H}, \mathrm{d}, J=16.4 \mathrm{~Hz}, \mathrm{H}_{1}\right), 3.71-3.40\left(5 \mathrm{H}, \mathrm{m}, \mathrm{H}_{1}\right.$, $\left.+\mathrm{H}_{8}\right), 3.01\left(3 \mathrm{H}, \mathrm{s}, \mathrm{H}_{2}\right)$.

${ }^{13}$ C NMR (101 MHz, Acetone- $\left.d_{6}\right) \delta 168.5$ (s, 2으), 160.5 (s, C 7 ), 155.9 (s, C 4 ), 146.7 (s, C 5 ), $142.6\left(\mathrm{~s}, \mathrm{C}_{12}\right), 130.3\left(\mathrm{~s}, 2 \mathrm{C}_{14}\right), 129.8\left(\mathrm{~s}, \mathrm{C}_{10}\right), 128.8\left(\mathrm{~s}, 2 \mathrm{C}_{13}\right), 127.9\left(\mathrm{~s}, \mathrm{C}_{15}\right), 120.7\left(\mathrm{~s}, \mathrm{C}_{11}\right), 114.2$ (s, $\left.\mathrm{C}_{9}\right), 113.4\left(\mathrm{~s}, \mathrm{C}_{6}\right), 62.7\left(\mathrm{~s}, 2 \mathrm{C}_{1}\right), 55.5\left(\mathrm{~s}, \mathrm{C}_{8}\right), 47.5\left(\mathrm{~s}, \mathrm{C}_{2}\right)$.

GCMS (EI) $m / z$ (rel intensity): 121 (6), 122 (1), 145 (13), 205 (100), 206 (15).

HRMS (ESI-TOF): Calculated for $\mathrm{C}_{20} \mathrm{H}_{20} \mathrm{BNO}_{5} \mathrm{Na}[\mathrm{M}+\mathrm{Na}]^{+}:$388.1327. Found: 388.1328. 
(E)-(1-(3-methoxyphenyl)-2-phenylvinyl)boronic acid MIDA ester, (4.41.8):

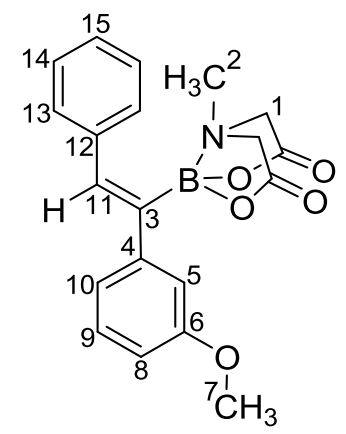

4.41.8 was prepared according to the general procedure A using 3-iodoanisole 4.41.2 (107 mg, $0.46 \mathrm{mmol})$. Purification by flash chromatography on silica gel $\left(\mathrm{Et}_{2} \mathrm{O} /\right.$ Acetone $\left.=85: 15\right)$ to give 4.41.8 $(6 \mathrm{mg}, 9 \%)$ as a colorless oil $(E / Z=>50: 1)$.

Formula: $\mathrm{C}_{20} \mathrm{H}_{20} \mathrm{BNO}_{5}$

Mass: $365.2 \mathrm{~g} / \mathrm{mol}$

IR $v_{\max }$ (neat): $1769,1594,1575,1483,1459,1336,1285,1195,1166,1102,1072,1031 \mathrm{~cm}^{-1}$. ${ }^{1}$ H NMR (400 MHz, Acetone- $\left.d_{6}\right) \delta 7.56-7.18\left(7 \mathrm{H}, \mathrm{m}, \mathrm{H}_{9}+\mathrm{H}_{11}+2 \mathrm{H}_{13}+2 \mathrm{H}_{14}+\mathrm{H}_{15}\right), 7.09-$ $6.94\left(2 \mathrm{H}, \mathrm{m}, \mathrm{H}_{8}+\mathrm{H}_{10}\right), 6.80\left(1 \mathrm{H}, \mathrm{m}, \mathrm{H}_{5}\right), 4.02\left(2 \mathrm{H}, \mathrm{d}, J=16.9 \mathrm{~Hz}, \mathrm{H}_{1}\right), 3.80\left(3 \mathrm{H}, \mathrm{s}, \mathrm{H}_{7}\right), 3.47$ $\left(2 \mathrm{H}, \mathrm{d}, J=16.8 \mathrm{~Hz}, \mathrm{H}_{1^{\prime}}\right), 2.97\left(3 \mathrm{H}, \mathrm{s}, \mathrm{H}_{2}\right)$.

${ }^{13}$ C NMR (101 MHz, Acetone- $\left.d_{6}\right) \delta 168.3$ (s, 2므), 160.3 (s, C 6 ), 149.1 (s, C 4 ), $146.1\left(\mathrm{~s}, \mathrm{C}_{11}\right)$, $140.2\left(\mathrm{~s}, \mathrm{C}_{12}\right), 129.8\left(\mathrm{~s}, \mathrm{C}_{9}\right), 129.4\left(\mathrm{~s}, 2 \mathrm{C}_{14}\right), 128.6\left(\mathrm{~s}, 2 \mathrm{C}_{13}\right), 127.5\left(\mathrm{~s}, \mathrm{C}_{15}\right), 121.9\left(\mathrm{~s}, \mathrm{C}_{10}\right), 115.0$ $\left(\mathrm{s}, \mathrm{C}_{8}\right), 112.8\left(\mathrm{~s}, \mathrm{C}_{5}\right), 63.2\left(\mathrm{~s}, 2 \mathrm{C}_{1}\right), 55.4\left(\mathrm{~s}, \mathrm{C}_{7}\right), 47.7\left(\mathrm{~s}, \mathrm{C}_{2}\right)$

GCMS (EI) m/z (rel intensity): 253 (80), 254 (100), 255 (24), 256 (7), 364 (12), 365 (47), 366 (12).

HRMS (ESI-TOF): Calculated for $\mathrm{C}_{20} \mathrm{H}_{20} \mathrm{BNO}_{5} \mathrm{Na}[\mathrm{M}+\mathrm{Na}]^{+}: 388.1327$. Found: 388.1328 


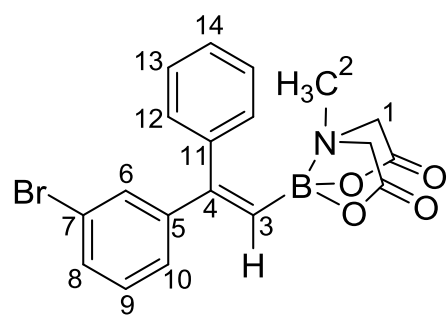

4.41.9 was prepared according to the general procedure A using 3-bromoiodobenzene 4.41.3 $(164 \mathrm{mg}, 0.58 \mathrm{mmol})$. Purification by flash chromatography on silica gel $\left(\mathrm{Et}_{2} \mathrm{O} /\right.$ Acetone $=83: 17$ to $80: 20)$ to give $4.41 .9(59 \mathrm{mg}, 73 \%)$ as a colorless oil $(E / Z=15: 1)$.

Formula: $\mathrm{C}_{19} \mathrm{H}_{17} \mathrm{BBrNO}_{4}$

Mass: $414.1 \mathrm{~g} / \mathrm{mol}$

IR $v_{\max }$ (neat): 1758, 1708, 1611, 1594, 1556, 1455, 1420, 1336, 1285, 1223, 1156, 1108, 1083, $1024,1005 \mathrm{~cm}^{-1}$.

${ }^{1}$ H NMR (400 MHz, Acetone- $\left.d_{6}\right) \delta 7.52-7.41\left(2 \mathrm{H}, \mathrm{m}, \mathrm{H}_{8}+\mathrm{H}_{9}\right), 7.40-7.18\left(7 \mathrm{H}, \mathrm{m}, \mathrm{H}_{6}+\mathrm{H}_{10}\right.$ $\left.+2 \mathrm{H}_{12}+2 \mathrm{H}_{13}+\mathrm{H}_{14}\right), 6.24\left(1 \mathrm{H}, \mathrm{s}, \mathrm{H}_{3}\right), 4.08\left(2 \mathrm{H}, \mathrm{d}, J=16.7 \mathrm{~Hz}, \mathrm{H}_{1}\right), 3.72(2 \mathrm{H}, \mathrm{d}, J=16.7 \mathrm{~Hz}$, $\left.\mathrm{H}_{1}{ }^{\cdot}\right), 3.17\left(3 \mathrm{H}, \mathrm{s}, \mathrm{H}_{2}\right)$.

${ }^{13}$ C NMR (101 MHz, Acetone- $\left.d_{6}\right) \delta 168.5(\mathrm{~s}, 2 \mathrm{CO}), 154.5\left(\mathrm{~s}, \mathrm{C}_{4}\right), 147.8\left(\mathrm{~s}, \mathrm{C}_{5}\right), 141.9\left(\mathrm{~s}, \mathrm{C}_{11}\right)$,

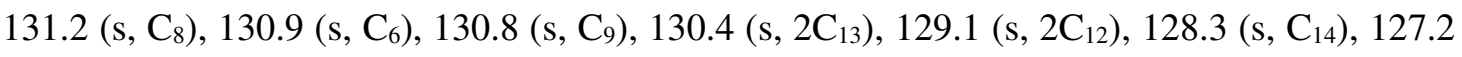
$\left(\mathrm{s}, \mathrm{C}_{10}\right), 122.9\left(\mathrm{~s}, \mathrm{C}_{7}\right), 62.7\left(\mathrm{~s}, 2 \mathrm{C}_{1}\right), 47.5\left(\mathrm{~s}, \mathrm{C}_{2}\right)$.

GCMS (EI) $m / z$ (rel intensity): 253 (93), 254 (100), 255 (28), 258 (3), 412 (51), 413 (6), 414 (92), 415 (16) $416(52)$.

HRMS (ESI-TOF): Calculated for $\mathrm{C}_{19} \mathrm{H}_{17} \mathrm{~B}^{79} \mathrm{BrNO}_{4} \mathrm{Na}[\mathrm{M}+\mathrm{Na}]^{+}$: 436.0326. Found: 436.0327.

HRMS (ESI-TOF): Calculated for $\mathrm{C}_{19} \mathrm{H}_{17} \mathrm{~B}^{81} \mathrm{BrNO}_{4} \mathrm{Na}[\mathrm{M}+\mathrm{Na}]^{+}$: 438.0305. Found: 436.0306. 


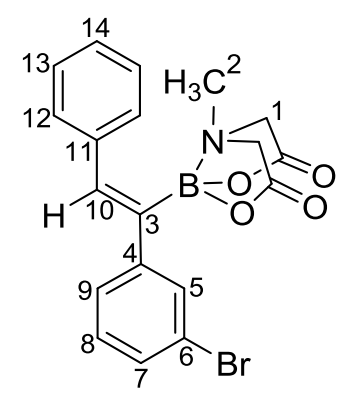

4.41.10 was prepared according to the general procedure A using 3-bromoiodobenzene 4.41.3 $(164 \mathrm{mg}, 0.58 \mathrm{mmol})$. Purification by flash chromatography on silica gel $\left(\mathrm{Et}_{2} \mathrm{O} /\right.$ Acetone $=$ 85:15) to give 4.41.10 (28 $\mathrm{mg}, 26 \%)$ as a colorless oil $(E / Z=>50: 1)$.

Formula: $\mathrm{C}_{19} \mathrm{H}_{17} \mathrm{BBrNO}_{4}$ Mass: $414.1 \mathrm{~g} / \mathrm{mol}$

IR $v_{\max }$ (neat): $1766,1708,1588,1556,1455,1336,1286,1244,1223,1100,1067,1030 \mathrm{~cm}^{-1}$. ${ }^{1}$ H NMR (400 MHz, Acetone- $\left.d_{6}\right) \delta 7.62\left(1 \mathrm{H}, \mathrm{s}, \mathrm{H}_{5}\right), 7.55-6.83\left(9 \mathrm{H}, \mathrm{m}, \mathrm{H}_{7}+\mathrm{H}_{8}+\mathrm{H}_{9}+\mathrm{H}_{10}+\right.$ $\left.2 \mathrm{H}_{12}+2 \mathrm{H}_{13}+\mathrm{H}_{14}\right), 4.03\left(\mathrm{~d}, 2 \mathrm{H}, J=16.9 \mathrm{~Hz}, \mathrm{H}_{1}\right), 3.49\left(\mathrm{~d}, 2 \mathrm{H}, J=16.9 \mathrm{~Hz}, \mathrm{H}_{1^{\prime}}\right), 2.97(3 \mathrm{H}, \mathrm{s}$, $\left.\mathrm{H}_{2}\right)$.

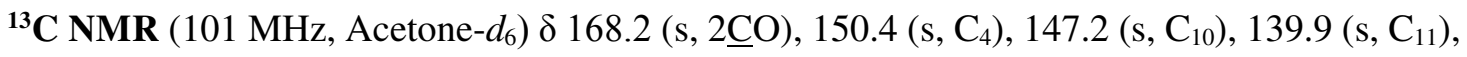
$132.4\left(\mathrm{~s}, \mathrm{C}_{7}\right), 130.6\left(\mathrm{~s}, \mathrm{C}_{5}\right), 129.9\left(\mathrm{~s}, \mathrm{C}_{8}\right), 129.4\left(\mathrm{~s}, 2 \mathrm{C}_{13}\right), 128.9\left(\mathrm{~s}, 2 \mathrm{C}_{12}\right), 128.7\left(\mathrm{~s}, \mathrm{C}_{14}\right), 127.8$ $\left(\mathrm{s}, \mathrm{C}_{9}\right), 122.5\left(\mathrm{~s}, \mathrm{C}_{6}\right), 63.4\left(\mathrm{~s}, 2 \mathrm{C}_{1}\right), 47.9\left(\mathrm{~s}, \mathrm{C}_{2}\right)$.

GCMS (EI) $m / z$ (rel intensity): 91 (30), 92 (2), 156 (1), 157 (25), 158 (1), 183 (100), 184 (9), 185 (96).

HRMS (ESI-TOF): Calculated for $\mathrm{C}_{19} \mathrm{H}_{17} \mathrm{~B}^{79} \mathrm{BrNO}_{4} \mathrm{Na}[\mathrm{M}+\mathrm{Na}]^{+}$: 436.0326. Found: 436.0325. HRMS (ESI-TOF): Calculated for $\mathrm{C}_{19} \mathrm{H}_{17} \mathrm{~B}^{81} \mathrm{BrNO}_{4} \mathrm{Na}[\mathrm{M}+\mathrm{Na}]^{+}$: 438.0304. Found: 436.0306. 


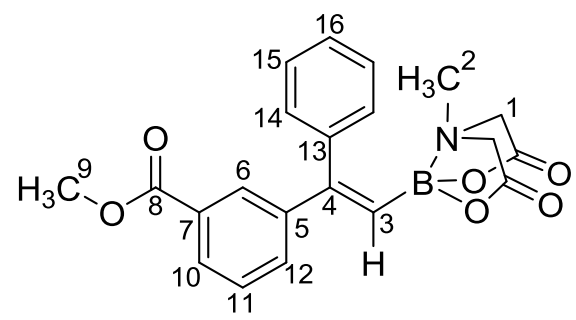

4.41.11 was prepared according to the general procedure $Y$ using methyl 3 -iodobenzoate 4.41.4 $(118 \mathrm{mg}, 0.45 \mathrm{mmol})$. Purification by flash chromatography on silica gel $\left(\mathrm{Et}_{2} \mathrm{O} /\right.$ Acetone $=83: 17$ to $80: 20)$ to give $\mathbf{4 . 4 1 . 1 1}(42 \mathrm{mg}, 72 \%)$ as a colorless oil $(E / Z=>50: 1)$.

Formula: $\mathrm{C}_{21} \mathrm{H}_{20} \mathrm{BNO}_{6}$ Mass: $393.2 \mathrm{~g} / \mathrm{mol}$

IR $v_{\max }$ (neat): 1762, 1709, 1612, 1596, 1440, 1358, 1336, 1283, 1221, 1108, $1083 \mathrm{~cm}^{-1}$.

${ }^{1}$ H NMR $\left(400 \mathrm{MHz}\right.$, Acetone- $\left.d_{6}\right) \delta 8.03-7.84\left(2 \mathrm{H}, \mathrm{m}, \mathrm{H}_{6}+\mathrm{H}_{11}\right), 7.54\left(1 \mathrm{H}, \mathrm{m}, \mathrm{H}_{12}\right), 7.47-$ $7.40\left(2 \mathrm{H}, \mathrm{m}, \mathrm{H}_{14}\right), 7.38-7.22\left(4 \mathrm{H}, \mathrm{m}, \mathrm{H}_{10}+2 \mathrm{H}_{15}+\mathrm{H}_{16}\right), 6.26\left(1 \mathrm{H}, \mathrm{s}, \mathrm{H}_{3}\right), 4.06(2 \mathrm{H}, \mathrm{d}, J=16.8$ $\left.\mathrm{Hz}, \mathrm{H}_{1}\right), 3.83\left(3 \mathrm{H}, \mathrm{s}, \mathrm{H}_{9}\right), 3.72\left(2 \mathrm{H}, \mathrm{d}, J=16.8 \mathrm{~Hz}, \mathrm{H}_{1}{ }^{\prime}\right), 3.13\left(3 \mathrm{H}, \mathrm{s}, \mathrm{H}_{2}\right)$.

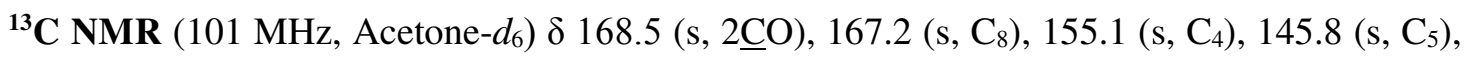
$142.1\left(\mathrm{~s}, \mathrm{C}_{13}\right), 132.7\left(\mathrm{~s}, \mathrm{C}_{12}\right), 131.1\left(\mathrm{~s}, \mathrm{C}_{7}\right), 130.3\left(\mathrm{~s}, 2 \mathrm{C}_{15}\right), 129.3\left(\mathrm{~s}, \mathrm{C}_{11}\right), 129.2$ (s, $\left.\mathrm{C}_{10}\right), 129.1$ $\left(\mathrm{s}, 2 \mathrm{C}_{14}\right), 128.8\left(\mathrm{~s}, \mathrm{C}_{6}\right), 128.2\left(\mathrm{~s}, \mathrm{C}_{16}\right), 62.7\left(\mathrm{~s}, 2 \mathrm{C}_{1}\right), 52.4\left(\mathrm{~s}, \mathrm{C}_{9}\right), 47.5\left(\mathrm{~s}, \mathrm{C}_{2}\right)$.

GCMS (EI) $m / z$ (rel intensity): 168 (3), 194 (29), 195 (18), 210 (100), 211 (18), 238 (75), 239 (14).

HRMS (ESI-TOF): Calculated for $\mathrm{C}_{21} \mathrm{H}_{20} \mathrm{BNO}_{6} \mathrm{Na}[\mathrm{M}+\mathrm{Na}]^{+}$: 416.1276. Found: 416.1276. 


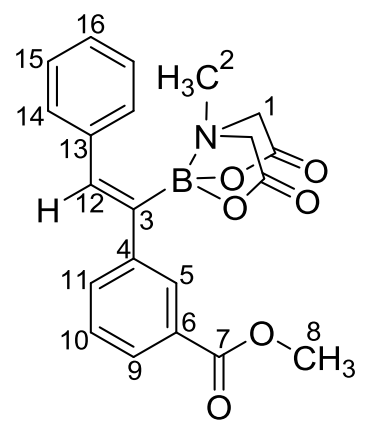

4.41.12 was prepared according to the general procedure A using 3-iodobenzoate 4.41.4 (118 $\mathrm{mg}, 0.45 \mathrm{mmol})$. Purification by flash chromatography on silica gel $\left(\mathrm{Et}_{2} \mathrm{O} /\right.$ Acetone $\left.=85: 15\right)$ to give 4.41.12 $(19 \mathrm{mg}, 25 \%)$ as a colorless oil $(E / Z=>50: 1)$.

Formula: $\mathrm{C}_{21} \mathrm{H}_{20} \mathrm{BNO}_{6}$ Mass: $393.2 \mathrm{~g} / \mathrm{mol}$

IR $v_{\max }$ (neat): 1767, 1712, 1439, 1335, 1285, 1223, 1112, 1070, $1032 \mathrm{~cm}^{-1}$.

${ }^{1} \mathbf{H}$ NMR (400 MHz, Acetone- $\left.d_{6}\right) \delta 8.05\left(1 \mathrm{H}, \mathrm{m}, \mathrm{H}_{9}\right), 7.94\left(1 \mathrm{H}, \mathrm{m}, \mathrm{H}_{5}\right), 7.73\left(1 \mathrm{H}, \mathrm{m}, \mathrm{H}_{11}\right), 7.54$ $-7.38\left(3 \mathrm{H}, \mathrm{m}, \mathrm{H}_{10}+2 \mathrm{H}_{15}\right), 7.38-7.20\left(4 \mathrm{H}, \mathrm{m}, \mathrm{H}_{12}+2 \mathrm{H}_{14}+\mathrm{H}_{16}\right), 4.04\left(2 \mathrm{H}, \mathrm{d}, J=16.9 \mathrm{~Hz}, \mathrm{H}_{1}\right)$, $3.90\left(3 \mathrm{H}, \mathrm{s}, \mathrm{H}_{8}\right), 3.47\left(2 \mathrm{H}, \mathrm{d}, J=16.9 \mathrm{~Hz}, \mathrm{H}_{1^{\prime}}\right), 3.00\left(3 \mathrm{H}, \mathrm{s}, \mathrm{H}_{2}\right)$.

${ }^{13}$ C NMR (101 MHz, Acetone- $\left.d_{6}\right) \delta 168.4(\mathrm{~s}, 2 \underline{\mathrm{CO}}), 167.6\left(\mathrm{~s}, \mathrm{C}_{7}\right), 148.5\left(\mathrm{~s}, \mathrm{C}_{4}\right), 147.1\left(\mathrm{~s}, \mathrm{C}_{12}\right)$, $140.2\left(\mathrm{~s}, \mathrm{C}_{13}\right), 134.5\left(\mathrm{~s}, \mathrm{C}_{11}\right), 131.1\left(\mathrm{~s}, \mathrm{C}_{6}\right), 130.6\left(\mathrm{~s}, \mathrm{C}_{9}\right), 129.5\left(\mathrm{~s}, 2 \mathrm{C}_{15}\right), 129.1\left(\mathrm{~s}, 2 \mathrm{C}_{14}+\mathrm{C}_{10}\right)$, $128.1\left(\mathrm{~s}, \mathrm{C}_{16}\right), 127.9\left(\mathrm{~s}, \mathrm{C}_{5}\right), 63.5\left(\mathrm{~s}, 2 \mathrm{C}_{1}\right), 52.5\left(\mathrm{~s}, \mathrm{C}_{8}\right), 48.1\left(\mathrm{~s}, \mathrm{C}_{2}\right)$.

GCMS (EI) $m / z$ (rel intensity): 205 (100), 206 (16), 220 (26).

HRMS (ESI-TOF): Calculated for $\mathrm{C}_{21} \mathrm{H}_{20} \mathrm{BNO}_{6} \mathrm{Na}[\mathrm{M}+\mathrm{Na}]^{+}$: 416.1276. Found: 416.1277. 


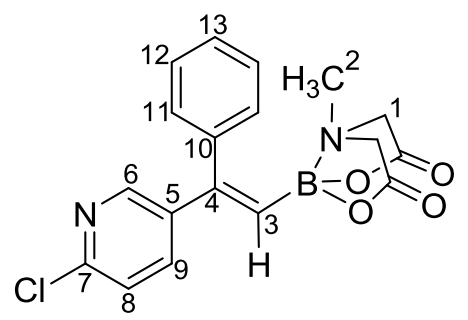

4.42.13 was prepared according to the general procedure A using 2-chloro-5-iodopyridine 4.42.6 $(70 \mathrm{mg}, 0.29 \mathrm{mmol})$. Purification by flash chromatography on silica gel $\left(\mathrm{Et}_{2} \mathrm{O} /\right.$ Acetone $=$ $83: 17$ to $80: 20)$ to give $\mathbf{4 . 4 2 . 1 3}(19 \mathrm{mg}, 53 \%)$ as a colorless oil $(E / Z=3: 1)$.

Formula: $\mathrm{C}_{18} \mathrm{H}_{16} \mathrm{BClN}_{2} \mathrm{O}_{4}$ Mass: $370.6 \mathrm{~g} / \mathrm{mol}$

IR $v_{\max }$ (neat): 1758, 1613, 1576, 1457, 1336, 1287, 1229, 1160, 1101, 1082, $1023 \mathrm{~cm}^{-1}$.

${ }^{1} \mathbf{H}$ NMR $\left(400 \mathrm{MHz}\right.$, Acetone- $\left.d_{6}\right) \delta 8.33\left(0.7 \mathrm{H}, \mathrm{m} \mathrm{H}_{6(E)}\right), 8.24\left(0.3 \mathrm{H}, \mathrm{m}, \mathrm{H}_{6(\mathrm{Z})}\right), 7.65(1 \mathrm{H}, \mathrm{dt}, J=$ 8.1, $\left.2.3 \mathrm{~Hz}, \mathrm{H}_{9(E)+(Z)}\right), 7.51-7.13\left(6 \mathrm{H}, \mathrm{m}, \mathrm{H}_{8}+2 \mathrm{H}_{11}+2 \mathrm{H}_{12}+\mathrm{H}_{13}\right), 6.36\left(0.3 \mathrm{H}, \mathrm{s}, \mathrm{H}_{3(Z)}\right), 6.32$ $\left(0.7 \mathrm{H}, \mathrm{s}, \mathrm{H}_{3(E)}\right), 4.18\left(0.6 \mathrm{H}, \mathrm{d}, J=16.9 \mathrm{~Hz}, \mathrm{H}_{1(Z)}\right), 4.09\left(1.4 \mathrm{H}, \mathrm{d}, J=16.7 \mathrm{~Hz}, \mathrm{H}_{1(E)}\right), 4.02(0.6 \mathrm{H}$, d, $\left.J=16.8 \mathrm{~Hz}, \mathrm{H}_{1\left(Z^{\prime}\right)}\right), 3.79\left(1.4 \mathrm{H}, \mathrm{d}, J=16.7 \mathrm{~Hz}, \mathrm{H}_{1\left(E^{\prime}\right)}\right), 3.23\left(0.9 \mathrm{H}, \mathrm{s}, \mathrm{H}_{2(\mathrm{Z})}\right), 3.16(2.1 \mathrm{H}, \mathrm{s}$, $\left.\mathrm{H}_{2(E)}\right)$.

${ }^{13}$ C NMR (101 MHz, Acetone- $\left.d_{6}\right) \delta 168.5$ (s, 2므), 151.7 (s, $\left.\mathrm{C}_{7}\right), 150.9,150.8,150.7,149.2$ $\left(\mathrm{s}, \mathrm{C}_{6}\right), 141.2\left(\mathrm{~s}, \mathrm{C}_{4}\right), 140.0\left(\mathrm{~s}, \mathrm{C}_{5}\right), 138.5\left(\mathrm{~s}, \mathrm{C}_{9}\right), 137.6\left(\mathrm{~s}, \mathrm{C}_{10}\right), 130.3\left(\mathrm{~s}, 2 \mathrm{C}_{12}\right), 129.2\left(\mathrm{~s}, 2 \mathrm{C}_{11}\right)$, $128.9,128.5\left(\mathrm{~s}, \mathrm{C}_{13}\right), 128.2,124.4\left(\mathrm{~s}, \mathrm{C}_{8}\right), 124.3,62.7\left(\mathrm{~s}, 2 \mathrm{C}_{1}\right), 62.5,47.5\left(\mathrm{~s}, \mathrm{C}_{2}\right)$.

A mixture of $(E / Z)$-isomers were obtained, unable to differentiate carbon peaks of $(E)$-isomer from $(Z)$-isomer.

GCMS (EI) $m / z$ (rel intensity): 150 (12), 151 (30), 152 (29), 153 (16), 200 (29), 201 (5), 202 (20), 214 (55), 215 (100), 216 (37), 217 (34).

HRMS (ESI-TOF): Calculated for $\mathrm{C}_{18} \mathrm{H}_{17} \mathrm{BClN}_{2} \mathrm{O}_{4}[\mathrm{M}+\mathrm{H}]^{+}$: 371.0964 . Found: 371.0967. 
(E)-(1-(2,3-dihydrobenzofuran-5-yl)-2-phenylvinyl)boronic acid MIDA ester, (4.42.15):

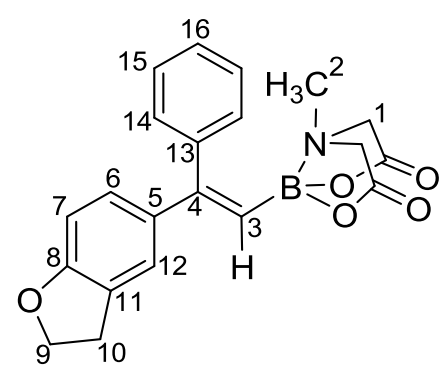

4.42.15 was prepared according to the general procedure A using 5-iodo-1-benzofuran 4.42.8 $(101 \mathrm{mg}, 0.41 \mathrm{mmol})$. Purification by flash chromatography on silica gel $\left(\mathrm{Et}_{2} \mathrm{O} /\right.$ Acetone $=83: 17$ to $80: 20)$ to give $\mathbf{4 . 4 2 . 1 5}$ (38 $\mathrm{mg}, 74 \%)$ as a colorless oil $(E / Z=13: 1)$.

Formula: $\mathrm{C}_{21} \mathrm{H}_{20} \mathrm{BNO}_{5}$

Mass: $377.2 \mathrm{~g} / \mathrm{mol}$

IR $v_{\max }$ (neat): 1761, 1606, 1490, 1453, 1337, 1286, 1236, 1082, $1025 \mathrm{~cm}^{-1}$.

${ }^{1}$ H NMR (400 MHz, Acetone- $d_{6} \delta 7.39-7.13\left(6 \mathrm{H}, \mathrm{m}, \mathrm{H}_{6}+2 \mathrm{H}_{14}+2 \mathrm{H}_{15}+\mathrm{H}_{16}\right), 6.99(2 \mathrm{H}$, brdd, $\left.J=8.4,2.1 \mathrm{~Hz}, \mathrm{H}_{12}\right), 6.62\left(1 \mathrm{H}, \mathrm{d}, J=8.3 \mathrm{~Hz}, \mathrm{H}_{7}\right), 6.05\left(1 \mathrm{H}, \mathrm{s}, \mathrm{H}_{3}\right), 4.53\left(2 \mathrm{H}, \mathrm{t}, J=8.7 \mathrm{~Hz}, \mathrm{H}_{9}\right)$, $4.00\left(2 \mathrm{H}, \mathrm{d}, J=16.7 \mathrm{~Hz}, \mathrm{H}_{1}\right), 3.65\left(2 \mathrm{H}, \mathrm{d}, J=16.7 \mathrm{~Hz}, \mathrm{H}_{1}\right), 3.14\left(2 \mathrm{H}, \mathrm{t}, J=8.7 \mathrm{~Hz}, \mathrm{H}_{10}\right), 3.08$ $\left(3 \mathrm{H}, \mathrm{s}, \mathrm{H}_{2}\right)$

${ }^{13}$ C NMR (101 MHz, Acetone- $\left.d_{6}\right) \delta 168.5$ (s, 2ㄷ), $160.93\left(\mathrm{~s}, \mathrm{C}_{8}\right), 156.0\left(\mathrm{~s}, \mathrm{C}_{4}\right), 143.0\left(\mathrm{~s}, \mathrm{C}_{5}\right)$, $137.9\left(\mathrm{~s}, \mathrm{C}_{13}\right), 130.3\left(\mathrm{~s}, 2 \mathrm{C}_{15}\right), 128.8\left(\mathrm{~s}, 2 \mathrm{C}_{14}\right), 128.2\left(\mathrm{~s}, \mathrm{C}_{16}\right), 128.1\left(\mathrm{~s}, \mathrm{C}_{11}\right), 127.8\left(\mathrm{~s}, \mathrm{C}_{6}\right), 124.7$ $\left(\mathrm{s}, \mathrm{C}_{12}\right), 109.1\left(\mathrm{~s}, \mathrm{C}_{7}\right), 72.10\left(\mathrm{~s}, \mathrm{C}_{9}+\mathrm{C}_{10}\right), 62.6\left(\mathrm{~s}, 2 \mathrm{C}_{1}\right), 47.4\left(\mathrm{~s}, \mathrm{C}_{2}\right)$.

HRMS (ESI-TOF): Calculated for $\mathrm{C}_{21} \mathrm{H}_{20} \mathrm{BNO}_{5} \mathrm{Na}[\mathrm{M}+\mathrm{Na}]^{+}: 400.1327$. Found: 400.1329 . 
(E)-(1-(2,3-dihydrobenzofuran-5-yl)-2-phenylvinyl)boronic acid MIDA ester, (4.42.15'):

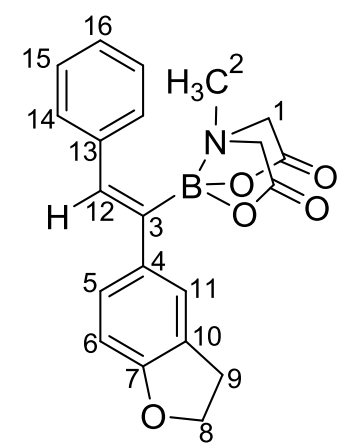

4.42.15'was prepared according to the general procedure A using 5-iodo-1-benzofuran 4.42.8 $(101 \mathrm{mg}, 0.41 \mathrm{mmol})$. Purification by flash chromatography on silica gel $\left(\mathrm{Et}_{2} \mathrm{O} /\right.$ Acetone $=$ $85: 15)$ to give $4.42 .15 '(4.0 \mathrm{mg}, 8 \%)$ as a colorless oil $(E / Z=>50: 1)$.

Formula: $\mathrm{C}_{21} \mathrm{H}_{20} \mathrm{BNO}_{5}$

Mass: $377.2 \mathrm{~g} / \mathrm{mol}$

IR $v_{\max }$ (neat): 1764, 1608, 1490, 1448, 1337, 1288, 1227, 1074, $1029 \mathrm{~cm}^{-1}$.

${ }^{1}$ H NMR (400 MHz, Acetone- $\left.d_{6}\right) \delta 7.38-7.32\left(2 \mathrm{H}, \mathrm{m}, 2 \mathrm{H}_{15}\right), 7.32-7.25\left(3 \mathrm{H}, \mathrm{m}, \mathrm{H}_{11}+2 \mathrm{H}_{14}\right)$, $7.23-7.12\left(3 \mathrm{H}, \mathrm{m}, \mathrm{H}_{5}+\mathrm{H}_{12}+\mathrm{H}_{16}\right), 6.67\left(1 \mathrm{H}, \mathrm{d}, J=8.2 \mathrm{~Hz}, \mathrm{H}_{6}\right), 4.53\left(2 \mathrm{H}, \mathrm{t}, J=8.7 \mathrm{~Hz}, \mathrm{H}_{8}\right)$, $4.00\left(2 \mathrm{H}, \mathrm{d}, J=16.8 \mathrm{~Hz}, \mathrm{H}_{1}\right), 3.46\left(2 \mathrm{H}, \mathrm{d}, J=16.8 \mathrm{~Hz}, \mathrm{H}_{1}{ }^{\prime}\right), 3.20\left(2 \mathrm{H}, \mathrm{t}, J=8.7 \mathrm{~Hz}, \mathrm{H}_{9}\right), 2.96$ $\left(3 \mathrm{H}, \mathrm{s}, \mathrm{H}_{2}\right)$.

${ }^{13}$ C NMR (101 MHz, Acetone- $\left.d_{6}\right) \delta 168.4$ (s, 2드), 159.8 (s, C 7 ), $145.4\left(\mathrm{~s}, \mathrm{C}_{12}\right), 140.5\left(\mathrm{~s}, \mathrm{C}_{13}\right)$, $139.9\left(\mathrm{~s}, \mathrm{C}_{4}\right), 129.5\left(\mathrm{~s}, 2 \mathrm{C}_{15}\right), 129.2\left(\mathrm{~s}, \mathrm{C}_{11}\right), 128.6\left(\mathrm{~s}, 2 \mathrm{C}_{14}\right), 127.8\left(\mathrm{~s}, \mathrm{C}_{10}\right), 127.3\left(\mathrm{~s}, \mathrm{C}_{16}\right), 126.5$ $\left(\mathrm{s}, \mathrm{C}_{5}\right), 109.2\left(\mathrm{~s}, \mathrm{C}_{6}\right), 71.85\left(\mathrm{~s}, \mathrm{C}_{8}+\mathrm{C}_{9}\right), 63.2\left(\mathrm{~s}, 2 \mathrm{C}_{1}\right), 47.7\left(\mathrm{~s}, \mathrm{C}_{2}\right)$.

HRMS (ESI-TOF): Calculated for $\mathrm{C}_{21} \mathrm{H}_{20} \mathrm{BNO}_{5} \mathrm{Na}[\mathrm{M}+\mathrm{Na}]^{+}:$: 400.1327. Found: 400.1330 . 


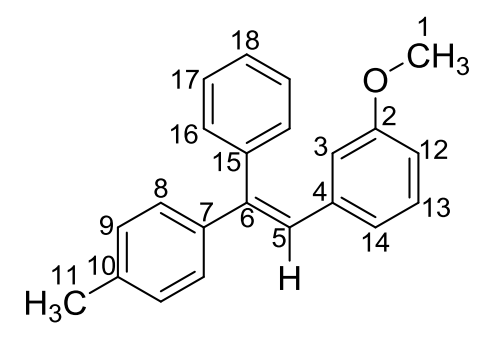

THF (2 ml) was added to an oven-dried vial containing Sphos (11 $\mathrm{mg}, 0.027 \mathrm{mmol})$ and $\operatorname{Pd}(\mathrm{OAc})_{2}(3 \mathrm{mg}, 0.013 \mathrm{mmol})$ under argon. The mixture was stirred for $10 \mathrm{mins}$ to form the catalytic solution. The catalytic solution followed by $\mathrm{NaOH}(1 \mathrm{M}, 0.50 \mathrm{~mL})$ were then added to a solution of 4.29 .1 ( $65 \mathrm{mg}, 0.19 \mathrm{mmol})$ and 3-bromoanisole (30 $\mathrm{mg}, 0.16 \mathrm{mmol})$ and left to stir for 4 hours at room temperature under a subdued light environment. To the reaction mixture was added phosphate buffer $\mathrm{pH} 7(4 \mathrm{~mL})$ and extracted with $\mathrm{Et}_{2} \mathrm{O}(3 \times 2 \mathrm{~mL})$. The combined organic phases were dried over $\mathrm{MgSO}_{4}$, filtered, and concentrated in vacuo. The crude material was purified by flash chromatography on silica (Pentane/ $\mathrm{CH}_{2} \mathrm{Cl}_{2}=100: 0$ to 80:20) to give 4.48.2 (38 mg, 80\%) as a colorless oil. (Tetrahedron $672011 \mathrm{pp} 4342$ )

Formula: $\mathrm{C}_{22} \mathrm{H}_{20} \mathrm{O}$

Mass: $300.4 \mathrm{~g} / \mathrm{mol}$

IR $v_{\max }$ (neat): $1595,1576,1509,1486,1463,1443,1431,1377,1315,1266,1237,1197,1164$, $1137,1073,1041 \mathrm{~cm}^{-1}$.

${ }^{1}$ H NMR (400 MHz, Acetone- $\left.d_{6}\right) \delta 7.39-7.27\left(3 \mathrm{H}, \mathrm{m}, 2 \mathrm{H}_{17}+\mathrm{H}_{13}\right), 7.26-7.18\left(4 \mathrm{H}, \mathrm{m}, 2 \mathrm{H}_{8}+\right.$ $\left.2 \mathrm{H}_{16}\right), 7.14-7.09\left(2 \mathrm{H}, \mathrm{m}, \mathrm{H}_{9}\right), 7.09-7.01\left(1 \mathrm{H}, \mathrm{m}, \mathrm{H}_{18}\right), 6.93\left(1 \mathrm{H}, \mathrm{s}, \mathrm{H}_{5}\right), 6.73-6.61(2 \mathrm{H}, \mathrm{m}$, $\left.\mathrm{H}_{3}+\mathrm{H}_{14}\right), 6.49\left(1 \mathrm{H}, \mathrm{m}, \mathrm{H}_{12}\right), 3.49\left(3 \mathrm{H}, \mathrm{s}, \mathrm{H}_{1}\right), 2.35\left(3 \mathrm{H}, \mathrm{s}, \mathrm{H}_{11}\right)$.

${ }^{13}$ C NMR (101 MHz, Acetone- $\left.d_{6}\right) \delta 158.8\left(\mathrm{~s}, \mathrm{C}_{2}\right), 142.4\left(\mathrm{~s}, \mathrm{C}_{6}\right), 140.4\left(\mathrm{~s}, \mathrm{C}_{15}\right), 140.2\left(\mathrm{~s}, \mathrm{C}_{7}\right)$, $138.5\left(\mathrm{~s}, \mathrm{C}_{10}\right), 137.2\left(\mathrm{~s}, \mathrm{C}_{4}\right), 130.1\left(\mathrm{~s}, 2 \mathrm{C}_{8}\right), 129.1\left(\mathrm{~s}, 2 \mathrm{C}_{9}+\mathrm{C}_{13}\right), 128.5\left(\mathrm{~s}, 2 \mathrm{C}_{17}\right), 127.6\left(\mathrm{~s}, 2 \mathrm{C}_{16}\right)$, 127.5(s, $\left.\mathrm{C}_{18}\right), 127.0\left(\mathrm{~s}, \mathrm{C}_{5}\right), 122.3\left(\mathrm{~s}, \mathrm{C}_{14}\right), 113.5\left(\mathrm{~s}, \mathrm{C}_{12}\right), 113.2\left(\mathrm{~s}, \mathrm{C}_{3}\right), 54.6\left(\mathrm{~s}, \mathrm{C}_{1}\right), 21.0\left(\mathrm{~s}, \mathrm{C}_{11}\right)$. 
GCMS (EI) $m / z$ (rel intensity): 286 (11), 287 (2), 299 (9), 300 (100), 301 (22), 302 (3).

HRMS (ESI-TOF): Calculated for $\mathrm{C}_{22} \mathrm{H}_{21} \mathrm{O}[\mathrm{M}+\mathrm{H}]^{+}:$301.1587. Found: 301.1588 . 
This page has been intentionally left blank. 


\section{$\underline{\text { References }}$}

1. Veeresham, C. Journal of Advanced Pharmaceutical Technology \& Research 2012, 3 (4), 200-201.

2. Newman, D. J.; Cragg, G. M. J. Nat. Prod. 2012, 75, 311.

3. $\quad$ Cragg, G. M.; Newman, D. J. Biochim. Biophys. Acta 2013, 1830 (6), 3670-3695.

4. (a) Newman, D. J.; Cragg, G. M. J. Nat. Prod. 2012, 75 (3), 311-335; (b) Roth, B. D. 1 The Discovery and Development of Atorvastatin, A Potent Novel Hypolipidemic Agent. In Prog. Med. Chem., F.D. King, A. W. O. A. B. R.; Scott, L. D., Eds. Elsevier: 2002; Vol. Volume 40, pp 1-22.

5. $\quad$ Tao, L.; Zhu, F.; Qin, C.; Zhang, C.; Chen, S.; Zhang, P.; Zhang, C.; Tan, C.; Gao, C.; Chen, Z.; Jiang, Y.; Chen, Y. Z. Scientific Reports 2015, 5, 9325.

6. $\quad$ Newman, D. J.; Cragg, G. M. J. Nat. Prod. 2016, 79 (3), 629-661.

7. Harvey, A. L.; Edrada-Ebel, R.; Quinn, R. J. Nat Rev Drug Discov 2015, 14 (2), 111129.

8. $\quad$ Schmid, I. I.; Sattler, I. I.; Grabley, S.; Thiericke, R. J Biomol Screen 1999, 4 (1), 1525.

9. $\quad$ Nicolaou, K. C. Proc. Natl. Acad. Sci. U. S. A. 2004, 101 (33), 11928.

10. Winter, J. M.; Jansma, A. L.; Handel, T. M.; Moore, B. S. Angew. Chem., Int. Ed. 2009, 48 (4), 767-770.

11. (a) Blair, L. M.; Sperry, J. J. Nat. Prod. 2013, 76 (4), 794-812; (b) Cheng, K. C.-C.; Cao, S.; Raveh, A.; MacArthur, R.; Dranchak, P.; Chlipala, G.; Okoneski, M. T.; Guha, R.; Eastman, R. T.; Yuan, J.; Schultz, P. J.; Su, X.-z.; Tamayo-Castillo, G.; Matainaho, T.; Clardy, J.; Sherman, D. H.; Inglese, J. J. Nat. Prod. 2015, 78 (10), 2411-2422.

12. Le Goff, G.; Ouazzani, J. Bioorg. Med. Chem. 2014, 22 (23), 6529-6544.

13. Maycock, C. D.; Barros, M. T.; Santos, A. G.; Godinho, L. S. Tetrahedron Lett. 1992, 33 (32), 4633-4636.

14. (a) Hamada, M.; Takeuchi, T.; Takeuchi, T.; Kondo, S.; Ikeda, Y.; Naganawa, H.; Maeda, K.; Okami, Y.; Umezawa, H. J. Antibiot. 1970, 23, 170; (b) Kondo, S.; Shibahara, S.; Takahashi, S.; Maeda, K.; Umezawa, H.; Ohno, M. J. Am. Chem. Soc. 1971, 93, 6305.

15. Shibahara, S.; Kondo, S.; Maeda, K.; Umezawa, H.; Ohno, M. J. Am. Chem. Soc. 1972, 94, 4353.

16. Kondo, S.; Yamamoto, H.; Maeda, K.; Umezawa, H. J. Antibiot. 1971, 24, 732.

17. (a) Wang, Y. F.; Izawa, T.; Kobayashi, S.; Ohno, M. J. Am. Chem. Soc. 1982, 104 (23), 6465-6466; (b) Uehara, Y.; Kondo, S.; Umezawa, H.; Suzukake, K.; Hori, M. J Antibiot (Tokyo) 1972, 25 (11), 685-8.

18. Mizuno, S.; Nitta, K.; Umezawa, H. J. Antibiot. 1970, 23, 581.

19. Pestka, S. Аnnu. Rev. Microbiol. 1971, 25, 487-562.

20. (a) Masters, J. J.; Hegedus, L. S. J. Org. Chem. 1993, 58 (17), 4547-4554; (b) Weigel, N. L.; Zhang, Y. J. Mol. Med. 1998, 76, 469; (c) Kasahara, K.; Iida, H.; Kibayashi, C. J. Org. Chem. 1989, 54 (9), 2225-2233; (d) Schmidt, U.; Stäbler, F.; Lieberknecht, A. Synthesis 1992, 1992 (05), 482-486; (e) Jain, R. P.; Williams, R. M. J. Org. Chem. 2002, 67 (18), 6361-6365; (f) Davies, S. G.; Ichihara, O. Tetrahedron: Asymmetry 1996, 7, 1919; (g) Davies, S. G.; Ichihara, O.; Roberts, P. M.; Thomson, J. E. Tetrahedron 2011, 67 (1), 216-227; (h) Nishiguchi, S.; Sydnes, M. O.; Taguchi, A.; Regnier, T.; Kajimoto, T.; Node, M.; Yamazaki, Y.; Yakushiji, F.; Kiso, Y.; Hayashi, Y. Tetrahedron 2010, 66 (1), 314-320; (i) Hayashi, Y.; Regnier, T.; Nishiguchi, S.; Sydnes, M. O.; Hashimoto, D.; Hasegawa, J.; Katoh, T.; Kajimoto, T.; Shiozuka, M.; Matsuda, R.; Node, M.; Kiso, Y. Chem. Commun. 2008, (20), 2379-2381; (j) Pierdet, A.; Nédélec, L.; Delaroff, V.; Allais, A. Tetrahedron 1980, 36, 1763; (k) Pasquet, G.; Boucherot, D.; Pilgrim, W. R.; Wright, B. Tetrahedron Lett. 1980, 21 (10), 931-934; (1) Iida, H.; Kasahara, K.; Kibayashi, C. J. Am. Chem. Soc. 1986, 108 (15), 4647-4648; (m) Tanner, D.; Somfai, P. 
Tetrahedron Lett. 1988, 29, 2373; (n) Socha, D.; Jurczak, M.; Chmielewski, M. Tetrahedron Lett. 1995, 36, 135; (o) Jang, J. L., Y.; Ahn, Y. Bull. Korean. Chem. Soc. 1997, 18, 254-256; (p) Makoto, S.; Asato, M.; Tamotsu, F. Chem. Lett. 1998, 27 (5), 467-468; (q) Naidu, S. V.; Kumar, P. Tetrahedron Lett. 2007, 48 (22), 3793-3796.

21. Yamada, K.; Kurokawa, T.; Tokuyama, H.; Fukuyama, T. J. Am. Chem. Soc. 2003, 125 (22), 6630-6631.

22. (a) Jadhav, P. K.; Bhat, K. S.; Perumal, P. T.; Brown, H. C. J. Org. Chem. 1986, 51 (4), 432-439; (b) Racherla, U. S.; Brown, H. C. J. Org. Chem. 1991, 56 (1), 401-404.

23. Node, M.; Hashimoto, D.; Katoh, T.; Ochi, S.; Ozeki, M.; Watanabe, T.; Kajimoto, T. Org. Lett. 2008, 10 (13), 2653-2656.

24. Kitamura, M.; Tokunaga, M.; Ohkuma, T.; Noyori, R. Tetrahedron Lett. 1991, 32 (33), 4163-4166.

25. (a) Schmidt, U.; Riedl, B.; Griesser, H.; Fitz, C. Synthesis 1991, 1991 (08), 655-657; (b) Knowles, W. S.; Sabacky, M. J.; Vineyard, B. D.; Weinkauff, D. J. J. Am. Chem. Soc. 1975, 97 (9), 2567-2568.

26. De Bernardo, S.; Tengi, J. P.; Sasso, G.; Weigele, M. Tetrahedron Lett. 1988, 29, 4077.

27. Boysen, M. M. K. Cycloadditions. In Carbohydrates - Tools for Stereoselective Synthesis, Wiley-VCH Verlag GmbH \& Co. KGaA: 2013; pp 65-106.

28. Miljkovic, M. 2014, (1), 304.

29. Barton, D. H. R.; Gero, S. D.; Maycock, C. D. Journal of the Chemical Society, Perkin Transactions 1 1982, (0), 1541-1551.

30. $\quad$ Grewe, R.; Nolte, E. Justus Liebigs Annalen der Chemie 1952, 575 (1), 1-17.

31. Hayashi, M.; Takezaki, H.; Hashimoto, Y.; Takaoki, K.; Saigo, K. Tetrahedron Lett. 1998, 39 (null), 7529.

32. (a) Bates, R. W.; Sa-Ei, K. Org. Lett. 2002, 4, 4225-4227; (b) Bates, R. W.; Boonsombat. J. Org. Biomol. Chem. 2005, 3, 520.

33. (a) Bates, R. W.; Nemeth, J.; Snell, R. Synthesis 2008, 1033; (b) Bates, R. W.; Lu, Y. J. Org. Chem. 2009, 74 (24), 9460-9465; (c) Bates, R. W.; Lim, C. J. Synlett 2010, 866; (d) Bates, R. W.; Lu, Y. Org. Lett. 2010, 12, 3938.

34. (a) Bates, R. W.; Snell, R.; Winbush. Synlett. 2008, 1042; (b) Bates, R. W.; Ping, S. Synthesis 2009, 655-659.

35. (a) Janza, B.; Studer, A. Synthesis 2002, 2117; (b) Moriyama, K.; Izumisawa, Y.; Togo, H. J. Org. Chem. 2011, 76, 7249-7255.

36. (a) Tay, B. Y.; Xiang, K.; Thiemann, T.; Dongol, K. G. Synth. Commun. 2006, 36 (9), 1247-1257; (b) Dongol, K. G.; Tay, B. Y. Tetrahedron Lett. 2006, 47 (6), 927-930; (c) LaLonde, R. L.; Wang, Z. J.; Mba, M.; Lackner, A. D.; Toste, D. F. Angew. Chem., Int. Ed. 2010, 49, 598601; (d) Peng, J.; Lin, W.; Yuan, S.; Chen, Y. J. Org. Chem. 2007, 72 (8), 3145-3148.

37. Kanemasa, S.; Oderaotoshi, Y.; Tanaka, J.; Wad, E. J. Am. Chem. Soc. 1998, 120, $12355-12356$.

38. Gothelf, K. V.; Jorgensen, K. A. Chem. Rev. 1998, 98, 863-909.

39. (a) Bates, R. W.; Snell, R. H.; Winbush, S. A. Synlett 2008, 2008 (07), 1042-1044; (b) Bates, R. W.; Boonsombat, J.; Lu, Y.; Nemeth, J.; Sa-Ei, K.; Song, P.; Cai, M.; Cranwell, P.; Winbush, S. A. Pure Appl. Chem. 2008, 8 (4), 681-685.

40. $\quad$ Bates, R. W.; Nemeth, J. A.; Snell, R. H. Synthesis 2008, 2008 (07), 1033-1038.

41. Bates, R. W.; Song, P. Synthesis 2009, 2009 (EFirst), 655-659.

42. Bates, R. W.; Tang, C. H.; Tan, Y.; Buang, S. N. b. Synlett 2012, 23 (15), 2266-2268.

43. (a) Schaus, S. E.; Brandes, B. D.; Larrow, J. F.; Tokunaga, M.; Hansen, K. B.; Gould, A. E.; Furrow, M. E.; Jacobsen, E. N. J. Am. Chem. Soc. 2002, 124 (7), 1307-1315; (b) Furrow, M. E.; Schaus, S. E.; Jacobsen, E. N. J. Org. Chem. 1998, 63 (20), 6776-6777.

44. Alam, M.; Wise, C.; Baxter, C. A.; Cleator, E.; Walkinshaw, A. Org. Process Res. Dev. 2012, $16(3), 435-441$.

45. Holub, N.; Neidhöfer, J.; Blechert, S. Org. Lett. 2005, 7 (7), 1227-1229. 
46. Wittmann, V. Angew. Chem., Int. Ed. 2006, 45 (21), 3399-3400.

47. (a) Mitsunobu, O. Synthesis 1981, 1-28; (b) Mitsunobu, O.; Yamada, Y. Bull. Chem. Soc. Jpn. 1967, 40, 2380.

48. Li, J. J. Ing-Manske procedure. In Name Reactions: A Collection of Detailed Reaction Mechanisms, Springer Berlin Heidelberg: Berlin, Heidelberg, 2003; pp 203-203.

49. $\quad$ Criegee, R.; Wenner, G. Annales de Chimie - Science des Materiaux 1949, 9, 564.

50. (a) Criegee, R.; Lohaus, G. Annales de Chimie - Science des Materiaux 1953, 6, 583; (b) Criegee, R.; Lohaus, G. Annales de Chimie - Science des Materiaux 1953, 12, 583.

51. Roy, R. K. The Journal of Physical Chemistry A 2003, 107 (3), 397-404.

52. Motorina, I. A.; Sviridova, L. A.; Golubeva, G. A.; Zelenin, K. N.; Bezhan, I. P.; Ershov, A. Y.; Bundel, Y. G. Chem. Het. Cpd. 1989, 1371-1377.

53. Bates, R. W.; Khanizeman, R. i. N.; Hirao, H.; Tay, Y. S.; Sae-Lao, P. Org. Biomol. Chem. 2014, 12 (27), 4879-4884.

54. $\quad$ Yang, H. W.; Zhao, C.; Romo, D. Tetrahedron 1997, 53 (48), 16471-16488.

55. Djerassi, C.; Engle, R. R. J. Am. Chem. Soc. 1963, 75, 3838.

56. $\quad$ Carlsen, P. J.; Katsuki, T.; Martin, V. S.; Sharpless, K. B. J. Org. Chem. 1981, 46, 3936.

57. Bäumer, U. S.; Schäfer, H. J. Electrochim. Acta 2003, 48 (5), 489-495.

58. Albert, J. S.; Hamilton, A. D. 1,3-Dicyclohexylcarbodiimide-4Dimethylaminopyridine. In Encyclopedia of Reagents for Organic Synthesis, John Wiley \& Sons, Ltd: 2001.

59. Montalbetti, C. A. G. N.; Falque, V. Tetrahedron 2005, 61 (46), 10827-10852.

60. Pinho e Melo, T. M. V. D. Synthesis of Azides. In Organic Azides, John Wiley \& Sons, Ltd: 2010; pp 53-94.

61. Fry, A. J. J. Org. Chem. 2015, 80 (8), 3758-3765.

62. Olivier, N. B.; Altman, R. B.; Noeske, J.; Basarab, G. S.; Code, E.; Ferguson, A. D.; Gao, N.; Huang, J.; Juette, M. F.; Livchak, S.; Miller, M. D.; Prince, D. B.; Cate, J. H. D.; Buurman, E. T.; Blanchard, S. C. Proceedings of the National Academy of Sciences 2014, 111 (46), 16274-16279.

63. Kashiwagi, T.; Kotani, S.; Nakajima, M.; Sugiura, M. Tetrahedron Lett. (0).

64. (a) Kung, H. F.; Guo, Y. Z.; Yu, C. C.; Billings, J.; Subramanyam, V.; Calabrese, J. C. J. Med. Chem. 1989, 32 (2), 433-437; (b) Kempf, D. J.; Marsh, K. C.; Paul, D. A.; Knigge, M. F.; Norbeck, D. W.; Kohlbrenner, W. E.; Codacovi, L.; Vasavanonda, S.; Bryant, P.; Wang, X. C.; et al. Antimicrob. Agents Chemother. 1991, 35 (11), 2209-14; (c) Vickery, K.; Bonin, A. M.; Fenton, R. R.; O'Mara, S.; Russell, P. J.; Webster, L. K.; Hambley, T. W. J. Med. Chem. 1993, 36 (23), 3663-3668; (d) Kammermeier, T.; Wiegrebe, W. Arch Pharm (Weinheim) 1995, 328 (5), 409-15; (e) Jahn, T.; König, G. M.; Wright, A. D.; Wörheide, G.; Reitner, J. Tetrahedron Lett. 1997, 38 (22), 3883-3884; (f) Bergeron, R. J.; Feng, Y.; Weimar, W. R.; McManis, J. S.; Dimova, H.; Porter, C.; Raisler, B.; Phanstiel, O. J. Med. Chem. 1997, 40 (10), 1475-1494; (g) Franklin, A. S.; Ly, S. K.; Mackin, G. H.; Overman, L. E.; Shaka, A. J. J. Org. Chem. 1999, 64 (5), 1512-1519; (h) Cohen, F.; Overman, L. E. J. Am. Chem. Soc. 2001, 123 (43), 10782-10783; (i) Wehn, P. M.; Du Bois, J. J. Am. Chem. Soc. 2002, 124 (44), 12950-12951.

65. (a) Vedejs, E.; Lee, N.; Sakata, S. T. J. Am. Chem. Soc. 1994, 116 (5), 2175-2176; (b) Vedejs, E.; Kruger, A. W.; Suna, E. J. Org. Chem. 1999, 64 (21), 7863-7870; (c) Vedejs, E.; Kruger, A. W.; Lee, N.; Sakata, S. T.; Stec, M.; Suna, E. J. Am. Chem. Soc. 2000, 122 (19), 4602-4607; (d) Sergeyev, S. Helv. Chim. Acta 2009, 92 (3), 415-444.

66. (a) Kammermeier, T.; Wiegrebe, W. Archiv der Pharmazie 1994, 327 (9), 563-570; (b) Grasa, G. A.; Zanotti-Gerosa, A.; Hems, W. P. J. Organomet. Chem. 2006, 691 (10), 23322334; (c) Hems, W. P.; Groarke, M.; Zanotti-Gerosa, A.; Grasa, G. A. Acc. Chem. Res. 2007, 40 (12), 1340-1347.

67. (a) Lu, S.-F.; Du, D.-M.; Xu, J.; Zhang, S.-W. J. Am. Chem. Soc. 2006, 128 (23), 74187419; (b) Braun, W.; Calmuschi-Cula, B.; Englert, U.; Höfener, K.; Alberico, E.; Salzer, A. Eur. J. Org. Chem. 2008, 2008 (12), 2065-2074; (c) Giampietro, N. C.; Wolfe, J. P. J. Am. Chem. 
Soc. 2008, 130 (39), 12907-11; (d) Ríos-Lombardía, N. s.; Busto, E.; García-Urdiales, E.; GotorFernández, V.; Gotor, V. J. Org. Chem. 2009, 74 (6), 2571-2574; (e) Dagousset, G.; Drouet, F.; Masson, G. r.; Zhu, J. Org. Lett. 2009, 11 (23), 5546-5549; (f) Terada, M.; Machioka, K.; Sorimachi, K. Angew. Chem., Int. Ed. 2009, 48 (14), 2553-2556; (g) Kurokawa, T.; Kim, M.; Du Bois, J. Angew. Chem., Int. Ed. 2009, 48 (15), 2777-2779.

68. Kizirian, J.-C. Chem. Rev. 2007, 108 (1), 140-205.

69. Cannarsa, M. J. Chemistry and Industry 1996, 374 - 378.

70. (a) Alexakis, A.; Lensen, N.; Tranchier, J. P.; Mangeney, P. J. Org. Chem. 1992, 57 (17), 4563-4565; (b) Denmark, S. E.; Kim, J.-H. Synthesis 1992, 1992 (01/02), 229-234.

71. Barluenga, J.; Olano, B.; Fustero, S. J. Org. Chem. 1983, 48 (13), 2255-2259.

72. Enders, D.; Jegelka, U.; Dücker, B. Angew. Chem., Int. Ed. Engl. 1993, 32 (3), 423-425.

73. Barluenga, J.; Tomás, M.; Kouznetsov, V.; Jardón, J.; Rubio, E. Synlett 1991, 1991 (11), 821-823.

74. $\quad$ Merla, B.; Arend, M.; Risch, N. Synlett 1997, 1997 (02), 177-178.

75. Zhao, C.-H.; Liu, L.; Wang, D.; Chen, Y.-J. Eur. J. Org. Chem. 2006, 2006 (13), $2977-$ 2986.

76. Morgen, M.; Bretzke, S.; Li, P.; Menche, D. Org. Lett. 2010, 12 (20), 4494-4497.

77. Trost, B. M.; Malhotra, S.; Olson, D. E.; Maruniak, A.; Du Bois, J. J. Am. Chem. Soc. 2009, 131 (12), 4190-4191.

78. Bosque, I.; González-Gómez, J. C.; Guijarro, A.; Foubelo, F.; Yus, M. J. Org. Chem 2012, 77 (22), 10340-10346.

79. $\quad$ Kumar, P.; Jha, V.; Gonnade, R. J. Org. Chem. 2013, 78 (23), 11756-11764.

80. He, Z.-Q.; Zhou, Q.; Wu, L.; Chen, Y.-C. Adv. Synth. Catal. 2010, 352 (11-12), 19041908.

81. Thottumkara, A. P.; Parsons, W. H.; Du Bois, J. Angew. Chem., Int. Ed. 2014, 53 (23), $5760-5784$.

82. Umezawa, S. Structures and Syntheses of Aminoglycoside Antibiotics. In $A d v$. Carbohydr. Chem. Biochem., Tipson, R. S.; Derek, H., Eds. Academic Press: 1974; Vol. Volume 30, pp 111-182.

83. Hanessian, S.; Roy, R. J. Am. Chem. Soc. 1979, 101 (19), 5839-5841.

84. Rodríguez, J.; Jiménez, C.; Blanco, M.; Tarazona, G.; Fernández, R.; Cuevas, C. Org. Lett. 2016, 18 (22), 5832-5835.

85. (a) Heathcock, C. H.; Kleinman, E. F.; Binkley, E. S. J. Am. Chem. Soc. 1982, 104 (4), 1054-1068; (b) Kitajima, M.; Takayama, H. Lycopodium Alkaloids: Isolation and Asymmetric Synthesis. In Alkaloid Synthesis, Knölker, H.-J., Ed. Springer Berlin Heidelberg: Berlin, Heidelberg, 2012; pp 1-31.

86. Morita, H.; Hirasawa, Y.; Shinzato, T.; Kobayashi, J. i. Tetrahedron 2004, 60 (33), 7015-7023.

87. Bödeker, K. Justus Liebigs Annalen der Chemie 1881, 208 (3), 363-367.

88. Ma, X.; Gang, D. R. Nat. Prod. Rep. 2004, 21 (6), 752-772.

89. Ishida, H.; Kimura, S.; Kogure, N.; Kitajima, M.; Takayama, H. Org. Biomol. Chem. 2015, 13 (28), 7762-7771.

90. (a) Ayer, W. A.; Bowman, W. R.; Joseph, T. C.; Smith, P. J. Am. Chem. Soc. 1968, 90 (6), 1648-1650; (b) Maclean, D. B. Chapter 4 The Lycopodium Alkaloids. In The Alkaloids: Chemistry and Physiology, Manske, R. H. F., Ed. Academic Press: 1968; Vol. Volume 10, pp 305-382; (c) Stork, G.; Kretchmer, R. A.; Schlessinger, R. H. J. Am. Chem. Soc. 1968, 90 (6), 1647-1648; (d) Kraus, G. A.; Hon, Y. S. J. Am. Chem. Soc. 1985, 107 (14), 4341-4342; (e) Padwa, A.; Brodney, M. A.; Marino, J. P.; Sheehan, S. M. J. Org. Chem. 1997, 62 (1), 78-87; (f) Grieco, P. A.; Dai, Y. J. Am. Chem. Soc. 1998, 120 (20), 5128-5129; (g) Mori, M.; Hori, K.; Akashi, M.; Hori, M.; Sato, Y.; Nishida, M. Angew. Chem. 1998, 110 (5), 659-661; (h) Mori, M.; Hori, K.; Akashi, M.; Hori, M.; Sato, Y.; Nishida, M. Angew. Chem., Int. Ed. 1998, 37 (5), 636-637. 
91. Evans, D. A.; Scheerer, J. R. Angew. Chem., Int. Ed. 2005, 44 (37), 6038-6042.

92. Marion, L.; Manske, R. H. F. Canadian Journal of Research 1948, $26 b$ (1), 1-2.

93. Nishikawa, Y.; Kitajima, M.; Takayama, H. Org. Lett. 2008, 10 (10), 1987-1990.

94. Ayer, W. A.; Jenkins, J. K.; Valverde-Lopez, S.; Burnell, R. H. Can. J. Chem. 1967, 45 (5), 433-443.

95. Ayer, W. A.; Jenkins, J. K.; Piers, K.; Valverde-Lopez, S. Can. J. Chem. 1967, 45 (5), 445-450.

96. (a) Ayer, W. A.; Jenkins, J. K.; Valverde-Lopez, S.; Burnell, R. H. Tetrahedron Lett. 1964, 5 (32), 2201-2209; (b) Ayer, W. A.; Piers, K. Can. J. Chem. 1967, 45 (5), 451-459.

97. Chandra, A.; Pigza, J. A.; Han, J.-S.; Mutnick, D.; Johnston, J. N. J. Am. Chem. Soc. 2009, 131 (10), 3470-3471.

98. Knölker, H.-J. A. S. K. Alkaloid Synthesis. www.springerlink.com/content/978-3-64225528-1/contents/.

99. Nishikawa, Y.; Kitajima, M.; Kogure, N.; Takayama, H. Tetrahedron 2009, 65, 1608.

100. Veerasamy, N.; Carlson, E. C.; Carter, R. G. Org. Lett. 2012, 14 (6), 1596-1599.

101. Enamorado, M. F.; Connelly, C. M.; Deiters, A.; Comins, D. L. Tetrahedron Lett. 2015, 56 (23), 3683-3685.

102. Fürst, A.; Plattner, P. A. Helv. Chim. Acta 1949, 32 (1), 275-283.

103. Sugiura, M.; Mori, C.; Kobayashi, S. J. Am. Chem. Soc. 2006, 128 (34), 11038-11039.

104. Veerasamy, N.; Carter, R. G. Tetrahedron 2016, 72 (33), 4989-5001.

105. Comins, D. L.; LaMunyon, D. H. J. Org. Chem. 1992, 57 (22), 5807-5809.

106. (a) Khau, V. V.; Martinelli, M. J. Tetrahedron Lett. 1996, 37 (25), 4323-4326; (b) Hong, X.; Küçük, H. B.; Maji, M. S.; Yang, Y.-F.; Rueping, M.; Houk, K. N. J. Am. Chem. Soc. 2014, 136 (39), 13769-13780; (c) K. Gallos, J.; E. Koumbis, A.; E. Apostolakis, N. Journal of the Chemical Society, Perkin Transactions 1 1997, (17), 2457-2460; (d) Mish, M. R.; Guerra, F. M.; Carreira, E. M. J. Am. Chem. Soc. 1997, 119 (35), 8379-8380; (e) Jäger, V.; Bierer, L.; Dong, H.-Q.; Palmer, A. M.; Shaw, D.; Frey, W. J. Heterocycl. Chem. 2000, 37 (3), 455-465; (f) Kobayashi, S.; Shimizu, H.; Yamashita, Y.; Ishitani, H.; Kobayashi, J. J. Am. Chem. Soc. 2002, 124 (46), 13678-13679; (g) Kobayashi, S.; Hirabayashi, R.; Shimizu, H.; Ishitani, H.; Yamashita, Y. Tetrahedron Lett. 2003, 44 (16), 3351-3354; (h) Huisgen, R.; Grashey, R.; Sauer, J. Cycloaddition reactions of alkenes. In The Alkenes (1964), John Wiley \& Sons, Ltd.: 2010; pp 739-953; (i) Yamashita, Y.; Kobayashi, S. J. Am. Chem. Soc. 2004, 126 (36), 11279-11282. 107. Ellis, J. M.; King, S. B. Tetrahedron Lett. 2002, 43 (33), 5833-5835.

108. (a) Pyrazolidine Chemistry. In Chemistry of Heterocyclic Compounds, John Wiley \& Sons, Inc.: 2008; pp 279-285; (b) Lykke, L.; Carlsen, B. D.; Rambo, R. S.; Jørgensen, K. A. J. Am. Chem. Soc. 2014, 136 (32), 11296-11299; (c) Izquierdo, C.; Esteban, F.; Parra, A.; Alfaro, R.; Alemán, J.; Fraile, A.; Ruano, J. L. G. J. Org. Chem. 2014, 79 (21), 10417-10433.

109. Ma, S.; Jiao, N.; Zheng, Z.; Ma, Z.; Lu, Z.; Ye, L.; Deng, Y.; Chen, G. Org. Lett. 2004, 6 (13), 2193-2196.

110. (a) Starostin, E. K.; Furman, D. B.; Ignatenko, A. V.; Barkova, A. P.; Nikishin, G. I. Russian Chemical Bulletin 2006, 55 (11), 2016-2019; (b) Michalak, M.; Wicha, J. Org. Biomol. Chem. 2011, 9 (9), 3439-3446; (c) De La Mare, H. E.; Kochi, J. K.; Rust, F. F. J. Am. Chem. Soc. 1963, 85 (10), 1437-1449.

111. Bates, R. W.; Sridhar, S. J. Org. Chem. 2011, 76 (12), 5026-5035.

112. Hayashi, Y.; Sumiya, T.; Takahashi, J.; Gotoh, H.; Urushima, T.; Shoji, M. Angew. Chem., Int. Ed. 2006, 45 (6), 958-961.

113. (a) Michaelis, A.; Kaehne, R. Berichte der deutschen chemischen Gesellschaft 1898, 31 (1), 1048-1055; (b) Arbusow, B. A. Michaelis-Arbusow- und Perkow-Reaktionen. In Pure Appl. Chem., 1964; Vol. 9, p 307.

114. Jitsuo, K.; Tamon, O.; Yukiko, H.; Yasuyuki, F. Bull. Chem. Soc. Jpn. 1996, 69 (4), 1029-1031. 
115. Knudsen, C. G.; Chandraratna, R. A. S.; Walkeapaa, L. P.; Chauhan, Y. S.; Carey, S. C.; Cooper, T. M.; Birge, R. R.; Okamura, W. H. J. Am. Chem. Soc. 1983, 105 (6), 1626-1631. 116. (a) Wang, Z. Wohl-Ziegler Bromination. In Comprehensive Organic Name Reactions and Reagents, John Wiley \& Sons, Inc.: 2010; (b) Carreno, M. C.; Garcia Ruano, J. L.; Sanz, G.; Toledo, M. A.; Urbano, A. The Journal of Organic Chemistry 1995, 60 (16), 5328-5331.

117. (a) Paterson, I.; Anderson, E. A.; Dalby, S. M.; Lim, J. H.; Maltas, P.; Moessner, C. Chem. Commun. 2006, (40), 4186-4188; (b) Paterson, I.; Yeung, K.-S.; Smaill, J. B. Synlett 1993, 1993 (10), 774-776; (c) Paterson, I.; Haslett, G. W. Org. Lett. 2013, 15 (6), 1338-1341; (d) O'Nei, G. W.; Phillips, A. J. J. Am. Chem. Soc. 2006, 128 (16), 5340-5341; (e) Sinisterra, J. V.; Mouloungui, Z.; Delmas, M.; Gaset, A. Synthesis 1985, 1985 (12), 1097-1100.

118. Hayashi, Y.; Kanayama, J.; Yamaguchi, J.; Shoji, M. J. Org. Chem. 2002, 67 (26), 9443-9448.

119. (a) Aponick, A.; Li, C.-Y.; Biannic, B. Org. Lett. 2008, 10 (4), 669-671; (b) White, J. D.; Kranemann, C. L.; Kuntiyong, P. Org. Lett. 2001, 3 (25), 4003-4006; (c) Cossy, J.; Guérinot, A. Chapter Five - Natural Products Containing Oxygen Heterocycles-Synthetic Advances Between 1990 and 2015. In Adv. Heterocycl. Chem., Eric, F. V. S.; Christopher, A. R., Eds. Academic Press: 2016; Vol. Volume 119, pp 107-142.

120. Yeung, K.-S.; Paterson, I. Chemical Reviews 2005, 105 (12), 4237-4313.

121. Ai, Y.; Kozytska, M. V.; Zou, Y.; Khartulyari, A. S.; Smith, A. B. J. Am. Chem. Soc. 2015, 137 (49), 15426-15429.

122. Lowe, J. T.; Wrona, I. E.; Panek, J. S. Org. Lett. 2007, 9 (2), 327-330.

123. Kozmin, S. A. Org. Lett. 2001, 3 (5), 755-758.

124. Skepper, C. K.; Quach, T.; Molinski, T. F. J. Am. Chem. Soc. 2010, 132 (30), 1028610292.

125. Kang, S. H.; Kang, S. Y.; Kim, C. M.; Choi, H.-w.; Jun, H.-S.; Lee, B. M.; Park, C. M.; Jeong, J. W. Angew. Chem., Int. Ed. 2003, 42 (39), 4779-4782.

126. (a) Williams, D. R.; Kiryanov, A. A.; Emde, U.; Clark, M. P.; Berliner, M. A.; Reeves, J. T. Angew. Chem., Int. Ed. 2003, 42, 1258; (b) Smith, A. B.; Zhu, W.; Shirakami, S.; Sfouggatakis, C.; Doughty, V. A.; Bennett, C. S.; Sakamoto, Y. Org. Lett. 2003, 5, 761; (c) Pattenden, G.; González, M. A.; Little, P. B.; Millan, D. S.; Plowright, A. T.; Tornos, J. A.; Ye, T. Org. Biomol. Chem. 2003, 1, 4173.

127. Lee, E. C., S. J.; Kim. H.; Han, H. O.; Kim, Y. K.; Min, S. J.; Son, S .H.; Lim, S. M.; Jang, S. W. Angew. Chem. Int. Ed. 2002, 41 (1), 176-178.

128. Duan, S.; Moeller, K. D. J. Am. Chem. Soc. 2002, 124 (32), 9368-9369.

129. (a) Ding, F.; Jennings, M. P. Org. Lett. 2005, 7 (12), 2321-2324; (b) Boulard, L.; BouzBouz, S.; Cossy, J.; Franck, X.; Figadère, B. Tetrahedron Lett. 2004, 45 (35), 6603-6605; (c) Evans, P. A.; Cui, J.; Gharpure, S. J. Org. Lett. 2003, 5 (21), 3883-3885; (d) Carreño, M. C.; Des Mazery, R.; Urbano, A.; Colobert, F.; Solladié, G. J. Org. Chem. 2003, 68 (20), 7779-7787. 130. (a) Nicolaou, K. C.; Prasad, C. V. C.; Somers, P. K.; Hwang, C. K. J. Am. Chem. Soc. 1989, 111, 5330; (b) Larrosa, I.; Romea, P.; Urpí, F. Tetrahedron 2008, 64 (12), 2683-2723.

131. (a) Crimmins, M. T.; Siliphaivanh, P. Org. Lett. 2003, 5, 4641; (b) Evans, D. A.; Ripin, D. H. B.; Halstead, D. P.; Campos, K. R. J. Am. Chem. Soc. 1999, 121, 6816; (c) Fettes, A.; Carreira, E. J. Org. Chem. 2003, 68, 9274; (d) Paterson, I.; Chen, D. Y. K.; Coster, M. J.; Aceña, J. L.; Bach, J.; Gibson, K. R.; Keown, L. E.; Oballa, R. M.; Trieselmann, T.; Wallace, D. J.; Hodgson, A. P.; Norcross, R. D. Angew. Chem., Int. Ed. 2001, 40, 4055; (e) Paterson, I.; Arnott, E. A. Tetrahedron Lett. 1998, 39, 7185.

132. (a) Paterson, I.; Tudge, M. Angew. Chem., Int. Ed. 2003, 42, 343; (b) Forsyth, C. J.; Ahmed, F.; Cink, R. D.; Lee, C. S. J. Am. Chem. Soc. 1998, 120, 5597; (c) Smith, A. B.; Minbiole, K. P.; Verhoest, P. R.; Schelhaas, M. J. Am. Chem. Soc. 2001, 123 (44), 10942-10953. 133. (a) Marumoto, S.; Jaber, J. J.; Vitale, J. P.; Rychnovsky, S. D. Org. Lett. 2002, 4 (22), 3919-3922; (b) Rychnovsky, S. D.; Thomas, C. R. Org. Lett. 2000, 2, 1217; (c) Vitale, J. P.; Wolckenhauer, S. A.; Do, N. M.; Rychnovsky, S. D. Org. Lett. 2005, 7, 3255. 
134. Perry, M. A.; Rychnovsky, S. D.; Sizemore, N. Synthesis of Saturated Tetrahydropyrans. In Synthesis of Saturated Oxygenated Heterocycles I: 5- and 6-Membered Rings, Cossy, J., Ed. Springer Berlin Heidelberg: Berlin, Heidelberg, 2014; pp 43-95.

135. Trost, B. M.; Toste, F. D. J. Am. Chem. Soc. 1999, 121, 4545.

136. Hansen, E. C.; Lee, D. Tetrahedron Lett. 2004, 45 (38), 7151-7155.

137. Semmelhack, M. F.; Zhang, N. J. Org. Chem. 1989, 54 (19), 4483-4485.

138. White, J. D.; Kuntiyong, P.; Lee, T. H. Org. Lett. 2006, 8 (26), 6039-6042.

139. Marshall, J. A.; Yanik, M. M. Tetrahedron Lett. 2000, 41, 4717.

140. Gallagher, P. O.; McErlean, C. S. P.; Jacobs, M. F.; Watters, D. J.; Kitching, W. Tetrahedron Lett. 2002, 43, 531.

141. Kawai, N.; Mahadeo Hande, S.; Uenishi, J. i. Tetrahedron 2007, 63 (37), 9049-9056.

142. Yang, C.-G.; Reich, N. W.; Shi, Z.; He, C. Org. Lett. 2005, 7 (21), 4553-4556.

143. Coulombel, L.; Favier, I.; Dunach, E. Chem. Commun. 2005, (17), 2286-2288.

144. Marotta, E.; Foresti, E.; Marcelli, T.; Peri, F.; Righi, P.; Scardovi, N.; Rosini, G. Org. Lett. 2002, 4 (25), 4451-4453.

145. Qian, H.; Han, X.; Widenhoefer, R. A. J. Am. Chem. Soc. 2004, 126 (31), 9536-9537.

146. Guérinot, A.; Serra-Muns, A.; Gnamm, C.; Bensoussan, C.; Reymond, S.; Cossy, J. Org. Lett. 2010, 12 (8), 1808-1811.

147. Oku, N.; Takada, K.; Fuller, R. W.; Wilson, J. A.; Peach, M. L.; Pannell, L. K.; McMahon, J. B.; Gustafson, K. R. J. Am. Chem. Soc. 2010, 132 (30), 10278-10285.

148. (a) Roskoski, R., Jr. Biochem. Biophys. Res. Commun. 2005, 338 (3), 1307-15; (b) Ronnstrand, L. Cell. Mol. Life Sci. 2004, 61 (19-20), 2535-48.

149. Taylor, M. L.; Metcalfe, D. D. Hematol. Oncol. Clin. North Am. 2000, 14, 517.

150. Ahlers, A.; de Haro, T.; Gabor, B.; Fürstner, A. Angew. Chem., Int. Ed. 2016, 55 (4), 1406-1411.

151. Kishi, T. F., Y.; Takamura, H.; Kadota, I. HETEROCYCES 2014, 89 (2), 515.

152. Negishi, E.; Van Horn, D. E.; King, A. O.; Okukado, N. Synthesis 1979, 501.

153. (a) Anderson, B. A.; Becke, L. M.; Booher, R. N.; Flaugh, M. E.; Harn, N. K.; Kress, T. J.; Varie, D. L.; Wepsiec, J. P. J. Org. Chem. 1997, 62, 8634; (b) Bobeck, D. R.; Warner, D. L.; Vedejs, E. J. Org. Chem. 2007, 72, 8506; (c) Mulder, R. J.; Shafer, C. M.; Molinski, T. F. J. Org. Chem. 1999, 64, 4995.

154. Krasovskiy, A.; Malakhov, V.; Gavryushin, A.; Knochel, P. Angew. Chem., Int. Ed. 2006, 45, 6040 .

155. (a) Duboudin, J. G.; Jousseaume, B.; Bonakdar, A. J. Organomet. Chem. 1979, 168, 227; (b) Wiemer, D. F.; Han, Q. J. Am. Chem. Soc. 1992, 114, 7692; (c) Larock, R. C.; Doty, M. J.; Han, X. J. Org. Chem. 1999, 64, 8770.

156. (a) Kim, H. Y.; Lurain, A. E.; Gacia-Garcia, P.; Carroll, P. J.; Walsh, P. J. J. Am. Chem. Soc. 2005, 127, 13138; (b) Nugent, W. A. J. Chem Soc., Chem. Commun. 1997, 1369; (c) A. Nugent, W. Chem. Commun. 1999, (15), 1369-1370.

157. Corey, E. J.; Imwinkelried, R.; Pikul, S.; Xiang, Y. B. J. Am. Chem. Soc. 1989, 111 (14), 5493-5495.

158. Narasaka, K.; Pai, F. C. Tetrahedron 1984, 40, 2233.

159. (a) Chen, K. M.; Hardtmann, G. E.; Prasad, K.; Repic, O.; Shapiro, M. J. Tetrahedron Lett. 1987, 28, 155; (b) Rychnovsky, S. D.; Rogers, B.; Yang, G. J. Org. Chem. 1993, 58, 3511.

160. (a) Roush, W. R.; Walts, A. E.; Hoong, L. K. J. Am. Chem. Soc. 1985, 107, 8186; (b) Roush, W. R.; Palkowitz, A. D.; Ando, K. J. Am. Chem. Soc. 1990, 112, 6348.

161. Hirayama, L. C.; Gamsey, S.; Knueppel, D.; Steiner, D.; DeLaTorre, K.; Singaram, B. Tetrahedron Lett. 2005, 46, 2315.

162. Hafner, A.; Duthaler, R. O.; Marti, R.; Rihs, G.; Rothe Streit, P.; Schwarzenbach, F. J. Am. Chem. Soc. 1992, 114, 2321.

163. Dossetter, A. G.; Jamison, T. F.; Jacobsen, E. N. Angew. Chem., Int. Ed. 1999, 38, 2398. 164. Mukaiyama, T.; Usui, M.; Saigo, K. Chem. Lett. 1976, 49. 
165. Inanaga, J.; Hirata, K.; Saeki, H.; Katsuki, T.; Yamaguchi, M. Bull. Chem. Soc. Jpn. 1979, 52, 1989.

166. Hikota, M.; Tone, H.; Horita, K.; Yonemitsu, O. J. Org. Chem. 1990, 55, 7.

167. Shiina, I.; Kubota, M.; Oshiumi, H.; Hashizume, M. J. Org. Chem. 2004, 69, 1822.

168. (a) Smith, A. B.; Boldi, A. M. J. Am. Chem. Soc. 1997, 119, 6925; (b) Smith, A. B.; Pitram, S. M.; Boldi, A. M.; Gaunt, M. J.; Sfouggatakis, C.; Moser, W. H. J. Am. Chem. Soc. 2003, 125, 14435; (c) Smith, A. B.; Xian, M. J. Am. Chem. Soc. 2006, 128, 66; (d) Smith, A. B.; Wuest, W. M. Chem. Commun. 2008, 5883.

169. Smith, I. I. I. A. B.; Wuest, W. M. Chem. Commun. 2008, (45), 5883-5895.

170. (a) Hicks, D. R.; Fraser-Reid, B. Synthesis 1974, 1974, 203; (b) Hicks, D. R.; FraserReid, B. Synthesis 1974, 1974 (03), 203-203; (c) Corey, E. J.; Weigel, L. O.; Chamberlin, A. R.; Lipshutz, B. J. Am. Chem. Soc. 1980, 102 (4), 1439-1441.

171. (a) Smith, A. B.; Safonov, I. G.; Corbett, R. M. J. Am. Chem. Soc. 2002, 124, 11102; (b) Smith, A. B.; Mesaros, E. F.; Meyer, E. A. J. Am. Chem. Soc. 2006, 128, 5292; (c) Smith, A. B.; Simov, V. Org. Lett. 2006, 8, 3315; (d) Smith, A. B.; Basu, K.; Bosanac, T. J. Am. Chem. Soc. 2007, 129, 14872.

172. Corey, E. J.; Lee, T. W. Chem. Commun. 2001, (15), 1321-1329.

173. Schmitt, D. C.; Dechert-Schmitt, A.-M. R.; Krische, M. J. Org. Lett. 2012, 14 (24), 6302-6305.

174. (a) Mukaiyama, T.; Banno, K.; Narasaka, K. J. Am. Chem. Soc. 1974, 96 (24), $7503-$ 7509; (b) Evans, D. A.; Dart, M. J.; Duffy, J. L.; Yang, M. G. J. Am. Chem. Soc. 1996, 118 (18), 4322-4343.

175. Pavia, D. L.; Lampman, G. M.; Kriz, G. S.; Vyvyan, J. A. Introduction to Spectroscopy. Cengage Learning: 2008.

176. (a) Petrier, C.; Luche, J. L. J. Org. Chem. 1985, 50 (6), 910-912; (b) Luche, J. L. J. Am. Chem. Soc. 1978, 100 (7), 2226-2227.

177. Wipf, P.; Spencer, S. R. J. Am. Chem. Soc. 2005, 127 (1), 225-235.

178. (a) Basireddy, V. S. R.; V, H. R.; Medaboina, D.; Balasubramanian, S.; Reddy, Y. V. R. Org. Biomol. Chem. 2016; (b) Aubele, D. L.; Wan, S.; Floreancig, P. E. Angew. Chem., Int. Ed. 2005, 44 (22), 3485-3488.

179. Likhtenshtein, G. Stilbene Materials. In Stilbenes, Wiley-VCH Verlag GmbH \& Co. KGaA: 2009; pp 159-188.

180. Likhtenshtein, G. Stilbenes: Applications in Chemistry, Life Sciences and Materials Science. 2010.

181. Itami, K.; Tonogaki, K.; Nokami, T.; Ohashi, Y.; Yoshida, J.-i. Angew. Chem., Int. Ed. 2006, 45 (15), 2404-2409.

182. (a) Hart, J. H. Annu. Rev. Phytopathol. 1981, 19, 437; (b) Burns, J.; Yokota, T.; Ashihara, H.; Lean, M. E. J.; Crozier, A. J. Agr. Food Chem. 2002, 50, 3337.

183. (a) Jordan, V. C. J. Med. Chem. 2003, 46 (7), 1081-1111; (b) Jordan, V. C. J. Med. Chem. 2003, 46 (6), 883-908; (c) Salih, A. K.; Fentiman, I. S. Cancer Treatment Reviews 27 (5), 261-273; (d) Fisher, B.; Costantino, J. P.; Wickerham, D. L.; Redmond, C. K.; Kavanah, M.; Cronin, W. M.; Vogel, V.; Robidoux, A.; Dimitrov, N.; Atkins, J.; Daly, M.; Wieand, S.; Tan-Chiu, E.; Ford, L.; Wolmark, N.; Breast, o. N. S. A.; Investigators, B. P. Journal of the National Cancer Institute 1998, 90 (18), 1371-1388; (e) Kaur, G.; Mahajan, M. P.; Pandey, M. K.; Singh, P.; Ramisetti, S. R.; Sharma, A. K. Bioorg. Med. Chem. Lett. 2016, 26 (8), $1963-$ 1969.

184. Uddin, M. J.; Rao, P. N. P.; Knaus, E. E. Bioorg. Med. Chem. 2004, 12 (22), 5929-5940.

185. Itami, K.; Tonogaki, K.; Ohashi, Y.; Yoshida, J.-i. Org. Lett. 2004, 6 (22), 4093-4096.

186. Zhang, X.; Chi, Z.; Xu, B.; Li, H.; Yang, Z.; Li, X.; Liu, S.; Zhang, Y.; Xu, J. Dyes Pigm. 2011, 89 (1), 56-62.

187. Zhang, X.-q.; Chi, Z.-g.; Xu, B.-j.; Li, H.-y.; Zhou, W.; Li, X.-f.; Zhang, Y.; Liu, S.-w.; $\mathrm{Xu}$, J.-r. Journal of Fluorescence 2011, 21 (1), 133-140. 
188. Flynn, A. B.; Ogilvie, W. W. Chemical Reviews 2007, 107 (11), 4698-4745.

189. (a) Mc Murry, J. E. Chem. Rev. 1989, 89, 1513; (b) Ephritikhine, M. Chem. Commun. 1998, 2549.

190. (a) Meerwein, H.; Buchner, E.; van Emster, K. J. Prakt. Chem. 1939, 152, 237; (b) Rondestvedt, C. S. Org. React. 1976, 24, 225; (c) Hari, D. P.; König, B. Angew. Chem., Int. Ed. 2013, 52, 2.

191. (a) Hilt, G.; Hengst, C. J. Org. Chem. 2007, 72, 7337; (b) Mc Nulty, J.; Mc Leod, D. Tetrahedron Lett. 2013, 54, 6303; (c) Saiyed, A. S.; Patel, K. N.; Kamath, B. V.; Bedekar, A. V. Tetrahedron Lett. 2012, 53, 4692.

192. Orelli, L. R.; Bisceglia, J. Á. Curr. Org. Chem. 2012, 16, 2206.

193. (a) Blakemore, P. R. J. J. Chem. Soc., Perkin Trans. 1 2002, 2563; (b) Aïssa, C. Eur. J. Org. Chem. 2009, 1831; (c) Markó, I. E.; Pospíšil, J.; de Meijere, A. Science of Synthesis, Vol 47a: Alkenes. 2009; p 105.

194. Hojo, M.; Murakami, Y.; Aihara, H.; Sakuragi, R.; Baba, Y.; Hosomi, A. Angew. Chem., Int. Ed. 2001, 40, 621.

195. Shimizu, M.; Fujimoto, T.; Minezaki, H.; Hata, T.; Hiyama, T. J. Am. Chem. Soc. 2001, $123,6947$.

196. Itami, K.; Nokami, T.; Ishimura, Y.; Mitsudo, K.; Kamei, T.; Yoshida, J. J. Am. Chem. Soc. 2001, 123, 11577.

197. Marino, J. P.; Nguyen, H. N. J. Org. Chem. 2002, 67, 6291.

198. (a) Shindo, M.; Sato, Y.; Shishido, K. Tetrahedron Lett. 1998, 39, 4857; (b) Shindo, M.; Sato, Y.; Shishido, K. J. Org. Chem. 2000, 65, 5443; (c) Shindo, M.; Sato, Y.; Yoshikawa, T.; Koretsune, R.; Shishido, K. J. Org. Chem. 2004, 69, 3912; (d) Shindo, M.; Matsumoto, K.; Mori, S.; Shishido, K. J. Am. Chem. Soc. 2002, 124, 6840.

199. (a) Spino, C.; Thibault, C.; Gingras, S. J. Org. Chem. 1998, 63, 5283; (b) Nakatani, K.; Izawa, T.; Isoe, S. J. Org. Chem. 1994, 59, 5961; (c) Nakatani, K.; Izawa, T.; Odagaki, Y.; Isoe, S. J. Chem. Soc., Chem. Commun. 1993, 1365; (d) Gansauer, A.; Pierobon, M.; Bluhm, H. Angew. Chem., Int. Ed. 2002, 41, 3206.

200. de Meijere, A.; Diederich, F. Metal-Catalyzed Cross-Coupling Reactions. 2004.

201. Cherney, A. H.; Kadunce, N. T.; Reisman, S. E. Chemical Reviews 2015, 115 (17), 9587-9652.

202. Suzuki, A. Pure. Appl. Chem. 1985, 57, 1749.

203. Heck, R. F. Org. React. (N.Y.) 1982, 27, 345.

204. Stille, J. K. Angew. Chem., Int. Ed. Engl. 1986, 25, 508.

205. Tamao, K.; Sumitani, K.; Kumada, M. J. Am. Chem. Soc. 1972, 94, 4374.

206. King, A. O.; Okukado, N.; Negishi, E. i. J. Chem. Soc., Chem. Commun. 1977, 683.

207. Hiyama, T.; Wakasa, N. Tetrahedron Lett. 1985, 26, 3259.

208. Sonogashira, K.; Tohda, Y.; Hagihara, N. Tetrahedron Lett. 1975, 16, 4467.

209. (a) Miller, R. B.; Al-Hassan, M. I. J. Org. Chem. 1985, 50, 2121; (b) Cummins, C. H. Synth. Commun. 1995, 25, 4071; (c) Studemann, T.; Ibrahim-Ouali, M.; Knochel, P. Tetrahedron 1998, 54, 1299; (d) Brown, S. D.; Armstrong, R. W. J. Org. Chem. 1997, 62, 7076. 210. Takahashi, T.; Xi, C.; Ura, Y.; Nakajima, K. J. Am. Chem. Soc. 2000, 122 (13), 32283229 .

211. Shimizu, K.; Takimoto, M.; Mori, M.; Sato, Y. Synlett 2006, 2006 (18), 3182-3184.

212. Xi, C.; Huo, S.; Mahmaoud, A.; Hara, R.; Takahashi, T. Tetrahedron Lett. 1997, 38, 4099.

213. Kamei, T.; Itami, K.; Yoshida, J.-i. Adv. Synth. Catal. 2004, 346 (13-15), 1824-1835.

214. Wipf, P.; Jahn, H. Tetrahedron 1996, 52, 12853.

215. Tsuji, H.; Ueda, Y.; Ilies, L.; Nakamura, E. J. Am. Chem. Soc. 2010, 132 (34), 1185411855. 
216. (a) Corriu, R. J. P.; Masse, J. P. J. Chem. Soc., Chem. Commun. 1972, 144a; (b) Murahashi, S.; Yamamura, M.; Yanagisawa, K.; Mita, N.; Kondo, K. J. Org. Chem. 1979, 44, 2408.

217. (a) Yeung, C. S.; Dong, V. M. Chem. Rev. 2011, 111, 1215; (b) Arockiam, P. B.; Bruneau, C.; Dixneuf, P. H. Chemical Reviews 2012, 112 (11), 5879-5918; (c) Lyons, T. W.; Sanford, M. S. Chem. Rev. 2010, 110, 1147; (d) Ackermann, L. Chem. Rev. 2011, 111, 1315; (e) Colby, D. A.; Bergman, R. G.; Ellman, J. A. Chem. Rev. 2010, 110, 624; (f) Ackermann, L. Acc. Chem. Res. 2014, 47 (2), 281-295; (g) Jia, C.; Piao, D.; Oyamada, J.; Lu, W.; Kitamura, T.; Fujiwara, Y. Science 2000, 287 (5460), 1992-1995; (h) Yamamoto, Y. Chem. Soc. Rev. 2014, 43 (5), 1575-1600; (i) Min, M.; Kim, D.; Hong, S. Chem. Commun. 2014, 50 (59), 8028-8031. 218. Nevado, C.; Echavarren, A. M. Synthesis 2005, 2005 (02), 167-182.

219. Xu, X.; Chen, J.; Gao, W.; Wu, H.; Ding, J.; Su, W. Tetrahedron 2010, 66 (13), $2433-$ 2438.

220. Jia, C.; Lu, W.; Oyamada, J.; Kitamura, T.; Matsuda, K.; Irie, M.; Fujiwara, Y. J. Am. Chem. Soc. 2000, 122 (30), 7252-7263.

221. Reetz, Manfred T.; Sommer, K. Eur. J. Org. Chem. 2003, 2003 (18), 3485-3496.

222. Padala, K.; Jeganmohan, M. Chem. Commun. 2014, 50 (93), 14573-14576.

223. Manikandan, R.; Jeganmohan, M. Org. Biomol. Chem. 2015, 13 (42), 10420-10436.

224. Hu, F.; Szostak, M. Chem. Commun. 2016, 52 (62), 9715-9718.

225. Kakiuchi, F.; Yamamoto, Y.; Chatani, N.; Murai, S. Chem. Lett. 1995, 681.

226. Zhou, C.; Emrich, D. E.; Larock, R. C. Org. Lett. 2003, 5 (9), 1579-1582.

227. (a) Larock, R. C.; Yum, E. K.; Refvik, M. D. J. Org. Chem. 1998, 63, 7652; (b) Larock, R. C. Pure Appl. Chem. 1999, 71, 1453; (c) Roesch, K. R.; Zhang, H.; Larock, R. C. J. Org. Chem. 2001, 66, 8042.

228. (a) Cacchi, S. J. Organomet. Chem. 1999, 576, 42; (b) Arcadi, A.; Cacchi, S.; Fabrizi, G.; Marinelli, F.; Pace, P. Eur. J. Org. Chem. 2000, 4099; (c) Arcadi, A.; Cacchi, S.; Fabrizi, G.; Marinelli, F.; Pace, P. Eur. J. Org. Chem. 1999, 3305, 5.

229. Zhou, C.; Larock, R. C. Org. Lett. 2005, 7 (2), 259-262.

230. Jiao, J.; Hyodo, K.; Hu, H.; Nakajima, K.; Nishihara, Y. J. Org. Chem. 2014, 79 (1), 285-295.

231. (a) Eliel, E. L.; Wilen, S. H. Stereochemistry of Organic Compounds. 1994; p 696; (b) Kitching, W.; Olszowy, H. A.; Drew, G. M.; Adcock, W. J. Org. Chem. 1982, 47, 5153.

232. Shimizu, M.; Nakamaki, C.; Shimono, K.; Schelper, M.; Kurahashi, T.; Hiyama, T. J. Am. Chem. Soc. 2005, 127 (36), 12506-12507.

233. (a) Hata, T.; Kitagawa, H.; Masai, H.; Kurahashi, T.; Shimizu, M.; Hiyama, T. Angew. Chem., Int. Ed. 2001, 40, 790; (b) Kurahashi, T.; Hata, T.; Masai, H.; Kitagawa, H.; Shimizu, M.; Hiyama, T. Tetrahedron 2002, 58, 6381.

234. Nagao, K.; Ohmiya, H.; Sawamura, M. Org. Lett. 2015, 17 (5), 1304-1307.

235. Bauer, A.; Miller, M. W.; Vice, S. F.; McCombie, S. W. Synlett 2001, 2001 (02), 02540256.

236. Kotek, V.; Dvořáková, H.; Tobrman, T. Org. Lett. 2015, 17 (3), 608-611.

237. Kotek, V.; Polák, P.; Tobrman, T. Monatshefte für Chemie - Chemical Monthly 2016, 147 (2), 405-412.

238. Chen, J.; Chen, S.; Xu, X.; Tang, Z.; Au, C.-T.; Qiu, R. J. Org. Chem. 2016, 81 (8), 3246-3255.

239. Cahiez, G.; Moyeux, A.; Poizat, M. Chem. Commun. 2014, 50 (64), 8982-8984.

240. (a) Beller, M.; Riermeier, T. H. Tetrahedron Lett. 1996, 37, 6535; (b) Herrmann, W. A.; Brossmer, C.; Öfele, K.; Reisinger, C. P.; Priermeier, T.; Beller, M.; Fischer, H. Angew. Chem., Int. Ed. Engl. 1995, 34, 1844; (c) Littke, A. F.; Fu, G. C. Angew. Chem., Int. Ed. 1999, $38,2411$.

241. Morales-Morales, D.; Grause, C.; Kasaoka, K.; Redón, R. o.; Cramer, R. E.; Jensen, C. M. Inorg. Chim. Acta 2000, 300-302, 958-963. 
242. Ohff, M.; Ohff, A.; Boom, M. E.; Milstein, D. J. Am. Chem. Soc. 1997, 119, 11687.

243. Albisson, D. A.; Bedford, R. B.; Scully, P. N. Tetrahedron Lett. 1998, 39, 9793.

244. (a) Feuerstein, M.; Doucet, H.; Santelli, M. Tetrahedron Lett. 2002, 43 (12), 2191-2194;

(b) Berthiol, F.; Doucet, H.; Santelli, M. Eur. J. Org. Chem. 2003, 2003 (6), 1091-1096.

245. (a) Carri, W.; Candiani, I. Acc. Chem. Res. 1995, 28, 2; (b) de Meijere, A.; Meyer, F. E. Angew. Chem., Int. Ed. Engl. 1995, 33 (23-24), 2379-2411.

246. (a) Li, Y.; Liu, G.; Cao, C.; Wang, S.; Li, Y.; Pang, G.; Shi, Y. Tetrahedron 2013, 69 (30), 6241-6250; (b) Xu, D.; Lu, C.; Chen, W. Tetrahedron 2012, 68 (5), 1466-1474.

247. Nadri, S.; Joshaghani, M.; Rafiee, E. Organometallics 2009, 28 (21), 6281-6287.

248. Joshaghani, M.; Daryanavard, M.; Rafiee, E.; Nadri, S. J. Organomet. Chem. 2008, 693 , 3135 .

249. Beletskaya, I. P.; Cheprakov, A. V. Chemical Reviews 2000, 100 (8), 3009-3066.

250. Liu, P.; Pan, Y.-m.; Hu, K.; Huang, X.-c.; Liang, Y.; Wang, H.-s. Tetrahedron 2013, 69 (37), 7925-7930.

251. Redón, R.; García-Peña, N. G.; Ugalde-Saldivar, V. M.; García, J. J. J. Mol. Catal. A: Chem. 2009, 300 (1-2), 132-141.

252. Wu, S.; Ma, H.; Jia, X.; Zhong, Y.; Lei, Z. Tetrahedron 2011, 67, 250.

253. Sharma, D.; Kumar, S.; Shil, A. K.; Guha, N. R.; Bandna; Das, P. Tetrahedron Lett. 2012, 53 (52), 7044-7051.

254. Calò, V.; Nacci, A.; Monopoli, A.; Cotugno, P. Angew. Chem., Int. Ed. 2009, 48 (33), 6101-6103.

255. Woerly, E. M.; Struble, J. R.; Palyam, N.; O’Hara, S. P.; Burke, M. D. Tetrahedron 2011, 67 (24), 4333-4343.

256. Gillis, E. P.; Burke, M. D. Aldrichimica acta 2009, 42 (1), 17-27.

257. Uno, B. E.; Gillis, E. P.; Burke, M. D. Tetrahedron 2009, 65 (16), 3130-3138.

258. Lee, S. J.; Gray, K. C.; Paek, J. S.; Burke, M. D. J. Am. Chem. Soc. 2008, 130 (2), $466-$

468.

259. (a) Li, J.; Burke, M. D. J. Am. Chem. Soc. 2011, 133, 13774; (b) Nogushi, H.; Shioda, T.; Chou, C. M.; Suginome, M. Org. Lett. 2008, 10, 377; (c) Nogushi, H.; Hojo, K.; Suginome, M. J. Am. Chem. Soc. 2007, 129, 758; (d) Molander, G. A.; Sandrock, D. L. J. Am. Chem. Soc. 2008, 130, 15792; (e) Molander, G. A.; Felix, L. A. J. Org. Chem. 2005, 70, 3950; (f) Close, A. J.; Kemmitt, P.; Emmerson, M. K.; Spencer, J. Tetrahedron 2014, 70, 9125; (g) Knapp, D. M.; Gillis, E. P.; Burke, M. D. J. Am. Chem. Soc. 2009, 131, 6961; (h) Gillis, E. P.; Burke, M. D. J. Am. Chem. Soc. 2007, 129, 6716; (i) Gillis, E. P.; Burke, M. D. J. Am. Chem. Soc. 2008, 130, 14084; (j) Cornil, J.; Echeverria, P.-G.; Phansavath, P.; Ratovelomanana-Vidal, V.; Guérinot, A.; Cossy, J. Org. Lett. 2015, 17 (4), 948-951; (k) Gonzalez, J. A.; Ogba, O. M.; Morehouse, G. F.; Rosson, N.; Houk, K. N.; Leach, A. G.; Cheong, P. H. Y.; Burke, M. D.; Lloyd-Jones, G. C. Nat Chem 2016, advance online publication.

260. Reid, W. B.; Spillane, J. J.; Krause, S. B.; Watson, D. A. J. Am. Chem. Soc. 2016, 138 (17), 5539-5542.

261. Kappe, C. O. Angew. Chem., Int. Ed. 2004, 43 (46), 6250-6284.

262. Batsanov, A. S.; Knowles, J. P.; Whiting, A. J. Org. Chem. 2007, 72 (7), 2525-2532.

263. Jutand, A. Mechanisms of the Mizoroki-Heck Reaction. In The Mizoroki-Heck Reaction, John Wiley \& Sons, Ltd: 2009; pp 1-50.

264. Crisp, G. T. Chem. Soc. Rev. 1998, 27, 427.

265. Muller, T.; Bräse, S. Formation of Heterocycles. In The Mizoroki-Heck Reaction, John Wiley \& Sons, Ltd: 2009; pp 215-258.

266. (a) Reetz, M. T.; Breinbauer, R.; Wanninger, K. Tetrahedron Lett. 1996, 37, 4499; (b) Reetz, M. T.; de Vries, J. G. ChemInform 2004, 35 (46), no-no; (c) Jeffery, T. Tetrahedron Lett. 2000, 41 (44), 8445-8449.

267. Wrackmeyer, B. Prog. Nucl. Magn. Reson. Spectrosc. 1979, 12 (4), 227-259.

268. Calhorda, M. J.; Brown, J. M.; Cooley, N. A. Organometallics 1991, 10 (5), 1431-1438. 
269. Carey, F. A.; Sundberg, R. J. Advanced Organic Chemistry Part B: Reactions and Synthesis. 2007.

270. Nunes, C. M.; Limberger, J.; Poersch, S.; Seferin, M.; Monteiro, A. L. Synthesis 2009, 2009 (16), 2761-2765.

271. Okamura, H.; Kuroda, S.; Ikegami, S.; Tomita, K.; Sugimoto, Y.; Sakaguchi, S.; Ito, Y.; Katsuki, T.; Yamaguchi, M. Tetrahedron 1993, 49, 10531.

272. (a) Cossy, J.; Bauer, D.; Bellosta, V. Tetrahedron 2002, 58 (29), 5909-5922; (b) Ley, S. V.; Anthony, N. J.; Armstrong, A.; Brasca, M. G.; Clarke, T.; Culshaw, D.; Greck, C.; Grice, P.; Jones, A. B.; Lygo, B.; Madin, A.; Sheppard, R. N.; Slawin, A. M. Z.; Williams, D. J. Tetrahedron 1989, 45 (22), 7161-7194.

273. Trost, B. M.; Crawley, M. L. J. Am. Chem. Soc. 2002, 124 (32), 9328-9329.

274. Guérinot, A.; Lepesqueux, G.; Sablé, S.; Reymond, S.; Cossy, J. J. Org. Chem. 2010, 75 (15), 5151-5163.

275. BouzBouz, S.; Cossy, J. Org. Lett. 2004, 6 (20), 3469-3472.

276. Glänzer, B. I.; Faber, K.; Griengl, H. Tetrahedron 1987, 43 (24), 5791-5796.

277. Vilaivan, T.; Winotapan, C.; Banphavichit, V.; Shinada, T.; Ohfune, Y. J. Org. Chem. 2005, 70 (9), 3464-3471. 
This page has been intentionally left blank. 
This page has been intentionally left blank. 


\title{
Chapter 6
}

\author{
6.1 Appendix
}

6.1.1 ${ }^{1} \mathrm{H}$ and ${ }^{13} \mathrm{C}$ NMR Spectra and Energy Calculations for Chapter I

6.1.2 Selected ${ }^{1} \mathrm{H}$ and ${ }^{13} \mathrm{C}$ NMR Spectra For Chapter II

6.1.3 Selected ${ }^{1} \mathrm{H}$ and ${ }^{13} \mathrm{C}$ NMR Spectra For Chapter III

6.1.4 $\quad{ }^{1} \mathrm{H}$ and ${ }^{13} \mathrm{C}$ NMR Spectra For Chapter IV 
This page has been intentionally left blank. 
6.1.1 ${ }^{1} \mathrm{H}$ and ${ }^{13} \mathrm{C}$ NMR Spectra and Energy Calculations for Chapter I

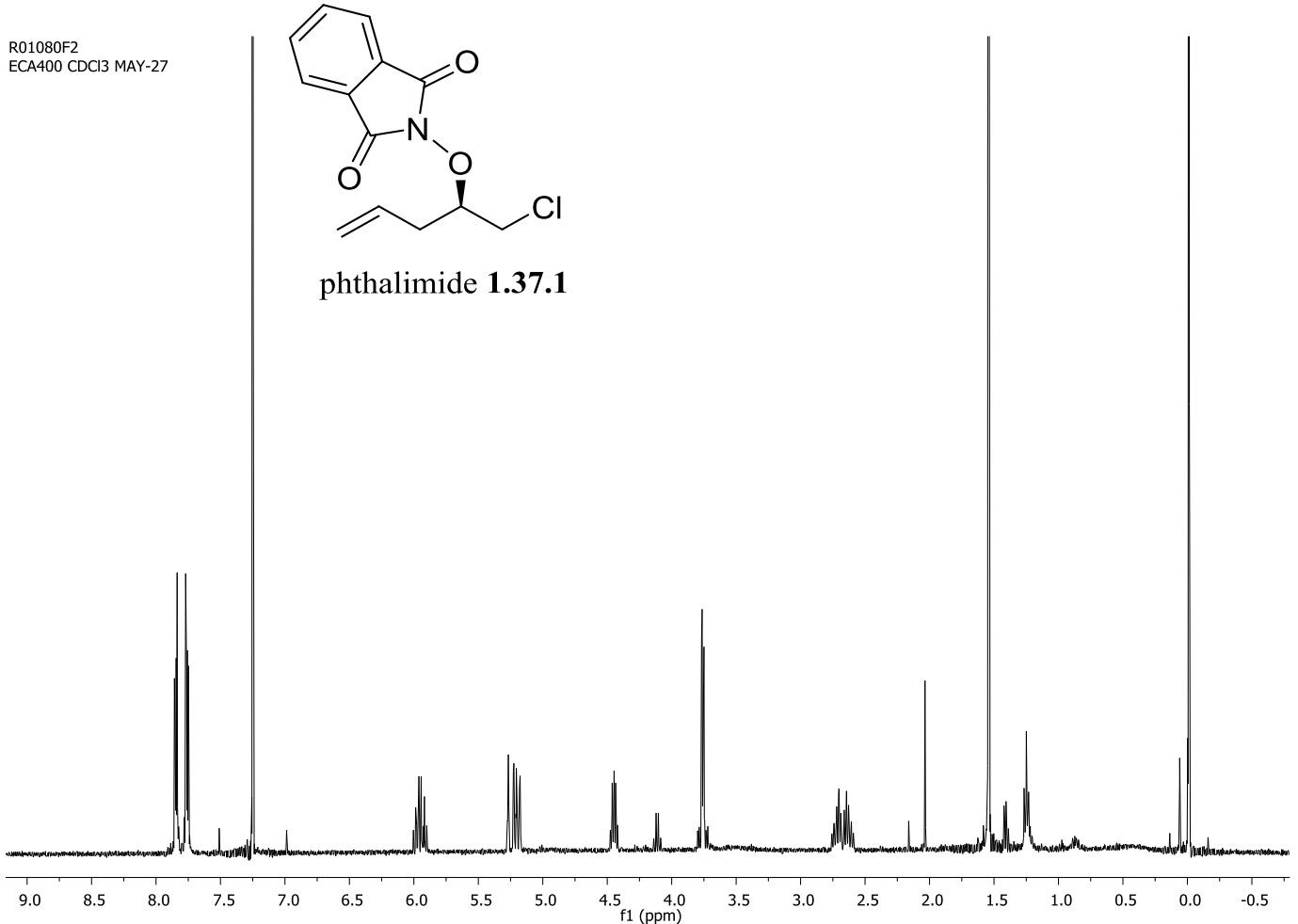

Phth-Cl-13C

Phth-Cl-13C, CDCl3, AV400, JAN-15-201

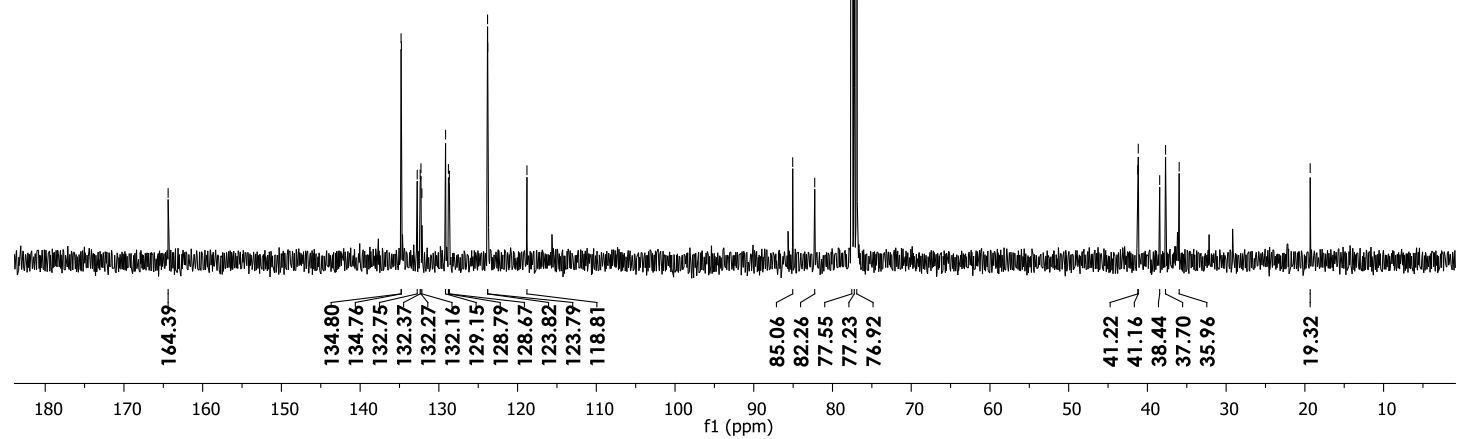




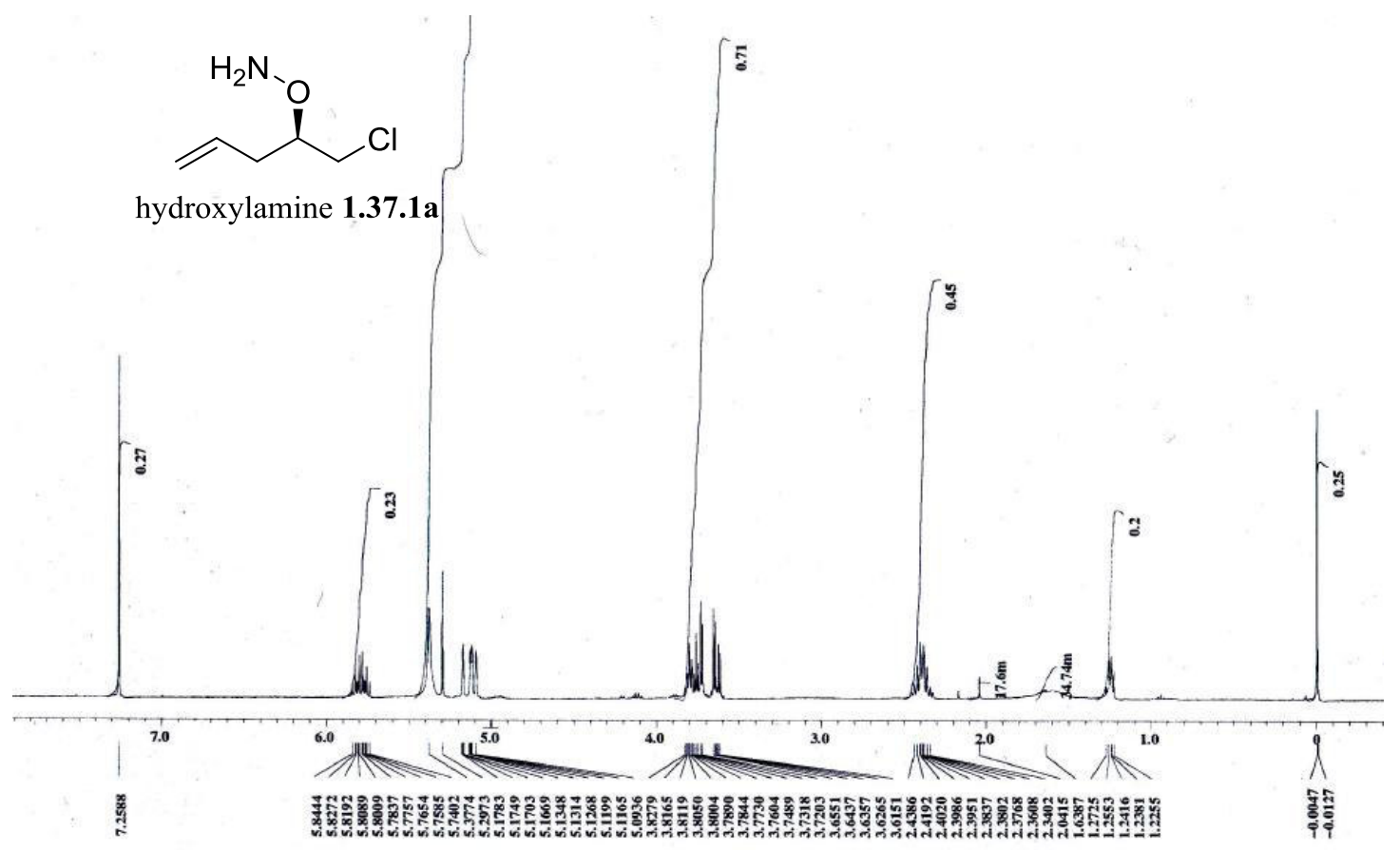

13CNH2-Cl
ECA400 CDCl3 JAN19-2014

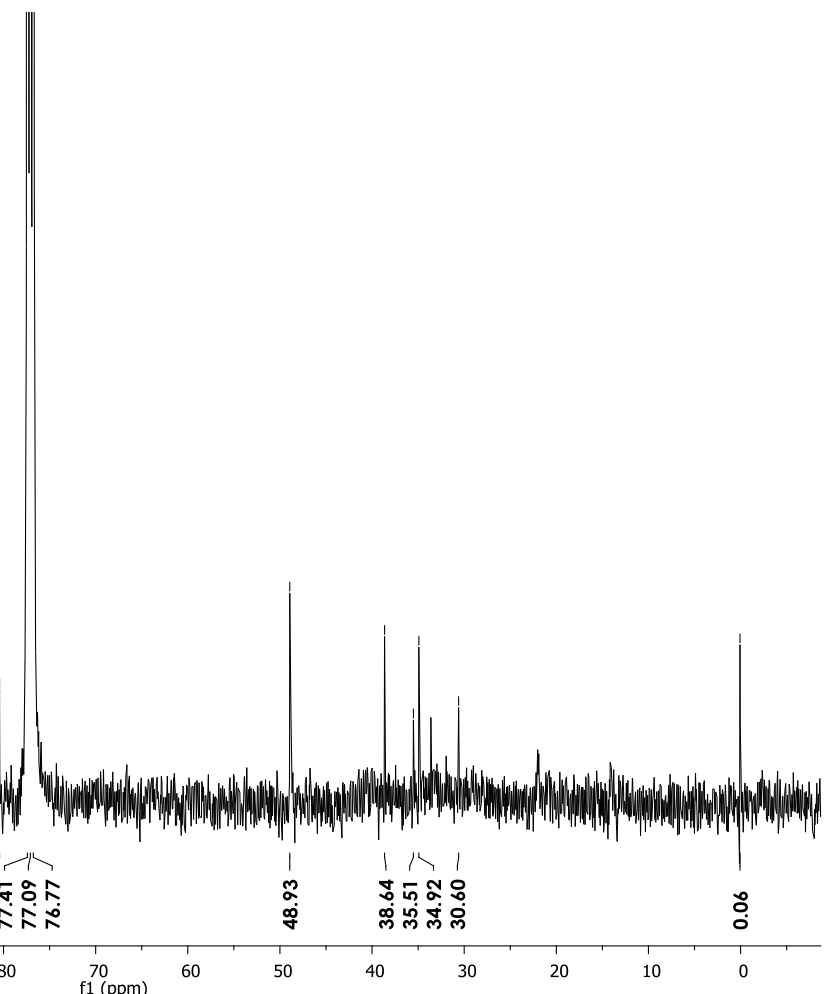




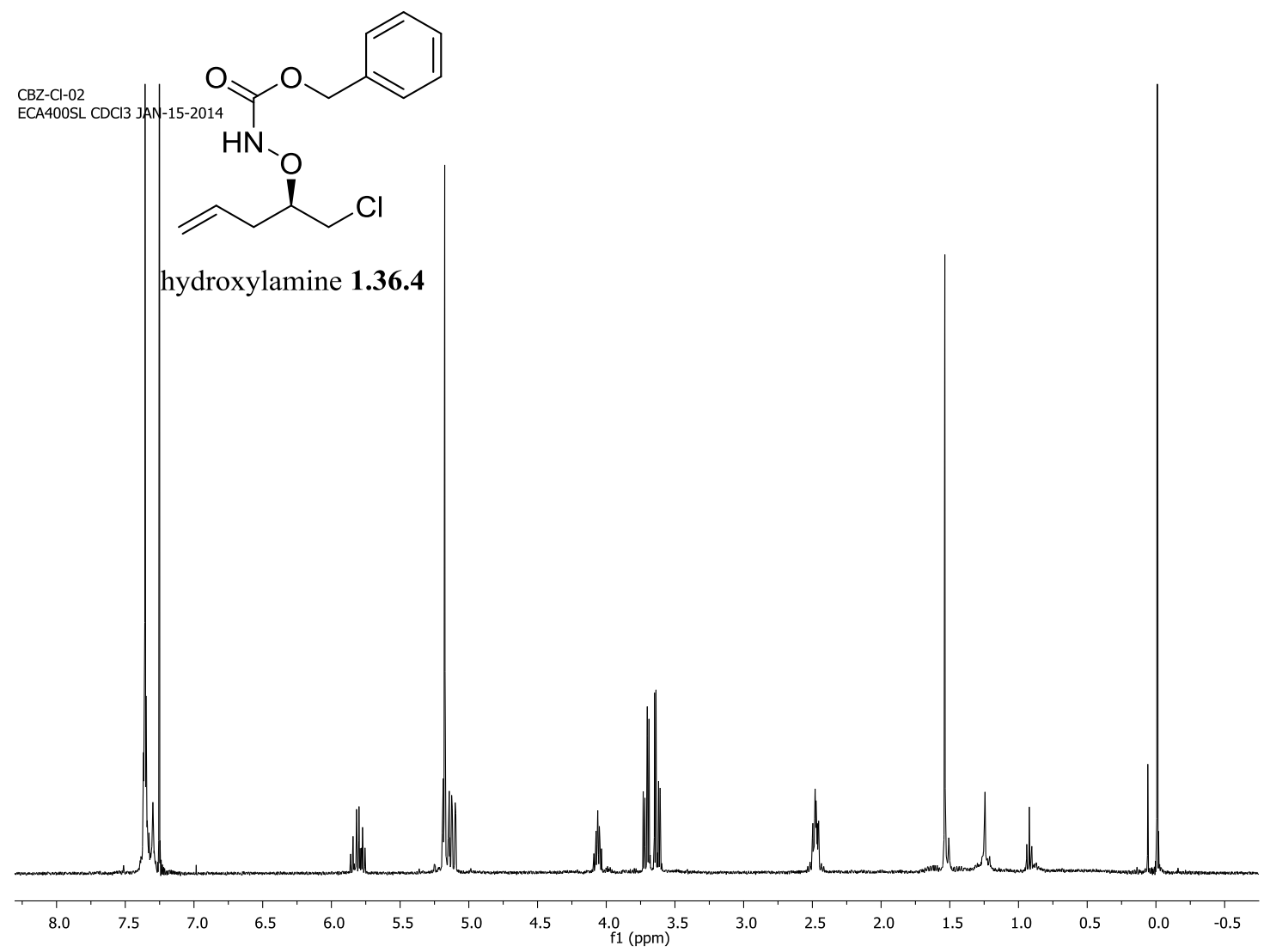

CBZ-Cl-13C-2

ECA400SL CDCI3 JAN 16-2014

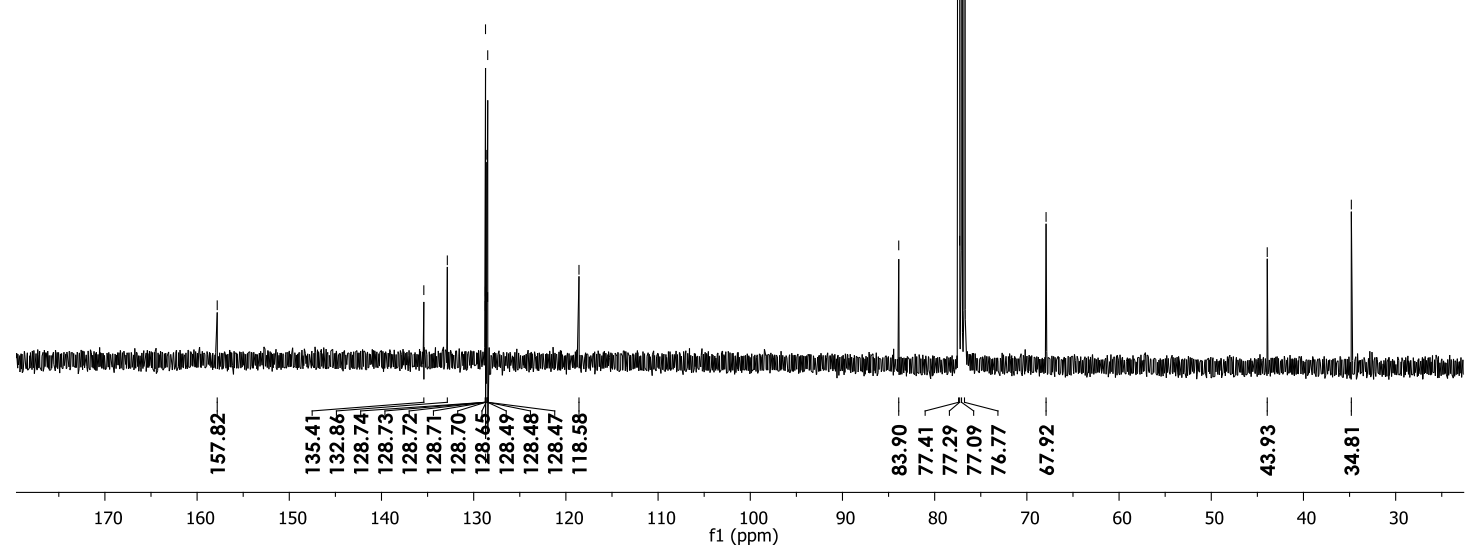




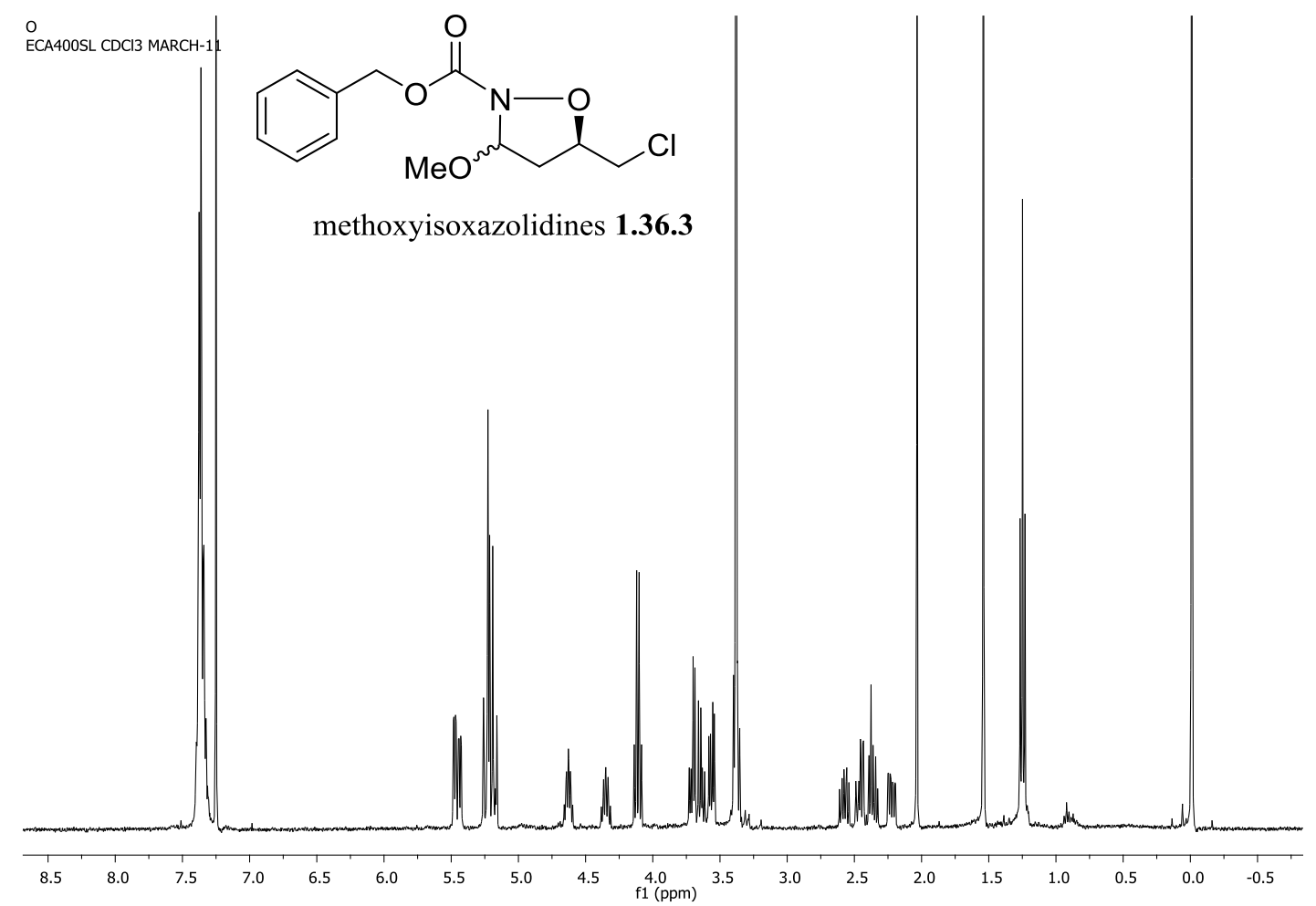

MeO-13C

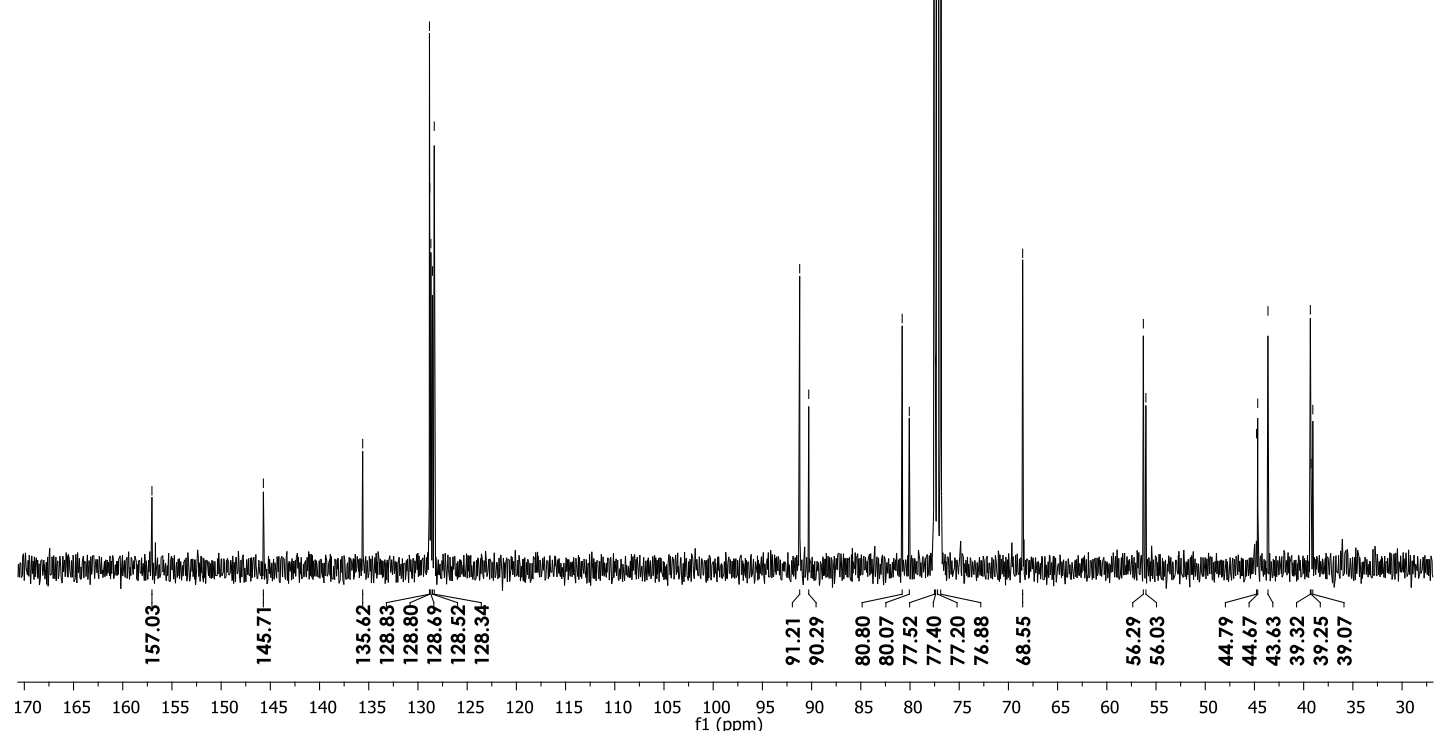


RSAK1

RSAK1, $1 \mathrm{H}$
AV $400 \mathrm{MHZ}$ CDCl3 JULY-1<smiles>C=CCC1CC(CCl)ON1C(=O)OCc1ccccc1</smiles>

allyl isoxazolidine $\mathbf{1 . 3 6 . 2}$

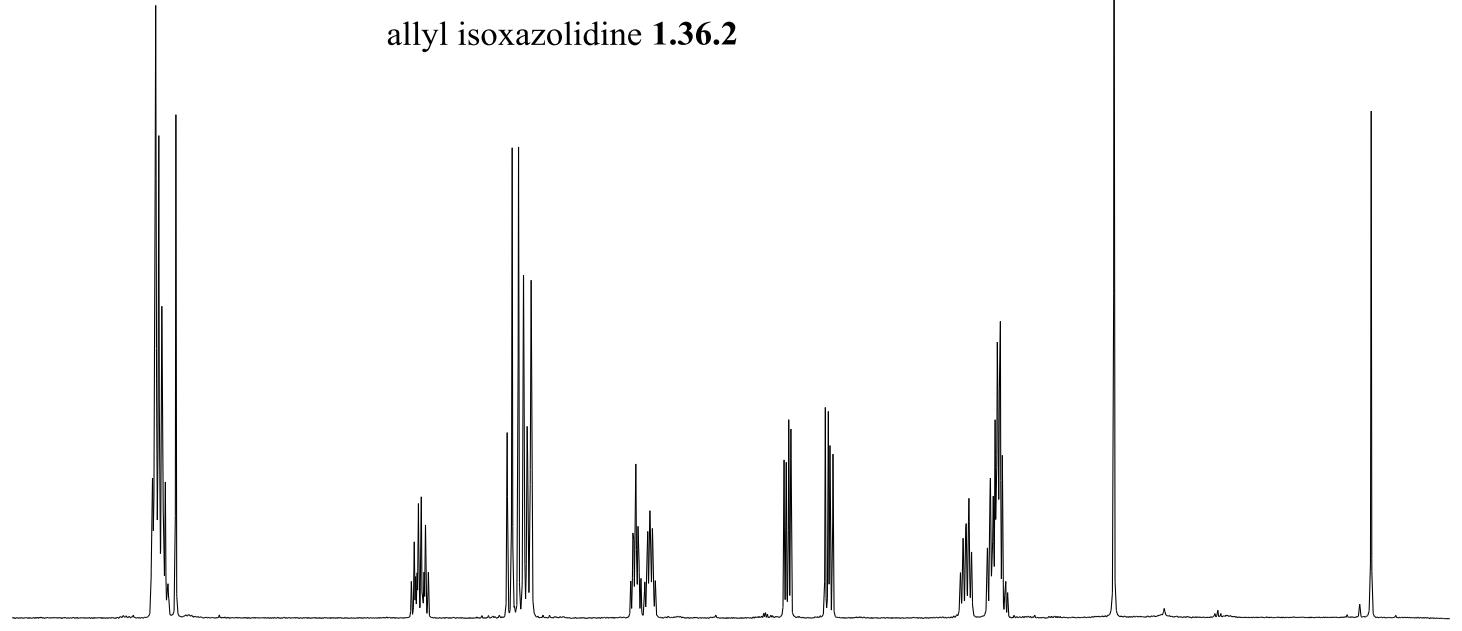

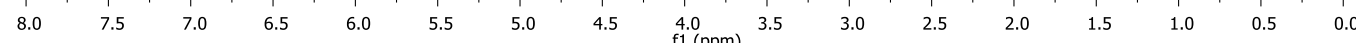

RSAK1-13C
RSAK1-13C

RSAK1-13C AV $400 \mathrm{MHz}$ CDCl3 JULY-1

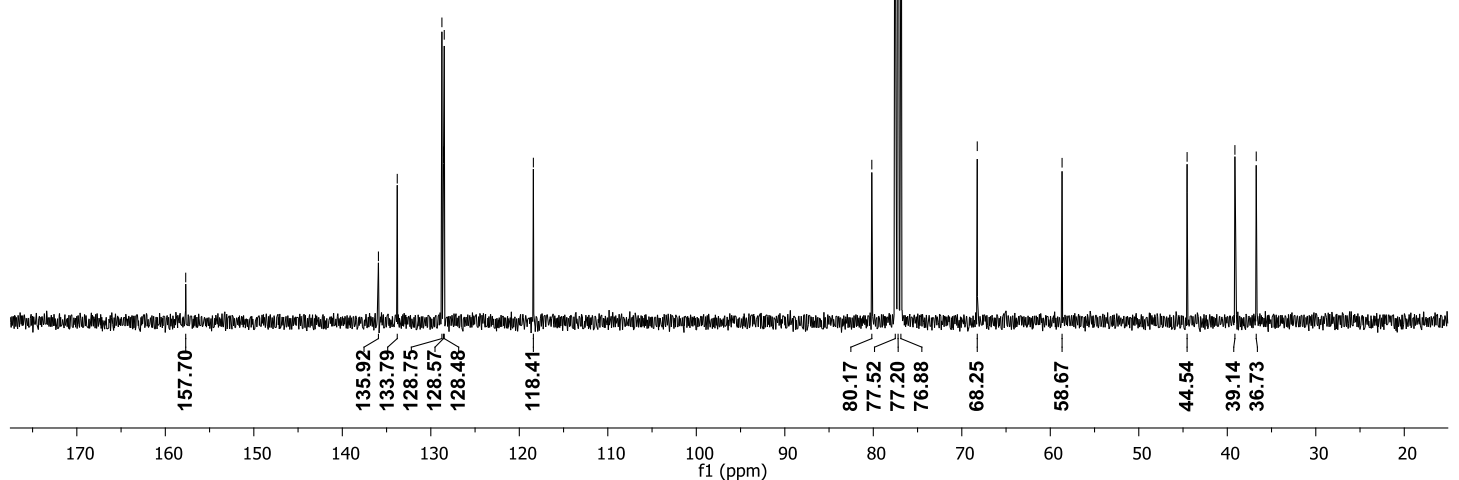




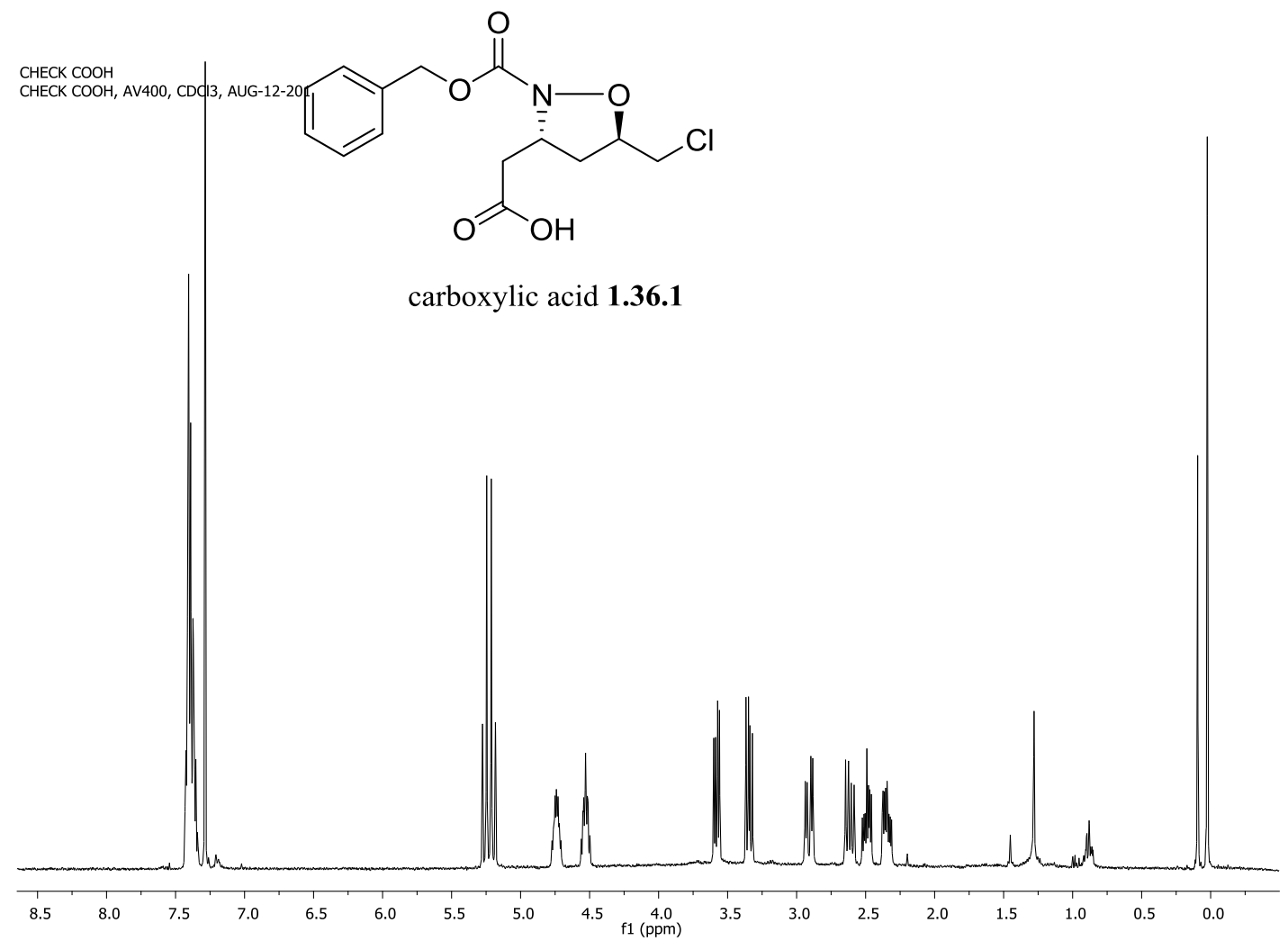

$\mathrm{COOH}-13$

ECA400SL CDC13 AUG-12-2013

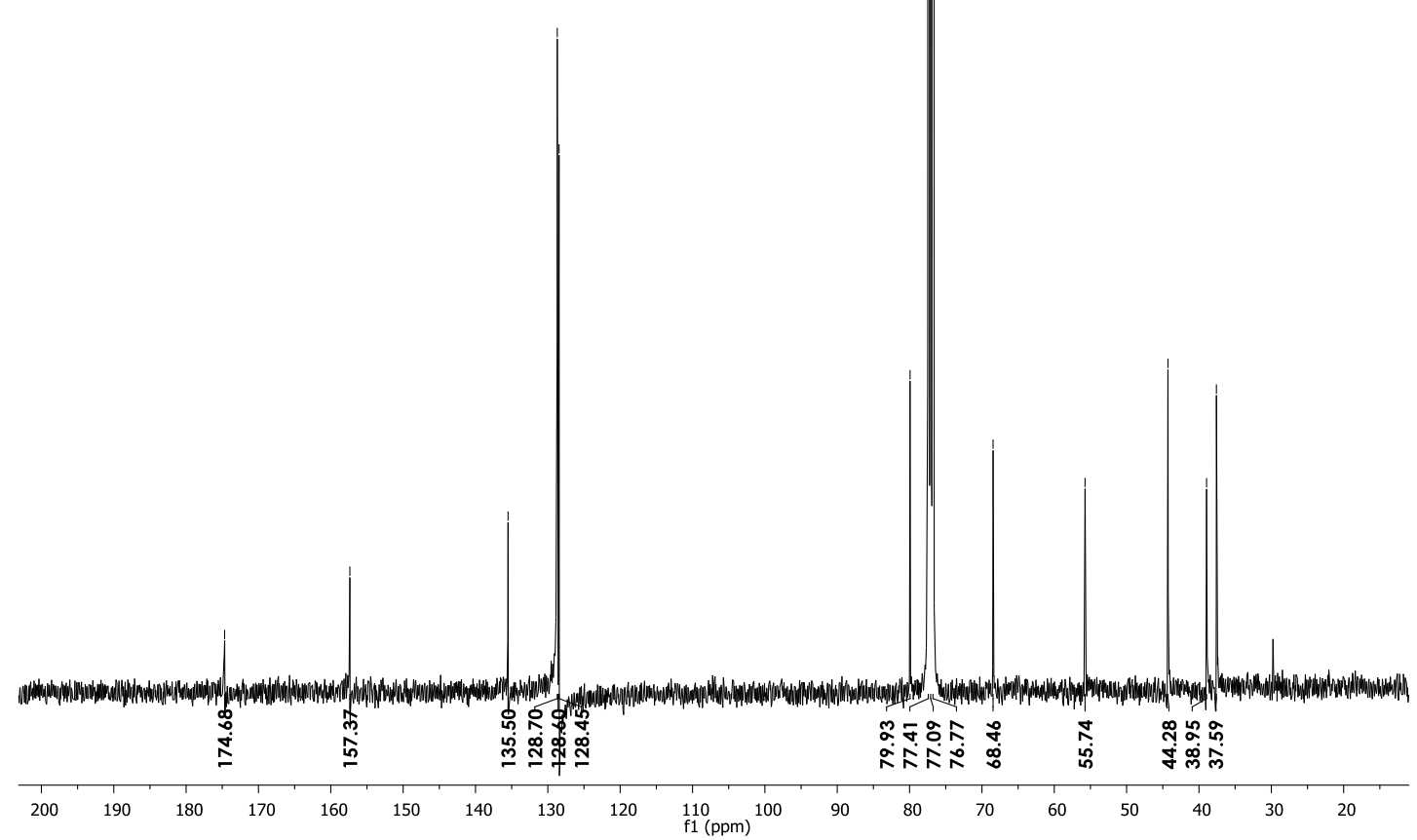




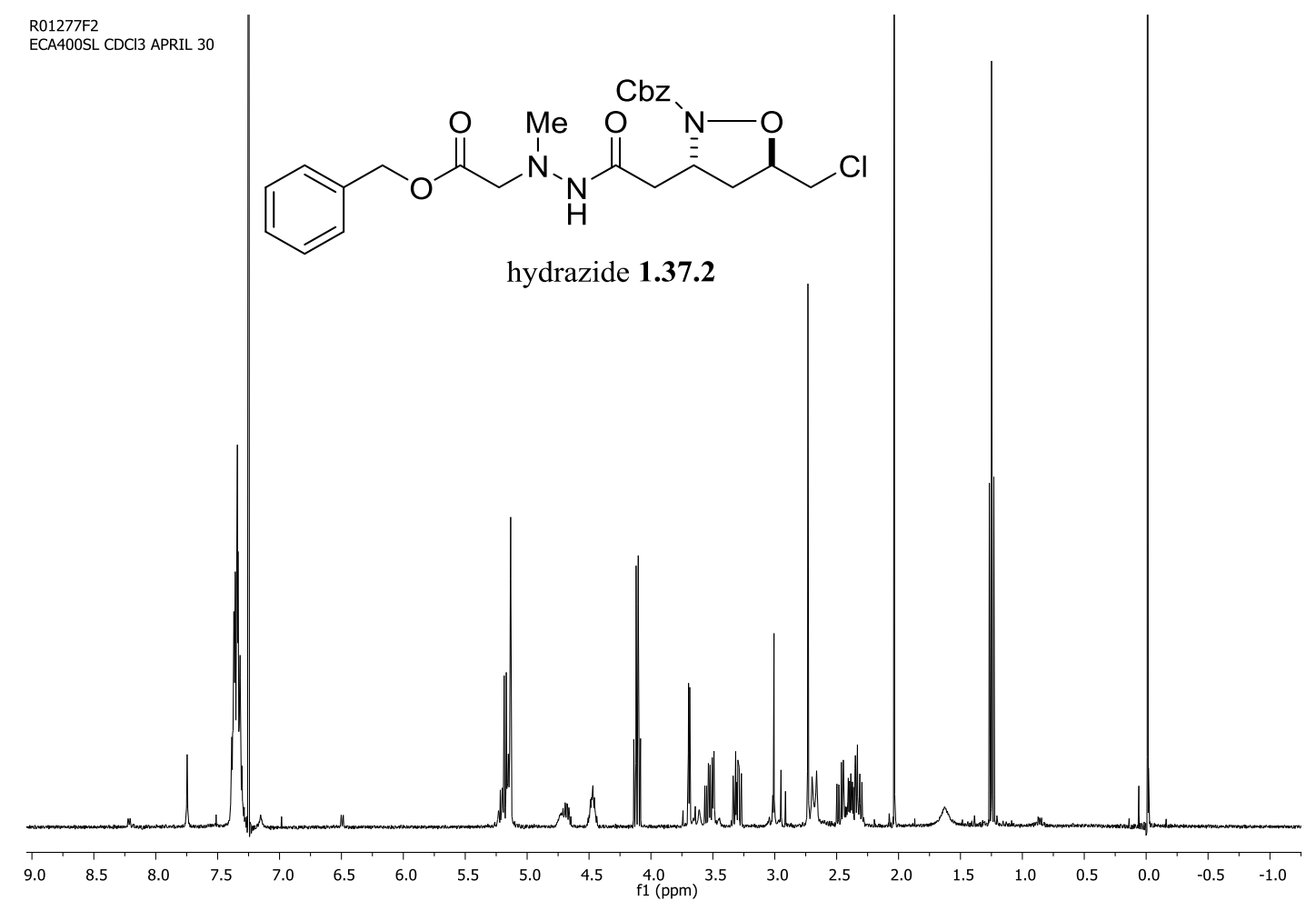

R01273F2-13C

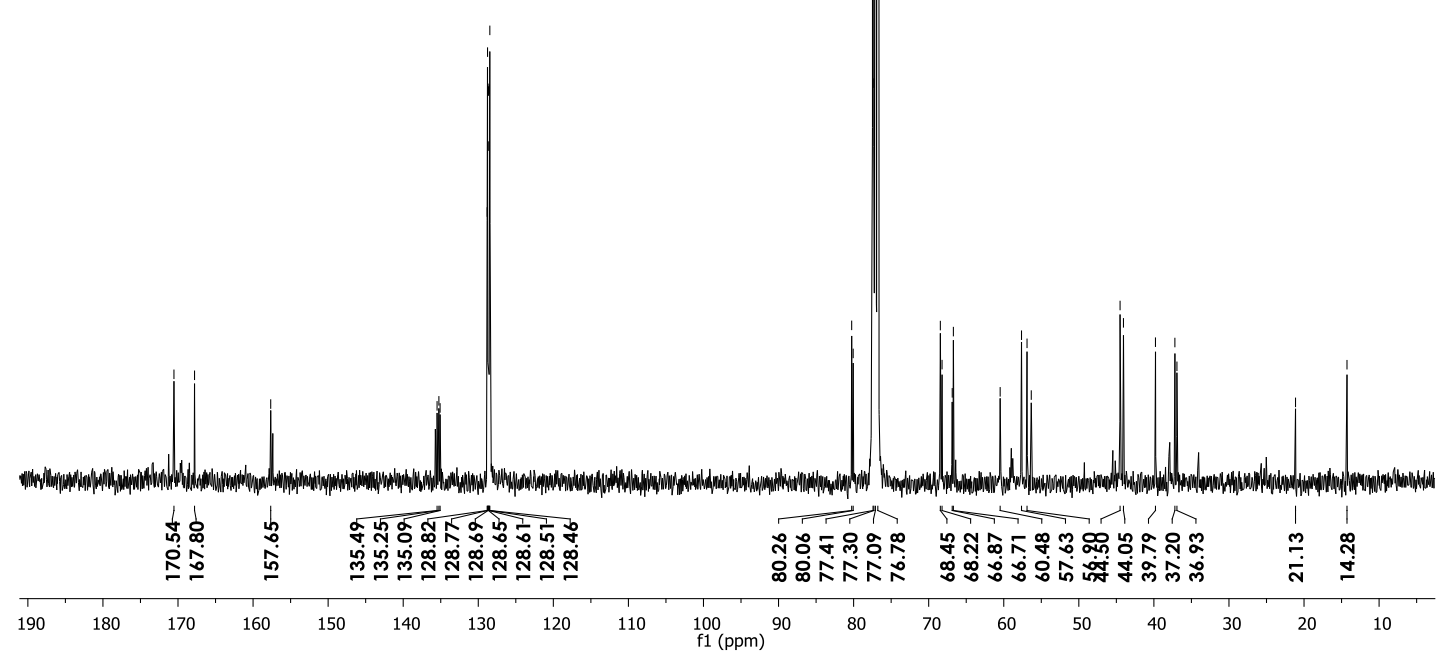




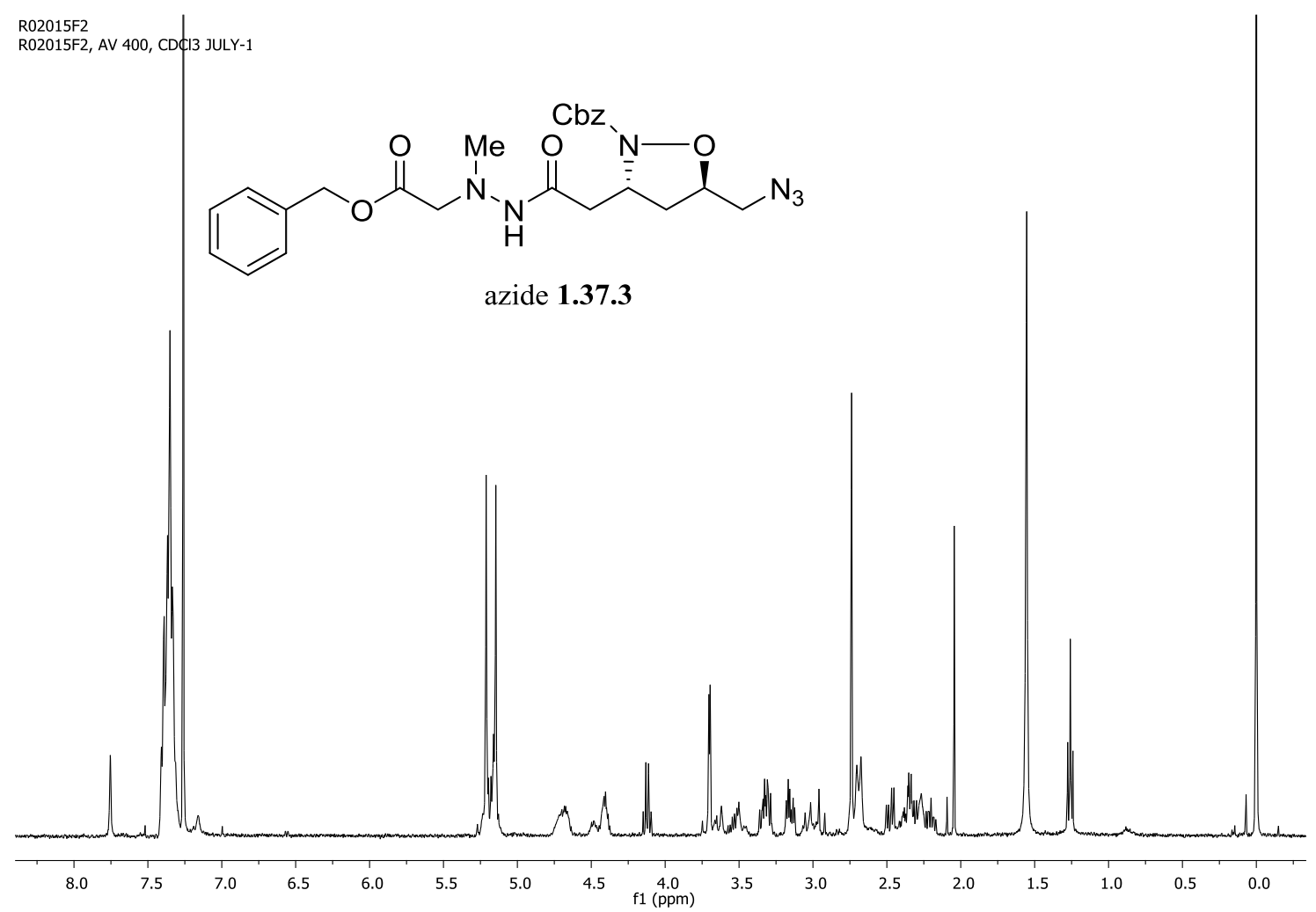

AZIDE-13C

AZIDE-13C, CDCl3, AV400, JAN-15-201

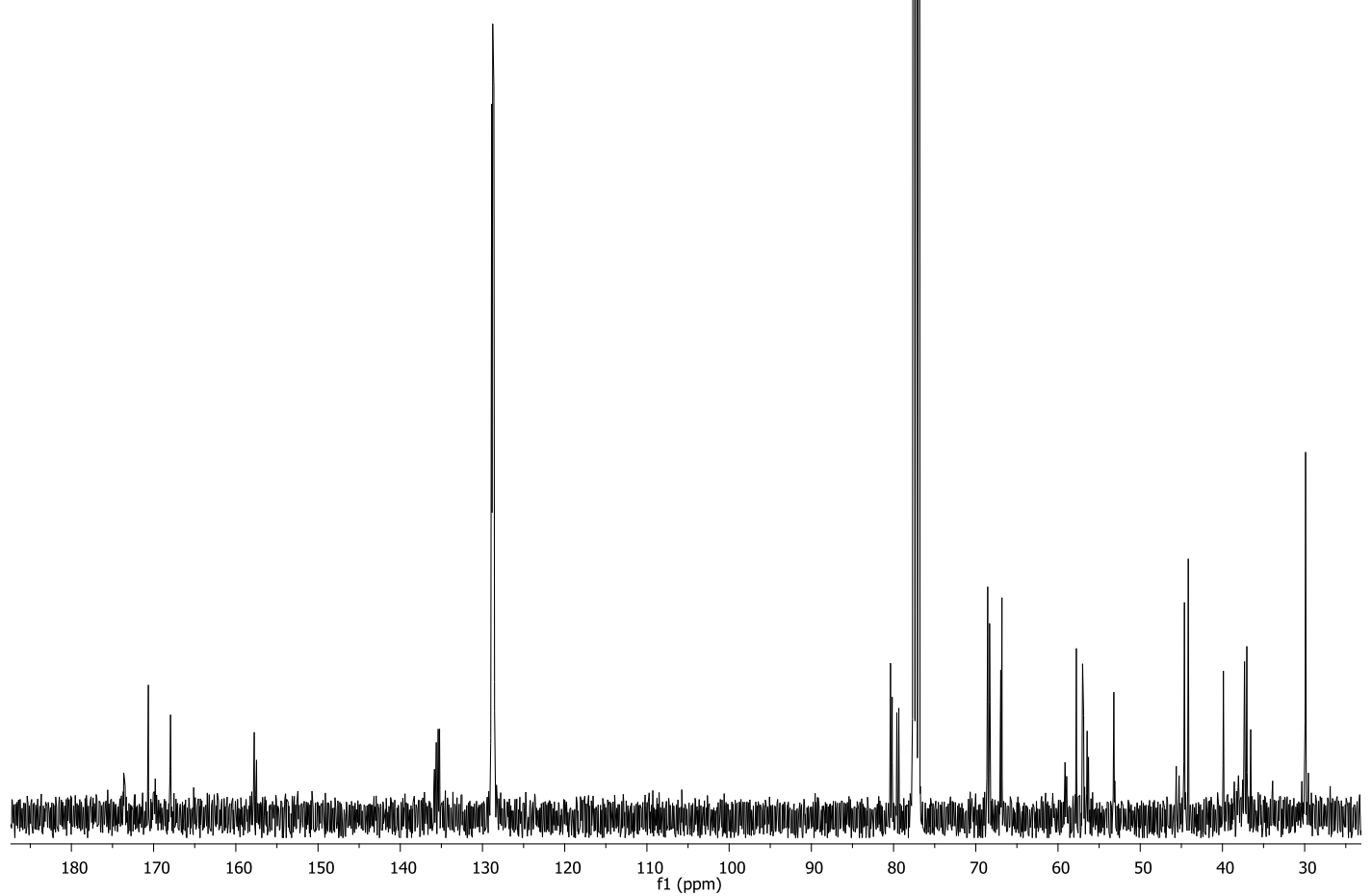




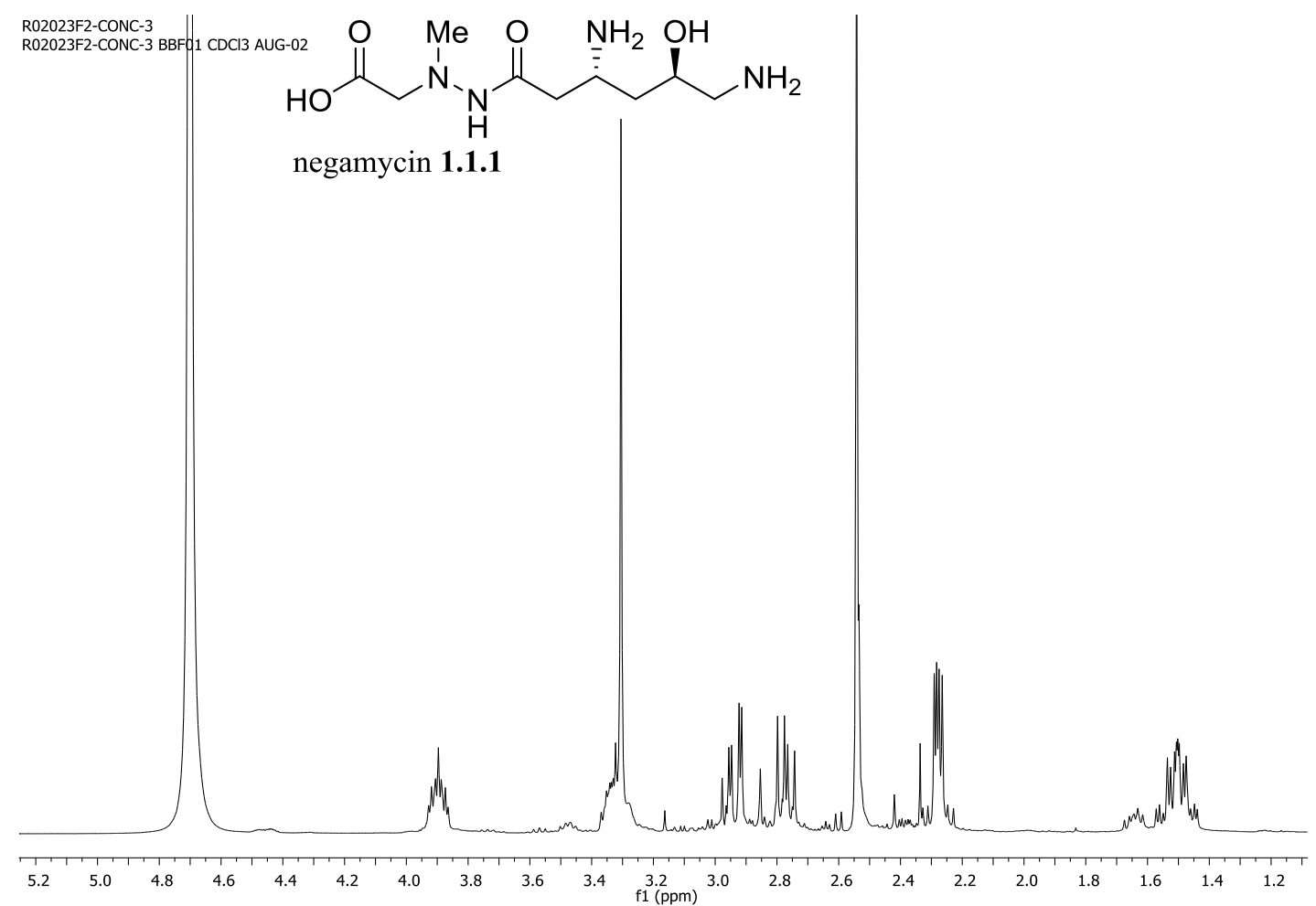

R02023F2-CONC-13C

R02023F2-CONC-13C BBF01 CDCl3 AUG-02

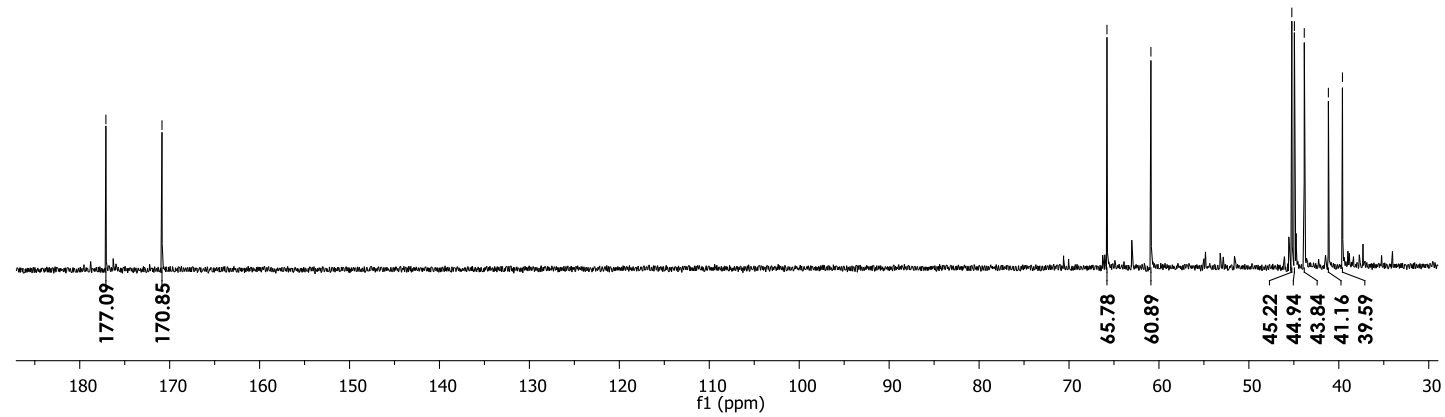


This page has been intentionally left blank. 
(a) $\mathrm{R}=\mathrm{Cl}$
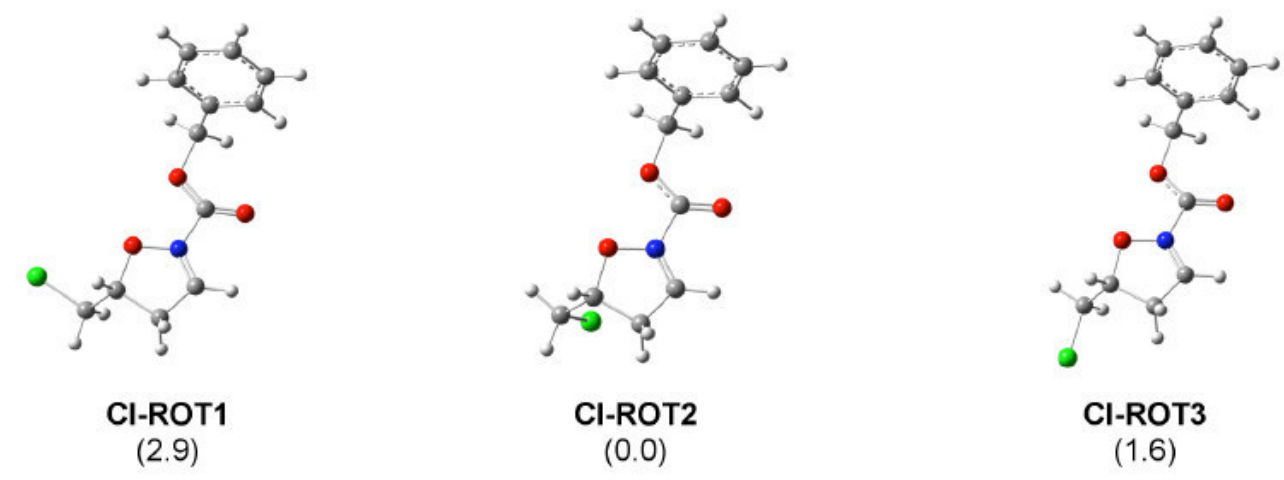

(b) $\mathrm{R}=\mathrm{CH}_{3}$

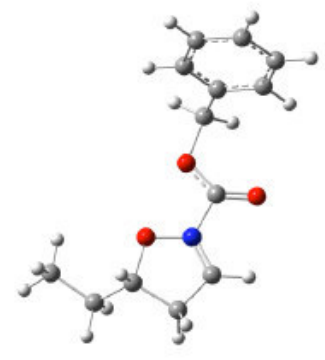

$\mathrm{CH}_{3}$-ROT1

$(0.0)$

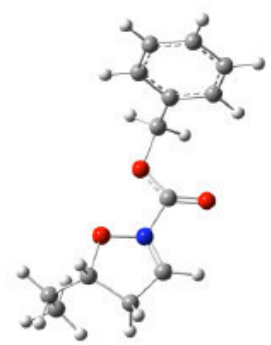

$\mathrm{CH}_{3}$-ROT2

$(0.7)$

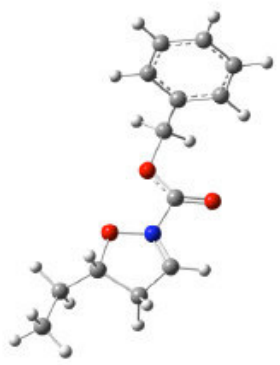

$\mathrm{CH}_{3}$-ROT3

$(0.2)$

Figure S1. Geometry and relative energy (in $\mathrm{kcal} / \mathrm{mol}$, in parentheses) of rotamers, as determined at the B3LYP/6-31G* + ZPE level. 


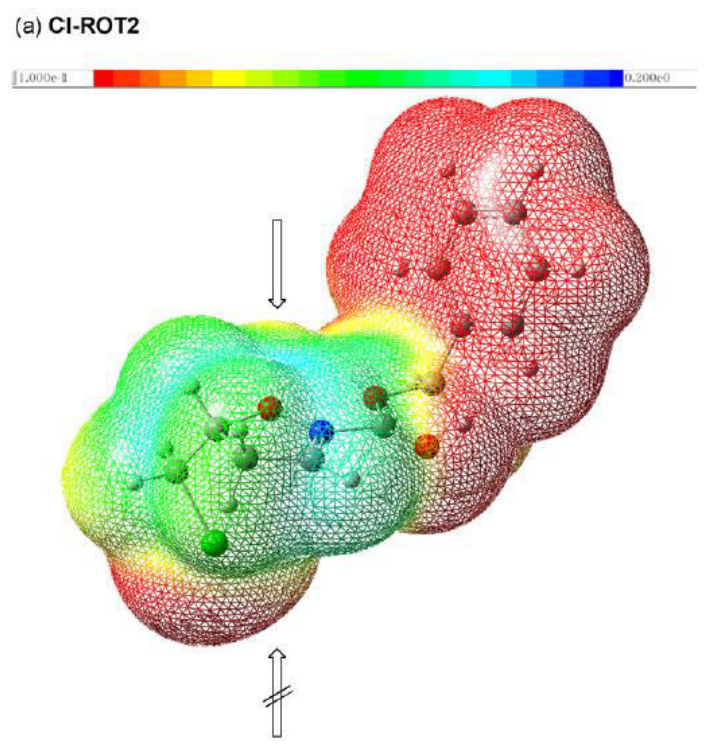

(b) $\mathrm{CH}_{3}-\mathrm{ROT1}$

Figure S2. Electrostatic potential maps for (a) Cl-ROT2 and (b) $\mathbf{C H}_{3}-\mathbf{R O T 1}$. 
Table S1. Raw energy data

(a) $\mathrm{Cl}$

\begin{tabular}{|l|c|c|r|r|}
\hline & $\begin{array}{c}\mathrm{E} \\
(\mathrm{au})\end{array}$ & $\begin{array}{l}\text { ZPE } \\
(\mathrm{au})\end{array}$ & \multicolumn{1}{c|}{$\begin{array}{c}\Delta \mathrm{E} \\
(\mathrm{kcal} / \mathrm{mol})\end{array}$} & \multicolumn{1}{|c|}{$\begin{array}{l}\Delta(\mathrm{E}+\mathrm{ZPE}) \\
(\mathrm{kcal} / \mathrm{mol})\end{array}$} \\
\hline Cl-ROT1 & -1205.414579 & 0.236988 & 2.9 & 2.7 \\
\hline Cl-ROT2 & -1205.419133 & 0.237250 & 0.0 & 0.0 \\
\hline Cl-ROT3 & -1205.416513 & 0.237168 & 1.6 & 1.6 \\
\hline
\end{tabular}

(b) $\mathrm{CH}_{3}$

\begin{tabular}{|l|c|c|r|r|}
\hline & $\begin{array}{c}\mathrm{E} \\
(\mathrm{au})\end{array}$ & $\begin{array}{c}\text { ZPE } \\
(\mathrm{au})\end{array}$ & $\begin{array}{c}\Delta \mathrm{E} \\
(\mathrm{kcal} / \mathrm{mol})\end{array}$ & $\begin{array}{c}\Delta(\mathrm{E}+\mathrm{ZPE}) \\
(\mathrm{kcal} / \mathrm{mol})\end{array}$ \\
\hline CH3-ROT1 & -785.146894 & 0.274512 & 0.0 & 0.0 \\
\hline CH3-ROT2 & -785.146005 & 0.274668 & 0.6 & 0.7 \\
\hline CH3-ROT3 & -785.146661 & 0.274548 & 0.1 & 0.2 \\
\hline
\end{tabular}


XYZ coordinates of optimized geometries

\begin{tabular}{|c|c|c|c|}
\hline \multicolumn{4}{|c|}{$===$ Cl-ROT1 === } \\
\hline C & -2.582344 & 0.916823 & 0.668539 \\
\hline 0 & -1.095747 & 0.851194 & 0.623918 \\
\hline C & -1.699323 & 1.244170 & -1.505582 \\
\hline C & -3.009418 & 1.137265 & -0.810109 \\
\hline $\mathrm{H}$ & -2.894884 & -0.053786 & 1.056320 \\
\hline $\mathrm{H}$ & -1.478920 & 1.430053 & -2.551002 \\
\hline $\mathrm{H}$ & -3.602310 & 2.049391 & -0.960547 \\
\hline $\mathrm{H}$ & -3.596692 & 0.306572 & -1.220204 \\
\hline $\mathrm{N}$ & -0.734802 & 1.083595 & -0.671534 \\
\hline C & -3.001185 & 2.039683 & 1.602373 \\
\hline $\mathrm{H}$ & -4.092857 & 2.109905 & 1.602700 \\
\hline $\mathrm{H}$ & -2.582790 & 2.996335 & 1.278698 \\
\hline C & 0.743521 & 1.130938 & -1.028576 \\
\hline 0 & 1.005375 & 1.333833 & -2.177943 \\
\hline 0 & 1.426702 & 0.938627 & 0.044646 \\
\hline C & 2.943679 & 0.867426 & -0.105844 \\
\hline $\mathrm{H}$ & 3.210008 & 1.628800 & -0.839267 \\
\hline $\mathrm{H}$ & 3.258792 & 1.164770 & 0.892883 \\
\hline C & 3.371840 & -0.509090 & -0.487151 \\
\hline C & 3.649818 & -0.819108 & -1.826852 \\
\hline C & 3.502172 & -1.501024 & 0.496864 \\
\hline C & 4.053086 & -2.107180 & -2.176399 \\
\hline $\mathrm{H}$ & 3.559172 & -0.051000 & -2.590274 \\
\hline C & 3.900825 & -2.788121 & 0.143903 \\
\hline $\mathrm{H}$ & 3.298957 & -1.261045 & 1.537783 \\
\hline C & 4.176278 & -3.090847 & -1.192639 \\
\hline $\mathrm{H}$ & 4.278360 & -2.340823 & -3.212591 \\
\hline $\mathrm{H}$ & 4.007402 & -3.551144 & 0.909010 \\
\hline $\mathrm{H}$ & 4.495980 & -4.092339 & -1.465528 \\
\hline $\mathrm{Cl}$ & -2.463893 & 1.731910 & 3.283695 \\
\hline
\end{tabular}

\begin{tabular}{|c|c|c|c|}
\hline \multicolumn{4}{|c|}{$===$ Cl-ROT2 === } \\
\hline C & -2.625015 & 0.925404 & 0.639751 \\
\hline 0 & -1.149779 & 0.757363 & 0.602056 \\
\hline C & -1.713562 & 1.256660 & -1.514021 \\
\hline C & -3.032864 & 1.120792 & -0.844003 \\
\hline $\mathrm{H}$ & -3.003918 & -0.011616 & 1.055521 \\
\hline $\mathrm{H}$ & -1.478306 & 1.493618 & -2.545639 \\
\hline $\mathrm{H}$ & -3.644655 & 2.015292 & -1.008828 \\
\hline $\mathrm{H}$ & -3.579353 & 0.264524 & -1.259365 \\
\hline $\mathrm{N}$ & -0.761412 & 1.046594 & -0.677501 \\
\hline C & -2.962356 & 2.065163 & 1.585940 \\
\hline $\mathrm{H}$ & -2.499293 & 1.897127 & 2.559850 \\
\hline $\mathrm{H}$ & -4.046622 & 2.127350 & 1.711712 \\
\hline C & 0.717395 & 1.109804 & -1.004693 \\
\hline 0 & 1.001710 & 1.347892 & -2.142110 \\
\hline 0 & 1.385602 & 0.887511 & 0.074115 \\
\hline C & 2.903056 & 0.840398 & -0.056765 \\
\hline $\mathrm{H}$ & 3.169660 & 1.620579 & -0.769992 \\
\hline $\mathrm{H}$ & 3.203148 & 1.120300 & 0.951644 \\
\hline C & 3.356502 & -0.522041 & -0.461123 \\
\hline C & 3.641609 & -0.804543 & -1.805358 \\
\hline C & 3.503295 & -1.528173 & 0.505784 \\
\hline C & 4.066976 & -2.079524 & -2.176179 \\
\hline $\mathrm{H}$ & 3.538684 & -0.025215 & -2.555678 \\
\hline C & 3.924824 & -2.802078 & 0.131898 \\
\hline $\mathrm{H}$ & 3.295703 & -1.309164 & 1.550515 \\
\hline C & 4.206340 & -3.077551 & -1.209183 \\
\hline $\mathrm{H}$ & 4.297160 & -2.291609 & -3.215930 \\
\hline $\mathrm{H}$ & 4.044403 & -3.575824 & 0.884254 \\
\hline $\mathrm{H}$ & 4.543560 & -4.068615 & -1.498573 \\
\hline $\mathrm{Cl}$ & -2.379962 & 3.656300 & 0.970738 \\
\hline
\end{tabular}

$\begin{array}{lrrr}\text { === Cl-ROT } 3=== & & \\ \text { C } & -2.621947 & 0.904917 & 0.662394 \\ \text { O } & -1.140938 & 0.757699 & 0.628555 \\ \text { C } & -1.711743 & 1.236516 & -1.491726 \\ \mathrm{C} & -3.028924 & 1.070314 & -0.824748 \\ \mathrm{H} & -2.993094 & -0.021994 & 1.100561 \\ \mathrm{H} & -1.478270 & 1.465806 & -2.525770 \\ \mathrm{H} & -3.685962 & 1.931541 & -0.995579 \\ \mathrm{H} & -3.547713 & 0.194935 & -1.237003 \\ \mathrm{~N} & -0.755027 & 1.055692 & -0.651547 \\ \mathrm{C} & -2.935177 & 2.097455 & 1.560470 \\ \mathrm{H} & -2.526748 & 3.026059 & 1.154206 \\ \mathrm{H} & -2.536066 & 1.934875 & 2.562621 \\ \mathrm{C} & 0.724818 & 1.128247 & -0.982074 \\ \mathrm{O} & 1.004458 & 1.373444 & -2.119047 \\ \mathrm{O} & 1.395534 & 0.904416 & 0.094371 \\ \mathrm{C} & 2.914729 & 0.846070 & -0.041983 \\ \mathrm{H} & 3.184017 & 1.630227 & -0.749827 \\ \mathrm{H} & 3.218559 & 1.115446 & 0.968129 \\ \mathrm{C} & 3.355284 & -0.516181 & -0.458650 \\ \mathrm{C} & 3.638743 & -0.788564 & -1.805430 \\ \mathrm{C} & 3.492097 & -1.532599 & 0.499138 \\ \mathrm{C} & 4.052904 & -2.063778 & -2.187780 \\ \mathrm{H} & 3.543778 & -0.001326 & -2.548527 \\ \mathrm{C} & 3.902119 & -2.806676 & 0.113529 \\ \mathrm{H} & 3.285724 & -1.321595 & 1.545747 \\ \mathrm{C} & 4.182231 & -3.071987 & -1.229960 \\ \mathrm{H} & 4.282151 & -2.268260 & -3.229235 \\ \mathrm{H} & 4.013873 & -3.588585 & 0.858566 \\ \mathrm{H} & 4.510568 & -4.063331 & -1.528400 \\ \mathrm{Cl} & -4.719836 & 2.273179 & 1.684357\end{array}$

$\begin{array}{lrrr}==\text { CH3-ROT } 1=== & & \\ \mathrm{C} & -2.668184 & 0.726163 & -0.056925 \\ \mathrm{O} & -1.229649 & 0.725352 & 0.372828 \\ \mathrm{C} & -1.480635 & 2.657992 & -0.745946 \\ \mathrm{C} & -2.866771 & 2.123825 & -0.700477 \\ \mathrm{H} & -2.727096 & -0.068696 & -0.806062 \\ \mathrm{H} & -1.100533 & 3.582285 & -1.166504 \\ \mathrm{H} & -3.498522 & 2.790698 & -0.095456 \\ \mathrm{H} & -3.312785 & 2.083049 & -1.700259 \\ \mathrm{~N} & -0.673099 & 1.855924 & -0.145988 \\ \mathrm{C} & -3.537895 & 0.421032 & 1.150992 \\ \mathrm{H} & -4.579703 & 0.481731 & 0.807460 \\ \mathrm{H} & -3.403518 & 1.219281 & 1.892193 \\ \mathrm{C} & -3.267874 & -0.952269 & 1.774297 \\ \mathrm{H} & -2.241413 & -1.027542 & 2.146024 \\ \mathrm{H} & -3.943430 & -1.120369 & 2.617438 \\ \mathrm{H} & -3.430872 & -1.757961 & 1.049851 \\ \mathrm{C} & 0.809296 & 2.099756 & 0.035551 \\ \mathrm{O} & 1.245756 & 3.119955 & -0.413184 \\ \mathrm{O} & 1.315481 & 1.102255 & 0.677474 \\ \mathrm{C} & 2.820182 & 1.125083 & 0.898522 \\ \mathrm{H} & 3.076766 & 2.156931 & 1.139705 \\ \mathrm{H} & 2.906772 & 0.490819 & 1.779268 \\ \mathrm{C} & 3.542005 & 0.593928 & -0.294805 \\ \mathrm{C} & 4.104114 & 1.472661 & -1.232578 \\ \mathrm{C} & 3.667075 & -0.791273 & -0.480146 \\ \mathrm{C} & 4.783119 & 0.970021 & -2.341890 \\ \mathrm{H} & 4.016270 & 2.546119 & -1.088396 \\ \mathrm{C} & 4.341520 & -1.290727 & -1.591893 \\ \mathrm{H} & 3.243725 & -1.475675 & 0.251236\end{array}$




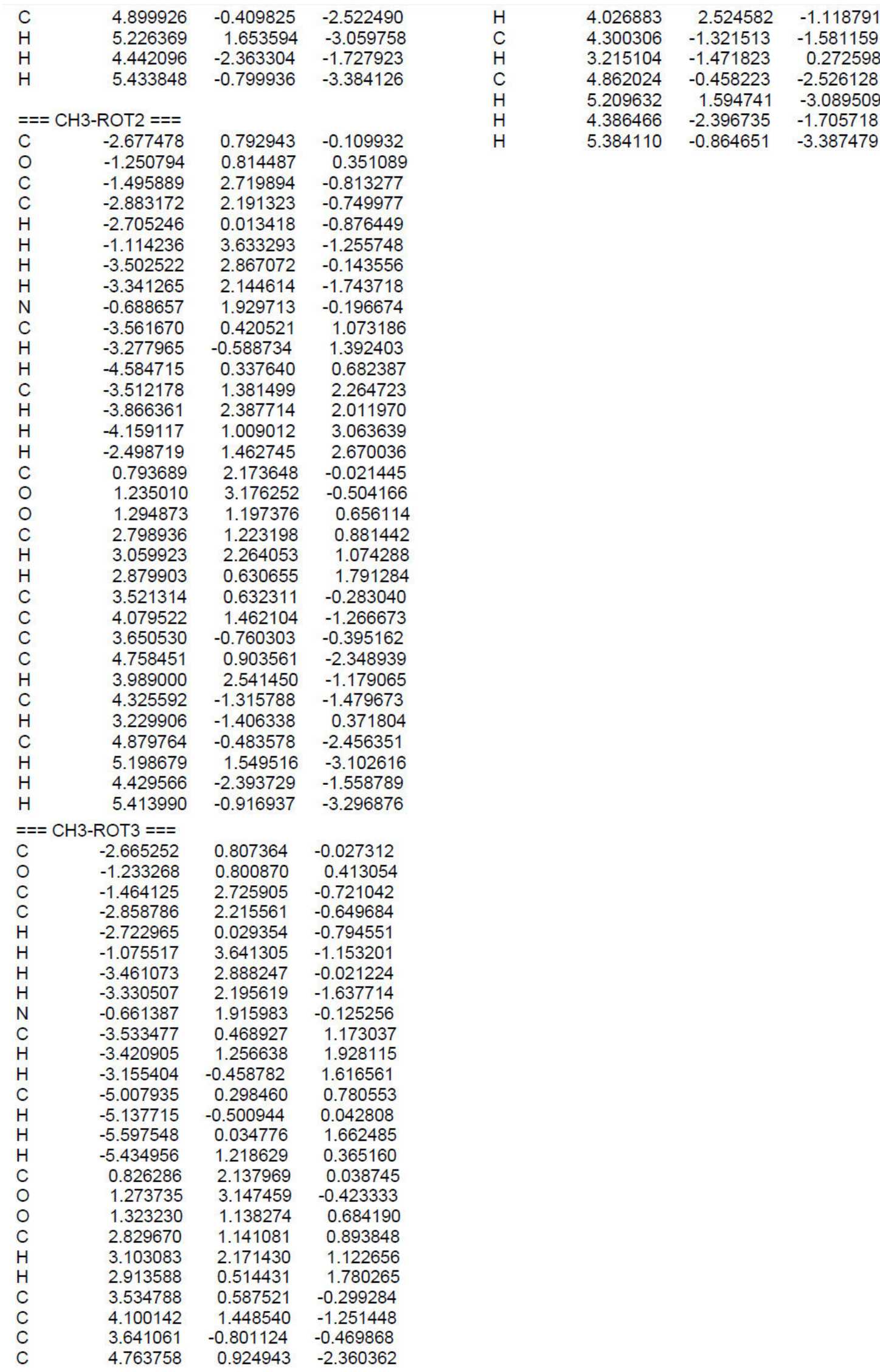


This page has been intentionally left blank. 


\subsubsection{Selected ${ }^{1} \mathrm{H}$ and ${ }^{13} \mathrm{C}$ NMR Spectra for Chapter II}

Substrate 2.14.7
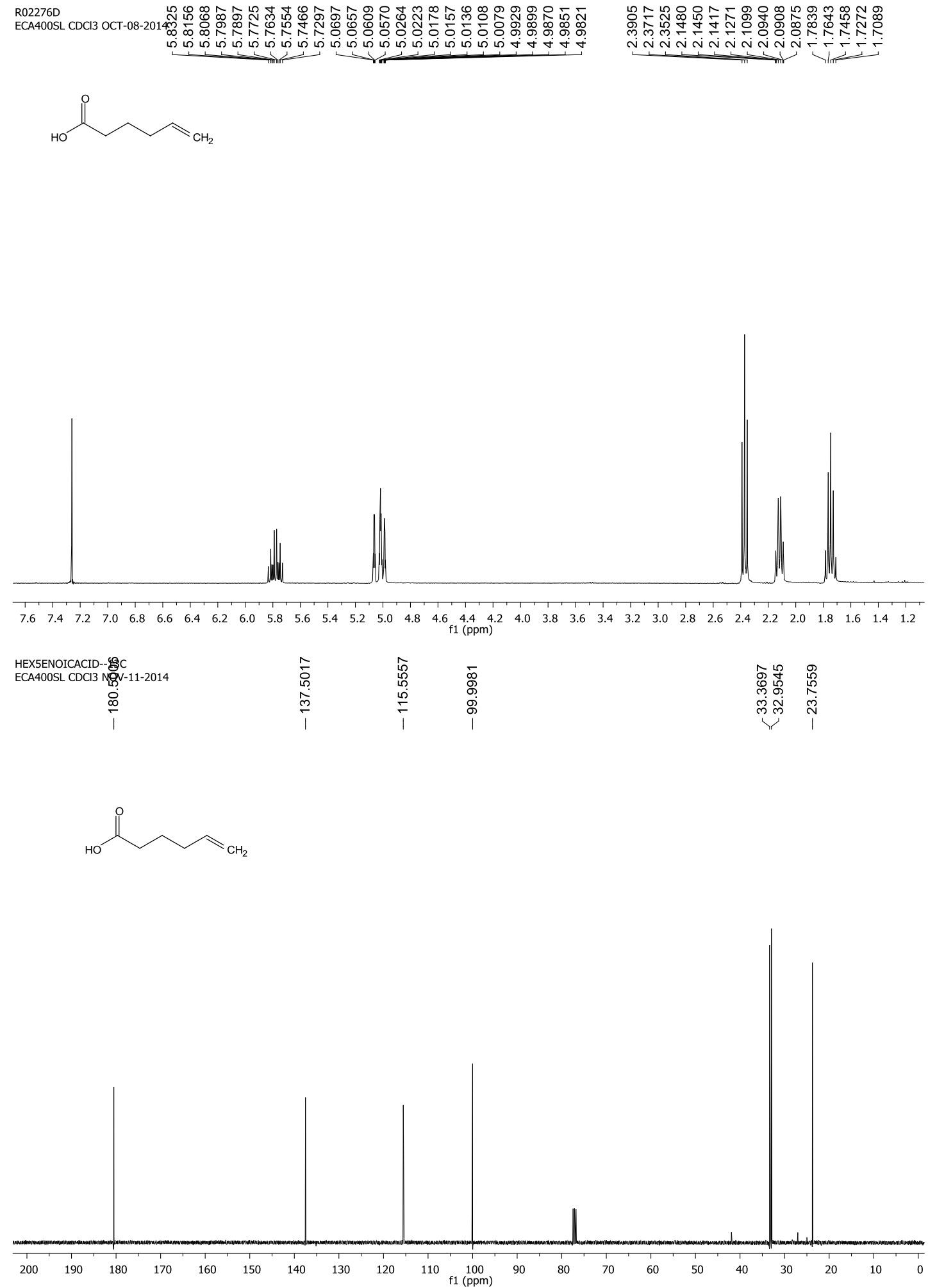


\section{Substrate 2.14.6}

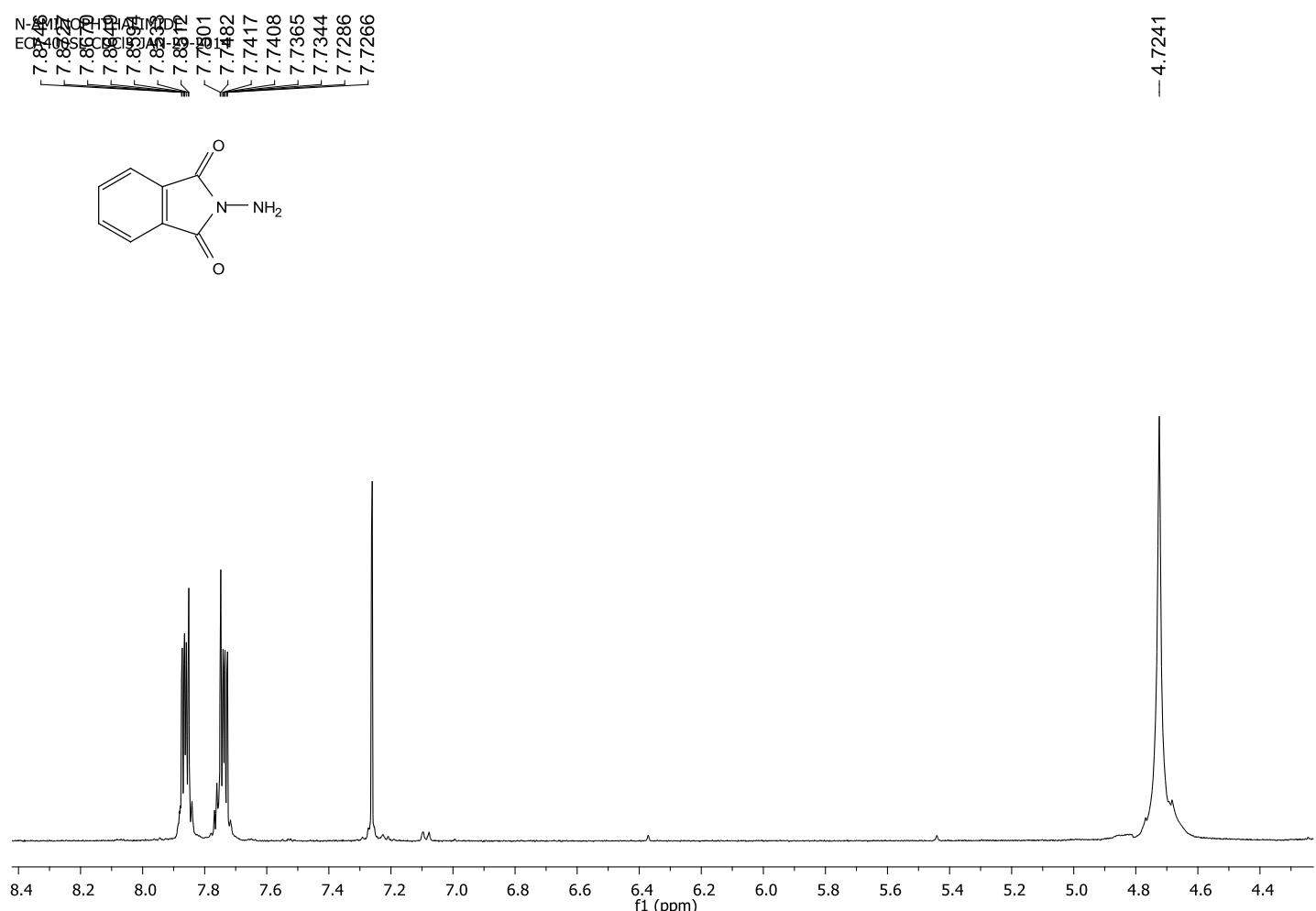

AMINOPHTHALIMIDE-13C ECA400SL CDCI3 JAN30-2014

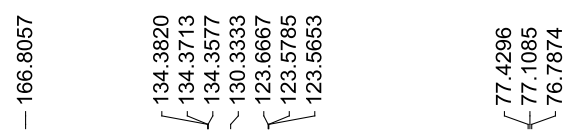<smiles>NN1C(=O)c2ccccc2C1=O</smiles>

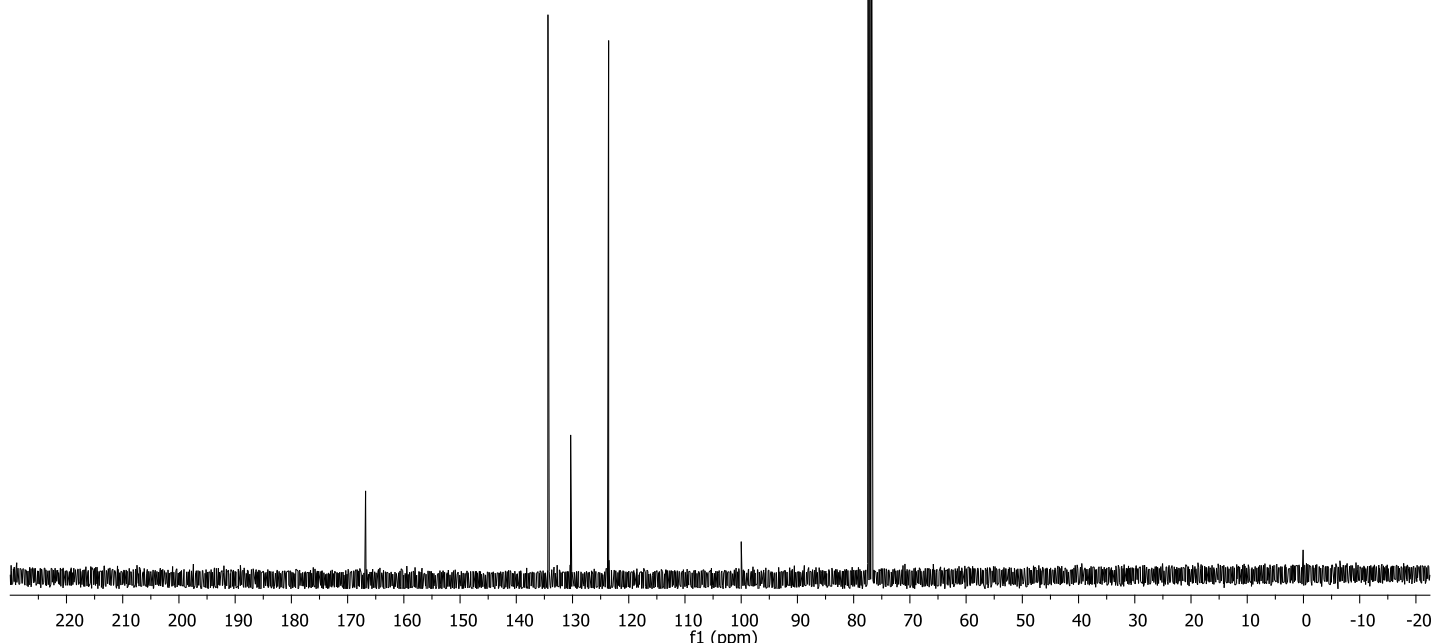


Substrate 2.14.5

R02084F1-disubstitutedaminophthalimide ECA400SL CDCI3 NOV-21-2013
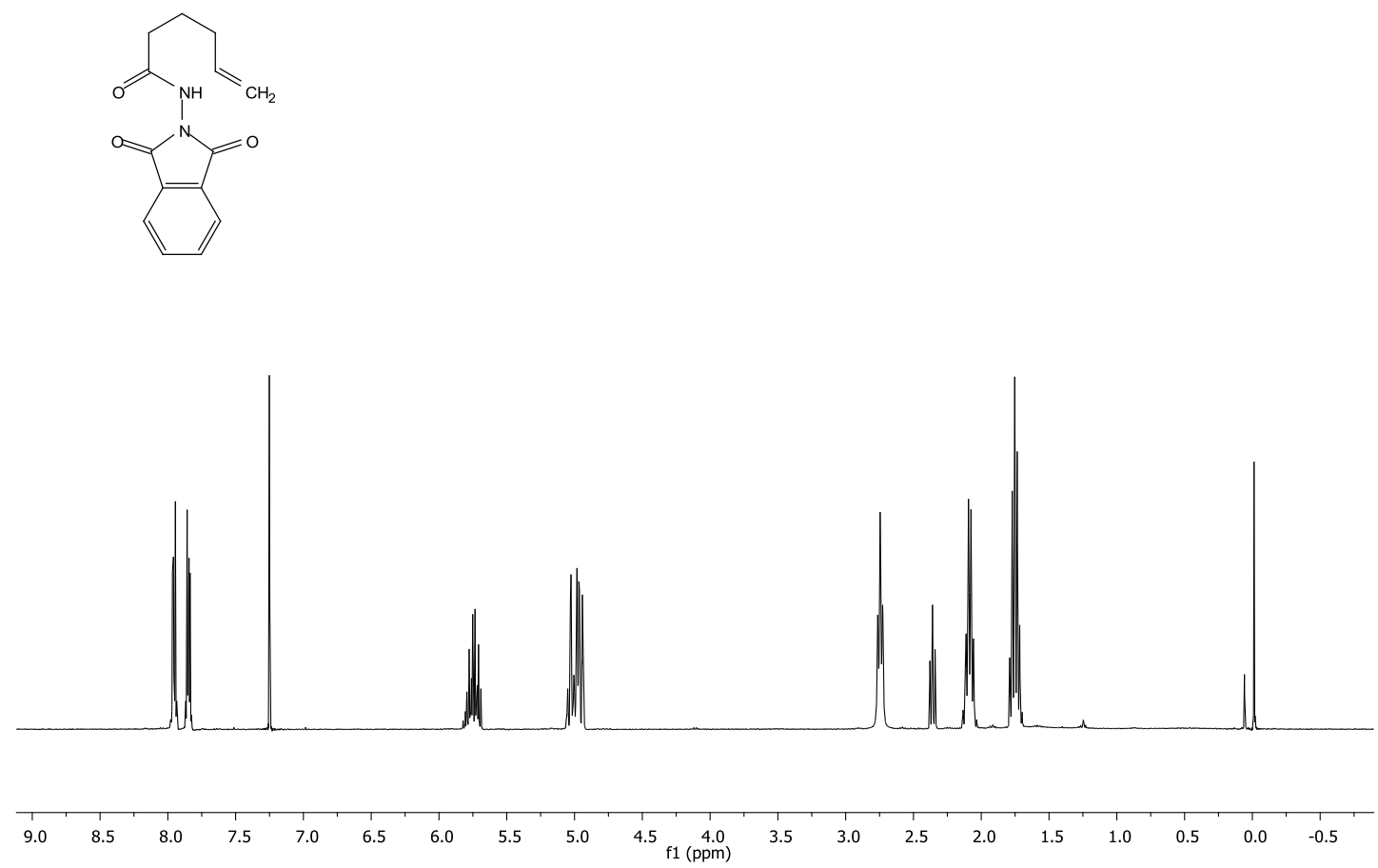

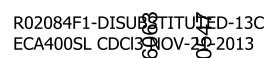
$\stackrel{i}{i} \stackrel{i}{i}$

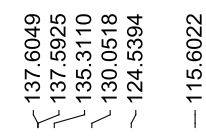

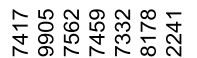

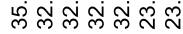
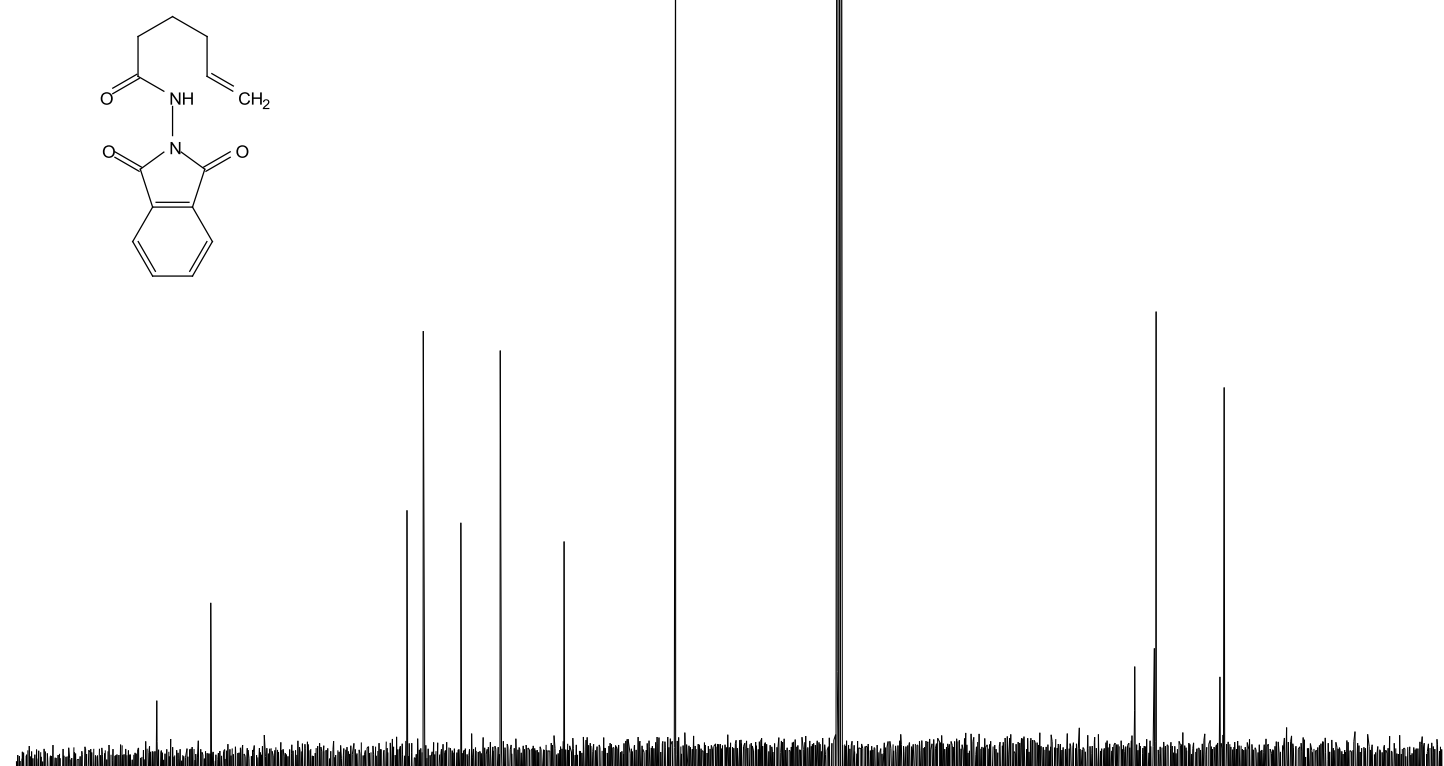
Substrate 2.14.3

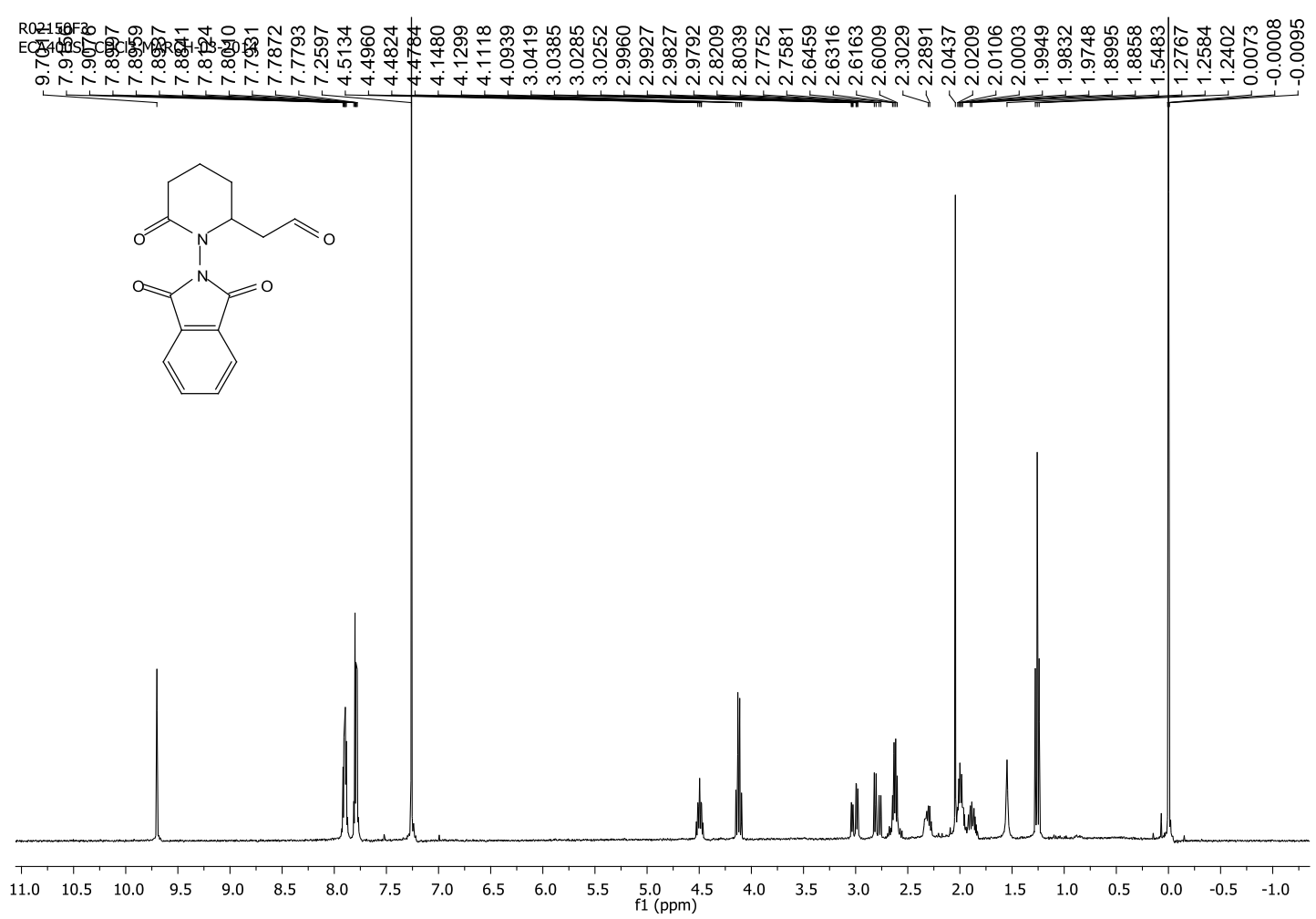

\begin{tabular}{|c|c|c|c|c|c|c|c|}
\hline 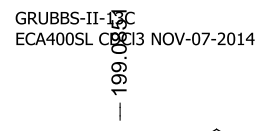 & 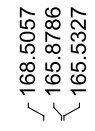 & 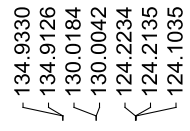 & 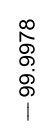 & 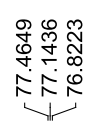 & $\begin{array}{l}\stackrel{\mathscr{m}}{+} \\
\stackrel{0}{0} \\
\dot{0} \\
1\end{array}$ & $\begin{array}{l}\frac{\infty}{0} \\
\stackrel{\infty}{+\infty} \\
\end{array}$ & 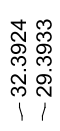 \\
\hline
\end{tabular}<smiles>O=CCC1CCCC(=O)N1N1C(=O)c2ccccc2C1=O</smiles>

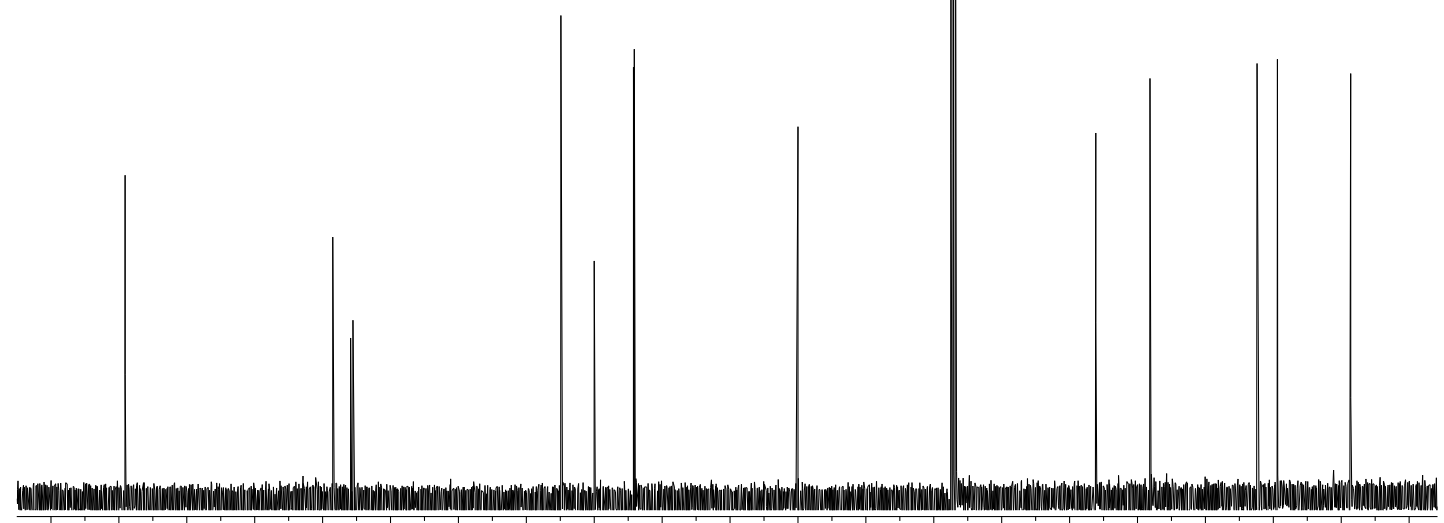

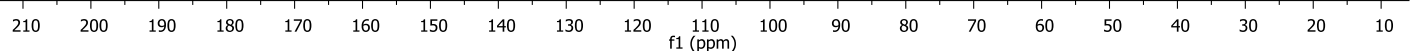


Substrate 2.16.12

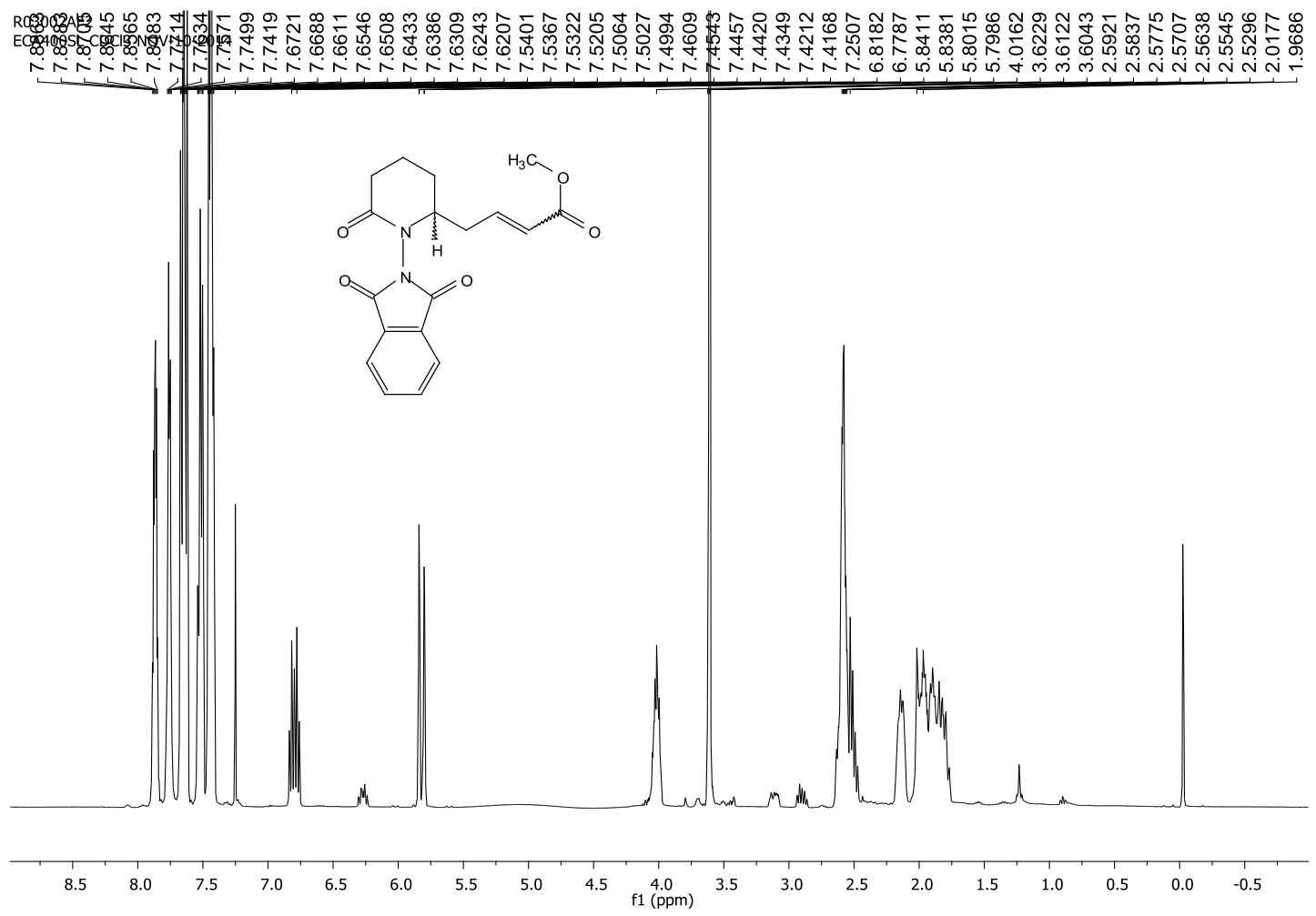

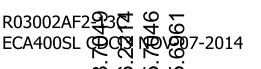
ஜ்

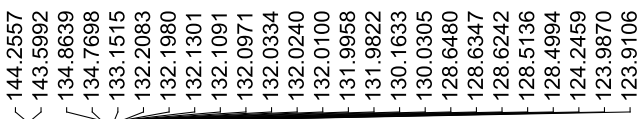

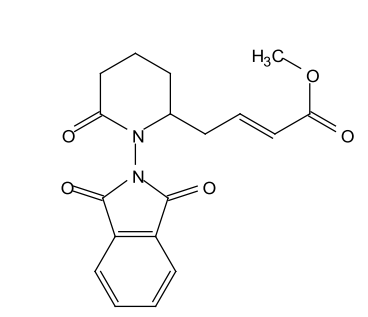

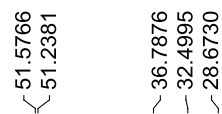

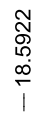

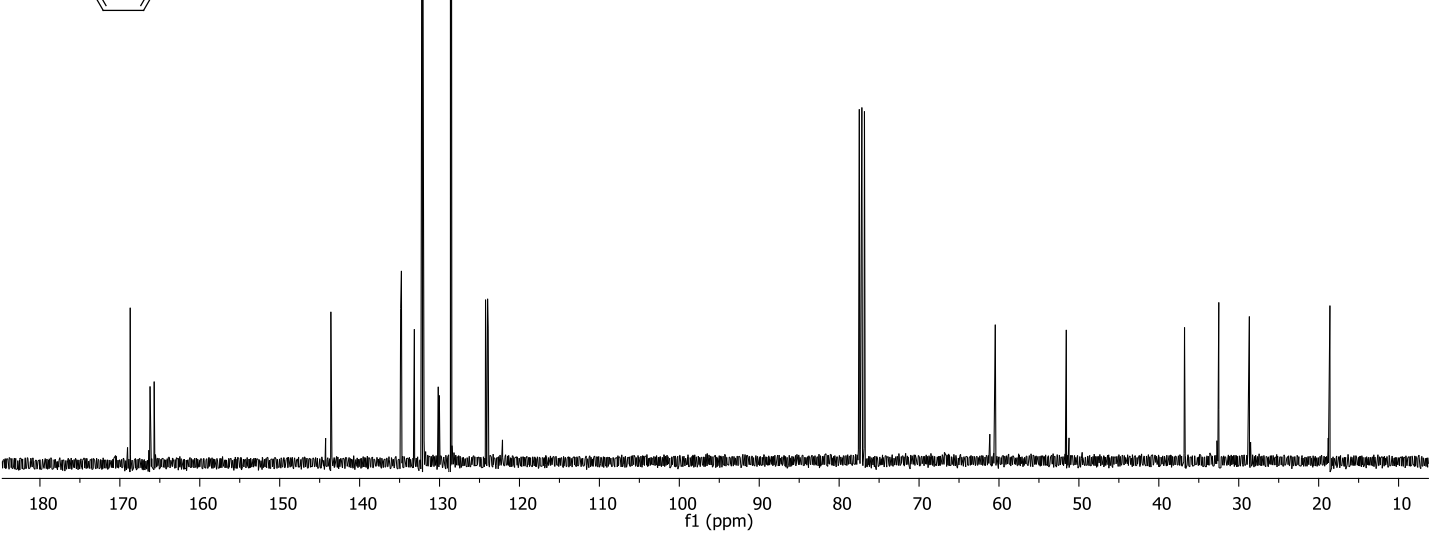

399 
Substrate 2.16.13

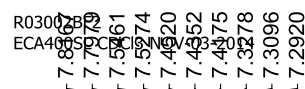

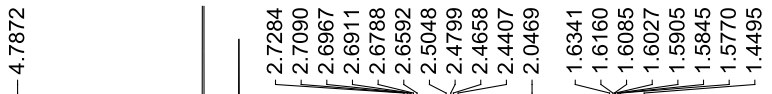

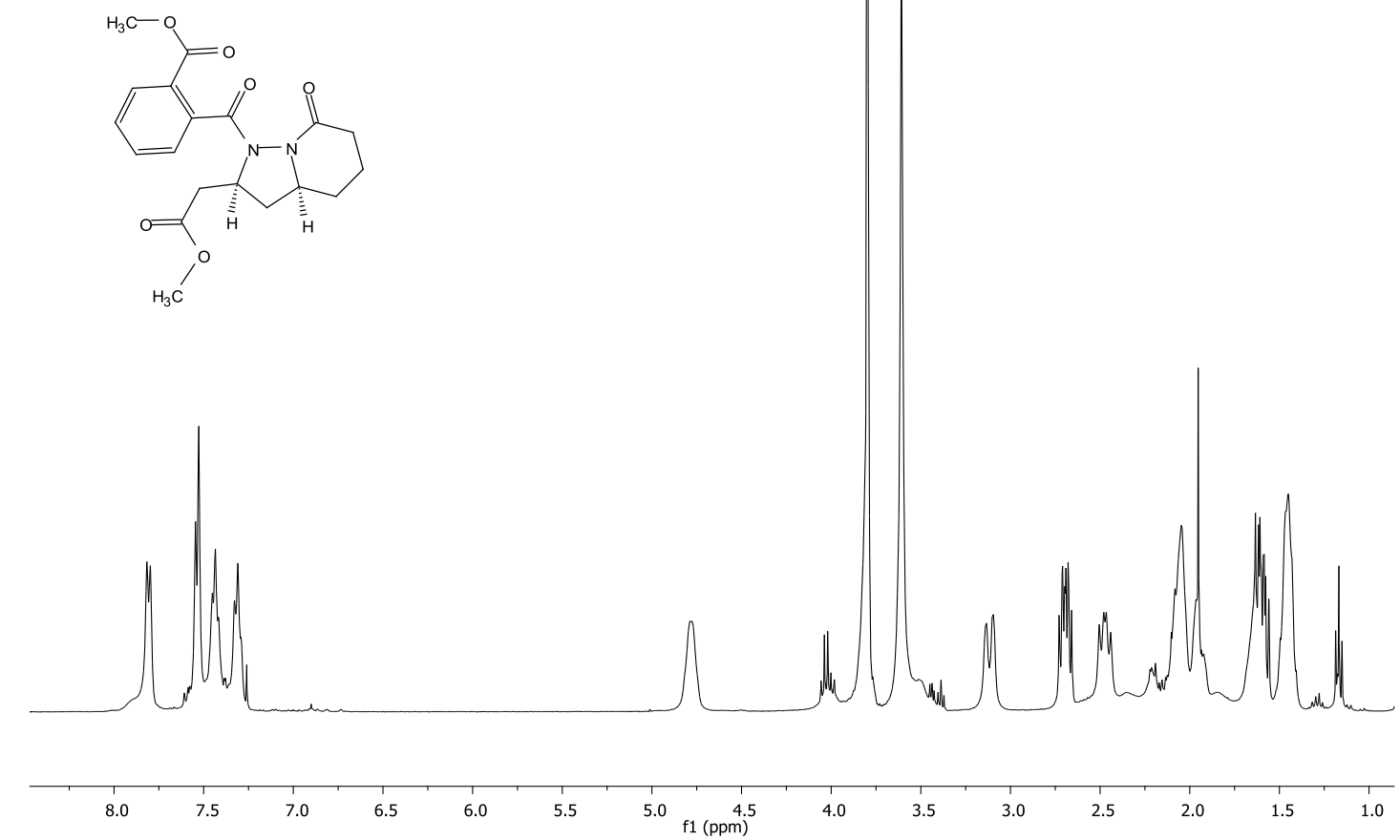

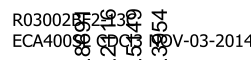

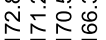

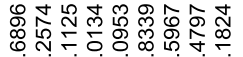

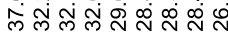

$<1$

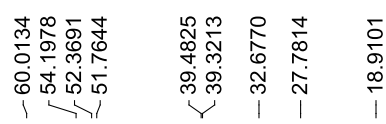

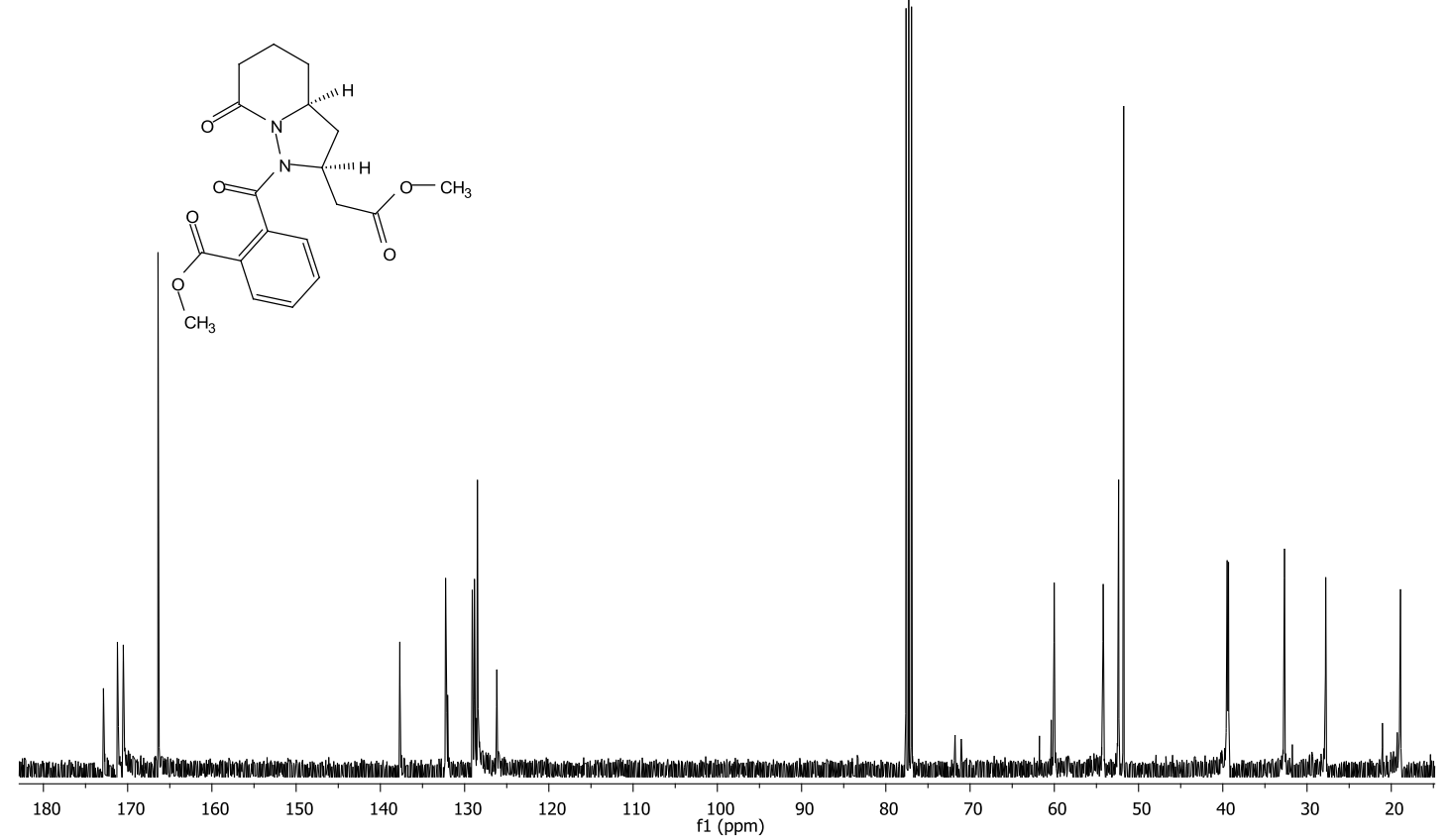




\subsubsection{Selected ${ }^{1} \mathrm{H}$ and ${ }^{13} \mathrm{C}$ NMR Spectra for Chapter III}
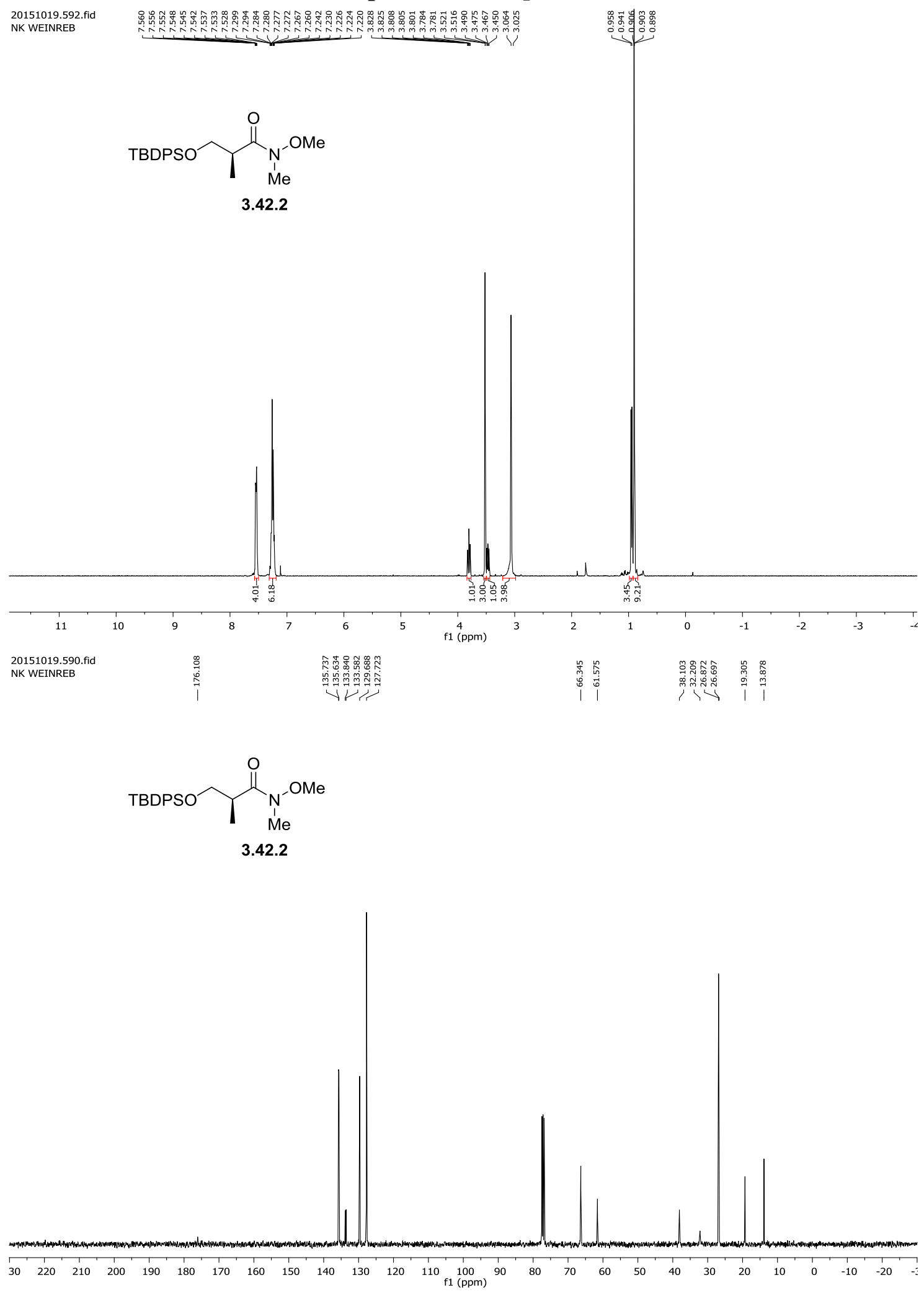

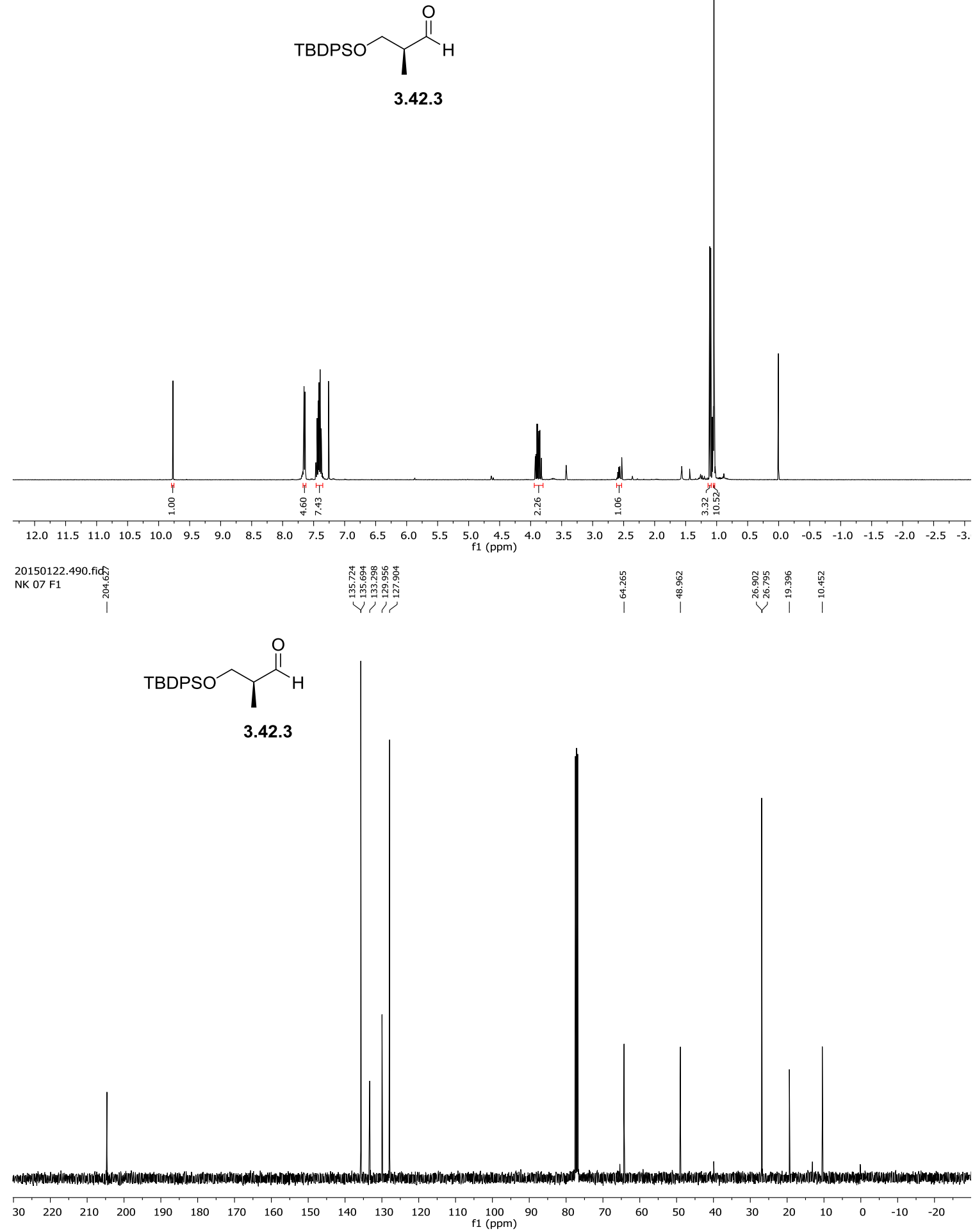

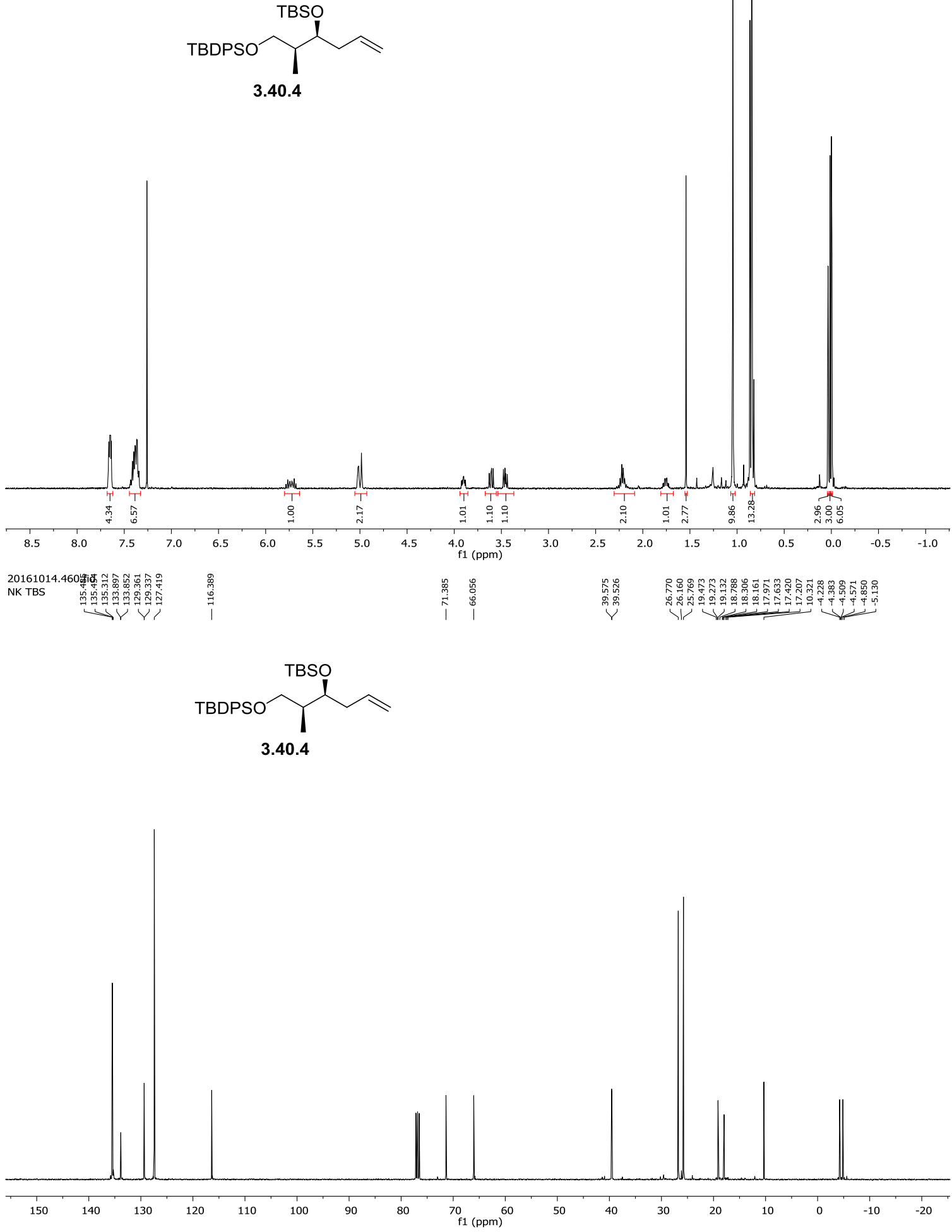

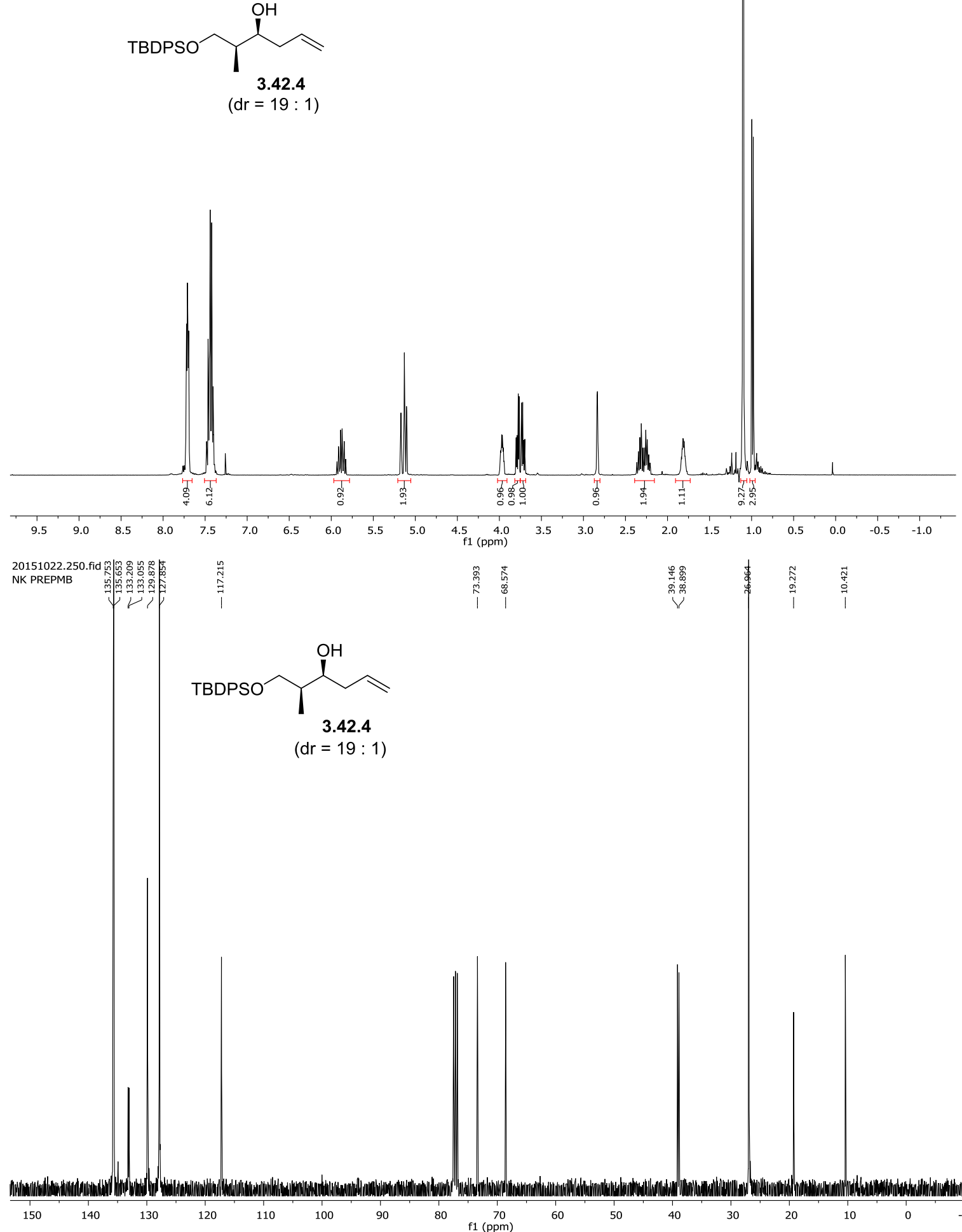

$40 \quad 30$ 

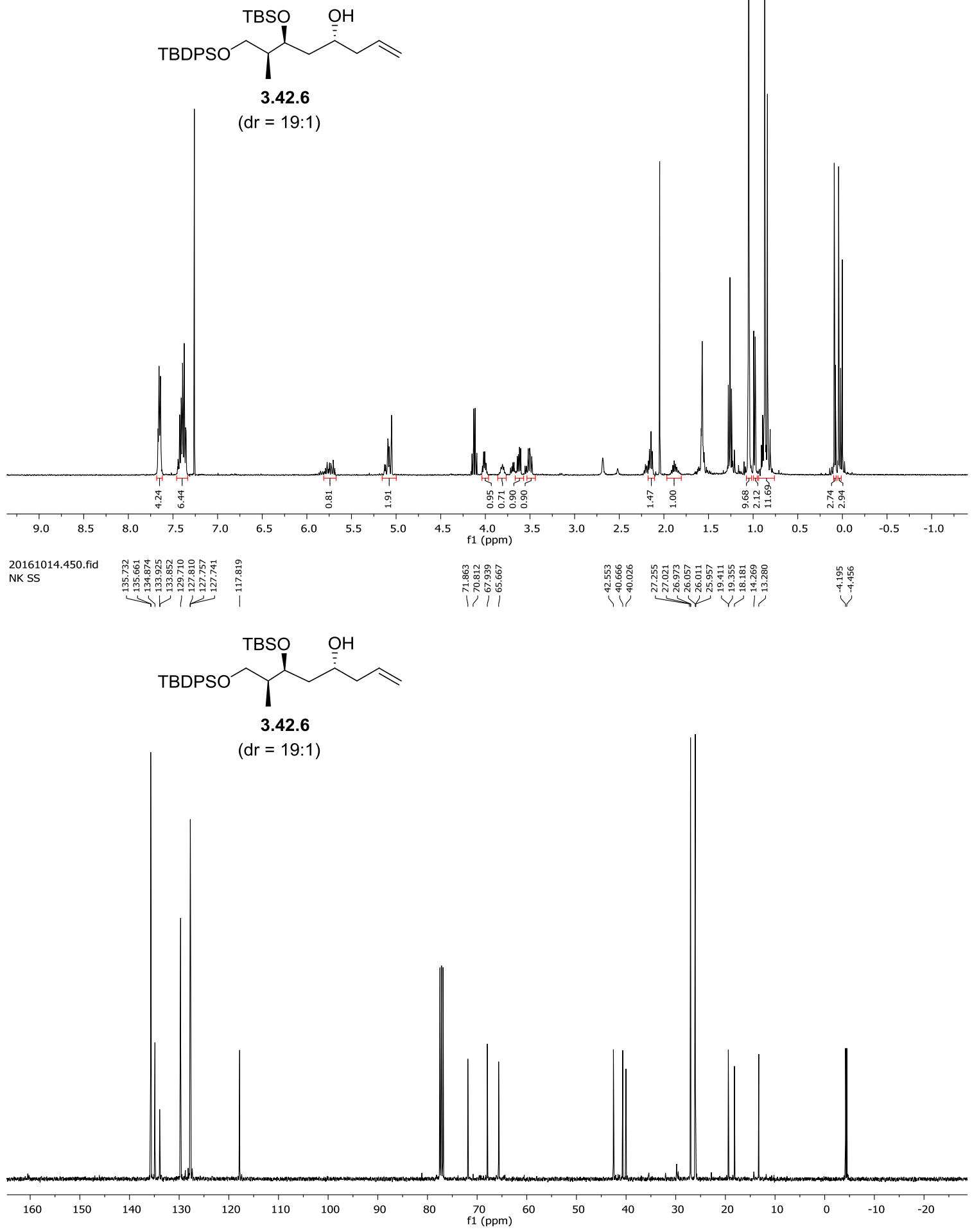

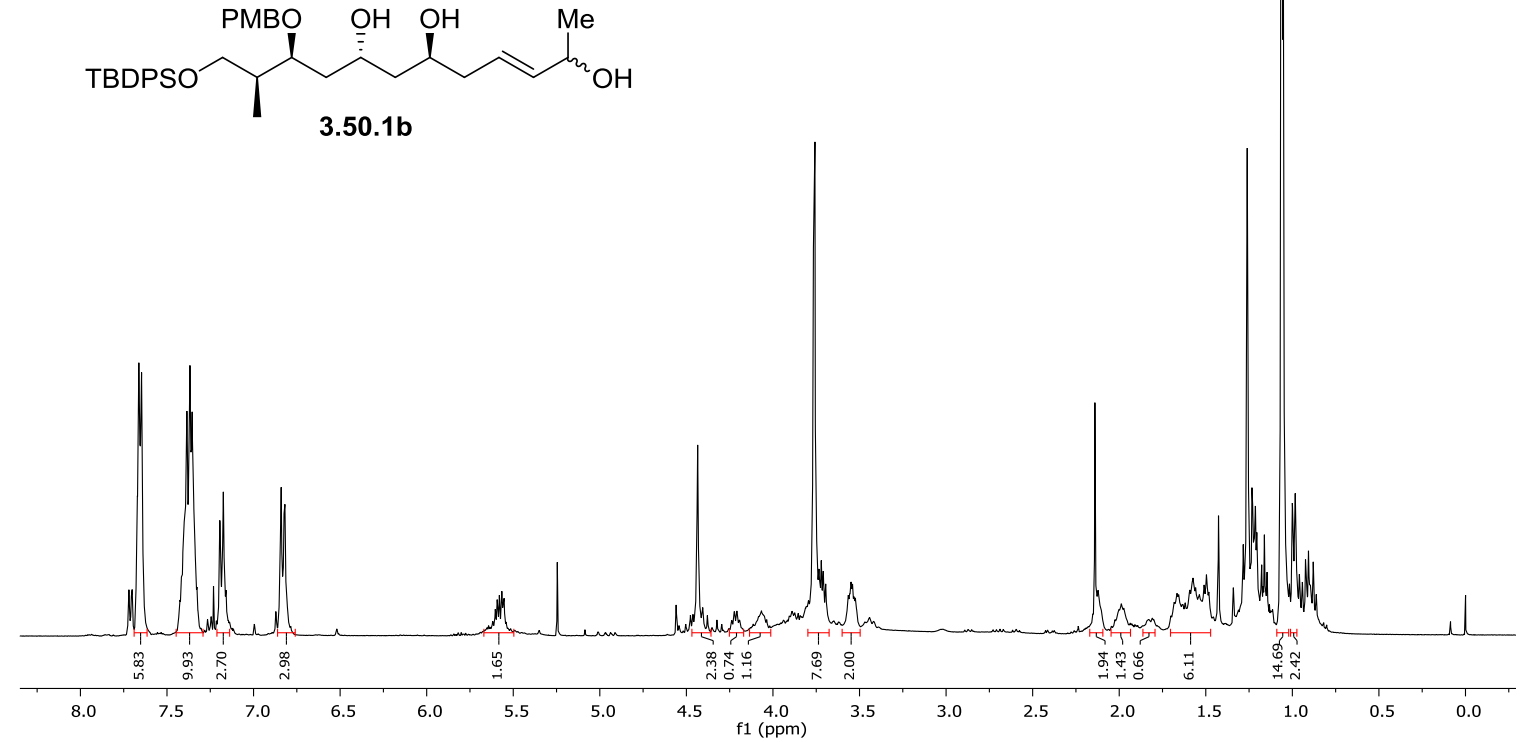


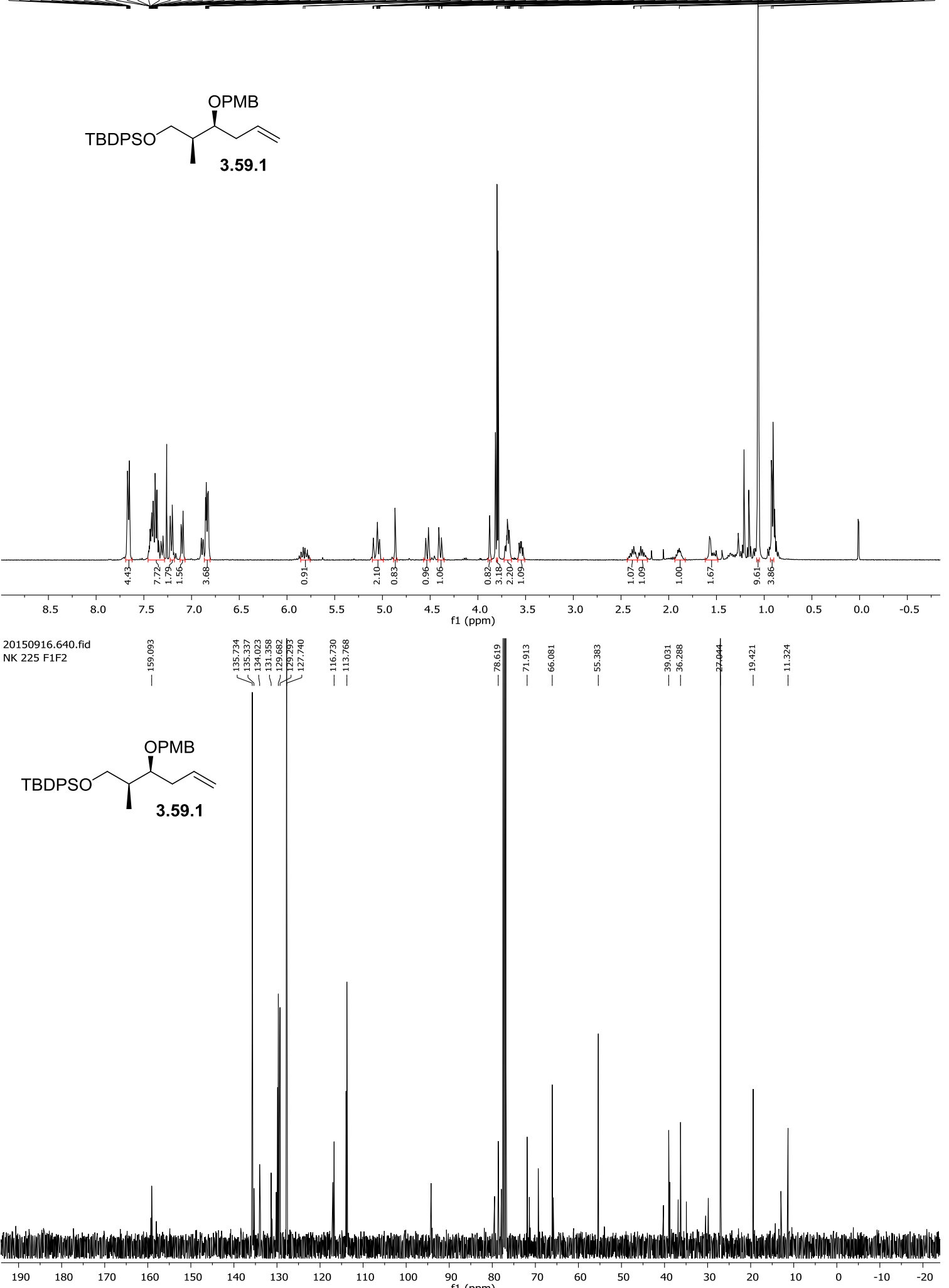

$\begin{array}{llllllllllll}190 & 180 & 170 & 160 & 150 & 140 & 130 & 120 & 110 & 100 & 90 & 90 \\ \mathrm{f} 1(\mathrm{ppm})\end{array}$ 

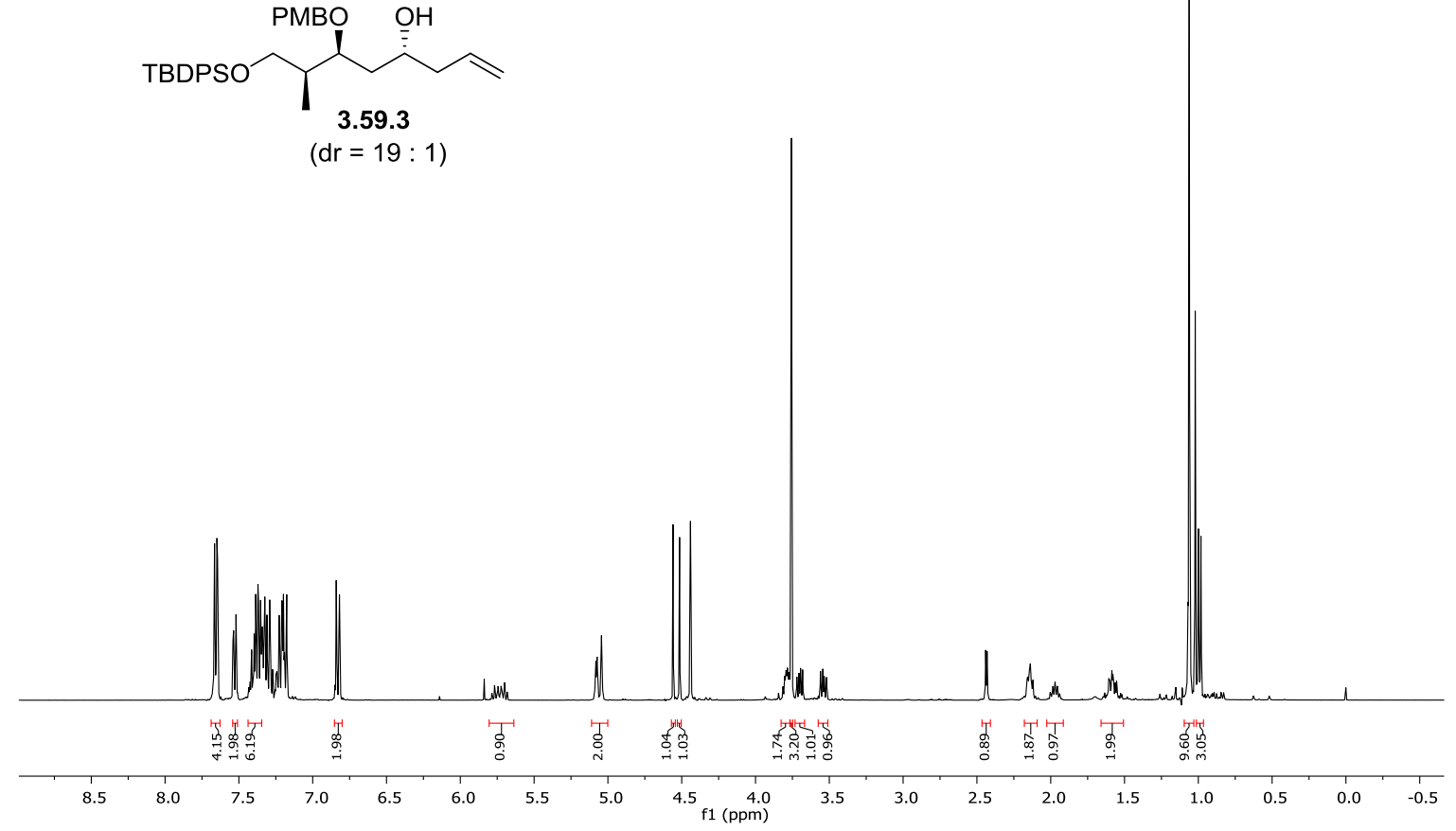

\begin{tabular}{|c|c|c|c|c|c|}
\hline $\begin{array}{l}20151020.490 . \text { fid } \\
\text { NK } 259 \text { F2 }\end{array}$ & 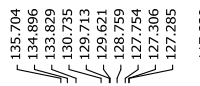 & | & 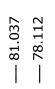 & 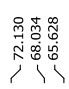 & \\
\hline
\end{tabular}
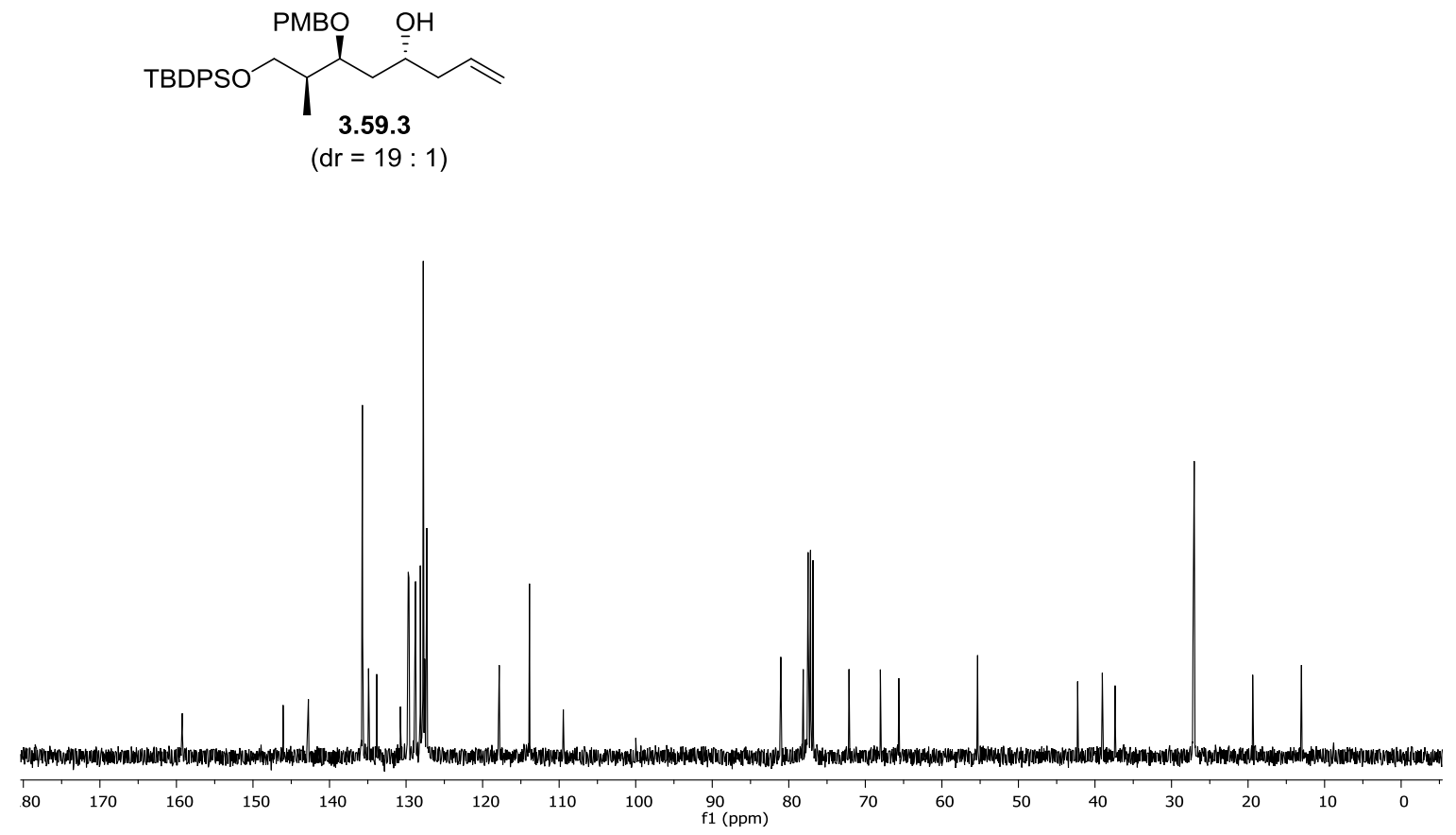


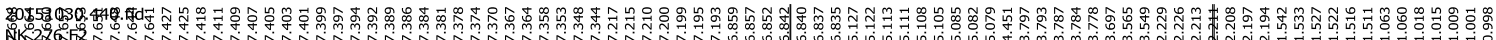

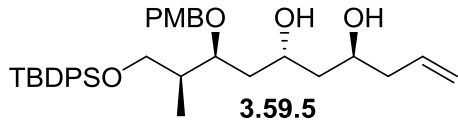

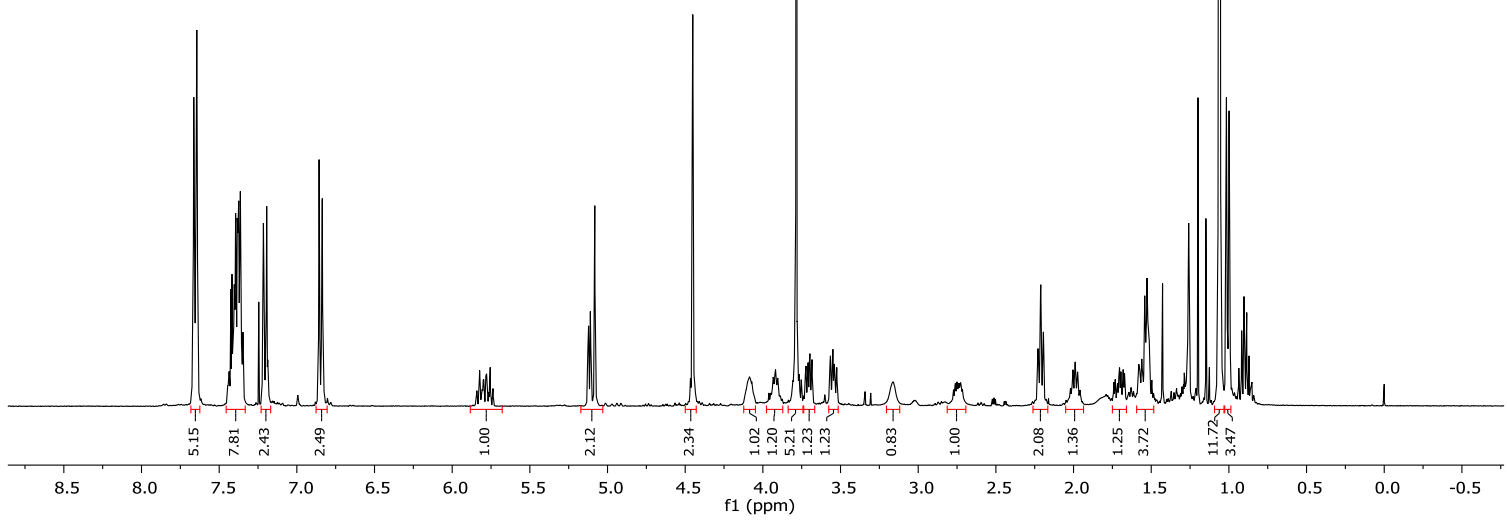

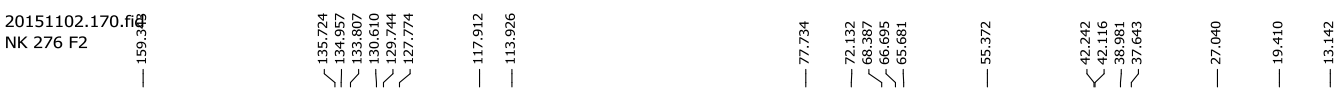
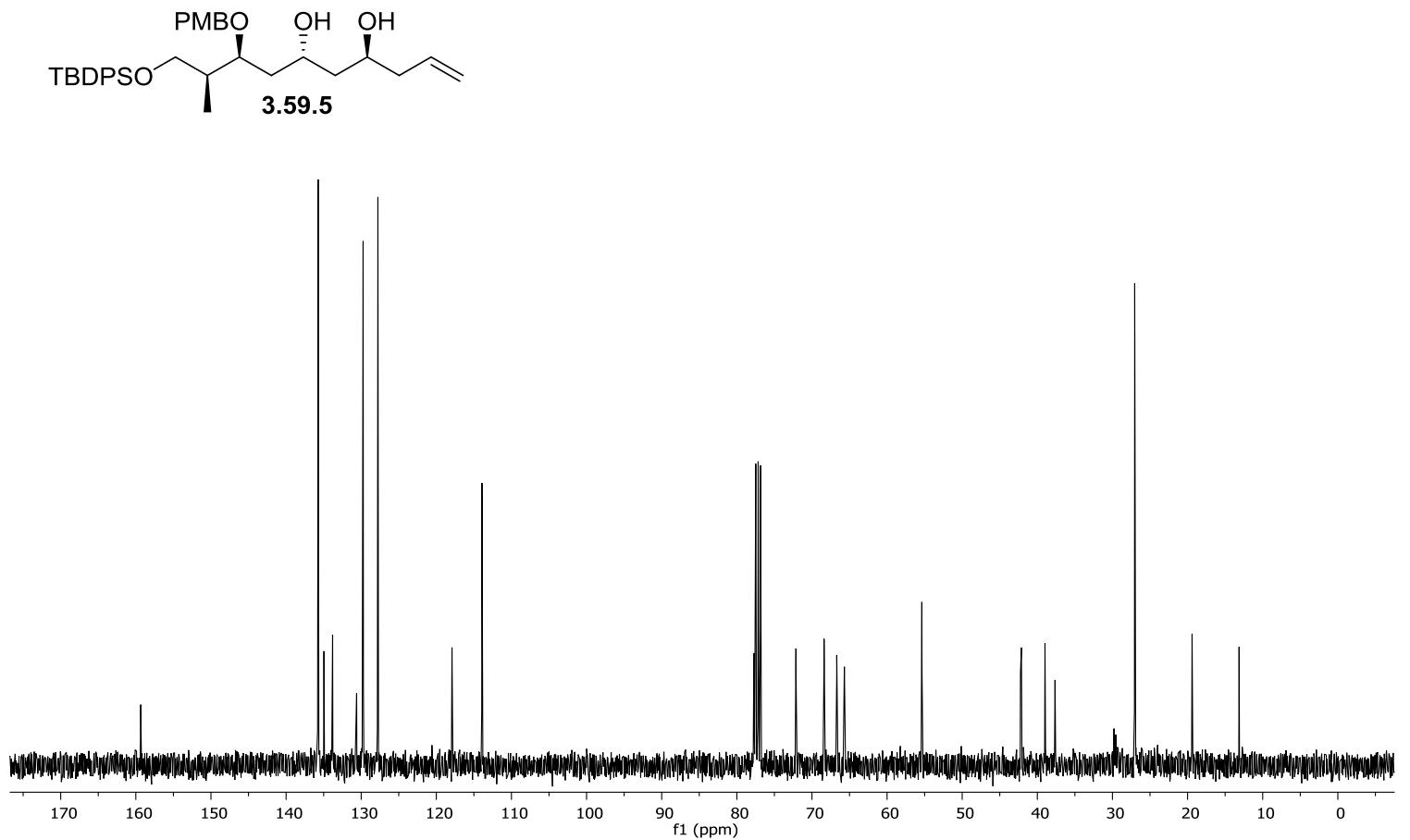


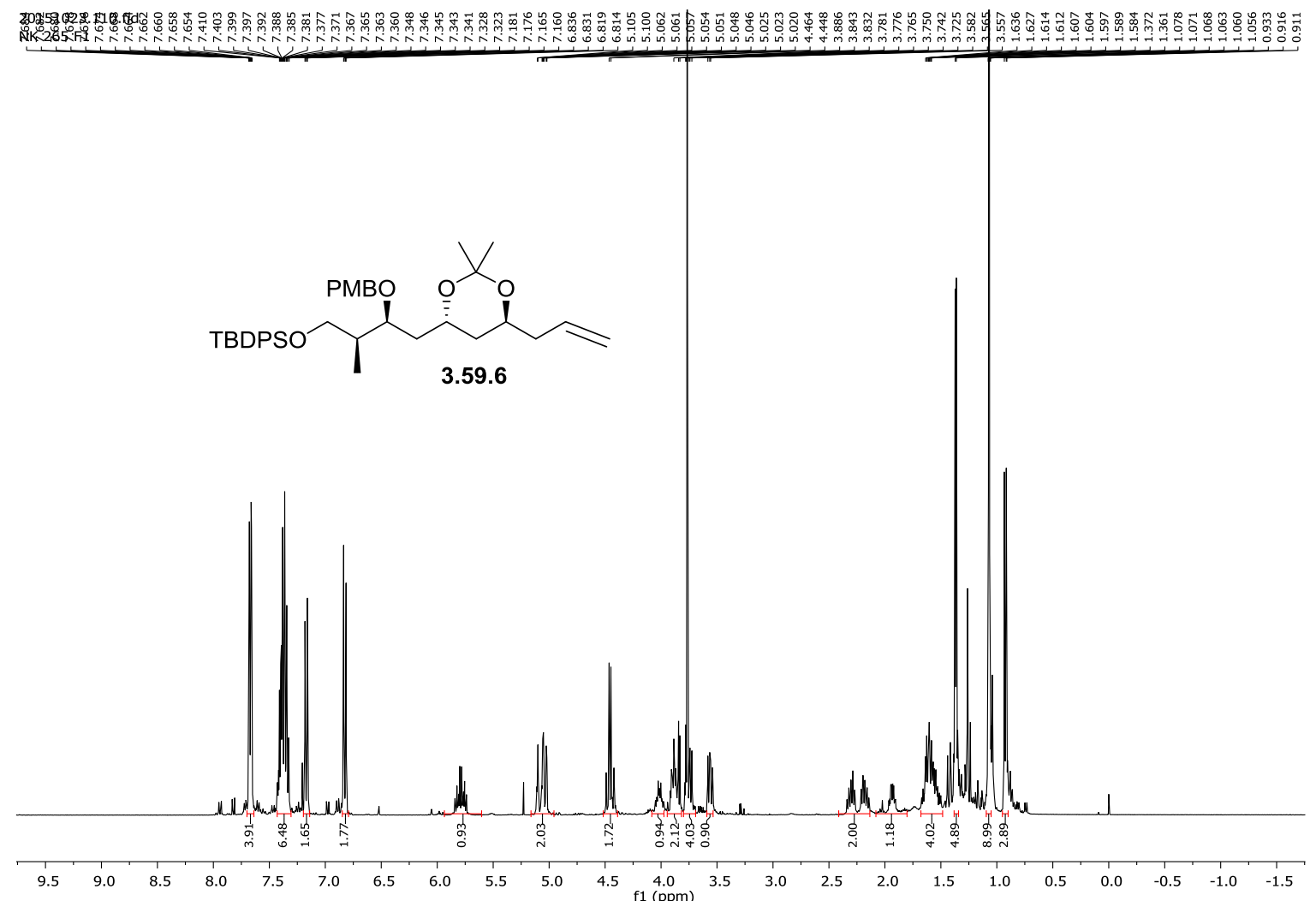

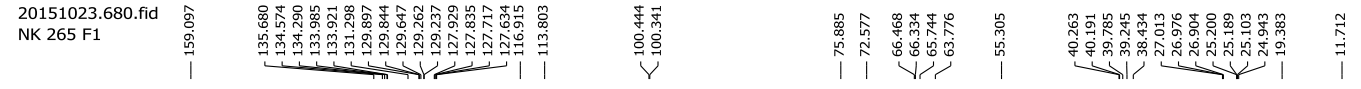

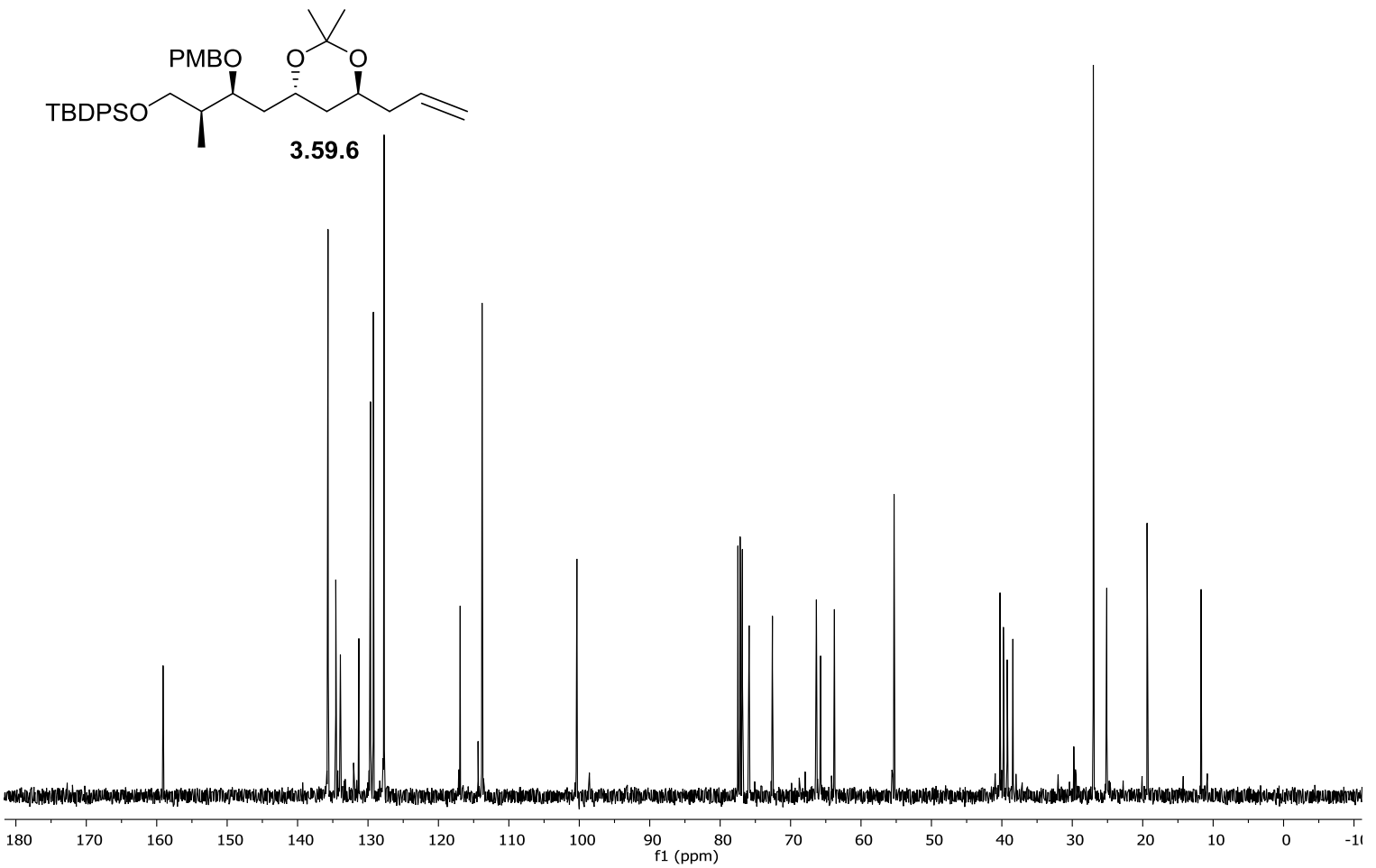




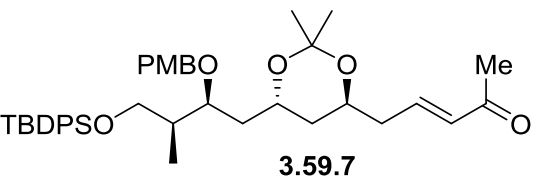

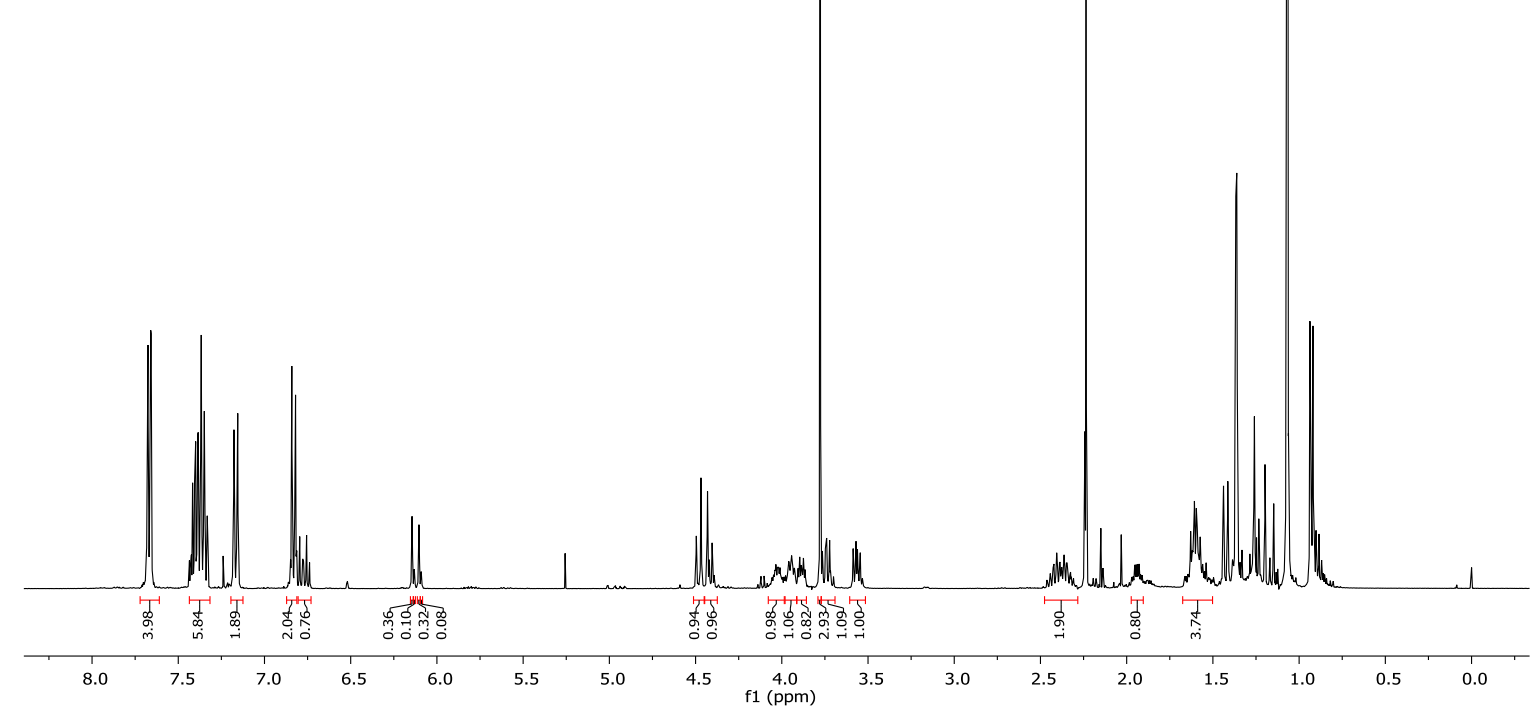

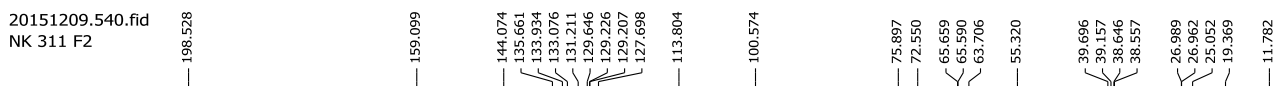

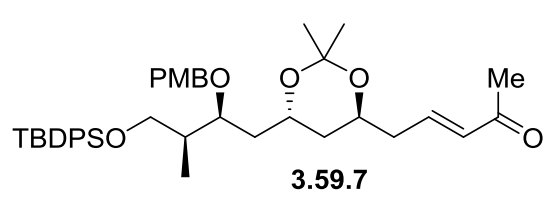




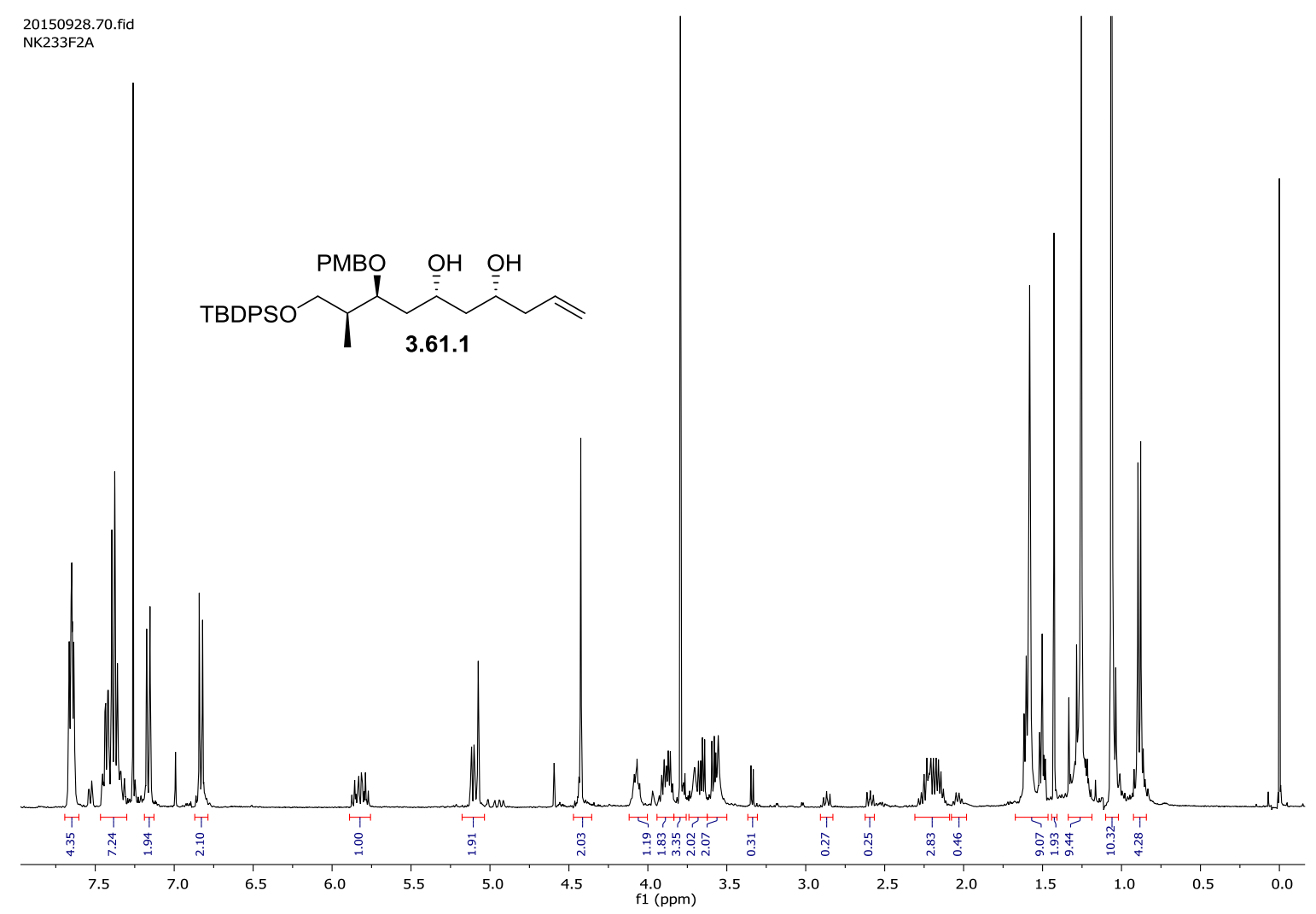




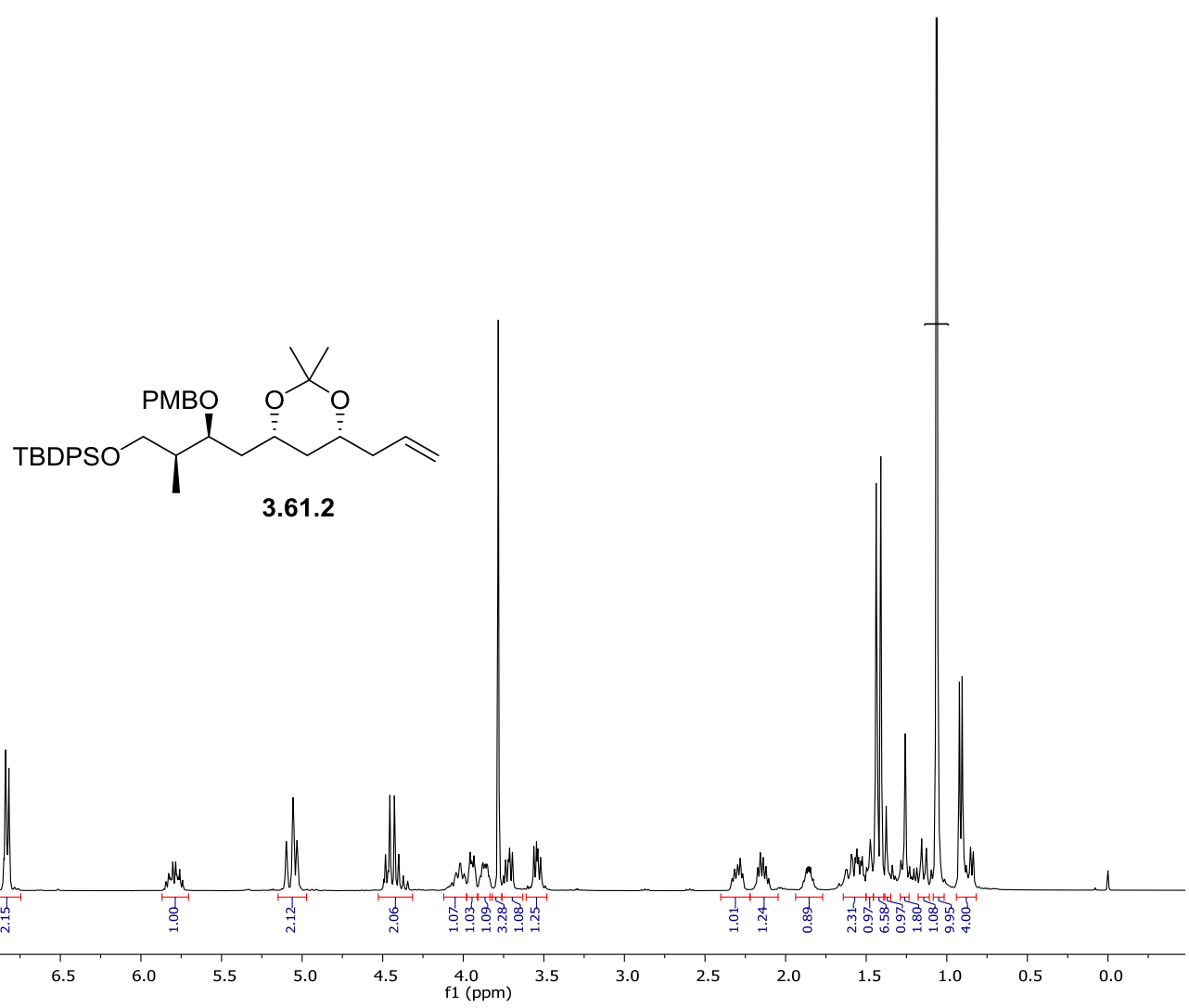

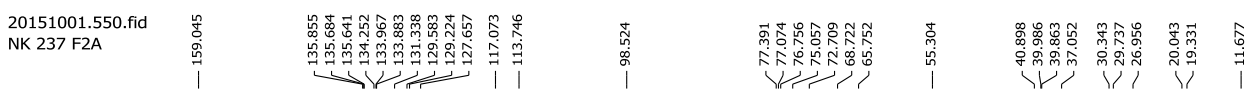
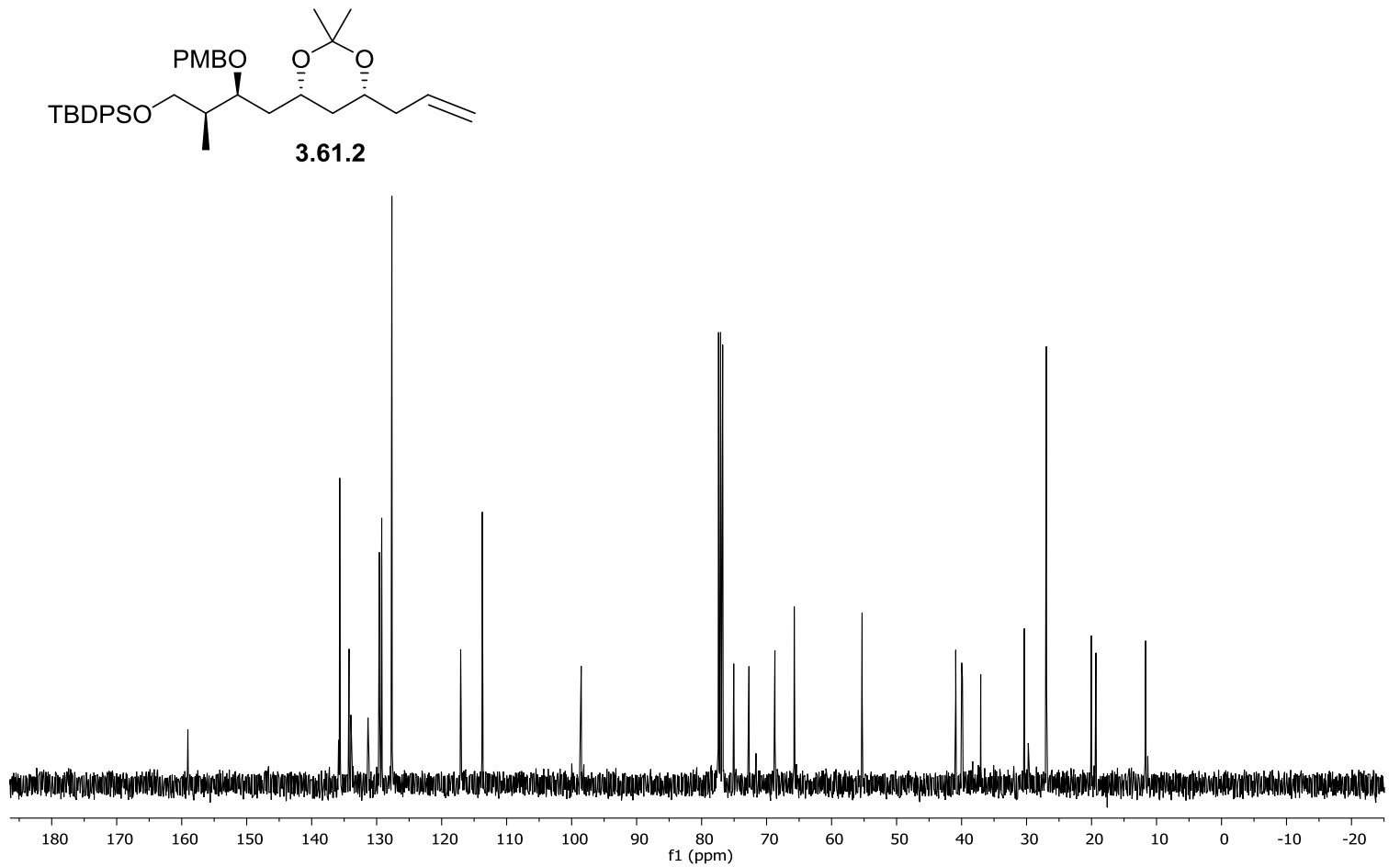
<smiles>CC(CO[R16](C)(C)C)C(C)CC(O)CC(O)C/C=C/C(=O)c1ccccc1</smiles>

3.64 .2

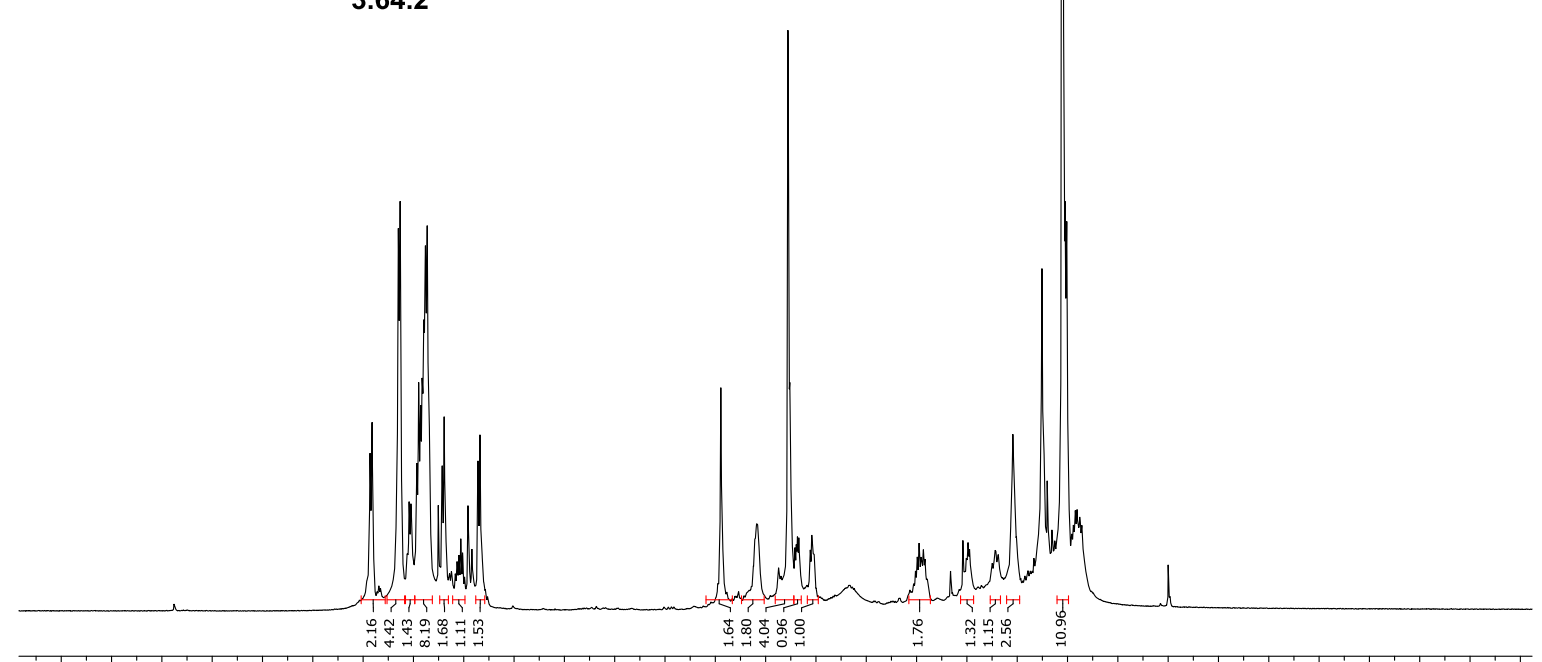

$\begin{array}{lllllllllllllllllllllllllllllllllllll}11.0 & 10.5 & 10.0 & 9.5 & 9.0 & 8.5 & 8.0 & 7.5 & 7.0 & 6.5 & 6.0 & 5.5 & 5.0 & 4.5 & 4.0 & 3.5 & 3.0 & 2.5 & 2.0 & 1.5 & 1.0 & 0.5 & 0.0 & -0.5 & -1.0 & -1.5 & -2.0 & -2.5 & -3.0 & -3.1\end{array}$

20160325.520.fid NK 425 F3

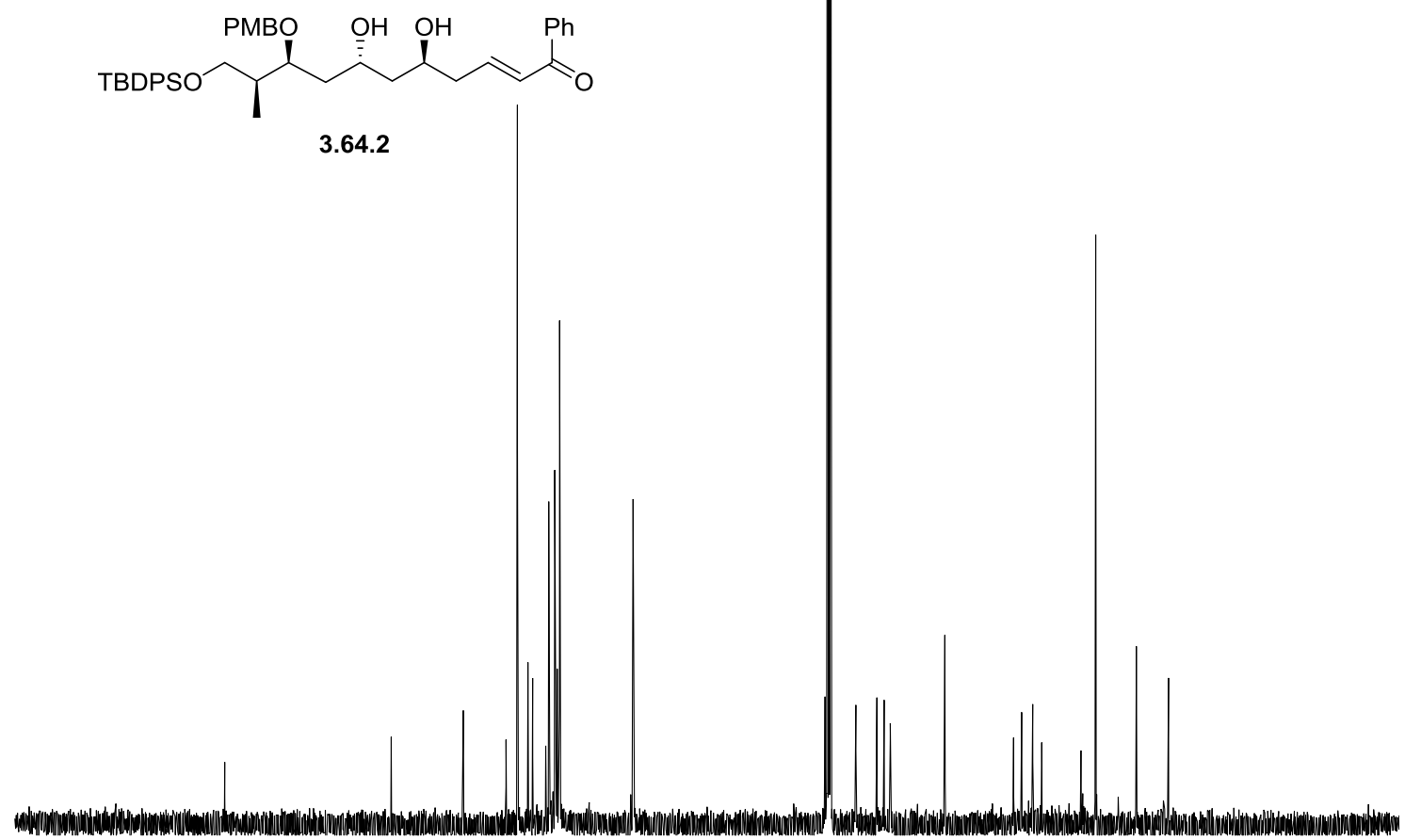

3.64.2

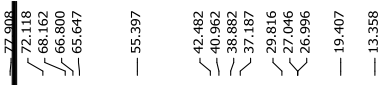

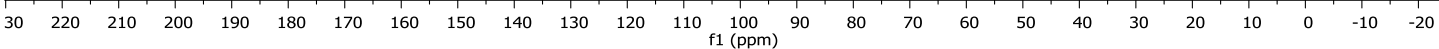




\subsubsection{Selected ${ }^{1} \mathrm{H}$ and ${ }^{13} \mathrm{C}$ NMR Spectra for Chapter IV}

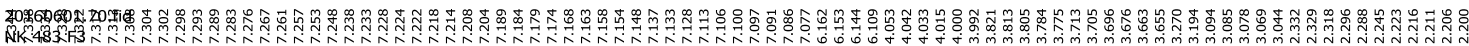

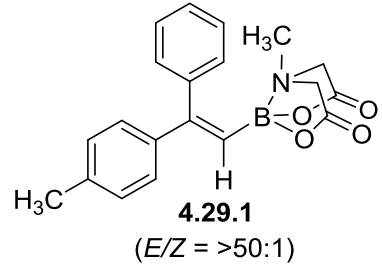
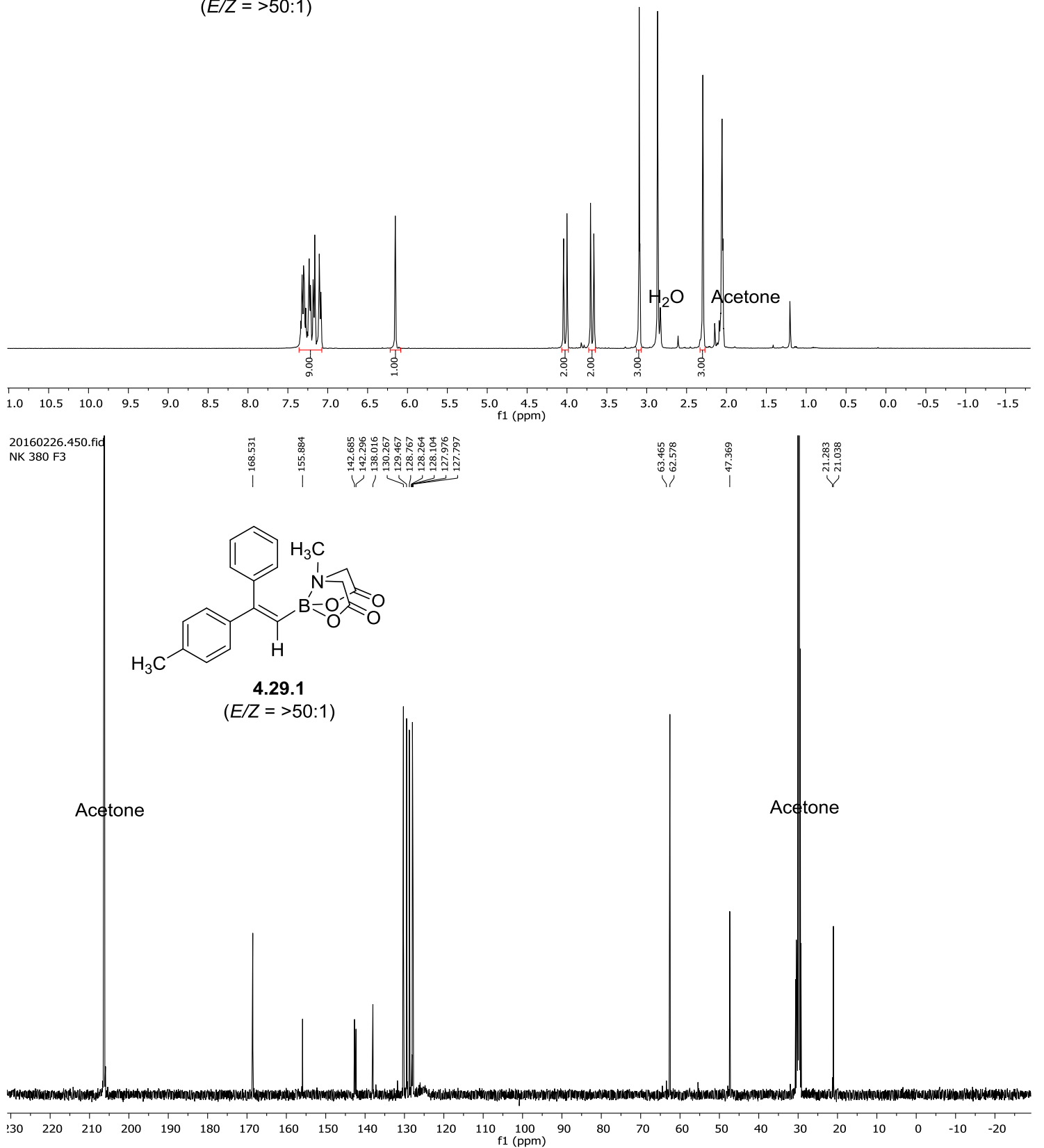

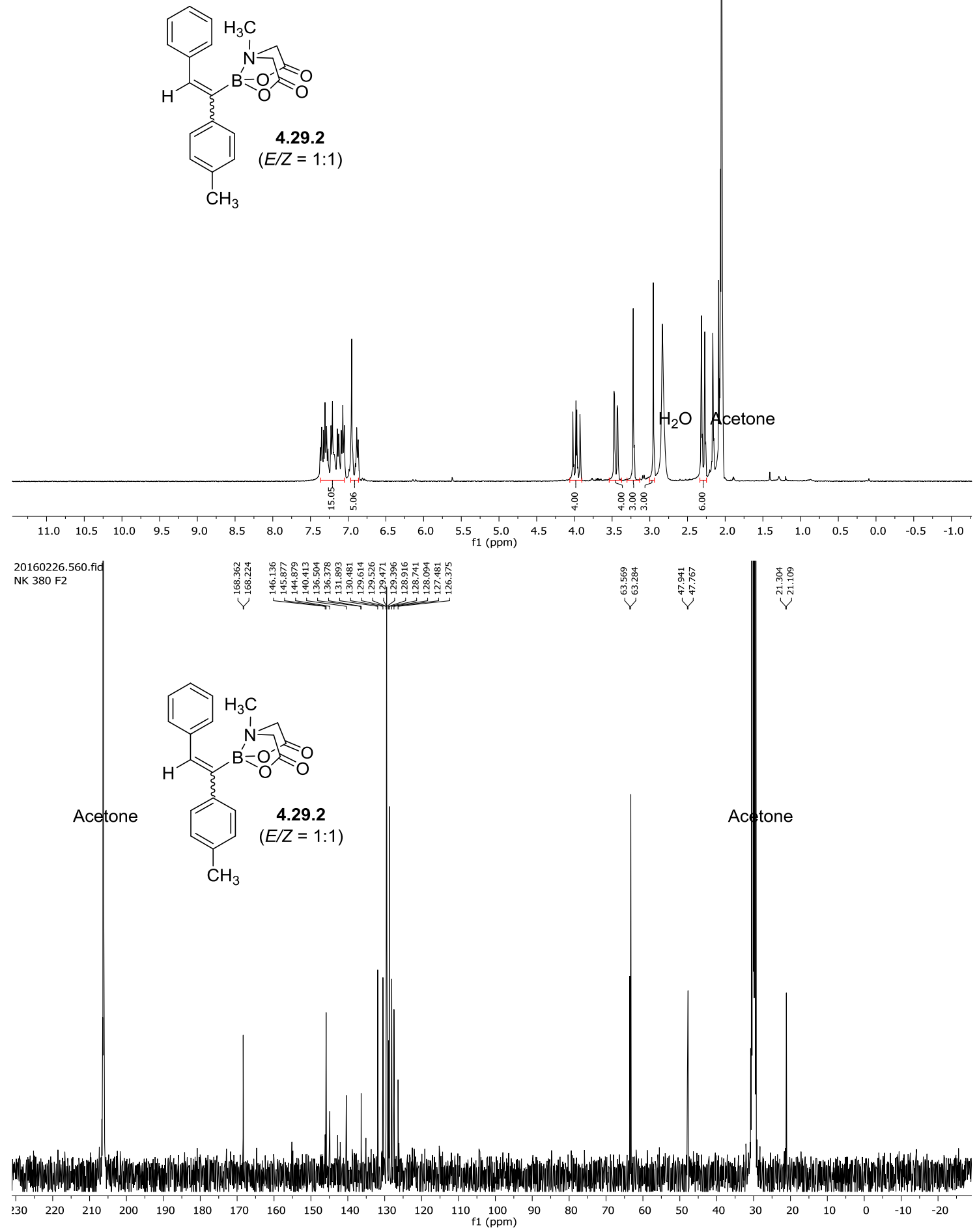

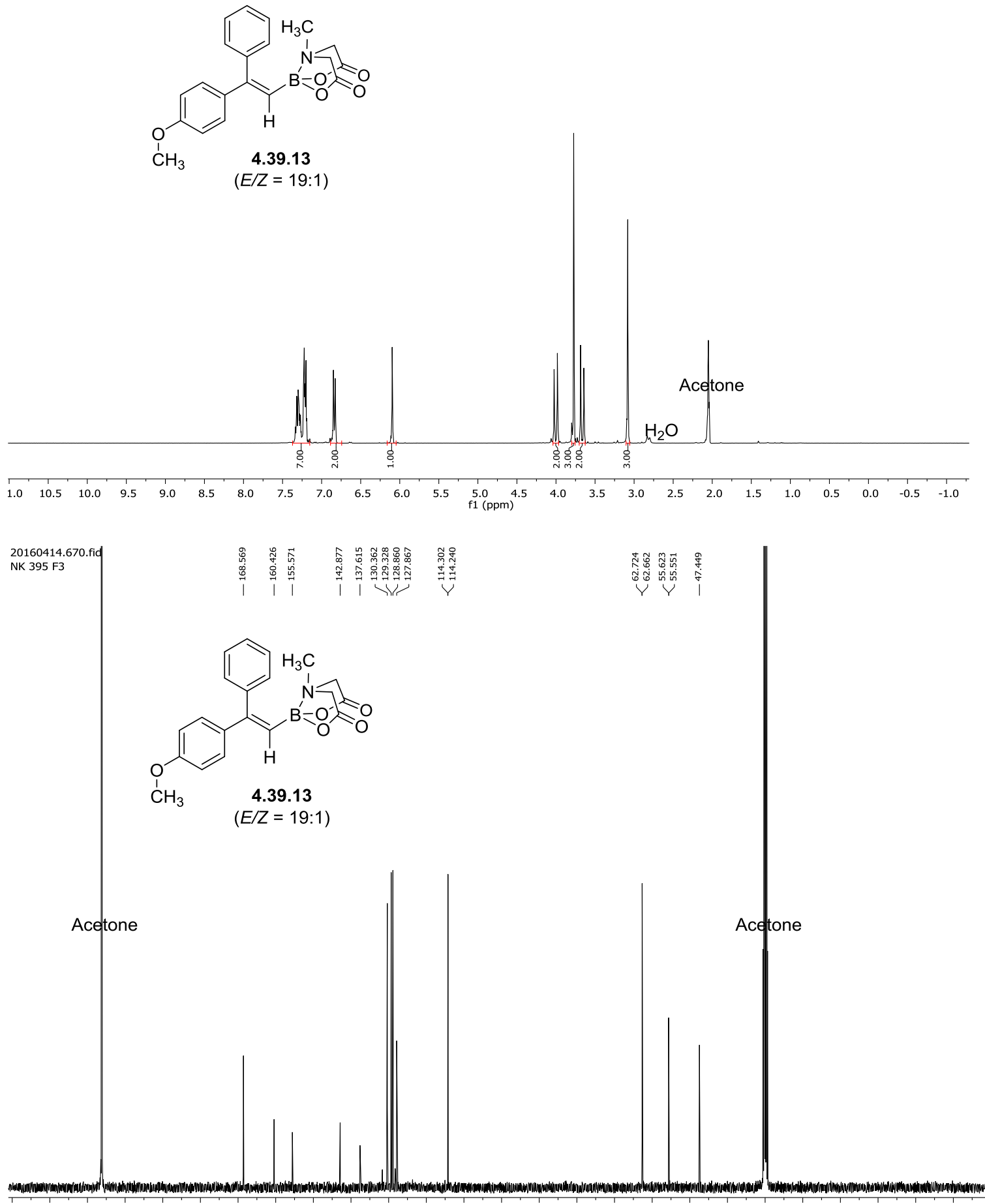

$\begin{array}{llllllllllllllllllllllllll}130 & 220 & 210 & 200 & 190 & 180 & 170 & 160 & 150 & 140 & 130 & 120 & 110 & 100 & 90 & 80 & 70 & 60 & 50 & 40 & 30 & 20 & 10 & 0 & -10 & -20\end{array}$ 

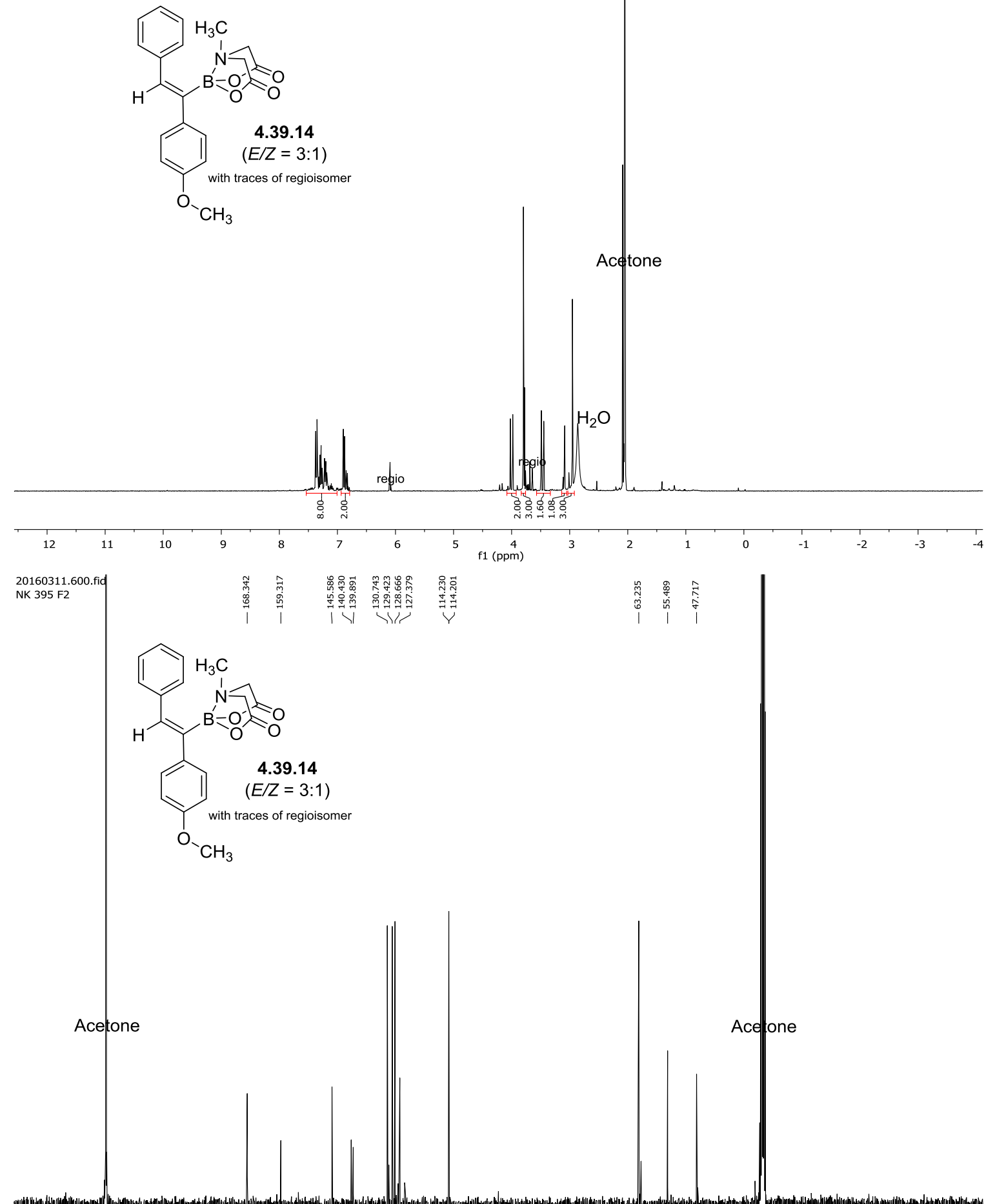


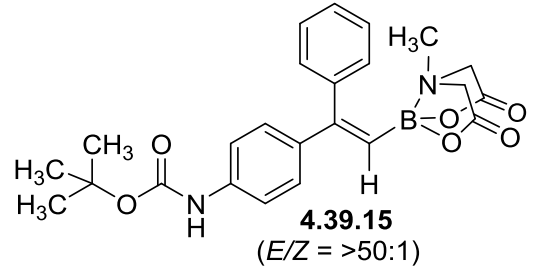
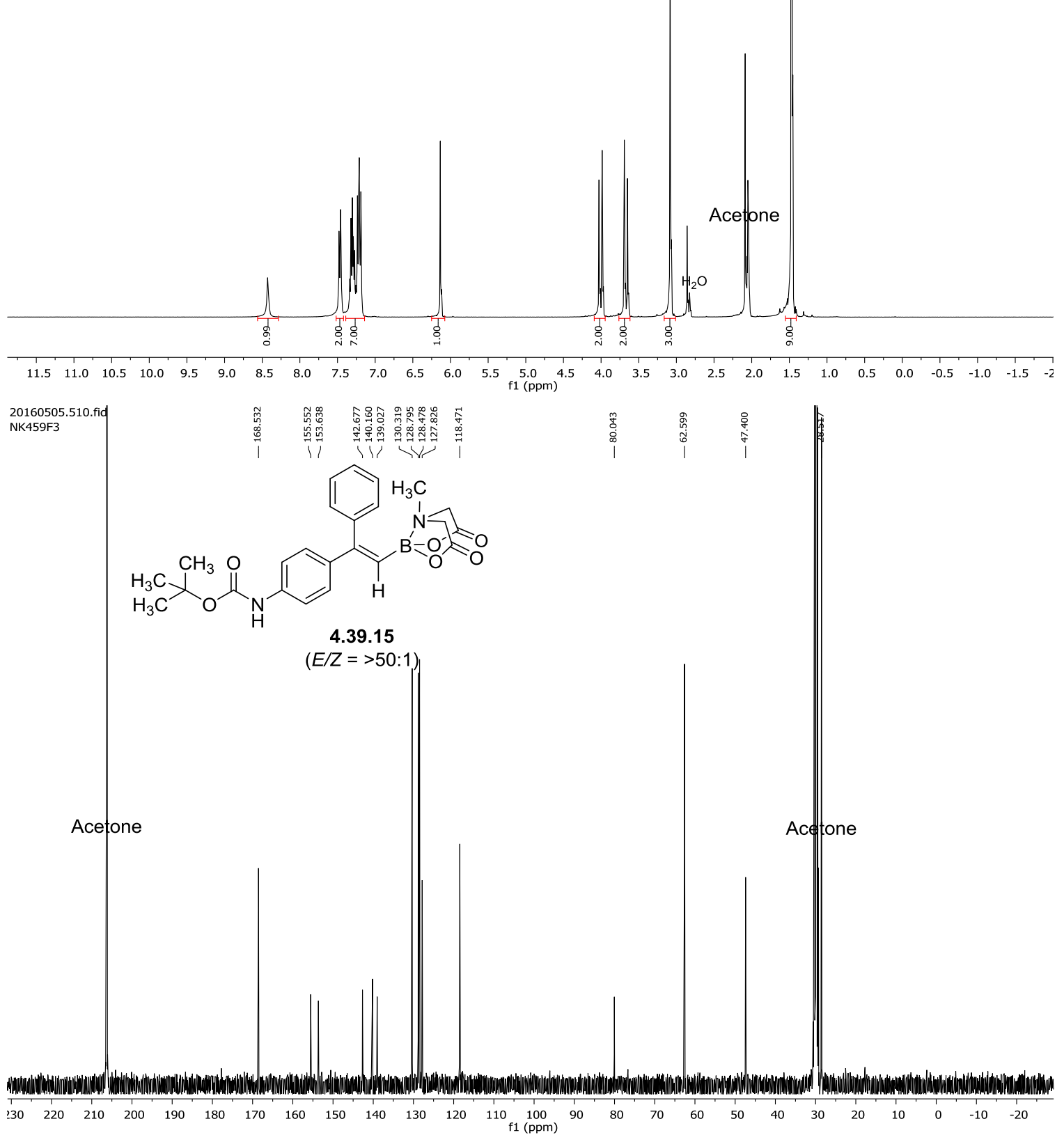
<smiles>CN1CC(=O)OB(C=C(c2ccccc2)c2ccc(C(F)(F)F)cc2)O1</smiles>
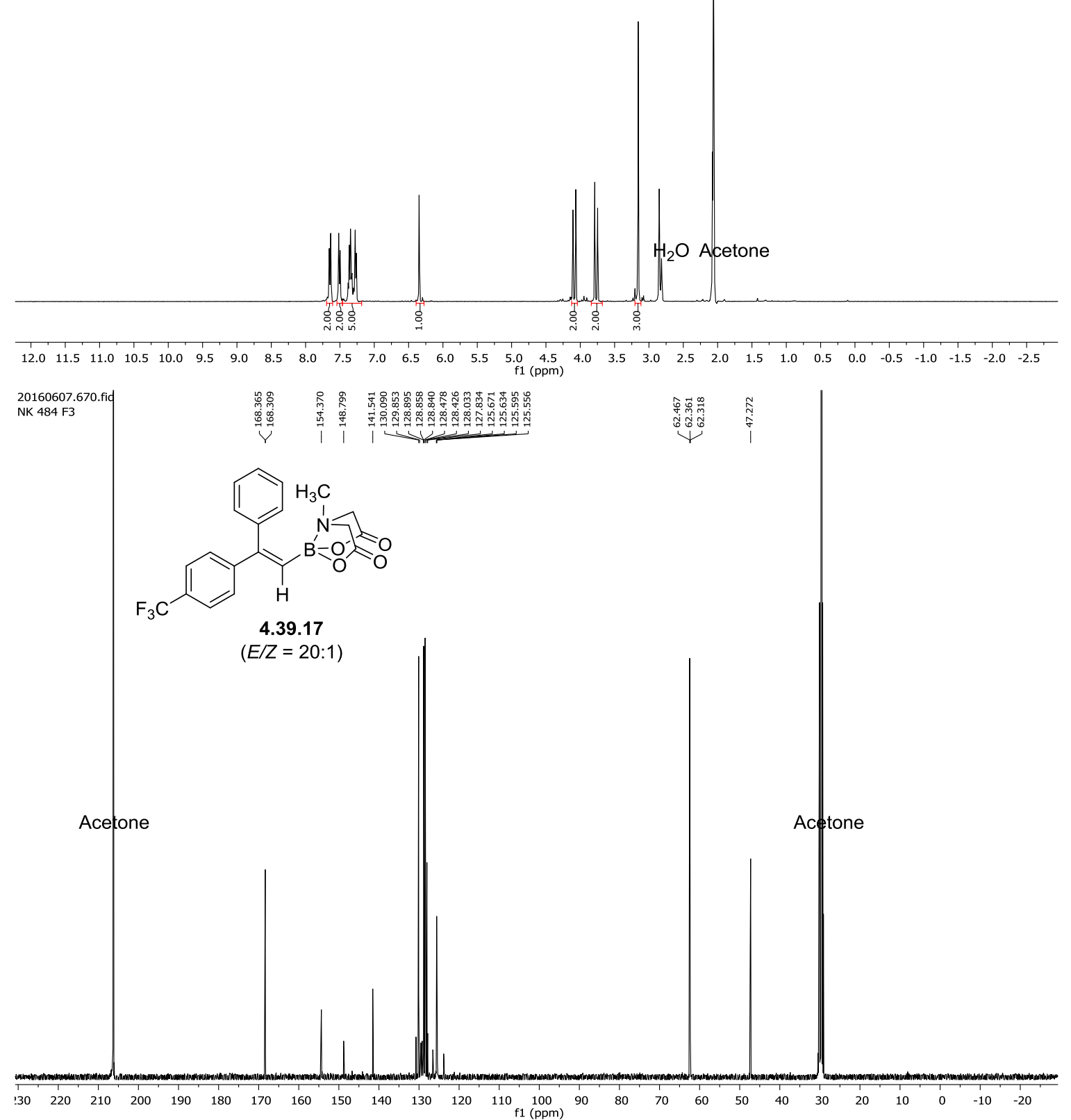


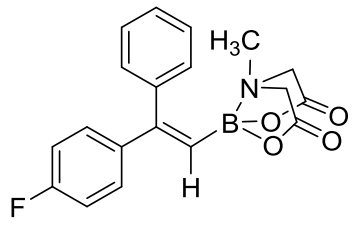

4.39.19

$(E / Z=11: 1)$
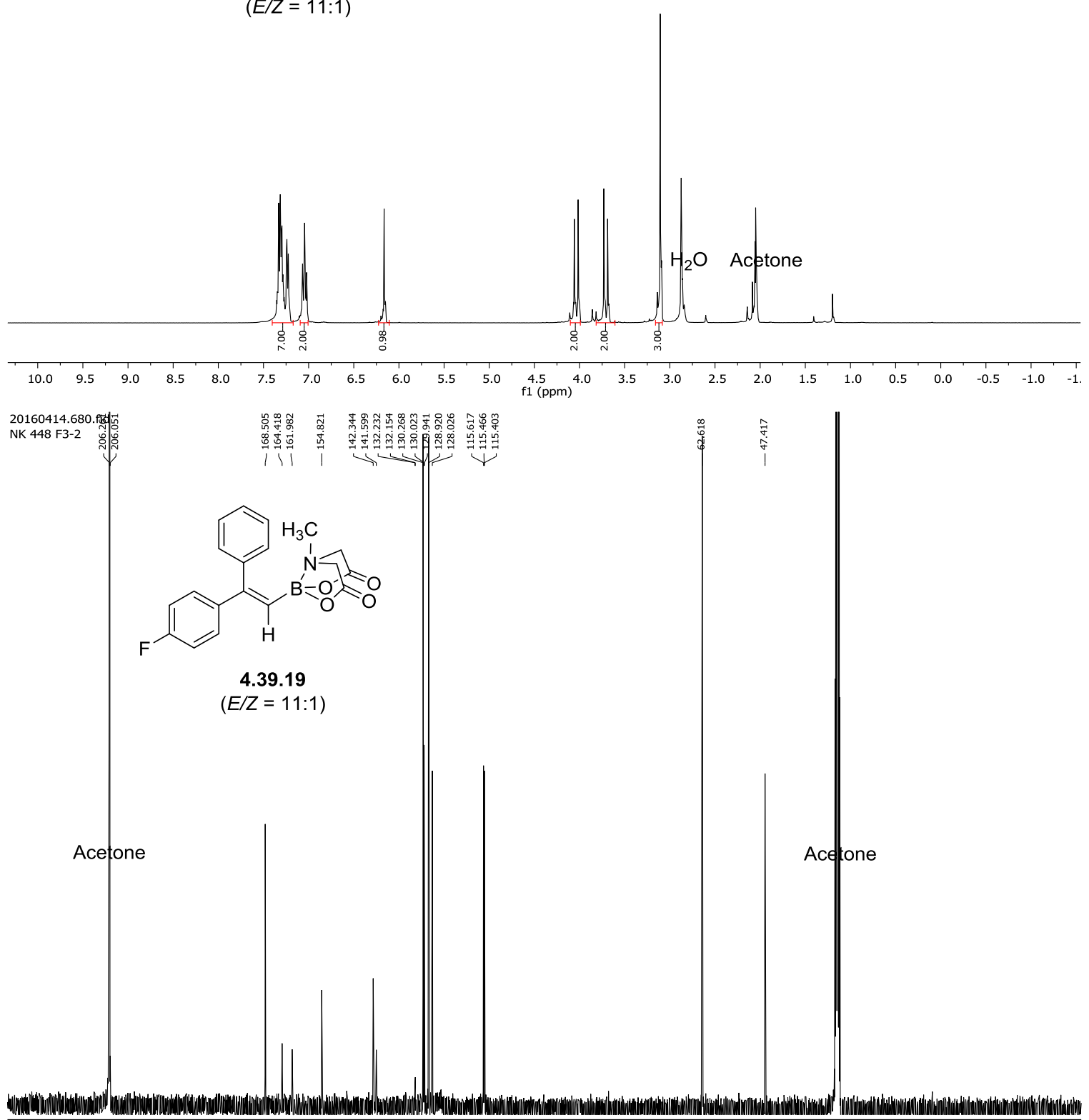

$\begin{array}{llllllllllllllllllllllllll}330 & 220 & 210 & 200 & 190 & 180 & 170 & 160 & 150 & 140 & 130 & 120 & 110 & 100 & 90 & 80 & 70 & 60 & 50 & 40 & 30 & 20 & 10 & 0 & -10 & -20\end{array}$ 


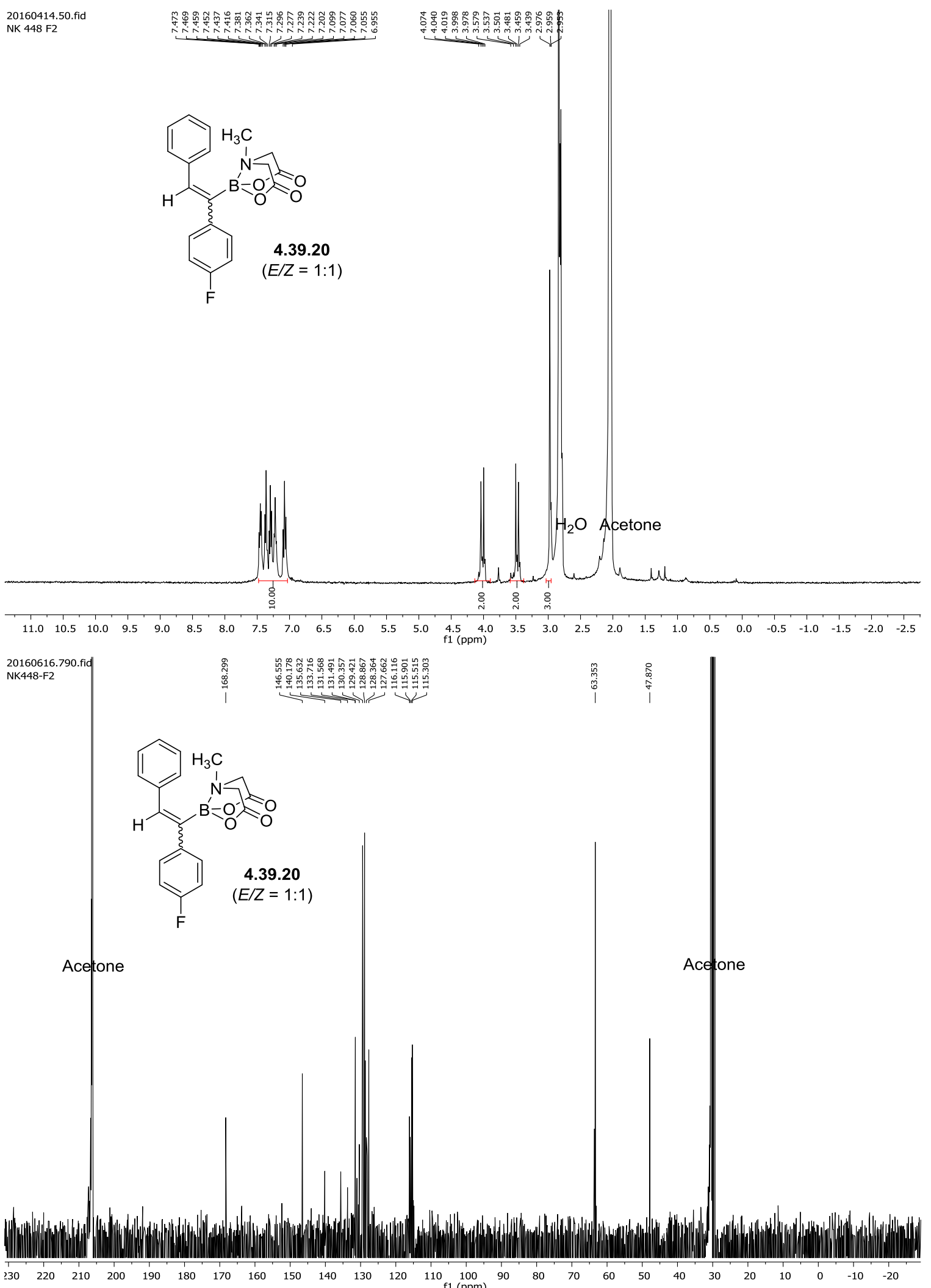



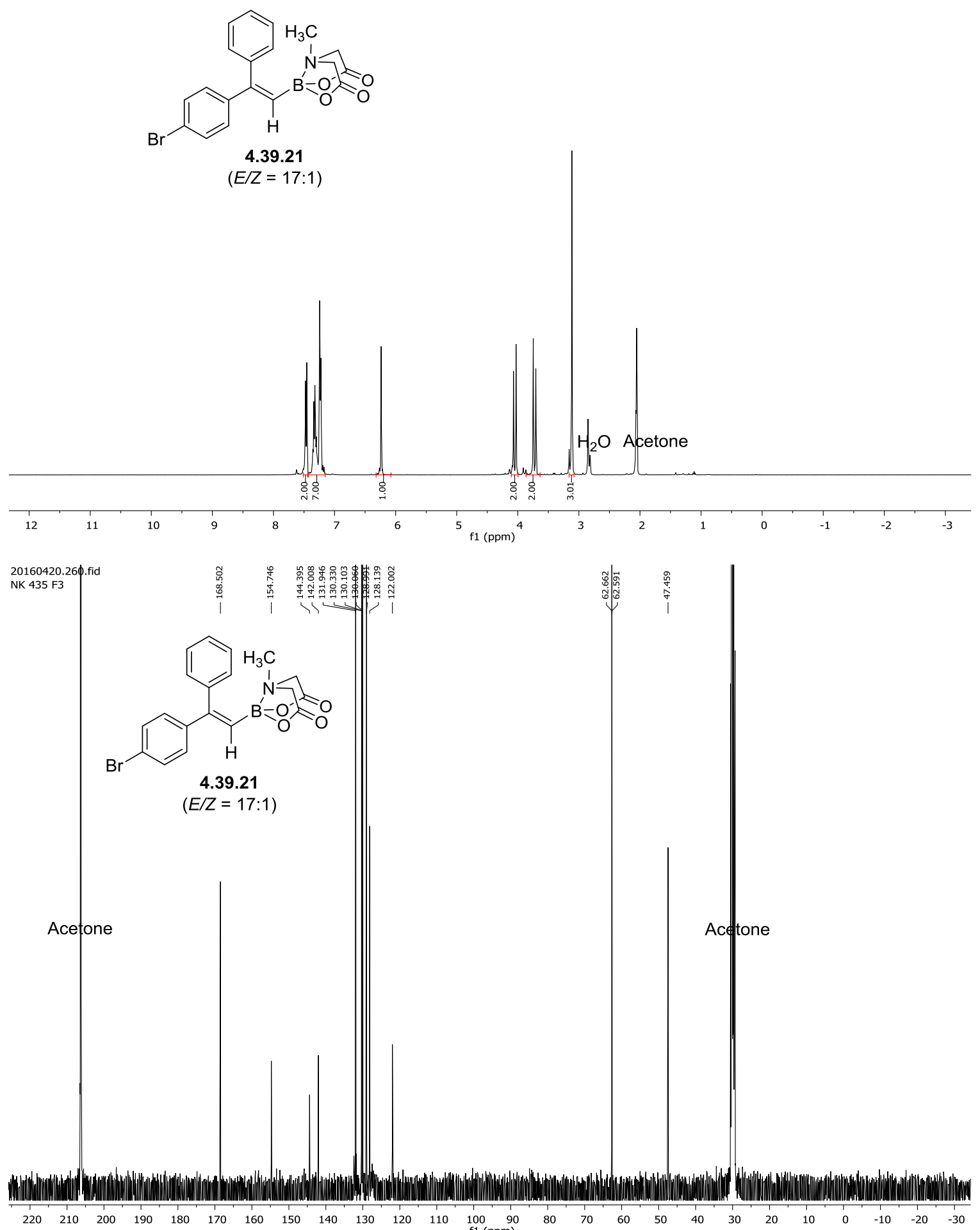


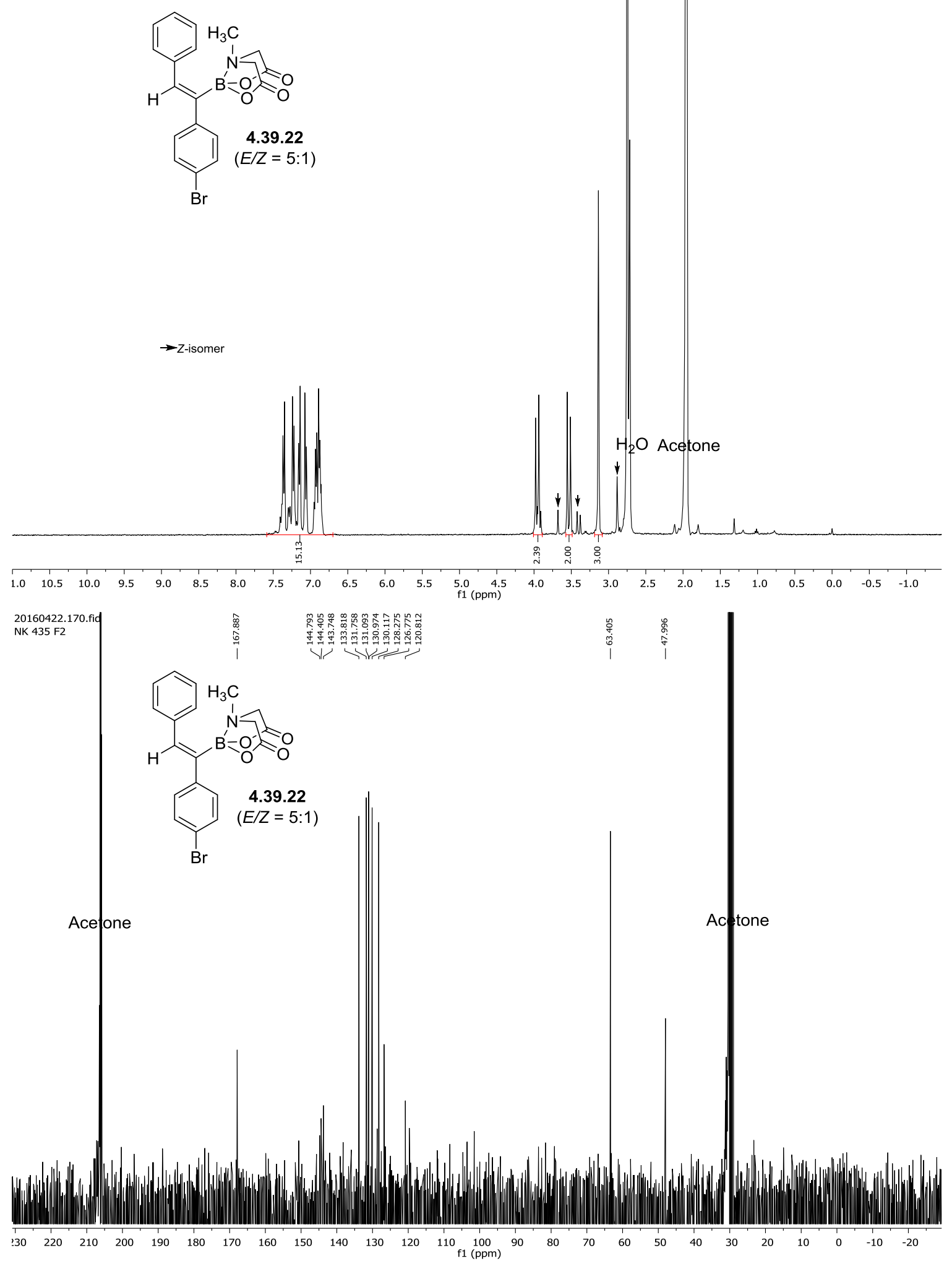




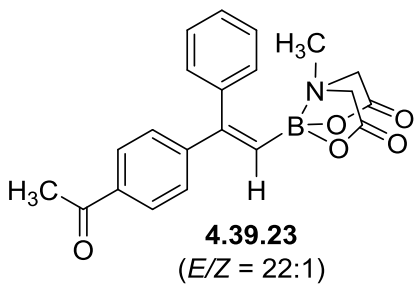
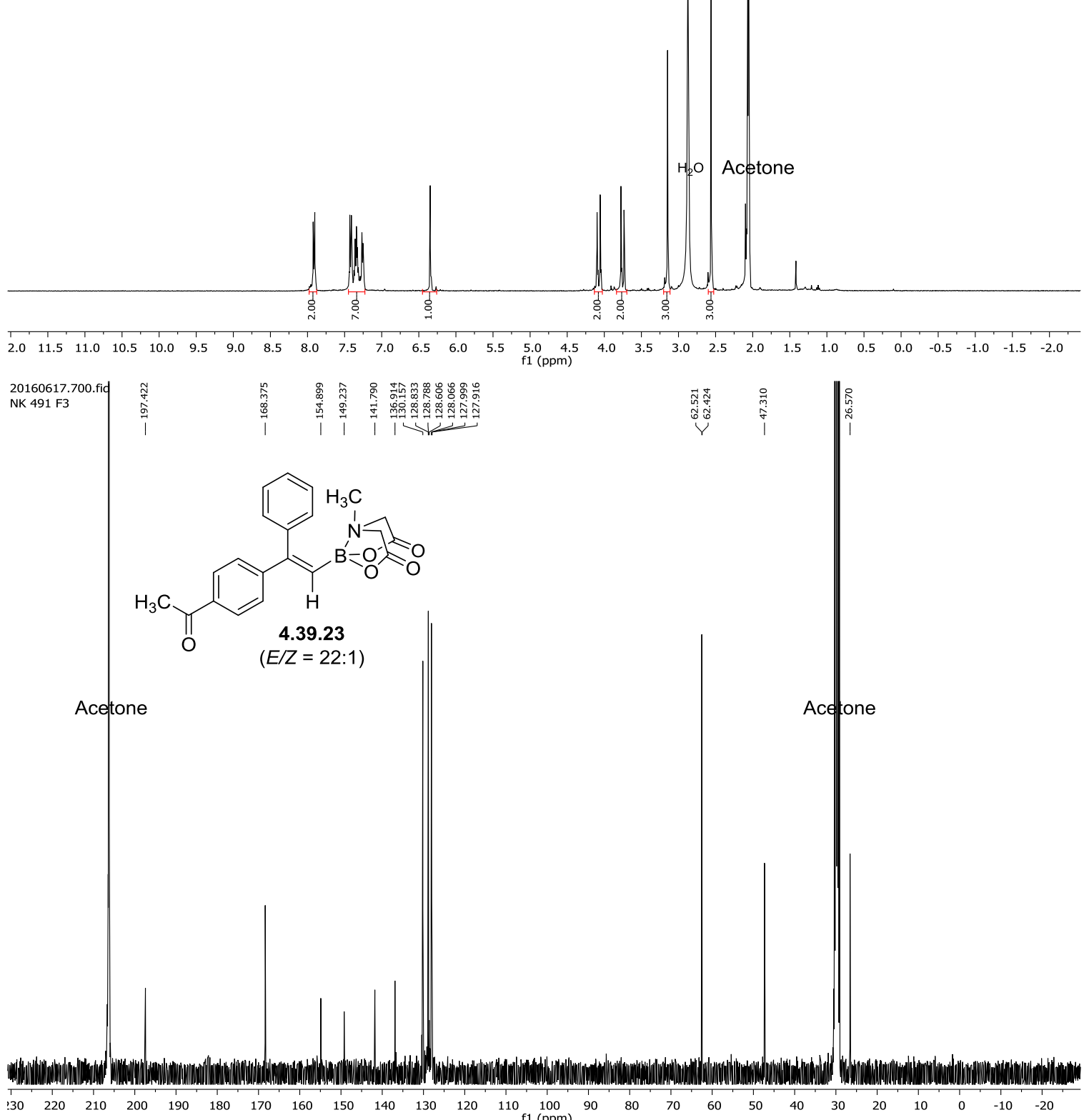

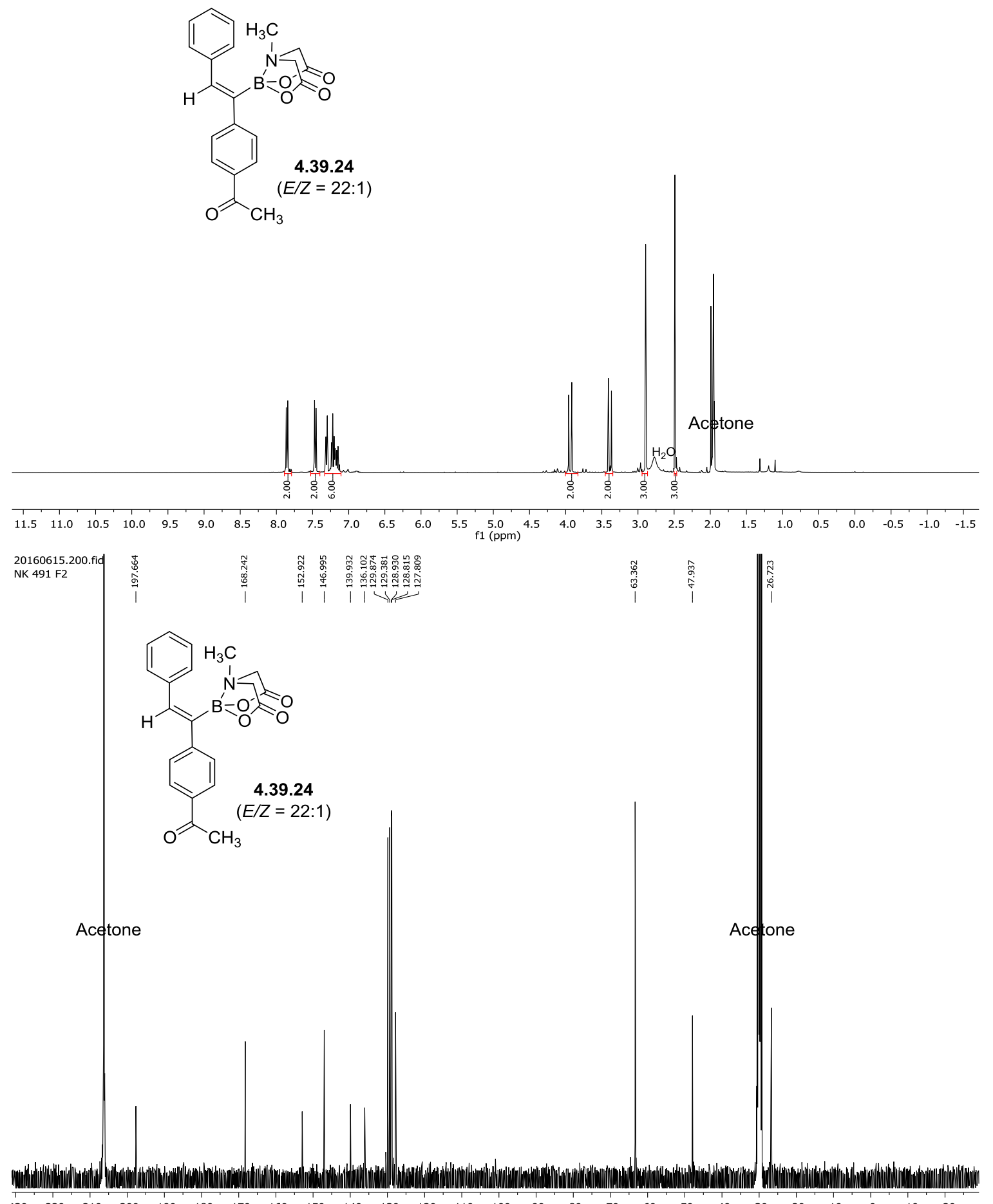

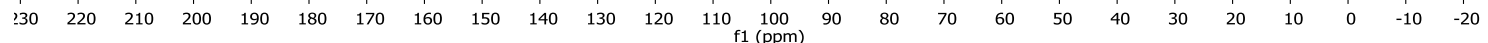



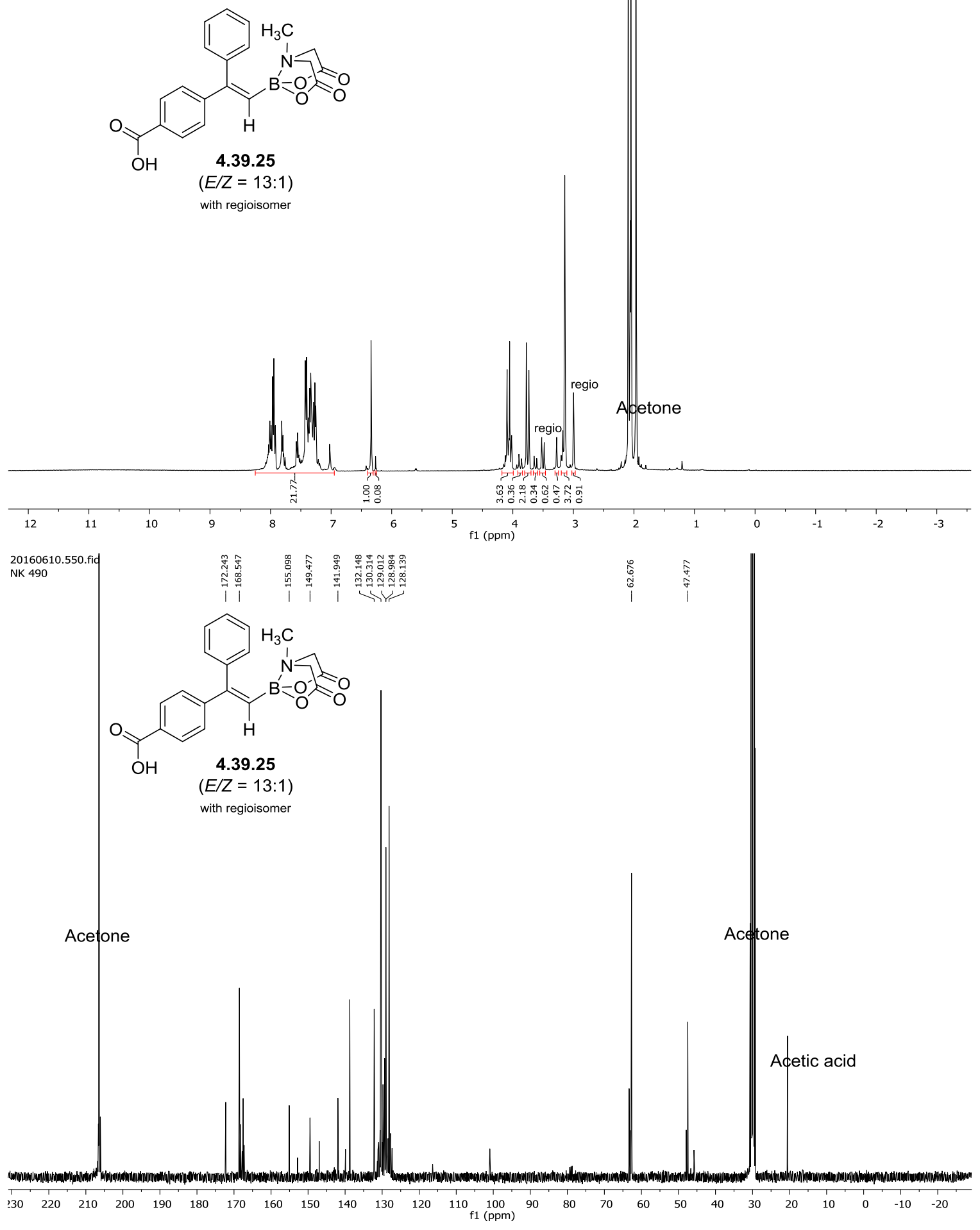


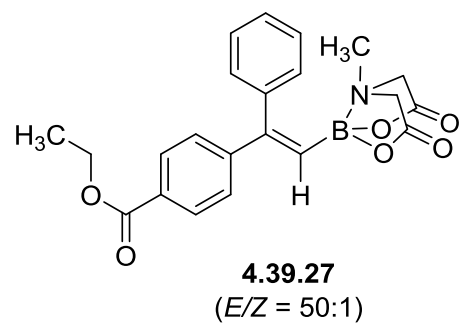

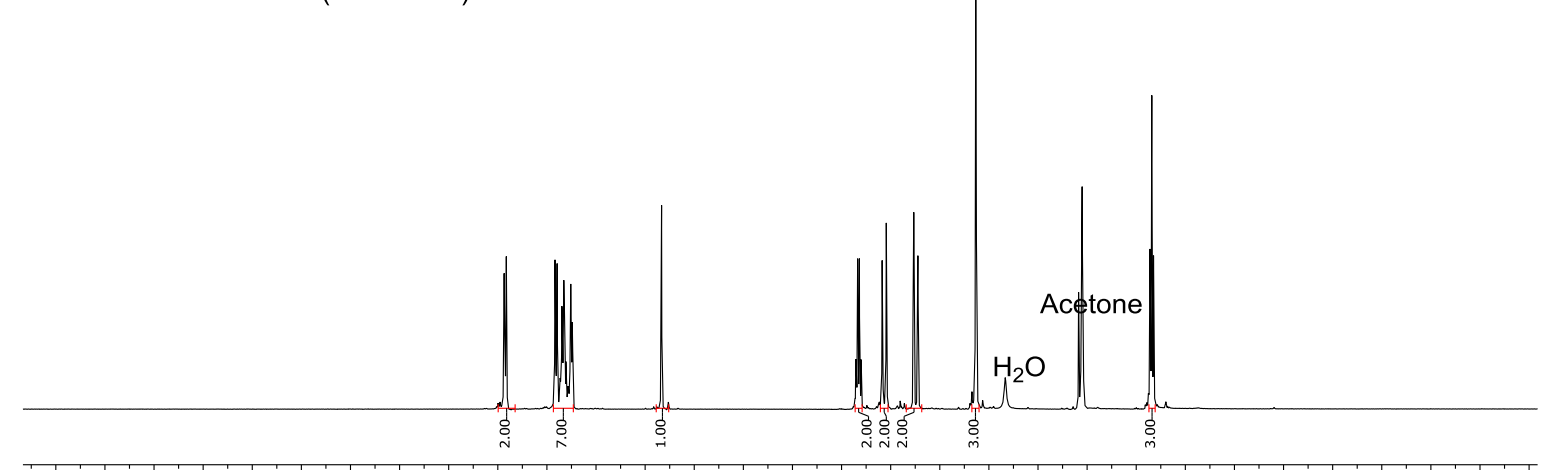
\begin{tabular}{lllllllllllllllllllllllllllllllllllll}
\hline 12.5 & 12.0 & 11.5 & 11.0 & 10.5 & 10.0 & 9.5 & 9.0 & 8.5 & 8.0 & 7.5 & 7.0 & 6.5 & 6.0 & 5.5 & 5.0 & 4.5 & 4.0 & 3.5 & 3.0 & 2.5 & 2.0 & 1.5 & 1.0 & 0.5 & 0.0 & -0.5 & -1.0 & -1.5 & -2.0 & -2.
\end{tabular}

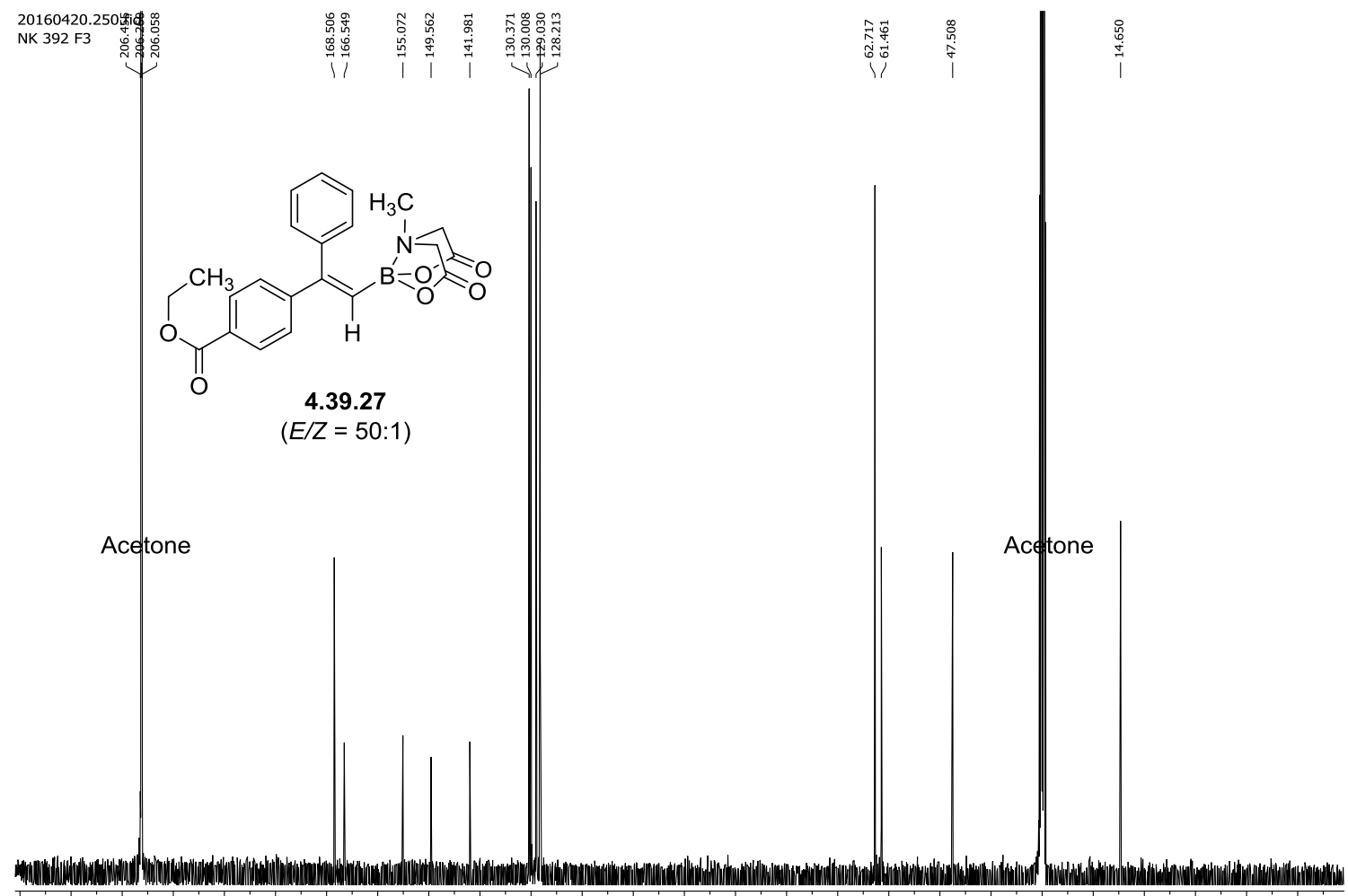

$\begin{array}{llllllllllllllllllllllllll}330 & 220 & 210 & 200 & 190 & 180 & 170 & 160 & 150 & 140 & 130 & 120 & 110 & 100 & 90 & 80 & 70 & 60 & 50 & 40 & 30 & 20 & 10 & 0 & -10 & -20\end{array}$ 

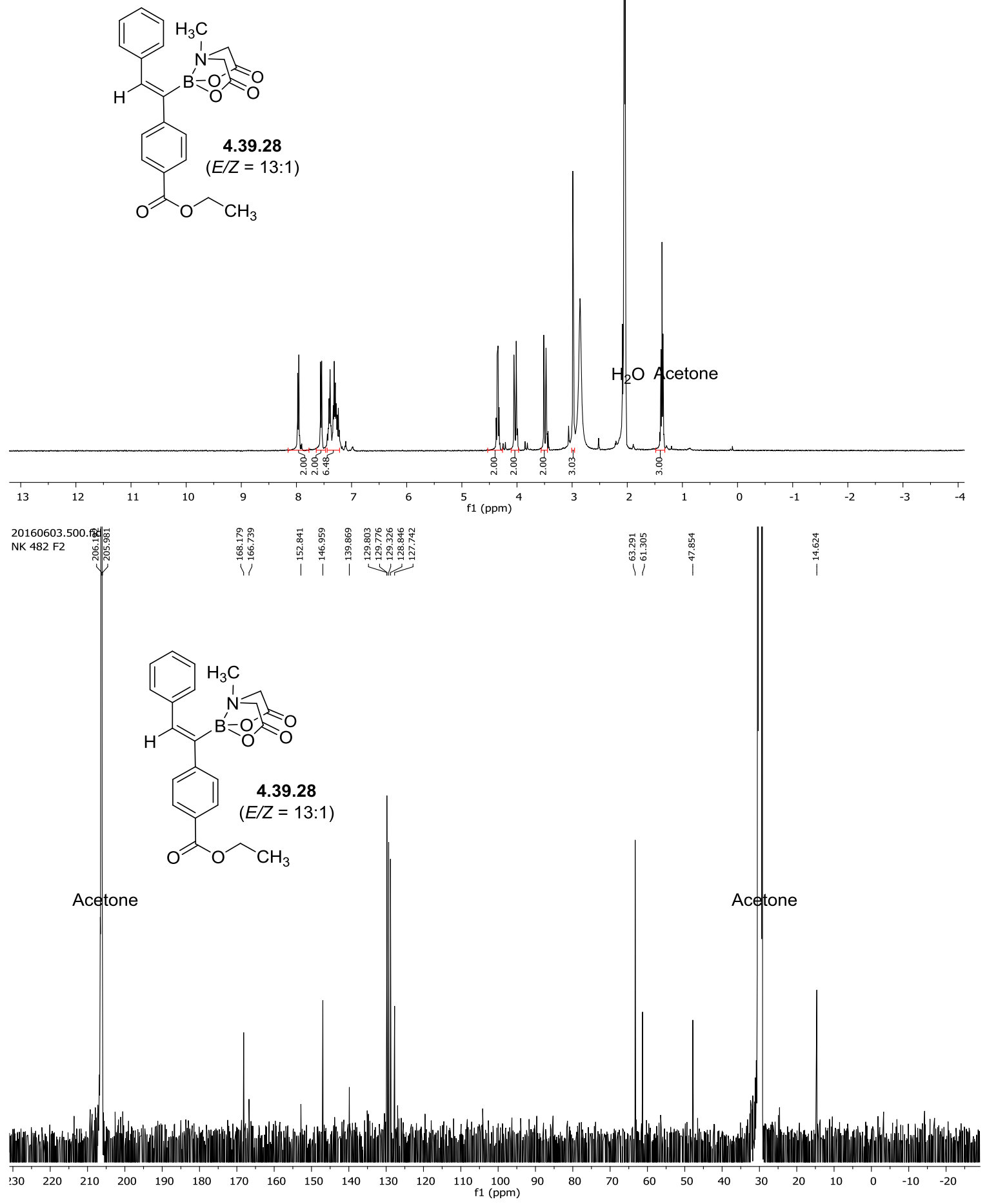


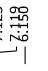

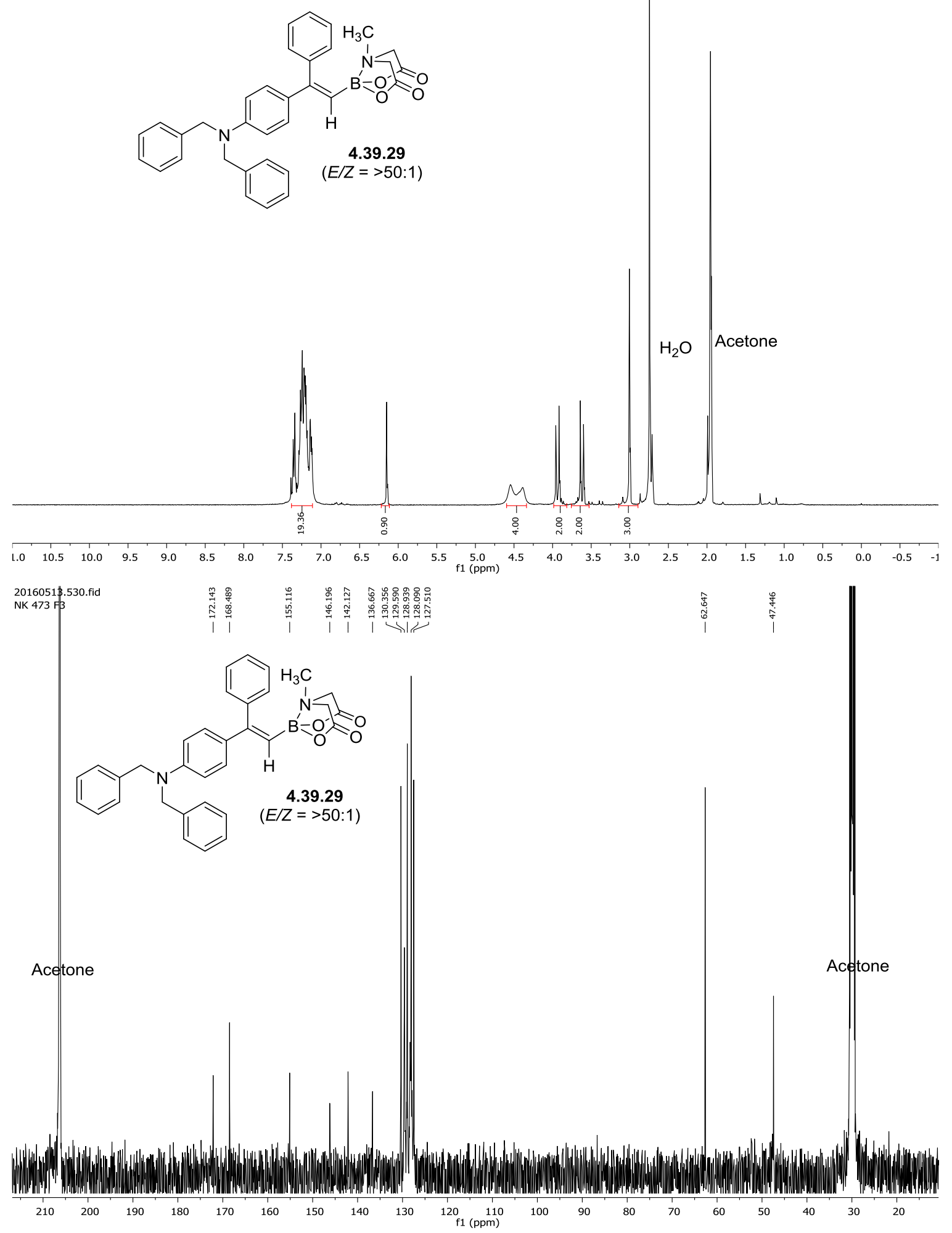


<smiles>Cc1cccc(/C(=C/c2ccccc2)B2OC(=O)CN(C)C2=O)c1</smiles>

4.41.5

$(E / Z=>50: 1)$
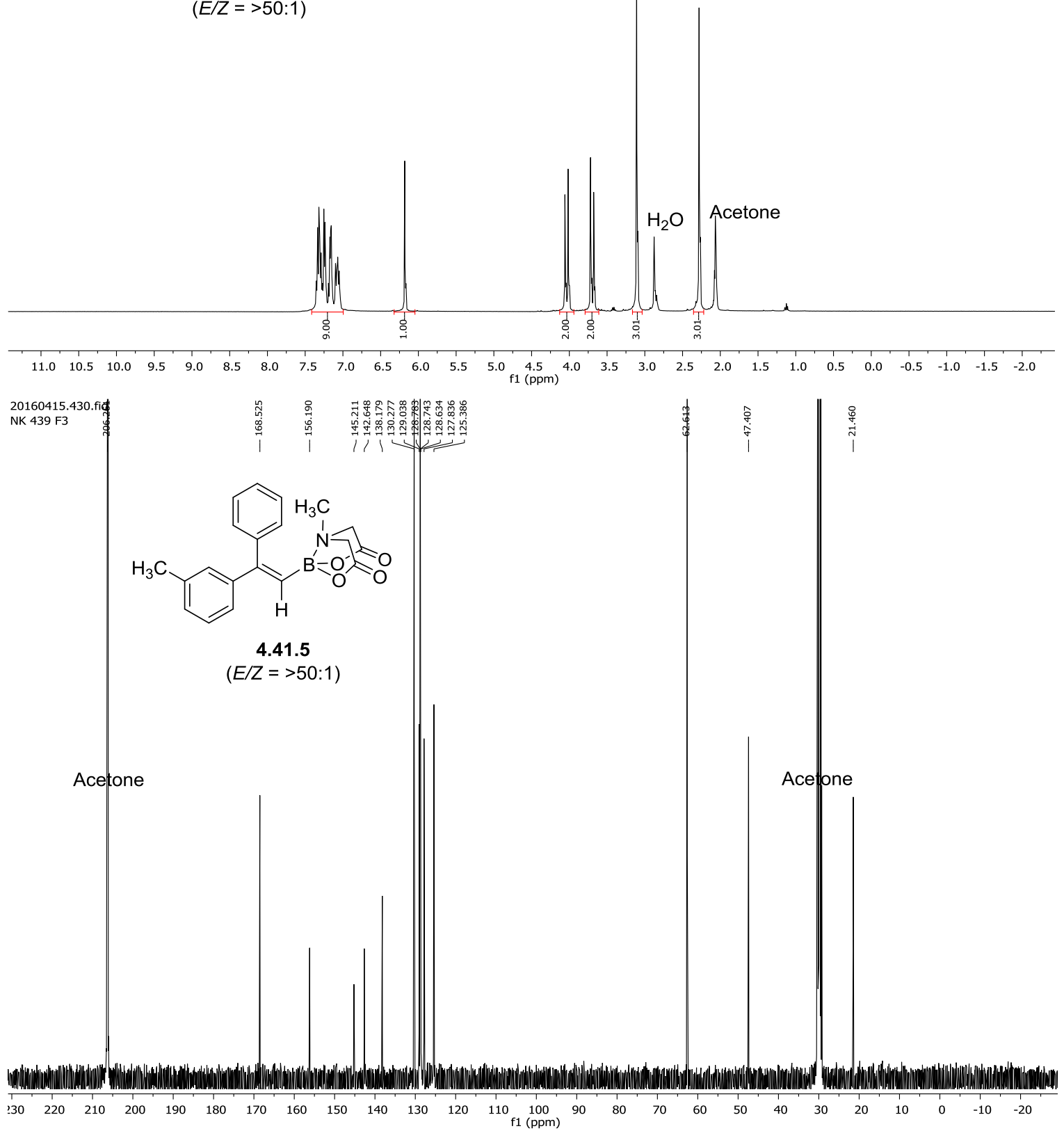


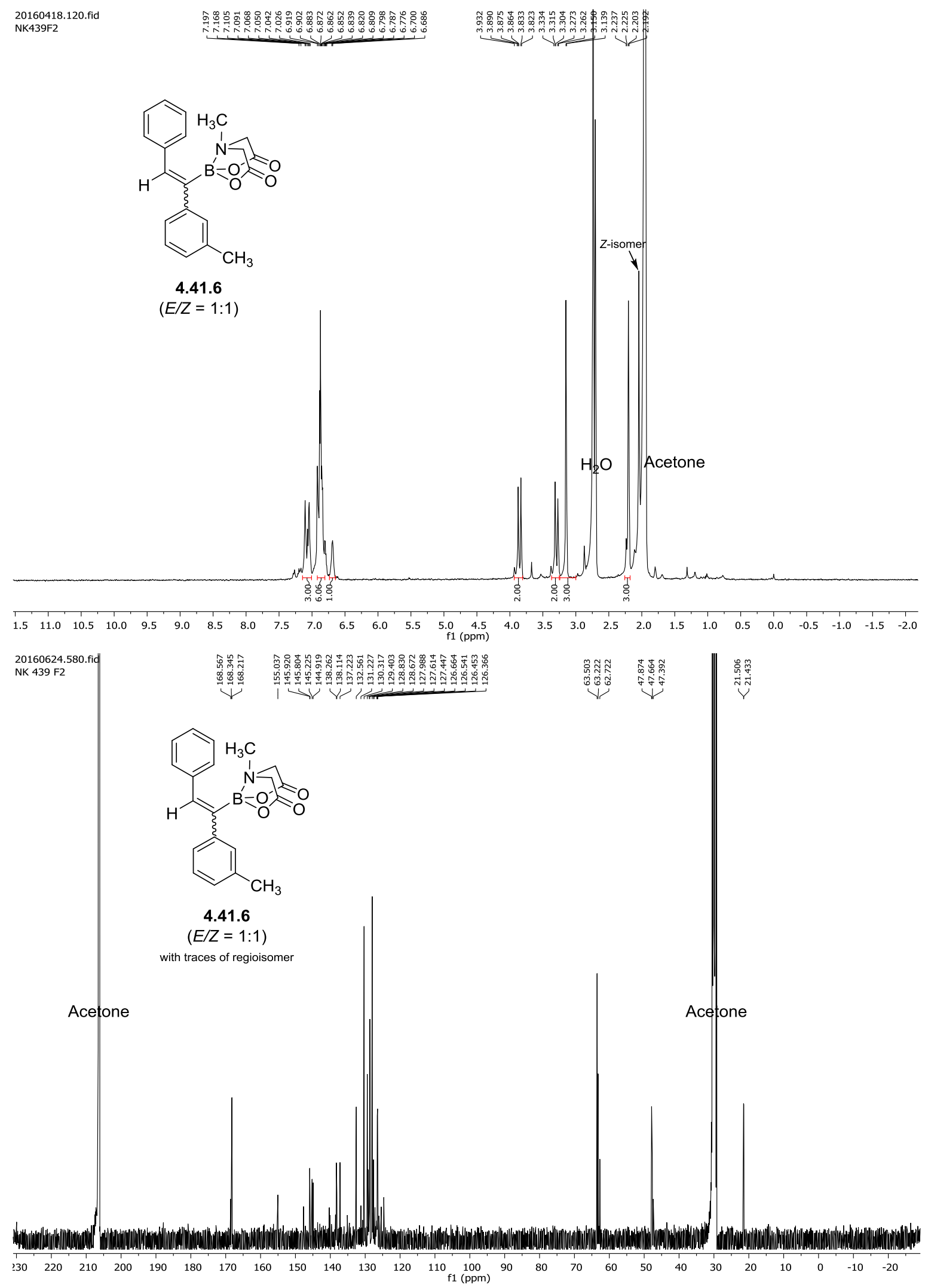


<smiles>COc1cccc(/C(=C/P2CN(C)CC(=O)O2)c2ccccc2)c1</smiles>

$(E / Z=>50: 1)$
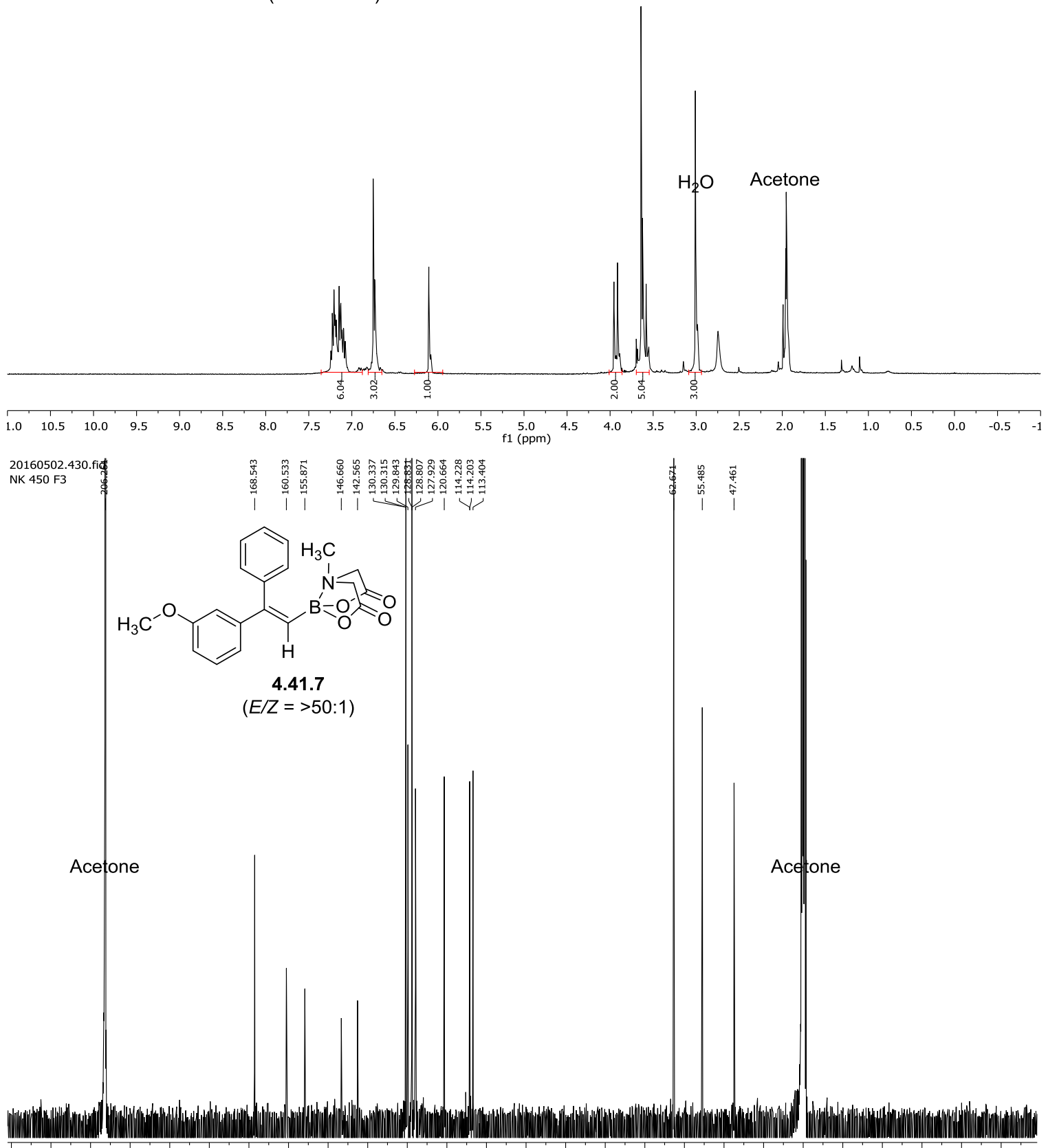

$\begin{array}{llllllllllllllllllllllllll}130 & 220 & 210 & 200 & 190 & 180 & 170 & 160 & 150 & 140 & 130 & 120 & 110 & 100 & 90 & 80 & 70 & 60 & 50 & 40 & 30 & 20 & 10 & 0 & -10 & -20\end{array}$ 

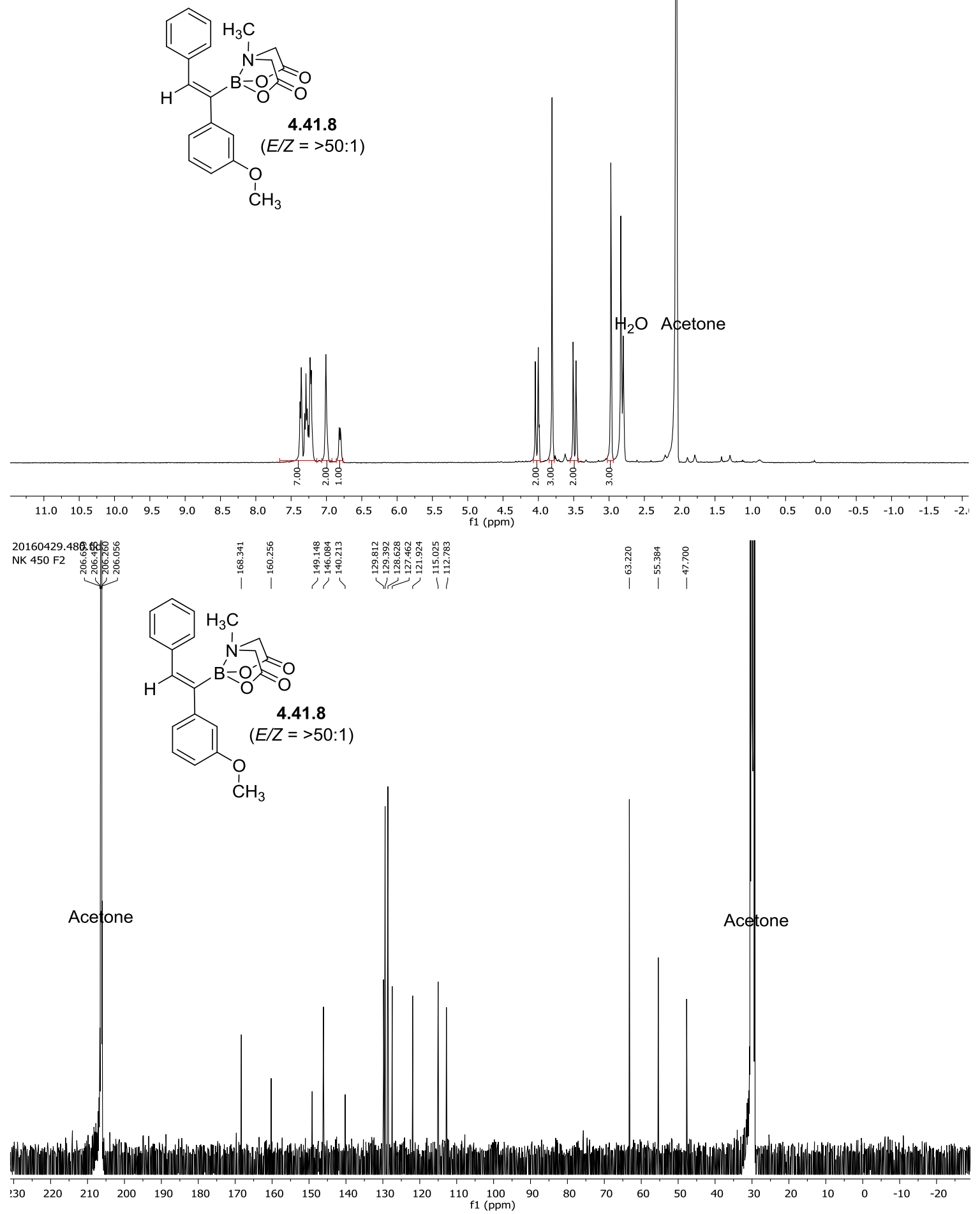
<smiles>CN1CC(=O)OB(C=C(c2ccccc2)c2cccc(Br)c2)O1</smiles>

4.41.9

$(E / Z=15: 1)$
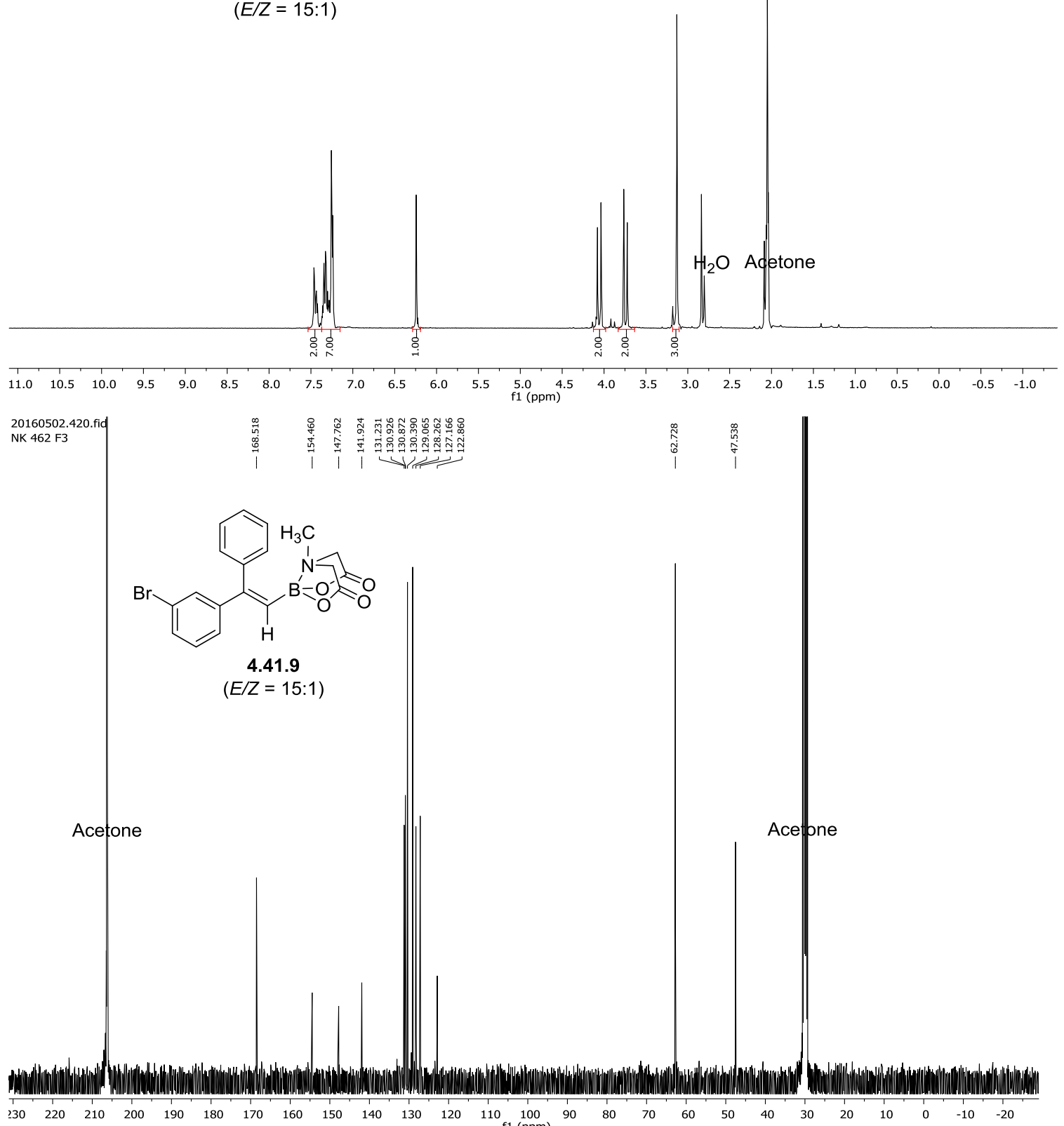

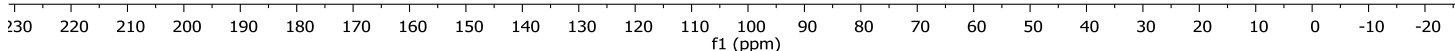



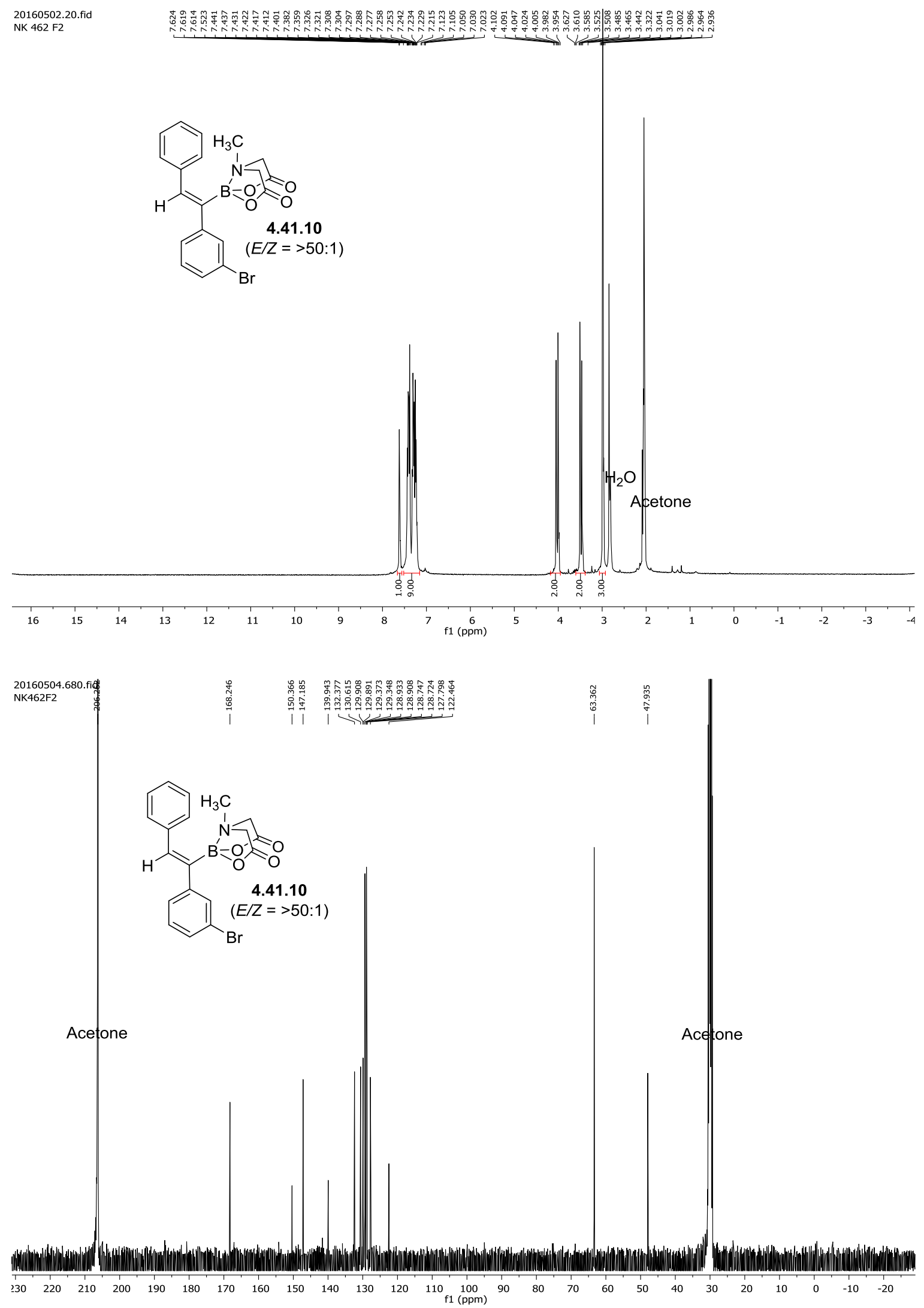
<smiles>COC(=O)c1cccc(/C=C\B2OC(=O)CN(C)C2=O)c1</smiles>

4.41.11

$(E / Z=>50: 1)$
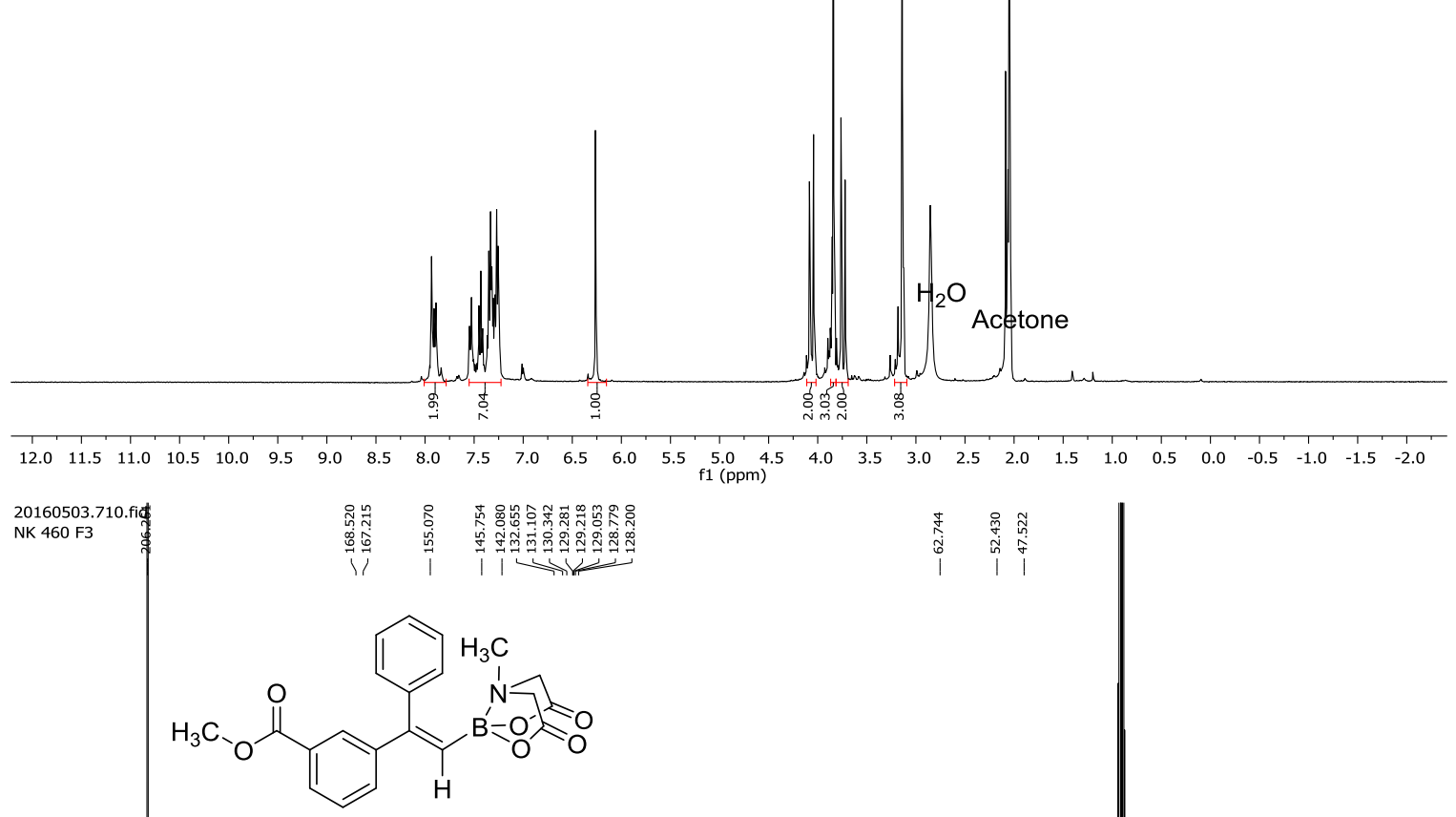

4.41.11

$(E / Z=>50: 1)$

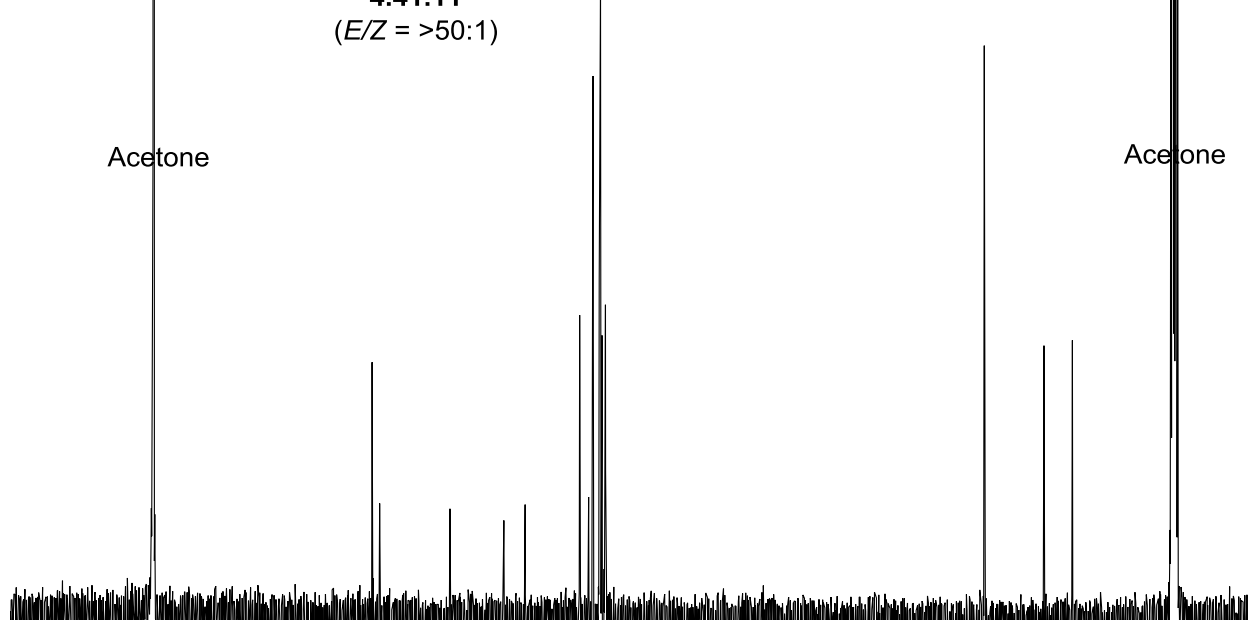

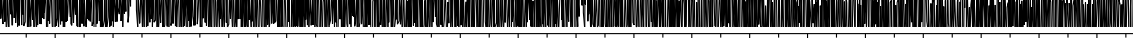

$\begin{array}{lllllllllllllllllllllllllll}3 & 30 & 220 & 210 & 200 & 190 & 180 & 170 & 160 & 150 & 140 & 130 & 120 & 110 & 100 & 90 & 80 & 70 & 60 & 50 & 40 & 30 & 20 & 10 & 0 & -10 & -20\end{array}$ 


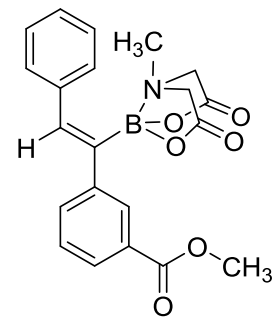

4.41.12

$(E / Z=>50: 1)$

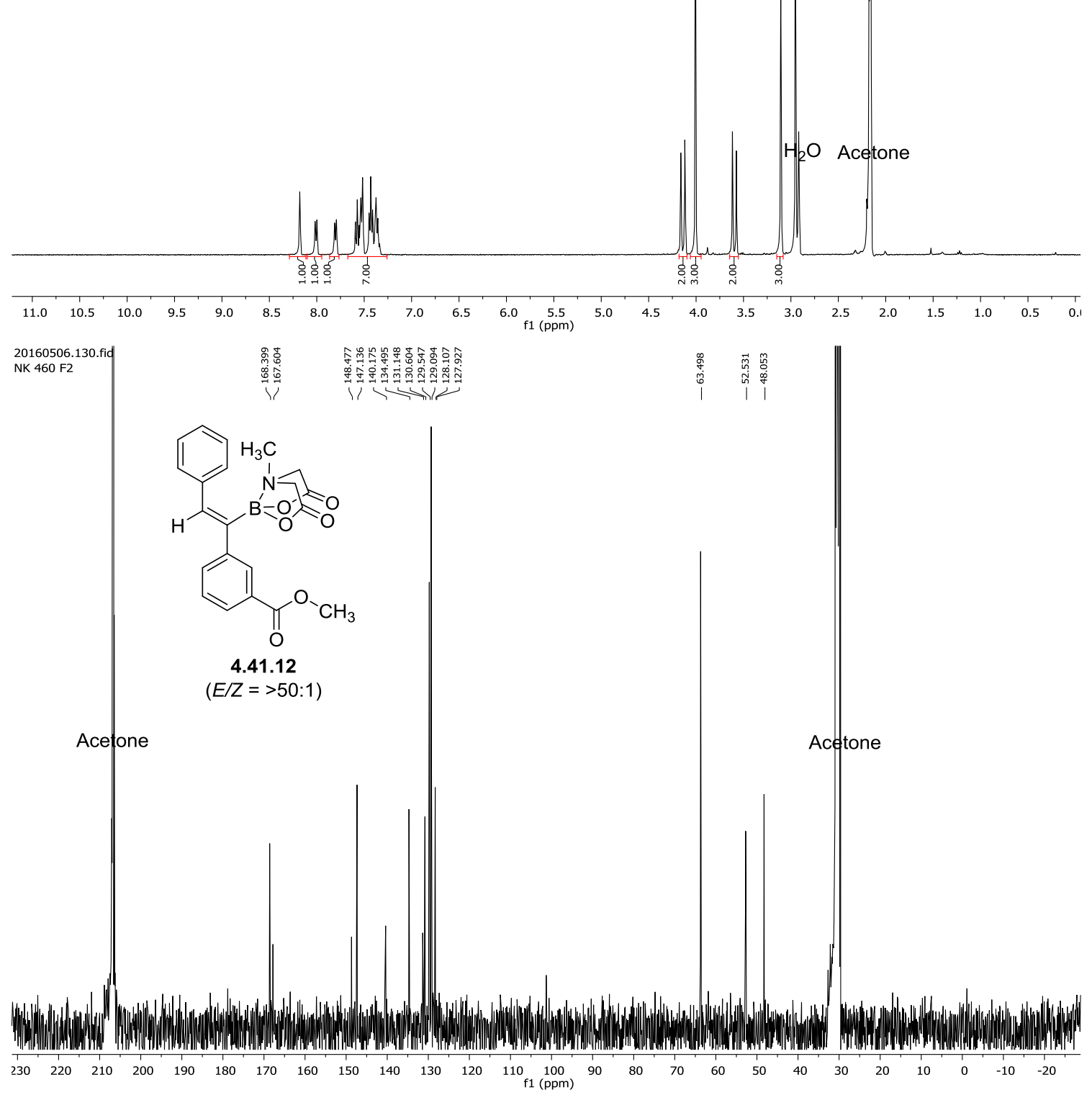



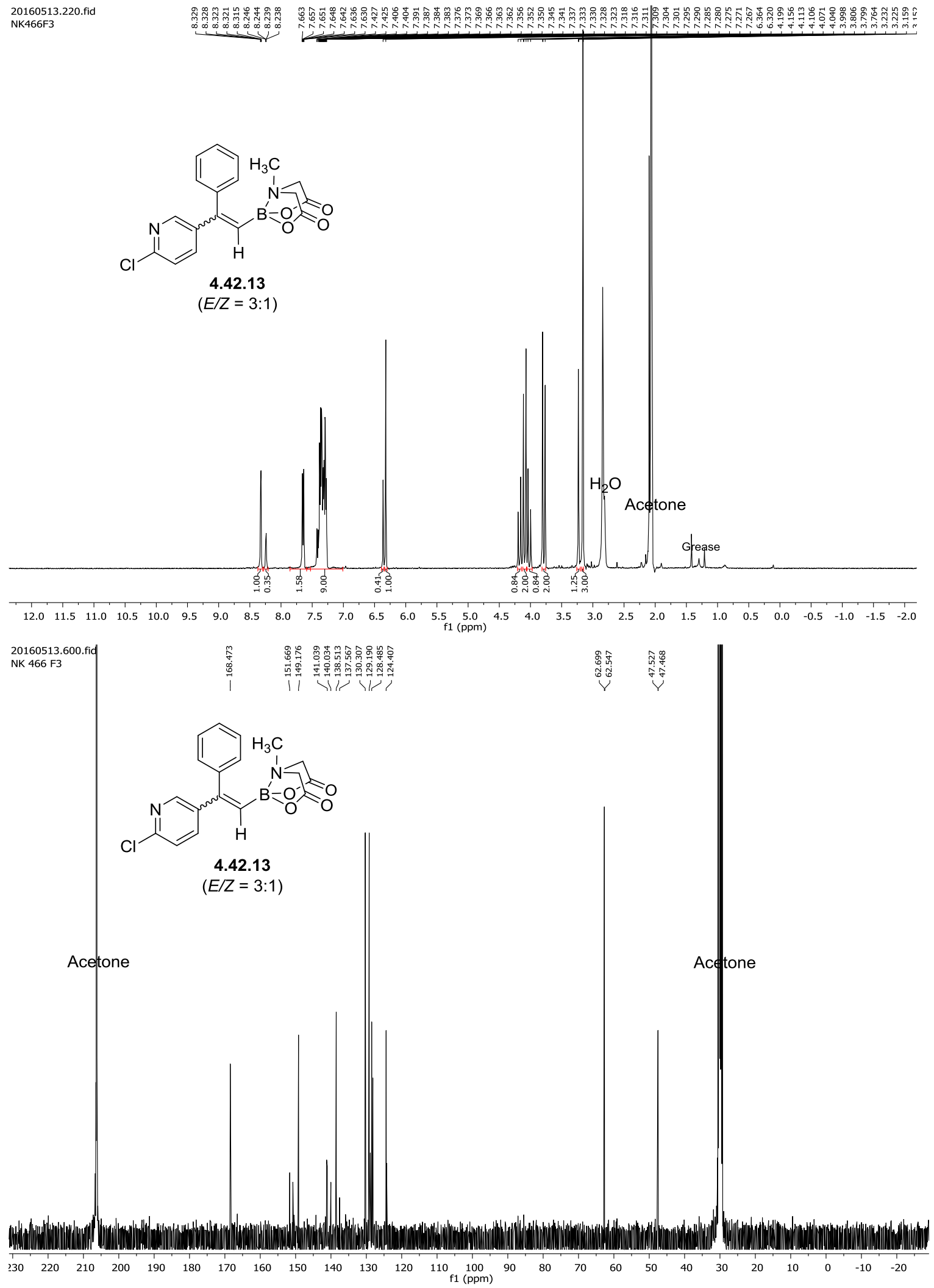


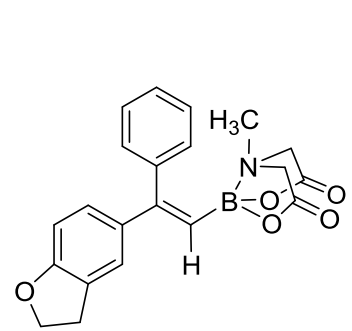

4.42.15

$(E / Z=13: 1)$

with traces of $Z$-regioisomer and starting material
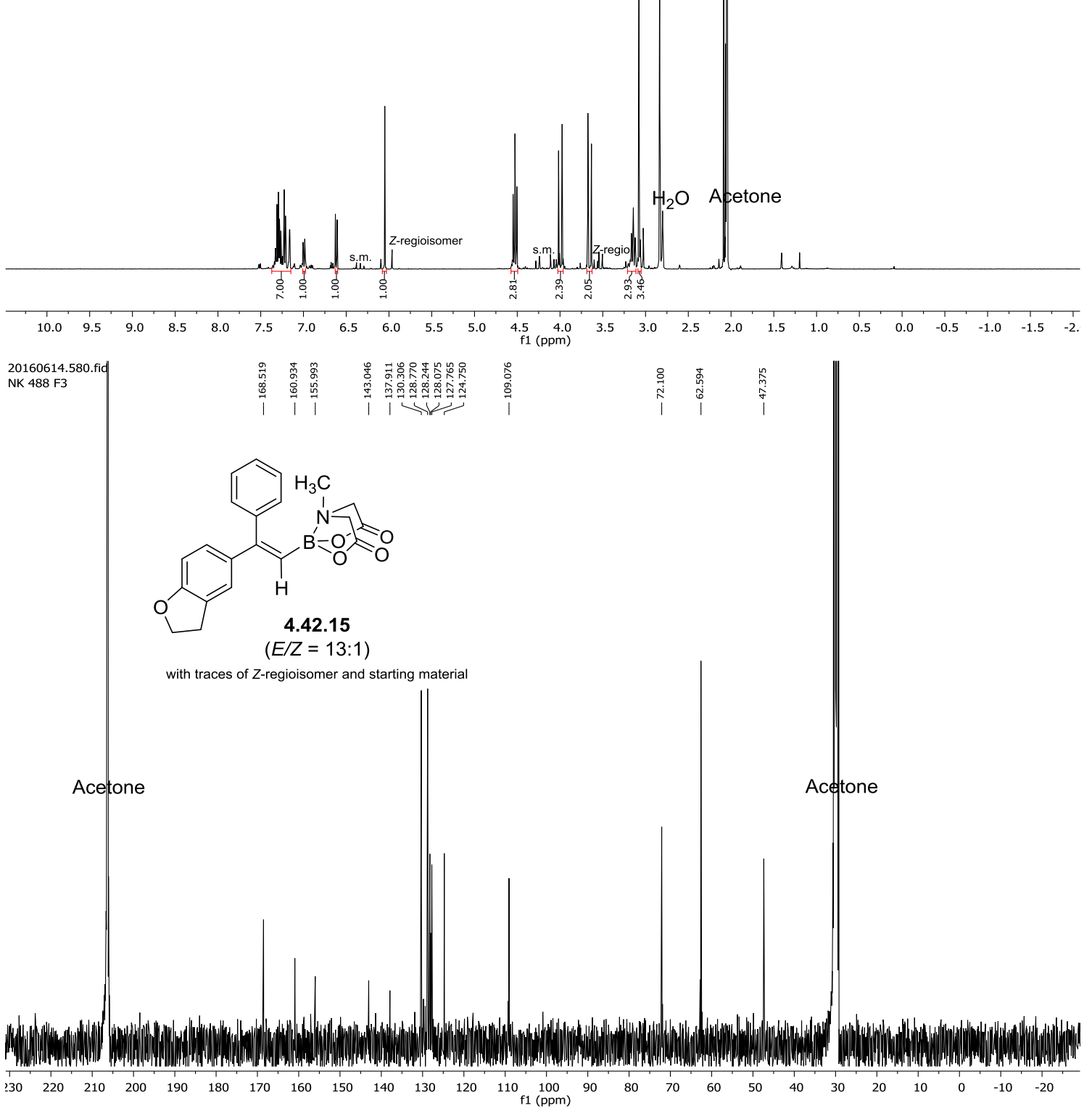


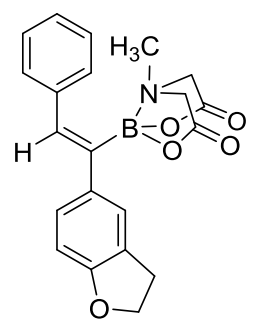

4.42.15'

$(E / Z=>50: 1)$

with traces of starting material
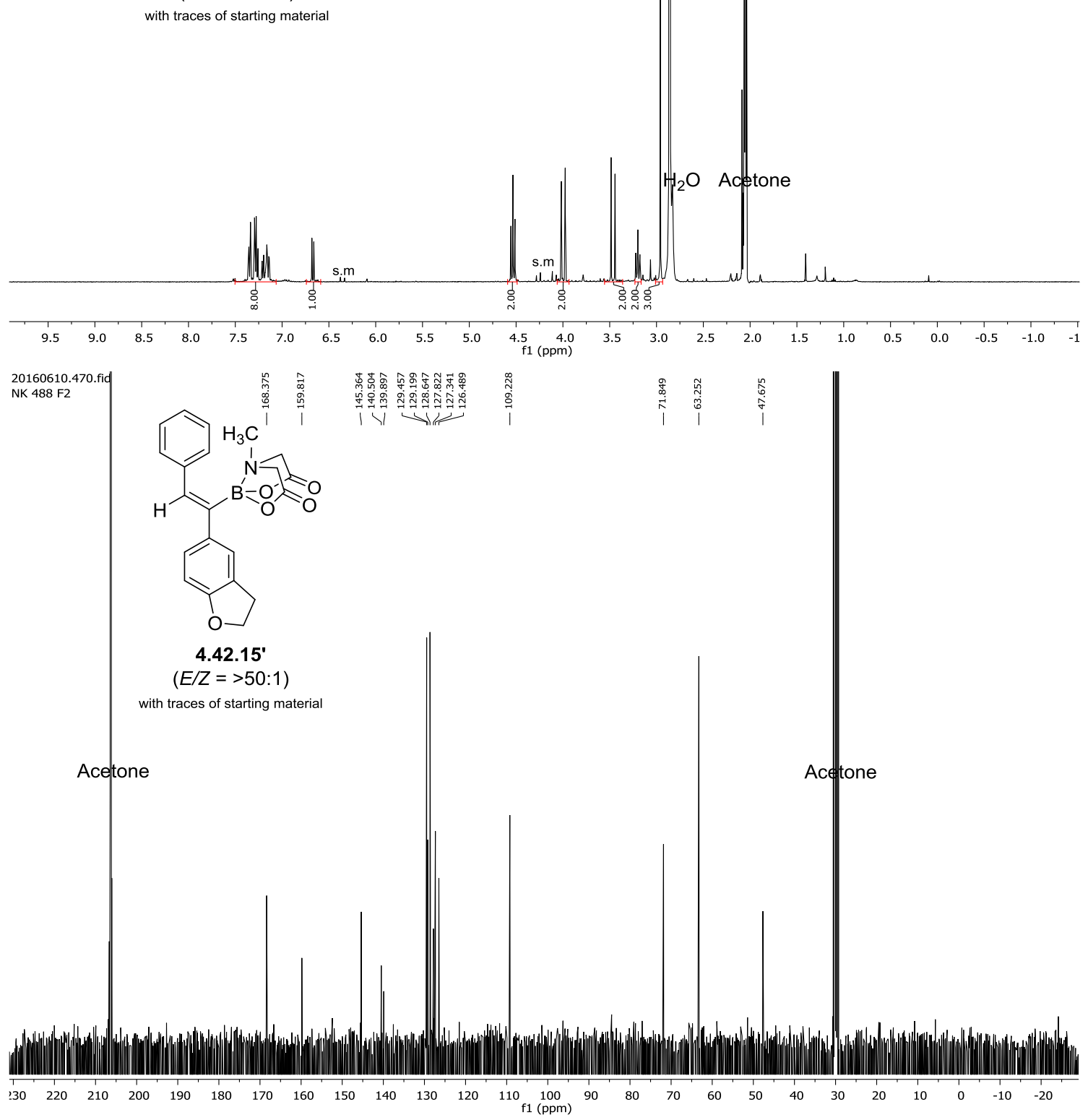
<smiles>COc1cccc(/C=C(\c2ccccc2)c2ccc(C)cc2)c1</smiles>

$(E)-4.48 .2$

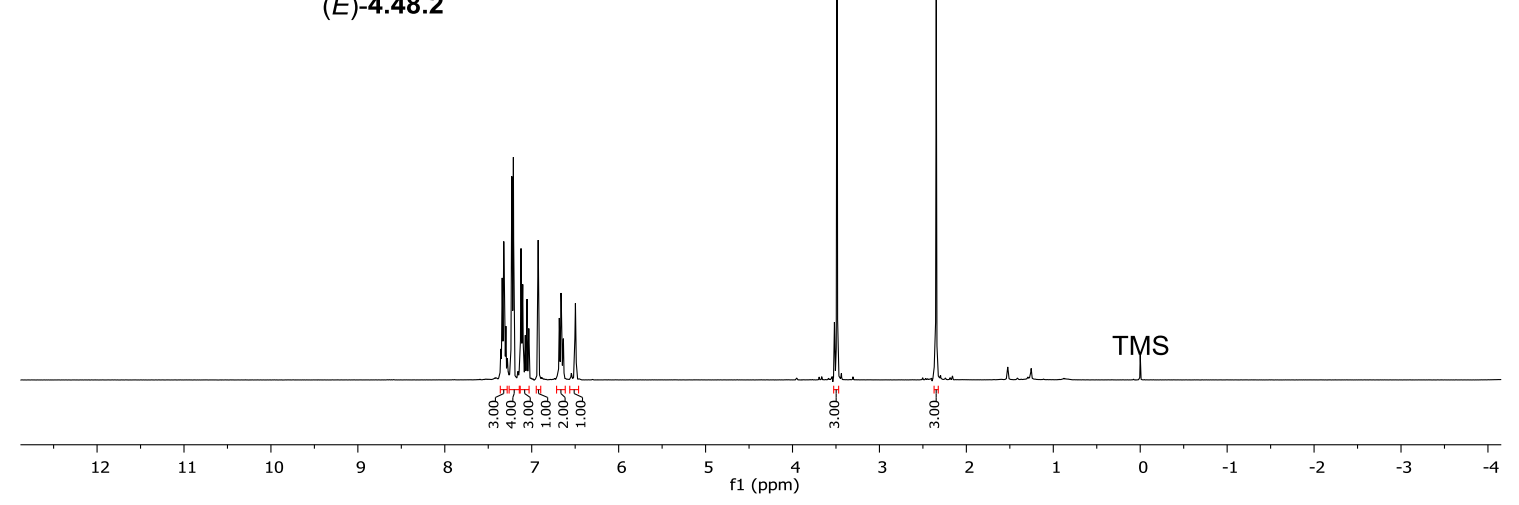

20160513.670.fid NK 467 F3

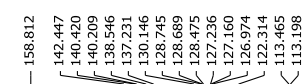<smiles>COc1cccc(/C=C(\c2ccccc2)c2ccc(C)cc2)c1</smiles>

$(E)-4.48 .2$

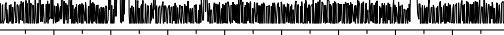

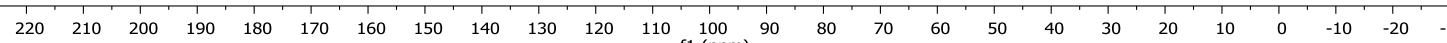




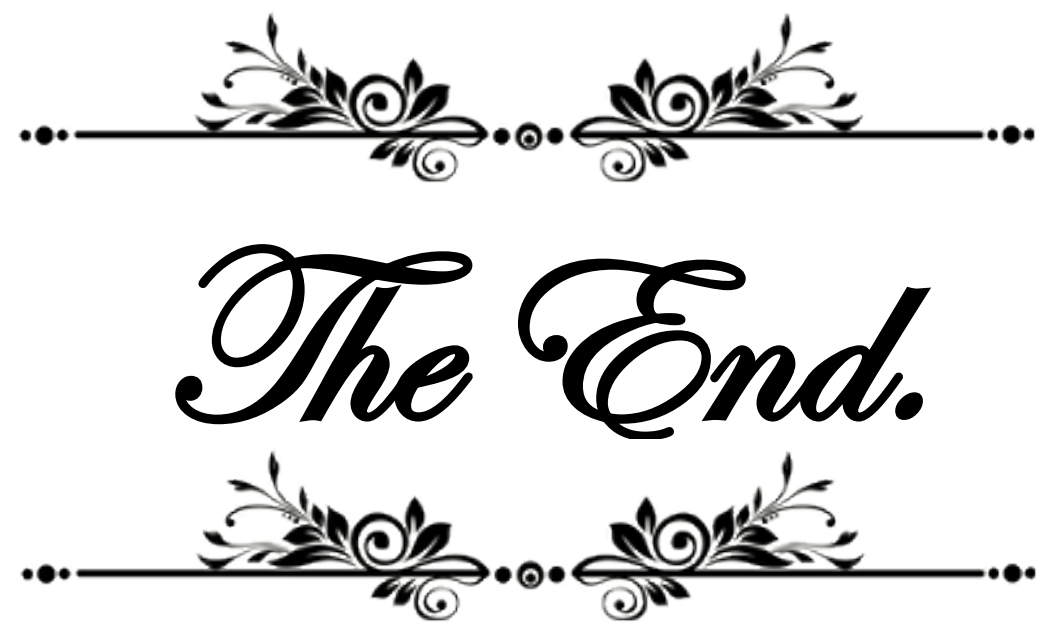

